\title{
GEOMAGNETICALLY INDUCED CURRENTS IN THE NEW ZEALAND POWER SYSTEM
}

\author{
BY \\ KAMRAN MUKHTAR
}

\begin{abstract}
A thesis
submitted to the Victoria University of Wellington in fulfilment of the requirements for the degree of Doctor of Philosophy
\end{abstract}

Victoria University of Wellington

(2021) 


\section{Abstract}

This thesis focuses on the use of magnetotelluric (MT) data from both the North Island and South Island of New Zealand to model Geomagnetically Induced Currents (GIC) in the New Zealand power network. The model results have been compared with those from a previously used thinsheet (TS) conductance model and with measured GIC.

Initially, a single station modelling approach using a uniform conductivity Earth model is used to model the measured GIC in a transformer at Islington (ISL). This model is further improved by separately modelling low and high frequency components of GIC and then combining these to give full GIC. The model reproduces most of the GIC variations and the correlation coefficient is $>70 \%$ for major magnetic storms from 2002-2015. As the model reproduces an average response of the network towards geoelectric fields it underestimates the most of extreme GIC. The analysis of GIC from other substations suggests that measured GIC depend on local geoelectric fields and the substation configuration within the network which cannot be captured using a single station approach. These limitations of single station model are addressed using more realistic geoelectric fields based on magnetotelluric data and consideration of the full network.

To compute geoelectric fields in the whole network the gaps between MT sites are filled using a Nearest Neighbor interpolation technique. As the northern part of the North Island has no MT data an equivalent circuit approach is followed to model GIC for only the lower part of the network. The MT model GIC are in the period range of 2-30 minutes, based on the available MT data period range. Both the MT and TS techniques are used to compute geoelectric fields and to model GIC for the St. Patrick's Day storm of 2015 and a 20 November 2003 magnetic storm. Both the MT and TS methods show the same transformers as experiencing large GIC during both storms. The primary difference between the models is that amplitudes of high frequency components of the TS model are significantly smaller than for the MT model. In particular they do not produce large GIC during the sudden storm commencement (SSC) of the St. Patrick's Day magnetic storm. For the 20 November 2003 storm the TS model effectively reproduces the low frequency components and extreme GIC. The model results show that the North Island power network could be at risk during adverse space weather conditions. 
Although the South Island has sparser MT data the same technique is used to model SI GIC during both the St. Patrick's Day and 2003 magnetic storms. Results are compared with measured data from ISL, South Dunedin (SDN) and Halfway Bush (HWB) transformers. The MT model effectively reproduces the measured GIC variations particularly during SSC during the St. Patrick's Day storm. The TS model gives a very small GIC magnitude during the SSC. During the 20 November 2003 storm both the MT and TS models reproduce strong amplitudes of low frequency components seen in the ISL measured data.

Both the MT and TS models show a substantial scale difference between measured and model GIC both for ISL and HWB transformers that needs to be further explored either in terms of better geoelectric interpolation or power network parameters. Overall, the MT model appears much more promising for future GIC modelling, particularly during a sudden storm commencement and for abrupt GIC variations. 


\section{Declaration}

This thesis has been completed by myself and has not been submitted before to qualify for any other academic degree. All the ethic procedures and guidelines have been followed properly while preparing the thesis. 


\section{Acknowledgment}

This work has been completed in the School of Chemical and Physical Sciences and I am grateful to everyone who helped me during the period of my PhD. First and foremost, I am extremely grateful to my supervisor, Dr Malcolm Ingham, for providing me guidance and the freedom to indulge in my work. He showed a great level of patience and brought me up to achieve something myself. I would also like to thank my secondary supervisor, Dr. Wiebke Heise from GNS Science, for her unwavering support and belief in me. It would be incomplete without the name of Prof. Craig Rodger from University of Otago for providing a superb collaboration and motivation, and everyone involved in this project. Especially, Daniel Mac Manus and Tim Divett who have been very helpful for discussions throughout my PhD. I also want to mention Dr. Gillian Turner for being very helpful within the School.

It would not be justice without mentioning my family who made sacrifices to allow me accomplish this research particularly during the hard times of Covid-19. I am deeply grateful to my beloved wife who has been with me during all the time and for her support in my research work. Finally, I would like to express my gratitude to my parents, brothers and sisters for their tremendous understanding and encouragement to me. 


\section{Contents}

Abstract

Declaration iv

Acknowledgement $\quad \mathrm{V}$

Contents $\quad$ vi

List of Tables $\quad$ xi

List of Figures $\quad$ xii

Symbols and Abbreviations $\quad$ xxiii

\section{Contents}

$\begin{array}{ll}\text { Chapter 1: Introduction } & 1\end{array}$

1.1 Geomagnetically Induced Currents (GIC) and their Impact 1

1.2 New Zealand GIC Data 3

$\begin{array}{ll}1.3 \text { GIC Studies in New Zealand } & 7\end{array}$

1.4 Structure and Aim of This Thesis $\quad 8$

$\begin{array}{ll}\text { Chapter 2: Background to GIC Studies } & 10\end{array}$

2.1 Geoeffective Interplanetary Structures 10

2.2 Calculation of Geoelectric Fields $\quad 19$

2.2.1 Calculation of Electric and Magnetic Fields Using Ionospheric Source Currents

2.2.2 Calculation of Geoelectric Field Using Ground Magnetic Data 26

2.2.3 Calculation of Geoelectric Field Using a Thin-sheet Conductance Model 29 
2.2.4 Calculation of Geoelectric Field Using Magnetotelluric Data

2.3 MT Perspective of GIC Modelling for New Zealand

2.4 Network Modelling

2.4.1 Substation Level Modelling

2.4.2 Transformer Level Modelling

2.5 Network Models for the New Zealand Network

2.5.1 Substation Level Network Model of New Zealand 45

2.5.2 Transformer Level Network Model of New Zealand 48

2.6 Discussion

Chapter 3: Spectral Approach to GIC Modelling at Islington Substation

3.1 Introduction

3.2 Dataset

3.3 Data Analysis

3.4 Methodology

3.4.1 Geoelectric Field Determination

3.4.2 GIC Modelling

3.5 Model Results

3.6 A Model Using Different Network Constants at High and Low Frequencies

3.7 Islington GIC Comparison with Other Substations

3.8 MT Geoelectric Field 86

3.9 Islington GIC Data and Solar Interplanetary Structures 88

3.10 Discussion

Chapter 4: Utilization of MT Data for GIC Modelling in the North Island, New Zealand

4.1 Introduction 
4.3 The St. Patrick's Day Magnetic Storm (2015)

4.4 Calculation of Geoelectric Field

4.5 Spatial Interpolation of Geoelectric Field

4.6 Uniform Electric Field and Transformer Response

4.7 Uniform Electric Field and Substation Response

4.8 Network Model and Equivalent Circuit Approach

4.9 MT Geoelectric Field and Transformer GIC

4.10 MT Geoelectric Fields and Substation GIC

4.11 Comparison with Measured GIC

4.12 Comparison with the Thin-sheet Conductance Model

4.12.1 Thin-sheet Geoelectric Field and Transformer GIC

4.12.2 Thin-sheet Geoelectric Field and Substation GIC

4.13 Discussion

Chapter 5: GIC Modelling of North Island for the Magnetic Storm 20 November2003

5.1 Introduction

5.2 The Solar Storm of 20 November2003

5.3 MT Model Geoelectric Field

5.4 MT Geoelectric Field and Transformer GIC

5.5 MT Model Substation GIC

5.6 Comparison of MT Model and Measured GIC

5.7 Thin-sheet Model Geoelectric Field 148

5.8 Thin-sheet Model Transformer GIC 150

5.9 Thin-sheet Model Substation GIC 153

5.10 Thin-sheet Model GIC and Measured Data Comparison 155

5.12 Discussion 
Chapter 6: MT Data GIC Modelling for the South Island During St. Patrick's Day 2015 Storm

6.1 Introduction

6.2 Magnetotelluric (MT) Data in the South Island

6.3 St. Patrick's Day Magnetic Storm (2015) and the South Island measured GIC

6.4 Calculation of Geoelectric Field

6.5 Spatial Interpolation of Geoelectric Field

6.6 Uniform Electric Field and Transformer Response

6.7 Uniform Electric Field and Substation Response

6.8 South Island Network Model and Equivalent Circuit Approach

6.9 GIC Results Using MT Data

6.10 Comparison with Measured GIC

6.11 Geoelectric Fields Using Thin-sheet Conductance Model

6.11.1 Model GIC using Thin-sheet Geoelectric fields

6.11.3 Thin-sheet Model GIC Comparison with Measured Data

6.12 Discussion

Chapter 7: GIC Modelling for the South Island for the Magnetic Storm 20 November2003

7.1 Introduction

7.2 MT Geoelectric Field 201

7.3 Transformer Level GIC Modelling Using MT Data 204

7.4 MT Model and Substation GIC 209

7.5 MT Model Comparison with Measured GIC

7.6 Thin-Sheet Model Geoelectric Field

7.7 Transformer Level GIC Modelling Using the Thin-sheet Model 
Chapter 8: Discussion and Conclusions 221

8.1 Single Site Modelling at ISL 221

8.2 Modelling North Island GIC 223

8.3 Modelling South Island GIC 226

8.4 Comparison with GIC studies in Other Countries 228

8.5 Summary and Suggestions 230

$\begin{array}{ll}\text { Appendix-Maxwell's Equations } & 232\end{array}$

Appendix-MT Measurements $\quad 235$

$\begin{array}{ll}\text { Appendix-Paper Published } & 240\end{array}$

$\begin{array}{ll}\text { References } & 241\end{array}$ 


\section{List of Tables}

1.1 Timeline of GIC measurements at different substations of New Zealand. The red bar shows no data or bad data, and green bar shows availability of GIC data. The substation GIC is based on availability of any of transformer data within that substation.

2.1 Interplanetary structures mainly responsible for geomagnetic storm and their features based on solar wind parameters.

2.2 The equivalent skin depth for a three-layer Earth model at different periods.

2.3 The skin depth in the thin-sheet at different periods.

2.4 Currents computed in lines and nodes for sample network using LP85.

2.5 Currents going through lines and transformer windings for Redclyffe substation using uniform fields of 1 $\mathrm{V} / \mathrm{km}$ in east direction.

3.1 Correlation comparison of magnetic field and its derivative with GIC data of ISL during major geomagnetic storms.

3.2 Network constant values using least regression method for ISL, SDN and INV during two magnetic storms.

3.3 Solar wind parameters considered along with GIC data of ISL.

4.1 Discrete periods of magnetotelluric response tensor of North Island available to model geoelectric field.

4.2 Currents in lines connected with NPL substation and transformer GIC.

4.3 The lines for which northern ends (third column) have been earthed to isolate network of lower North Island.

4.4 GIC in all earthed transformers of RDF and KAW substations, and the substation level GIC which is sum of GIC of all earthed transformers.

5.1 The line connection and the currents produced at 1844 UT and 1847 UT based on transformers experiencing large GIC.

6.1 Details of magnetotelluric data available for the South Island.

6.2 Line connection and currents for different substations experiencing large transformer level GIC.

7.1 Line connections and their currents for transformers experiencing large GIC. 


\section{List of Figures}

Figure 1. 1: Network model of the South Island showing different voltage power lines and the locations of GIC observations in 2019 based on Table 1.1.

4

Figure 1. 2: One-day measured GIC in Islington (ISL) transformer (T6H) for St. Patrick's Day magnetic storm 2015. The close-up view shows sampling intervals during sudden storm commencement.

Figure 2. 1: The smoothed sunspot number (red) and monthly sunspot number (blue) for solar cycles 23 and 24 starting from 1996. The inset picture above shows GIC data availability at Islington transformer T6.

Figure 2. 2: Contribution of different interplanetary structures in producing moderate magnetic storms ($100 n T<$ Dst <-50nT) during solar cycle 23 and solar cycle 24 . SC23 consists of total 196 storms whereas SC24 consists of total 130 storms.

Figure 2. 3: Contribution of interplanetary structures which includes CIR, ICME, SH+ICME and SH-producing intense geomagnetic storms (Dst $\leq-100 n$ T) during SC23 and SC24. SC23 consists of total 90 storms whereas SC24 consists of total 18 storms.

Figure 2. 4: Comparison of different interplanetary structures causing intense magnetic storms during different periods of solar cycles 23 and 24 based on available ISL GIC data. ICME dominates during 20022006 whereas SH+ICME dominates during 2010-2016.

Figure 2. 5: ISL GIC extreme values and Dst-minimum during both solar cycles. SC23 consists of total 43 and SC24 consists of 18 intense geomagnetic storms.

Figure 2. 6: Top six panels show solar wind one-minute data where the interplanetary structure is shock followed by a magnetic cloud, and the bottom panel shows one-minute interpolated measured GIC of Islington during magnetic storm of 18 August 2003.

Figure 2. 7: Top six panels show solar wind one-minute data where the shock is followed by sheath and magnetic cloud regions, and bottom panel shows one-minute interpolated measured GIC of Islington during 20 December 2015 storm.

Figure 2. 8: Schematic diagram (not on scale) showing how geoelectric fields are produced due an electrojet. The electrojet is taken as a line current in the direction of electric field, and corresponding horizontal and downward magnetic field components. The geoelectric field is the sum of both source fields and fields associated with telluric currents.

Figure 2. 9: Complex image method showing the source current above the surface of Earth and the image current at complex depth of $h+2 p$. The geoelectric field is sum of fields produced by both external (source) and image (internal/induced) currents.

Figure 2. 10: Conductivity-depth model obtained for Invercargill and Halfway Bush using the 3-D impedance tensor in the period range of 2-332 minutes.

Figure 2. 11: Magnetic field (Bx,Bz) and electric field (Ey) in response to an auroral electrojet calculated by CIM using the conductivity model of Invercargill. 
Figure 2. 12: Magnetic field $(B x, B z)$ and electric field $(E y)$ in response to an auroral electrojet calculated by CIM using the conductivity model of Halfway Bush.

Figure 2. 13: The consideration of correct loop that goes down to infinity at $B=0$ is necessary to accurately determine geoelectric fields as expressed by Faraday's Law of induction (adopted from Boteler and Pirjola, 2017).

Figure 2. 14: The conductance structure of the New Zealand using thin-sheet conductance model (adopted from Divett et al., 2017).

Figure 2. 15: The thin-sheet computed geoelectric field in response for a 5-minute period and an amplitude of $100 \mathrm{nT}$ magnetic field oriented 30o east of north, (a) for North Island. (b) for South Island. 34

Figure 2. 16: Magnetotelluric response tensor components (real and imaginary) at MT site HKB-803. Red circles show discrete periods of MT tensor ranging 1.4-30 minutes.

Figure 2. 17: (a) MT computed geoelectric field for magnetic field of $100 \mathrm{nT}$ at $300 \mathrm{E}$ of $\mathrm{N}$ having period of 5 minute for North Island. (b) Computed geoelectric fields for South Island.

Figure 2. 18: Left hand panel: magnetometer hole with the author sitting beside it. Right hand panel: data logger for recording electric and magnetic fields. The electrodes for electric field measurements are spread $\sim 50 \mathrm{~m}$ apart and are not shown in these pictures.

Figure 2. 19: (a) Network consisting of three phase connections of two-winding transformer and autotransformer. (b) Network representation of power lines and transformers for model development.

Figure 2. 20: A sample network consisting of four substations/nodes and a non-uniform electric field.

Figure 2. 21: (a) Transformer level network model for normal and auto transformer following Boteler and Pirjola (2014). (b) The nodal representation different voltage levels and transformer configuration, where horizontal lines show different voltage levels in the network.

Figure 2. 22: (a) North Island transmission network showing different voltage levels and dots showing substations. (b) Line resistance map of the network.

46

Figure 2. 23: The South Island of New Zealand showing transmission line resistances and substation node locations, all 220, 110 and $66 \mathrm{kV}$ lines are shown.

Figure 2. 24: Transformer-level representation of network of Redclyffe substation in the North Island. It consists of two normal transformers (T1 and T2) and two autotransformers (T3 and T4).

49

Figure 3. 1: Map of the South Island of New Zealand. Main urban centers are shown by filled circles; ISL and other substations having GIC measurements are shown by open circles. The EYR geomagnetic observatory is shown by the open square.

Figure 3. 2: (a) Comparison of GIC (red) and magnetic field (BX) in black, and (b) comparison of GIC and the time rate of change of magnetic field (Bx') for the magnetic storm of 23 May 2002.

Figure 3. 3: (a) Comparison of GIC (red) and magnetic field (BX) in black, and (b) comparison of GIC and the time rate of change of magnetic field $\left(B x^{\prime}\right)$ for the magnetic storm of 29 October2003. 
Figure 3. 4: (a) GIC and magnetic field (BX) and (b) shows GIC and the time rate of change of magnetic field $\left(B x^{\prime}\right)$ for the magnetic storm of $26 \mathrm{Sep} 2011$.

Figure 3. 5: (a) GIC and magnetic field (Bx), (b) shows GIC and the time rate of change of magnetic field $\left(B x^{\prime}\right)$ for the magnetic storm of 02 October2013.

Figure 3. 6: A spectral comparison of GIC measured for two magnetic storms, the period range of data starts from 2 minutes. Red curve (2002) shows small magnitude at shorter periods (high frequency) as compared to blue curve (2011).

Figure 3. 7: A spectral comparison of the magnetic field $(B x, B y)$ for the two magnetic storms for which GIC are shown in Figure 3.6.

60

Figure 3. 8: A comparison of slope values for both measured GIC (blue) and magnetic field Bx (red) during different years. The slope of GIC reduces as compared to Bx during 2011-2015.

Figure 3. 9: GIC data of ISL showing strong low frequency components during two magnetic storms (a) GIC raw data of for magnetic storms of 23 May 2002, (b) GIC raw data of 29 October2003 where (c) shows close-up view of data points in red ellipse.

Figure 3. 10: GIC data of ISL with abrupt variations only depicting only high frequency components (a) magnetic storm of 26 Sep 2011 and close-up view in (b). (c) magnetic storm of 02 October2013 and closeup in (d).

62

Figure 3. 11: Geoelectric fields computed using a uniform conductivity model for ISL and EYR magnetic field data for the magnetic storm of 23 May 2002.

64

Figure 3. 12: Geoelectric fields computed using a uniform conductivity model for ISL and EYR magnetic field data for a magnetic storm of 26 Sep 2011.

Figure 3. 13: The network constant $(a, b)$ during major geomagnetic storms from 2002-2006 and 20112015. The values are negative in 2002-2006 and positive during 2010-2015 showing a possible change in the network.

Figure 3. 14: Comparison of model and measured GIC both in time (top panel) and frequency domain (bottom panel) for the magnetic storm of 23 May 2002. The correlation is 0.89 .

68

Figure 3. 15: Comparison of model and measured GIC both in time (top panel) and frequency domain (bottom panel) for the magnetic storm of 29 October2003. The correlation is 0.91 .

Figure 3. 16: Comparison of model and measured GIC both in time (upper panel) and frequency domain (lower panel) for the magnetic storm of $26 \mathrm{Sep} 2011$. The correlation is 0.70.

Figure 3. 17: Comparison of model and measured GIC both in time (upper panel) and frequency domain (lower panel) for the magnetic storm of 02 October2013. The correlation is 0.79 .

Figure 3. 18: The correlation coefficient and P values between model and measured GIC data during major geomagnetic storms from 2002-2006 and 2011-2015.

Figure 3. 19: Comparison of measured (red) and model (black) GIC both in time (upper panel) and frequency domain (lower panel) for the magnetic storm of $26 \mathrm{Sep} 2011$ using geoelectric fields calculated using only the time rate of change of the magnetic field. The correlation is improved to 0.77 . 
Figure 3. 20: Comparison of model and measured GIC both in time (upper panel) and frequency domain (lower panel) for the magnetic storm of 02 October2013 using geoelectric fields calculated using only the time rate of change of the magnetic field. The correlation is improved to 0.92 .

Figure 3. 21: The correlation coefficient and P values between model and measured GIC during 2002-2006 and 2011-2015. The model GIC uses geoelectric fields computed without any previous values of the magnetic field.

Figure 3. 22: The flow chart of GIC modelling based on using different network constants for low and high frequency components of measured GIC.

Figure 3. 23: Comparison of measured (red) and model (black) GIC, in time domain (top panel), and frequency domain (bottom panel) using different network constants both for high and low frequency components of measured GIC for the magnetic storm of 23 May 2002. The correlation coefficient is 0.90.

Figure 3. 24: Model results time domain (top panel) and frequency domain (b) using different network constants for high and low frequency components of measured GIC for the magnetic storm of $26 \mathrm{Sep} 2011$. Correlation coefficient is 0.79 .

Figure 3. 25a: The correlation coefficient and performance parameter after using different network constants for both low and high frequency components of the measured GIC.

Figure 3.25b: The plots of network constants for (a) low frequency components and (b) for high frequency components.

Figure 3. 26: (a) Comparison of ISL (red) GIC with HWB (black) and SDN (blue) GIC for magnetic storm of 02 October2013. (b) shows normalized GIC when each of GIC has maximum value of 1.

80

Figure 3. 27: (a) Comparison of ISL (red) GIC with HWB (black) and SDN (blue) GIC for St. Patrick's Day magnetic storm of 17 March2015. (b) shows large amplitudes of slow variations after normalizing HWB and SDN data based on the extreme GIC value at ISL.

Figure 3. 28: The comparison of ISL (red) and INV (blue) GIC for magnetic storm of 02 October2013. The lower panel shows rescaling of INV data based on the largest GIC in the ISL data.

Figure 3. 29: The comparison of ISL (red) and INV (blue) GIC for magnetic storm of 17 March2015.

Figure 3. 30: Top panel shows time domain comparison of South Dunedin model (black) and measured (red) GIC for magnetic storm of 02 October2013 and bottom panel shows frequency domain comparison of the same.

Figure 3. 31: Top panel shows comparison of South Dunedin (SDN) model (black) and measured (red) GIC for magnetic storm of 17 March2015 where arrows show where discrepancy between model and data is high. Bottom panel shows comparison in frequency domain.

Figure 3. 32: Comparison of INV model (black) and measured (red) GIC for magnetic storm of 02 October2013 as top panel and bottom panel shows comparison in frequency domain.

Figure 3. 33: INV model (black) and measured (blue) GIC for magnetic storm of 17 March2015 in time domain (top panel) and frequency domain (bottom panel). Arrows highlight where discrepancy is high between model and data. 
Figure 3. 34: The time domain plot of geoelectric fields calculated using impedance tensor data from Invercargill (INV) and South Dunedin (SDN) during the magnetic storm of 17 March2015.

Figure 3. 35: Geoelectric fields at INV and SDN, calculated using the uniform conductivity model, during the magnetic storm of 17 March2015.

Figure 3. 36: Contribution of different interplanetary structures producing intense magnetic storms (Dst<100nT) during two different period of solar cycles 23 and 24. Total 43 geomagnetic storms were considered during 2002-2006 and 18 during 2011-2016.

Figure 3. 37a: shows relation between hourly mean values of absolute GIC and hourly mean values of different solar wind parameters for 20 geomagnetic storms during the period of 2002-2015. 91

Figure 3. 37b: shows relation between hourly mean values of absolute GIC and hourly average values of IMF-Bz for 20 geomagnetic storms during the period of 2002-2015.It shows GIC is highly correlated with negative values of IMF-Bz.

Figure 4. 1: MT sites shown on conductance map of North Island.

96

Figure 4. 2: The $K p$ index as a measure of geomagnetic activity downloaded from WDC (http://wdc.kugi.kyoto-u.ac.jp/kp/index.html) for 17 March 2015.

Figure 4. 3: Solar plasma and magnetic field parameters as observed by ACE satellite for 17 March 2015. The gaps show times with no data points, s1 and s2 marks the interplanetary shocks. The non-magnetic cloud (nonMC) and sheath region of ICME have been shown by horizontal bars.

98

Figure 4. 4: (a) Eyrewell (EYR) magnetic observatory data of horizontal field components during St. Patrick's Day storm on 17 March 2015; (b) Time derivatives of the magnetic field components; (c) Spectral power distribution of the EYR magnetic field and comparison of the period range available in the MT data.

100

Figure 4. 5: GIC observed data, interpolated to 1-minute sampling at Islington transformer (ISL-T6H) near Christchurch.

Figure 4. 6: Earth impedance tensor as function of frequency for CAR-119. Red circles show actual MT periods. A third-degree polynomial is used for interpolation purpose.

102

Figure 4. 7: Resulting geoelectric field using MT data and magnetic data of EYR observatory.

103

Figure 4. 8: Calculated vector geoelectric fields at MT sites at 04:47 UT. The white arrows show the smallest field ( $E=11.03 \mathrm{mV} / \mathrm{km}$ ) exists near the east coast (high conductance associated with the Hikurangi Margin) whereas the largest field ( $E=457.50 \mathrm{mV} / \mathrm{km}$ ) exists in the region close to the mountainous spine of the North Island. The upper part of island is not shown here because no MT data is available in that region.

Figure 4. 9: Interpolated geoelectric fields at 04:47 UT using the Nearest Neighbor method. It uses the original field values for extrapolation therefore the same fields exist in the form of patches.

107

Figure 4. 10: Interpolated geoelectric fields at 04:47 UT using Biharmonic Spline method. This gives smooth fields between MT sites, but results are worst in northern part.

108

Figure 4. 11: Transformer GIC produced in the North Island power network of after applying a uniform electric field of $1 \mathrm{~V} / \mathrm{Km}$ in the direction shown by the arrow. 
Figure 4. 12: Color map showing GIC at different substations and line currents for a uniform geoelectric field of $1 \mathrm{~V} / \mathrm{km}$ in the east direction.

Figure 4. 13: GIC computed in all substations of the North Island while applying uniform electric field of 1 $\mathrm{V} / \mathrm{km}$ in four different directions shown by arrow.

Figure 4. 14: (a) Equivalent Circuit configuration for the North Island power network. Green lines show normal connections with substations (shown by black dots). Blue lines show part of neighboring network and are regarded as having infinite resistances. The red lines are connections with normal resistance values except that their northern ends (shown by red dots) have been earthed. (b) shows close-up view of earthed connection substations.

Figure 4. 15: A comparison of GIC calculated using the full network (red) and calculated using the equivalent circuit approach (blue) using uniform fields of $1 \mathrm{~V} / \mathrm{km}$ in different orientations as shown in respective panels.

Figure 4. 16: MT model transformer GIC for all North Island substations during the SSC at 0447 UT. The Red bars show negative GIC values and blue bars show positive GIC values.

Figure 4. 17: Transformers (as mentioned on top of each panel) showing largest GIC during St. Patrick's Day storm calculated using MT geoelectric fields. All transformers show a strong peak of GIC at 04:47 UT (SSC).

Figure 4. 18: Substation-level GIC. Two substations for which transformer-level GIC are very small are BPE and HAY, both in the lower North Island. WHI is located on the east coast.

Figure 4. 19: MT model results. (a) Transformer locations experiencing the largest GIC and (b) locations of significant substation-level GIC during St. Patrick's Day storm. The size of circles is proportional to GIC magnitude.

Figure 4. 20: The network configurations for transformers (RDF-T1H) of Redclyffe (RDF) substation in left panel, and transformer (ISL-T6H) of Islington substation in right panel. The red dots show RDF and ISL transformers connected to other substations by power lines.

Figure 4. 21a: Individual time domain plots of measured GIC of Islington transformer and MT model GIC of two transformers of Redclyffe substation as mentioned on top of each panel. The model GIC shows different scale of magnitudes but the time variations are same.

Figure 4.21b: A comparison of model GIC of Redclyffe transformer (RDF-T1H) with observed GIC at Islington (ISL-T6H). The correlation coefficient is 0.64 . The model GIC effectively reproduces the observed GIC during the SSC as shown in close-up view (right-hand panel).

Figure 4. 22: Thin-sheet electric fields at 0447 UT during Sudden Storm Commencement. The geoelectric fields are on the same scale as used for MT geoelectric fields (Figure 4.8). The spatial distribution of geoelectric fields is smooth over different conductive regions. The yellow circles show location of largest and smallest geoelectric fields.

Figure 4. 23: Computed GIC during the Sudden Storm Commencement at 04:47 UT using TS conductance model.

Figure 4. 24: Transformers having large GIC at transformer-level during the St. Patrick's Day storm using TS conductance model. The effect of SSC is not very strong in these modelling results. 
Figure 4. 25: Substation GIC at transformer-level during the St. Patrick's Day storm using TS conductance model.

Figure 5. 1: (a) Kp-index values and (b) solar plasma and field parameters as observed by ACE satellite on 20 November2003. The dotted vertical line shows arrival of the shock and the horizontal arrow bar shows the boundaries of magnetic cloud (MC).

Figure 5. 2: (a) Magnetic field measured at EYR observatory, (b) time derivatives of the field, (c) One-minute averaged GIC in transformer T6H at Islington during the magnetic storm of 20 November2003. 136

Figure 5. 3: Time domain geoelectric field north (Ex) and east (Ey) components computed using magnetotelluric response tensor of an MT site shown in the map.

137

Figure 5. 4: Computed geoelectric fields vectors at the location of MT sites on the North Island grid at 1844 and 1847 UT during the magnetic storm of 20 November2003. The white arrows show the maximum fields.

Figure 5. 5: Geoelectric fields after applying Nearest Neighbor interpolation technique to MT geoelectric fields shown in Figure 5.4.

Figure 5. 6: Model transformer GIC at 1844 and 1847 UT using MT data. Redclyffe (RDF) and New Plymouth (NPL) transformers, on the horizontal axis, show largest response to geoelectric fields.

140

Figure 5. 7: Currents through power lines and transformers at 1844 and 1847 UT respectively. Left hand panel shows large GIC in RDF is caused by line (in red) connected with Wairakei (WRK) substation. Similarly, right hand panel shows large GIC in NPL transformer is caused by lines (in red) connected with Huntly (HLY) and Taumarunui (TMN) substations.

142

Figure 5. 8: Transformers (as shown on top of each panel) identified experiencing large GIC during the magnetic storm of 20 November2003 using MT modelling. Largest GIC occurs during the main phase of magnetic storm when magnetic field variations are maximum.

Figure 5. 9: Snapshot of substation GIC during two different times. Substation (on horizontal axis) showing largest GIC here also have large number of transformers.

Figure 6. 1: Locations of MT sites superimposed on the conductance map of South Island. Sites are distributed in three main groups. The conductivity structure varies from medium to high resistive regions based upon shades of yellow and green, respectively.

160

Figure 6. 2: Eyrewell magnetic observatory data of the horizontal field components (top panel) and their time derivative (bottom panel) during the St. Patrick's Day storm, 2015.

162

Figure 6. 3: One-minute interpolated GIC measured data at Islington (ISL-T6H), Halfway Bush (HWB-T4L) and South Dunedin (SDN-T2L) transformers, respectively during the St. Patrick's Day storm.

Figure 6. 4: Plot of real and imaginary components of the magnetotelluric response function against period range for an MT site. Red circles show discrete MT periods and blue line is a third-degree polynomial fit.

Figure 6. 5: Resulting geoelectric field for an MT site in Southland (shown as dot in the inset picture) depicting an abrupt change in geoelectric field value during the SSC of the solar storm.

165

Figure 6. 6: Resultant vector geoelectric fields calculated from the MT data at 0447 UT. The smallest field value is on the east coast whereas the largest field value is in the north-west. 
Figure 6. 7: Interpolated geoelectric fields at 0447 UT calculated using the Nearest Neighbor method. NN uses original field values for extrapolation therefore the same fields exist in form of patches. A large area of the South Island is interpolated with very little information and this could affect GIC modelling results.

Figure 6. 8: Interpolated geoelectric fields at 0447 UT using the Delaunay triangulation method. This gives smooth fields between MT sites but results are unreliable elsewhere.

Figure 6. 9: (a) Transformer level GIC produced in the South Island power network by a uniform electric field of $1 \mathrm{~V} / \mathrm{km}$ in east direction. (b) Line currents and the transformer GIC shown on the map where current magnitude is given by the color bar.

Figure 6. 10: (a) Transformer level GIC produced in the South Island power network by a uniform electric field of $1 \mathrm{~V} / \mathrm{km}$ in north direction. (b) Line currents and the transformer GIC shown on the map where current magnitude is given by the color bar.

Figure 6. 11: Transformer level GIC produced in the South Island power network by a uniform electric field of $1 \mathrm{~V} / \mathrm{km}$ in northeast direction.

Figure 6. 12: Transformer level GIC produced in the South Island power network by a uniform electric field of $1 \mathrm{~V} / \mathrm{km}$ in northwest direction.

172

Figure 6. 13a: Substation GIC produced in the South Island power network for uniform electric field of 1 $\mathrm{V} / \mathrm{km}$ in an east direction. The right-hand panel shows the location of substations and currents in power lines.

Figure 6.13b: Substation GIC produced in the South Island power network for uniform electric field of 1 $\mathrm{V} / \mathrm{km}$ in a north direction. The right-hand panel shows the location of substations and currents in power lines.

Figure 6.13c: Substation GIC produced in the South Island power network for uniform electric field of 1 $\mathrm{V} / \mathrm{km}$ in a northeast direction. The right-hand panel shows the location of substations and currents in power lines.

Figure 6.13d: Substation GIC produced in the South Island power network for uniform electric field of 1 $\mathrm{V} / \mathrm{km}$ in a northwest direction. The right-hand panel shows the location of substations and currents in power lines.

177

Figure 6. 14: The application of the approach of using two neighboring networks for the South Island power network. All connection points at Roxburgh (ROX) substation shown as a red dot have been earthed to isolate both parts of the networks. The blue lines and green lines now represent two independent networks without any electric connection between them.

178

Figure 6. 15: A comparison of GIC calculated using full network (orange) and neighboring network/ equivalent circuit approach (blue) using uniform fields of $1 \mathrm{~V} / \mathrm{km}$ at different orientations as shown in respective panels.

Figure 6. 16: Transformer level GIC in the South Island power network during the Sudden Storm Commencement (SSC) using MT computed geoelectric fields.

Figure 6. 17: MT GIC modelling results showing transformers (as shown on top of each panel) experiencing the largest GIC during St. Patrick's Day storm. 
Figure 6. 18: Substation-level GIC magnitude during Sudden Storm Commencement (SSC) at 0447 UT (left hand panel) and location of substations and power line currents (right hand panel).

Figure 6. 19: Left panel shows transformers experiencing large GIC and right panel shows large substations level GIC for St. Patrick's Day storm.

Figure 6. 20a: Top: observed GIC in Islington transform ISL-T6H in the period range of 2-30 minutes. Bottom: MT model GIC. The variations are quite similar both in observed and model GIC but the magnitude of model GIC is almost four times smaller than observed GIC.

Figure 6.20b: A comparison of model and observed GIC in Islington transform ISL-T6H showing correlation coefficient of 0.86 . Although the model GIC is very small in magnitude but it effectively reproduces GIC time variations during both the SSC (on right) and the rest of the storm.

187

Figure 6. 21: How interpolation affects the GIC results at Islington transformer (ISL-T6H).

188

Figure 6. 22a: Top: observed GIC in South Dunedin transform SDN-T2H in the period range of 2-83 minutes; bottom MT model GIC. The model GIC match very well both in magnitude and time variations.

189

Figure 6.22b: A comparison of model and observed GIC in South Dunedin transform SDN-T2H showing correlation coefficient of 0.79 . The model GIC effectively reproduces variations during both the SSC (on right) and the rest of storm.

189

Figure 6. 23a: Top: observed GIC in Halfway Bush transform HWB-T4L in the period range of 2-83 minutes; bottom MT model GIC. The model GIC has very small magnitude compared to the observed GIC. 190

Figure 6.23b: A comparison of model and observed GIC in Halfway Bush transform HWB-T4L showing correlation coefficient of 0.83. The model GIC during the SSC is very small compared to measured data.

190

Figure 6. 24: TS electric fields computed during the Sudden Storm Commencement at 0447 UT. 191

Figure 6. 25: Transformer GIC during Sudden Storm Commencement at 0447 UT using the thin-sheet conductance model.

Figure 6. 26: Thin-sheet conductance model results of transformer having largest GIC during St. Patrick's Day storm.

Figure 6. 27: Substation level GIC at 0447 UT using thin-sheet conductance model during St. Patrick's Day storm.

Figure 6. 28a: Top: observed GIC in Islington transform ISL-T6H, bottom: TS model GIC.

Figure 6.28b: A comparison of TS model and observed GIC in Islington transform ISL-T6H ( $r=0.66)$.

Figure 6. 29a: Top: observed GIC in South Dunedin transform-T2H; bottom TS model GIC.

Figure 6.29b: A comparison of TS model and observed GIC in South Dunedin transform-T2H $(r=0.61)$.

Figure 6. 30a: Top: observed GIC in Halfway Bush transform HWB-T4L; bottom TS model GIC. 
Figure 6.30b: A comparison of TS model and observed GIC of Halfway Bush transform HWB-T4 where both plots are on different scale $(r=0.64)$.

Figure 6. 31a: Top: observed GIC in Manapouri transform MAN-T6H; bottom TS model GIC.

Figure 6.31b: A comparison of TS model and observed GIC of Manapouri transform MAN-T6H where both plots are on different scale $(r=0.68)$.

Figure 7. 1a: Time domain geoelectric field computed using the impedance response tensor of an MT site in Otago (also shown by dot on the map) during magnetic storm of 20 November2003.

202

Figure 7.1b: Computed geoelectric field for MT site in the region of Marlborough covering the comparatively shorter period (1.9-15.2 minutes).

Figure 7. 2: Computed geoelectric fields vectors at the locations of MT sites on the South Island grid at 1844 and 1847 UT during the magnetic storm of 20 November2003.

Figure 7. 3: Geoelectric fields after applying Nearest Neighbor interpolation technique to MT fields shown in Figure 7.2. Nearest Neighbor fills the empty cells using the given field values.

203

Figure 7. 4: Transformer level GIC at 1844 and 1847 UT calculated using MT data.

205

Figure 7. 5: Color intensity plot of transformers and line currents at 1844 and 1847 UT. Transformer and line currents are on the same scale where red color shows highest current and blue color shows small currents.

Figure 7. 6: Transformers identified (shown top of each panel) experiencing large GIC during the magnetic storm of 20 November 2003 using MT modelling. Significant GIC occur during the main phase of the magnetic storm when the magnetic field variations are maximum.

208

Figure 7. 7: Substation level GIC at 1844 and 1847 UT using MT model.

209

Figure 7. 8: Left panel (red circles) shows transformers experiencing largest GIC whereas right panel shows (blue circles) substations experiencing largest GIC. The size of the circle on scale of maximum GIC magnitude.

Figure 7. 9a: Plots of measured (upper panel) and model GIC calculated using MT data (lower panel) of transformer-T6H at Islington.

Figure 7.9b: A comparison plot of measured (red) and model (black) GIC at Islington T6H. The bottom panel shows a zoom view during the main phase of the magnetic storm ( $r=0.84)$. 212

Figure 7.9c: A spectral comparison of measured and model GIC.

Figure 7. 10: TS computed horizontal components of the geoelectric field for a site in Otago during the magnetic storm of 2003.

Figure 7. 11: Thin-sheet geoelectric field vectors on conductance map of the South Island at 1844 and 1847 UT, respectively. 
Figure 7. 12: Transformer GIC at 1844 and 1847 UT respectively using TS geoelectric fields.

Figure 7. 13: Transformers identified (as shown in top of each panel) experiencing large GIC during the magnetic storm of 20 November 2003 using TS fields. The existence of low frequency components is quite evident using the TS model.

Figure 7. 14: Substations identified as experiencing large GIC during the magnetic storm of 20 November 2003 using TS geoelectric fields.

Figure 7. 15a: Individual plots of measured (top panel) and model (bottom panel) GIC of Islington transformer (ISL-T6H).

218

Figure 7.15b: Comparison of measured (red) and thin-sheet model (black) GIC. Although, model GIC has very small magnitude as compared to measured data, but it effectively reproduces variations particularly in longer period range $(r=0.84)$. 


\section{Symbols and Abbreviations}

The symbols and abbreviations used in this thesis are listed below in different sections based on their application. Bold letters are for vector quantities. Physical quantities are given in SI units which have also been used in the thesis.

\section{Geo-Electromagnetism}

\begin{tabular}{|c|c|c|c|}
\hline Physical quantity & Symbol & Units & Definition \\
\hline $\begin{array}{l}\text { Geomagnetic } \\
\text { field vector }\end{array}$ & $B$ & $n T$ & $B_{x}$-North; $B_{y}$-East and $B_{z}$-downward \\
\hline $\begin{array}{l}\text { Geoelectric field } \\
\text { vector }\end{array}$ & $\boldsymbol{E}$ & $\mathrm{mV} / \mathrm{km}$ & $E_{x}$-North; $E_{y}$-East and $E_{z}$-downwards \\
\hline Frequency & $f$ & $H z$ & Frequency of electromagnetic wave \\
\hline Current & $I$ & $A$ & Flow of charge per unit time \\
\hline Current density & $\mathrm{j}$ & A. $m^{-2}$ & Current per unit area \\
\hline Magnetotelluric & MT & & Measured magnetotelluric data \\
\hline Thin-sheet & TS & & Thin Sheet conductance model \\
\hline $\begin{array}{l}\text { Permeability of } \\
\text { free space }\end{array}$ & $\mu_{0}$ & $N \cdot m^{-2}$ & $\begin{array}{l}\text { Free space value }\left(4 \pi * 10^{-7}\right) \text { is used for geo- } \\
\text { electromagnetic induction }\end{array}$ \\
\hline $\begin{array}{l}\text { Permittivity of } \\
\text { free space }\end{array}$ & $\varepsilon_{0}$ & $N \cdot m^{2} \cdot C^{-2}$ & $\begin{array}{l}\text { Free space value }\left(9 * 10^{9}\right) \text { is } \\
\text { used for geo-electromagnetic induction }\end{array}$ \\
\hline $\begin{array}{l}\text { Magnetotelluric } \\
\text { response tensor }\end{array}$ & $\underline{\underline{Z}}$ & $m V k m^{-1} n T^{-1}$ & $\begin{array}{l}\text { The tensor for measured geoelectric and } \\
\text { geomagnetic field variations in frequency } \\
\text { domain }\end{array}$ \\
\hline $\begin{array}{l}\text { Angular } \\
\text { frequency }\end{array}$ & $\omega$ & rad.sec ${ }^{-1}$ & $\omega=2 \pi f$ \\
\hline $\begin{array}{l}\text { Complex skin } \\
\text { depth }\end{array}$ & $p$ & & $\begin{array}{l}\text { It is related to complex wave number } \\
\text { appearing in the wave equation }\end{array}$ \\
\hline Skin depth & $\delta$ & $\mathrm{km}$ & $\begin{array}{l}\text { The distance a wave travels before its } \\
\text { amplitude decay by } 37 \%\end{array}$ \\
\hline
\end{tabular}




\begin{tabular}{|c|c|c|c|}
\hline Conductivity & $\sigma$ & S. $m^{-1}$ & Conductivity of the Earth \\
\hline Resistivity & $\rho$ & S. $m$ & Resistivity of the Earth \\
\hline Magnetic flux & $\varnothing$ & V.sec & $\begin{array}{l}\text { Number of magnetic field lines through a } \\
\text { surface }\end{array}$ \\
\hline $\begin{array}{l}\text { Skin depth in the } \\
\text { thin-sheet }\end{array}$ & $\eta$ & $\mathrm{km}$ & $\begin{array}{l}\text { Skin depth used in the thin-sheet } \\
\text { conductance model }\end{array}$ \\
\hline
\end{tabular}

Network Model

\begin{tabular}{|c|c|}
\hline Abbreviation & Definition \\
\hline$a, b$ & Network constants in response to geoelectric fields \\
\hline $\mathrm{AC}$ & Alternating Current \\
\hline BP17 & Boteler and Pirjola (2017) Network model \\
\hline DC & Direct Current \\
\hline emf & Electromotive force causing current to flow in transmission lines \\
\hline GIC & $\begin{array}{l}\text { Geomagnetically Induced Current going through individual transformer } \\
\text { windings. GICs have been used as plural }\end{array}$ \\
\hline$G I C_{\text {sub }}$ & $\begin{array}{l}\text { Substation GIC which is sum of GICs through all earthed transformers within } \\
\text { a substation }\end{array}$ \\
\hline HV & High Voltage node of power network \\
\hline HVDC & High Voltage DC link between North and South Island \\
\hline ISL-T6H & Transformer level GIC (High voltage winding of T6 transformer of Islington) \\
\hline$J$ & $\begin{array}{l}\text { The currents produced in power lines are called source currents and in } \\
\text { network model represented in form of a matrix }\end{array}$ \\
\hline LP85 & Lehtinen and Pirjola (1985) Network model \\
\hline LV & Low Voltage node of power network \\
\hline$R_{L}$ & Resistance value of a transmission line connecting two substations \\
\hline
\end{tabular}




$$
\begin{array}{ll}
R_{T} & \text { Transformer winding resistance } \\
R_{e} & \text { Earth/ground resistance } \\
\text { THD } & \text { Total harmonic distortion } \\
V_{t h} & \text { Thevenin voltage when using a Thevenin circuit in network model } \\
Y & \text { Network admittance matrix which includes line resistance values } \\
Z & \quad \text { Network earthing impedance matrix which contains earthing resistances }
\end{array}
$$

\title{
Space Weather
}

\author{
Abbreviation \\ Definition \\ ACE $\quad$ Advanced Composition Explorer (Solar monitoring satellite) \\ $\mathrm{Bz} \quad$ IMF-B component in south direction \\ CIM Complex Image Method- It is used to map geoelectric and geomagnetic fields \\ based on ionospheric current system \\ CME Coronal Mass Ejection \\ Dst Geomagnetic activity index based on equatorial magnetic observatories and \\ given in units of nano Tesla \\ EYR Eyrewell Magnetic observatory near Christchurch \\ FAC Field Aligned Currents-A system of ionospheric current parallel to geomagnetic \\ field at polar region. \\ IMF-B Interplanetary Magnetic Field-B \\ IMF-Ey Interplanetary Electric field component Ey \\ ICME Interplanetary Coronal Mass Ejection \\ Kp Geomagnetic activity index based on high-latitudes magnetic observatories and \\ value varies from 1 to 9 \\ MC Magnetic Cloud \\ OMNI Solar wind database website (https://omniweb.gsfc.nasa.gov/) from where one- \\ minute data of different interplanetary structure is downloaded \\ $\mathrm{SH} \quad$ Sheath region of solar wind
}


SSC Sudden Storm Commencement

SC Solar cycle

SSN Sunspot Number

\section{Mathematics}

\begin{tabular}{|c|c|}
\hline Symbol & Definition \\
\hline$d / d t$ & Time derivative \\
\hline FFT & Fast Fourier Transformation \\
\hline$i$ & Imaginary part of complex number $(i=\sqrt{-1})$ \\
\hline$P$ & $\begin{array}{l}\text { Performance parameter to determine accuracy of model results with measured } \\
\text { GIC data }\end{array}$ \\
\hline$r$ & Linear correlation coefficient also known as Pearson correlation coefficient \\
\hline RMSE & Root Mean Square Error \\
\hline $\boldsymbol{\nabla}$ & Del operator to calculate Divergence or Curl of vector field \\
\hline$x$ & Vector cross product \\
\hline$\oint$ & Integral around a close path \\
\hline
\end{tabular}

\section{Coordinate system}

$(\hat{x}, \hat{y}, \hat{z}) \quad$ Cartesian coordinate system where $\hat{x}=$ North, $\hat{y}=$ East and $\hat{z}=$ downward into the Earth 


\section{Chapter 1: Introduction}

\subsection{Geomagnetically Induced Currents (GIC) and their Impact}

Electric fields occurring at the Earth's surface in connection with geomagnetic storms, produce Geomagnetically Induced Currents (GIC) in the conductors of technological systems. These unwanted currents have been observed flowing through electric power transmission grids, oil and gas pipelines, telecommunication cables and railway equipment (Molinski, 2002; Pulkkinen et al., 2001; Ingham and Rodger, 2018; Boteler, 2006). During severe magnetic storms currents can reach up to several hundred Amperes and could adversely affect modern power transmission systems and contribute to enhanced corrosion of pipelines. For example, GIC can interfere with the operation of electric power grids, damage transformers, and even cause blackouts. GIC can flow to ground at substations of the power system causing partial saturation of the power transformers (Kappenman, 2007; Rodger et al., 2020). This produces increased power consumption and heating of the transformer and distortion of the alternating current (AC) waveform, which leads to a variety of effects on the power system e.g., mis-operation of protective relays, voltage instability, and in a worst-case scenario leading towards power blackouts and transformer destruction.

The impact of GIC on ground technology was first observed on 02 September 1859 during what is known as the Carrington Event, when a white light solar flare caused an intense geomagnetic storm (Cliver and Svalgaard, 2004). This remains the most intense magnetic distortion ever recorded on the Earth (Carrington, 1859) causing a widespread problem for the existing technology of the time i.e., the telegraph system (Boteler, 2006). The impact of geomagnetic storms on a power network was significantly observed during 1940 magnetic storms when the power systems in the northeast of the US and Canada experienced malfunctions of equipment (Davidson, 1940). The most prominent impact in terms of modern technology happened on 13 March 1989 in Canada, when a magnetic storm caused collapse of the Hydro-Québec system (Boteler, 2019; Bolduc, 2002). The power grid system of North America and Europe also 
experienced the impact of this storm. Similarly, major geomagnetic disturbances in 1958, 1972, 1982, 1989, 2003, and 2006 caused problems for power systems in different parts of the world (Bolduc, 2002; Wik et al., 2009).

The impact of geomagnetic storms is strongest in auroral regions, but this does not mean there is no impact on power systems of mid latitudes countries. There has been strong evidence of GIC impacts at low and middle latitudes e.g., South Africa (Koen and Gaunt, 2003), New Zealand (Mac Manus et al., 2017), Brazil (Trivedi et al., 2007), China (Liu et al., 2009) and Japan (Watari et al., 2009). Gaunt and Coetzee (2007) showed increased transformer problems occurred after large geomagnetic storms in South Africa and resulted in transformer deterioration and eventual failure. GIC damage to the New Zealand electrical grid has also occurred e.g., one significant GIC impact was the destruction of a transformer at Dunedin/Halfway Bush (Béland \& Small, 2004; Marshall et al., 2012). Significant magnetic field rates of change have also been found in equatorial latitudes during coronal mass ejection (CME) arrival times, when the local magnetic signature is amplified by the equatorial electrojet (Carter et al., 2016). Therefore, GIC measurements and modelling studies are of growing interest in many countries.

To protect a power grid from GIC different mitigation strategies have been adopted during severe magnetic storms. Since GIC frequencies are less than $1 \mathrm{~Hz}$ whereas the typical operating frequency of a power system is 50 or $60 \mathrm{~Hz}$, devices to block the flow of GIC have been investigated (Bolduc et al., 2005). For example, devices like capacitors which block the flow of direct current (DC) but allow AC to pass, can be installed. However, GIC can flow throughout the network and the installation of such devices may simply cause current flow to be diverted and lead to a larger GIC elsewhere (Pirjola, 2002; Arajarvi et al., 2011). The cost of treating a whole network is not an optimal solution. Kappenman et al. (1997) estimated the cost to be several billion dollars for the United States network alone. Given the prohibitive cost and difficulties of an engineering solution, operational mitigation of GIC is considered as one of the best strategies to manage the GIC risk. To assess the level of risk, we need to establish how often significant GIC are likely to occur, and how possibly it could affect the network. 


\subsection{New Zealand GIC Data}

New Zealand, the focus of this thesis, consists of two major islands. The power network of both Islands is owned and operated by Transpower New Zealand Limited and the electricity supply in both islands is connected through an underground High Voltage DC (HVDC) link. This link does not allow GIC to flow between the networks of the two islands, therefore networks of both islands are treated separately. There are extensive GIC measurements in the South Island transmission network acquired by Transpower New Zealand Ltd. at many substations and individual transformers (Mac Manus et al., 2017). Historically, GIC measurements were made to monitor stray currents entering the $\mathrm{AC}$ transmission network during the Earth return operation of the HVDC link joining the South and North Islands. The impact of the HVDC earth return currents from the data have been removed prior use of data for GIC studies (Mac Manus et al., 2017).

In many cases multiple transformers in the same substation are independently monitored. Usually, GIC measurements are limited to single station or small number of transformers, but the South Island of New Zealand is rich in having a long record of measurements on multiple transformers/ substations (Marshall et al., 2012). The South Island high-voltage transmission network consists of transmission lines with three different voltage ranges: 50/ $66 \mathrm{kV}, 110 \mathrm{kV}$, and $220 \mathrm{kV}$. The network consists of 64 substations connected by 121 transmission lines (Divett et al., 2017). Figure 1.1 shows the South Island network and the location of all GIC observations (red squares). Different line colors show different voltage power lines connecting substations. The Eyrewell (EYR) magnetic observatory is also shown as the magenta diamond near the Islington (ISL) substation. GIC observation in the North Island exist only near Wellington close to the northern termination of the HVDC link.

Geoelectric fields produces electromotive force $(e m f)$ in the main power lines of network therefore GIC can flow throughout the network while entering the Earth through transformer neutral/ground points (Boteler and Pirjola, 1998a). Since transformers are most effected by DC, usually GIC measurement devices are installed on the ground connections of individual transformers. The GIC monitoring devices installed are Hall effect current transducers (LEM model LT 505-S), installed onto the transformer neutral line connection to Earth. Table 1.1 shows 
a timeline of GIC data acquisition for different substations of South Island, where the green shows availability of data and red shows no or bad availability of data. Substation GIC is based on the availability of any transformer data within that substation. GIC measurements started in 2001 at 12 substations. Multiple sensors were installed to monitor GIC in different transformers within a substation such that a total 36 sensors were deployed in 2001 (Mac Manus et a., 2017). This number initially increased slowly, with measurements being made at 36-40 transformers in 12 distinct locations until 2009. However, additional LEM began to be installed at additional substations with a specific focus of monitoring GIC during space weather events. The expansion included measurements at the Halfway Bush (HWB) substation and specifically the transformer HWB-T4 which had been replaced after the 6 November 2001 storm. And by February 2015 a total of 58 transformers were being monitored at 17 distinct locations.

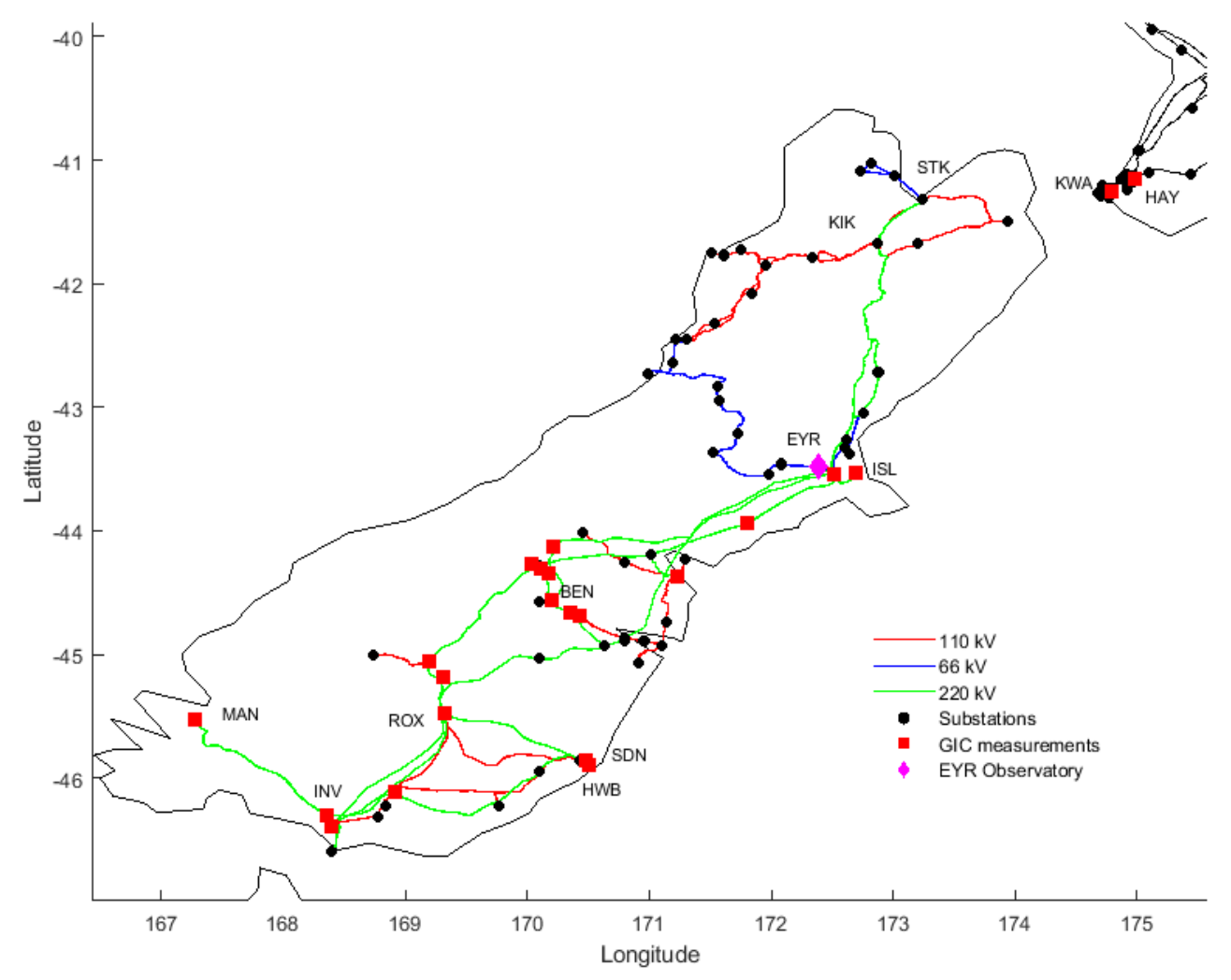

Figure 1. 1: Network model of the South Island showing different voltage power lines and the locations of GIC observations in 2019 based on Table 1.1. 
In recent years additional measurements have been added with the specific goal of space weather oriented GIC monitoring (Rodger et al., 2020). The time resolution of the LEM DC measurement is determined by the archiving software. This degrades the time resolution of the data set when the DC values are changing slowly but stores high time resolution data when the DC values are changing rapidly. In practice, this means that during geomagnetic storms when GIC are present the data have the highest time resolution ( $4 \mathrm{sec})$, while at other times when the values are slowly changing the time resolution can be considerably longer (as much as 1 hour or 1 minute).

\section{Table 1.1}

Timeline of GIC measurements at different substations of New Zealand. The red bar shows no data or bad data, and green bar shows availability of GIC data. The substation GIC is based on availability of any of transformer data within that substation.

\begin{tabular}{|c|c|c|c|c|c|c|c|c|c|c|c|c|c|c|c|c|c|c|c|}
\hline Substation & $\begin{array}{l}20 \\
01\end{array}$ & $\begin{array}{l}20 \\
02\end{array}$ & $\begin{array}{l}20 \\
03\end{array}$ & $\begin{array}{l}20 \\
04\end{array}$ & $\begin{array}{l}20 \\
05\end{array}$ & $\begin{array}{l}20 \\
06\end{array}$ & $\begin{array}{l}20 \\
07\end{array}$ & $\begin{array}{l}20 \\
08\end{array}$ & $\begin{array}{l}20 \\
09\end{array}$ & $\begin{array}{l}20 \\
10\end{array}$ & $\begin{array}{l}20 \\
11\end{array}$ & $\begin{array}{l}20 \\
12\end{array}$ & $\begin{array}{l}20 \\
13\end{array}$ & $\begin{array}{l}20 \\
14\end{array}$ & \begin{tabular}{|l|}
20 \\
15 \\
\end{tabular} & $\begin{array}{l}20 \\
16\end{array}$ & $\begin{array}{l}20 \\
17\end{array}$ & $\begin{array}{l}20 \\
18 \\
\end{array}$ & $\begin{array}{l}20 \\
19 \\
\end{array}$ \\
\hline Haywards (HAY) & & & & & & & & & & & & & & & & & & & \\
\hline $\begin{array}{l}\text { Kaiwharawhara } \\
\text { (KAW) }\end{array}$ & & & & & & & & & & & & & & & & & & & \\
\hline Bromley (BRY) & & & & & & & & & & & & & & & & & & & \\
\hline Islington (ISL) & & & & & & & & & & & & & & & & & & & \\
\hline Ashburton (ASB) & & & & & & & & & & & & & & & & & & & \\
\hline Timaru (TIM) & & & & & & & & & & & & & & & & & & & \\
\hline Tekapo B (TKB) & & & & & & & & & & & & & & & & & & & \\
\hline Ohau A (OHA) & & & & & & & & & & & & & & & & & & & \\
\hline Ohau B (OHB) & & & & & & & & & & & & & & & & & & & \\
\hline Ohau C (OHC) & & & & & & & & & & & & & & & & & & & \\
\hline Benmore (BEN) & & & & & & & & & & & & & & & & & & & \\
\hline Aviemore (AVI) & & & & & & & & & & & & & & & & & & & \\
\hline Waitaki (WTK) & & & & & & & & & & & & & & & & & & & \\
\hline Cromwell (CML) & & & & & & & & & & & & & & & & & & & \\
\hline Clyde (CYD) & & & & & & & & & & & & & & & & & & & \\
\hline Roxburgh (ROX) & & & & & & & & & & & & & & & & & & & \\
\hline $\begin{array}{l}\text { Halfway Bush } \\
\text { (HWB) }\end{array}$ & & & & & & & & & & & & & & & & & & & \\
\hline $\begin{array}{l}\text { South Dunedin } \\
\text { (SDN) }\end{array}$ & & & & & & & & & & & & & & & & & & & \\
\hline Manapouri (MAN) & & & & & & & & & & & & & & & & & & & \\
\hline Gore (GOR) & & & & & & & & & & & & & & & & & & & \\
\hline $\begin{array}{l}\text { North Makarewa } \\
\text { (NMA) }\end{array}$ & & & & & & & & & & & & & & & & & & & \\
\hline Invercargill (INV) & & & & & & & & & & & & & & & & & & & \\
\hline
\end{tabular}

Figure 1.2 shows one day of measured raw GIC (without any interpolation) data of T6 transformer at Islington (ISL) substation for the St. Patrick's Day magnetic storm (17 March 2015). The total 
number of data points are 2524 showing the average sampling interval of about half a minute. One strong peak in the data is $-17.01 \mathrm{~A}$ at 04:47 during the Sudden Storm Commencement (SSC), shown on right hand panel, and during the 4 minutes period shown there are 29 measurements of GIC i.e., an average sampling interval of about 8 seconds.

GIC modelling is a common approach to assess power network risk during extreme magnetic storms but is not the only way of assessing risk to a network. Harmonic distortion observations provide a different way to look at the space weather impact on power transmission networks based on network wide harmonic observations of stressed transformers. Saturation of a transformer core resulting from GIC can lead to the generation of voltage harmonics in the power system (Girgis \& Vedante, 2015). Clilverd et al. (2018) have demonstrated the occurrence of this at HWB substation near Dunedin in South Island. Analysis by Rodger et al. (2020) of total harmonic distortion (THD) measurements shows significant THD occurred at several locations in the North Island during a magnetic storm suggesting the existence of previously undetected GIC in the North Island power network. Many of these locations are close to New Plymouth (NPL) in the western part of the North Island. As described earlier direct measurements of GIC in the North Island exist only near Wellington therefore the data of THD could be used for comparison with a GIC model for the North Island. THD data shows there is evidence of GIC impacts in the North Island. Total harmonic distortion measurements are made in both North and South islands at over 126 substations in the New Zealand transmission network (Rodger et al., 2020).
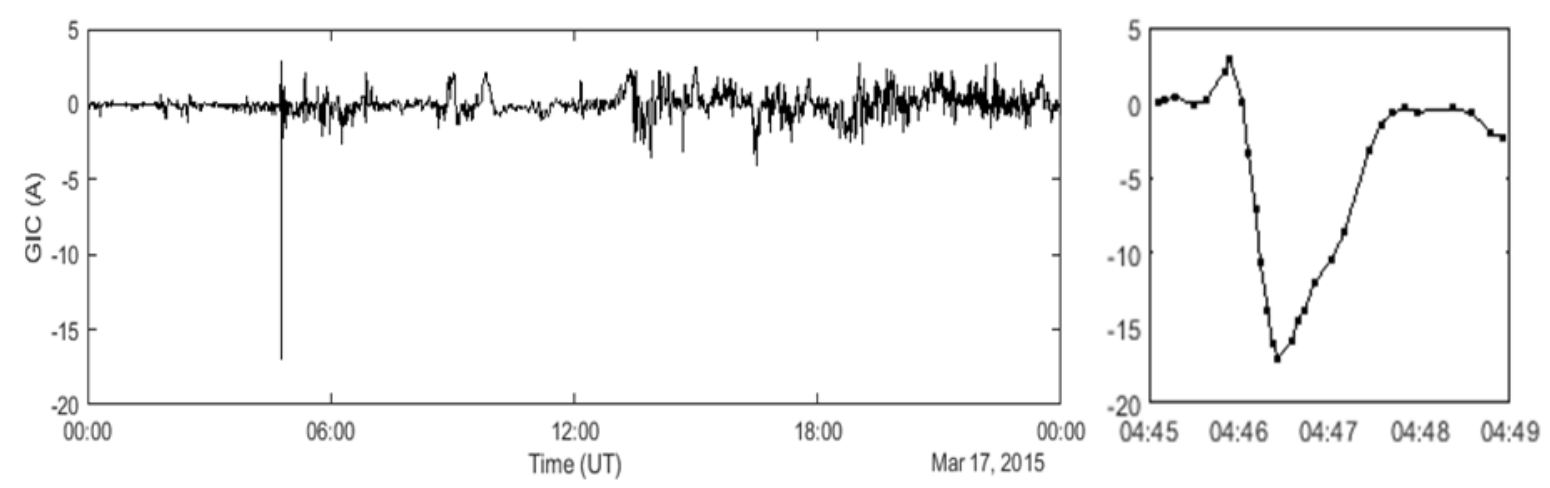

Figure 1. 2: One-day measured GIC in Islington (ISL) transformer (T6H) for St. Patrick's Day magnetic storm 2015. The close-up view shows sampling intervals during sudden storm commencement. 


\subsection{GIC Studies in New Zealand}

The large number of GIC and THD measurements available means that New Zealand is unusually well positioned to investigate and model GIC. New Zealand is also one of the few countries that has experienced direct transformer damage at Halfway Bush from a large geomagnetic storm (Béland \& Small, 2004; Marshall et al., 2012). This occurred on 6 November 2001 at 1:53 UT, within a few minutes of a storm sudden commencement, the network operator (Transpower New Zealand Ltd) received alarms from transformers across the South Island. Simultaneously, the voltage control equipment for Christchurch city tripped along with a transformer feeding Dunedin city. One of the phases of Dunedin and Halfway Bush substations failed within 1 min of the GIC onset time, and an internal inspection revealed that the transformer (HWB-T4) at Halfway Bush substation was beyond repair.

GIC modeling studies in New Zealand have thus far concentrated on the South Island, Iying between latitudes of $41^{\circ} \mathrm{S}$ and $47^{\circ} \mathrm{S}$, and in relative proximity to the auroral zone. The South Island has traditionally been presumed to be at higher risk from GIC than the North Island which lies at latitudes between $34^{\circ} \mathrm{S}$ and $42^{\circ} \mathrm{S}$. The long recorded GIC measurements have been utilized for GIC modelling and risk assessment e.g., Mac Manus et al., (2017) showed that the rate of change of the $\mathrm{H}$-component $\left(\mathrm{H}^{\prime}\right)$ of the local magnetic field is the best correlated driver of observed GIC magnitude, but not for every storm in every location. Investigations of likely extreme GIC magnitude within New Zealand has been given by Rodger et al., (2017), showing that an estimated peak GIC of approximately 150 to 600 A could occur with a 100-year return period at a particular transformer near Christchurch. A different approach based on magnetic field transfer functions found similarly large peak GIC magnitude at multiple New Zealand locations (Ingham et al., 2017). Divett et al., $(2017,2018)$ developed a network model of the New Zealand power system using a nodal network approach. The model uses geoelectric field as input to compute transformer level GIC in every transformer of the network. These studies used thin-sheet conductance model to derive the geoelectric fields used for GIC modelling for the South Island power network and results have been compared with measured data (Divett et al., 2020). 


\subsection{Structure and Aim of This Thesis}

As is described later, the thin-sheet modelling technique has several limitations in terms of being able to successfully model GIC. In this thesis an alternative approach of using magnetotelluric (MT) data is explored to model GIC in both North and South Island power networks. Since the raw GIC measurements are not on uniform intervals therefore in this thesis on-minute interpolated measured GIC has been used for comparison with model GIC. The structure of the thesis is as follows.

Chapter 2 gives further background on the origin of GIC and on techniques to model the induced electric fields that produce GIC. It also provides a comparison of thin-sheet geoelectric fields and geoelectric fields calculated using the magnetotelluric response tensor which suggest that the magnetotelluric calculated fields are more realistic than thin-sheet results.

Chapter 3 discusses the measured GIC data at Islington (ISL) from 2002-2015 for major geomagnetic storms (based on $K p$ index). These have been modelled using time domain geoelectric fields derived from uniform conductivity Earth model. Comparison of modelling results with measured data and the limitations of the single station approach are discussed. Solar interplanetary structures for the major geomagnetic storms and the relationship of different solar wind parameters to Islington GIC data are also given.

Chapter 4 describes the transformer level and substation level GIC modelling using MT geoelectric fields for the North Island during the St. Patrick's Day magnetic storm of 17 March 2015. Thin-sheet (TS) model GIC are also given and compared with the MT GIC model. Transformers and substations have been identified as experiencing large GIC using both (MT and TS) techniques. Since there are no measured GIC which can be compared with model GIC for the North Island, model GIC at Redclyffe (RDF) substation are compared with an appropriate substation (Islington) in South Island. The accuracy of both models with respect to measured data is discussed in terms of the correlation coefficient $(r)$ and performance parameter $(P)$. To further analyze the GIC modelling results another geomagnetic storm of 20 November2003 is considered in the same fashion and is presented as Chapter 5. 
Similarly, in Chapter 6, geoelectric fields have been computed using MT data from the South Island for the St. Patrick's Day magnetic storm. Since the measured GIC are available at multiple locations in the South Island the MT model GIC and thin-sheet model GIC have been compared with measured data for various transformers. Chapter 7 gives the same analysis and modelling results for the magnetic storm of 20 November 2003. The last chapter summarizes the results of both MT and TS techniques and gives suggestions for possible future work. 


\section{Chapter 2: Background to GIC Studies}

\subsection{Geoeffective Interplanetary Structures}

Solar events, such as coronal mass ejection (CME) create disturbances within the Earth's magnetosphere which can give rise to geomagnetic storms and substorms. The solar wind, a continuous stream of plasma emitted from the surface of the Sun, is the main source of energy transfer into the atmosphere of the Earth. The solar wind also carries the material emitted during coronal mass ejections which propagate into the interplanetary space. The interplanetary remnants of CME are commonly known as interplanetary coronal mass ejection (ICME) and are also the most important geoeffective interplanetary structures. Different interplanetary structures have been identified based upon solar wind plasma and field parameters e.g., interplanetary magnetic field strength (IMF-B), southward IMF-B component $(B z)$, proton density, proton temperature, solar wind speed etc. The high-resolution data of solar eruptions and interplanetary plasma/field parameters are available through spacecraft observations e.g., TRACE, WIND, ACE, SOHO and ULYSSES. Based on their interplanetary structures solar storms have been classified into different types. Many studies (Gonzalez et. at., 2007; Rawat et. al., 2018; Echer et al., 2008) have classified the interplanetary structures producing large geomagnetic storms during the solar cycles 23 and 24. Following the nomenclature of Echer et al., 2008, primarily solar storms are characterized in terms of corotating interaction region (CIR) and interplanetary coronal mass ejection (ICME). CIR is formed due to the interaction of low-speed streams and high-speed streams embedded in the solar wind. A major and geoeffective type of ICME is called magnetic cloud (MC). The common criteria of a MC is that the $B z$ component of the interplanetary magnetic field display a smooth $\sim 180^{\circ}$ rotation and low proton beta (the ratio of plasma pressure to magnetic pressure or energy density) (Huttunen et al., 2002). ICME not fulfilling the above conditions of $M C$ is considered as nonMC. Magnetic clouds are important in the context that it is well established that the interplanetary magnetic field $B z$ plays a crucial role for the efficient energy transfer from the solar wind into the magnetosphere through the magnetic reconnection process (Gonzalez et al., 1994). This is the reason why a MC is also 
regarded as effective in producing a magnetic storm. In this study, for simplicity, we regard both $M C$ and nonMC as ICME. It is important to mention that during a solar storm the interplanetary structures mostly occur along with other significant well recognized structures such as a shock $(s)$. A shock is formed ahead of ICME when the speed difference between the driving ICME and background solar wind exceeds magnetosonic wave speed. If a $M C$ is preceded by an interplanetary fast shock, it is mentioned as (SMC), otherwise, it is called as a MC with no shock (nsMC).

A third type of significant interplanetary structure is known as sheath (SH). SH is identified when the $B z$ fluctuates north and south several times and the solar wind dynamic pressure is usually high (Huttunen et al., 2008). The fourth category is a composite of sheath field and ICME regarded as $\mathrm{SH}+\mathrm{ICME}$ where a sheath is followed by the ICME. Similarly, SH+MC stands for a sheath followed by a magnetic cloud. Sheath is mostly present in the region between the interplanetary shock and the driver ICME and both are partly responsible for the main phase of a geomagnetic storm. These characteristics are given in short form in Table 2.1.

\section{Table 2.1}

Interplanetary structures mainly responsible for geomagnetic storm and their features based on solar wind parameters.

\begin{tabular}{|c|c|}
\hline $\begin{array}{l}\text { Interplanetary } \\
\text { structure }\end{array}$ & Main characteristics \\
\hline $\begin{array}{l}\text { Corotating interaction } \\
\text { region }(\mathrm{CIR})\end{array}$ & $\begin{array}{l}\text { Caused by low-speed stream and high-speed stream of } \\
\text { solar wind }\end{array}$ \\
\hline $\begin{array}{l}\text { Interplanetary coronal } \\
\text { mass ejection (ICME) }\end{array}$ & $\begin{array}{l}\text { Magnetic cloud }(\mathrm{MC}) \text { : Large value of interplanetary } \\
\text { magnetic field, smooth full rotation } 180 \text { in } B z \text {, low } \\
\text { proton temperature/ low proton beta } \sim 0.1 \text {, } \\
\text { NonMC: Does not fulfill any of above criteria of } \mathrm{MC}\end{array}$ \\
\hline Sheath (SH) & $\begin{array}{l}\text { The interplanetary magnetic field components are } \\
\text { highly fluctuating, flow pressure is high and driver of } \\
\text { storm's main phase }\end{array}$ \\
\hline SH+ICME & $\begin{array}{l}\text { Exhibiting both } \mathrm{SH} \text { and ICME structures and partly } \\
\text { responsible for geomagnetic storm }\end{array}$ \\
\hline
\end{tabular}

Solar cycles 23 and 24 have many different features and many studies have explored interplanetary structures causing geomagnetic storms during both cycles. The $D s t$ index which represents the strength of the ring current in the region of ionosphere, is widely used as a 
measure of geomagnetic activity. It is derived by measuring global variations in the horizontal components of the magnetic field at low-latitude. According to the peak Dst index, two broad categories, used here, are intense storms (Dst $\leq-100 \mathrm{nT})$ and moderate storms $(-100 \mathrm{nT}<D s t$ $\leq-50 \mathrm{nT}$ ). Figure 2.1 shows monthly sunspot numbers (SSN) for solar cycle 23 (SC23) and solar cycle 24 (SC24). The inset picture shows the availability of continuously measured GIC on T6 transformer at ISL. Solar cycle 23 maximum, has significantly greater sunspot numbers ( 200) in comparison with solar cycle 24 maximum which has only $\sim 150$. This resulted in different numbers of geomagnetic storms, e.g., solar cycle 23 witnessed 11 super magnetic storms (Dst $\leq-250 \mathrm{nT}$ ) whereas solar cycle 24 did not have any observed super magnetic events. There were also more intense magnetic storms ( $D s t \leq-100 \mathrm{nT}$ ) during solar cycle 23 compared to solar cycle 24 e.g., Echer et al., (2013) identified 90 intense storms during solar cycle 23 and Rawat et al., (2018) identified 18 during solar cycle 24.

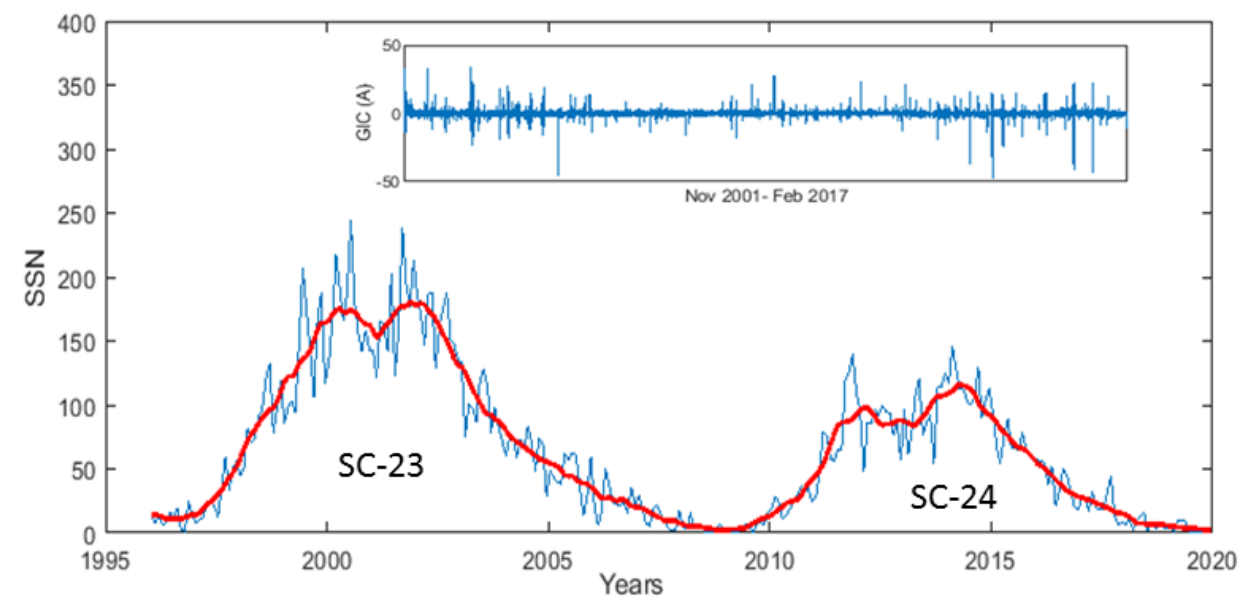

Figure 2. 1: The smoothed sunspot number (red) and monthly sunspot number (blue) for solar cycles 23 and 24 starting from 1996. The inset picture above shows GIC data availability at Islington transformer T6.

The magnetospheric response differs significantly for different interplanetary structures (Miyoshi and Kataoka, 2005) therefore it is hoped to be able to correlate interplanetary structures and GIC. However, GIC are the end-product of solar storms and there is a chain of mechanisms involved in their production which makes it difficult to make such a correlation. GIC are strongly impacted by ground conductivity structure, network configuration, and the current systems in the ionosphere/magnetosphere. Some studies have explored significantly different mechanisms of GIC production during ICME and CIR structures and showed that ICME produce large GIC 
compared to CIR (Kataoka and Pulkkinen, 2008; Borovsky and Denton, 2006; Huttunen et al., 2008). Many studies have considered Dst in relation with different interplanetary structures (Huttunen et al., 2002; Gopalswamy et al., 2015; Rawat et al., 2018; Echer et al., 2008).

Interplanetary structures have also been identified for moderate geomagnetic storms during both solar cycles. Solar cycle 23 consists of 196 moderate geomagnetic storms (Echer et al., 2013) and there were 130 during solar cycle 24 (Rawat et al., 2018). Figure 2.2 shows the contribution of each of the interplanetary structures (based on Table 2.1) causing moderate geomagnetic storms during both solar cycles. The Figure shows that during solar cycle $23,48 \%$ of storms were CIR driven, while $41.3 \%$ were driven by ICME and/or their SH. During solar cycle $24,41 \%$ of the moderate storms were driven by $\mathrm{CIR}$, and ICME and/or their SH contributed about $49 \%$. This suggests that the interplanetary structures causing moderate geomagnetic storms do not show any significant differences between the solar cycles. 'Others' include interplanetary structures outside the considered structures therefore have not been discussed here.
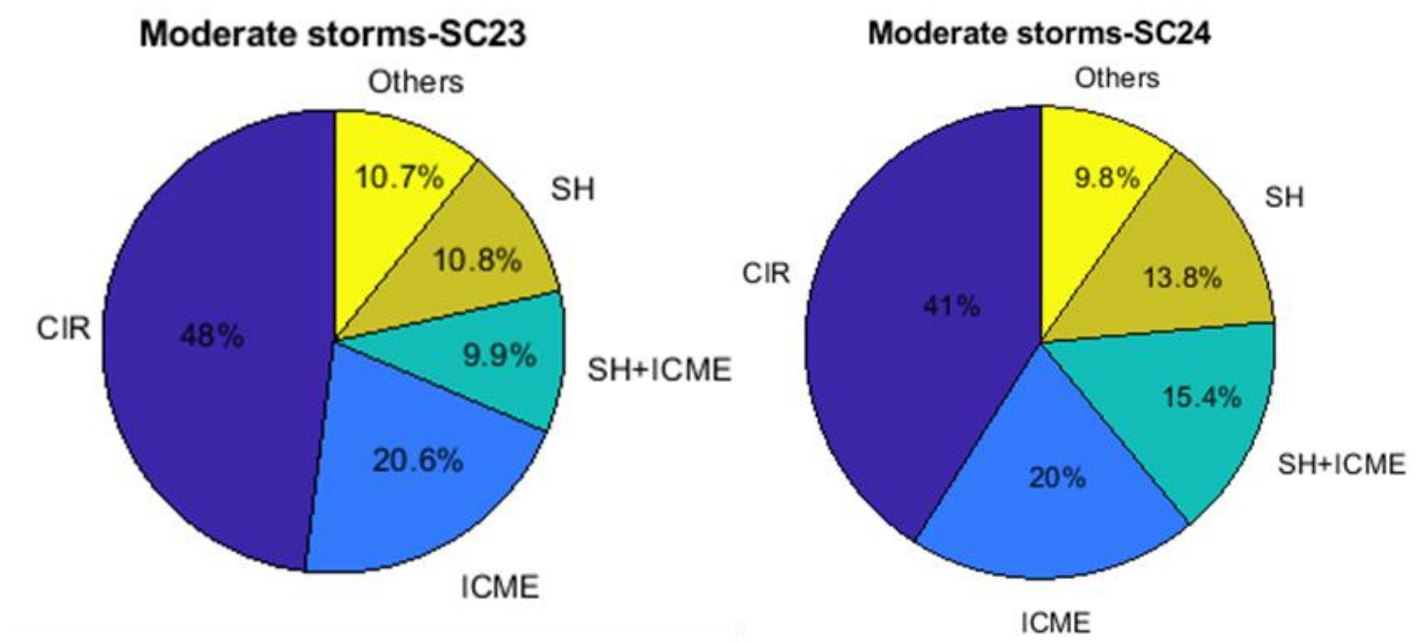

Figure 2. 2: Contribution of different interplanetary structures in producing moderate magnetic storms $(-100 n T<D s t<-50 n T)$ during solar cycle 23 and solar cycle 24 . SC23 consists of total 196 storms whereas SC24 consists of total 130 storms.

Both solar cycles have also been explored based on interplanetary structures causing intense geomagnetic storms (Dst $\leq-100 \mathrm{nT}$ ). Interplanetary structures based on Table 2.1 have been identified for intense geomagnetic storms both for solar cycles 23 and 24 and are shown as Figure 2.3. Solar cycle 23 consists of a total of 90 intense storms and solar cycle 24 consists of a total of 18 intense storms. It shows the contribution of ICME ( 38\%) is significant during solar cycle 23 
whereas during solar cycle 24 composite structure, $\mathrm{SH}+\mathrm{ICME}$ are related to $38.8 \%$ of such storms. The contribution of $\mathrm{SH}+\mathrm{ICME}$ increases substantially from $17 \%$ in solar cycle 23 to $38.8 \%$ during solar cycle 24. ICME driven geomagnetic storms include both $\mathrm{MC}$ and nonMC, of which $\mathrm{MC}$ are the major part of $\sim 24.4 \%$. The contribution of the remaining three interplanetary structures remains almost the same during both solar cycles. CIR contribution is minimum in both cycles with $13 \%$ and $11.1 \%$ during SC23 and SC 24 , respectively. SH driven storms also slightly change from $24 \%$ to $22.2 \%$ from SC23 to SC24. 'Others' can only be seen in SC 23 and include composite structures outside the considered four structures.
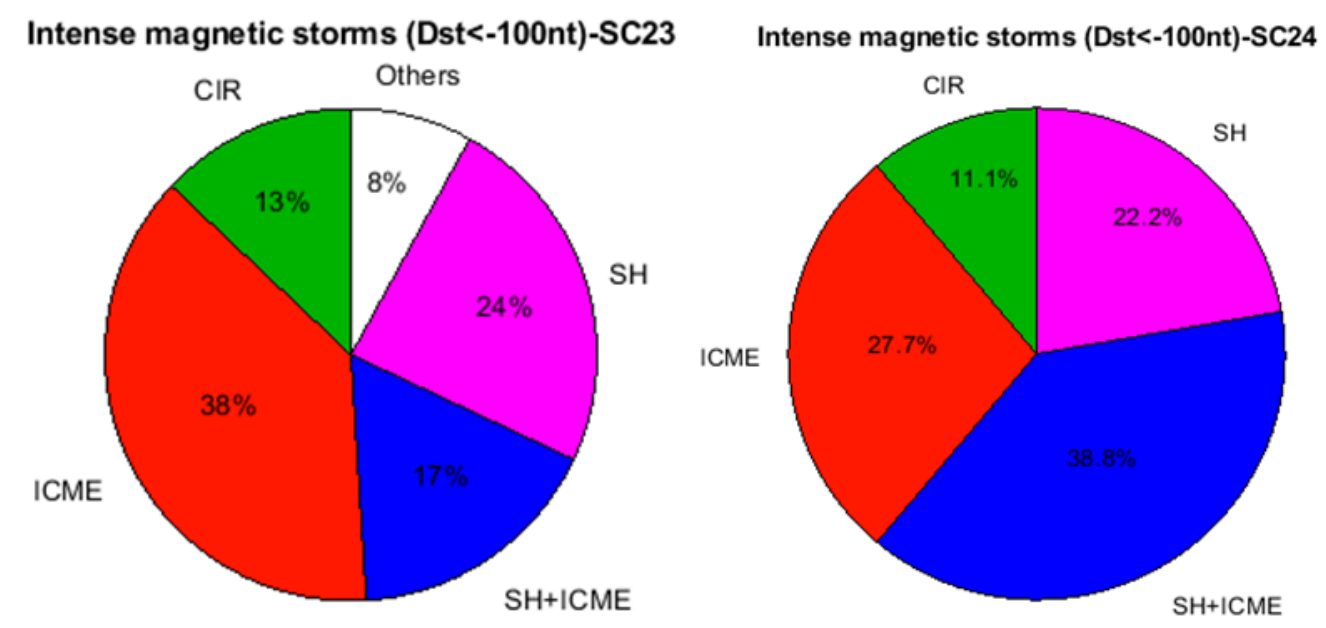

Figure 2. 3: Contribution of interplanetary structures which includes $C I R, I C M E, S H+I C M E$ and SHproducing intense geomagnetic storms (Dst $\leq-100 n T$ ) during SC23 and SC24. SC23 consists of total 90 storms whereas SC24 consists of total 18 storms.

So far, the contribution of interplanetary structures have been discussed during complete solar cycles. The contribution of each of these interplanetary structures has also been explored in terms of Islington GIC data (2002-2017) and is shown in Figure 2.4. During 2002-2006 a total of 43 intense geomagnetic storms have been identified, whereas during 2010-2016 there is a total of 18 intense geomagnetic storms. The same trend as seen during both solar cycles is seen i.e., during 2002-2005, ICME cause intense magnetic storms whereas during solar cycle 24 (2010 to 2016), SH+ICME produced intense magnetic storms. 


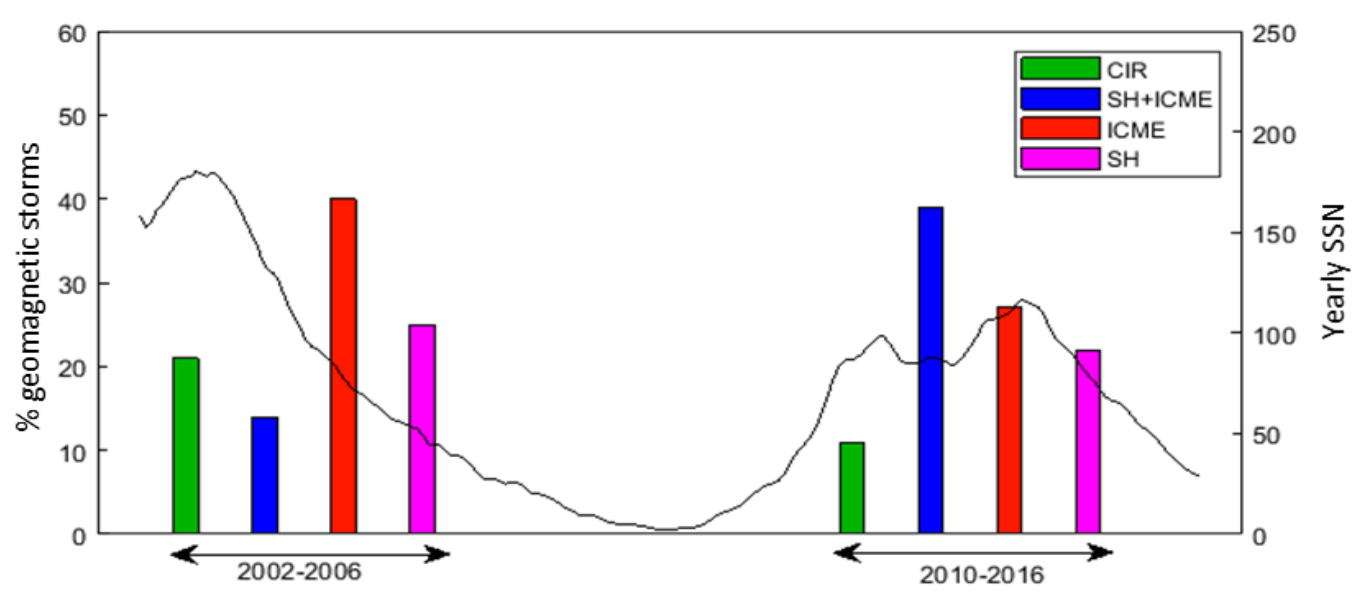

Figure 2. 4: Comparison of different interplanetary structures causing intense magnetic storms during different periods of solar cycles 23 and 24 based on available ISL GIC data. ICME dominates during 2002-2006 whereas SH+ICME dominates during 2010-2016.

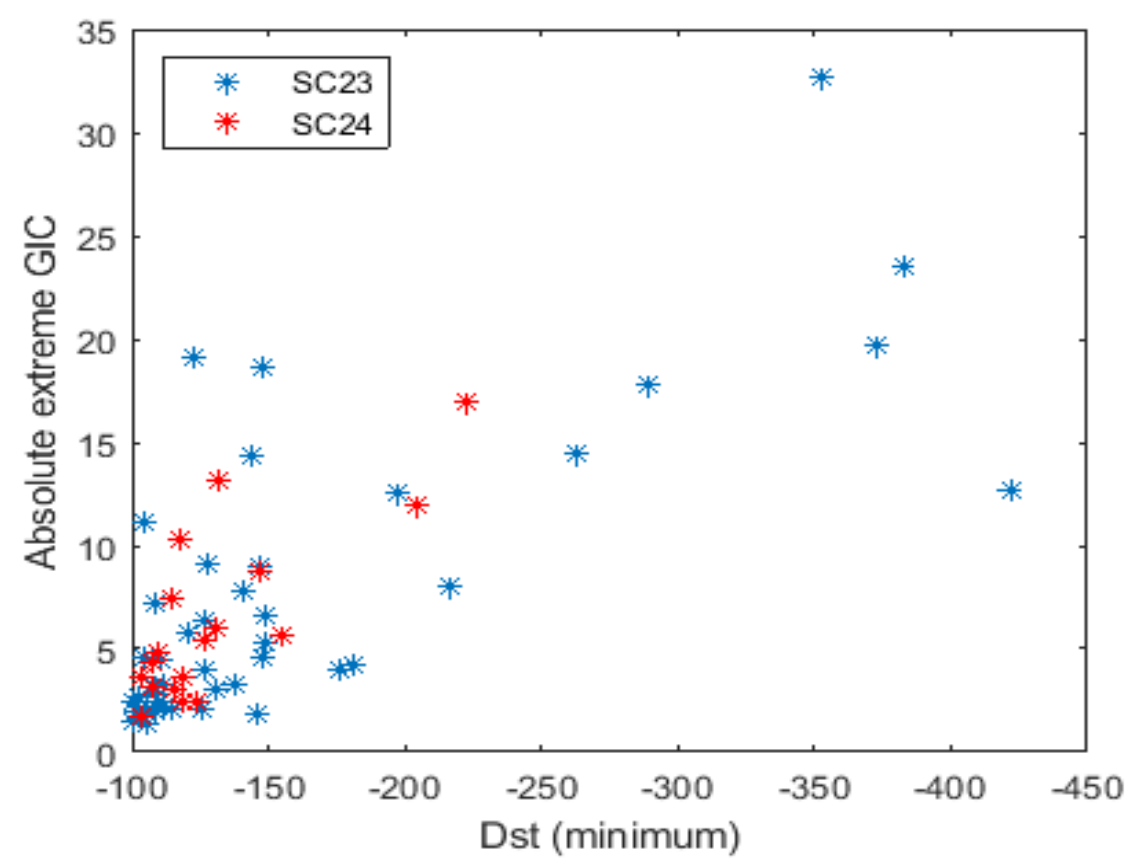

Figure 2. 5: ISL GIC extreme values and Dst-minimum during both solar cycles. SC23 consists of total 43 and SC24 consists of 18 intense geomagnetic storms.

To explore if this discrepancy of interplanetary structures has any contribution to observed GIC data at Islington, the GIC have been considered in correlation with the $D s t$ index for all intense geomagnetic storms. The extreme GIC value and the minimum Dst value have been taken into account for each geomagnetic storm. Figure 2.5 shows the relationship between Dst minimum 
and extreme GIC for all intense geomagnetic storms with the blue stars for solar cycle 23 (20022006) and the red stars for solar cycle 24 (2010-2016). Clearly GIC occur during storms with higher $D s t$, although there is a considerable spread particularly at low Dst. There are 6 points on the extreme right of Figure 2.5 which are due to the super storms of solar cycle 23 . There are no super storms for solar cycle 24. Although there is large scatter in this dataset and the number of solar storms is also different during the two solar cycles, this still shows a similar kind of the impact as ICME and SH+ICME on the measured ISL GIC data. The disparity seen in ISL GIC data cannot be directly associated with the considered interplanetary structures.

Apart from the geoeffectiveness of both ICME and SH+ICME as seen above in relation to Islington GIC, ICME and SH+ICME share a lot of common features in terms of solar wind parameters. As the name suggests $\mathrm{SH}+\mathrm{ICME}$ contain an ICME structure with the addition of a sheath (SH) region. Two intense geomagnetic storms of almost the same Dst index have been selected, one geomagnetic storm caused by ICME on 18 August 2003 of Dst $\sim-148$ nT, and the other geomagnetic storm caused by SH+ICME on 20 December 2015 of Dst -155 nT. Figure 2.6 shows a 3-day comparison of solar wind parameters and ISL GIC for the first of these storms, Figure 2.7 shows the same for the second storm. In Figure 2.6, Although the main phase of the magnetic storm is on 18 August 2003, to include the solar wind conditions before the storm and in the recovery phase, data from 17-19 August 2003 are shown. One-minute solar wind data is downloaded from OMNI database (https://omniweb.gsfc.nasa.gov/). The first six panels show solar wind parameters which include the interplanetary magnetic field, $B z$, the solar wind speed, the proton density, the temperature and the flow pressure. The bottom panel shows Islington GIC data. The shock $(s)$ can be by an abrupt increase in magnitude of all solar wind parameters before the arrival of the ICME and is shown as the dotted vertical line. GIC showed a significant response of 1.4 A on the arrival of the shock. The ICME is of MC type as can be seen by the long lasting and smooth rotation of $\mathrm{Bz}$ shown by the double arrow in the Figure. During the main phase of the magnetic storm large fluctuations can be seen in GIC when $B z$ has a large magnitude southward. The maximum GIC reaches $4.5 \mathrm{~A}$ during the main phase of the magnetic storm. 

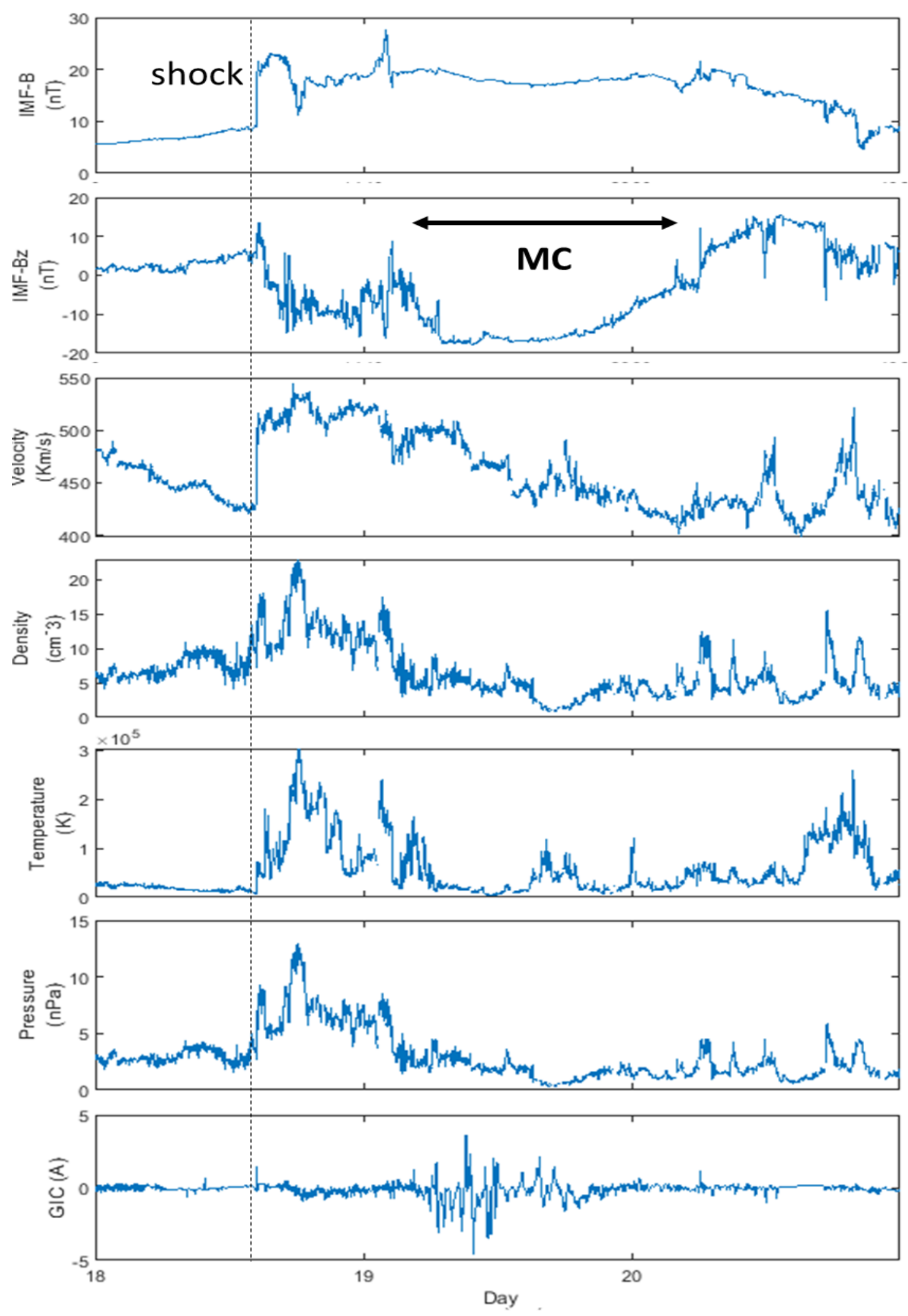

Figure 2. 6: Top six panels show solar wind one-minute data where the interplanetary structure is shock followed by a magnetic cloud, and the bottom panel shows one-minute interpolated measured GIC of Islington during magnetic storm of 18 August 2003. 

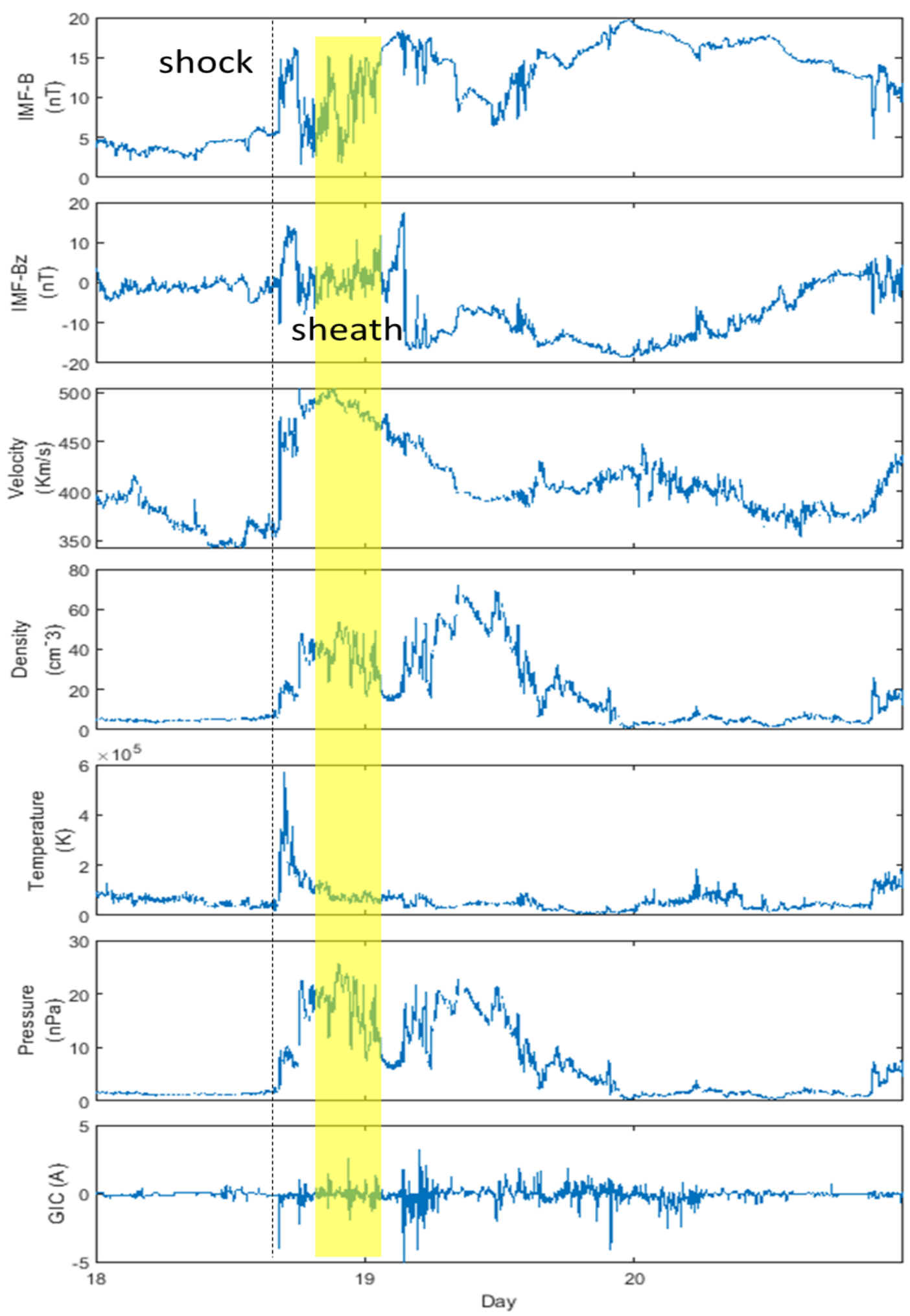

Figure 2. 7: Top six panels show solar wind one-minute data where the shock is followed by sheath and magnetic cloud regions, and bottom panel shows one-minute interpolated measured GIC of Islington during 20 December 2015 storm. 
Figure 2.7 shows the same comparison of solar wind parameters and GIC for the second storm (SH+ICME) on 20 December 2015. Shock compression intensified the IMF-B and after the shock the sheath region (yellow area) can be identified by a fluctuating $B z$ component and subsequent progress of the magnetic storm. The effect of the shock is significant as the GIC is 4 A. The ICME is nonMC therefore after the sheath region no smooth rotation in $B z$ component is seen. Both SH and ICME caused the main storm which is why it is classified as SH+ICME. The main phase of the magnetic storm caused large GIC fluctuations which reached to an extreme GIC of $\sim 5 \mathrm{~A}$. Thus, ICME and SH+ICME produced almost the same magnitude of GIC variations, and both are geoeffective. As analysis is limited to just these two magnetic storms it should not be extended more generally, but for these storms it shows, based on Islington GIC data, that the geoeffectiveness does not vary and is independent of the dominating interplanetary structures during both solar cycles.

\subsection{Calculation of Geoelectric Fields}

GIC are produced in response to the horizontal geoelectric field components $(\boldsymbol{E} \boldsymbol{x}, \boldsymbol{E} \boldsymbol{y})$, where $\boldsymbol{E} \boldsymbol{x}$ is in the geographic north direction and $\boldsymbol{E} \boldsymbol{y}$ in the east direction. $\boldsymbol{E z}$ is vertically downward and as it is perpendicular to power lines is not included in GIC modelling. To calculate GIC it is necessary to model electric fields produced on the surface of Earth in response to a geomagnetic storm. With a knowledge of the geoelectric fields, the GIC in a network can be determined using the geoelectric fields as an electromotive force $(e m f)$ source in power lines (Boteler and Pirjola, 2017). During geomagnetic storms, the compression of the magnetosphere by the solar wind, and the interaction of the solar wind with the Earth's geomagnetic field enhances the currents in both the magnetosphere and in the ionosphere (Bogdan, 2007) which are the principal cause of geoelectric fields. Along with these source fields the conductivity structure of the Earth is an important factor. Thus, knowledge of space currents and the Earth's conductivity structure permits the determination of the electric and magnetic fields at the Earth's surface by using Maxwell's equations and suitable boundary conditions (Pirjola and Viljanen, 1998). Initial work on determination of geoelectric fields from source currents was presented by Häkkinen and Pirjola (1986). Major systems of currents include the auroral electrojet and field aligned currents (FAC), which ave effectively been modelled and used in GIC studies. The modelling of the full three-dimensional system of ionospheric currents is not presently possible (Viljanen et al., 2004). 
Another commonly used approach for geoelectric field determination is using the Faraday's law and recorded magnetic field data from geomagnetic observatories. The geoelectric field is induced by the temporal variation of the magnetic field during a geomagnetic disturbance or storm. The following sections describe techniques of calculating geoelectric fields using both methods.

\subsubsection{Calculation of Electric and Magnetic Fields Using lonospheric Source Currents}

That a current carrying conductor deflects a nearby magnet was first observed experimentally by Hans Christian Oersted in 1820 and was quantified by Ampere. In 1831 Faraday observed that moving a magnet through a coil produces an electric current, but it was not until 1861 that James Clerk Maxwell proved explicitly, using his equations, that electric and magnetic fields are a manifestation of the same force. Maxwell's equations are fundamental in explaining the production of GIC which are caused by a system of currents in the ionosphere and magnetosphere. The ionospheric and magnetospheric field variations are superposed on the main geomagnetic field of the Earth. The highly conductive ionosphere and the conductive Earth can be considered as a large capacitor separated by the resistive atmosphere. The electric conductivity of the ground surface is of order $10^{-3} \mathrm{Sm}^{-1}$ but it generally increases with depth inside the Earth. Similarly, the atmosphere near the Earth's surface has poor conductivity of about $10^{-14} \mathrm{Sm}^{-1}$ but at an altitude of $100 \mathrm{~km}$, the start of the ionospheric region, the conductivity is about the same as that of the solid Earth. This explains how the electric environment of the upper atmosphere is coupled with the Earth inducing electric fields and telluric currents within the Earth (Constable, 2015).

GIC can be viewed as a result of the electromagnetic coupling between space currents and the conductors of the particular technological network at the Earth's surface as shown in Figure 2.8. The red arrow shows an eastward moving auroral electrojet which is the primary source of a time varying magnetic field in the Earth. Additionally, there is always a secondary contribution to the magnetic field from currents in the Earth (orange arrow) which are affected by the Earth's conductivity structure. The magnetic field can be calculated accurately enough by taking only the primary sources into account, that is, ignoring the induction in the Earth. However, the Earth's effect on the electric field can never be neglected. The space and Earth contributions to the 
horizontal electric field are nearly the same but opposite in sign making the total horizontal field very small in comparison to the two individual parts, whereas for the vertical electric field component, the two contributions are also practically equal but have the same sign (Hakkinen and Pirjola, 1986; Pirjola, 2002).

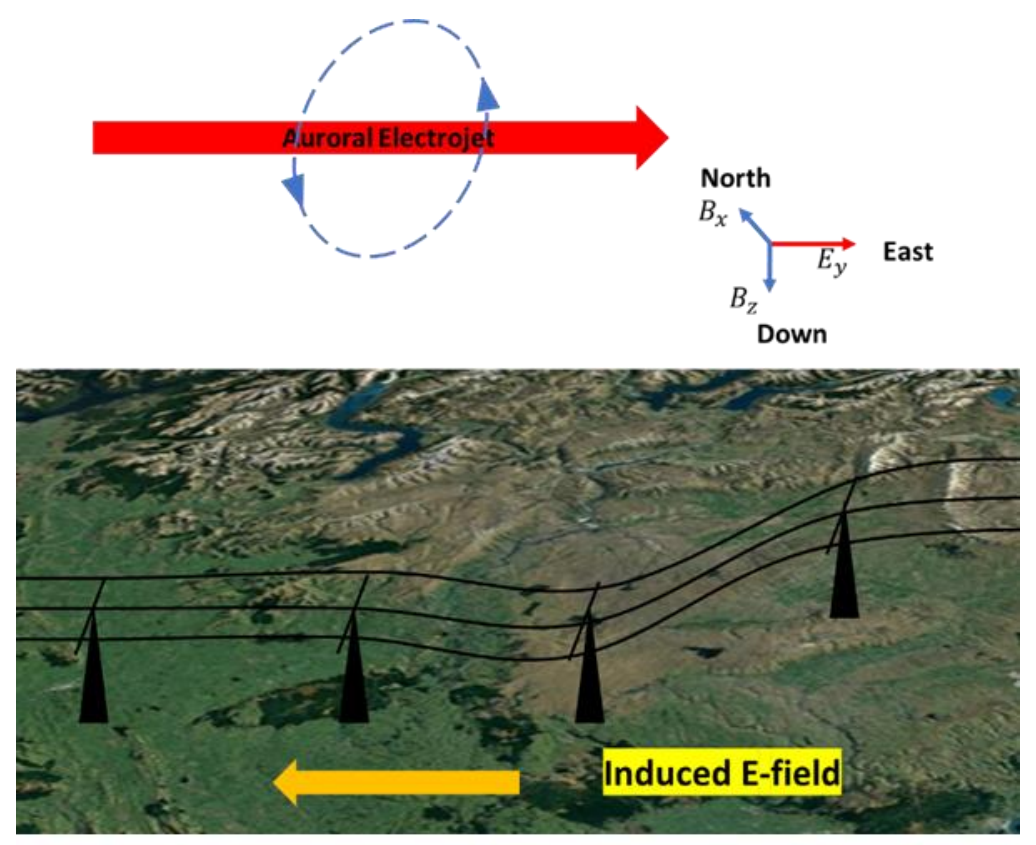

Figure 2. 8: Schematic diagram (not on scale) showing how geoelectric fields are produced due an electrojet. The electrojet is taken as a line current in the direction of electric field, and corresponding horizontal and downward magnetic field components. The geoelectric field is the sum of both source fields and fields associated with telluric currents.

The exact determination of the electromagnetic field based on Maxwell's equations at the surface of a layered Earth, using a general three-dimensional auroral current model was first presented by Hakkinen and Pirjola (1986). It is obtained by first determining the primary field (due to the electrojet) then solving Maxwell's equations in the air and the layered Earth with suitable boundary conditions. The total fields at the Earth's surface are the sum of the fields due to the external source plus the fields due to the currents induced in the Earth. However, the resulting formulae are complicated, and there is another, simpler, complex image method (CIM) which can equivalently be used for a current system not only consisting of an infinite length electrojet but is also successful for field aligned currents (FAC), a main process by which solar energy from the magnetosphere is transported into the ionosphere. The CIM in connection with 
GIC studies is very promising in this respect and gives the same results as using the exact equations (Pirjola and Viljanen, 1998; Boteler and Pirjola, 1998b).

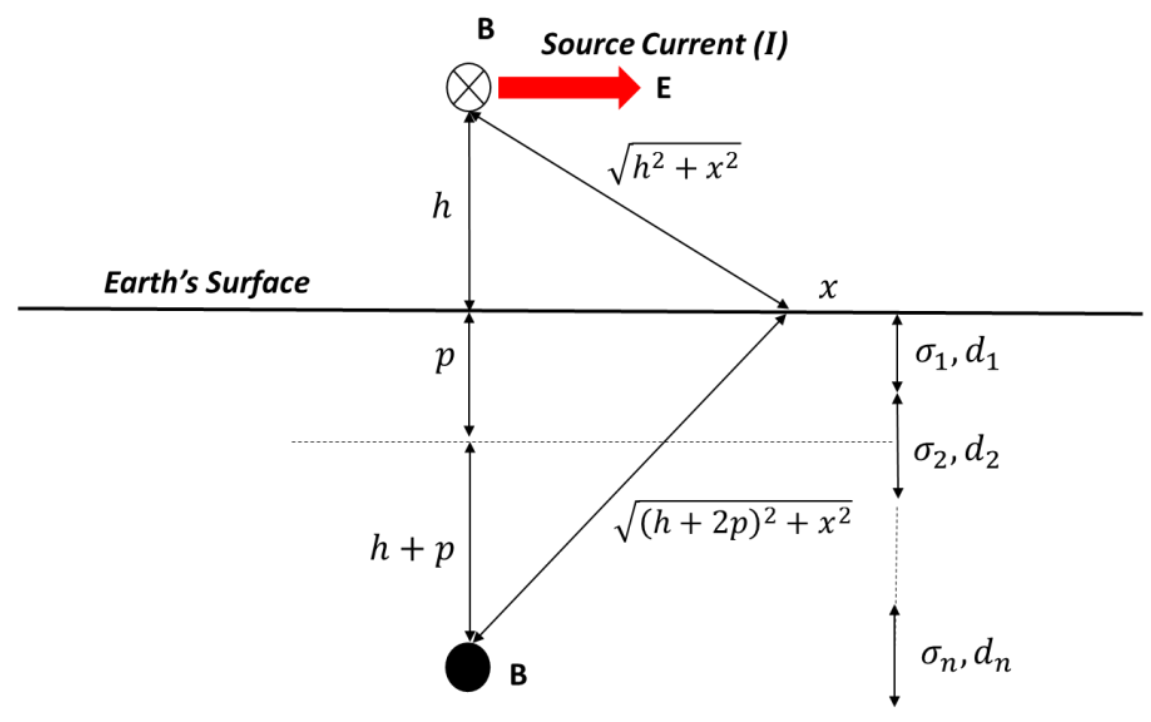

Figure 2. 9: Complex image method showing the source current above the surface of Earth and the image current at complex depth of $h+2 p$. The geoelectric field is sum of fields produced by both external (source) and image (internal/induced) currents.

Suppose an auroral electrojet of current $(I)$ is at height $h$ from the surface and we want to determine the electromagnetic field at a horizontal distance $x$ as shown in Figure 2.9 which also shows a layered conductivity Earth model. In the complex image method the internal (induced) fields are approximated by the fields due to an image current at a complex depth. Therefore, the complex skin depth, $p$, of wave penetrating the Earth can be represented as a reflecting surface so that the image current, opposite in direction, is the same distance below this level as the source current is above, as shown in the Figure. The simplest approximation to represent an idealized electrojet is an infinite long line current. The assertion of CIM is that the influence of the induced currents can be well represented by an image current at a complex depth $h+2 p$, where $p$ is the complex skin depth in the Earth (Boteler and Pirjola, 1998b). The magnetic and electric fields are then given by the sum of source and image currents, and their distances from the location on the surface. The magnetic fields due to both (source and image currents) can simply be calculated using Ampere's current Law and adding them. Since we are calculating fields at distance $x$ the distance from the sources would be $\sqrt{h^{2}+x^{2}}$ and $\sqrt{(h+2 p)^{2}+x^{2}}$. The electric 
field can be calculated using Faraday's Law by using the magnetic field as computed from Ampere's Law.

The CIM can further be extended to encompass a more realistic auroral electrojet by including the width of the electrojet. The auroral electrojet typically extends over $\sim 5^{\circ}$ of latitude and may be composed of several current filaments. A further approximation to include the width of the electrojet is that the electrojet current profile can be expressed as a Cauchy distribution. Kertz (1954) showed that magnetic fields at the Earth's surface due to a Cauchy distribution are equivalent to those due to a line current at a greater height. The Cauchy distribution looks like a normal distribution but has tails which do not vanish so quickly. This is a reasonable first approximation to the auroral electrojet which is known to be the cause of the major magnetic disturbances that occur at higher latitudes (Pirjola and Viljanen, 1998). It has been shown that the fields produced by this current profile with a half-width $a$ at a height $h$ are the same as the fields produced by a line current at a height $h+a$ (Boteler and Pirjola, 1998b). Therefore, in CIM the height of the source can be replaced with $h+a$, to include the width information of the electrojet. Using the above-mentioned method and approximations the electric and magnetic fields on the surface of Earth are therefore given by

$$
\begin{gathered}
E_{y}=-\frac{i \omega \mu_{o} I}{2 \pi} \ln \left(\frac{\sqrt{(h+a+2 p)^{2}+x^{2}}}{\sqrt{(h+a)^{2}+x^{2}}}\right) \\
B_{x}=\frac{\mu_{o} I}{2 \pi}\left(\frac{h+a}{(h+a)^{2}+x^{2}}+\frac{h+a+2 p}{(h+a+2 p)^{2}+x^{2}}\right) \\
B_{z}=-\frac{\mu_{O} I}{2 \pi}\left(\frac{x}{(h+a)^{2}+x^{2}}+\frac{x}{(h+a+2 p)^{2}+x^{2}}\right)
\end{gathered}
$$

where $x$ is the horizontal distance from the location of the electrojet. The electric field produced at the Earth's surface will have maximum amplitude directly underneath the current and the amplitude will decrease with increasing distance on either side. For a given frequency $(\omega)$ the complex skin depth, $p$, is related to the surface impedance $(Z)$ which can be calculated using a recursive relationship for any specified layered conductivity structure. $p$ is given by

$$
p=Z / i \omega \mu_{o}
$$


Therefore, for a given electrojet current $I$ of half width $a$ at height $h$, and complex skin depth $p$, the electromagnetic fields on the Earth's surface can be calculated using 2.1-2.3. To demonstrate CIM it has been applied to Earth conductivity models for Invercargill (INV) and Halfway Bush (HWB) two locations in the South Island. Simple resistivity models both for INV and HWB, based on 3-D impedance tensors, are shown in Figure 2.10. These are derived using a Bostick inversion (Goldberg and Rotstein, 1982) from the determinant impedance at each frequency. These simple models have been used to approximate layered earth models from which the surface impedance can be calculated and used to calculate the complex skin-depth using equation 2.4 .
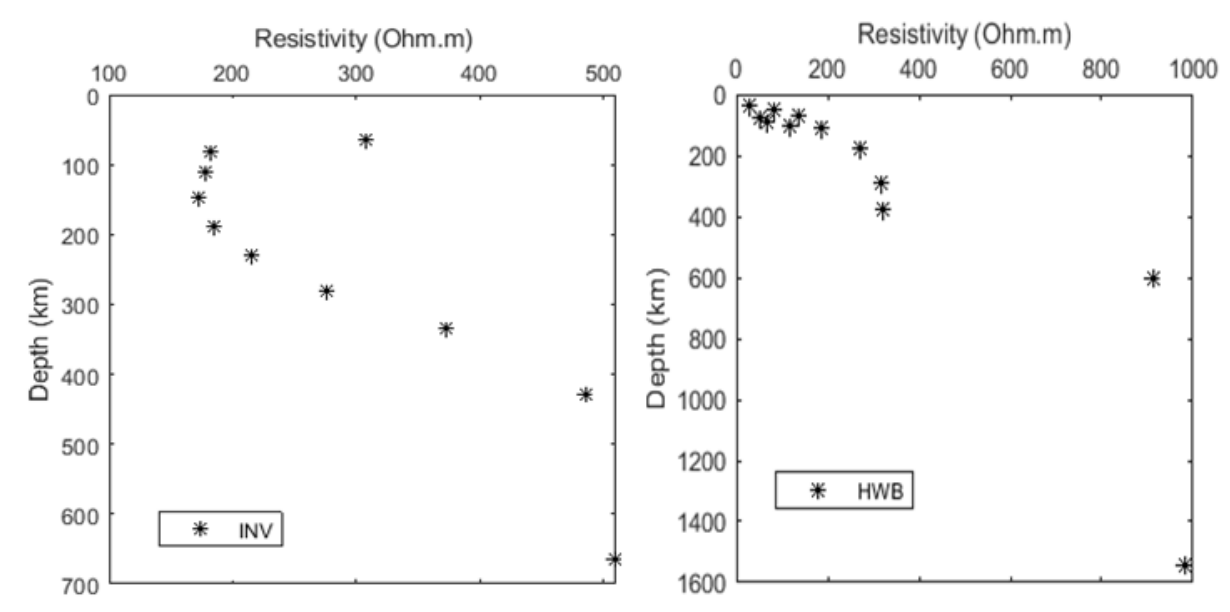

Figure 2. 10: Conductivity-depth model obtained for Invercargill and Halfway Bush using the 3-D impedance tensor in the period range of 2-332 minutes.

As an example, assume an aurora electrojet of line current 1 million A, varying with a period of 5 minutes, $100 \mathrm{~km}$ above the Earth, with current density half width $a=200 \mathrm{~km}$. The electric and magnetic fields calculated for Invercargill and Halfway Bush and are shown in Figure 2.11 and Figure 2.12, respectively. The location of the electrojet is at 0 on the $x$-axis and the values of electric and magnetic fields vary on either side of the electrojet.

The real and imaginary parts of both $\boldsymbol{E} \boldsymbol{y}$ and $\boldsymbol{B}$ fields have been shown separately. The contribution of internal and external fields has also been shown where possible. The magnetic field north component $(\boldsymbol{B} \boldsymbol{x})$ is significantly larger than the downward component $(\boldsymbol{B z})$. The magnetic field is similar for both INV and HWB, but the values of electric fields differ substantially due to the different conductivity structures at INV and HWB. INV geoelectric fields have a value of $\boldsymbol{E} \boldsymbol{y} \sim 700-2900 i$ and HWB has a value $\boldsymbol{E} \boldsymbol{y} \sim 900-1200 i \mathrm{mV} / \mathrm{km}$ at a horizontal distance of zero 
from the electrojet. This shows how the conductivity structure of the Earth can significantly impact the determination of geoelectric fields.
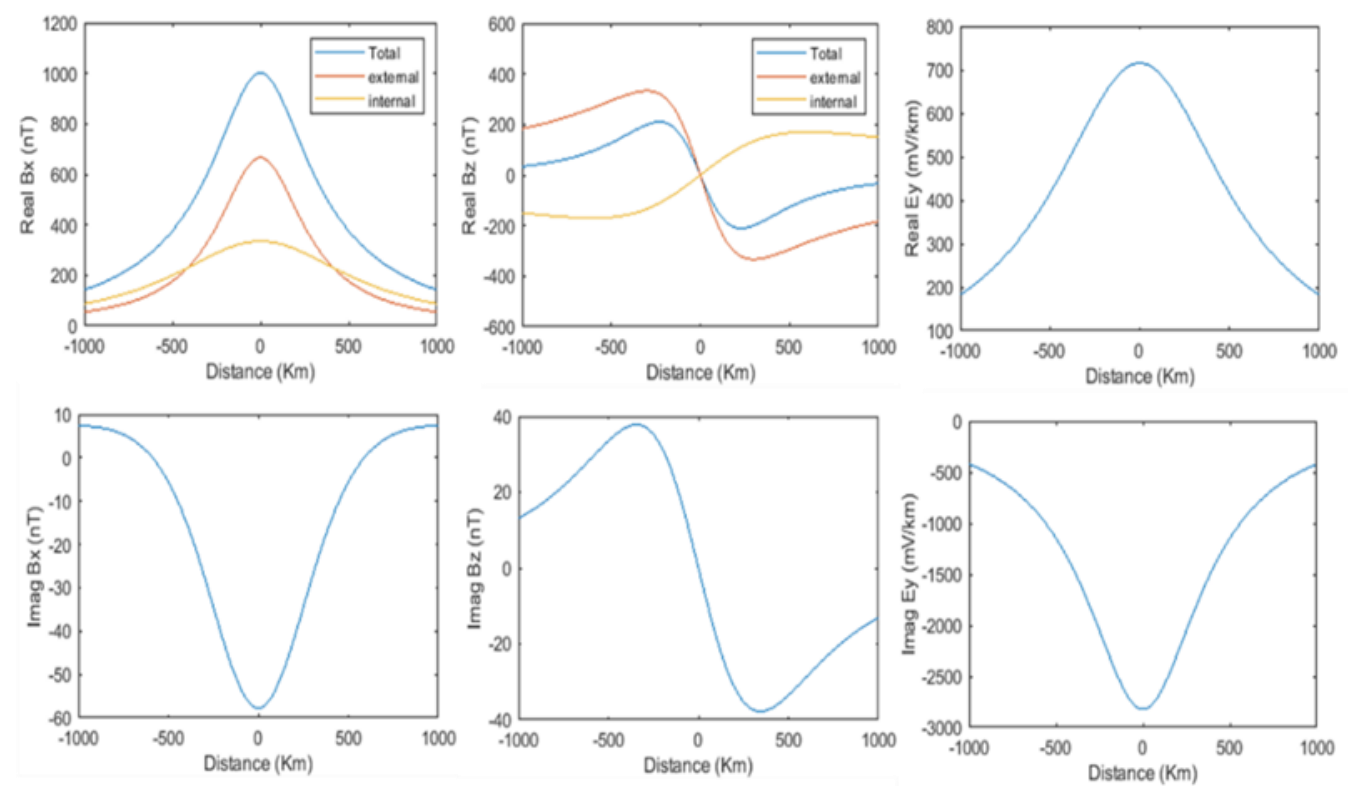

Figure 2. 11: Magnetic field $(\boldsymbol{B} \boldsymbol{x}, \boldsymbol{B z})$ and electric field $(\boldsymbol{E} \boldsymbol{y})$ in response to an auroral electrojet calculated by CIM using the conductivity model of Invercargill.
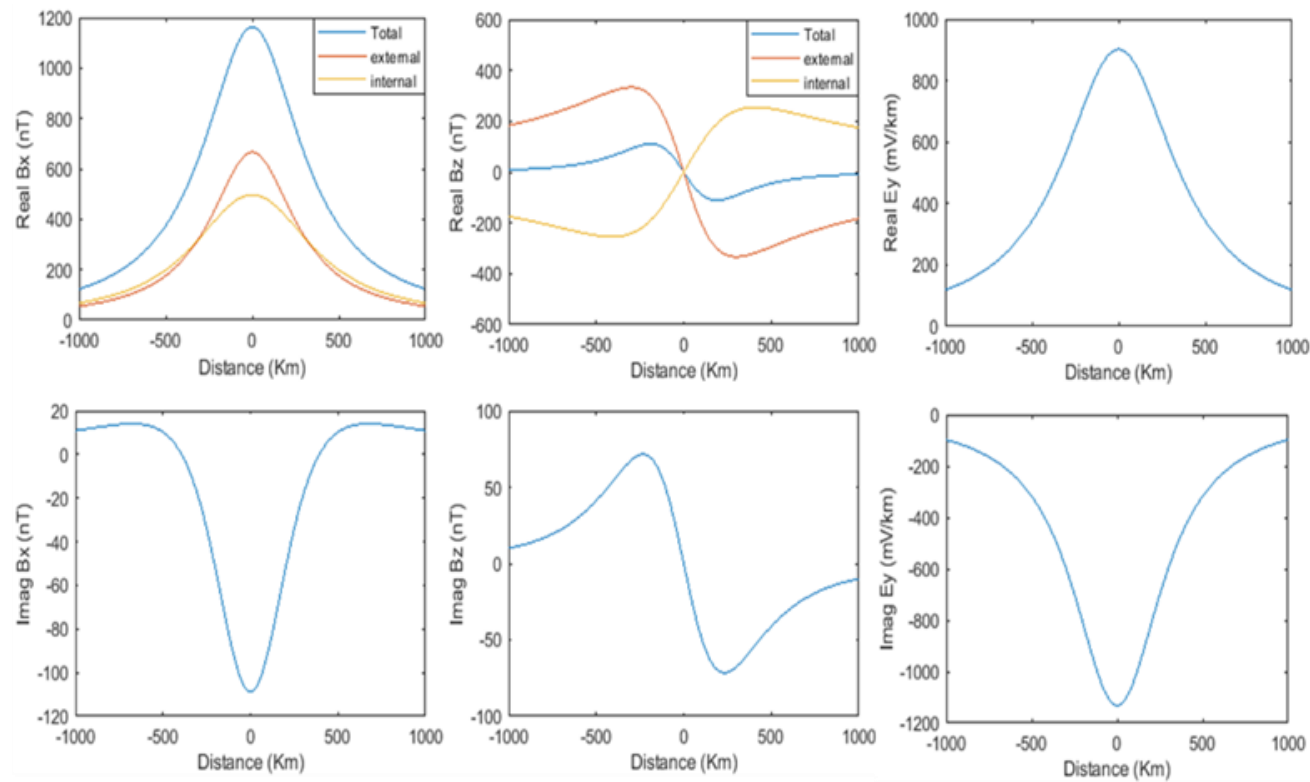

Figure 2. 12: Magnetic field $(\boldsymbol{B} \boldsymbol{x}, \boldsymbol{B z})$ and electric field $(\boldsymbol{E} \boldsymbol{y})$ in response to an auroral electrojet calculated by CIM using the conductivity model of Halfway Bush. 


\subsubsection{Calculation of Geoelectric Field Using Ground Magnetic Data}

Surface magnetic field measurements using the data from magnetic observatories can also be used for computation of geoelectric fields. The time derivative of the ground magnetic field $(\mathrm{dB} / \mathrm{dt})$ is also a reasonable proxy for GIC activity (Viljanen et al., 2001). Faraday's Law gives a simplistic approach to computing electric fields by the selection of a suitable loop around the power line as shown in Figure 2.13. As a loop enclosing only the power line and the surface of earth ( $\left.a b c^{\prime} d^{\prime}\right)$ does not result in sufficient emf to drive GIC, the loop has to be extended down to infinity where $B$ becomes zero (abcd) as shown in the Figure. This also implies that the electric field in the transmission line has the same magnitude and direction as the electric field along the Earth's surface (Boteler and Pirjola, 2017).

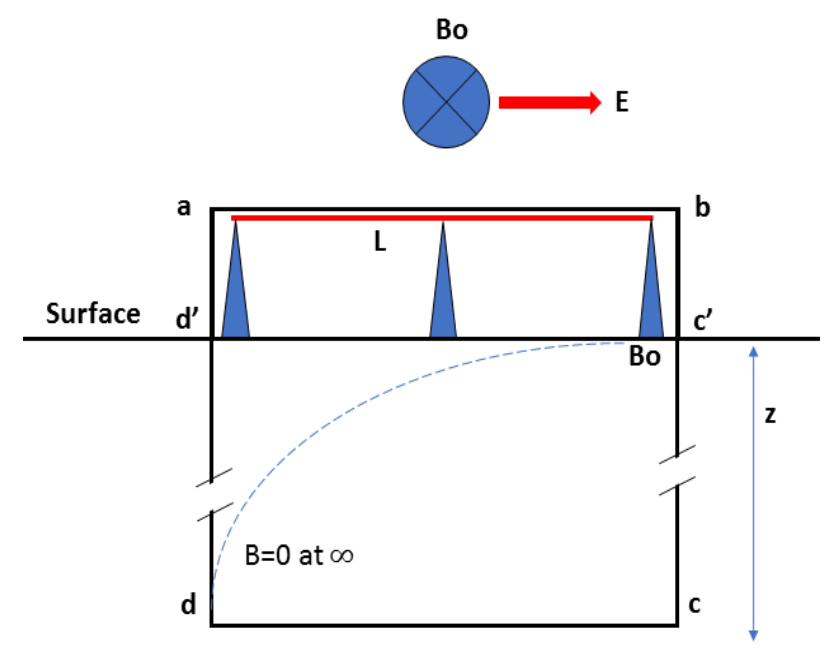

Figure 2. 13: The consideration of correct loop that goes down to infinity at $B=0$ is necessary to accurately determine geoelectric fields as expressed by Faraday's Law of induction (adopted from Boteler and Pirjola, 2017).

Referring to Figure 2.13 , the magnetic flux $(\varnothing)$ through the loop abcd can be found by using the line integral around the loop for depth $\mathrm{z}$ from 0 to infinity and multiplying by the line length $L$

$$
\emptyset=\int_{0}^{\infty} \boldsymbol{B} d z L
$$

Since the amplitude of magnetic field decreases exponentially depending on value of complex skin depth $(p)$, using $B=B_{0} e^{-z / p}$, and the definition of Faraday's Law 


$$
\begin{gathered}
\oint \boldsymbol{E} . \boldsymbol{d} \boldsymbol{l}=-d \emptyset / d t=-i \omega p \boldsymbol{B}_{\boldsymbol{o}} L \\
\boldsymbol{E}_{\mathbf{0}}=-i \omega p \boldsymbol{B}_{\mathbf{0}}
\end{gathered}
$$

where $\omega$ is angular frequency of the wave corresponding to complex skin depth $p$. $B_{0}$ is the magnitude of the magnetic field at the surface of the Earth. It is of interest to see if the same electric field results when using Maxwell's equations. Under the normal assumptions of geoelectromagnetics $\left(\sigma \gg \omega \varepsilon_{0}\right)$ the displacement current term can be ignored, and the magnetic permeability is taken as that of free space $\mu_{0}$. For a single frequency wave $\left(e^{i \omega t}\right)$ Maxwell's equations reduce to

$$
\nabla \times \boldsymbol{E}=-i \omega \boldsymbol{B}
$$

$$
\nabla \times \boldsymbol{B}=\mu_{0} \sigma \boldsymbol{E}
$$

Taking the Curl of the first equation, using the standard expression for Curl of the Curl of a vector, and then substituting into the second equation gives the diffusion equation.

$$
\begin{aligned}
& \nabla^{2} \boldsymbol{E}=i \omega \mu_{0} \sigma \boldsymbol{E} \\
& \nabla^{2} \boldsymbol{B}=i \omega \mu_{0} \sigma \boldsymbol{B}
\end{aligned}
$$

If we further consider that fields are horizontally polarized in the $x y$ directions and are propagating vertically $(z)$, in Cartesian coordinates the above equations can be simplified into

$$
\begin{aligned}
& \frac{\partial^{2} \boldsymbol{E}}{\partial z^{2}}+k^{2} \boldsymbol{E}=0 \\
& \frac{\partial^{2} \boldsymbol{B}}{\partial z^{2}}+k^{2} \boldsymbol{B}=0
\end{aligned}
$$

where $k^{2}=-i \omega \mu_{0} \sigma$ and the solution of these equations can be written as

$$
\begin{aligned}
& \boldsymbol{E}=\boldsymbol{E}_{\mathbf{0}} e^{-i k z} \\
& \boldsymbol{B}=\boldsymbol{B}_{\mathbf{0}} e^{-i k z}
\end{aligned}
$$


Since the $\boldsymbol{E}$ fields derived from Faraday's Law are expressed in terms of complex skin depth the above equations can equivalently be expressed as

$$
\begin{aligned}
& \boldsymbol{E}=\boldsymbol{E}_{\mathbf{0}} e^{-z / p} \\
& \boldsymbol{B}=\boldsymbol{B}_{\mathbf{0}} e^{-z / p}
\end{aligned}
$$

This shows the amplitude of the downward propagating wave decreases exponentially depending upon the value of complex skin depth $p$. The spatial variation of $\boldsymbol{E}$ is only along the $z$ direction therefore the curl of equation 2.6 can easily be solved by substituting $\boldsymbol{E}$ from (2.7) giving

$$
\boldsymbol{E}_{\mathbf{0}}=-i \omega p \boldsymbol{B}_{\mathbf{0}}
$$

showing that the diffusion equation and the Faraday's Law give the same result when considering a suitable loop around the power line.

Magnetic observatories have been installed worldwide for continuous acquisition of magnetic field data, and this can effectively be utilized for determination of electric fields. The simplest relation between surface electric and magnetic fields is obtained by making the plane wave assumption, which means that the primary electromagnetic field originating from an external current source is a vertically-downwards propagating plane wave. Assuming further that the Earth has a layered structure and operating in the frequency domain, the electric field is obtained by multiplying the magnetic field by the surface impedance. Thus, the plane wave method provides a good tool for the calculation of the geoelectric field if magnetic data are available (Cagniard, 1953; Wait, 1980).

GIC are usually considered in systems located over a limited area therefore a regional flat-Earth model is useful having a standard coordinate system of $x$ (North), $y$ (East) at the Earth's surface and $z$-axis pointing downwards. In the case of a simplistic approach of uniform conductivity of the Earth the geoelectric fields in the frequency domain are given by the following (Pirjola, 2002) 


$$
\begin{gathered}
\boldsymbol{E}_{\boldsymbol{x}}(f)=\sqrt{\frac{\omega}{\mu_{0} \sigma}} e^{i \pi / 4} \boldsymbol{B}_{\boldsymbol{y}}(\omega) \\
\boldsymbol{E}_{\boldsymbol{y}}(w)=-\sqrt{\frac{\omega}{\mu_{0} \sigma}} e^{i \pi / 4} \boldsymbol{B}_{\boldsymbol{x}}(\omega)
\end{gathered}
$$

This shows that the geoelectric fields $\left(\boldsymbol{E}_{\boldsymbol{x}}, \boldsymbol{E}_{\boldsymbol{y}}\right)$ are inversely proportional to the square root of the conductivity $(\sigma)$ and proportional to the square root of the frequency (inversely proportional to the square root of the period T). Also, the time-varying sinusoidal amplitude of the geoelectric field lags that of the geomagnetic field by a phase of $\pi / 4$. Plane wave calculations can be made using time series data from magnetic observatory recordings. If magnetic data are only available from one observatory in the area of a particular power system, then using this data for calculations across the whole power system involves the implicit assumption that the magnetic field variations are spatially uniform across the power system. If data are available from two magnetic observatories, at opposite ends of a power system, then the magnetic field variations at sites across the power system can be approximated by linear interpolation from the observatory recordings (Boteler, 2014). The following two sections describe determination of geoelectric fields by two different methods using ground magnetic field data.

\subsubsection{Calculation of Geoelectric Field Using a Thin-sheet Conductance Model}

Magnetotelluric (MT) measurements of geomagnetic and geoelectric field variations obtained from temporary deployments of sensors at individual geographic locations (Ferguson, 2012), are expressed as empirical impedance tensors (Chave, 2012). These impedance tensors can be inverted to obtain models of Earth conductivity to determine geoelectric fields. Many studies have employed the numerical thin-sheet modeling technique of Vasseur and Weidelt (1977) to calculate geoelectric fields across the extent of a power network. In this technique spatial variations in the ground electrical conductivity are embodied in a two-dimensional thin sheet with laterally varying conductance. The thin-sheet (TS) approach has been used by McKay (2003) and Beggan et al. (2013) to study GIC in the United Kingdom and by Kelly et al. (2017) and Bailey et al. $(2017,2018)$ in continental Europe. A thin-sheet conductance model has also been 
extensively used for GIC modelling in the South Island of New Zealand (Divett et al., 2017, 2018, 2020).

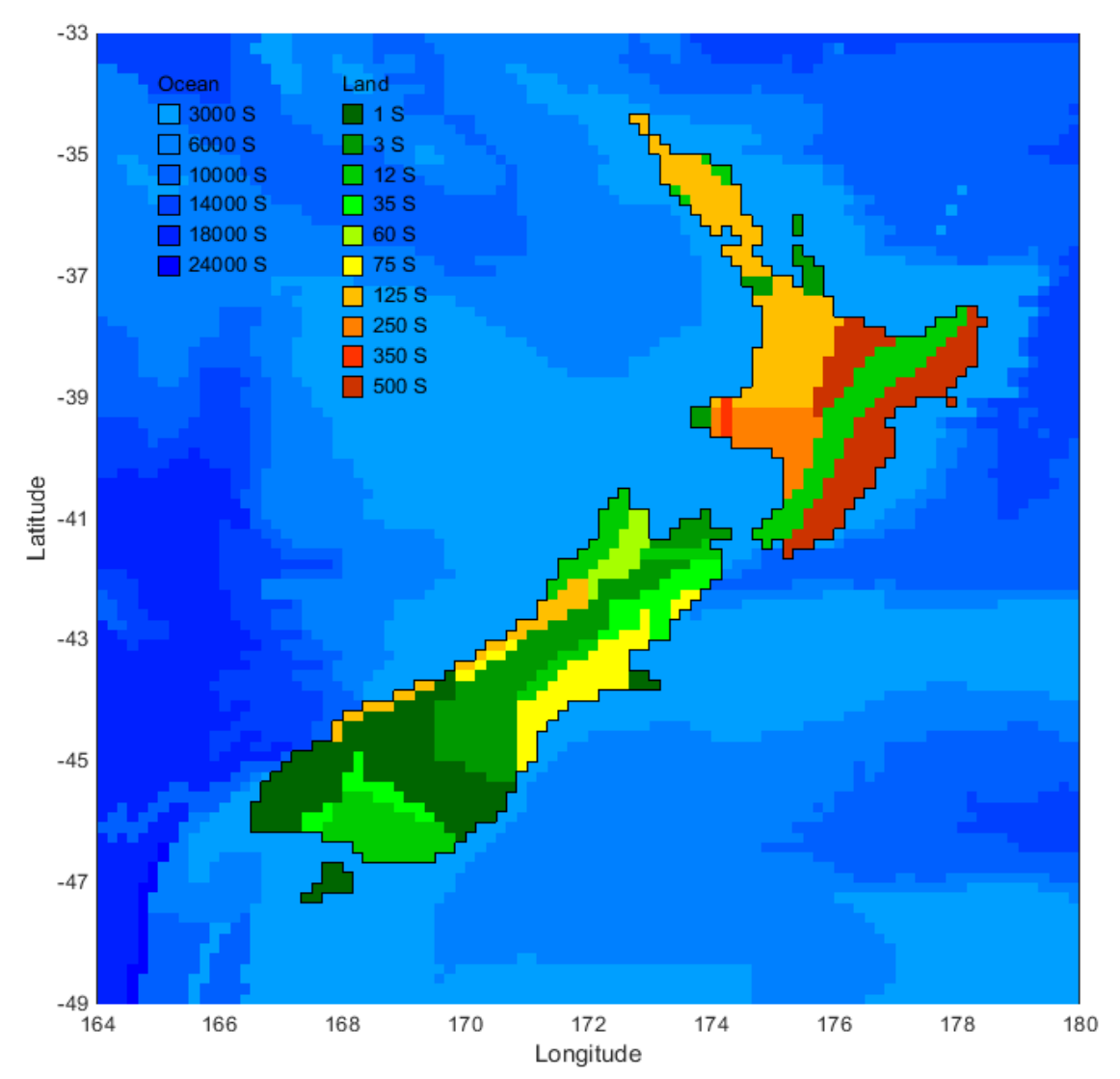

Figure 2. 14: The conductance structure of the New Zealand using thin-sheet conductance model (adopted from Divett et al., 2017).

Figure 2.14 shows the thin-sheet conductance model of New Zealand used by Divett et al. (2017). The conductance values on both for North and South Island range from 1 to $500 \mathrm{~S}$. Different colors show the corresponding values of conductance in different regions. The South Island has lower conductance (generally larger resistivity) compared to the North Island.

The thin sheet modeling technique of Vasseur and Weidelt (1977) was originally formulated to model the distortion by lateral variations in conductivity of currents induced in the Earth. The technique of representing three-dimensional conductive structure through two dimensional variations in the conductance of a thin sheet at the surface has several numerical restrictions. In these, quantities are generally expressed in terms of the skin depth $(\delta)$ of variations in the layered structure which underlies the thin sheet. The two principal conditions for validity are (Divett et al., 2017; Mukhtar et al., 2020) 


$$
\begin{gathered}
h \ll 1 \\
(h / \eta)^{2} \ll 1
\end{gathered}
$$

where $h$ is the thickness of the thin sheet (the depth range over which the conductance has been calculated), and $\eta$ is the skin depth in the thin-sheet. The concept of complex skin depth has already seen arises in the characteristic diffusion equations of the propagating waves in the Earth (Section 2.2.2). The term skin depth $(\delta)$, real part of complex skin depth, shows an attenuation factor, by which the amplitude of the wave propagating through the Earth reduce by a factor of $1 /$ e or about $37 \%$, and is given by

$$
\delta=0.5 \sqrt{\rho * T}
$$

where $\delta$ is in kilometers, $\rho$ is resistivity of layered Earth and $T$ is the period of the downward propagating wave in seconds. It shows that for a layered resistivity Earth model, the skin depth would be different depending upon period of wave and resistivity of layer. For example, Table 2.2 shows equivalent calculated skin depths $(\delta)$ at different periods for a layered Earth, having resistivities $\rho_{1}=1000 \Omega m, \rho_{2}=100 \Omega m$ and $\rho_{3}=1$, with boundaries at depths of $60 \mathrm{~km}$ and $320 \mathrm{~km}$ from the surface. Since $h$ is described in units $\delta, h$ is obtained by dividing the thickness of the thin-sheet i.e., $20 \mathrm{~km}$ by the skin-depth.

Table $\mathbf{2 . 2}$

The equivalent skin depth for a three-layer Earth model at different periods.

\begin{tabular}{ccc}
\hline $\mathbf{T}$ (minutes) & $\boldsymbol{\delta}(\mathbf{K m})$ & $\boldsymbol{h}$ \\
\hline 2 & 96 & 0.21 \\
5 & 128 & 0.156 \\
10 & 163 & 0.123 \\
20 & 214 & 0.093 \\
50 & 315 & 0.063 \\
90 & 408 & 0.049
\end{tabular}

The skin depths in the thin-sheet can be calculated both for North and South Islands using the maximum conductance values as given by the color legend in Figure 2.14. The maximum conductance for the South Island is $125 \mathrm{~S}(160 \Omega \mathrm{m})$ giving the values of $\eta_{\min }$ at different periods 
as shown in Table 2.3. The maximum conductance for North Island has a higher value of $500 \mathrm{~S}$ giving $\eta_{\min }$ at the different periods as shown in Table 3.1. This shows that the validity of the thinsheet approach is confirmed for longer periods for both the North and South Islands. The comparison of values of $h / \eta$ also shows that the thin-sheet model results for the South Island can be extended to shorter periods than they can for the North Island. This agrees with the conclusion reached by Divett et al., 2020. A third condition relates to the spacing of the numerical grid, which must be less than $\delta / 4$ which is also satisfied in the conductance grid selected (Divett et al., 2017).

Table 2.3

The skin depth in the thin-sheet at different periods.

\begin{tabular}{ccccc}
\hline $\mathbf{T}$ (minutes) & $\begin{array}{c}\boldsymbol{\eta}_{\min }(\boldsymbol{K m}) \\
\text { South Island }\end{array}$ & $\begin{array}{c}\boldsymbol{h} / \boldsymbol{\eta} \\
\text { South Island }\end{array}$ & $\begin{array}{c}\boldsymbol{\eta}_{\min }(\boldsymbol{K m}) \\
\text { North Island }\end{array}$ & $\begin{array}{c}\boldsymbol{h} / \boldsymbol{\eta} \\
\text { North Island }\end{array}$ \\
\hline 2 & 69 & 0.29 & 34.5 & 0.58 \\
5 & 110 & 0.18 & 55 & 0.36 \\
10 & 155 & 0.13 & 77.5 & 0.26 \\
20 & 219 & 0.09 & 109 & 0.18 \\
50 & 346 & 0.06 & 173 & 0.12 \\
90 & 465 & 0.04 & 232 & 0.09
\end{tabular}

In the thin-sheet modelling approach, GIC are assumed to be driven by the horizontal electric fields that are induced at the surface of the Earth by the temporal variation in horizontal components (north and east) of the linearly polarized magnetic field. For a given angular frequency of variation the electric field induced by the magnetic field is expressed as

$$
\begin{aligned}
& \boldsymbol{E}_{\boldsymbol{x}}=\left|\boldsymbol{E}_{\boldsymbol{x}}\right| e^{-i(\omega t-\alpha)} \\
& \boldsymbol{E}_{\boldsymbol{y}}=\left|\boldsymbol{E}_{\boldsymbol{y}}\right| e^{-i(\omega t-\beta)}
\end{aligned}
$$

where $\left|\boldsymbol{E}_{\boldsymbol{x}}\right|$ and $\left|\boldsymbol{E}_{\boldsymbol{y}}\right|$ are the complex field amplitudes in the north and east directions respectively, and $\alpha$ and $\beta$ are the phases of these fields relative to the inducing magnetic field. Using equations 2.9, Figure 2.15 shows thin-sheet computed geoelectric field for a uniform magnetic field (5-minute period) oriented 30 degrees east of north and of strength $100 \mathrm{nT}$, for both North and South Islands. Although, geoelectric fields can be computed for each cell of thinsheet grid they are shown only for locations of MT sites. The ellipses show both the polarization 
of the $\boldsymbol{E}$ field and the magnitude. The direction of the calculated fields is more scattered in the North Island compared to the South Island. Mostly fields are oriented in north-west/south-east or east/west directions.

Apart from the numerical limitations mentioned above the accuracy of calculated fields depends very much on the structure of the conductivity model. For example, the grid shown in Figure 2.14 is a very coarse grid and lateral variations in conductance are therefore very much approximated. Additionally, although the conductivity models on which the conductance map is based are themselves based on MT data, for the purpose of understanding deep structure the near-surface distortions are generally removed from the MT data. For example, prior to inverting $\underline{\underline{Z}}$ to obtain a 1-D or a 2-D ground conductivity model, shallow 3-D static distortion effects are typically removed from the impedance tensor (Groom and Bailey, 1989). The modified or stripped tensor is then used for inverse modeling of the ground conductivity structure. In the scheme proposed by Groom and Bailey (1989) the undistorted impedance tensor $\left(Z_{2}\right)$ is related to the measured impedance tensor $(\underline{\underline{Z}})$ by

$$
\underline{\underline{Z}}=g \operatorname{RTSAZ_{2}} R^{T}
$$

where, $g$ is a site gain, which is a scalar, $R$ is a rotation matrix, $T$ and $S$ are twist and shear tensors respectively, and $A$ is an anisotropy matrix. It is $Z_{2}$, the undistorted tensor, which is used in calculating conductivity models. The concept of distortion removal has an important consequence for those applying ground conductivity models to the GIC prediction problem. Methods that solve for the ground electric fields, given a known conductivity model that have first stripped out the effects of the shallow 3-D scattering layer will produce undistorted ground electric fields, with the effects of static distortion removed (Bonner and Schultz, 2017). Such electric fields differ from those measured at each site, since the real-world electric fields are distorted by fine-scale heterogeneities in near-surface conductivity structure, whereas the MT-derived conductivity models typically have such effects removed. Efforts to use ground conductivity models based on tensor stripped impedances will yield ground electric field predictions that will not, in general, match the measured electric fields on the ground. 

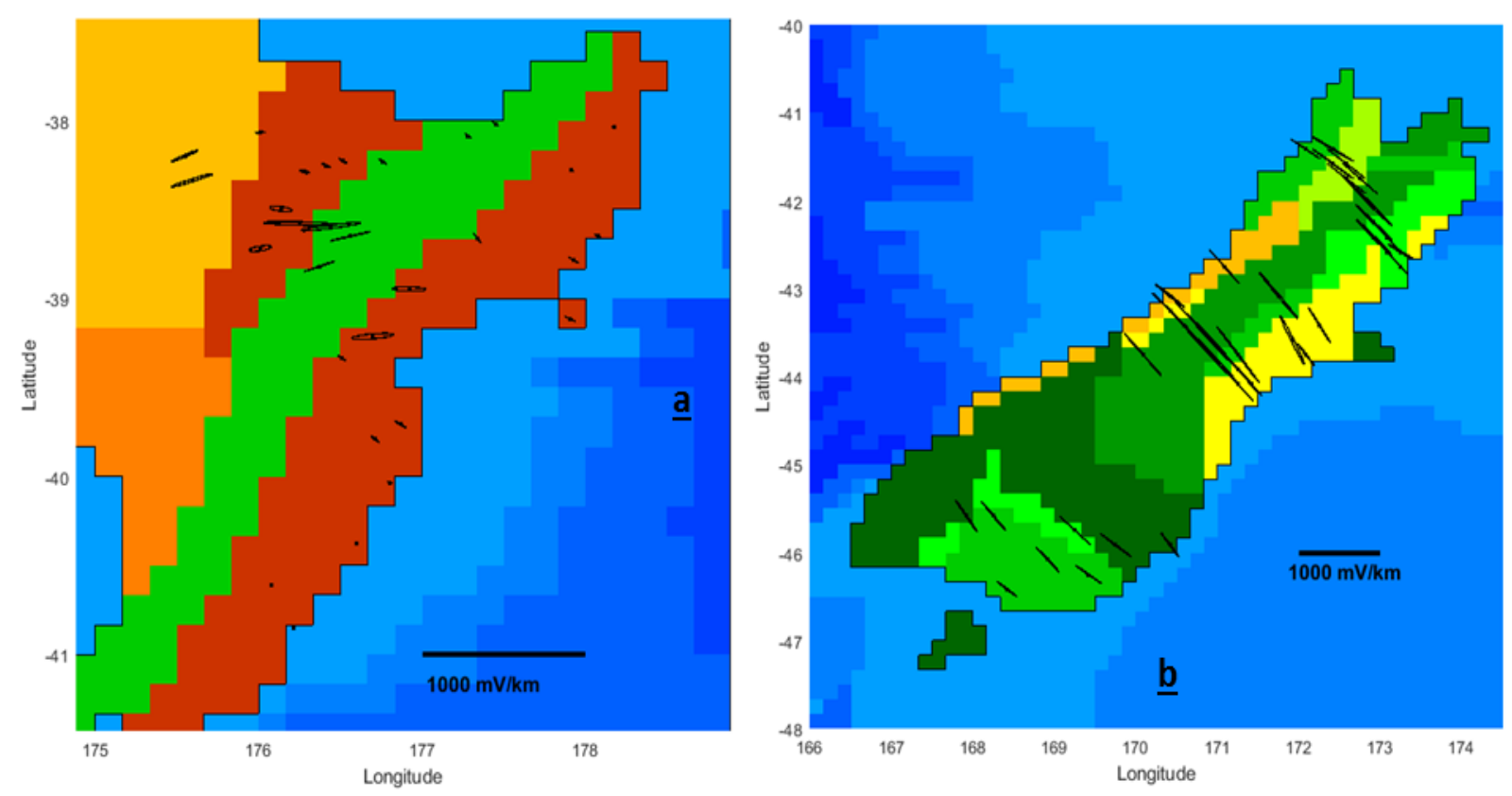

Figure 2. 15: The thin-sheet computed geoelectric field in response for a 5-minute period and an amplitude of $100 \mathrm{nT}$ magnetic field oriented $30^{\circ}$ east of north, (a) for North Island. (b) for South Island.

\subsubsection{Calculation of Geoelectric Field Using Magnetotelluric Data}

In above section it is seen that, although it is generally based on field measurements of electrical conductivity structure measured using magnetotelluric (MT) sounding, a thin-sheet model gives a very simplified picture of lateral variations. The advantage of estimating the electric field using MT tensors is that such estimates are based on actual measurements of the electric field rather than a conductivity model derived from both the electric and magnetic data. Direct use of an MT impedance tensor is able to estimate electric fields in the frequency domain from geomagnetic field spectra, as has been done by Blake et al. (2016) to calculate GIC in the Irish power network, by Torta et al. (2017) in Spain, and extensively by Bonner and Schultz (2017), Kelbert et al. (2017) and Love et al. (2018) in the United States. The use of the MT impedance tensor allows a much broader range of frequencies to be explored than is possible using the thin-sheet model and also allows incorporation of much finer detail than the thin-sheet modeling approach. The conductivity of the Earth varies both laterally and with depth and in recent studies more advanced and complex models of conductivity of the Earth have been used based on the Earth response function or complex valued impedance tensor $(\underline{\underline{Z}})$ from magnetotelluric measurements. In the GIC community the MT response tensor $(\underline{\underline{Z}})$ is used along with magnetic field data $\left(\boldsymbol{B}_{\boldsymbol{x}}, \boldsymbol{B}_{\boldsymbol{y}}\right)$ 
of a geomagnetic observatory to calculate geoelectric fields as given below (Torta et al., 2017; Bedrosian and Love, 2015)

$$
\underline{\boldsymbol{E}_{\boldsymbol{h}}}(f, x, y)=\underline{\underline{Z}}(f, x, y) \cdot \underline{\boldsymbol{B}_{\boldsymbol{h}}}(f, x, y)
$$

where the horizontal geomagnetic field $\boldsymbol{B}_{\boldsymbol{h}}$ and geoelectric field $\boldsymbol{E}_{\boldsymbol{h}}$ are in the frequency domain $(f)$ at a surface location $(x, y)$. The magnetotelluric response tensor defined in this way is related to the normal MT impedance tensor through the permeability $\mu$ and has practical units of $\mathrm{mV} / \mathrm{km} / \mathrm{nT}$. At each frequency $f$ and location $(x, y), \underline{\underline{Z}}$ is a $2 \times 2$ complex-valued matrix, therefore, the term $\underline{\underline{Z}} . \mu$ has units of Ohms.

For an idealized Earth conductivity that varies only with depth (1-D) the response tensor is antisymmetric (Simpson and Bahr, 2005)

$$
Z=\left(\begin{array}{cc}
0 & Z \\
-Z & 0
\end{array}\right)
$$

where each non-zero element $\underline{\underline{Z}}$ is complex-valued and frequency-dependent. If the conductivity varies in 3-D then, in general, none of the response tensor elements are zero i.e.,

$$
Z=\left(\begin{array}{ll}
Z_{x x} & Z_{x y} \\
Z_{y x} & Z_{y y}
\end{array}\right)
$$

No element of $\underline{\underline{Z}}$ for example, $Z_{x x}$ has a simple mathematical relationship to any other of the response tensor elements, $Z_{x y}, Z_{y x}$, and $Z_{y y}$. The equation which gives the relation between electric and magnetic fields using the above 3-D magnetotelluric response tensor is given by (Bedrosian and Love, 2015),

$$
\begin{aligned}
& \boldsymbol{E}_{\boldsymbol{x}}(f)=Z_{x x}(f) \boldsymbol{B}_{x}(f)+Z_{x y}(f) \boldsymbol{B}_{y}(f) \\
& \boldsymbol{E}_{\boldsymbol{y}}(f)=Z_{y x}(f) \boldsymbol{B}_{x}(f)+Z_{y y}(f) \boldsymbol{B}_{y}(f)
\end{aligned}
$$

$\boldsymbol{B}_{\boldsymbol{x}}$ and $\boldsymbol{B}_{\boldsymbol{y}}$ are horizontal components of the magnetic field. Using these equations, we can calculate the electric field at the periods of MT data. Figure 2.16 shows plots of the real and 
imaginary parts of $Z_{x x}, Z_{x y}, Z_{y x}$, and $Z_{y y}$ at one MT site HKB-803 (-39.50 N, 176.38 E). The red circles show MT measurements at different periods which ranges from 1.4-30 minutes.
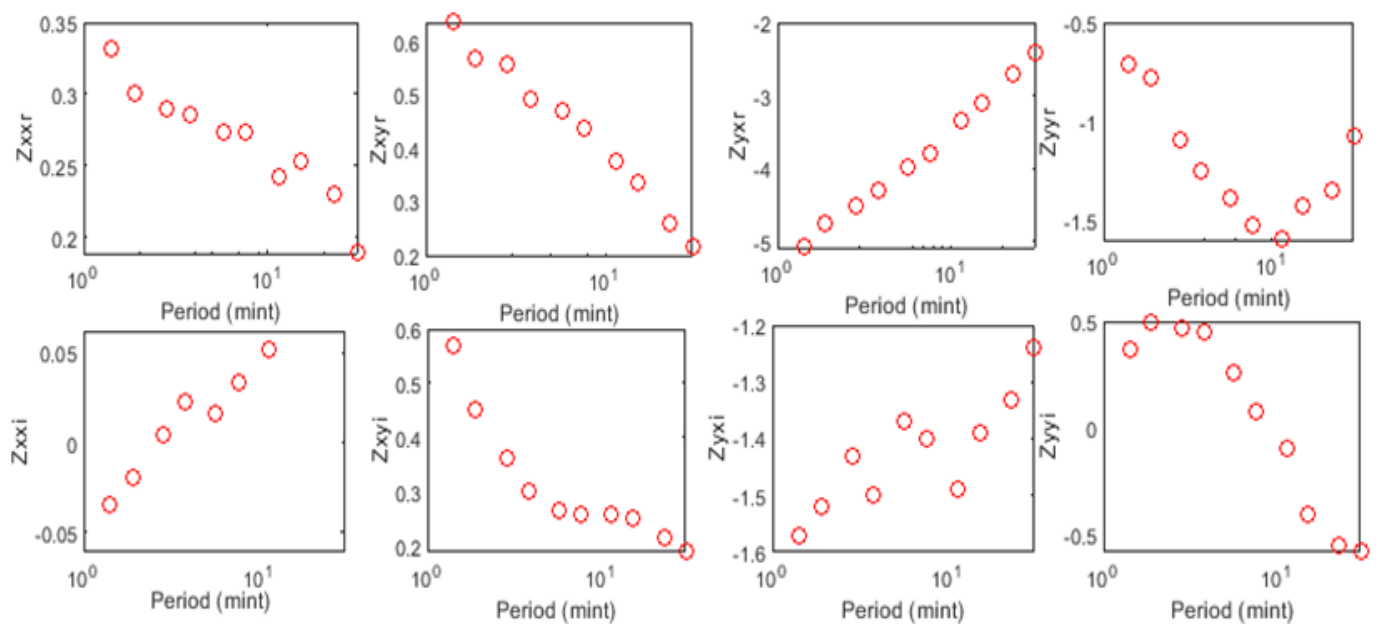

Figure 2. 16: Magnetotelluric response tensor components (real and imaginary) at MT site HKB803. Red circles show discrete periods of MT tensor ranging 1.4-30 minutes.

Using equation 2.11 geoelectric fields have been computed as for the thin-sheet model (uniform magnetic field in orientation $30^{\circ} \mathrm{E}$ of $\mathrm{N}$ of amplitude $100 \mathrm{nT}$ and having a period of 5 minutes). The resulting geoelectric fields are shown as Figure 2.17. The MT geoelectric fields show much more variations both in polarization, direction and magnitude compared to the thin-sheet computed geoelectric fields.

Although the numerical constraints on the thin-sheet model are a factor, a major difference between thin-sheet model and MT derived geoelectric fields is primarily is due to the removal/inclusion of galvanic effects, i.e. the build-up of electric charges along near-surface, small-scale conductivity contrasts or topographic inhomogeneities (Jiracek, 1990). The effect of galvanic distortion on the near surface electric field can be seen clearly in comparing Figures 2.15 and 2.17. The variation in electric fields over short distances also shows that use of a dense network of MT sites will allow a much more accurate picture of spatial variations in electric fields. Calculating electric fields directly from the measured impedance tensor means that distortions are taken into account and therefore giving a much more realistic picture than does a thin-sheet conductance model. 

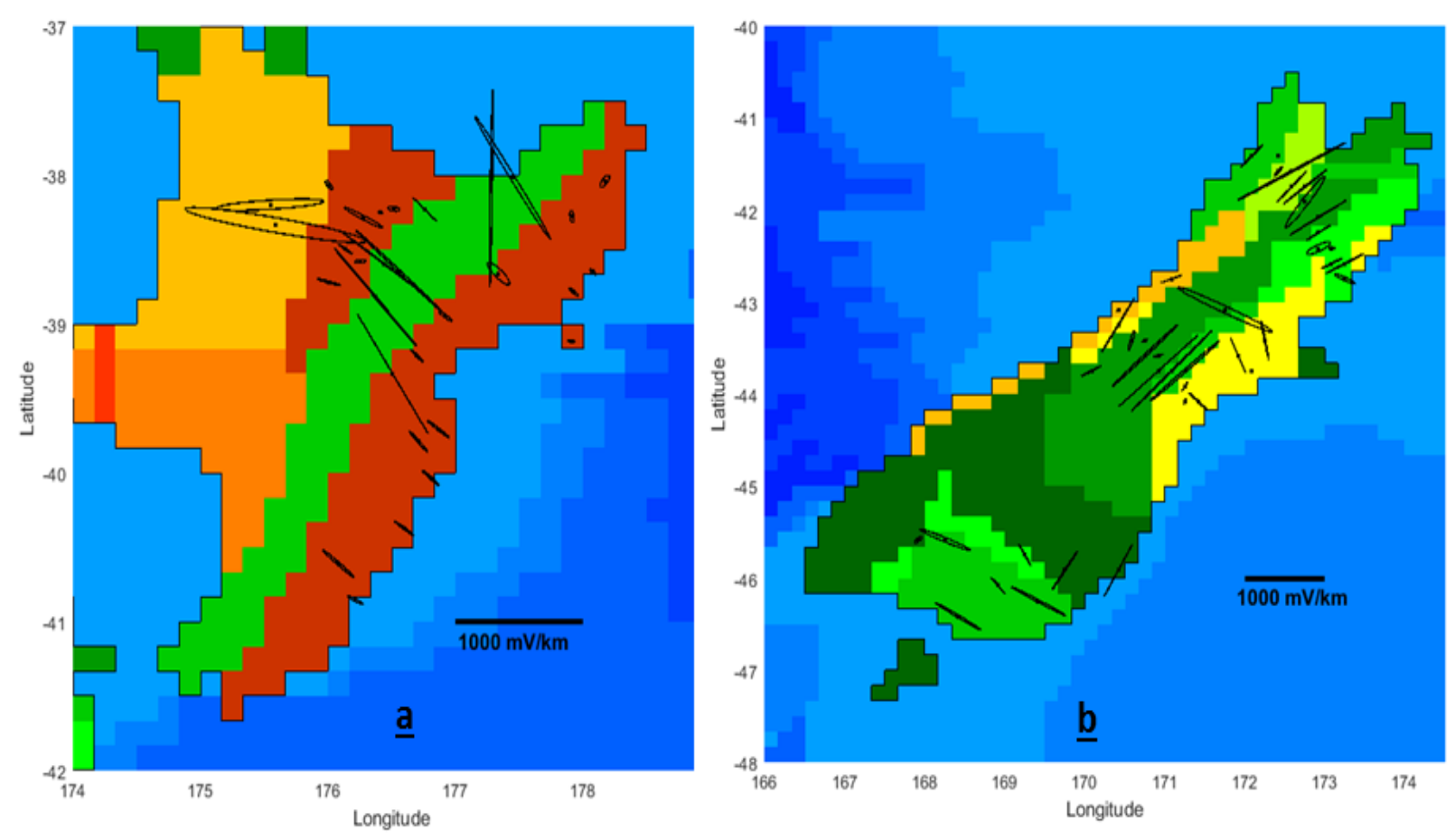

Figure 2. 17: (a) MT computed geoelectric field for magnetic field of $100 \mathrm{nT}$ at $30^{\circ} \mathrm{E}$ of $\mathrm{N}$ having period of 5 minute for North Island. (b) Computed geoelectric fields for South Island.

\subsection{MT Perspective of GIC Modelling for New Zealand}

As can be seen from the previous two sections, given the limitations associated with thin-sheet conductance modelling, the direct use of MT data is likely to provide more realistic geoelectric fields based on available magnetotelluric response tensors. The knowledge of the Earth's geoelectrical structures beneath the network becomes more important where there are abrupt variations in the derived geoelectric field either because of the ocean-land interfaces (Pirjola, 2013; Gilbert, 2015) or because of near-surface distortions. The juxtaposition of provinces with old and cold rocks that are largely devoid of electrical conducting mineral phases with deep intracontinental basins filled with conductive sedimentary rocks can result in major changes in geoelectric fields over short distances (Bedrosian and Love, 2015). Further, estimates of Earth conductivity based on simplistic assumptions about geology and structure to calculate synthetic impedance tensors may not fully represent the MT data. In particular, given that electrical conductivity may have a complicated three-dimensional variation (Ferguson et al., 2012), the induced geoelectric field at one site does not always correlate with that at another nearby site (McKay and Whaler, 2006). For example, one-dimensional estimate of conductivity were used to 
estimate geoelectric fields by Gannon et al. (2012); Viljanen et al. (2013); Wei et al. (2013) and more recently, geoelectric fields have been calculated using synthetic models of threedimensional conductivity (Beggan et al., 2013; Beggan, 2015). However, few have used impedance tensors obtained directly from magnetotelluric measurements (McKay and Whaler, 2006).

Thus, to obtain a good picture of the spatial variation of fields a large, dense network of MT sites is necessary. In the North Island of New Zealand, there are a large number of MT sites. These include studies by Bertrand et al. (2012, 2013), Heise et al. (2008, 2010, 2014), and Ingham (2005) in the Central Volcanic Region, by Cassidy et al. (2009), Ingham et al. (2009), and Stagpoole et al. (2009) on the volcanic systems, and by Heise et al. (2012), Ingham et al. (2001), and McLoughlin et al. (2002) along the east coast of the Island. In total well over 200 separate MT measurements have been made. Of these sites many are in very close geographic proximity but, overall, MT sites have been measured in 115 of the 463 m cells which make up the North Island in the thin-sheet conductance model used by Divett et al. (2017). Mostly sites are located in lower part of the North Island and along the east coast. No data exist in northern part of the North Island.

Unlike the North Island, the South Island has very sparse MT measurements. Only a total of 42 cells of the conductance model can be filled using available MT data, although some cells contain multiple MT sites. The MT site distribution in the South Island can broadly be divided into sites in three main regions from south to north. Furthest south, Southland/Otago (shown by shades of green on the conductance map in Figure 2.17) is a region mainly of medium to low conductance and MT data at 8 different locations was acquired as part of this PhD thesis. This usually covered the broader range of periods from 2-83 minutes, and was extended to 2-332 minutes for one site. The data for this MT survey is also attached as Appendix-MT measurements. Figure 2.18 shows a glimpse of MT field survey and MT equipment.

The second group of MT sites is in the center of South Island including measurements made by Wannamaker et al. (2002) and Ingham (1995, 1996a, 1996b). This region has a range of conductance variations from the east to west coasts shown by shades of yellow and green. Relatively high conductance (shown by shades of yellow) exists at both the east and west coast whereas a highly resistive region associated with the Southern Alps exists between them. Mostly 
MT sites are in the period range of 2-30 minutes but a few sites in the high conductive region have longer period range of 2-60 minutes. The third group of MT measurements is in the north of the Island and were reported by Wannamaker et al. (2009). Although there are significant differences in the geology the conductance variations in this region are similar to those in the central region. The total number of MT sites across Marlborough is 15 and these have a shorter period range of 2-15 minutes. In subsequent chapters the available MT data from both North and South Islands is used to model GIC in the New Zealand power network.
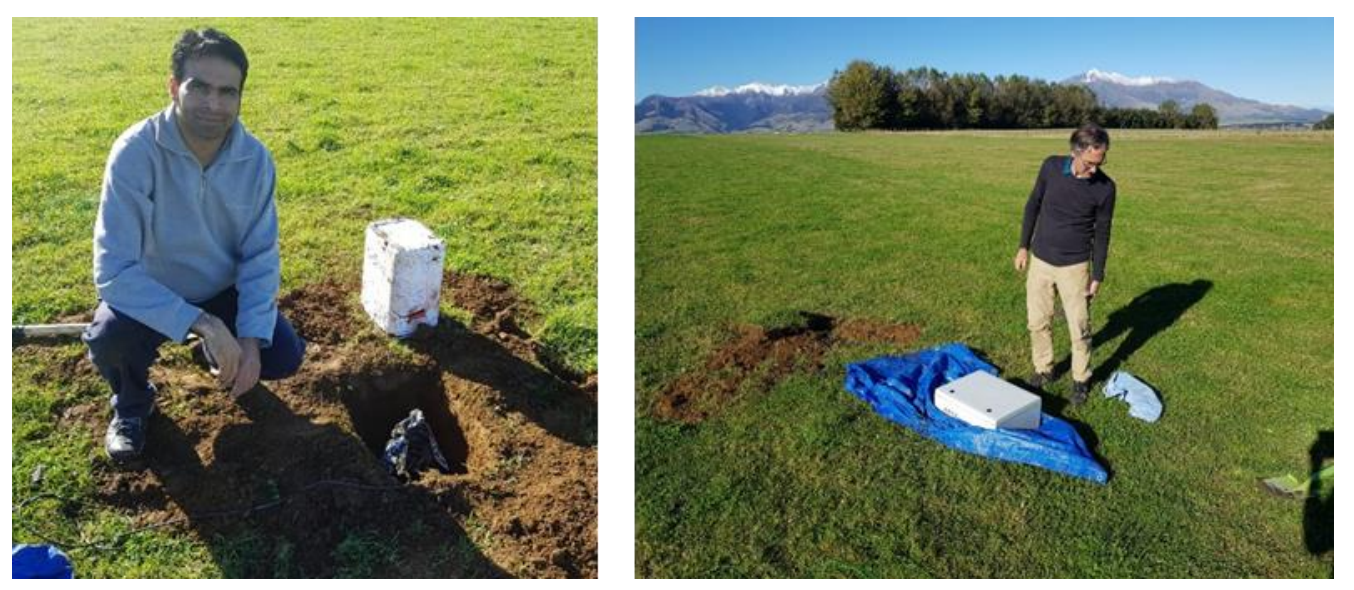

Figure 2. 18: Left hand panel: magnetometer hole with the author sitting beside it. Right hand panel: data logger for recording electric and magnetic fields. The electrodes for electric field measurements are spread $\sim 50 \mathrm{~m}$ apart and are not shown in these pictures.

In principle, once a geoelectric field map is constructed from measured storm time magnetic fields it can be used in to assess induction hazards for power grids (Burstinghaus et al., 2013; Zheng et al., 2013) and in scenario simulations (Pulkkinen et al., 2005; Viljanen et al., 2013; Torta et al., 2014) to evaluate the vulnerability of power grids for extreme event magnetic storms (Boteler, 2001; Overbye et al., 2013).

\subsection{Network Modelling}

The determination of geoelectric fields in the domain of the power network enables computation of GIC in the network using a network model. A power transmission system can be viewed as a network of conductors discretely grounded at certain nodes. Kirchhoff's and Ohm's laws are generally used to calculate currents going through transformers and power lines. Geoelectric fields produce an electromotive force in long power lines and the current flows between different 
substations. The transformer neutrals connected to ground provide a complete path to ground for GIC (Lehtinen and Pirjola, 1985; Boteler, 1999). GIC enter the power system via the transformer neutral connected to Earth, flow along the transmission lines and exit at another earthed transformer. Network models which calculate induced current at each node or substation on an electrical network have often been based on one of two methods: the Lehtinen and Pirjola (1985) matrix method (LP85 in short form) or the Nodal Admittance matrix method. Boteler and Pirjola (2014) present a description of both methods and show that the two are mathematically equivalent. LP85 was further extended by Boteler and Pirjola $(2014,2017)$; to incorporate multiple voltage levels in a network and different transformer types to compute GIC through individual transformer windings. LP85 is commonly used in the GIC community for computation of GIC. It has successfully been implemented by Beggan et al. (2013), Kelly et al. (2017), Blake et al. (2016) and Divett et al. (2017) in the UK, Ireland, France, and New Zealand networks respectively. These modelling techniques are discussed separately below.

\subsubsection{Substation Level Modelling}

A substation-level GIC is the total GIC flowing into or out of a substation through all earthed transformers within the substation. Lehtinen and Pirjola (1985) used the term node to represent a substation in a network. To construct a network model for calculating GIC in a power system it is convenient to consider that the resistance of each phase of the power system is identical and so experiences the same levels of GIC, as shown in Figure 2.19. This means we need to make calculations for only a single phase as shown in Figure 2.19(b), where $R_{L}$ represents the line resistance, and $R_{T}$ the transformer winding resistance. Since the currents from all three phases flow through the substation earthing or grounding resistance $R_{e}$, the equivalent resistance for one phase would be $R_{e} / 3$. In a two-winding/normal transformer the windings for the three phases are connected together at a neutral point, which is connected to ground as shown in Figure 2.19(a). In normal operation the AC currents in the three phases have the same amplitude but are 120 out of phase and sum to zero at the neutral point, so there is normally no AC flow from the neutral point to ground. 


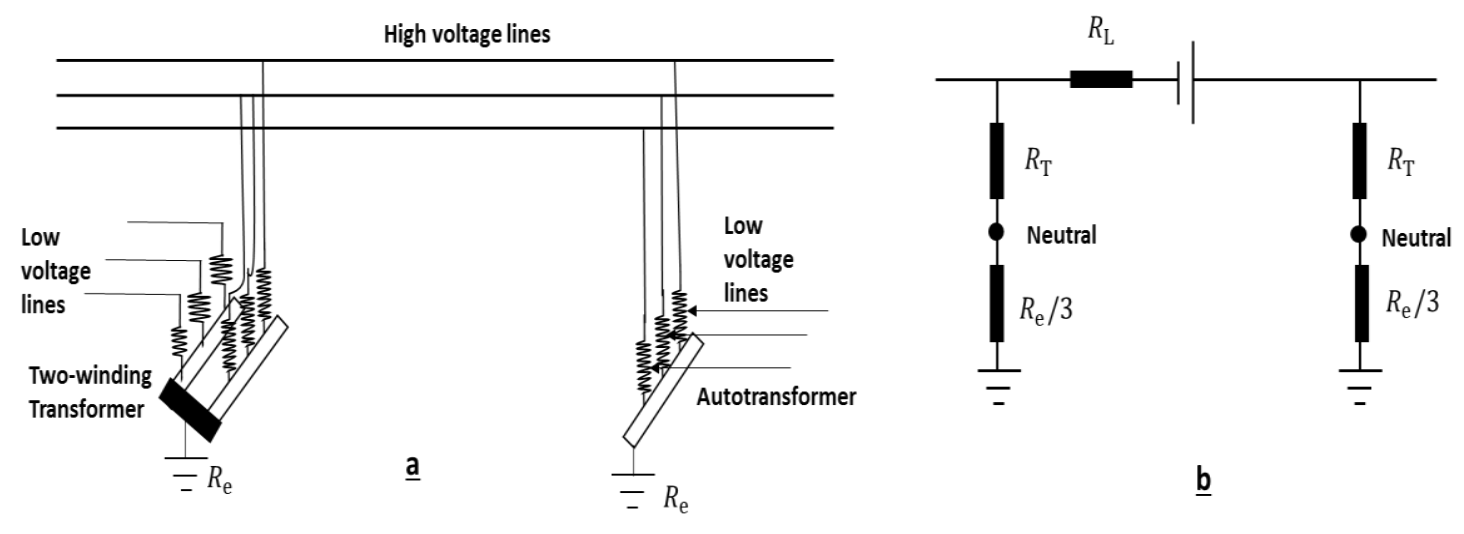

Figure 2. 19: (a) Network consisting of three phase connections of two-winding transformer and autotransformer. (b) Network representation of power lines and transformers for model development.

Knowing that GIC are slowly varying compared to the $50-\mathrm{Hz}$ mains, a DC treatment is acceptable, and Ohm's and Kirchhoff's laws in the DC form are used to formulate a network matrix model from the transmission line and earthing resistances information. LP85 provides all the expressions for the determination of the GIC flowing into the Earth at each substation given by

$$
G I C_{s u b}^{n}=(I+Y Z)^{-1} J
$$

$G I C_{\text {sub }}^{n}$ is the GIC at any particular substation for a total of $n$ nodes within the network, $I$ is the identity matrix, $Y$ is an $n \times n$ network admittance matrix (line resistances) matrix and $Z$ is the earthing impedance (earthing resistances) matrix. The consideration of network resistances instead of impedances is due to the fact that at GIC frequencies the reactive impedances are assumed to be negligible. The $n$-element $J$ matrix contains information on the geoelectric fields. The elements of the $Y$ and $Z$ matrices are given by

$$
Y_{i j}=\left\{\begin{array}{c}
-1 / R_{i j} \quad(i \neq j) \\
\sum_{k=1, k \neq i}^{n} 1 / R_{i k} \quad(i=j)
\end{array}\right.
$$

$R_{i j}$ is the line resistance $\left(R_{i j=} R_{j i}\right)$ between the nodes $i$ and $j$

$$
Z_{i j}= \begin{cases}0 & (i \neq j) \\ R_{i}^{e} & (i=j)\end{cases}
$$


$R_{i}^{e}$ is the resistance through which a substation is earthed. The $J$ matrix in equation 2.12 represents the source currents produced in the transmission lines due to geoelectric fields and is given by

$$
J_{i}=\sum_{j \neq i}^{n} \frac{V_{i j}}{R_{i j}}
$$

$V_{i j}$ is the geovoltage from node $i$ to node $j$, which is given by

$$
V_{i j}=\int_{i}^{j} E . d l
$$

where $\boldsymbol{E}$ is the electric field and $\boldsymbol{d} \boldsymbol{l}$ is the differential vector path length. The integration over the complete path of the line gives the voltage difference produced between the end points of the transmission lines.

The above method has been demonstrated with the example of four earthed substations shown as Figure 2.20. Each earthed resistor has value of $0.5 \Omega$ whereas the line resistance is $5 \Omega$. Suppose a non-uniform electric field is applied in direction such that it has value of $1 \mathrm{~V} / \mathrm{km}$ and $2 \mathrm{~V} / \mathrm{km}$ as shown in the Figure. Field lines are parallel to transmission lines from nodes 1 to 4 , and 2 to 3 , whereas they are perpendicular to lines from nodes 1 to 2 and 3 to 4 . For this simple network, the admittance matrix $(Y)$ and earthing matrix $(Z)$ can be written

$$
\begin{aligned}
& Y=\left(\begin{array}{cccc}
-1 / R_{12}+R_{14} & -1 / R_{12} & 0 & -1 / R_{14} \\
-1 / R_{21} & -1 / R_{21}+R_{23} & -1 / R_{23} & 0 \\
0 & -1 / R_{32} & -1 / R_{32}+R_{34} & -1 / R_{34} \\
-1 / R_{41} & 0 & -1 / R_{43} & -1 / R_{41}+R_{43}
\end{array}\right) \\
& Z=\left(\begin{array}{cccc}
R_{1}^{e} & 0 & 0 & 0 \\
0 & R_{2}^{e} & 0 & 0 \\
0 & 0 & R_{3}^{e} & 0 \\
0 & 0 & 0 & R_{4}^{e}
\end{array}\right)
\end{aligned}
$$

It can be noted that the line resistances are zero for substations having no direct connections e.g., there is no direct line between node 1 and 3 therefore $R_{13}$ zero. The geovoltages have been computed using the equation 2.15 and are also shown in Figure 2.20. Solving the matrix equation for $G I C_{\text {sub }}$ would give currents going through each node of the network as shown in Table 2.2. 
The positive GIC shows current going out of substation and negative GIC shows currents into a substation.

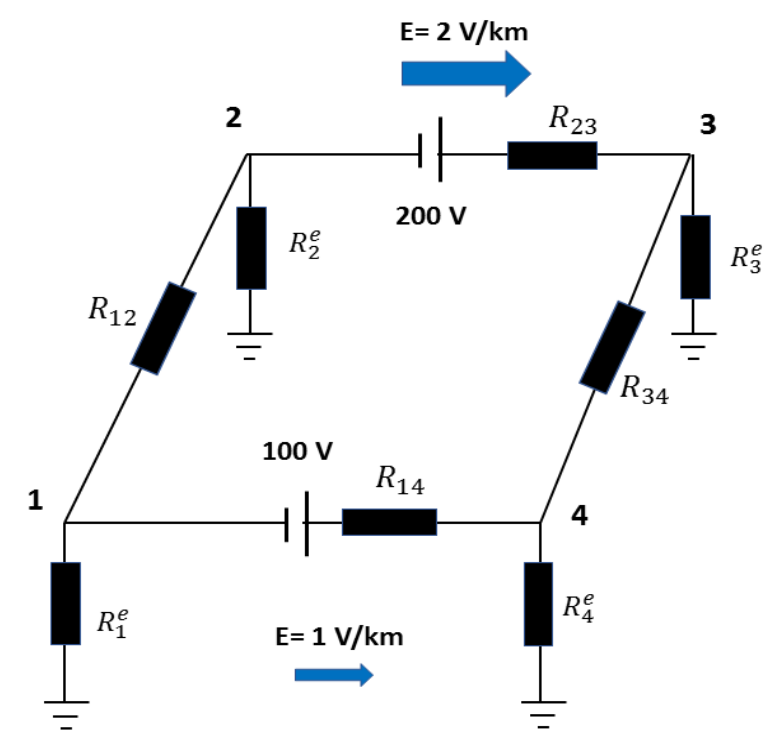

Figure 2. 20: A sample network consisting of four substations/nodes and a non-uniform electric field.

\section{Table 2.4}

Currents computed in lines and nodes for sample network using LP85.

\begin{tabular}{llll}
\hline Lines & Source currents & Nodes & GIC \\
\hline 1 to 2 & $0 \mathrm{~A}$ & 1 & $17.86 \mathrm{~A}$ \\
1 to 4 & 20 & 2 & $32.14 \mathrm{~A}$ \\
2 to 3 & $40 \mathrm{~A}$ & 3 & $-32.14 \mathrm{~A}$ \\
3 to 4 & $0 \mathrm{~A}$ & 4 & $-17.86 \mathrm{~A}$
\end{tabular}

\subsubsection{Transformer Level Modelling}

The transformer level GIC is the current that flows through the windings of a specific transformer of a substation. The LP85 method is basically for a single voltage level and in which all transformer resistances were considered together to give a single resistance to ground within the substation, each substation was represented by a single node, and all nodes were connected to a local ground. Boteler and Pirjola $(2014,2017)$ have described the necessary steps required in LP85 to obtain a transformer level model. This includes the type of transformers and their connections 
with different voltage levels of the network. Since there are different voltage levels within a substation the term node, is extended to High voltage (HV), Low voltage (LV) or earthed in transformer level modelling. When modelling the GIC flow in multiple voltage levels of a power system it is also necessary to consider the flow of GIC between different voltage levels. High voltage power transmission networks use three-phase AC with transformers to convert between voltage levels. The path for GIC flow between voltage levels is through the windings of the transformers at each substation. The type of transformer used determines the nature of the path for flow of GIC. Two types of transformer provide a path for GIC and are shown in Figure 2.21(a). The first type is a normal or two-winding transformer where separate windings are used for the high-voltage and low-voltage sides. The second type is an autotransformer, in which the highvoltage and low voltage sides share a winding with the low-voltage connection made part way along the winding. Since, the delta-connected windings do not have a connection to the ground and do not allow GIC to flow there is no need to consider them further. Figure 2.21(b) shows a substation consisting of both a two-winding transformer and an autotransformer and connecting with two other substations. When normal and autotransformers are in use for the same voltage levels at a substation, there are connections between all voltage levels and between those voltage levels and the neutral point, as shown in Figure 2.21(b). The series winding of the autotransformers connect the high voltage (HV) and low voltage (LV) buses shown in red and blue colors. The HV windings of the two-winding transformers connect the HV bus to the neutral point, and the LV windings of the transformers and common windings of the autotransformers connect the LV bus to the neutral point. The neutral point (shown in green) is a branch point between the connections from the HV and LV buses and the connection to the ground and so has to be kept as a node in the network model. Thus, both the HV bus and LV bus are ungrounded nodes in the network model. With this configuration, although there are three substations, GIC can be computed for all five nodes and the admittance would be a $5 \times 5$ matrix, and the earthing impedance would be a $5 \times 1$ matrix. GIC partly flow between the different voltage nodes and towards the Earth. The equation can be solved in a similar way as was done in the previous section giving GIC through each winding of the transformers. 


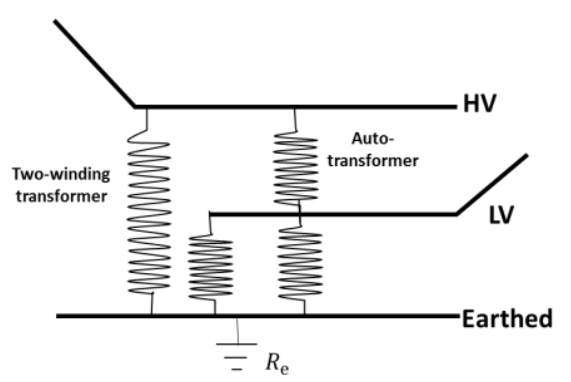

a

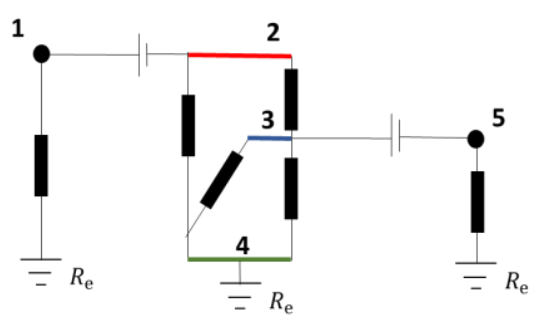

$\underline{\mathbf{b}}$

Figure 2. 21: (a) Transformer level network model for normal and auto transformer following Boteler and Pirjola (2014). (b) The nodal representation different voltage levels and transformer configuration, where horizontal lines show different voltage levels in the network.

\subsection{Network Models for the New Zealand Network}

The following sections briefly introduce important features of the power network model of New Zealand that have been used in previous studies of GIC in New Zealand (Divett et al., 2017, 2018 and 2020) and are also used in this thesis.

\subsubsection{Substation Level Network Model of New Zealand}

Initially the substation level GIC modelling approach for the network model of New Zealand was developed by Divett et al. (2017) following the nodal network approach of LP85, as discussed in section 2.4.1. In the substation approach each substation is represented as a node and a resistor. The resistor represents the combined resistance of every transformer within that substation and each substation is earthed through the earth ground resistance $\left(R_{e}\right)$. It is assumed that the current through each of the three phases of transformers and transmission lines is the same, and only one of the phases is modeled. Nodes are connected by a single transmission line resistor, representing a single phase of the three-phase transmission lines (e.g., $R_{m, n}$ line connecting nodes $m$ and $n$ ). Since GIC vary slowly compared to the $50 \mathrm{~Hz}$ AC power, a DC treatment is sufficient where $Y$ and $Z$ are taken as real matrices.

The transmission network of the North Island is shown as Figure 2.22(a) where (b) shows line resistance values for the North Island power network where the crosses show earthed substations. The transmission network principally consists of transmission lines with voltage ranges of 110 and $220 \mathrm{kV}$. However, a $400 \mathrm{kV}$ line connects Whakamaru (WKM), to the north of Lake Taupo, with Auckland. Within the North Island the majority of power is generated at a series 
of hydroelectric stations along the Waikato River which flows from Lake Taupo in the center of the Island through Hamilton (HAM) reaching the west coast to the south of Auckland. Other power stations based on geothermal, coal, gas, and wind are spread around the Island. The HVDC link which brings power from the major hydro lakes in the South Island terminates at Haywards (HAY) just to the north of Wellington. In total there are 33 power stations and 84 separate substations.
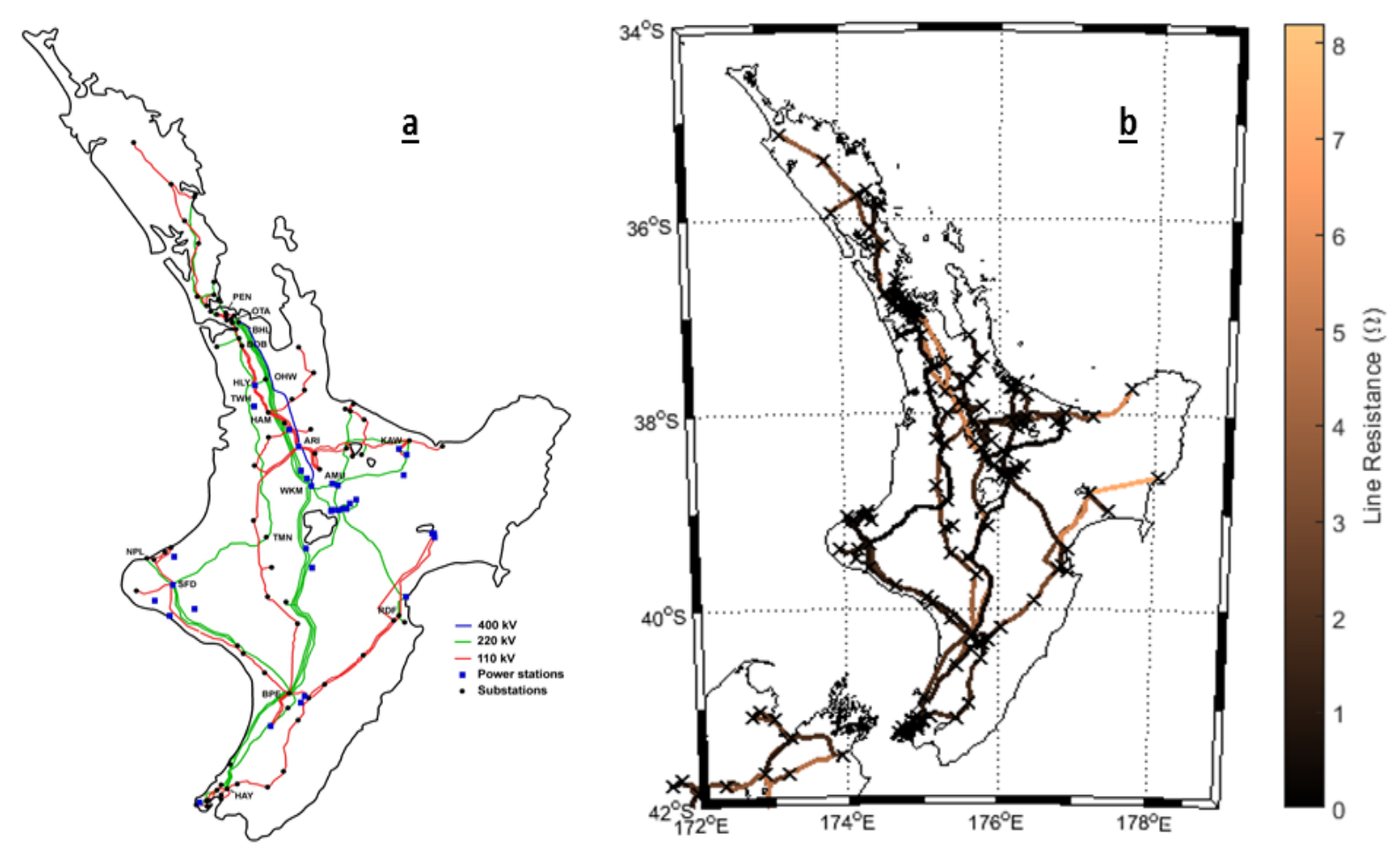

Figure 2. 22: (a) North Island transmission network showing different voltage levels and dots showing substations. (b) Line resistance map of the network.

The South Island high-voltage transmission network consists of transmission lines with three different voltage ranges: 50 or $66 \mathrm{kV}, 110 \mathrm{kV}$, and $220 \mathrm{kV}$ and is shown in Figure 1.1 of Chapter 1. Figure 2.23 shows line resistances by varying colors. The network consists of 64 substations connected by 121 transmission lines. The resistance of a single phase of each of the three-phase transmission line varies from $0.039 \Omega$ for the $220 \mathrm{kV}$ line between Ohau-B (OHB) and the nearest unearthed substation to $7.1 \Omega$ for the $66 \mathrm{kV}$ line connecting Coleridge (COL) to the unearthed substation directly north of COL. 


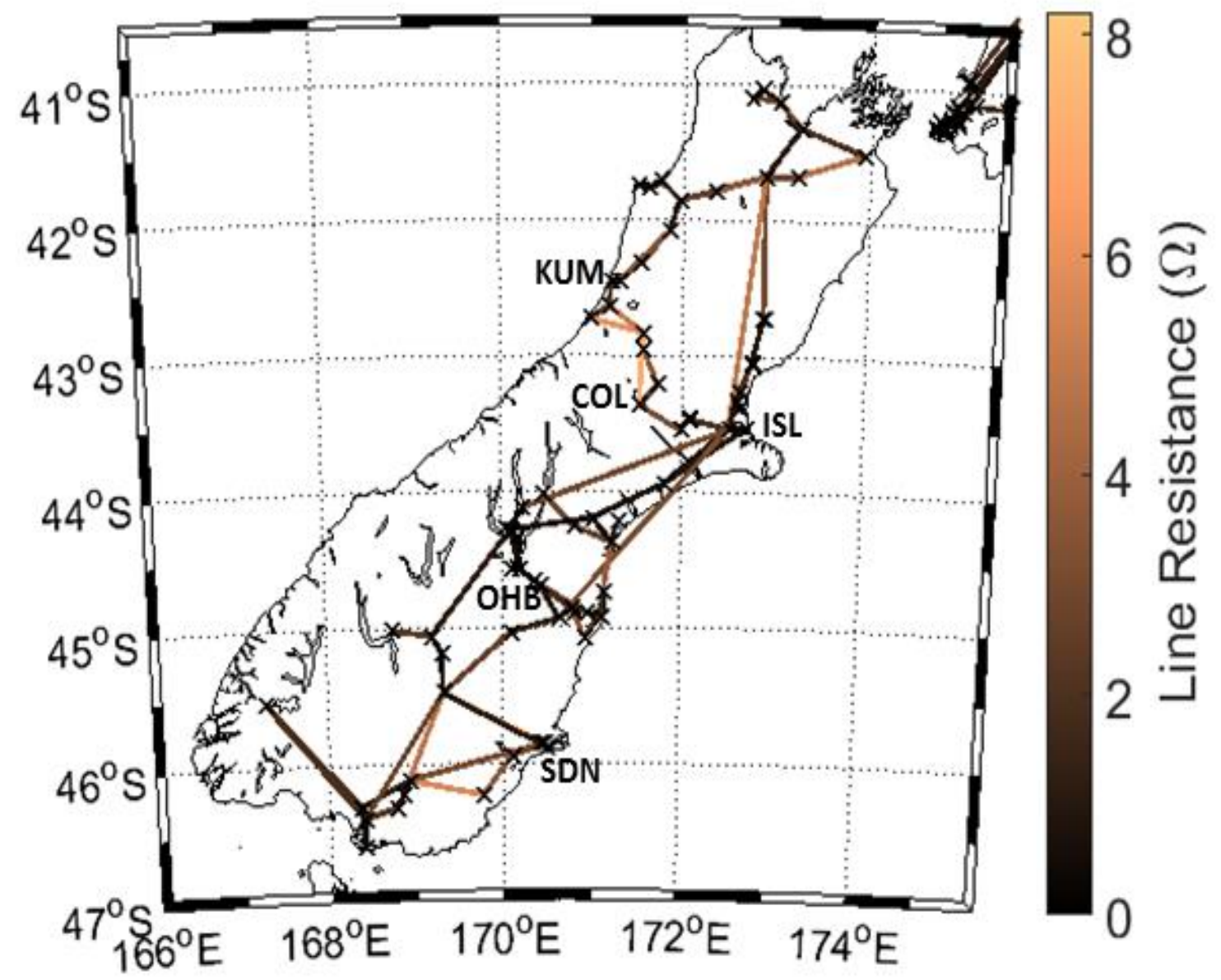

Figure 2. 23: The South Island of New Zealand showing transmission line resistances and substation node locations, all 220, 110 and $66 \mathrm{kV}$ lines are shown.

The resistances for parallel transmission lines connecting the same substations are added in parallel when building the network impedance matrix. The earthing impedance matrix is built from the $D C$ resistance of a single phase of the transformers at each substation, where it is assumed that the resistance of each substation is $R_{\text {tran }}=0.5 \Omega$. Earth ground resistance is the resistance between the Earth mat (grid of horizontal conductors buried inside Earth) at a substation and a remote ground measured and provided by Transpower. The value of this resistance at different substations can be as different as an order of magnitude depending on local soil type, underlying rock conductance, soil moisture content, and Earth mat size. $R_{e}$ ranges from $0.04 \Omega$ at South Dunedin (SDN) to $4 \Omega$ at Kumara (KUM), with a mean of $0.63 \Omega$. It is assumed that the $R_{e}$ is infinite at unearthed nodes. 


\subsubsection{Transformer Level Network Model of New Zealand}

A substation level model is useful to determine the total current going in or out of a substation through all earthed transformers but in the transformer level approach (section 2.4.2) it is seen that GIC still flow through transformer windings not earthed, and are potentially as harmful as for earthed transformers. GIC through individual transformer windings are also important as GIC monitoring devices are installed on individual transformer windings and different transformers have different GIC magnitudes within a substation (Divett et al., 2018). Therefore, calculating GIC through individual transformer windings is an important aspect of GIC modelling. Divett et al. (2018) developed the transformer level model of New Zealand following additional steps such as inclusion of different transformer types and their connections with different voltage levels of the network (Boteler and Pirjola, 2014, 2017).

To fully describe the transformer-level approach, Redclyffe (RDF) substation of North Island has been selected as shown in Figure 2.24. RDF consists of two autotransformers and two normal transformers therefore having all possible connections within a substation. High and low voltage buses (nodes) are shown as red (220 kV) and blue (110 kV), and the earthed node (0 V) is shown by the green line. T3 and T4 are autotransformer with the series winding connected to $220 \mathrm{kV}$, the common winding connected to the $0 \mathrm{~V}$ node, and a tap at $110 \mathrm{kV}$. Their function is to convert $220 \mathrm{kV}$ power to $110 \mathrm{kV}$ but from the perspective of GIC, the DC resistance of the windings provide a path for GIC to flow between each of the three nodes. T1 and T2 are normal where T1H and $\mathrm{T} 2 \mathrm{H}$ are the high-voltage sides of two-winding transformers, which convert $220 \mathrm{kV}$ to the local distribution voltage. Each of these high-voltage nodes is connected to local Earth by an infinite virtual resistor following Boteler and Pirjola (2014). The GIC through high-side windings of the autotransformers (RDF-T3H and RDF-T4H) would not enter the Earth, but the low-side windings (RDF-T3L and RDF-T4L) of the same transformers would allow GIC to enter the Earth. Therefore, the GIC flowing through high-side windings of the autotransformers do not contribute to substation GIC as these GIC would not be entering the Earth. In contrast, each winding of a normal transformer (RDF-T1 and RDF-T2) only provides a direct connection between one highvoltage node and Earth. It is important to note that in our example the substation shows only the high-voltage side of two normal transformers (RDF-T1H and RDF-T2H) connecting $220 \mathrm{kV}$ to $0 \mathrm{~V}$. The low-voltage side of these transformers connects to the local distribution ( $33 \mathrm{kV}$ ) side of the 
substation and is not modeled in our representation. The significance of these two types of transformer to GIC is that autotransformers provide a direct path for GIC to couple between two different voltage lines than normal transformers. In the case of a normal transformer connecting the $220 \mathrm{kV}$ network to the $110 \mathrm{kV}$ network, the GIC entering the substation on a $220 \mathrm{kV}$ transmission line must flow through the high-voltage side of the transformer, then through the Earth mat, before flowing through the low-voltage side of the transformer and into the transmission lines on the $110 \mathrm{kV}$ network. This shows the electrical connectivity inside a substation can make a significant difference to the GIC magnitudes in individual transformers.

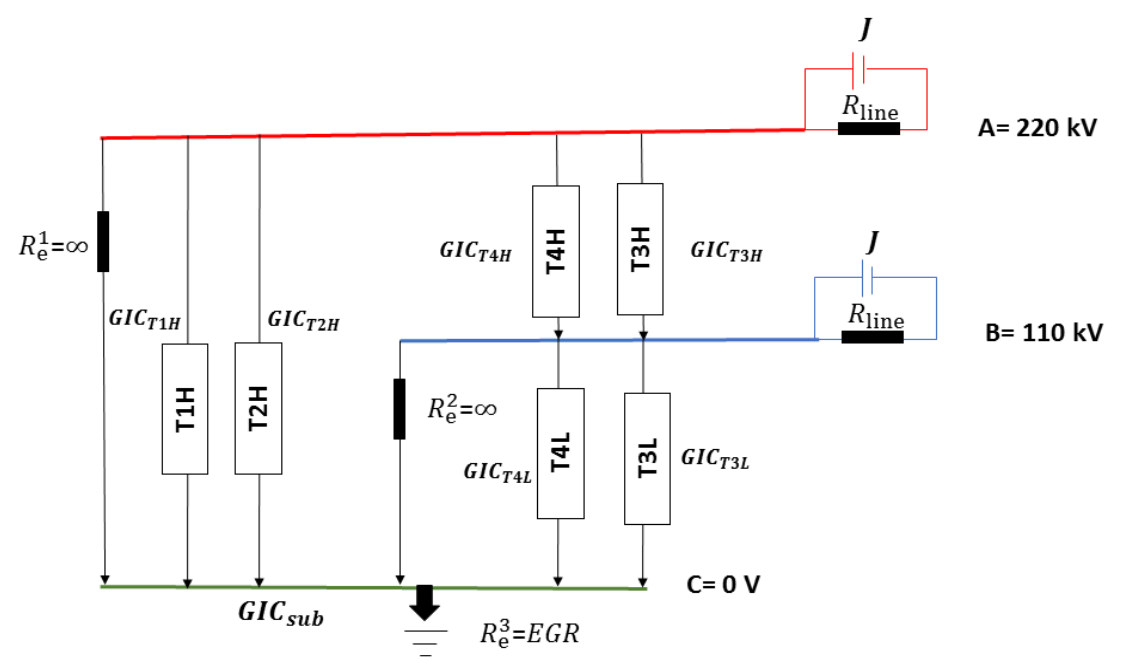

Figure 2. 24: Transformer-level representation of network of Redclyffe substation in the North Island. It consists of two normal transformers (T1 and T2) and two autotransformers (T3 and T4).

GIC have been computed for RDF substation using a uniform electric field of $1 \mathrm{~V} / \mathrm{km}$ in the east direction. The table shows total line currents to other substations and GIC in each winding of the transformers in the substation. RDF high voltage node has connection with three substations Whirinaki (WHI), Wairakei (WRK) and Whakatu (WTU). The low voltage node is connected with two substations, Tuai (TUI) and Fernhill (FHL). Where there is more than one line connecting nodes at different substations the Table shows the sum of all line currents for a substation. Since four transformers are earthed the sum of these would be the substation GIC which is $46.5 \mathrm{~A}$. The same nomenclature of GIC through autotransformers and normal transformers has been adopted throughout this thesis. 


\section{Table 2.5}

Currents going through lines and transformer windings for Redclyffe substation using uniform fields of $1 \mathrm{~V} / \mathrm{km}$ in east direction.

\begin{tabular}{cccccc}
\hline Node 1 & Node 2 & $\begin{array}{c}\text { Line current } \\
\text { (A) }\end{array}$ & $\begin{array}{c}\text { Transformer } \\
\text { windings }\end{array}$ & GIC (A) & $\begin{array}{c}\text { Earthed/ } \\
\text { Unearthed }\end{array}$ \\
\hline RDF110_2 & TUI110_1 & -7.2 & HVR_RDF_T1 & 46.42 & Earthed \\
RDF110_1 & FHL110_1 & 16.1 & HVR_RDF_T2 & 0.04 & Earthed \\
RDF220_1 & WHI220_1 & -3.7 & LVR_RDF_T3 & 0.02 & Earthed \\
RDF220_1 & WRK220_1 & 39.46 & LVR_RDF_T4 & 0.02 & Earthed \\
RDF220_1 & WTU220_1 & 0 & HVR_RDF_T3 & 18.80 & Unearthed \\
& & & HVR_RDF_T4 & 18.80 & Unearthed
\end{tabular}

\subsection{Discussion}

GIC pose a threat to transformers and whole networks during severe geomagnetic storms. The availability of measured GIC data provides an opportunity for model development and validation of model results. The South Island of New Zealand has a long GIC record for multiple transformers. In particular, GIC measurements on T6 transformer at Islington substation have a continuous record from 2001-2017. This partially covers solar cycle 23 and solar cycle 24 almost completely, as shown in Figure 2.1. Solar wind interplanetary structures causing intense geomagnetic storms (Dst $\leq-100 \mathrm{nT}$ ), have been explored in the context of the available Islington GIC data. Four interplanetary structures (CIR, ICME, SH+ICME and SH) have been considered as causing the intense geomagnetic storms during both solar cycles. It is seen that ICME dominates during solar cycle 23 whereas SH+ICME dominates during solar cycle 24 (Figure 2.3). Both these structures are geoeffective at producing large GIC as shown by the Islington GIC data (Figure 2.4).

The geoelectric fields on the surface of Earth in response to geomagnetic storms is the key parameter in modelling GIC. Geoelectric fields act as an electromotive force in power lines connecting different substations and drive currents through the power network (Figure 2.19). The current flows into or out of ground through transformer neutral/ground connections. A network model uses Kirchhoff's and Ohm's laws to compute the current going through different parts of the network. The most widely used approach is of Lehtinen and Pirjola (1985), which has been followed for model development of the New Zealand network. The model has also been updated as suggested by Boteler and Pirjola $(2014,2017)$ to compute transformer level GIC. 
Different techniques have been adopted to compute geoelectric fields. The complex image method determines both electric and magnetic fields on the surface of Earth from the source currents in the upper highly ionized atmosphere, and the ground magnetic field observatory data using Faraday's Law. Regardless of the techniques to be followed the conductivity structure of the Earth plays an important role and can impact the geoelectric field values significantly. Previously, a thin-sheet conductance model has been used to determine the geoelectric field in the vicinity of the South Island and to model GIC using magnetic field data from Eyrewell observatory. Geoelectric fields derived from a thin-sheet conductance model have limitations both in spatial variations and in frequencies involved in producing large GIC. Thin-sheet models also generally have had near-surface distortions/ galvanic distortions removed. In contrast, geoelectric fields computed directly from the magnetotelluric response tensor contains those distortion and therefore gives more realistic fields in response to magnetic variations. This has been demonstrated by computing thin-sheet and MT computed geoelectric fields for a 5-minute period for a magnetic field variation of $100 \mathrm{nT}$ amplitude directed $30^{\circ}$ East of North. Magnetotelluric geoelectric fields are significantly different both in polarization and magnitude and have more spatial variations compared to thin-sheet calculated geoelectric fields (Figure 2.15 and 2.17). However, the computation of geoelectric fields in the vicinity of a whole network would require a dense array of magnetotelluric data. The availability of MT data both in North and South Island has been discussed. The North Island has plenty of magnetotelluric measurements compared to South Island and these present an alternative technique for GIC modelling for New Zealand. 


\section{Chapter 3: Spectral Approach to GIC Modelling at Islington Substation}

\subsection{Introduction}

During a magnetic storm, intense currents produced in the magnetosphere and ionosphere create a time-dependent magnetic field at the Earth's surface. According to Faraday's law of induction this time varying magnetic field produces geoelectric fields which act as a voltage source at the end points of power lines, producing large currents which can enter the highvoltage power transmission grid and disrupt and damage transformers. Modelling these geomagnetically induced currents (GIC) in a power network is treated in two different steps. The first step is an extension of geophysical studies and ultimately leads to evaluation of surface geoelectric fields. It depends on the evolution of systems of plasma currents/electrojets in the magnetosphere and ionosphere, and a knowledge of the ground conductivity structure. The second step is about developing a model of the power transmission network which take these geoelectric fields as input and computes the GIC going to individual transformer windings. This second step deals with the resistive parameters of the power lines and transformers, their topology in the substations, and the orientation of the power lines. Once all network parameters are known a network model can be developed (Boteler and Pirjola, 2014; 2017). Usually, a lot more effort is required in mapping the geoelectric fields, and different techniques for geoelectric field computation have been developed. These include the thin sheet approach of Vasseur and Weidelt (1977), plane wave model using a uniform conductivity of the Earth (Viljanen and Pirjola, 1989), the impedance tensor approach (Bonner \& Schultz, 2017) and other empirical methods such as that of Trichtchenko et al. 2004. It is important to mention that different techniques are applicable under different situations.

The South Island of New Zealand is almost unique in having a long record of GIC measurements since 2001 and hence provides a good opportunity for GIC modelling (Mac Manus et al., 2017; Rodger et al., 2017). This chapter utilizes measured GIC data from a transformer at Islington (ISL) 
substation from 2002-2015 to model GIC for a single station. Magnetic field data from the geomagnetic observatory at Eyrewell (EYR) and observed GIC measurements have been used in development of an empirical model. The long record of measurements, which partially encompass two solar cycles (23 and 24), makes it reasonable to directly use magnetic field data for modelling purposes. In this chapter time domain geoelectric fields have been computed and related to measured GIC using scalar constants $a$ and $b$ (Pulkkinen et al., 2007). These network constants represent a linear response of the power network to geoelectric fields at an individual location. This modelling technique is applied to the whole GIC dataset at ISL from 2002-2015. A sign change in the network constants $(a$ and $b$ ) is observed between 2002-2006 and 2011-2015. The spectral components in the measured GIC also do not remain the same during the whole dataset. To better model the high frequency GIC during 2011-2015 we modify the modelling technique based on using two sets of network constants giving a different response of the network to low and high frequency components of the geoelectric field. It is then shown that this use of two sets of constants although required to model Islington GIC data, is not required at other substations, suggesting local changes to the network at Islington are responsible for the observed change in the sign of the network constants.

\subsection{Dataset}

Transpower New Zealand Ltd. manages the national transmission network and has continuously monitored GIC in individual transformers at different substations over many years. These measurements provide a significant and unique database (Mac Manus et al., 2017) for analysis and modelling purposes. For example, Ingham et al. (2017) related measured GIC with recorded geomagnetic variations using frequency dependent transfer functions for different substations. Islington substation has a continuous record of GIC measurements, starting from 2001. ISL lies on the western edge of Christchurch as shown in Figure 3.1. It has multiple transformers, and all are earthed. Since GIC measurement devices are installed on individual transformer windings a letter - ' $\mathrm{H}$ ' or ' $\mathrm{L}$ ', representing high or low connections as explained in Chapter 2.5.2, is used along with a transformer name/number. For example, the long record of measurements starting in 2001 was available for the high voltage winding of T6 transformer therefore denoted as ISL-T6H. The GIC monitoring device is a Hall effect current transducer (LEM model LT 505-S) installed onto the transformer neutral line connection to the Earth (Divett et al., 2017). The GIC record has a 
variable sampling interval that is generally some tens of seconds. However, during periods when the current is changing rapidly the sampling rate increases to approximately one sample every 4 sec (Ingham et al., 2017). For modelling purpose raw GIC data has been interpolated to oneminute uniform intervals. It is important to mention that one-minute interpolate $\mathrm{GIC}$ can significantly reduce GIC values during abrupt GIC variations.

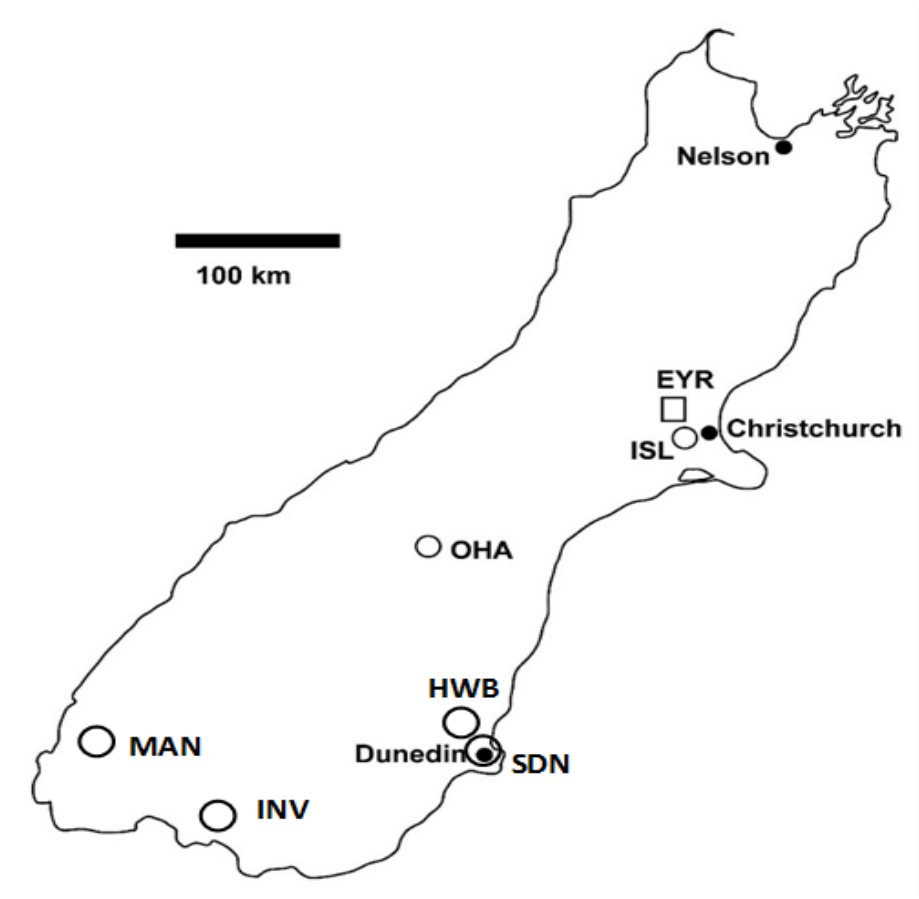

Figure 3. 1: Map of the South Island of New Zealand. Main urban centers are shown by filled circles; ISL and other substations having GIC measurements are shown by open circles. The EYR geomagnetic observatory is shown by the open square.

The magnetic field data used is from Eyrewell (EYR) geomagnetic observatory (43.42 S, 172.35 E), located at West Eyreton near Islington as shown in Figure 3.1. Islington substation and EYR are approximately $20 \mathrm{~km}$ apart therefore the magnetic field data from EYR is appropriate for GIC modelling at Islington. EYR is part of INTERMAGNET (http://www.intermagnet.org/) and is operated by GNS Science, New Zealand. This station provides 1-minute magnetic field data with coordinates $x$ (positive to geographic north), $y$ (positive to east), and $z$ (positive vertically downward) to the INTERMAGNET collaboration, with a resolution of $0.1 \mathrm{nT}$.

For GIC modelling major geomagnetic storms, based on their $K p$ index, have been selected from spaceweatherlive.com (https://www.spaceweatherlive.com/). For each storm, 2048 data points of interpolated GIC and magnetic field data have been selected. As raw GIC measurements are 
at variable sampling intervals, interpolation has been used to produce uniform one-minute interval data. The standard data length of 2048 points (minutes) which covers almost 1.5 days, was selected as it contains both slow and rapid variations of the magnetic field during a geomagnetic storm. It also provides convenience for Fourier Transformation during spectral analysis. GIC measurements during the period of solar minimum have not been included in modelling as GIC values are very small and the data quality is reduced due to noise. There are also no major geomagnetic storms and the period of 2007-2010 has therefore been excluded in our modelling. Since GIC were not available during the whole year of 2001 the dataset considered is from 2002-2006, and, after the solar minimum, from 2011-2015. Table 3.1 shows some selected major geomagnetic storms, the linear correlation coefficient $(r)$ between GIC and both the horizontal components of the magnetic field (denoted by $B_{x}$ and $B_{y}$ ) and the rates of change of the magnetic field (denoted by $B_{x}^{\prime}$ and $B_{y}{ }^{\prime}$ ). Since GIC and the magnetic field are on different scales normalized values have been used to compute the correlation coefficient. The extreme GIC values and the number of data points above a $3 \mathrm{~A}$ threshold during each storm have also been shown.

Table 3.1

Correlation comparison of magnetic field and its derivative with GIC data of ISL during major geomagnetic storms.

\begin{tabular}{llllllll}
\hline $\begin{array}{l}\text { Magnetic } \\
\text { storm }\end{array}$ & $\begin{array}{l}\boldsymbol{K} \boldsymbol{p} \\
\text { Max }\end{array}$ & $\begin{array}{l}\text { GIC above } \\
\text { 3 A }\end{array}$ & $\begin{array}{l}\text { Extreme } \\
\text { GIC values }\end{array}$ & $\boldsymbol{r ( B \boldsymbol { x } )}$ & $\boldsymbol{r}(\boldsymbol{B y})$ & $\boldsymbol{r}\left(\boldsymbol{B}_{x}^{\prime}\right)$ & $\boldsymbol{r ( \boldsymbol { B } _ { \boldsymbol { y } } ^ { \prime } )}$ \\
\hline 23 May 2002 & $8+$ & 13 & 5.6 & 0.52 & 0.21 & 0.54 & 0.56 \\
29 Oct 2003 & 9 & 247 & $+16.7,-12.5$ & 0.38 & -0.11 & 0.64 & 0.49 \\
27 Jul 2004 & $9-$ & 70 & $+10.7,-16.3$ & 0.31 & 0.28 & 0.60 & 0.71 \\
24 Aug 2005 & $9-$ & 68 & $+6.4,-8.1$ & 0.33 & 0.21 & 0.57 & 0.52 \\
26 Sep 2011 & $6+$ & 20 & $+7.4,-6.6$ & -0.21 & -0.14 & -0.79 & -0.52 \\
09 Mar 2012 & 8 & 20 & $+4.6,-5.25$ & -0.19 & 0.09 & -0.70 & -0.40 \\
02 Oct 2013 & $8-$ & 22 & $+7.8,-17.2$ & -0.25 & -0.31 & -0.94 & -0.70 \\
23 Jun 2015 & $8-$ & 50 & $+4.5,-11.6$ & -0.37 & -0.007 & -0.69 & -0.49
\end{tabular}

Table 3.1 depicts GIC characteristics during different magnetic storms. It is evident that GIC characteristics do not appear to be the same for the two time periods 2002-2006 and 2011-2015. In particular, GIC have a negative correlation with the rate of change of the magnetic field for magnetic storms during 2011-2015. This is true for all magnetic storms during this time period, 
not simply those given in Table 3.1. This change in sign of the correlation is believed to be due to a change in the network connections at ISL and is investigated further in the following sections.

\subsection{Data Analysis}

It is well known that large GIC are usually associated with geomagnetic disturbances that have a high rate of change, particularly the rate of change of the horizontal magnetic components e.g., Viljanen, 1998; Bolduc et al., 1998, but this is not always true. For example, Trichtchenko \& Boteler (2006) presented an example from Canada in which GIC resembled the geomagnetic field variation and another example, also from Canada, in which a correspondence between GIC and the time derivative of the geomagnetic field existed. Watari et al., (2009) demonstrated that in a recording in the Japanese power network GIC showed a high correlation with the geomagnetic field variation rather than with the time derivative. However, it is important to note that GIC are not directly proportional to either the geomagnetic field or its time derivatives, but that the relationship is more complicated. GIC measurements at ISL can effectively be utilized to investigate the kind of correlation which exists.

A comparison of GIC with magnetic field $\left(B_{x}\right.$ and $\left.B_{y}\right)$ and the rate of change of magnetic field $\left(B_{x}^{\prime}\right.$ and $\left.B_{y}{ }^{\prime}\right)$ is shown in Figures 3.2 and 3.3 for the magnetic storms of 23 May 2002 and 29 October2003 (given in Table 3.1). The comparison of both panels in each Figure shows that GIC variations appear to be related to both the magnetic field $\left(B_{x}, B_{y}\right)$ and the rate of change of the magnetic field $\left(B_{x}^{\prime}, B_{y}{ }^{\prime}\right)$. The slow time variations or low frequency components (frequency domain) in the GIC appear closely related to changes in the magnetic field itself, whereas abrupt time variations or high frequency components are highly correlated with the rate of change of the magnetic field. It suggests that GIC are related not only to the time rate of change of magnetic field but also to the magnetic field itself. Figure 3.2 and 3.3 shows GIC have a positive correlation with magnetic field, and this is also observed for all other geomagnetic storms during 2002-2006.

The same analysis has also been conducted for the magnetic storms during the period from 20112015. Figures 3.4 and 3.5 shows the same comparison for magnetic storms of 26 September 2011 and 02 October2013. In both these cases the GIC data shows only sharp fluctuations, without any slow time variation, and a strong correlation exists only with the time rate of change of the magnetic field. Note that the GIC and magnetic field variations depict a negative correlation as 
can also be seen in the Figures 3.4 and 3.5, which is also observed for all magnetic storms in 20112015. The flip in the direction of GIC during these periods is attributed to a change in polarity of the current measuring device, LEM, installed on transformer winding.
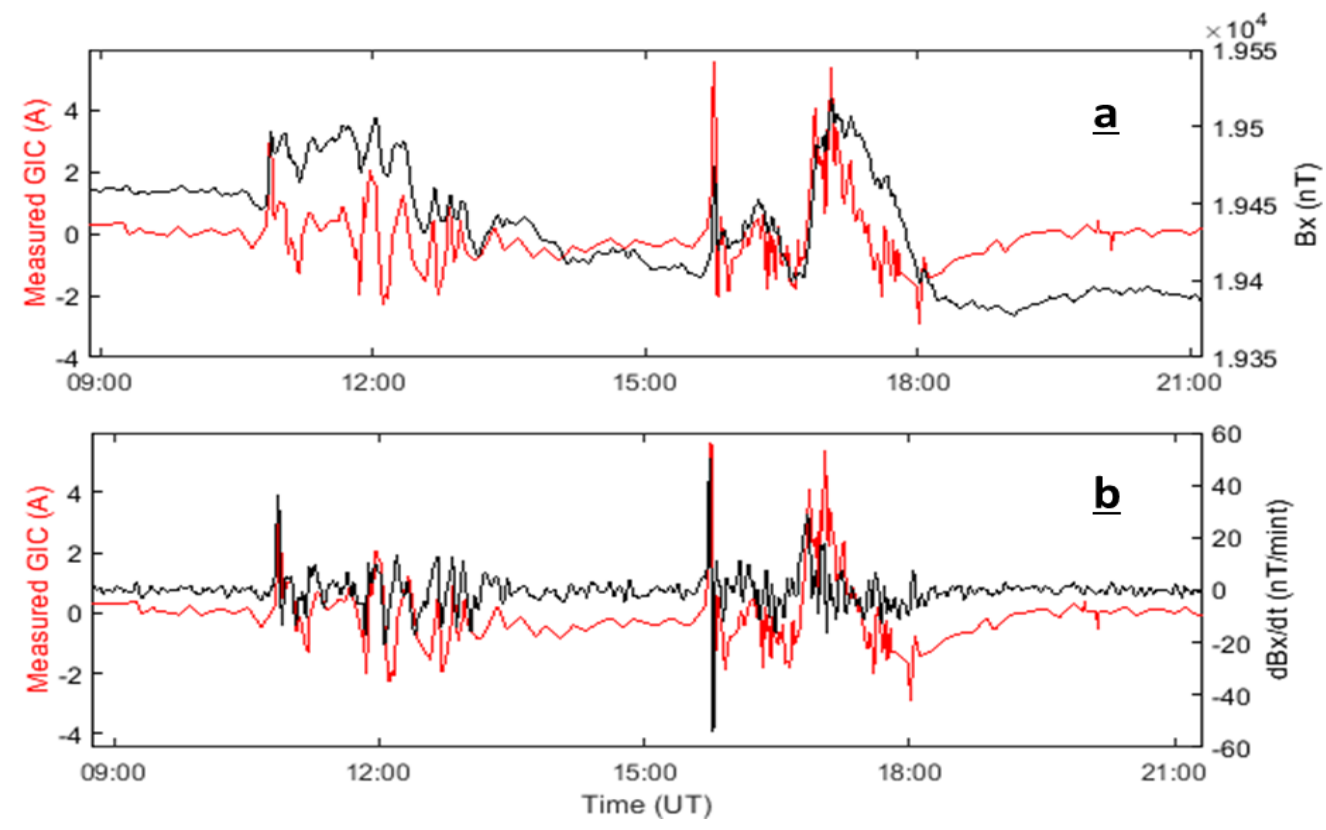

Figure 3. 2: (a) Comparison of GIC (red) and magnetic field $\left(B_{x}\right)$ in black, and (b) comparison of GIC and the time rate of change of magnetic field $\left(B_{x}^{\prime}\right)$ for the magnetic storm of 23 May 2002.
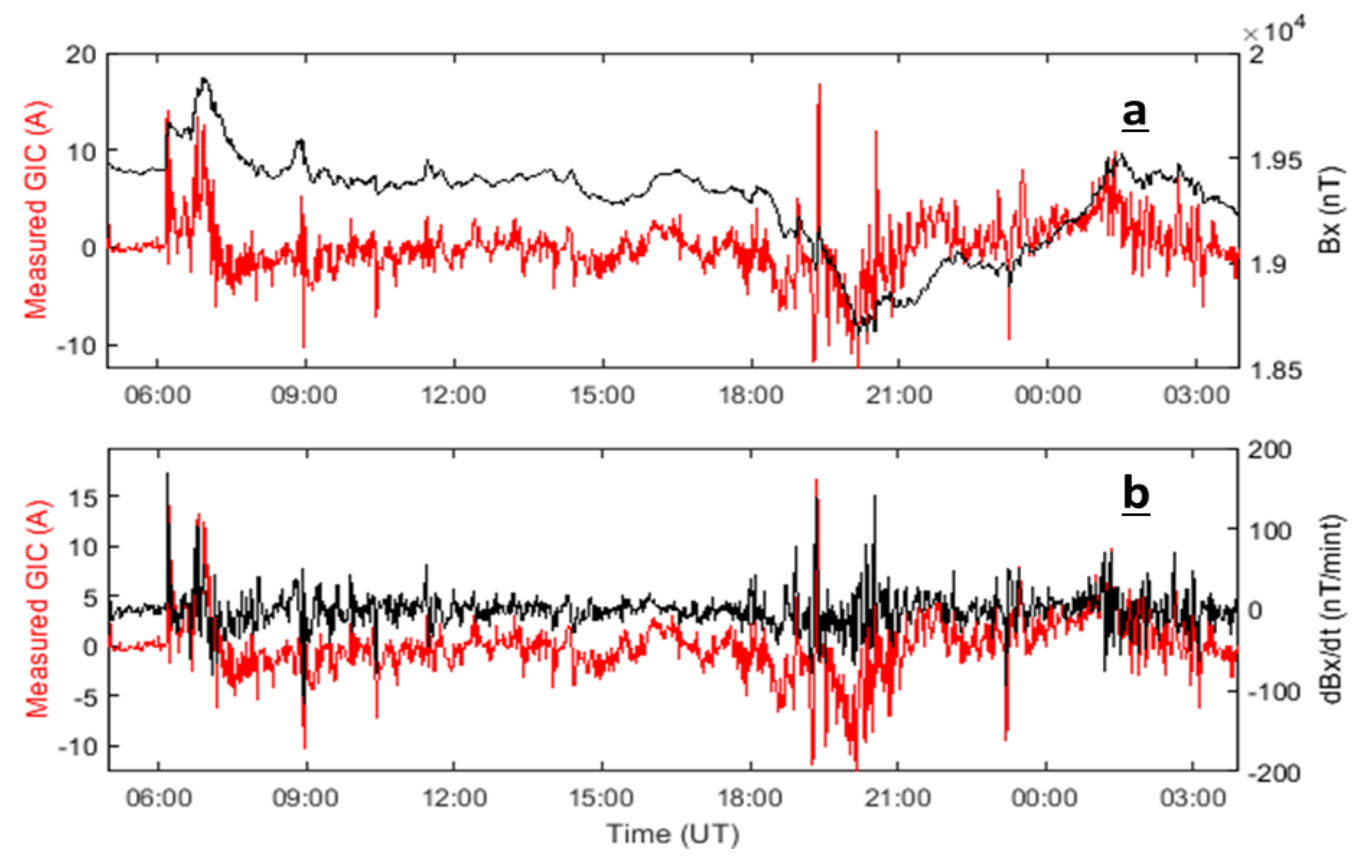

Figure 3. 3: (a) Comparison of GIC (red) and magnetic field $\left(B_{x}\right)$ in black, and (b) comparison of GIC and the time rate of change of magnetic field $\left(B_{x}^{\prime}\right)$ for the magnetic storm of 29 October2003. 

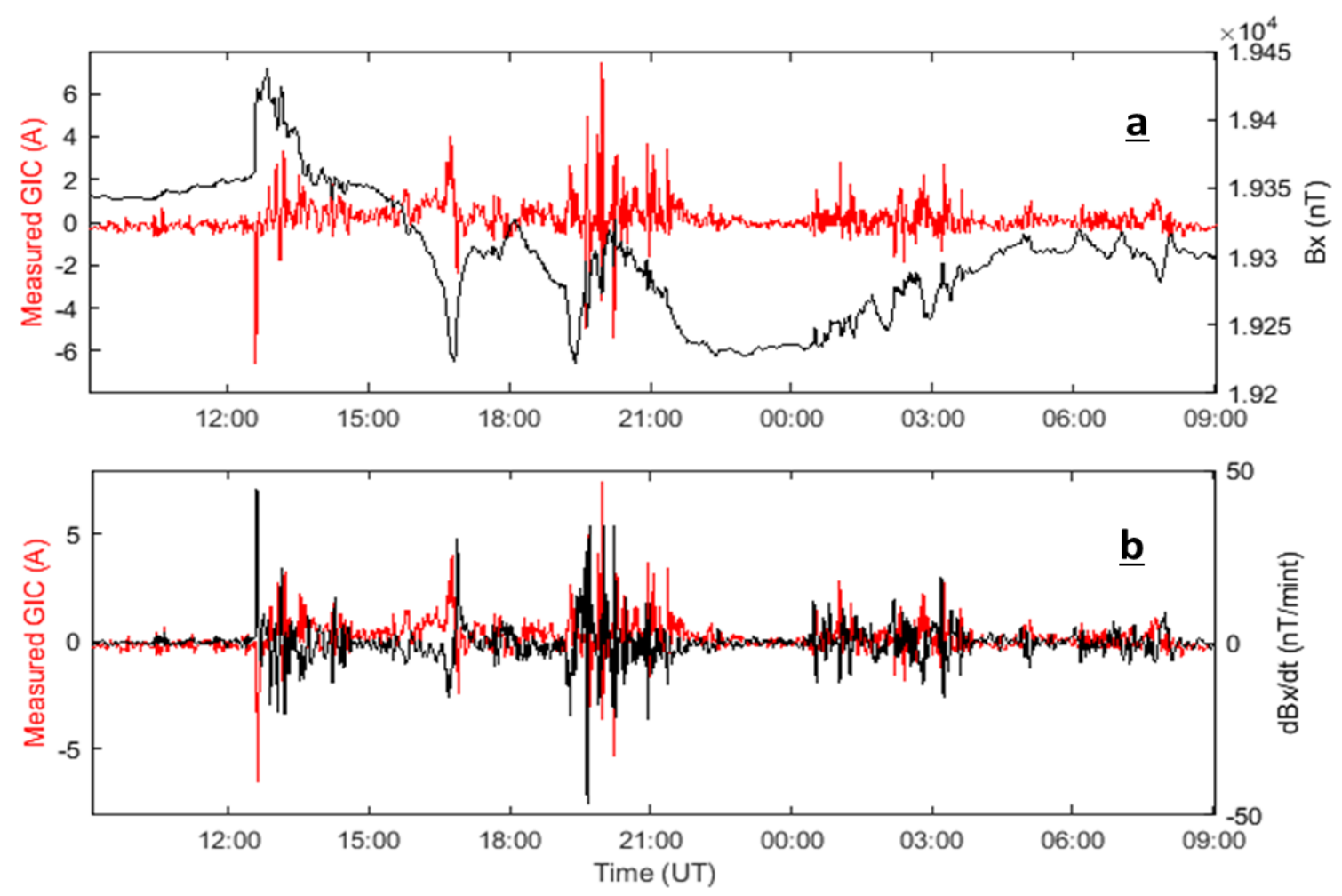

Figure 3. 4: (a) GIC and magnetic field $\left(B_{x}\right)$ and (b) shows GIC and the time rate of change of magnetic field $\left(B_{x}^{\prime}\right)$ for the magnetic storm of 26 Sep 2011.

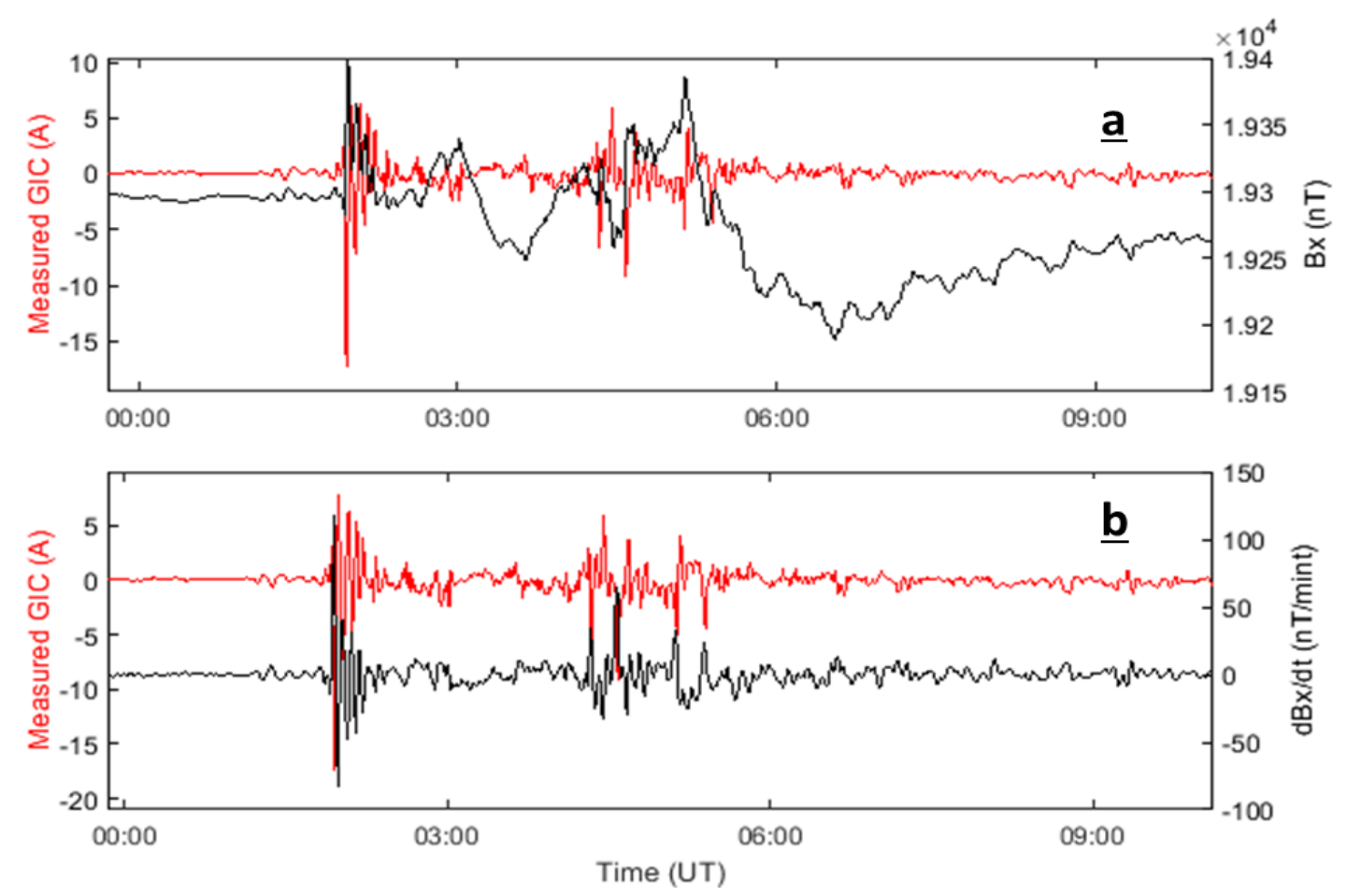

Figure 3. 5: (a) GIC and magnetic field $\left(B_{x}\right)$, (b) shows GIC and the time rate of change of magnetic field $\left(B_{x}^{\prime}\right)$ for the magnetic storm of 02 October 2013. 
The figures 3.2-3.5 indicate that the time variation of measured GIC vary significantly during different magnetic storms, suggesting the amplitudes of frequency components may differ significantly. A frequency domain plot of GIC for a magnetic storm of 23 May 2002 (red) and 26 September 2011 (blue), is shown in Figure 3.6. It shows that the high frequency components of GIC for 26 September 2011 have large amplitudes compared to the 23 May 2002 storm. A linear regression fit (straight line) has been performed to compare the spectral components of both magnetic storms. The slopes of these lines can be used to identify the differences in frequency components in the GIC data. For example, the magnetic storm of 26 September 2011, which has higher amplitudes of high frequency components, has a small slope value compared to the 23 May 2002 storm. To see if the same pattern exists in the geomagnetic data a similar plot for magnetic field component $\left(B_{x}, B_{y}\right)$ is shown as Figure 3.7. The spectra of the magnetic field do not show such a clear change in magnitude of spectral components as seen for the GIC data. This suggests that the difference in frequency components between different years, only prevails in GIC and not in the magnetic field data.

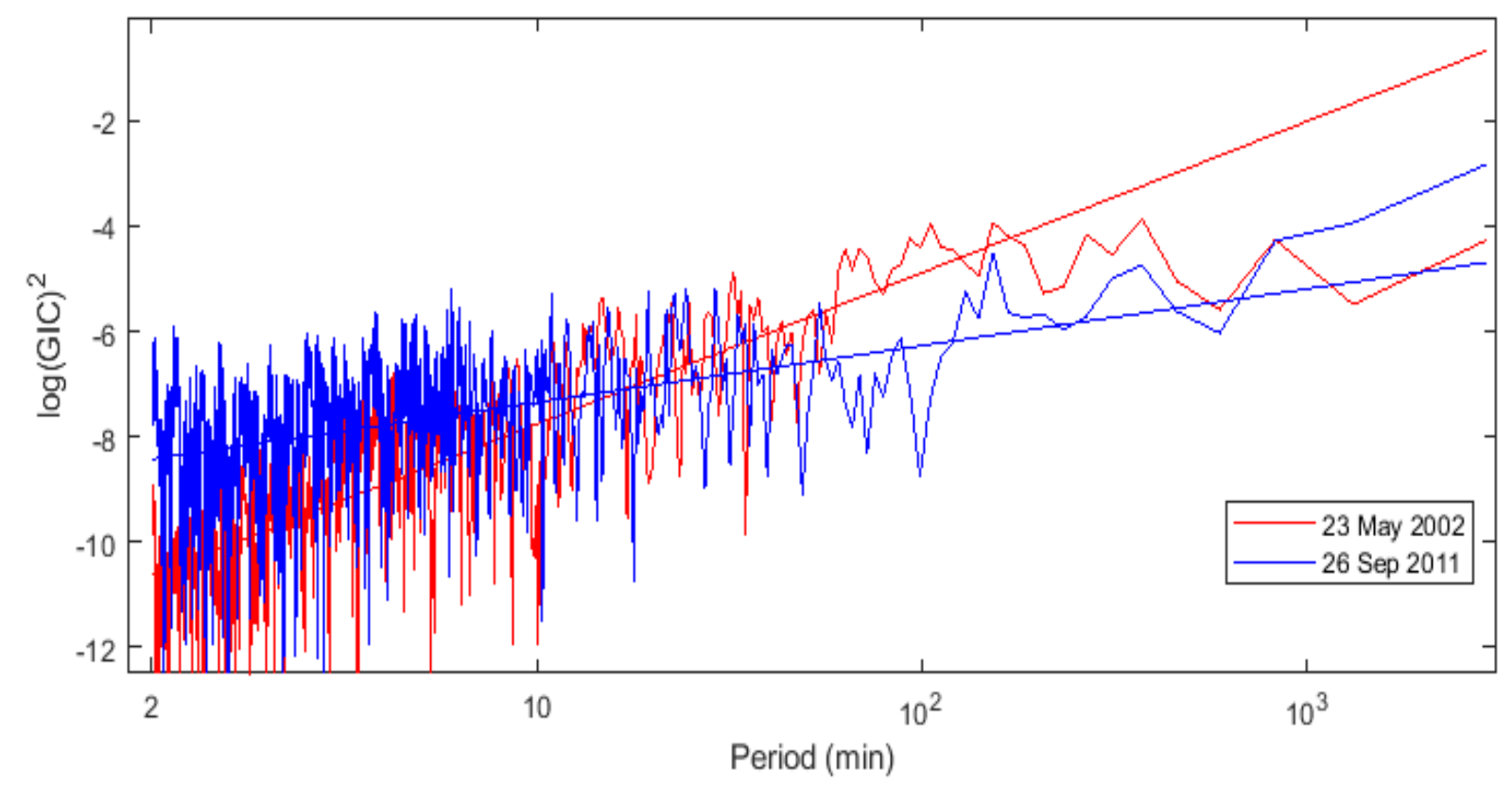

Figure 3. 6: A spectral comparison of GIC measured for two magnetic storms, the period range of data starts from 2 minutes. Red curve (2002) shows small magnitude at shorter periods (high frequency) as compared to blue curve (2011). 


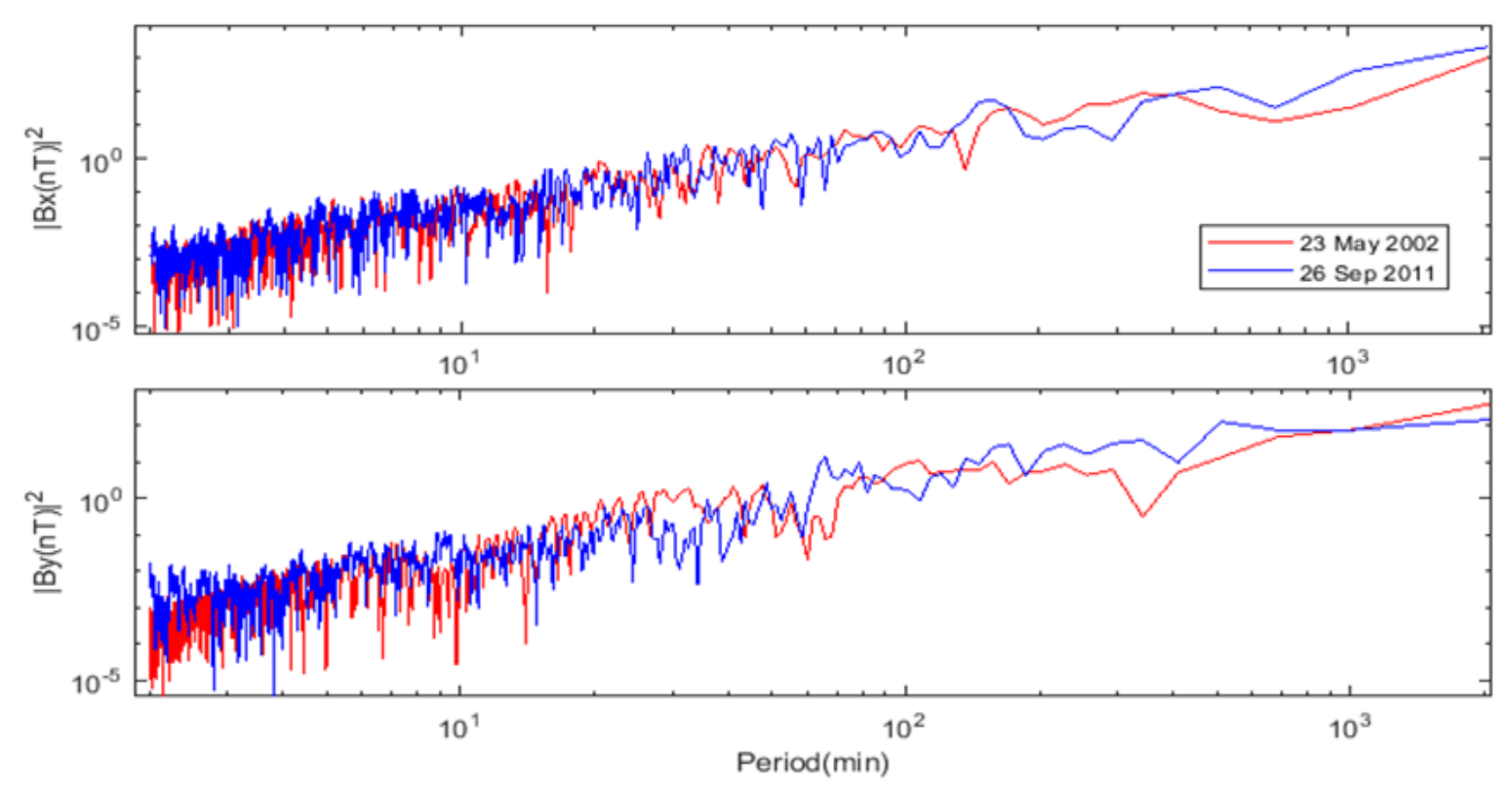

Figure 3. 7: A spectral comparison of the magnetic field $\left(B_{x}, B_{y}\right)$ for the two magnetic storms for which GIC are shown in Figure 3.6.

To see if this situation prevails for all magnetic storms, the spectral slope values for both GIC and the north component of magnetic field $\left(B_{x}\right)$ for all magnetic storms of the dataset have been evaluated and are shown as Figure 3.8. The left-hand axis shows the GIC slope values whereas the right-hand axis shows the slope values for $B_{x}$. The slope values vary throughout the years for both $B_{x}$ and GIC and it is not clear that any patterns exist. A horizontal line is drawn to help visualization of data points above and below the line. It becomes clear that most GIC spectral slope values lying below the line are during 2011-2015. For example, 21 slope values lie below the line during 2011-2016 whereas only 10 do so during 2002-2006. The average slope values are 0.83 and 1.3 for the two intervals, respectively. The spectral slope of $B_{x}$ does not vary much during different years. For example, there are $13 B_{x}$ slope values below the line during 20022006 and 16 during 2011-2015, with mean values .48 and 2.52, respectively.

It is important to remember that the raw GIC data exists in random sampling intervals (varying from fraction of seconds to minutes) and an interpolation was carried out to convert the raw data into a uniform one-minute sampling interval. The above analysis was presented based on the interpolated GIC results. It could be argued that the difference in frequency content may be due to interpolation. 


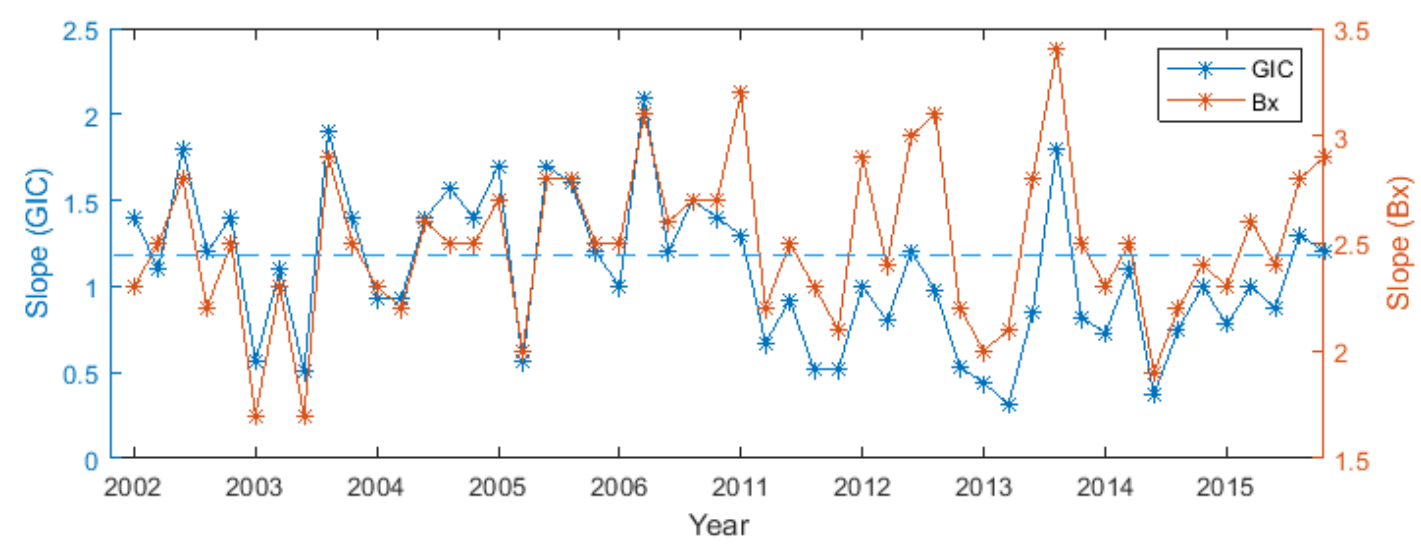

Figure 3. 8: A comparison of slope values for both measured GIC (blue) and magnetic field $B_{x}$ (red) during different years. The slope of GIC reduces as compared to $B_{x}$ during 2011-2015.

To see if slow time variations really exist in GIC, we have taken raw GIC and plotted them as shown in Figure 3.9 and 3.10 for the four magnetic storms under discussion. The first and second panels of Figure 3.9 shows two days of raw GIC measurements for magnetic storms in 2002 and 2003, respectively. The number of data points for the 2002 magnetic storm is smaller ( 470) compared to the 2003 magnetic storm for which there are 9795 data points. The raw GIC data also shows the existence of strong low frequency components as the raw data remain positive or negative for longer durations of time. This can be clearly seen in the close-up view of the GIC data points during the 2003 magnetic storm. Therefore, regardless of the number of data points, low frequency components exist in both storms.

Figure 3.10 shows raw data points for later magnetic storms in 2011 and 2013. The number of data points is 3888 and 2995 for the 2011 and 2013 storms, respectively. Both plots only show rapid variations without any smooth longer period variations. The enlarged veiw, on the right side, for both storms shows data points distribute vertically rather than horizontally as seen for the earlier magnetic storms. The above analysis of GIC measured data shows that the spectral content of the GIC data varies over years for different magnetic storms. The spectral differences between high and low frequency components is quite noticeable between 2002-2006 and 20112015. Further, the spectral content of the magnetic field data of EYR does not show up any significant corresponding difference. Since the magnetic field data is an essential input for computation of geoelectric fields, the above analysis is further extended and investigated by modelling of the GIC. 

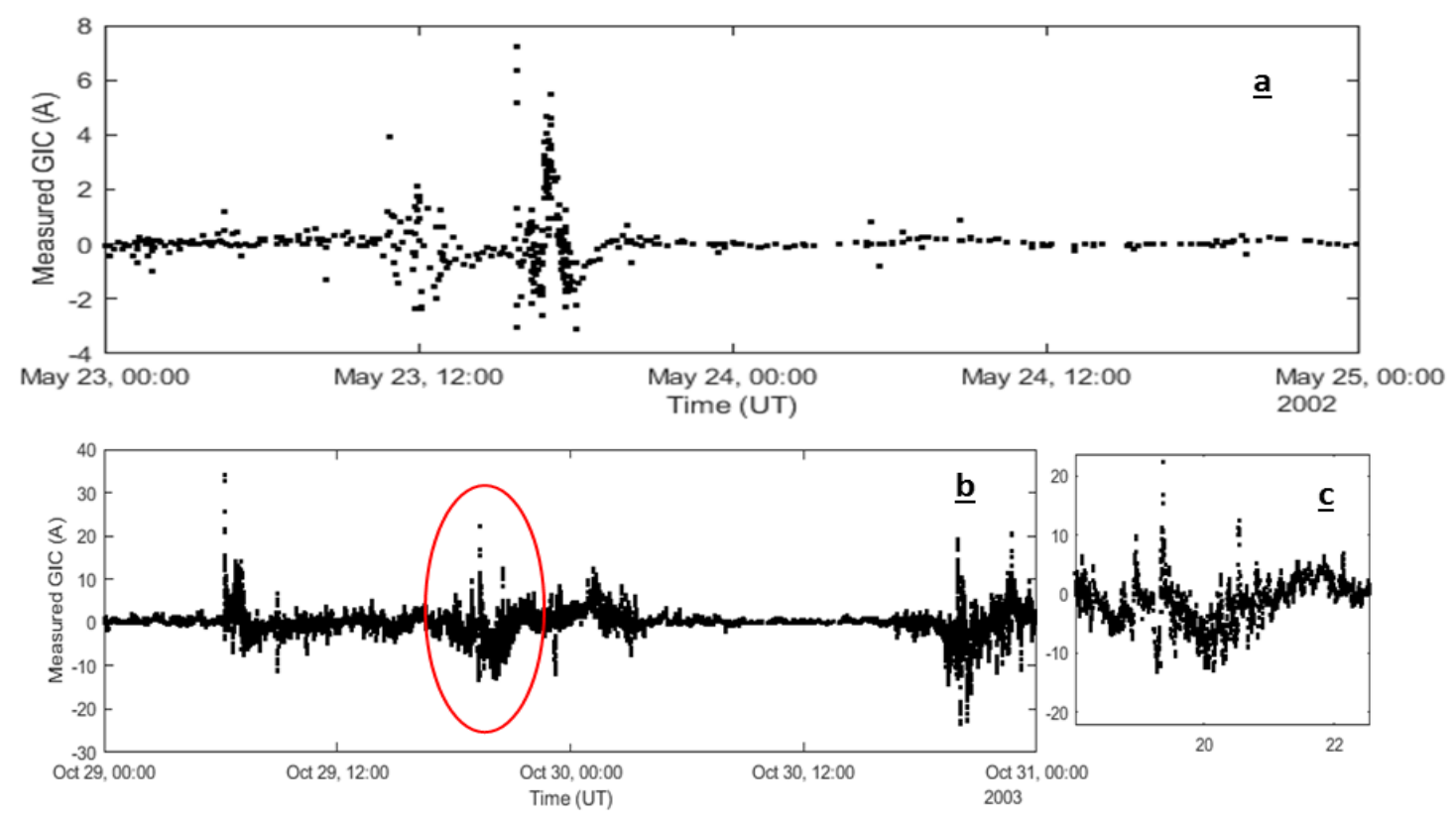

Figure 3. 9: GIC data of ISL showing strong low frequency components during two magnetic storms (a) GIC raw data of for magnetic storms of 23 May 2002, (b) GIC raw data of 29 October2003 where (c) shows close-up view of data points in red ellipse.
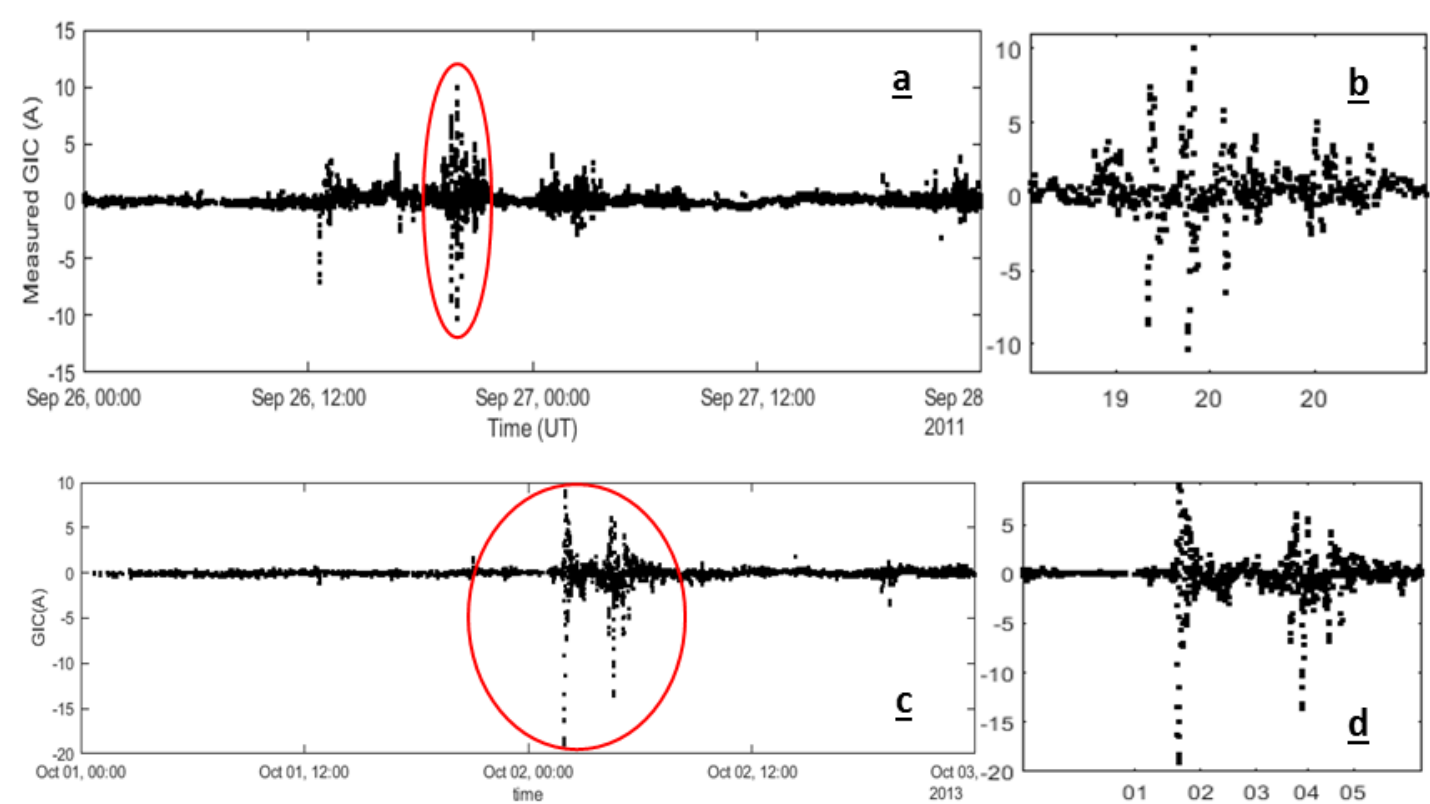

Figure 3. 10: GIC data of ISL with abrupt variations only depicting only high frequency components (a) magnetic storm of $26 \mathrm{Sep} 2011$ and close-up view in (b). (c) magnetic storm of 02 October2013 and close-up in (d). 


\subsection{Methodology}

\subsubsection{Geoelectric Field Determination}

GIC modelling results largely depend on the accuracy of determination of geoelectric fields. Geoelectric fields serve as the input to the power network model. In geoelectric field modelling, surface magnetic fields and the conductive structure of the Earth play an important role. We have followed a simple method given by Viljanen \& Pirjola (1989), which is based on the assumption that the primary magnetic field is a plane wave that propagates vertically downwards on a uniform conductive Earth. In the plane wave approach, the North and East components of the geoelectric field can be computed in terms of the time derivative of the East and North geomagnetic field components, respectively (Pirjola, 2002; Ngwira et al., 2011)

$$
\begin{aligned}
& E_{x}(t)=\frac{1}{\sqrt{\pi \mu_{0} \sigma}} \int_{-\infty}^{t} \frac{1}{\sqrt{t-u}} \frac{d B_{y}(u)}{d t} d u \\
& E_{y}(t)=-\frac{1}{\sqrt{\pi \mu_{0} \sigma}} \int_{-\infty}^{t} \frac{1}{\sqrt{t-u}} \frac{d B_{x}(u)}{d t} d u
\end{aligned}
$$

where $\mu_{0}$ is the permeability of free space, and $\sigma$ is a representative uniform conductivity of the Earth at the site of a substation. The above equations allow that the electric field not only depends upon the time derivative of the geomagnetic field (shown inside the integral), but also on the previous values of the magnetic field. The weighting of previous values decreases with time by the $\sqrt{t-u}$ factor in the denominator. Since we have discrete time domain magnetic field data a numerical solution of the above equations can be utilized to calculate discrete, uniform interval, geoelectric fields. The numerical solution of the above equations given by Vilajanen \& Pirjola (1989) is as follows

$$
\begin{gathered}
E\left(t_{n}\right)=\frac{2}{\left(\sqrt{\pi \mu_{0} \sigma \Delta}\right)\left(R_{n-1}-R_{n}-\sqrt{m} b_{n}-m\right)} \\
R_{n}=\sum_{i=n-m+1}^{n} b_{i} \sqrt{n-i+1}
\end{gathered}
$$

where $n$ shows the time at which the geoelectric field is to be computed. And $b_{n}=B_{n}-B_{n-1}$, where $B_{n}$ is the $\mathrm{n}^{\text {th }}$ value of the magnetic field component, and $\Delta$ is the sampling interval (i.e., 1 minute). The value of $m$ impacts on how much the previous magnetic field values affect the 
current electric field value. As the Islington GIC data appear, at least for the period 2002-2006, to depend upon not only the time rate of change of magnetic field but also previous values of the magnetic field, $\sim 90$ minutes prior values of magnetic field have been selected i.e., $m=90$ minutes.
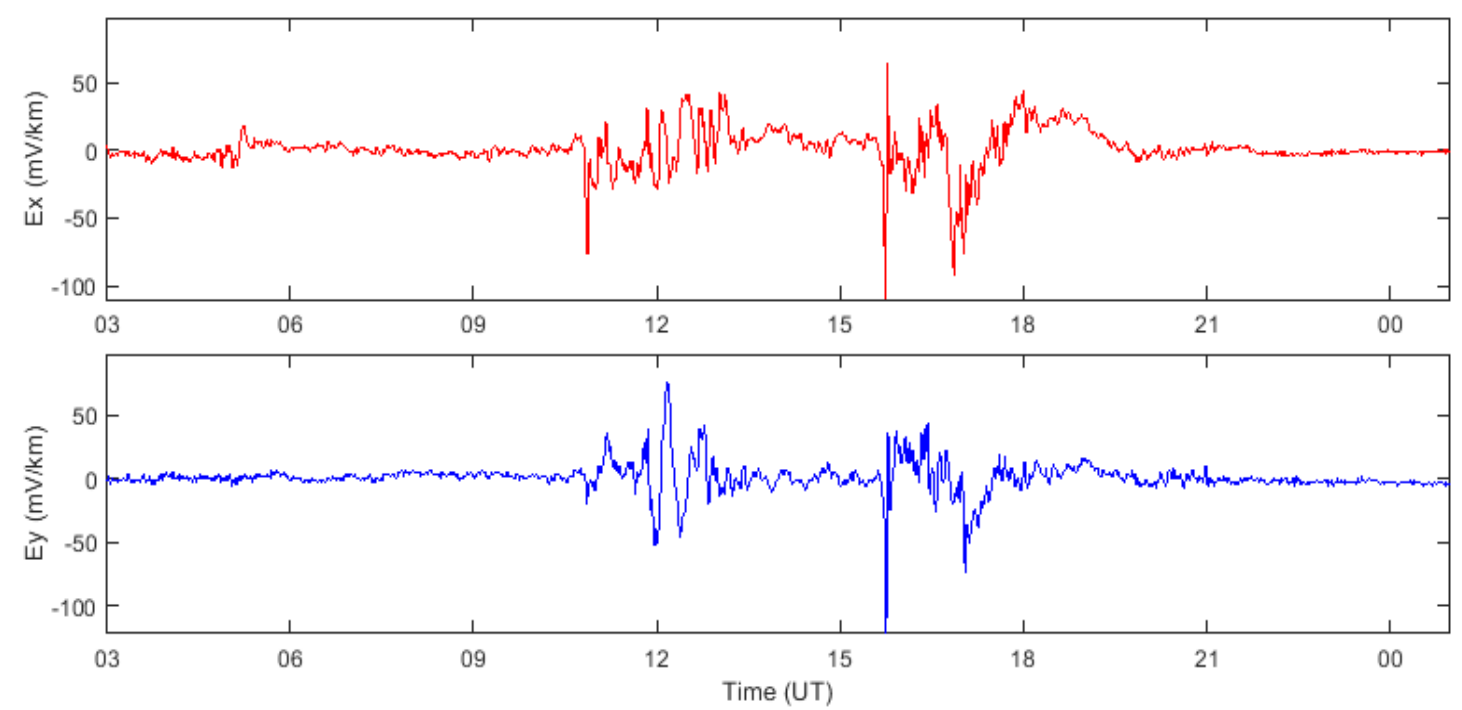

Figure 3. 11: Geoelectric fields computed using a uniform conductivity model for ISL and EYR magnetic field data for the magnetic storm of 23 May 2002.
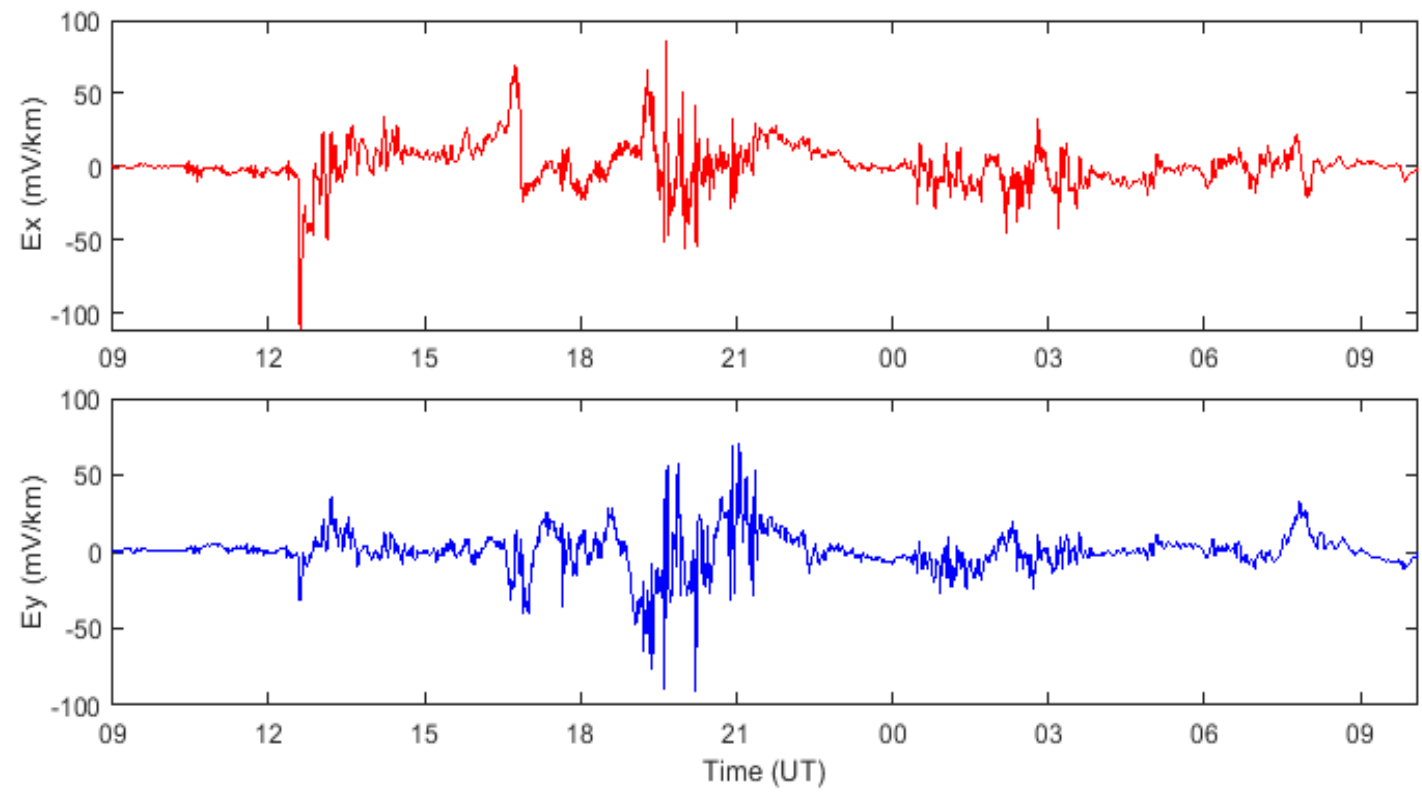

Figure 3. 12: Geoelectric fields computed using a uniform conductivity model for ISL and EYR magnetic field data for a magnetic storm of 26 Sep 2011. 
The conductivity of the Earth $(\sigma)$, is best described using a frequency dependent impedance tensor as has been recently followed by, for example, Torta et al. (2017) and Bedrosian \& Love (2015). However, the available impedance tensor at the closest location to Islington, an MT site measured close to the Eyrewell observatory, does not cover a wide period range, but only the range 2-30 minutes. In comparison, the GIC period range is 2-2048 minutes. For GIC analysis between different years and for a single station it does not in principle matter if a uniform conductivity or the impedance tensor approach is used. Therefore, given the limited period range of the available impedance tensor, we have used a uniform conductivity to represent structure at ISL. We have used an integrated conductivity model based on magnetotelluric measurements across the Canterbury Plains. The conductivity structure of the Canterbury Plains as a whole is relatively uniform and low to considerable depth (Wannamaker et al., 2002). Islington lies in this region and a uniform value of $0.001 \mathrm{~S} / \mathrm{m}$ is regarded as reasonable and has been used in the computation of geoelectric fields.

Using the above equations allows us to compute time domain discrete geoelectric fields corresponding to the magnetic field data from EYR observatory during major geomagnetic storms. For example, Figure 3.11 and 3.12, show plots of both geoelectric field components for the magnetic storms of 2002 and 2011. Geoelectric field magnitude is given in $\mathrm{mV} / \mathrm{km}$, where $E_{x}$ is positive north and $E_{y}$ positive in the east direction. $E_{x}$ depends upon rate of change of $B_{y}$ and vice versa as given by equations (3.1) and (3.2) above.

\subsubsection{GIC Modelling}

The transformer GIC data at Islington and the calculated geoelectric fields can be used to determine the response of the transformer during a magnetic storm. Considering that the electric field is spatially constant in the region of analysis, GIC can be expressed in terms of the relationship to the northward and eastward components of the electric fields (Pulkkinen et al., 2007). This leads to determination of scalar network constants $(a, b)$. Since the horizontal components of the geoelectric fields are mainly responsible for producing GIC, a simple equation relating GIC to electric fields is established as follows

$$
\operatorname{GIC}(t)=a \cdot E_{x}(t)+b \cdot E_{y}(t)
$$


$E_{x}$ and $E_{y}$ are time domain horizontal components of the local geoelectric field and GIC is the measured current going through an individual transformer or substation, $a$ and $b$ are sitedependent system parameters which depend on the electrical characteristics of the system. The units of $a$ and $b$ have been expressed as [A $\mathrm{km} / \mathrm{mV}$ ] based on the units used for geoelectric fields $(\mathrm{mV} / \mathrm{km})$. Network constants define the response of a particular site experiencing GIC to the driving geoelectric field. In this retrospective approach, the values of $a$ and $b$ can be determined by a least squares method given by

$$
\begin{aligned}
& a=\frac{\text { mean }\left(\operatorname{GIC} \cdot E_{y}\right) \cdot \operatorname{mean}\left(E_{x} \cdot E_{y}\right)-\text { mean }\left(\operatorname{GIC} \cdot E_{x}\right) \cdot \operatorname{mean}\left(E_{y}\right)^{2}}{\left(\operatorname{mean}\left(E_{x} \cdot E_{y}\right)\right)^{2}-\left(\operatorname{mean}\left(E_{x}\right)\right)^{2} \cdot\left(\operatorname{mean}\left(E_{y}\right)\right)^{2}} \\
& b=\frac{\text { mean }\left(\operatorname{GIC} \cdot E_{x}\right) \cdot \operatorname{mean}\left(E_{x} \cdot E_{y}\right)-\text { mean }\left(\operatorname{GIC} \cdot E_{y}\right) \cdot \operatorname{mean}\left(E_{x}\right)^{2}}{\left(\operatorname{mean}\left(E_{x} \cdot E_{y}\right)\right)^{2}-\left(\operatorname{mean}\left(E_{x}\right)\right)^{2} \cdot\left(\operatorname{mean}\left(E_{y}\right)\right)^{2}}
\end{aligned}
$$

Therefore, $a$ and $b$, should be treated more like algebraic constants rather than parameters depending on the network characteristics. The values of $a$ and $b$ are computed based on the measured GIC and magnetic field data. Their values could be different during different magnetic storms. Although, values of network constants show the response of the network towards the geoelectric fields, these should not be associated with substation admittance/resistance values.

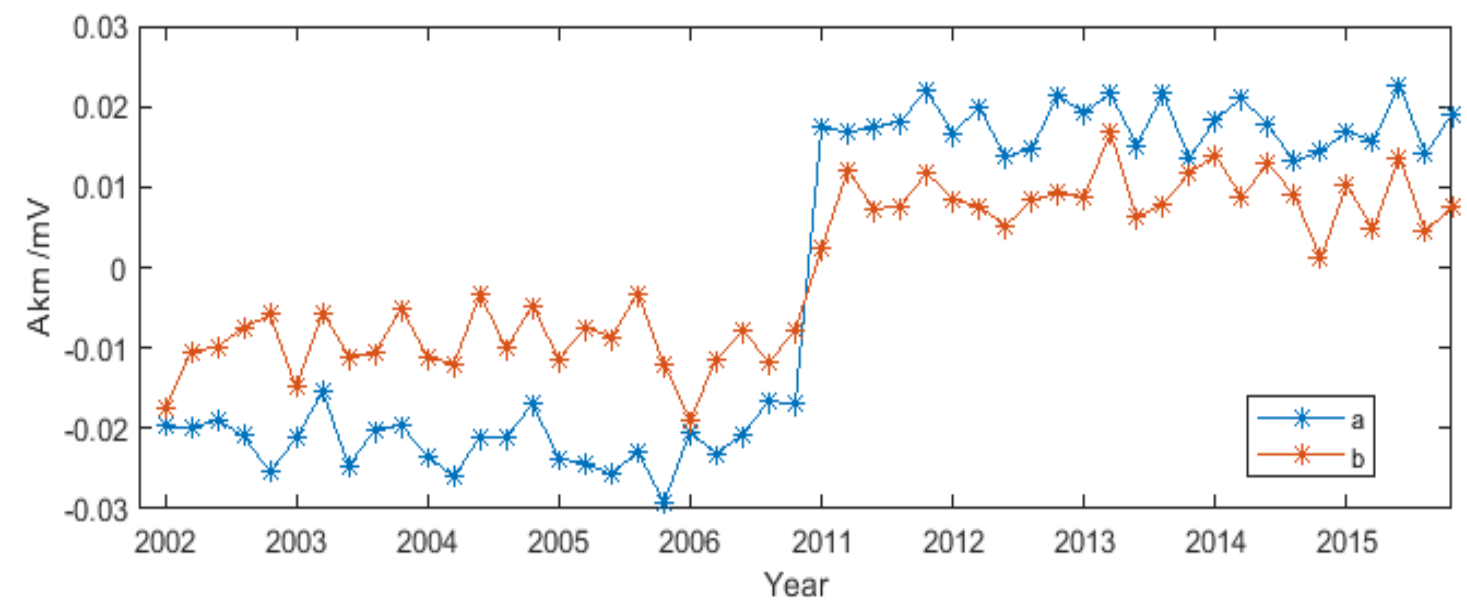

Figure 3. 13: The network constant $(a, b)$ during major geomagnetic storms from 2002-2006 and 2011-2015. The values are negative in 2002-2006 and positive during 2010-2015 showing a possible change in the network. 
The GIC dataset at ISL and model geoelectric fields have been used to determine the values of these constants for a series of geomagnetic storms. Figure 3.13 shows a plot of $a$ and $b$ for 50 geomagnetic storms based on the selected datasets. The values of $a$ and $b$ vary slightly between different storms but overall remain stable. The average values of $a$ and $b$ during 2002-2006 are -0.022 and -0.020 respectively whereas for $2011-2015$ these are 0.018 and 0.009 . This is consistent with the observation that GIC and the magnetic field have negative correlation during 2002-2006 and positive correlation during 2011-2015. The change in sign of $(a, b)$ is attributed to the GIC measuring device (LEM) installed on the transformer windings (Mike, Dalzell, pers. Comm., 2018). This sign change in GIC was also observed in transfer functions given by Ingham et al., (2017).

The robustness of the method is demonstrated by the stability of the network constants, as it can be seen that both constants remain stable during all years. Therefore, the average values of $(a$ and $b$ ) can effectively be utilized for GIC prediction with the information of only the magnetic field data. The slight variation in network values from storm to storm may be due to the presence of noise in the data. For example, among the selected 5 magnetic storms each year the GIC data tend to be dominated by noise during weak magnetic storms. It is important to note that values of $a$ and $b$ may be different for different transformers at the same substation even though the geoelectric fields are essentially the same. This is primarily because of different resistance values in transformer windings and different connections to the network leading to different GIC values for different transformers within the same substation. For example, Divett et al., (2017) examined observations of peak GIC at the Islington substation for different transformers and observed that there is a significant difference in the GIC in individual transformers within the same substation during geomagnetic storms.

\subsection{Model Results}

Model GIC can be calculated using the values of the network constants $(a, b)$, determined during each storm. Here, we have not used average value of $(a, b)$ to compute model GIC but individual values of the network constant, as given in Figure 3.13, have been used. A comparison of model and measured GIC gives an assessment of how well the single station approach performs. Although the correlation coefficient can be used as an index for determining the accuracy of 
model results compared with measured data, it does not account for any scale difference between model and measured data. Therefore, another comparison parameter, the performance parameter $(P)$, has been used by many in the GIC community e.g., Torta et al. (2014, 2017) and Marsal et al. (2017). The advantage of $P$ over the correlation coefficient $r$ is that it takes into account any scale differences between model and measured data. It is defined as

$$
P=1-\frac{1}{\sigma_{m}} \sqrt{\frac{\sum\left[\left(o_{i}-\bar{o}\right)-\left(m_{i}-\bar{m}\right)\right]}{N}}
$$

where $o_{i}$ and $m_{i}$ are discrete values of observed and model GIC, respectively, and $\bar{o}$ and $\bar{m}$ are mean values. The summation is done over the entire time series of $N$ points, and $\sigma_{m}$ is the standard deviation of the measured values.
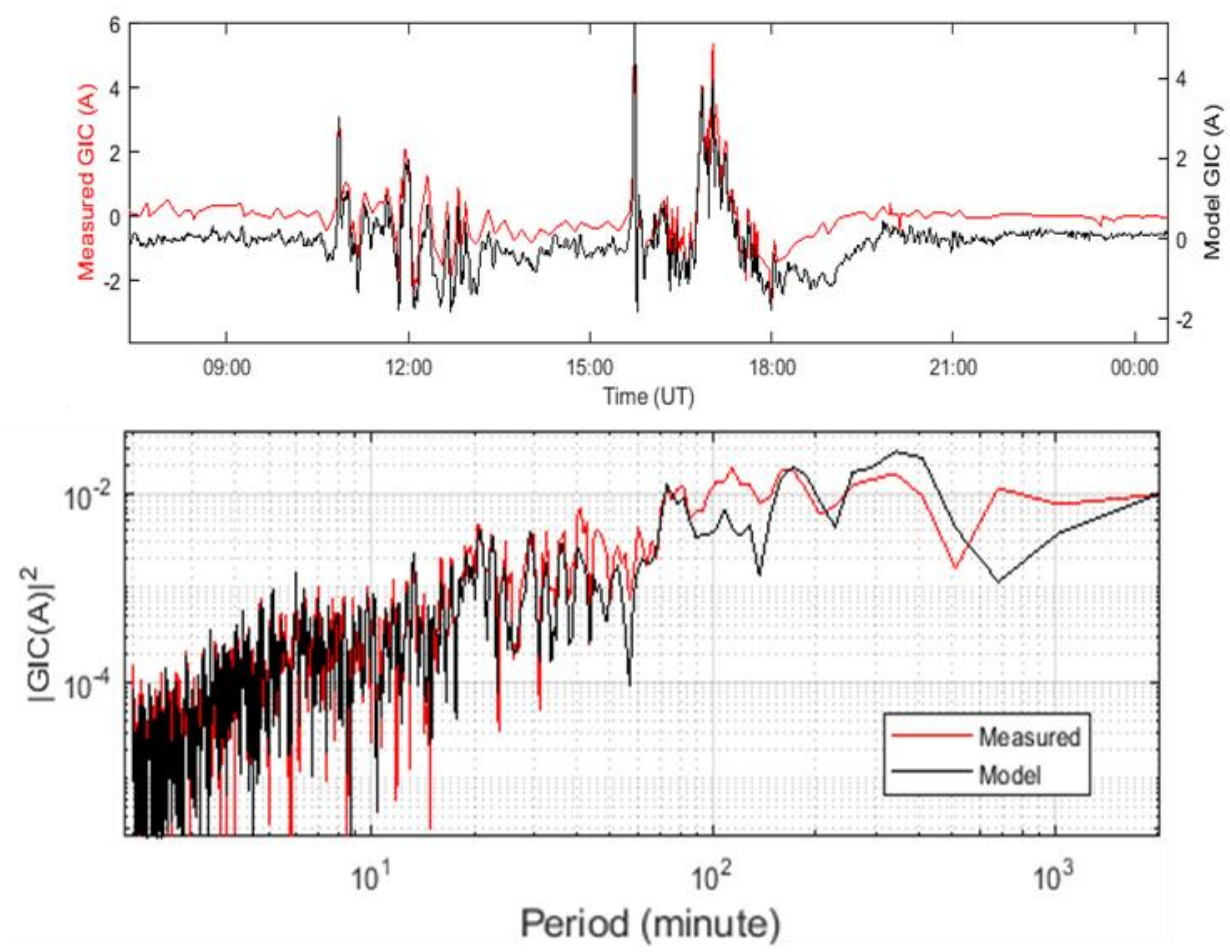

Figure 3. 14: Comparison of model and measured GIC both in time (top panel) and frequency domain (bottom panel) for the magnetic storm of 23 May 2002. The correlation is 0.89 .

Figures 3.14 and 3.15 show the model results and a comparison with measured GIC for the magnetic storms of 2002 and 2003, respectively. The lower panel of each figure shows a spectral comparison of the model and measured GIC. The $x$-axis shows period, given in minutes, and the $y$-axis shows spectral power or magnitude at each period. The upper panel shows that the model 
effectively reproduces most of the observed GIC variations, and this is also depicted by high values of $r=0.89$ and 0.91 and $P=0.42$ and 0.44 , respectively. The model fits both slow and fast time domain variations. The spectral plot also shows that the model spectrum matches well with the measured GIC spectrum for most frequencies, both in high and low frequency bands.
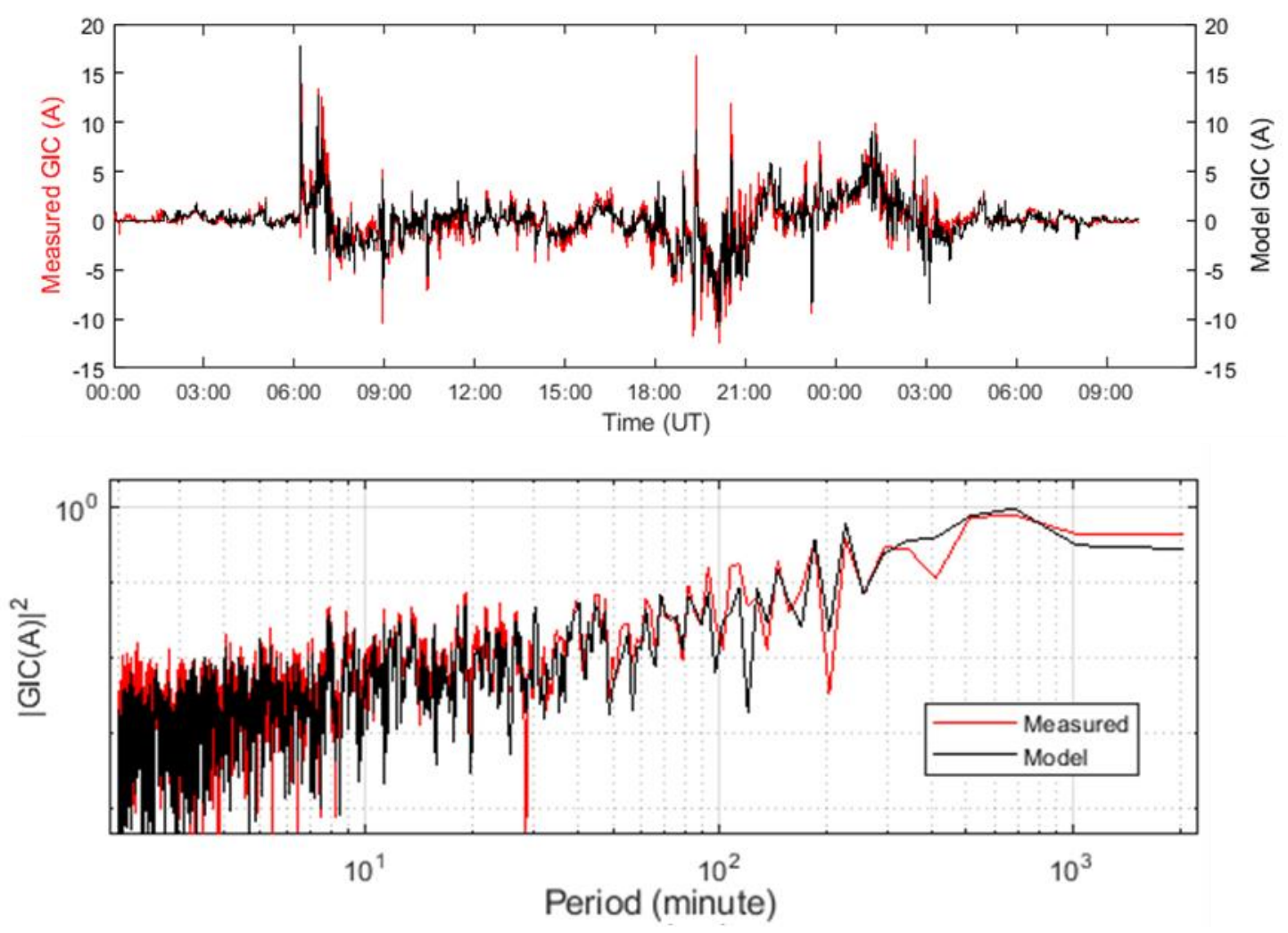

Figure 3. 15: Comparison of model and measured GIC both in time (top panel) and frequency domain (bottom panel) for the magnetic storm of 29 October2003. The correlation is 0.91.

Figure 3.16 shows a comparison of measured and model GIC, both in time domain and frequency domain (first and second panel respectively), for the magnetic storm of 2011. The correlation is 0.70 and the performance parameter is 0.29 . The time domain comparison shows that the model could not effectively reproduce the sharp fluctuations seen in the measured data, particularly during the main phase of the magnetic storm. This can be seen in the spectral comparison as a discrepancy between the magnitudes of model and measured GIC at higher frequencies. The model spectral components (black) are lower in amplitude than the measured GIC (red) at high frequency/ shorter periods. The model fits well at low frequencies. 
Figure 3.17 shows the same comparison of measured and model GIC for the magnetic storm of 2013. The correlation is 0.79 and the performance parameter is 0.32 . The first panel shows that model cannot reproduce amplitudes of sharp/abrupt variations seen in measure data, as was also seen in the Figure 3.16. The second panel shows a clear discrepancy between the magnitudes of model and measured GIC at higher frequencies. The model spectral components (black) are lower in amplitude than the measured GIC (red) at high frequency/ shorter periods. The model also does not fit well at low frequencies when the model amplitude is great than measured data. But the effect due to high frequency components is much more prominent, as seen in the first panel, because the large magnitude GIC are caused by abrupt fluctuations.
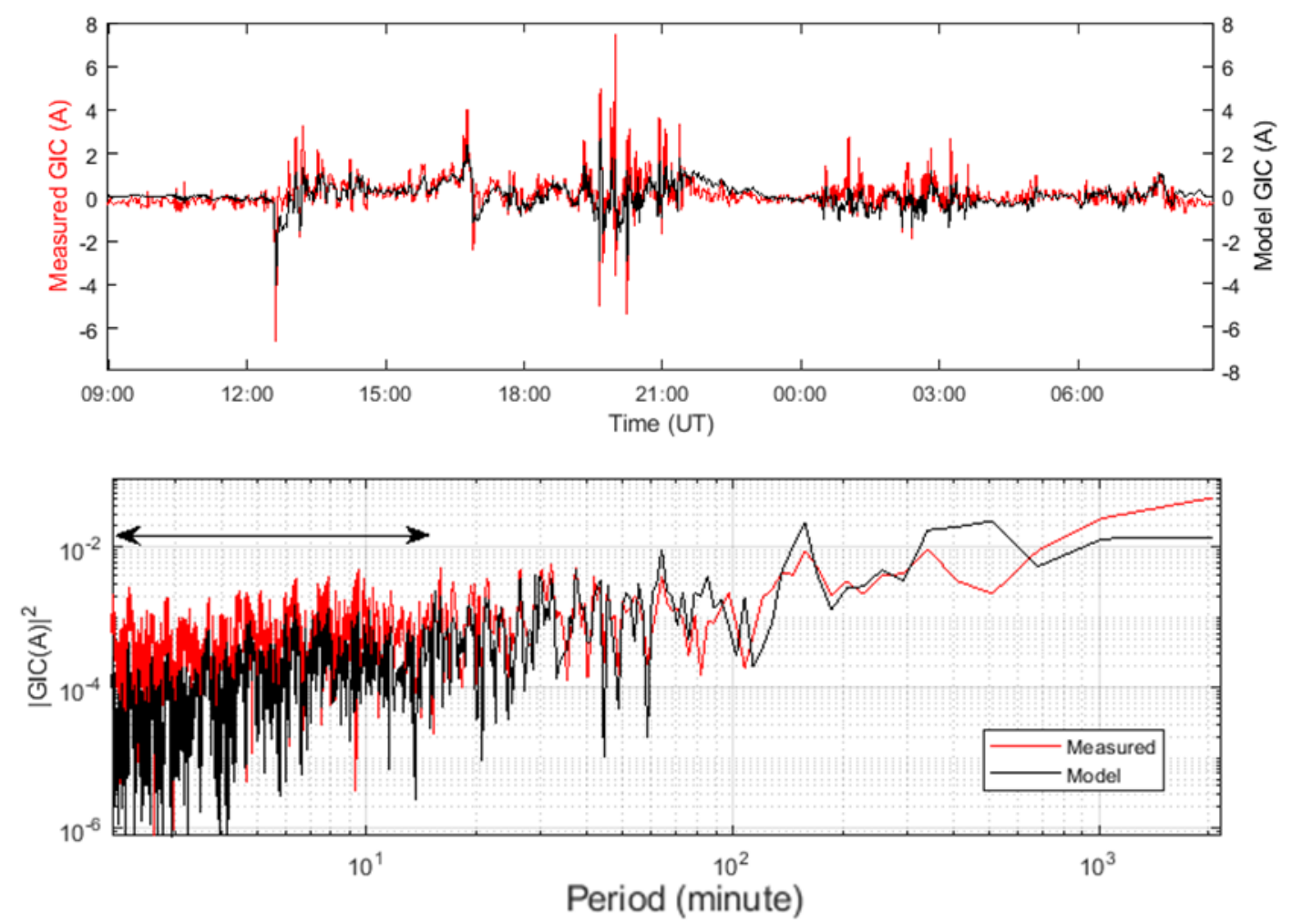

Figure 3. 16: Comparison of model and measured GIC both in time (upper panel) and frequency domain (lower panel) for the magnetic storm of $26 \mathrm{Sep} 2011$. The correlation is 0.70 .

The analysis of the above two magnetic storms shows that the model underestimates the measured GIC in the period range of 2-13 minutes (shown by arrows in Figure 3.16 and 3.17). This is the reason the model cannot effectively reproduce large currents seen in the measured GIC. The spectral comparison also shows that discrepancies could exist in any part of the spectrum, but the high frequency range is important in the context that, large currents in GIC are 
due to abrupt variations during both magnetic storms. Although, there is therefore a non-linear response of the network towards different frequency components of the geoelectric field, it is also important to remember that the geoelectric field itself strongly depends upon conductivity structure of the Earth. The discrepancy seen in geoelectric field and measured GIC may therefore be attributed to both electromagnetic induction and the network response.
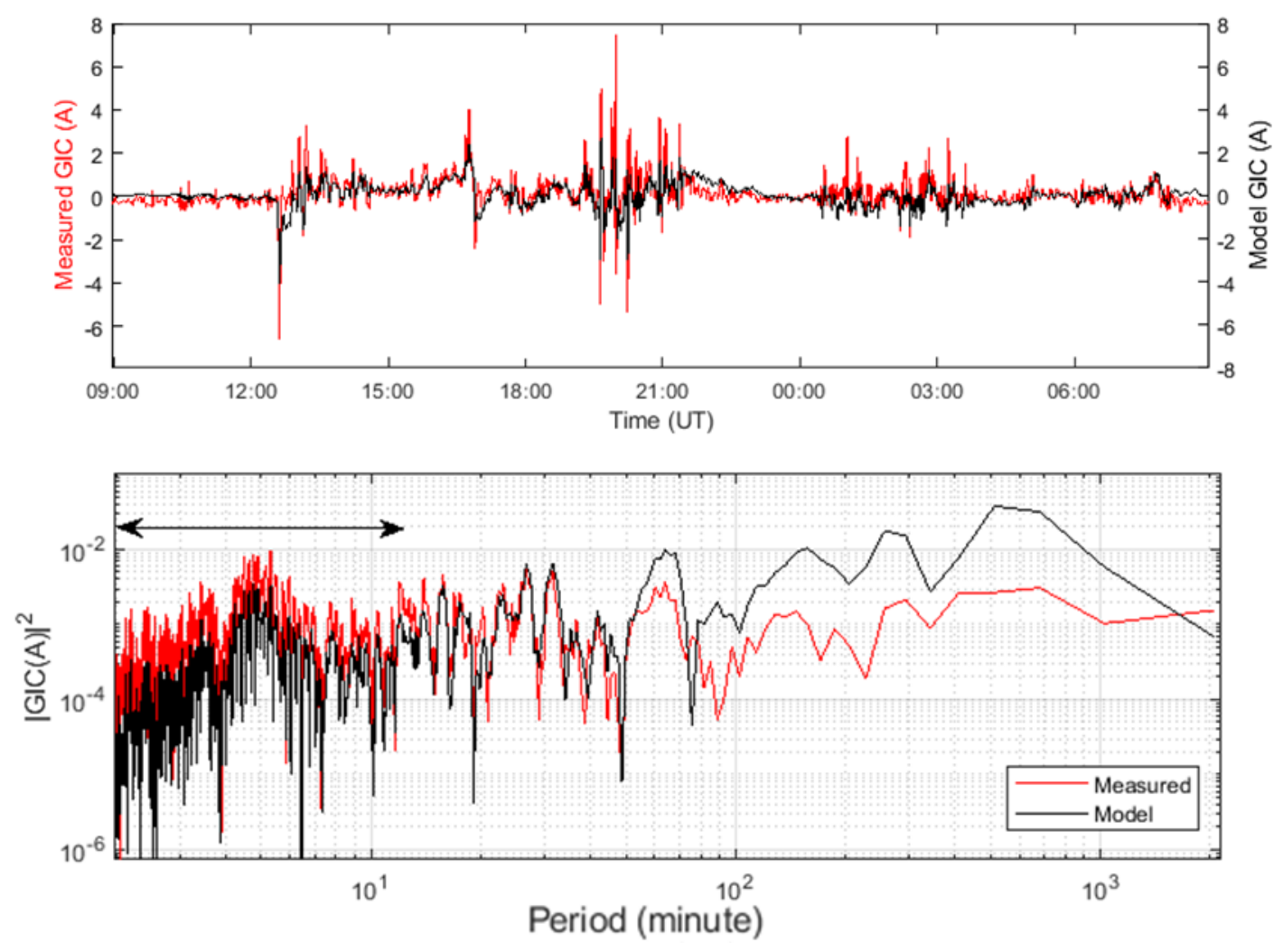

Figure 3. 17: Comparison of model and measured GIC both in time (upper panel) and frequency domain (lower panel) for the magnetic storm of 02 October2013. The correlation is 0.79.

The above results are consistent with the previous observation of GIC slope values which, as shown in Figure 3.8, appeared to be reduced during the years from 2011-2015. Thus, not only is there a change in sign of the network constants between 2002-2006 and 2011-2015, but the pattern of spectral components also changes. The correlation and performance parameter values for all 50 magnetic storms have been computed and are shown as Figure 3.18. Geomagnetic storms occurring from 2002-2007 show a high degree of accuracy in reproducing the model GIC, with an average correlation and $P$ of 0.85 and 0.49 respectively. However, during 2011-2015 the $r$ and $P$ values reduce significantly with average values of 0.72 and 0.32 , respectively. The 
inability of the model to reproduce such high amplitudes corresponding to high frequencies leads to a reduction in both accuracy parameters.

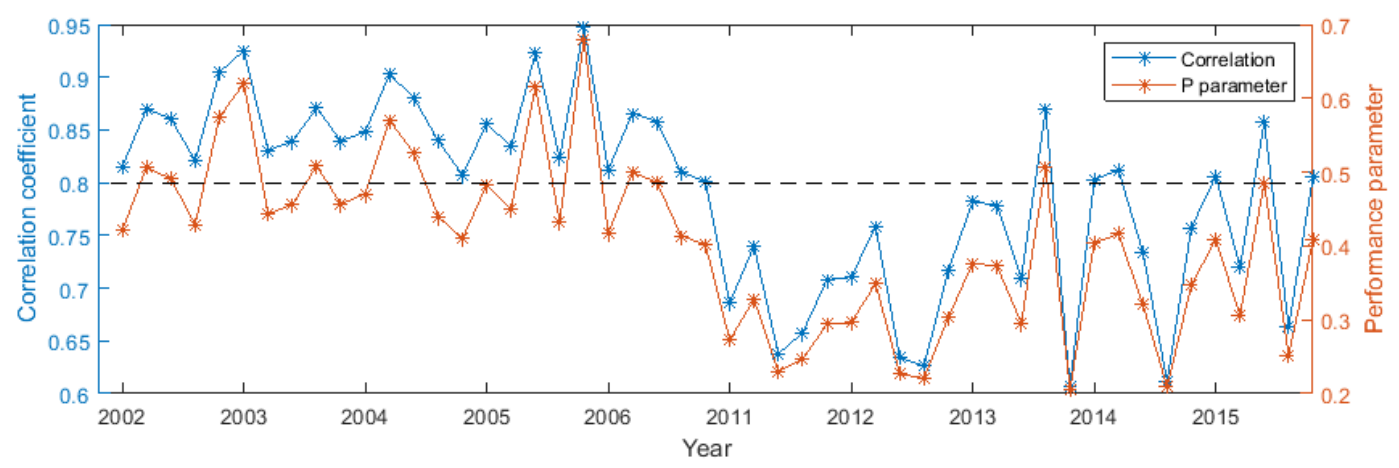

Figure 3. 18: The correlation coefficient and $P$ values between model and measured GIC data during major geomagnetic storms from 2002-2006 and 2011-2015.

In the above modelling technique significant previous values of magnetic field have been included, which results in geoelectric fields embedded with low frequency components. For example, referring to equation 3.3 and 3.4, the value of parameter $m$ is $\sim 90$ minutes, i.e., 90 minutes of prior values of magnetic field are included in the model. Although this gives good results during 2002-2006 magnetic storms, it is seen that the same modelling technique does not work well for 2011-2015 for which measured GIC are dominated by abrupt variations. To try and better model the high frequency variations, we have excluded any previous values of magnetic field (i.e., $m=0$ ) so that calculated geoelectric fields are effectively only dependent on the time derive of the magnetic field. Model GIC have been computed again for the magnetic storms of 2011 and 2013 and the same comparison is given in Figure 3.19 and Figure 3.20. The correlation coefficients are improved from 0.70 and 0.79 to 0.77 and 0.92 , respectively. The model now reproduces the high frequency components seen in the measured data during both storms. The accuracy for the magnetic storm in 2013 is greater than the 2011 storm as the model GIC not only match at high frequency but also at low frequency. Although, for the magnetic storm of 2011 a difference between model and measured spectra appears in low frequency band the large GIC variations are due to high frequency components and the model effectively reproduces the large GIC present in the measured data. The model GIC still carries the low frequency variations seen in the measured data, but the scale is reduced. 

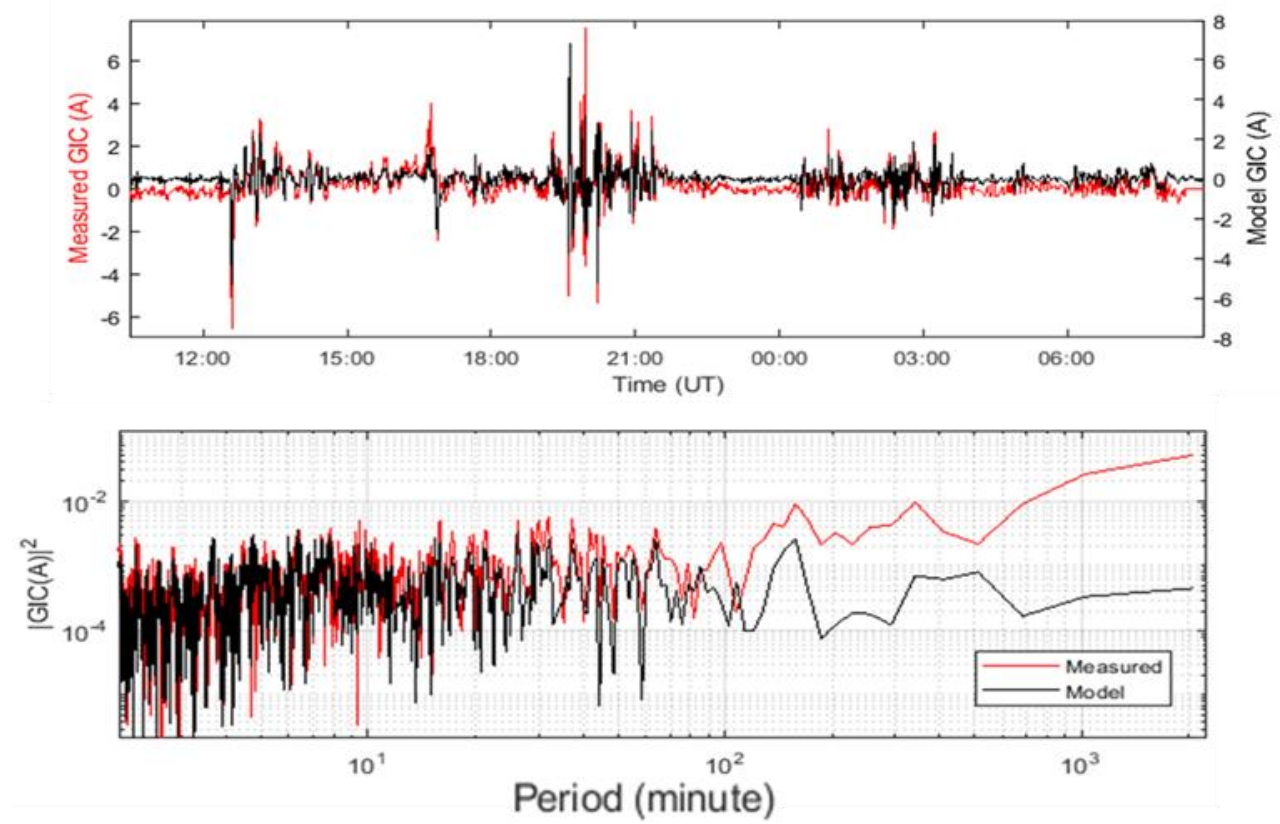

Figure 3. 19: Comparison of measured (red) and model (black) GIC both in time (upper panel) and frequency domain (lower panel) for the magnetic storm of 26 Sep 2011 using geoelectric fields calculated using only the time rate of change of the magnetic field. The correlation is improved to 0.77 .
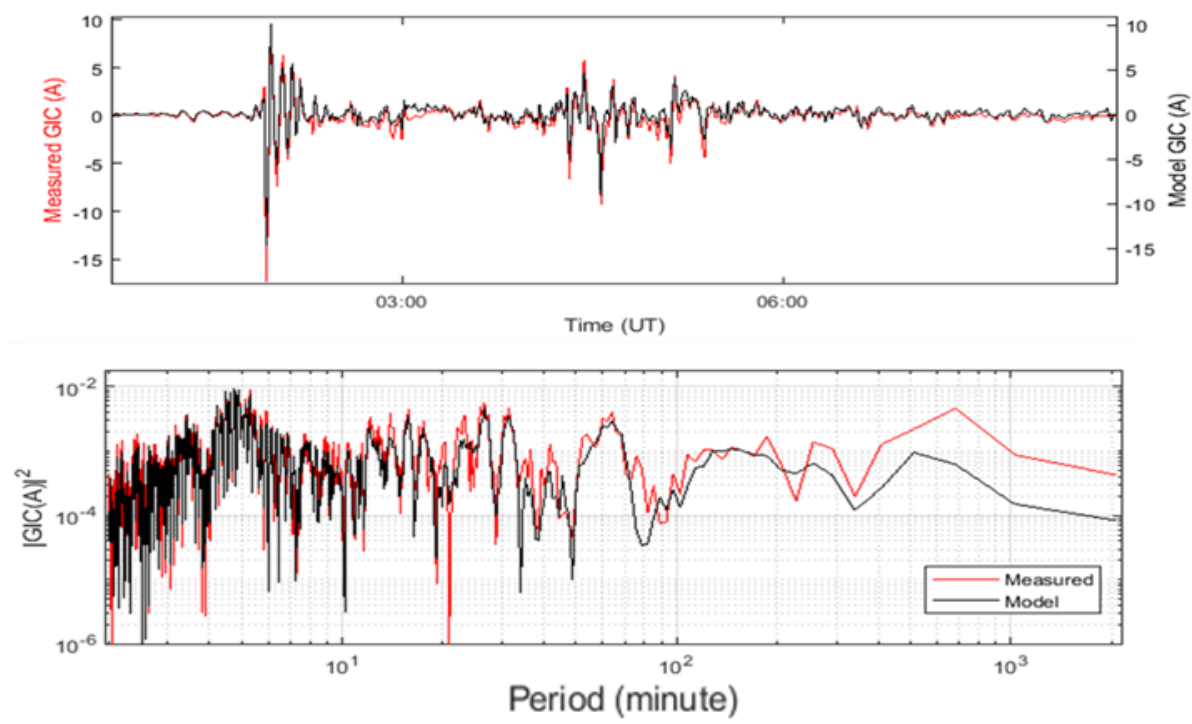

Figure 3. 20: Comparison of model and measured GIC both in time (upper panel) and frequency domain (lower panel) for the magnetic storm of 02 October2013 using geoelectric fields calculated using only the time rate of change of the magnetic field. The correlation is improved to 0.92 . 
We have applied this model, which only contains the time rate of change of the magnetic field, to compute GIC for all magnetic storms of the dataset, and the correlation and $P$ parameter are determined between the model and measured GIC and shown as Figure 3.21. The correlation and performance parameter are improved for later years i.e., 2011-2015, but in doing so the model accuracy for magnetic storms between 2002-2006 is reduced. Now the average value of correlation coefficient for $2002-2006$ is reduced to 0.67 whereas it is enhanced to a value of 0.78 in the period 2011-2015. This essentially confirms a change in the network constants $(a, b)$ over time such that the same site parameters cannot be utilized effectively for the whole dataset.

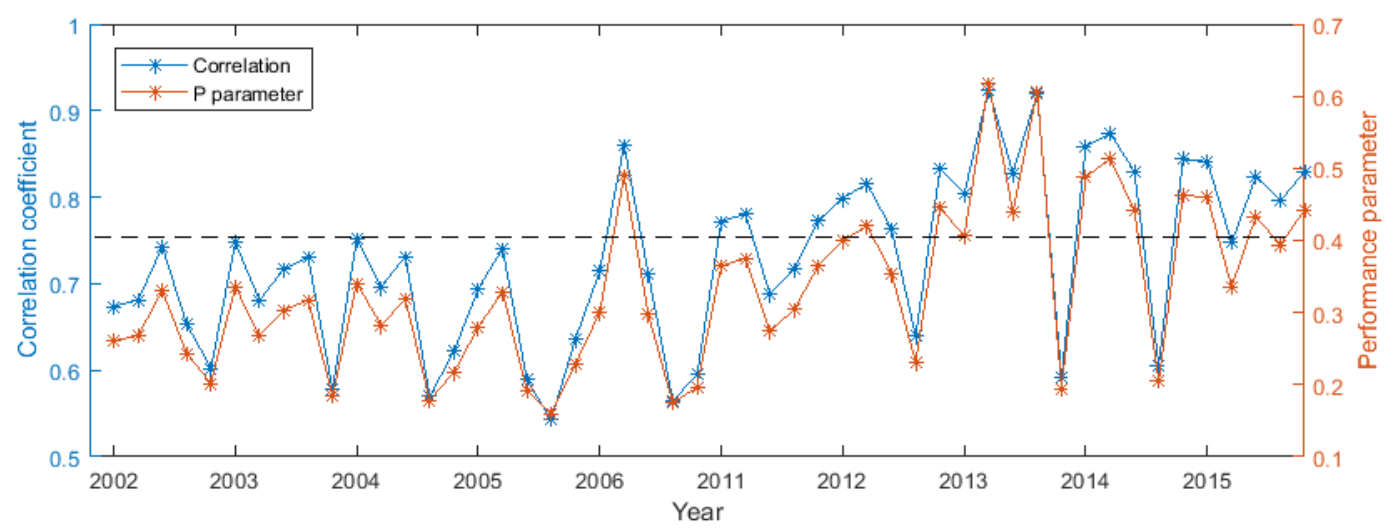

Figure 3. 21: The correlation coefficient and $P$ values between model and measured GIC during 2002-2006 and 2011-2015. The model GIC uses geoelectric fields computed without any previous values of the magnetic field.

To summarize, the same method of computing geoelectric fields is followed for all geomagnetic storms, but a difference in behavior in the GIC data is observed for different time spans. The GIC data show strong components of low frequency variations from 2002-2006 whereas during 20112015 more rapid variations in the GIC data are dominant. This is reflected as a change in the frequency spectra. The frequency change between 2002-2006 and 2011-2015 is only evident in the GIC data and not in the geomagnetic field or geoelectric fields. This behavior, as is the change in sign of $a$ and $b$, is therefore attributed to changes in the network. If the average values of $a$ and $b$ for each period are used for prediction purposes, the model reproduces GIC with almost identical results as using the actual values of $a$ and $b$ for each storm. The model gives an accuracy greater than $70 \%$ which is comparable with the results of the transfer function approach used by Ingham et al. (2017). 


\subsection{A Model Using Different Network Constants at High and Low Frequencies}

In comparing model results with the measured GIC it has been observed that the frequency components of the observed GIC data differ over different periods of time. This is reflected in the network constants which change signs between 2002-2006 and 2011-2015. The accuracy of the model also varies between these two periods. The measured GIC data at Islington shows large values of currents in the GIC data can be in either the low frequency or high frequency components. The model which includes previous values of the magnetic field fits the measured data when low frequency components in measured GIC dominate. The second model, based on only the time derivative of the magnetic field, fits when high frequency components dominate in the measured GIC. The network constants give a linear response of the network to all frequency components of the geoelectric field. However, that is not the case, as has been seen in the detailed modelling results. Therefore, to improve the model results and to develop a single model which fits for all magnetic storms we need to use non-linear network constants or, in other words, network constants which are different for low and high frequency components.

Since each dataset has 2048 data points with one-minute sampling interval, 1024 frequency components are generated using the Fourier Transformation. The frequencies of these components range from DC to $0.0083 \mathrm{~Hz}$, or in terms of periods from DC to 2 minutes. Based upon our analysis we assume that the initial 150 frequency components (DC to 13 minutes period) can be considered as low frequency and the rest are the high frequency components (213 minutes period). To develop a single model suitable for all magnetic storms, the measured GIC and computed geoelectric fields are split into two frequency bands: one regarded as high frequency and other regarded as low frequency. GIC modelling is carried out separately for the low and high frequency bands generating two sets of network constants $(a 1, b 1)$ and $(a 2, b 2)$. Finally, both high and low model spectra of the model GIC are combined, and an Inverse Fourier Transformation is applied to covert back into the time domain GIC. In this way a single time domain GIC is modelled twice using different network constants. The following flow diagram (Figure 3.22) describes the above-mentioned method. 


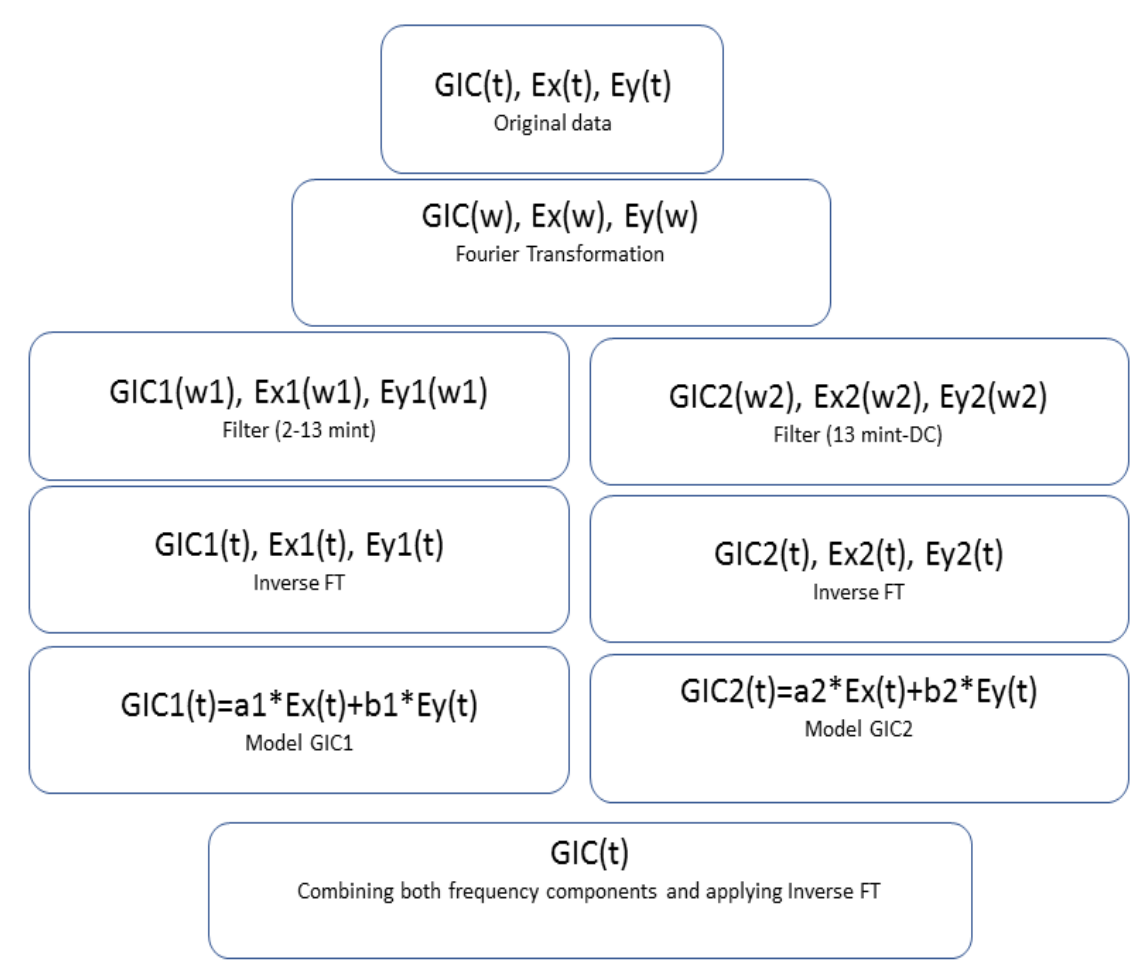

Figure 3. 22: The flow chart of GIC modelling based on using different network constants for low and high frequency components of measured GIC.

The model GIC calculated by following the above-described algorithm are shown in Figures 3.23 and 3.24, for the magnetic storms of 2002 and 2011, respectively. Both panels of the Figure show that the model effectively reproduces both the low and high frequency components showing a single model produces good results for both magnetic storms. The correlation coefficient is good for both magnetic storms and given by 0.90 and 0.79 with the performance parameters 0.55 and 0.37 , respectively. The spectral plots show that the model frequency components match well all the frequency components of the measured GIC, and the differences seen previously do not exist in these modelling results. 

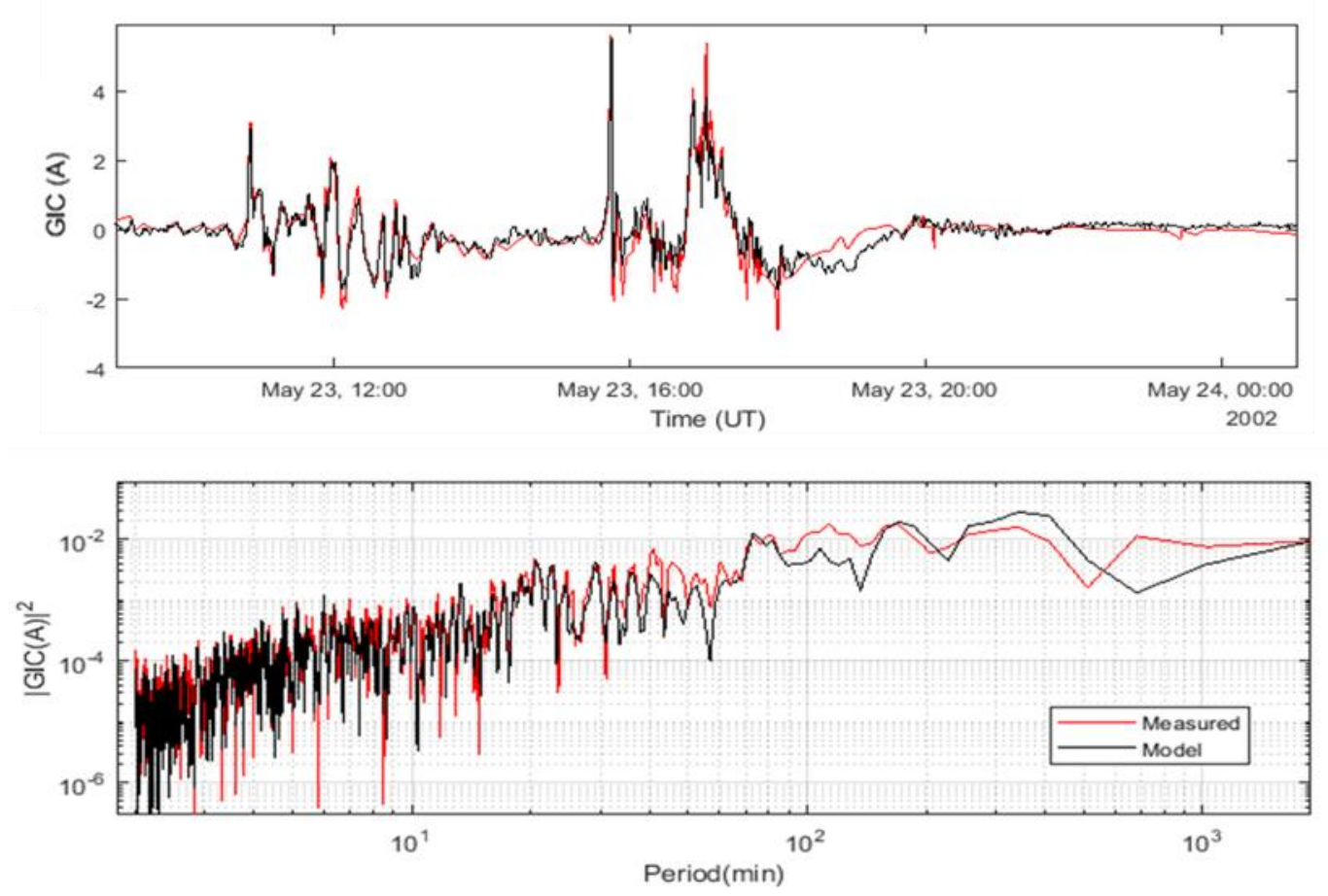

Figure 3. 23: Comparison of measured (red) and model (black) GIC, in time domain (top panel), and frequency domain (bottom panel) using different network constants both for high and low frequency components of measured GIC for the magnetic storm of 23 May 2002. The correlation coefficient is 0.90 .
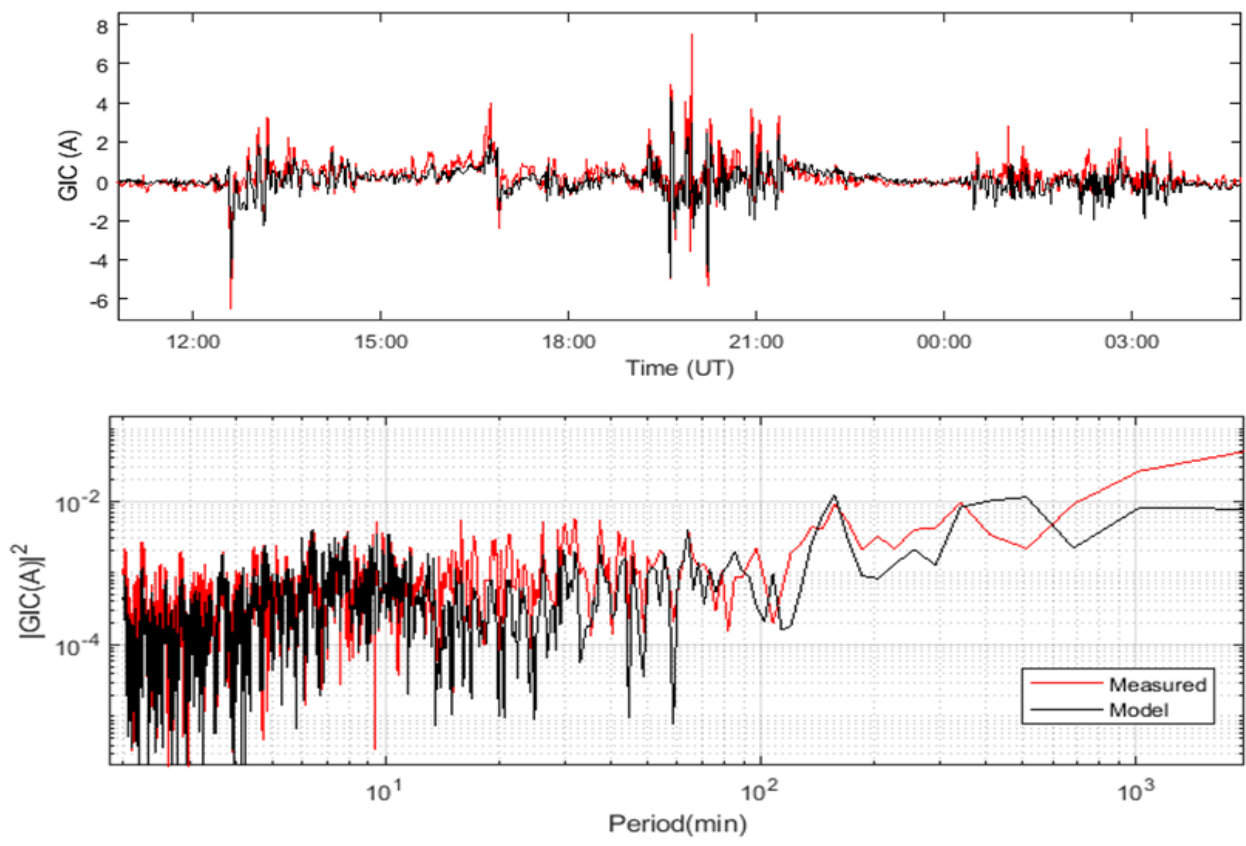

Figure 3. 24: Model results time domain (top panel) and frequency domain (b) using different network constants for high and low frequency components of measured GIC for the magnetic storm of 26 Sep 2011. Correlation coefficient is 0.79. 
This technique has been extended to the rest of the magnetic storms in the dataset and the correlation coefficients and performance parameters calculated. Figure 3.25a shows a plot of correlation coefficient and performance parameter for the 2002-2006 and 2011-2015 magnetic storms. It shows that overall the correlation coefficient is improved for all magnetic storms. For 2002-2006 the accuracy is about the same as for geoelectric fields calculated using previous values of the magnetic field, and for 2011-2015 the accuracy is the same as using geoelectric fields calculated using no previous magnetic field values.

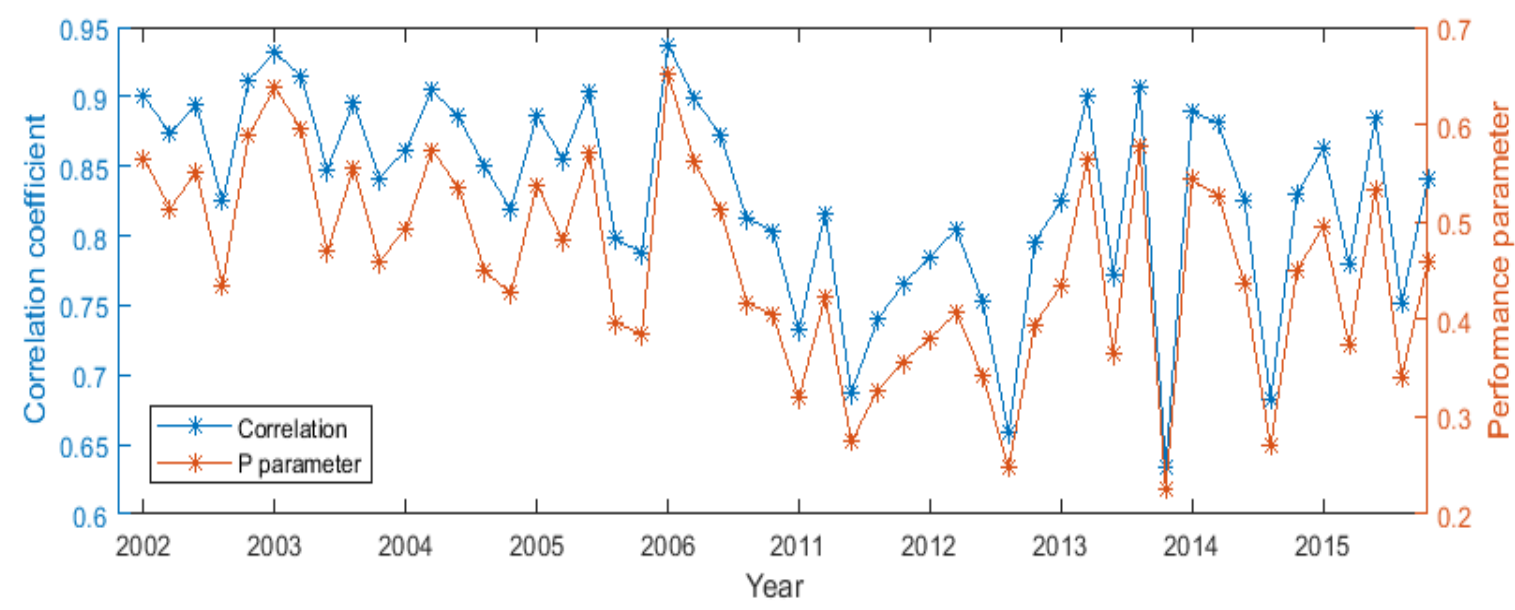

Figure 3. 25a: The correlation coefficient and performance parameter after using different network constants for both low and high frequency components of the measured GIC.
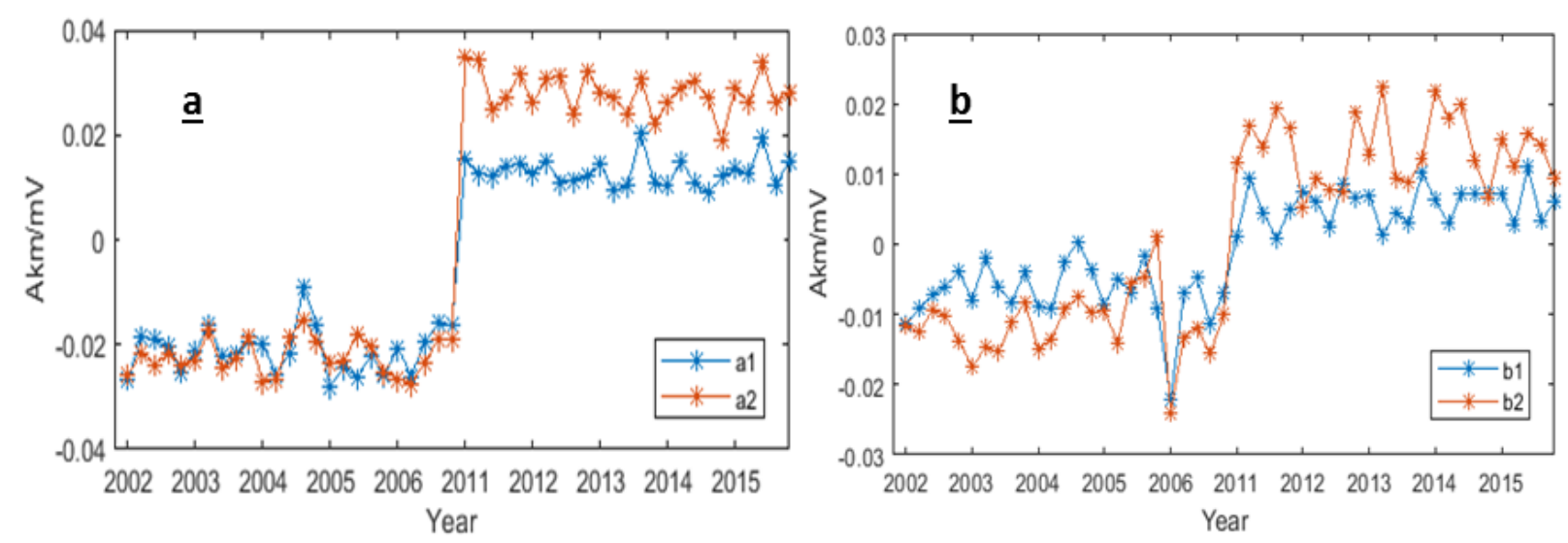

Figure 3.25b: The plots of network constants for (a) low frequency components and (b) for high frequency components. 
The flow diagram in Figure 3.22 shows two different sets of network constant corresponding to high and low frequency bands. For high frequency band these are (a1, b1) and for low frequency band these are $(a 2, b 2)$. Figure $3.25 b$ shows plot of these network constants. The left side panel shows values of $a 2$ are larger than $a 1$ during 2011-2015, which is consistent with the fact that low frequency amplitude in GIC data reduces during this period. Coefficients a1 and a2 are related to the Ex component and these are more stable than b1 and b2 which show much more fluctuation. This may indicate that the Ex (north-component) of the geoelectric field is more closely related to GIC. It could also be due to the orientation of line connections around the ISL substation. Referring to Figure 1.1 of Chapter 1, the transmission line connections of ISL with other substations extends both in north-south and east-west direction. Kikiwa (KIK) substation is north of ISL and the line ( $230 \mathrm{~km})$ follows a route mostly in a north-south direction. Similarly, another line ( $233 \mathrm{~km}$ ) goes south from ISL to Livingstone (LIV) and is also mostly oriented in a north-south direction. Only one major line $(213 \mathrm{~km})$ from ISL to Tekapo-B (TKB) extends equally both north-south and east-west.

\subsection{Islington GIC Comparison with Other Substations}

The above analysis of measured GIC at ISL shows different behavior over different years. To see if the spectral discrepancy seen in the ISL measured GIC data exists in other parts of the power network GIC data from other substations has been considered. As shown in Figure 3.1, ISL substation lies on the east coast in the middle of the South Island. No such long record of GIC measurements are available at any nearby substation. Although Ohau $\mathrm{A}(\mathrm{OHA})$ in the middle of the South Island (Figure 3.1), also has a long record of GIC measurements unfortunately these are not of good quality. Most GIC measurements are available in the southern part of the South Island e.g., South Dunedin (SDN), Halfway Bush (HWB), Invercargill (INV) and Manapouri (MAN) as also shown on the map in Figure 3.1. However, these GIC measurements mostly start only from 2012. Since the measured GIC at Islington exhibit only strong high frequency components during 2011-2015, these substations could be investigated to see if only high frequency components exist in their GIC dataset. Two magnetic storms of 02 October2013 and 17 March2015 have been selected for comparison purpose. Note that SDN and HWB are very close to each other and share many of the same network connections. As a result, the GIC variations at these sites are very similar and differ only in magnitude. Figure 3.26 shows ISL GIC comparison 
with both SDN and HWB for the magnetic storm of 2013. The largest magnitude of current is at 0157 UT and given by $-17.3,-34.1$ and -48.7 A for ISL, SDN and HWB, respectively. To compare low frequency components, it is important that all GIC should be on the same scale. Therefore, all three GIC have been normalized based on their maximum values and are shown as the second panel of the same Figure where the maximum value of each GIC is $1 \mathrm{~A}$. The bottom panel shows both SDN and HWB have low frequency components. Although these are very weak, they are not equal.
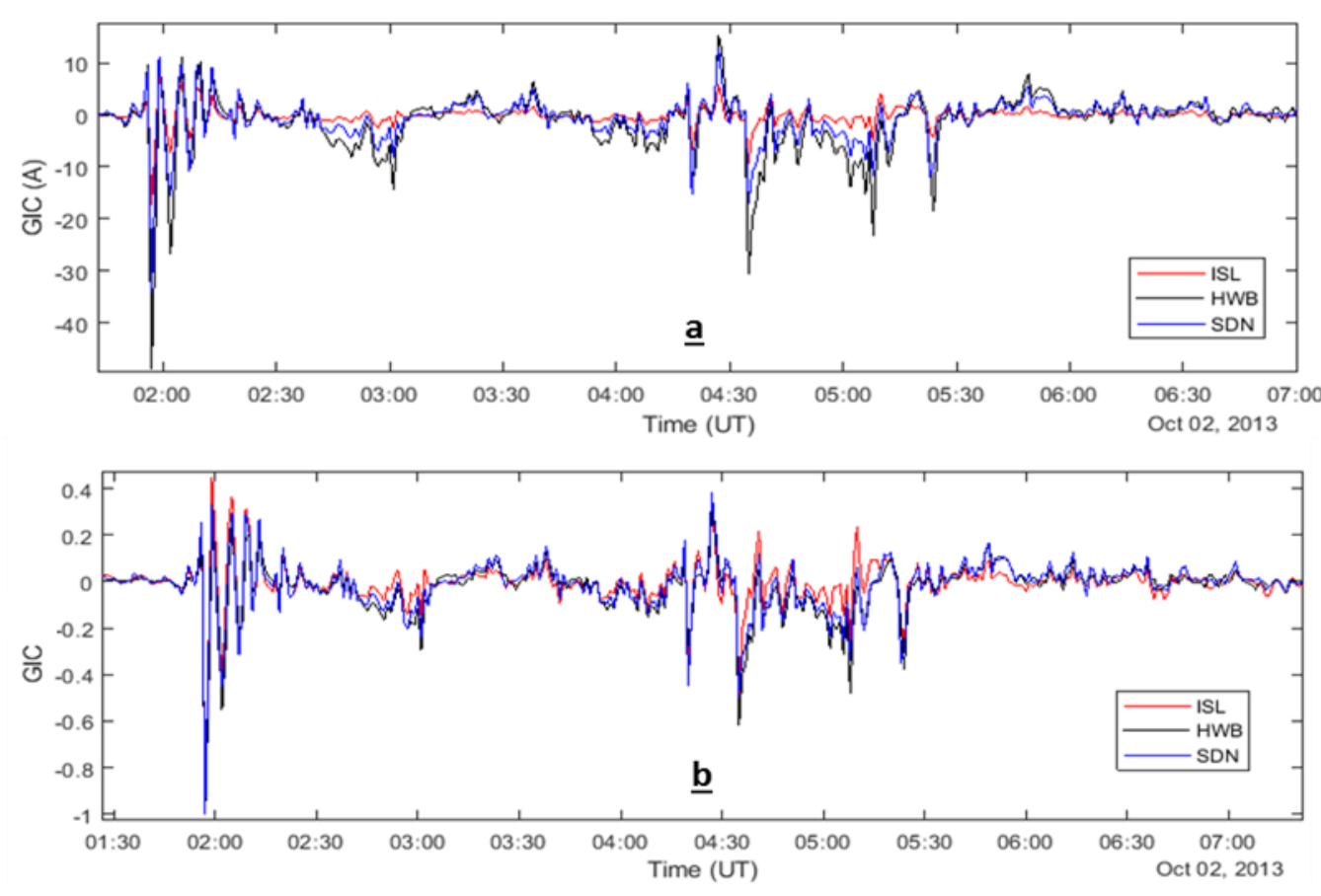

Figure 3. 26: (a) Comparison of ISL (red) GIC with HWB (black) and SDN (blue) GIC for magnetic storm of 02 October2013. (b) shows normalized GIC when each of GIC has maximum value of 1.

The magnetic storm of 17 March2015 has been considered for further comparison and given as Figure 3.27. The GIC have largest magnitude during the Sudden Storm Commencement at 0446 UT and given by -10.6, -15.8 and -28.9 A for ISL, SDN and HWB, respectively. The second panel of Figure 3.27 shows normalized GIC, showing strong low frequency components in SDN and HWB compared to ISL.

As mentioned SDN and HWB are close to each other, therefore another substation, Invercargill has been selected for further comparison. Invercargill (INV) substation lies close to the southern coast of the South Island. The measured GIC at INV is inverted therefore we have multiplied it by 
-1 before comparison. Figure 3.28 and 3.29 gives the same comparison of ISL with INV as was done for HWB and SDN. The comparison shows that INV also has strong low frequency components compared to ISL data during both storms.
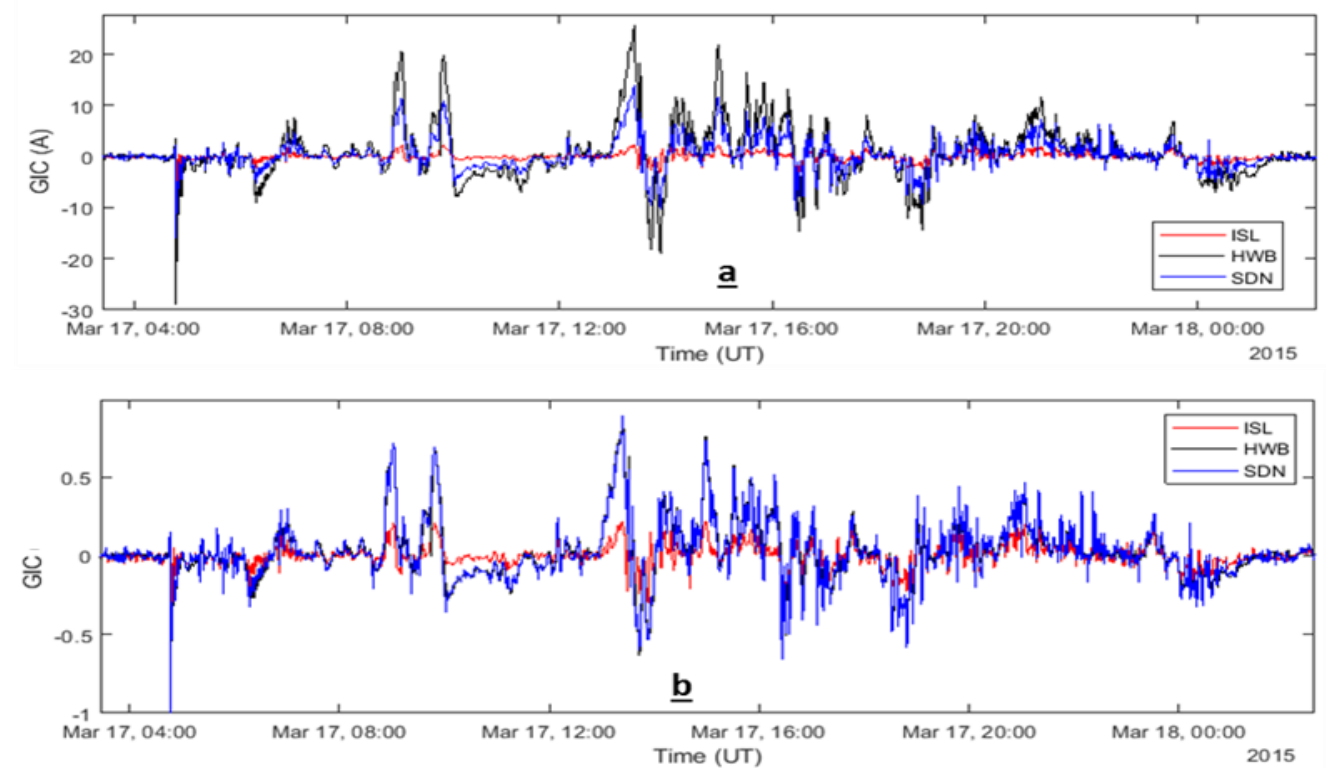

Figure 3. 27: (a) Comparison of ISL (red) GIC with HWB (black) and SDN (blue) GIC for St. Patrick's Day magnetic storm of 17 March2015. (b) shows large amplitudes of slow variations after normalizing HWB and SDN data based on the extreme GIC value at ISL.
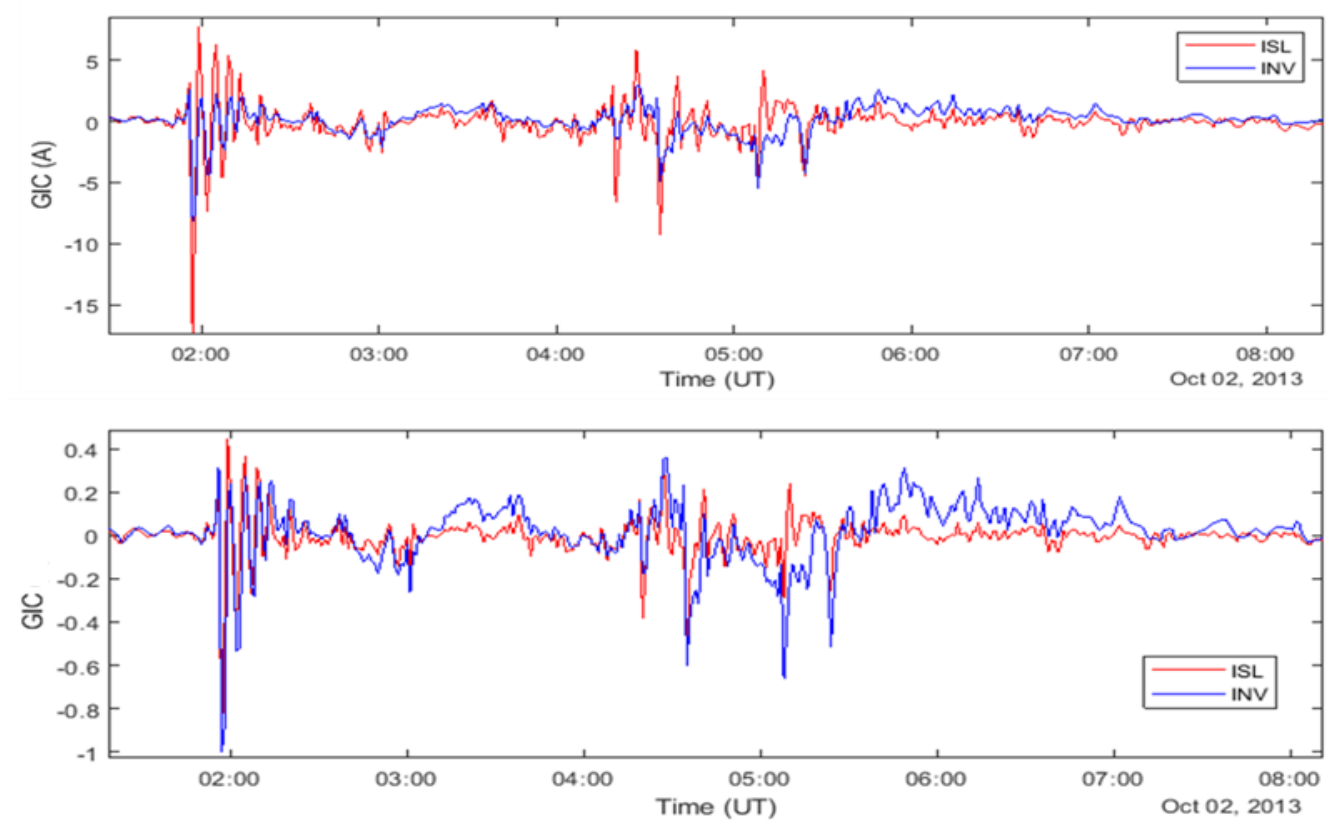

Figure 3. 28: The comparison of ISL (red) and INV (blue) GIC for magnetic storm of 02 October2013. The lower panel shows rescaling of INV data based on the largest GIC in the ISL data. 

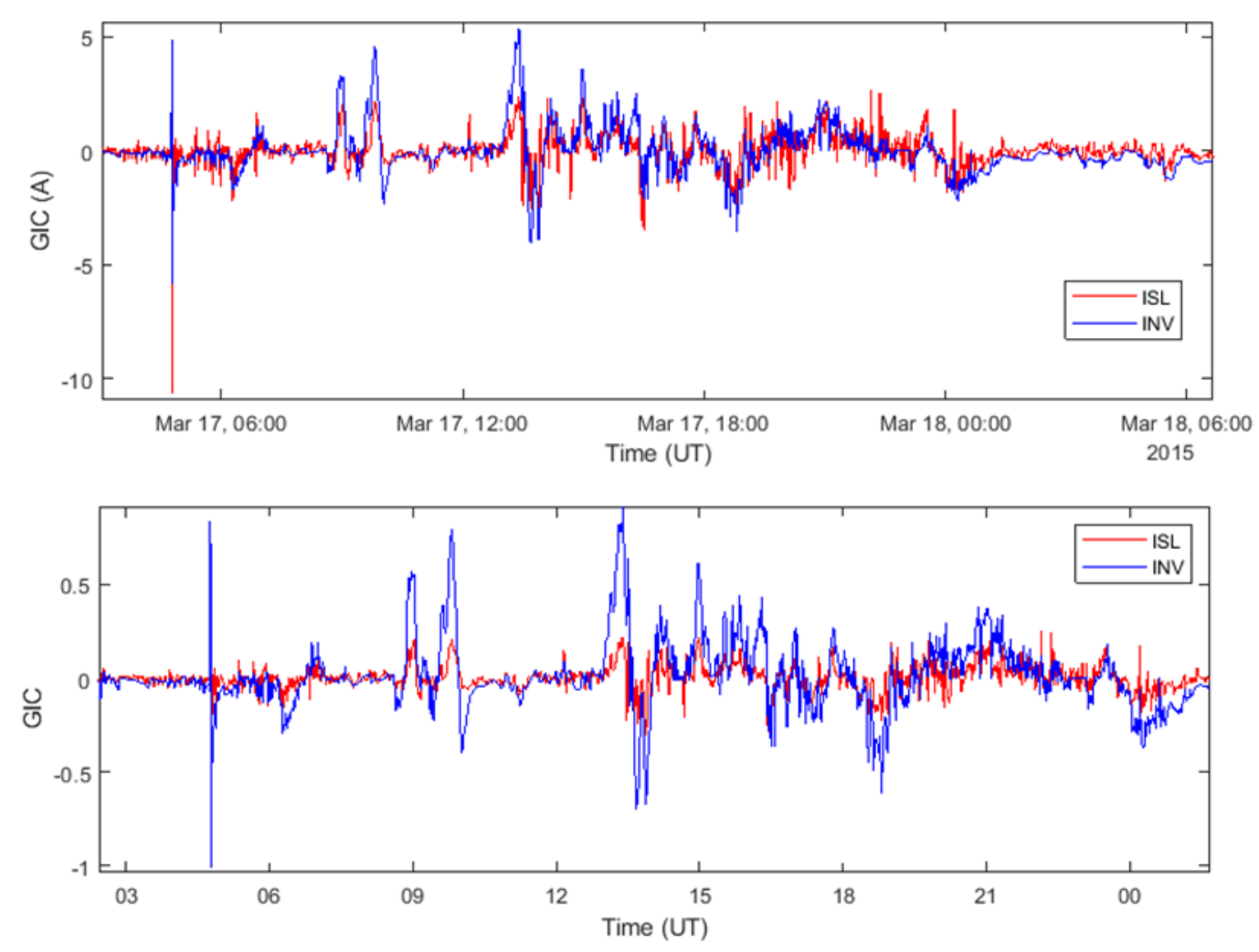

Figure 3. 29: The comparison of ISL (red) and INV (blue) GIC for magnetic storm of 17 March2015.

The above analysis shows that SDN, HWB and INV transformer GIC patterns are different to that from ISL in terms of frequency components. It is also seen that GIC signatures also vary for substations in different parts of the network. For example, the INV GIC during 17 March2015 (particularly during the SSC) storm is different to those of ISL, SDN and HWB. INV has clearly two opposite peaks ( $\pm 5 \mathrm{~A}$ ) during the SSC whereas others (ISL, SDN and HWB) only show one strong negative peak during the SSC. This indicates that INV substation experiences an equal amount of current going into and out of the transformers during the SSC whereas ISL, SDN and HWB only experience current going into the transformers during the SSC. It shows during a geomagnetic storm, transformers in different parts of the network are impacted differently.

To further investigate we have modelled the GIC of SDN and INV using network constants $(a, b)$ for the magnetic storms of 2013 and 2015. The same conductivity value (as for ISL) has been used as any value of uniform conductivity would not impact the spectral content of the geoelectric field. EYR magnetic field data is used again because no magnetic field measurements are available close to these substations. Figure 3.30 and 3.31 show model and measured GIC comparisons for SDN transformer $(\mathrm{T} 2 \mathrm{H})$. The lower panel of each figure shows the spectral 
comparison of measured and model GIC. For the 2013 magnetic storm, the correlation coefficient is 0.81 and performance parameter is 0.42 . For the magnetic storm 2015, the correlation coefficient and performance parameter are 0.60 and 0.20 , respectively. The model GIC do not show the consistent difference in the period range 2-13 minutes as seen in ISL data. Although differences exist in different parts of the spectrum these are random and occur in both high and low frequency components. For the magnetic storm 2013 model GIC have a good match for all frequency components with the only discrepancy being at end of high frequency components as shown by double arrow. For magnetic storm of 2015 , the difference is more prominent in the medium range frequency components as shown by the double arrows in the time domain comparison plot. The values of model network constants for SDN are also given along with ISL in Table 3.2. The values have same sign as of ISL but are relatively larger because the GIC magnitude for SDN is larger than ISL.

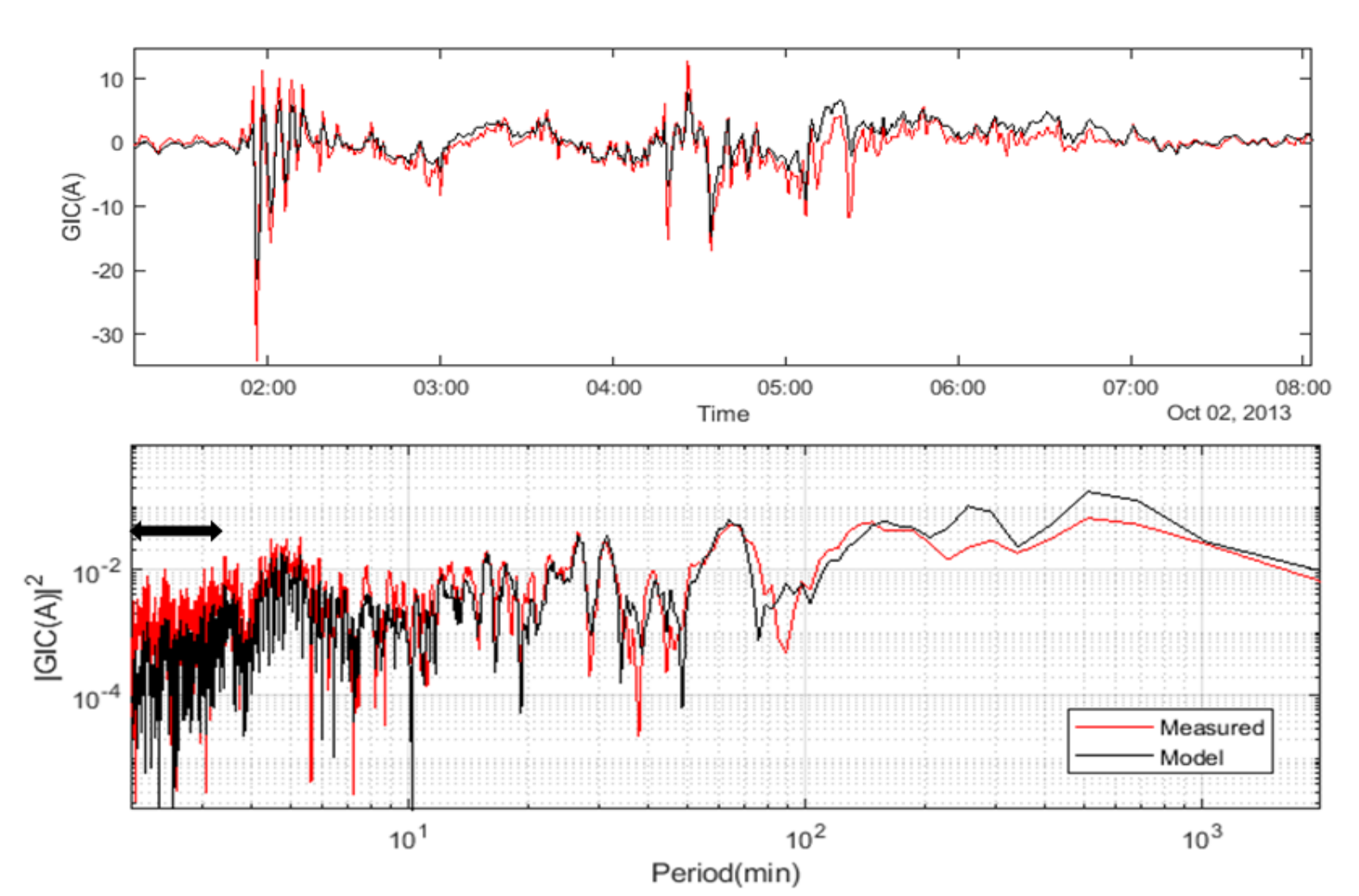

Figure 3. 30: Top panel shows time domain comparison of South Dunedin model (black) and measured (red) GIC for magnetic storm of 02 October2013 and bottom panel shows frequency domain comparison of the same. 


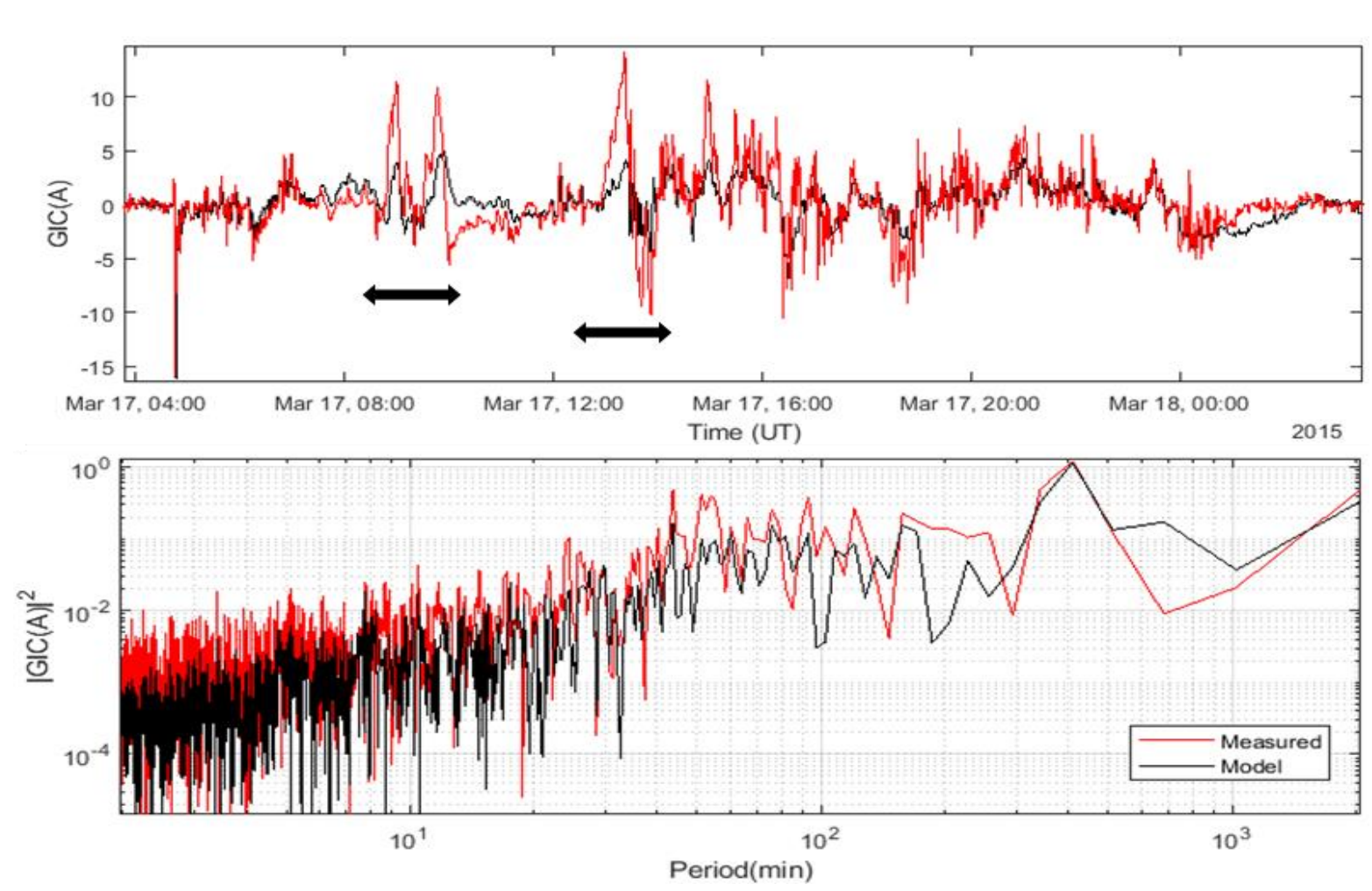

Figure 3. 31: Top panel shows comparison of South Dunedin (SDN) model (black) and measured (red) GIC for magnetic storm of 17 March2015 where arrows show where discrepancy between model and data is high. Bottom panel shows comparison in frequency domain.

Figure 3.32 and 3.33 shows the comparison of measured and model GIC at INV for the magnetic storms in 2013 and 2015. For the 2013 magnetic storm, the correlation coefficient is 0.84 and performance parameter is 0.44 . For the 2015 magnetic storm, the correlation coefficient and performance parameter are 0.70 and 0.28 , respectively. Comparison of both figures show that the model produces slightly better results for the magnetic storm of 2013 compared to 2015. For 2015 , the model results are not good in the medium range frequencies leading to a decrease in the correlation coefficient and performance parameter. This is also shown by the double arrow bars in the first panel of Figure 3.33. The network constant values for INV are also shown in Table 3.2. The negative sign shows GIC have anticorrelation with geoelectric fields. The value of $a$ is same for ISL, but the value of $b$ is very small. 


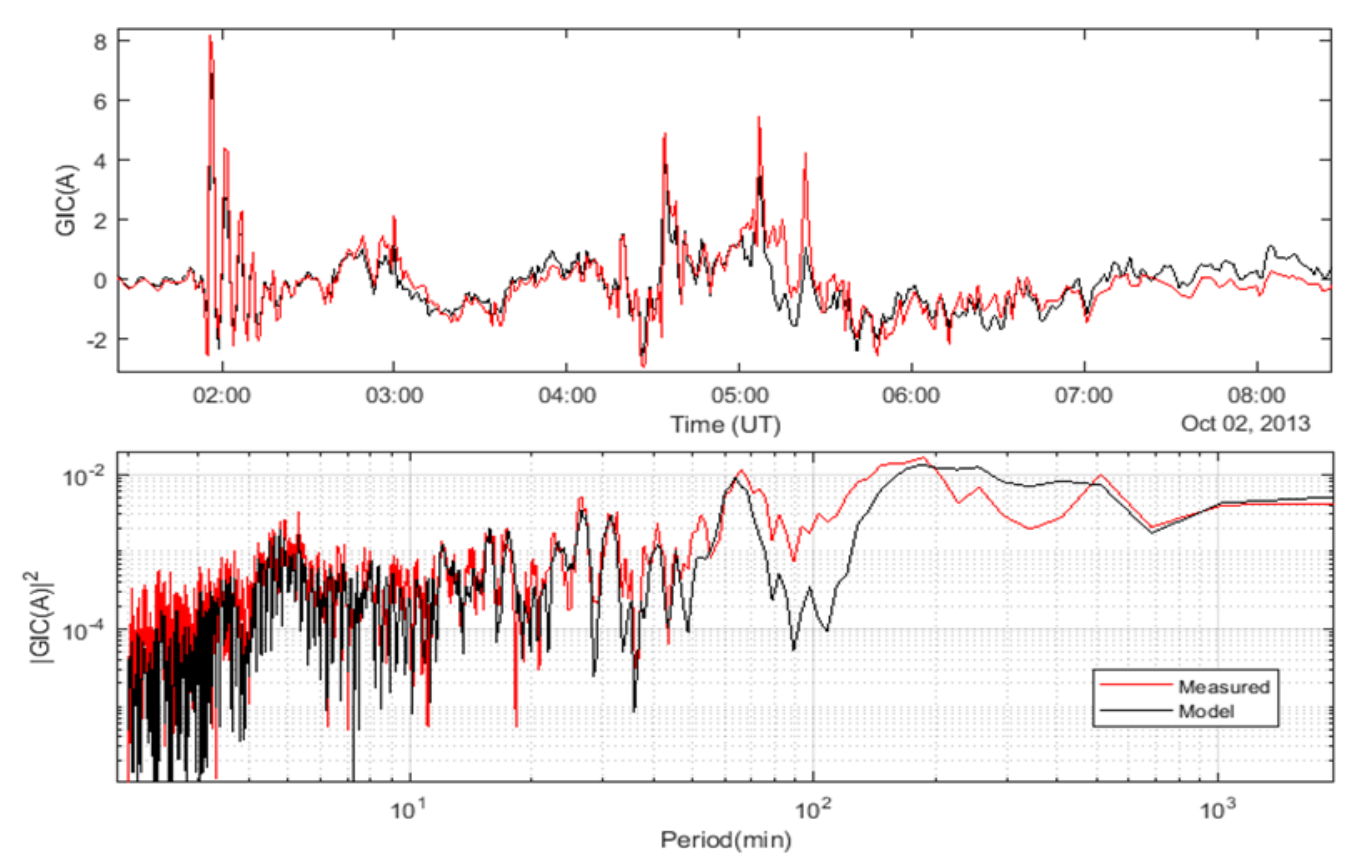

Figure 3. 32: Comparison of INV model (black) and measured (red) GIC for magnetic storm of 02 October2013 as top panel and bottom panel shows comparison in frequency domain.

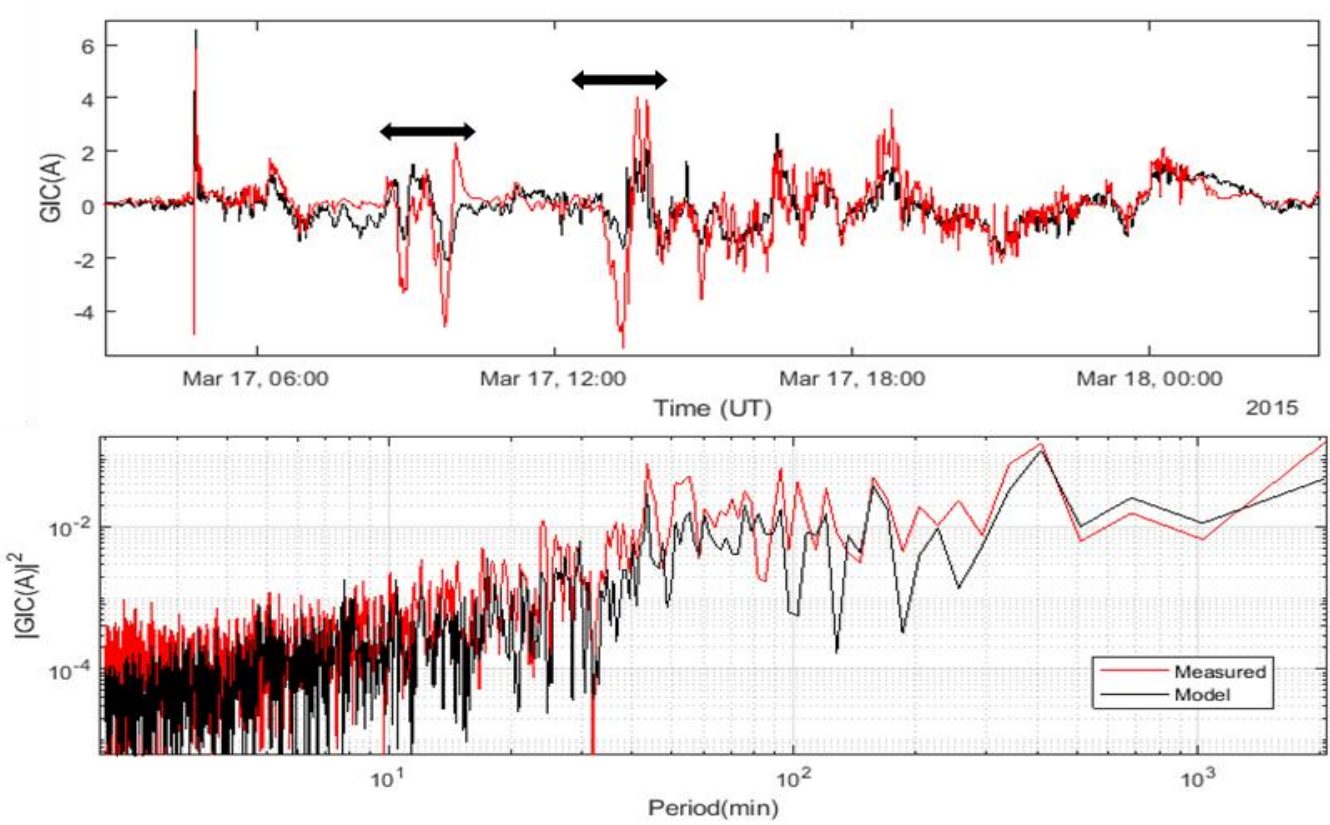

Figure 3. 33: INV model (black) and measured (blue) GIC for magnetic storm of 17 March2015 in time domain (top panel) and frequency domain (bottom panel). Arrows highlight where discrepancy is high between model and data. 


\section{Table 3.2}

Network constant values using least regression method for ISL, SDN and INV during two magnetic storms.

\begin{tabular}{ccccccc}
\hline Transformer & \multicolumn{2}{c}{ ISL $(\boldsymbol{a}, \boldsymbol{b})$} & \multicolumn{2}{c}{ SDN $(\boldsymbol{a}, \boldsymbol{b})$} & \multicolumn{2}{c}{ INV $(\boldsymbol{a}, \boldsymbol{b})$} \\
\hline 02 Oct 2013 & 0.021 & 0.020 & 0.055 & 0.032 & -0.021 & -0.005 \\
17 Mar 2015 & 0.016 & 0.010 & 0.047 & 0.024 & -0.021 & -0.006
\end{tabular}

The spectral signature at INV is quite different from ISL and SDN for both magnetic storms. For example, INV GIC are inverted. For the magnetic storm of 2015 INV GIC have two sharp peaks whereas for ISL and HWB both have only one sharp peak during the SSC, and the GIC shape at ISL is very similar to that at SDN and HWB. All three of these substations are on the east side of the network whereas INV is on the central south coast. This shows the flow of the current going out (positive) of one side of the network and going into (negative) the other side of the network and emphasizes the importance of considering the whole network in GIC modelling. The GIC at a substation is not only controlled by local geomagnetic variations but orientation of lines and connections to other substations.

\subsection{MT Geoelectric Field}

The preceding analysis shows some of the limitations of the single station modelling approach. Although it gives an accuracy of $\sim 70 \%$ it does not always accurately account for large values of GIC. The network constants are based on linear regression and the best fit therefore represents the general pattern which is dominant in the data rather than extreme GIC values. This is also the reason why the same geoelectric fields give almost the same results for different substations. There are also spectral discrepancies seen during different magnetic storms. For effective GIC mitigation strategies, it is more important to have accurate information on extreme values of GIC. The analysis also shows that the geoelectric field computed using a uniform conductivity approach does not satisfy the measured GIC for all spectral components. A more detailed conductivity structure should be considered for more realistic geoelectric fields, as Earth itself acts as a filter for geomagnetic variations.

The most obvious improvement is to use the impedance tensor approach (based on magnetotelluric sounding data) for more detailed geoelectric field modelling. This is 
demonstrated with the help of magnetotelluric derived geoelectric fields for INV and SDN. Figure 3.34 shows time domain plots of geoelectric fields for INV (first panel) and SDN (second panel) substations calculated from MT impedance data for the 17 March2015 magnetic storm. The INV geoelectric field is calculated in the period range 2-209 minutes whereas SDN geoelectric fields are calculated using a slightly longer period range of 2-332 minutes. The geoelectric fields at the two substations are significantly different as can clearly be seen during the SSC. For SDN both field components $(\boldsymbol{E} \boldsymbol{x}, \boldsymbol{E} \boldsymbol{y})$ are negative (i.e., in south and west directions) direction whereas for INV, $\boldsymbol{E} \boldsymbol{x}$ is positive (north) and $\boldsymbol{E} \boldsymbol{y}$ is negative (west). The relative locations of INV and SDN also effect the electric fields in that the largest component of the electric field is that parallel to the coast. Thus, at INV, on the south coast, variations in $\boldsymbol{E} \boldsymbol{y}$ are much larger than those in $\boldsymbol{E} \boldsymbol{x}$, while at SDN, on the south-east coast, the opposite is true. This is consistent with the fact that, close to a coastline, magnetic field variations perpendicular to the coast tend to be larger than those parallel to the coast - a manifestation of the so-called 'coast effect' (e.g., Liu et al., 2018).
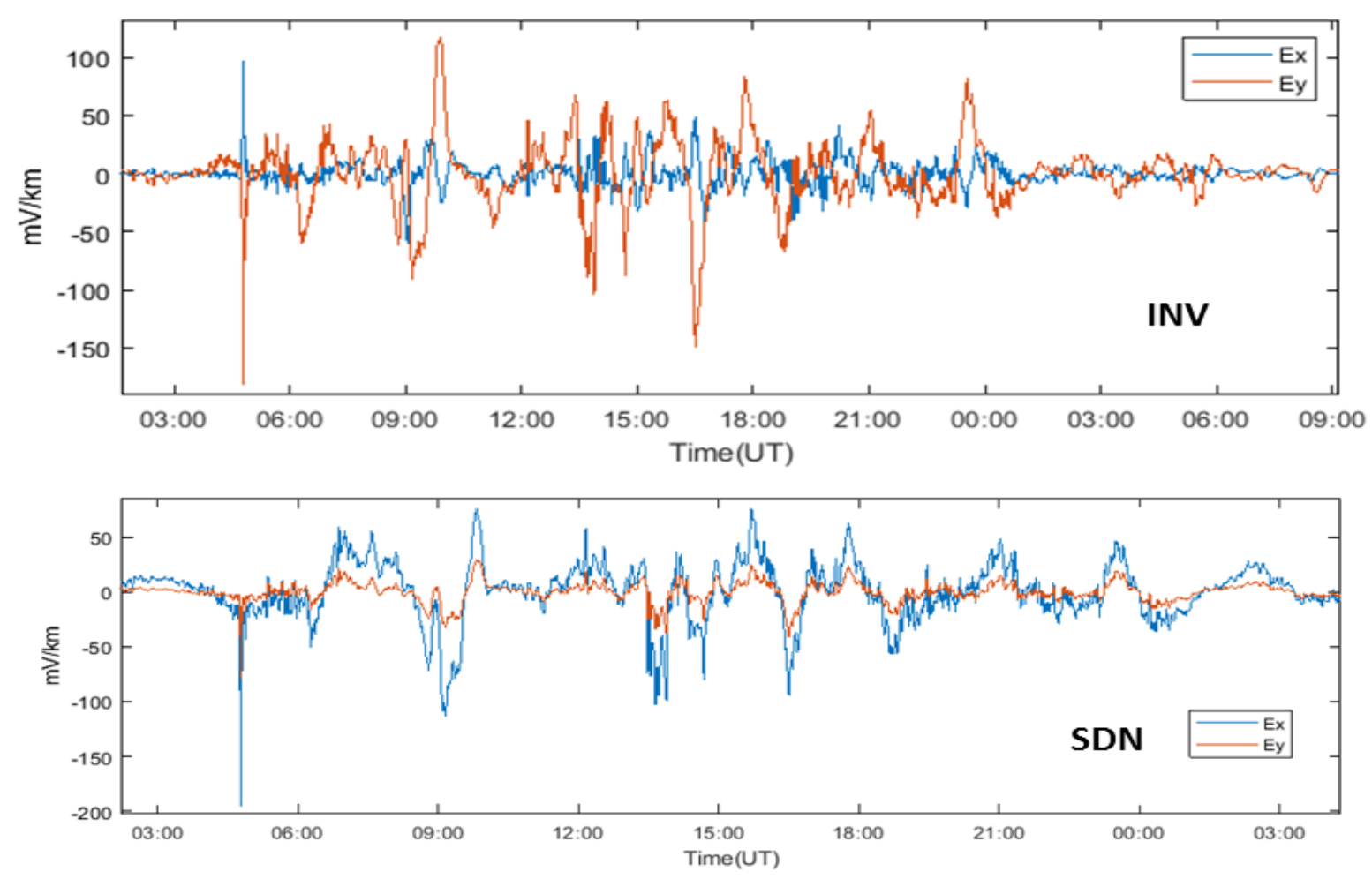

Figure 3. 34: The time domain plot of geoelectric fields calculated using impedance tensor data from Invercargill (INV) and South Dunedin (SDN) during the magnetic storm of 17 March2015. 
Figure 3.35 shows a plot of geoelectric fields calculated using the uniform conductivity model as has been used in the above GIC modelling. The uniform conductivity geoelectric fields are very different from those calculated from the MT impedance tensor at both SND and INV, again illustrating that consideration of local geoelectric fields is an important aspect of correctly modelling GIC.

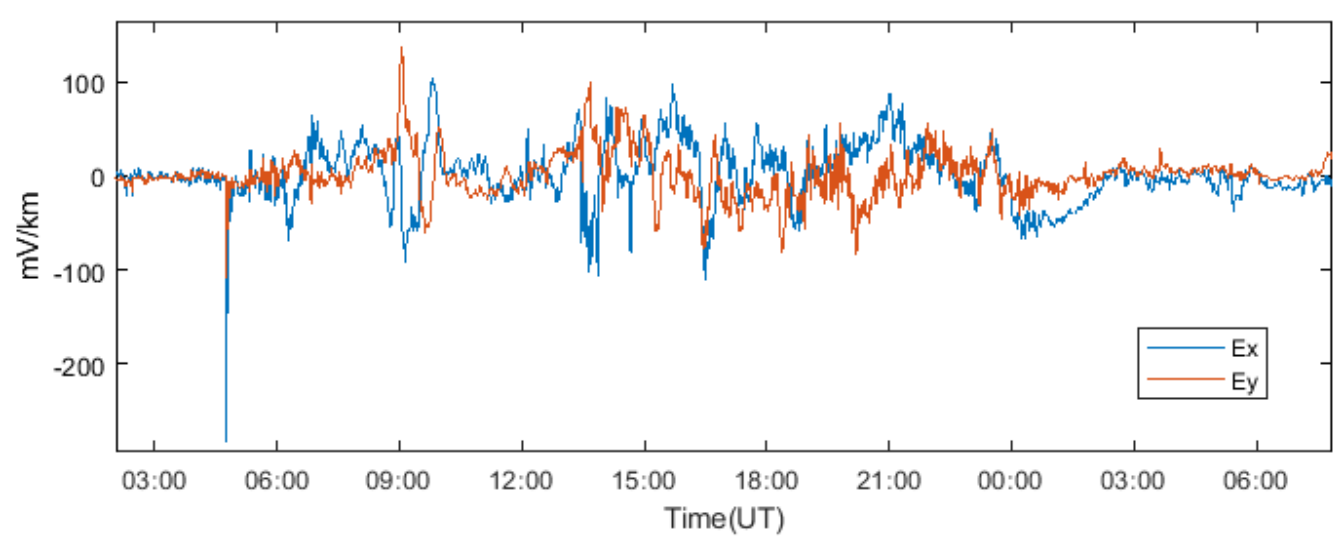

Figure 3. 35: Geoelectric fields at INV and SDN, calculated using the uniform conductivity model, during the magnetic storm of 17 March2015.

\subsection{Islington GIC Data and Solar Interplanetary Structures}

The GIC data at Islington considered in this study span from 2002-2015 and relate to geomagnetic storms caused by different interplanetary structures during solar cycles 23 and 24 (as discussed in Chapter 2). The spectral discrepancy seen in the GIC data of ISL might also be anticipated to be due to these different interplanetary structures during both solar cycles. Although, in the previous two sections it was seen that consideration of the full network and MT geoelectric fields are important in the context of GIC modelling, we have also investigated if there could be any influence due to these interplanetary structures. Referring to Chapter 2, Figure 3.36 shows the percentage contribution of each of interplanetary structures based on 43 intense geomagnetic storms in 2002-2006 (Echer et al., 2008; Gonzalez, et al., 2007), and 18 intense geomagnetic storms from 2011-2016 (Rawat et al., 2018; Watari, 2017). The Figure shows the contribution of interplanetary structures is not the same during both periods and a major trade-off can be seen between ICME and SH+ICME. Interplanetary coronal mass ejection (ICME) plays a major role 
(40\%) causing intense magnetic storms during 2002-2006, whereas during 2011-2016 SH+ICME (composite of sheath and ICME) dominates (39\%). The corotating interaction region (CIR) contribution also reduces significantly from 20 to $10 \%$ between the two periods. It is logical that two different dominating structures might have caused the spectral discrepancy seen in the GIC data at ISL. In this chapter two geomagnetic storms (23 May 2002 and 29 October2003) have been considered from SC23 which depict strong low frequency components. These geomagnetic storms were caused by sheath and magnetic cloud ( $\mathrm{SH}+\mathrm{MC})$ type structures, respectively. Remembering from Chapter 2 that a $\mathrm{MC}$ is the one type of interplanetary coronal mass ejection this suggests that $\mathrm{SH}+\mathrm{ICME}$ can produce low frequency GIC. However, these are also the dominating interplanetary structures during solar cycle 24 , and the geomagnetic storms of 26 Sep 2011 and 02 October2013 only showed high frequency components and were caused by sheath structures. This suggests that it is not possible to explore the spectral discrepancy of GIC data using the four broad interplanetary structures shown in Figure 3.36.
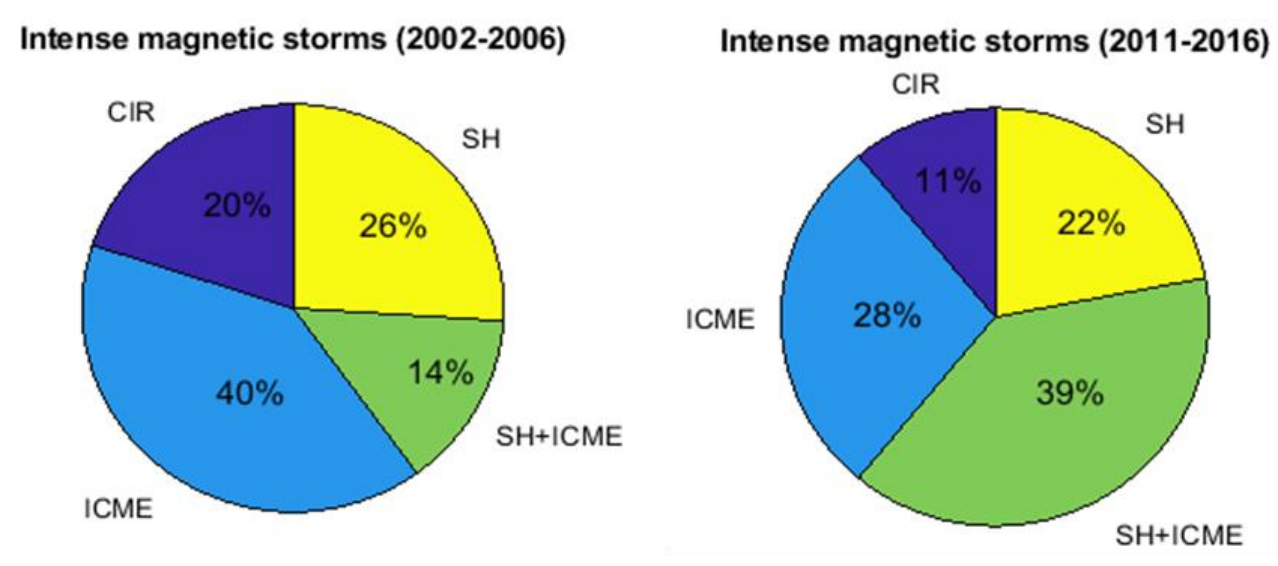

Figure 3. 36: Contribution of different interplanetary structures producing intense magnetic storms (Dst<-100nT) during two different period of solar cycles 23 and 24. Total 43 geomagnetic storms were considered during 2002-2006 and 18 during 2011-2016.

The long-recorded Islington GIC measurements can also be analyzed in relation with different solar wind parameters e.g., interplanetary magnetic field, proton density, temperature, solar wind pressure etc. Solar wind parameters can have significantly different kinds of variations within a single interplanetary structure that may affect the GIC (Huttunen et al., 2008). Although, the solar wind causes the energy to deposit in the atmosphere of Earth, leading to initiation and 
evolution of a geomagnetic storm, direct modelling is always difficult due to the complex chain of mechanisms involved. To make comparison simple, the hourly average values of solar wind parameters have been considered against hourly mean values of GIC data. It is also that highresolution solar data e.g., one minute data, are not available for all geomagnetic storms. The solar and GIC data for a total of 20 major geomagnetic storms from 2002-2015 is considered and presented here. Since a period of approximately 1.5 days was selected for each geomagnetic storm the hourly mean GIC above a threshold of $0.5 \mathrm{~A}$ was selected. This ensures that the high GIC values are plotted against individual solar wind parameters. Table 3.3 shows list of solar field and plasma parameters.

Table 3.3

Solar wind parameters considered along with GIC data of ISL.

\begin{tabular}{lll}
\hline Category & Name of parameters & Units \\
\hline Magnetic field & 1. Interplanetary magnetic field (IMF-B) & $\mathrm{nT}$ \\
& 2. Bz in GSE & $\mathrm{nT}$ \\
Plasma & 3. Temperature & $\mathrm{K}$ \\
& 4. Flow speed & $\mathrm{Km} / \mathrm{s}$ \\
& 5. Proton density & $\mathrm{cm}^{-3}$ \\
Derived parameter & 6. Flow pressure & $\mathrm{nPa}$ \\
& 7. Interplanetary electric field (IMF-Ey) & $\mathrm{mV} / \mathrm{m}$
\end{tabular}

Figure 3.37a shows plots of hourly values of solar wind parameters and the absolute hourly mean GIC at ISL. The yellow lines show the linear fits. Although there are broad positive correlations between both IMF-Ey and IMF-B with GIC there is a considerable degree of scatter which leads to large root mean square errors (RMSE) in the fits ( $\sim 9.5)$. Scatter plots for the solar wind pressure and proton density are similar with RSME given by $\sim 10.5$. Solar wind speed and temperature show very poor correlation with GIC magnitude. The $B z$ component of solar magnetic field for the same dataset has also been considered and is strongly linked to producing large GIC. More than $75 \%$ of large GIC ( $\geq \pm 4 \mathrm{~A}$ ) in all magnetic storms happened when $B z$ was in the south direction as shown in Figure 3.37b. Nonetheless, clear relations between solar wind parameters and GIC at ISL are not apparent. 

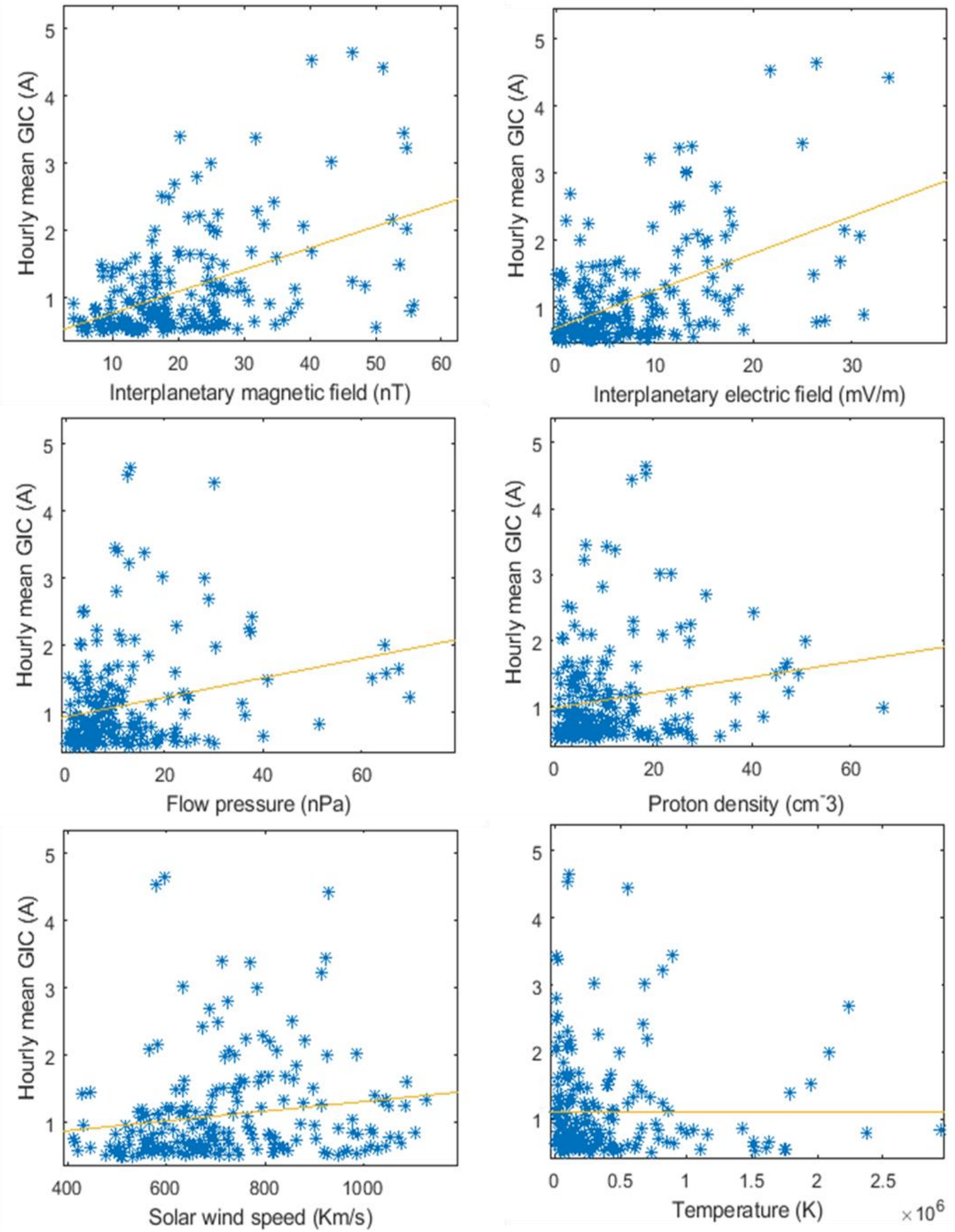

Figure 3. 37a: shows relation between hourly mean values of absolute GIC and hourly mean values of different solar wind parameters for 20 geomagnetic storms during the period of 2002-2015. 


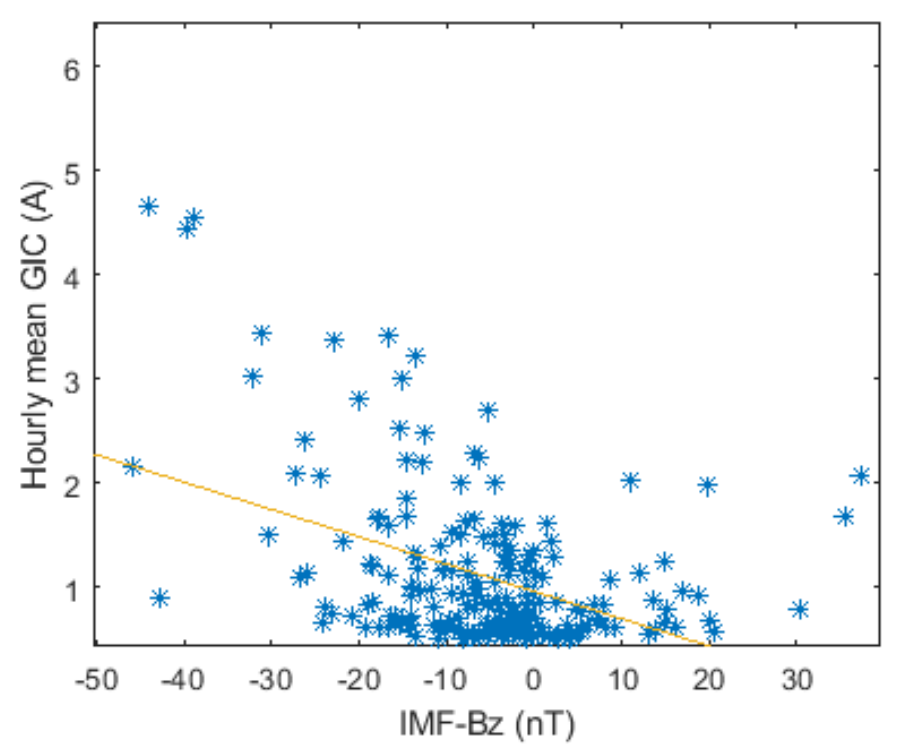

Figure 3. 38b: shows relation between hourly mean values of absolute GIC and hourly average values of IMF-Bz for 20 geomagnetic storms during the period of 2002-2015. It shows GIC is highly correlated with negative values of IMF-Bz.

\subsection{Discussion}

Measured GIC at Islington from 2002-2015, have been discussed in relation to Eyrewell magnetic field data for major magnetic storms. Geoelectric fields computed using a uniform conductivity time domain formula have been used for GIC modelling. The geoelectric fields have been related to GIC using network constants $(a, b)$, where the values of $a$ and $b$ are determined using a least squares method. The network constant values remain stable for all magnetic storms, showing that average values of the constants could be used for modelling GIC, given a knowledge of magnetic field data. The network constants $(a, b)$ which represent a uniform response of the network to all frequencies of geoelectric fields, produce differences in the accuracy of model GIC over different years, particularly between the periods of 2002-2006 and 2011-2015. The distribution of spectral components of GIC also differs for the two time periods. There is a dominance of high frequency components in the later years, although no such change in spectral content is seen in the magnetic field data at EYR observatory. A quantitative analysis using slope values of GIC spectra shows that the amplitudes of high frequency components increase during 2011-2016, whereas the spectral components of the magnetic field remain almost the same 
(Figure 3.8). To develop a better model for all magnetic storms different network constant have been used for low and high frequency components. Periods shorter than 13 minutes are regarded as high frequency, whereas longer periods are regarded as low frequency. Using this approach, calculated model GIC give good accuracy for all years.

GIC data from other substations (SND, HWB and INV) have been explored to compare ISL GIC with other parts of the network. The analysis shows the existence of low frequency components using two magnetic storms from 2013 and 2015. This suggests that the spectral discrepancy seen in Islington GIC data is local and does not exist in other parts of the network. Whereas observed GIC at INV and SDN are significantly different from those at ISL, GIC modelling shows no significant differences in correlation coefficients. This shows the limitations of single station modelling. Furthermore, geoelectric fields calculated using magnetotelluric response tensors gives very different fields both for INV and SDN. It is clear, that rather than a uniform conductivity model, a more detailed conductivity structure should be considered for more realistic geoelectric fields.

Exploration of the spectral discrepancy seen in the GIC data at ISL in connection with dominating interplanetary structures shows no correlation. The correlation of solar wind parameters with hourly mean values of GIC shows, solar wind $E_{y}$ and $B$ have the strongest correlation while proton density and flow pressure have lower correlation, and solar wind speed and temperature shows essentially no correlation with GIC. ISL GIC data also show that the southward $B z$ component plays a key role in production of GIC. 


\section{Chapter 4: Utilization of MT Data for GIC Modelling in the North Island, New Zealand}

\subsection{Introduction}

Previously, the thin-sheet conductance modelling technique proposed by Vasseur and Weidelt (1977), has been used for determination of geoelectric fields in New Zealand (Divett et al, 2017, 2018). This has been a milestone in GIC studies in New Zealand and many useful results have been derived. However, the thin-sheet technique comes with some serious limitations as it is valid over only a limited period range and therefore unable to reproduce the complete range of variations in model GIC. It also has spatial limitations as the model itself is a crude approximation of variations in conductive structure and not able to produce locally accurate surface geoelectric fields. This has been demonstrated by comparing the results of predictions of GIC for the South Island between the thin-sheet model (Divett et al., 2017), and a transfer function analysis (Ingham et al., 2017) which does not use the thin-sheet approach. There are significant differences in the predictions for GIC in the lower South Island.

In the previous chapter it was suggested that geoelectric fields derived from magnetotelluric (MT) sounding data at different South Island sites could be used for modelling GIC. Based upon this MT data an alternative approach for GIC modelling can be explored, but requires a dense array of MT sites. The South Island is very sparse in MT data so GIC modelling for the whole network would be rather uncertain and difficult. However, the North Island is rich in terms of having many MT sites and, as a result, we can utilize the MT data for calculation of GIC for the whole power network in the North Island. This is also important in that the North Island has not yet been studied in the context of GIC studies and there are no measured GIC data available for it.

Therefore, in this chapter we have employed an alternative approach for computing geoelectric fields for the North Island based on MT data. The magnetotelluric method (e.g., Cagniard, 1953; 
Chave and Jones, 2012) which gives a complex valued Earth response function is used to model the conductivity structure of the Earth. At a given location a frequency dependent impedance tensor relates geoelectric fields and geomagnetic fields. The North Island MT data therefore can be utilized to map geoelectric fields in response to a given geomagnetic variation. To compute geoelectric fields we have considered the magnetic variations during the St. Patrick's Day magnetic storm (17 March 2015). Since MT data are available in only a limited range of frequencies the geoelectric fields can only be derived in that range. Furthermore, MT sites are generally concentrated in the lower part of the North Island (at higher latitudes) and there are many gaps where no MT data are available. Spatial interpolation is performed to fill these gaps in the data. However, extrapolation to areas outside the main region of coverage of the MT data is found not to be effective due to the lack of data for the upper part of the North Island. To overcome this problem of extrapolated geoelectric fields, Thevenin's Equivalent circuit approach (Boteler et al., 2013) has been used for that part of the transmission network. The results of using this technique were verified using uniform electric fields of $1 \mathrm{~V} / \mathrm{km}$. Finally, the computed geoelectric fields are input to the power network model to calculate GIC in individual transformers and substations during the St. Patrick's Day storm.

\subsection{Magnetotelluric (MT) Data in the North Island}

Magnetotelluric sounding is generally concerned with ground level geomagnetic and geoelectric field measurements to estimate the electrical conductivity of the Earth. The conductivity models are obtained from a derived data product known as the MT response/impedance tensor $Z(f, x, y)$, which is also sometimes referred to as the MT transfer function. The MT transfer function is a complex valued $2 \times 2$ matrix and is given in the discrete frequency domain. In the GIC community the MT transfer function is used with magnetic field data during a magnetic storm to map geoelectric fields in the region of concern.

Geographically, the North Island extends between latitudes of $34^{\circ}$ and $42^{\circ} \mathrm{S}$. MT data in the North Island were acquired at various locations during studies reported by Bertrand et al. $(2012,2013)$, Heise et al. (2008, 2010, 2014) and Ingham (2005) in the Central Volcanic Region; by Cassidy et al. (2009), Ingham et al. (2009) and Stagpoole et al. (2009) on the volcanic systems; and by Heise et al. (2012), Ingham et al. (2001) and McLoughlin et al. (2002) along the east coast of the island. 
Figure 4.1 shows the sites of selected MT measurements (black dots) superimposed on the thinsheet conductance map of the North Island of New Zealand used by Divett et al. (2017). There is a total of 115 cells which can be filled using available MT data. Although some cells contain multiple MT sites each grid cell is represented by a single MT site. The map is based on metric units where each cell has dimensions of $20 \times 20 \mathrm{~km}$, corresponding to 0.1667 degrees in geographic coordinates. Magnetotelluric measurements are basically carried out to map lateral and depth variations in the conductivity structure of the Earth and the resulting Earth response function can be used to determine geoelectric fields. The conductance map of the North Island can broadly be divided into three main regions from east to west. The east coast is mainly of high conductance associated with the Hikurangi Margin, and a dense array of MT data exists in this region. Low conductance (shown by the green color) exists to the west of this region and is associated with the mountainous spine of the North Island. Very few measurements have been carried out in this area. Further west is assumed to be mainly dominated by intermediate conductance, although a dense array of MT sites exists in the center of the island where high conductance values are associated with the Central Volcanic Region. A small number of sites are distributed along the west coast from the Manawatu to Taranaki. The MT sites along the west coast were measured as part of this study to cover he empty area around the west coast and details can be found in the Appendix-MT Measurements.

\section{Table 4.1}

Discrete periods of magnetotelluric response tensor of North Island available to model geoelectric field.

\begin{tabular}{llll}
\hline $\begin{array}{l}\text { S. } \\
\text { No. }\end{array}$ & $\begin{array}{l}\text { Frequency } \\
(\mathbf{H z})\end{array}$ & $\begin{array}{l}\text { Period } \\
(\mathbf{s e c})\end{array}$ & $\begin{array}{l}\text { Period } \\
\text { (min) }\end{array}$ \\
\hline 1 & 0.017 & 57.0 & 0.95 \\
2 & 0.012 & 85.2 & 1.42 \\
3 & 0.0088 & 114.0 & 1.90 \\
4 & 0.0058 & 170.4 & 2.84 \\
5 & 0.0044 & 227.4 & 3.79 \\
6 & 0.0029 & 341.4 & 5.69 \\
7 & 0.0022 & 455.4 & 7.59 \\
8 & 0.0015 & 682.8 & 11.38 \\
9 & 0.0011 & 910.2 & 15.17 \\
10 & 0.00073 & 1365.6 & 22.76 \\
11 & 0.00054 & 1820.4 & 30.34
\end{tabular}


Since the Earth response function is best represented in the frequency domain, time domain magnetic field data for the St. Patrick's Day Storm is converted into the frequency domain prior to computation of electric fields. The MT data have a broad range of discrete frequencies from which the frequencies useful in GIC studies have been selected. Table 4.1 shows these 11 discrete frequencies in the lower frequency range of the MT data. The shortest period of MT data in this range is $\sim 60$ seconds which is the sampling interval of the magnetic field time series.

\subsection{The St. Patrick's Day Magnetic Storm (2015)}

This section gives a comparison of solar wind parameters and the geomagnetic field measured during the St. Patrick's Day magnetic storm. This was the largest magnetic storm of solar cycle 24 (Navia et al., 2018; Kamide and Kusano, 2015) and started on 17 March 2015. Magnetic observatories installed on the ground measure magnetic field variations during these storms. The planetary K-index $(K p)$ is a useful index for scaling the intensity of a magnetic storm particularly at higher latitudes. It is derived from a 3-hour mean standardized K-index from 13 geomagnetic observatories between 44 degrees and 60 degrees northern or southern geomagnetic latitudes (https://www.ngdc.noaa.gov/stp/GEOMAG/kp_ap.html). The $K p$-index value ranges from 0 to 9 where a value of 0 means that there is very little geomagnetic activity and a value of 9 means extreme geomagnetic activity. Space Weather Live (https://www.spaceweatherlive.com/) provides data for all past storms based upon the $K p$ index. The St. Patrick's Day magnetic storm reached a maximum $K p$ index of 8 as shown in Figure 4.2.

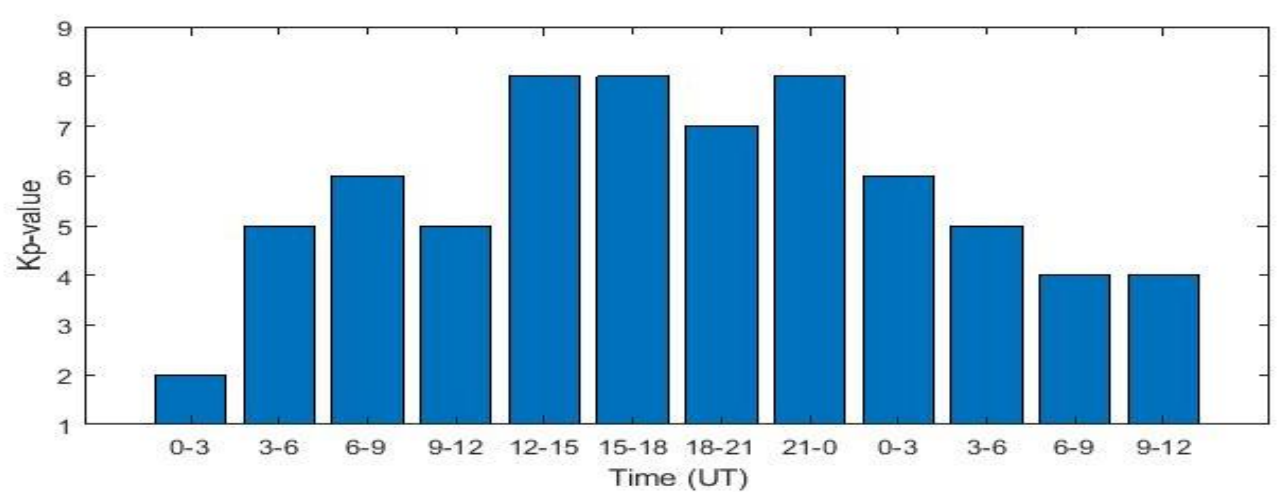

Figure 4. 2: The $K p$ index as a measure of geomagnetic activity downloaded from WDC (http://wdc.kugi.kyoto-u.ac.jp/kp/index.html) for 17 March 2015. 
As discussed in Chapter 2, the SH+ICME was the dominating interplanetary structure causing intense geomagnetic storms during solar cycle 23. The St. Patrick's Day magnetic storm was also caused by Sheath followed by non-magnetic cloud ICME (Rawat et. al. 2018). Figure 4.3 shows one-minute solar plasma and field parameters observed by ACE/WIND satellites and downloaded from the NASA OMNI2 database. There are some gaps in the plots showing no data. Referring to Figure 4.3, the interplanetary first shock (s1) preceding the Coronal Mass Ejection arrival is detected at 0445 UT (Kozyreva, 2018).

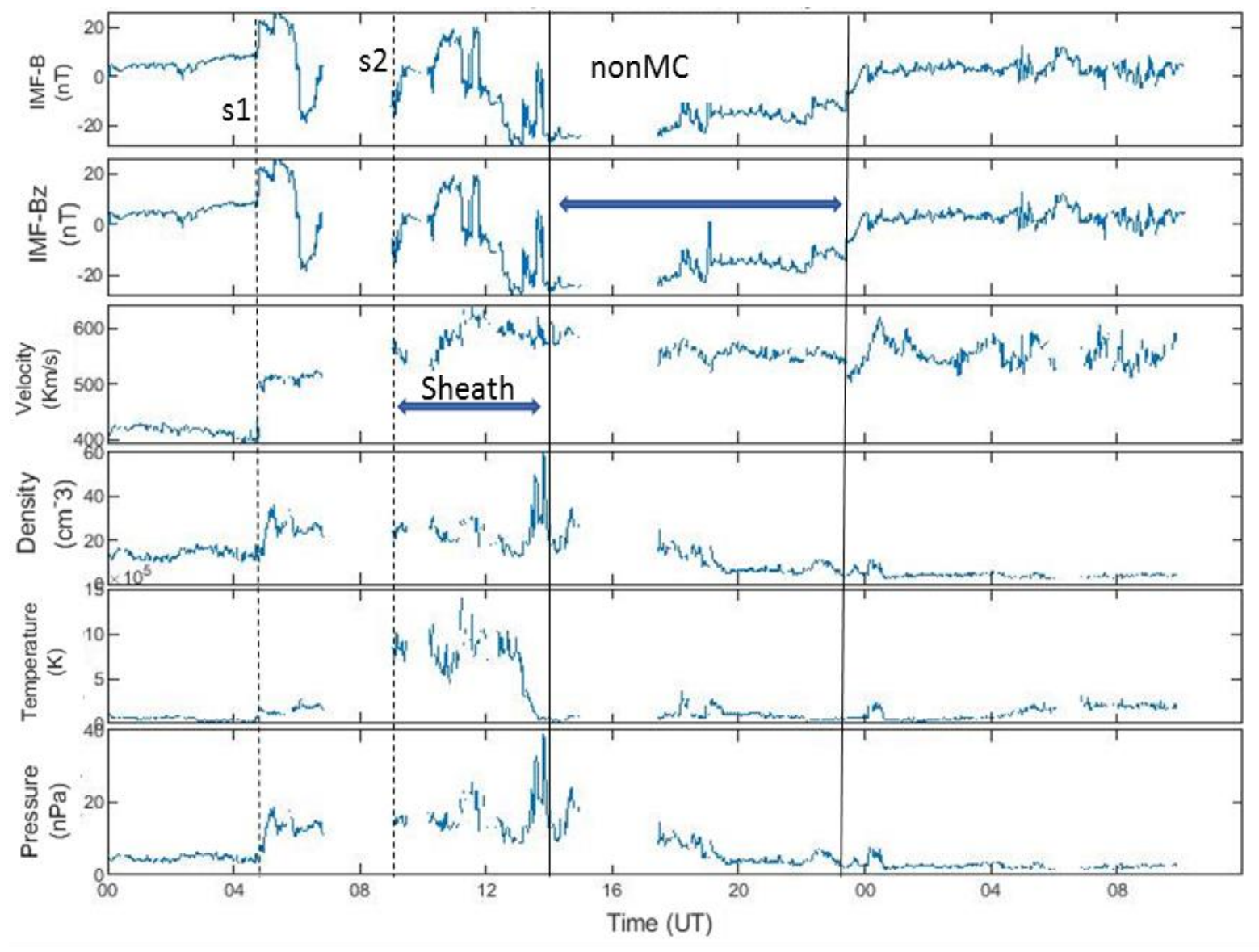

Figure 4. 3: Solar plasma and magnetic field parameters as observed by ACE satellite for 17 March 2015. The gaps show times with no data points, s1 and s2 marks the interplanetary shocks. The non-magnetic cloud (nonMC) and sheath region of ICME have been shown by horizontal bars.

During the shock, the interplanetary magnetic field (IMF-B) experiences strong variations and $B z$ becomes more strongly positive. The shock hits the magnetosphere at $\sim 0446$ UT and induces the Sudden Storm Commencement as can be seen in the magnetic field data of Figure 4.4. The 
$\mathrm{Bz}$ and By components of magnetic field during the SSC reach 19435 and $8316 \mathrm{nT}$ respectively, with a maximum rate of change of $71.86 \mathrm{nT} / \mathrm{min}$ and $35.10 \mathrm{nT} / \mathrm{min}$ respectively. At the same time, the largest GIC were observed on the Islington transformer (ISL-T6H) reaching a value of -10.5 A as shown in Figure 4.5. A sudden increase in solar wind parameters can be seen in Figure 4.3 just after the shock at $\sim 0500$ UT. The solar wind plasma density reaches up to $\sim 35 \mathrm{~cm}^{-3}$, the solar dynamic pressure $(\mathrm{P})$ up to $\sim 15 \mathrm{nPa}$ and the velocity increase from $\sim 400$ to $\sim 500 \mathrm{~km} / \mathrm{s}$ (Kozyreva et al., 2018). The $K p$-index reaches a value of 6 . The storm's main phase follows the rotation of the interplanetary magnetic field (IMF-Bz) southward at $\sim 0600$ UT after which it starts to fluctuate between north and south from 09:00-15:00 UT showing a sheath (SH) region, where the secondary shock (s2) preceding the sheath region is shown by another dotted line (Echer et al., 2008). The solar wind temperature reaches to its maximum value of $1.4 * 10^{6} \mathrm{~K}$ during the sheath region. The nonMC (interplanetary coronal mass ejections which do not have any identifiable magnetic cloud structure), shown by the arrow bars, causes a very gradual storm growth and $K p$ reaches it maximum value of 8 at which it stays for a couple of hours. The $K p$ index shows that the main phase lasts $\sim 12$ hours reaching a maximum at the end of March 17 and then declining on the next day.

Figure 4.4 (c) shows a logarithm plot of the power spectrum of the magnetic field (Bx) at different periods, like other magnetic storms, it can be seen that more power is embedded at longer periods. Since MT impedance data are given over a specific period range (Table 4.1) the double arrow shows the range of MT measurements available based on one-minute interval geomagnetic data. The geoelectric field that can be calculated using the MT impedance tensor would be in this common range of periods. This storm is also important in the context that it produced significant GIC which were measured in many transformers in the South Island of New Zealand (Mac Manus et al. 2017; Rodger et al. 2017), for example as shown in Figure 4.5 for a transformer at Islington. 

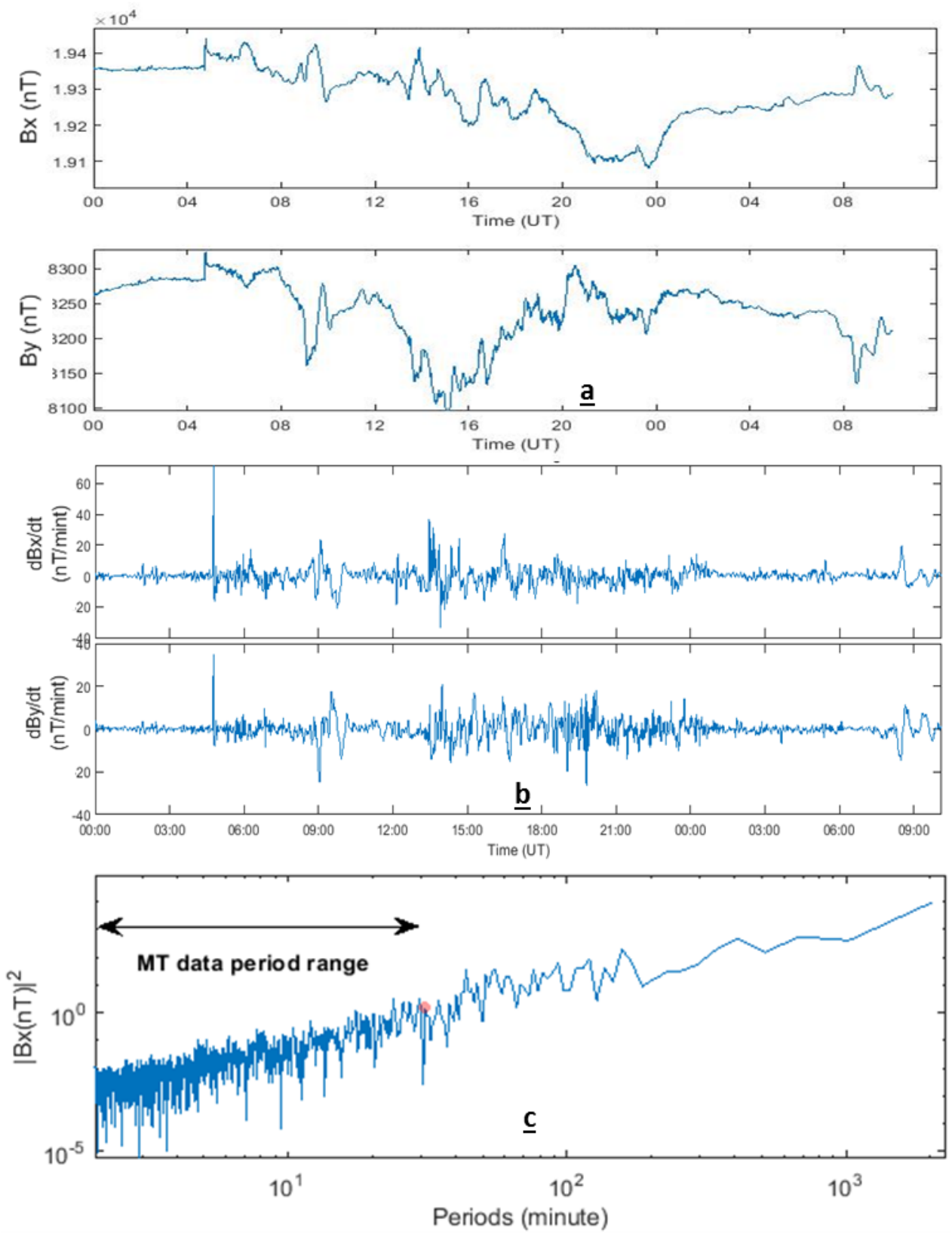

Figure 4. 4: (a) Eyrewell (EYR) magnetic observatory data of horizontal field components during St. Patrick's Day storm on 17 March 2015; (b) Time derivatives of the magnetic field components; (c) Spectral power distribution of the EYR magnetic field and comparison of the period range available in the MT data. 


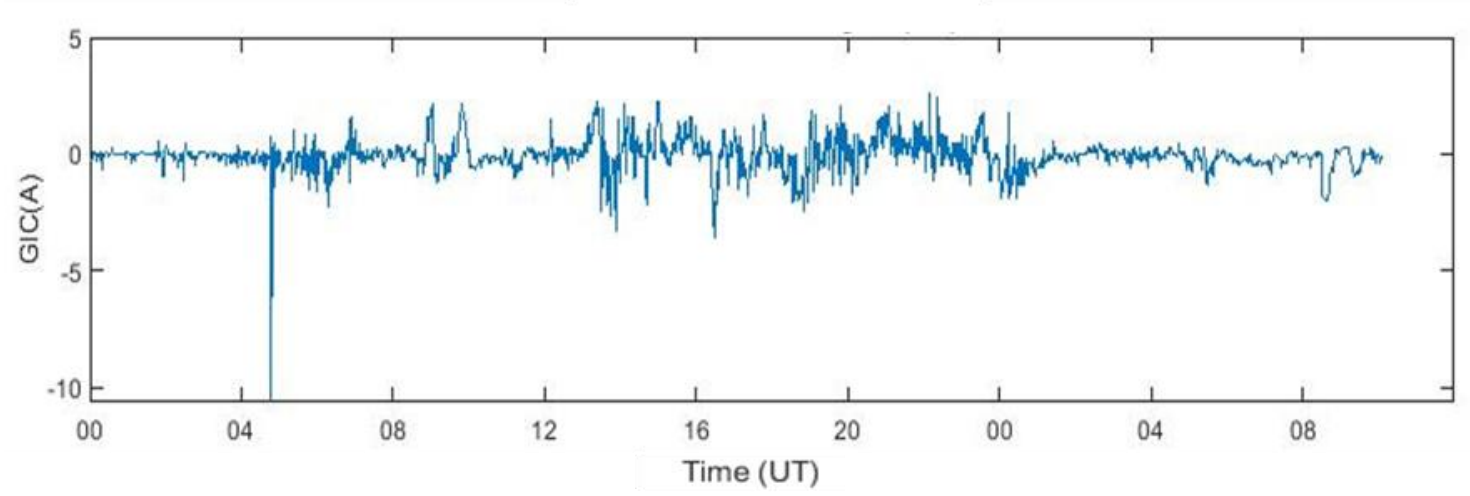

Figure 4. 5: GIC observed data, interpolated to 1-minute sampling at Islington transformer (ISLT6H) near Christchurch.

\subsection{Calculation of Geoelectric Field}

The knowledge of geoelectric fields is the most important parameter in GIC studies. In the previous chapter the simplistic uniform conductivity Earth model was used to estimate time domain geoelectric fields. The primary electromagnetic field originating from ionospheric and magnetospheric sources is a plane wave propagating vertically downwards. Therefore, a flatEarth model and the standard coordinate system, $x y$-plane at the Earth's surface and $z$-axis pointing downwards, can be used to model GIC in a power network. The uniform conductivity geoelectric fields, equations 3.1 and 2.2 used in Chapter 3, in the frequency domain are given by (e.g., Pirjola, 2002)

$$
\begin{gathered}
\boldsymbol{E}_{\boldsymbol{y}}(f)=-\sqrt{\frac{2 \pi}{\mu_{0} \sigma T}} e^{i \pi / 4} \boldsymbol{B}_{x}(f) \\
\boldsymbol{E}_{\boldsymbol{x}}(f)=\sqrt{\frac{2 \pi}{\mu_{0} \sigma T}} e^{i \pi / 4} \boldsymbol{B}_{\boldsymbol{y}}(f)
\end{gathered}
$$

This shows that the geoelectric fields $\left(\boldsymbol{E}_{\boldsymbol{x}}, \boldsymbol{E}_{\boldsymbol{y}}\right)$ are inversely proportional to the square root of the conductivity $(\sigma)$ and proportional to the square root of the frequency (inversely proportional to the square root of the period T). Also, the time-varying sinusoidal amplitude of the geoelectric field lags that of the geomagnetic field by a phase of $\pi / 4$. 
Since the conductivity of the Earth varies both laterally and with depth in recent studies more advanced and complex models of conductivity structure of the Earth have been used (more details are given in section 2.2.4 of Chapter 2). These models are based on the Earth response function or complex valued impedance tensor $(\underline{\underline{Z}})$ from magnetotelluric measurements. MT measurements themselves, which relate the induced electric field to the time-varying magnetic field can be used to directly predict the electric fields resulting from any geomagnetic variation. The magnetotelluric response tensor defined in this way is related to the normal MT impedance tensor through the permeability $\mu$, and has units of $\mathrm{mV} / \mathrm{km} / \mathrm{nT}$. At each frequency $f$ and location $(x, y), \underline{\underline{Z}}$ is a $2 \times 2$ complex-valued matrix. If the conductivity varies in 3-D then, in general

$$
Z=\left(\begin{array}{ll}
Z_{x x} & Z_{x y} \\
Z_{y x} & Z_{y y}
\end{array}\right)
$$

No element of $\underline{\underline{Z}}$ for example, $Z_{x x}$ has a simple mathematical relationship to any other of the response tensor elements, $Z_{x y}, Z_{y x}$, and $Z_{y y}$. In this study we have used MT data as given in the above matrix of four non-zero complex elements.
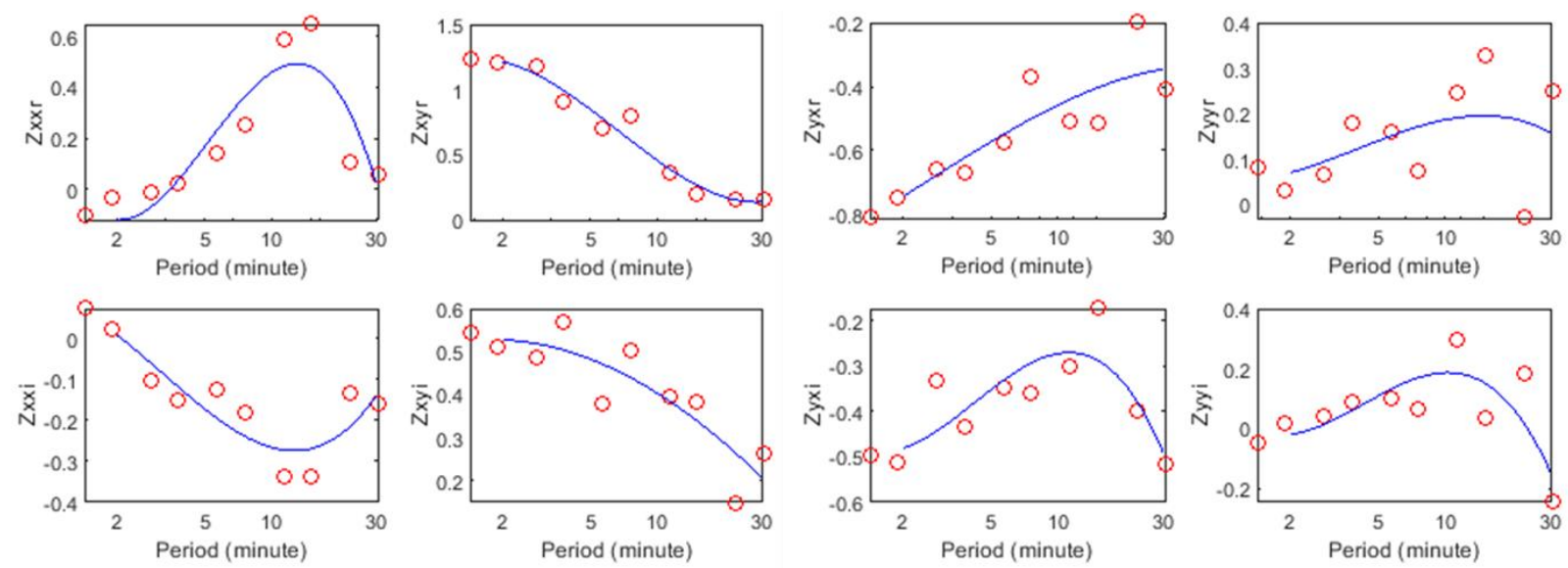

Figure 4. 6: Earth impedance tensor as function of frequency for CAR-119. Red circles show actual $M T$ periods. A third-degree polynomial is used for interpolation purpose.

Figure 4.6 shows plots of the real and imaginary parts of $Z_{x x}, Z_{x y}, Z_{y x}$, and $Z_{y y}$ at a MT site named CAR-119 (-41.07 N, 175.64 E), for which data were acquired on 16 March 2014. The $x$-axis is on a logarithmic scale where the red circles show the measured periods of MT data from approximately 2 to 30 minutes as given in Table 4.1. These periods have been interpolated using third-degree polynomials (blue curve) so that values of the components of the impedance tensor 
can be estimated at all periods/frequencies over the period range of the data. The equation which gives the relation between electric and magnetic fields in terms of periods $(T)$, using the above 3-D magnetotelluric response tensor, is given by (Bedrosian and Love, 2015)

$$
\begin{aligned}
& \boldsymbol{E}_{x}(T)=Z_{x x}(T) \boldsymbol{B}_{x}(T)+Z_{x y}(T) \boldsymbol{B}_{y}(T) \\
& \boldsymbol{E}_{y}(T)=Z_{y x}(T) \boldsymbol{B}_{x}(T)+Z_{y y}(T) \boldsymbol{B}_{y}(T)
\end{aligned}
$$

where $T$ is the period of variation and $\left(\boldsymbol{B}_{\boldsymbol{x}}, \boldsymbol{B}_{\boldsymbol{y}}\right)$ are horizontal components of the magnetic field. Using equations 4.1a and 4.1b, the expected electric field during the St. Patrick's Day storm can be calculated using the magnetic field data from Eyrewell magnetic observatory. Since the magnetic field data for the St Patrick's Day storm consists of 2048 data points with a one-minute interval the frequency transformation gives a range of periods from 120 to 2048 seconds. Based upon the MT data point distribution, to calculate the electric field at all intermediate periods a $3^{\text {rd }}$ degree polynomial is applied t the impedance data using MATLAB (blue curves in Figure 4.6). The use of interpolated values enables us to compute geoelectric field for all periods corresponding to the magnetic field data. These frequency domain geoelectric fields are converted into the time domain by applying an inverse Fast Fourier Transform (FFT).

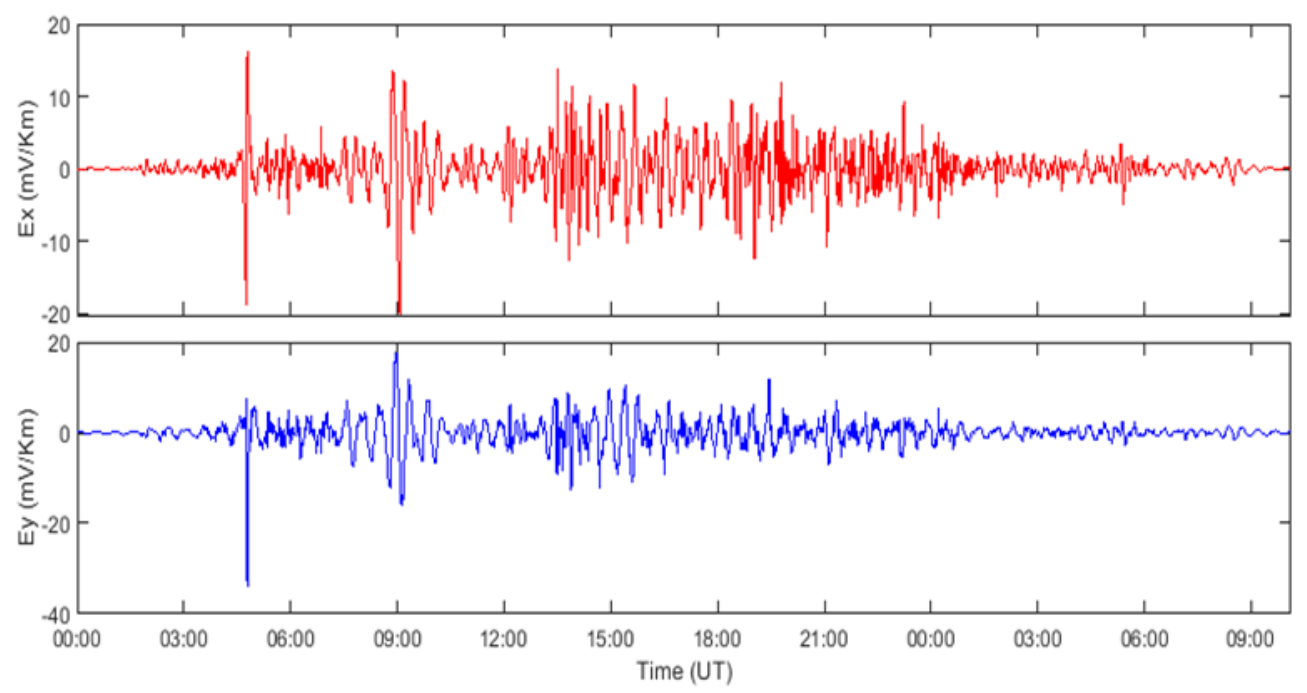

Figure 4. 7: Resulting geoelectric field using MT data and magnetic data of EYR observatory. 
Figure 4.7 shows computed time domain real-valued geoelectric fields for an example MT site by following this procedure. $\boldsymbol{E}_{\boldsymbol{x}}$ points in the North and $\boldsymbol{E}_{\boldsymbol{y}}$ in the East direction. The vector sum of both components of the electric field gives the total electric field.

\subsection{Spatial Interpolation of Geoelectric Field}

Referring to Figure 4.1 it is evident that MT data are not available for each cell of the thin-sheet model grid. MT sites are also largely distributed in the southern part of the North Island whereas the northern part is completely empty of MT sites. The application of MT data for calculating geoelectric fields requires a dense array of such measurements (Ingham et al., 2017). To run the power network model, horizontal geoelectric fields at each cell of the grid in the vicinity of substations and power lines are required. In the thin-sheet grid the North Island consists of a total of 436 cells among which 115 cells are filled with MT data. Since no magnetic observatory data are available for the North Island the magnetic data from Eyrewell (EYR) magnetic observatory in the South Island have been used for modelling purposes. Although it is expected that the magnetic field strength in the North Island would be smaller than in the EYR data, and would also not be the same throughout the North Island, the EYR data can still be used to derive some very useful results for the North Island. This argument is supported by Divett et al. (2020) for the South Island during the St Patrick's Day magnetic storm of 2015, who found that the geoelectric fields calculated using the thin-sheet approach showed only a minor differences between using a spatially varying magnetic field and a uniform magnetic field across the South Island. It has also been validated through the analysis of the magnetic field observations from four separate locations: two close to Dunedin, the Eyrewell observatory, and at a single location (Te Wharau) in the lower North Island (Rodger et al., 2020). In analyzing Total Harmonic Distortion occurring between 6-9 September 2017, time periods were selected where there were GIC associated with significant rates of change of the horizontal magnetic field. For a SSC occurring at 2302 UT on 7 September, the rates of change of the field were about $10 \mathrm{nT} / \mathrm{min}$ at Dunedin dropping to $6.6 \mathrm{nT} / \mathrm{min}$ at Te Wharau. At 0145 UT (8 September) observed rates of change of the horizontal field were $10 \mathrm{nT} / \mathrm{min}$ at Dunedin, $5.3 \mathrm{nT} / \mathrm{min}$ at EYR, and $3.7 \mathrm{nT} / \mathrm{min}$ at Te Wharau. A large rate of change of $30.6 \mathrm{nT} / \mathrm{min}$ at Dunedin between 1200 and 1300 UT 8 September saw smaller rates of change at EYR (12.3 nT/min) and Te Wharau (7.9 nT/min). Thus, in accordance with the result of Divett et al. (2020), rates of change during the St. Patrick's Day 
storm were relatively uniform across all four locations. Additionally, a comparison of rates of change $(d B x / d t)$ of the northward component of the magnetic field at an MT site close to New Plymouth and at the Eyrewell geomagnetic observatory is given by Mukhtar et al., (2020). These were calculated every minute over a period of 5 hours on 11 November 2019, showing a good correlation between $d B x / d t$ at the two locations. This further supports the suggestion that field variations at Eyrewell do indeed give a reasonable estimate of those in the North Island although how good this comparison is will vary from storm to storm with, on occasion, rates of change of the field in the lower North Island perhaps being only $60-70 \%$ of those at EYR.

Figure 4.8 shows a vector representation of computed geoelectric fields corresponding to all 115 MT sites at 04:47 UT when the geoelectric fields have a maximum value during the Sudden Storm Commencement (SSC). The Figure shows that geoelectric fields vary both in magnitude and direction throughout the North Island. The minimum geoelectric field $E=11.03 \mathrm{mV} / \mathrm{km}$ occurs near the east coast $(-38.74,177.58)$ in the high conductance region associated with the Hikurangi Margin and has a north-west direction. The maximum geoelectric field of $E=457.50 \mathrm{mV} / \mathrm{km}$ exists in the region $(-39.58,175.58)$ just to the west of the part of the thin-sheet model that represents the mountainous spine of the North Island, it has a south-west direction.

The geoelectric field corresponding to each cell having MT data, have been computed directly. But in order to fill empty cells with geoelectric fields, a 2-D interpolation technique is adopted. Interpolation is a statistical method of filling gaps in a dataset based upon the information contained in the given data points. Data can be interpolated in different ways based upon their uses and field of work. Many useful interpolation methods have been developed to fill spatial gaps in a 2-D dataset e.g., Longley et al., (2005). If there are $N$ number of MT sites of geoelectric field values $E_{i}$ at random locations of $r_{i}$ in a grid (where $i=1,2,3 \ldots N$ ) then

$$
F\left(r_{i}\right)=E_{i}
$$

where the determination of $F\left(r_{i}\right)$ which passes through all known data points is imposed with additional conditions, defining the character of various interpolation techniques. MATLAB offers different interpolation techniques for 2-D scattered data e.g. Linear, Nearest Neighbor, Natural and Spline. All these techniques have been tested on the available MT data. The Nearest Neighbor Interpolation seems to be most relevant as empty cells are filled based upon the 
neighboring vectors of geoelectric fields and there is no need to calculate weight values (Ashraf et al., 2017). Figure 4.9 shows interpolation results using the Nearest Neighbor method. The empty cells are filled with existing MT geoelectric fields with no weighting of geoelectric fields. The interpolation results are reliable between the cells containing MT sites, but the extrapolation produces the same fields for whole of the north-western part of North Island making extrapolation results very unrealistic.

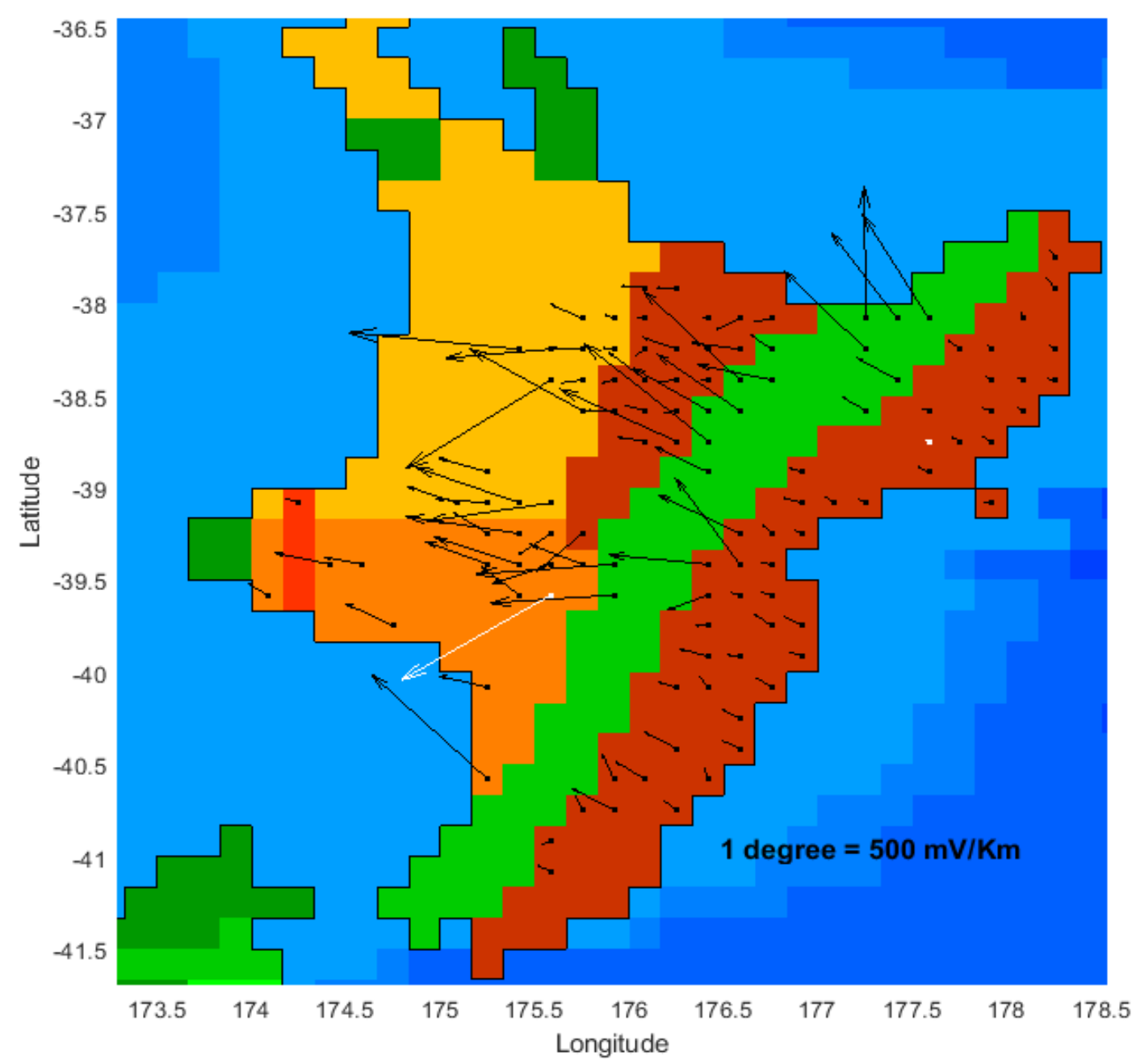

Figure 4. 8: Calculated vector geoelectric fields at MT sites at 04:47 UT. The white arrows show the smallest field ( $E=11.03 \mathrm{mV} / \mathrm{km}$ ) exists near the east coast (high conductance associated with the Hikurangi Margin) whereas the largest field ( $E=457.50 \mathrm{mV} / \mathrm{km}$ ) exists in the region close to the mountainous spine of the North Island. The upper part of island is not shown here because no MT data is available in that region. 


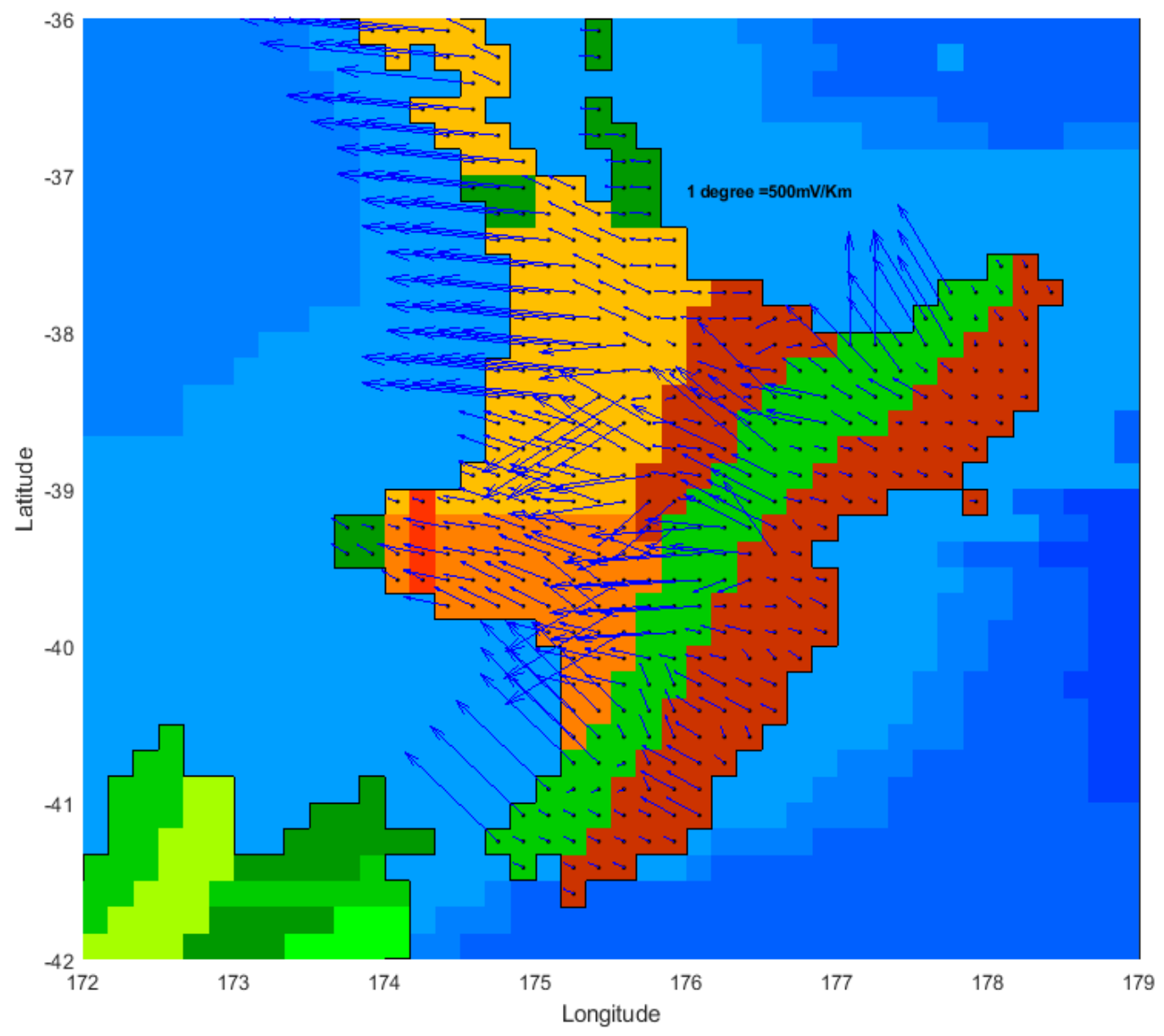

Figure 4. 9: Interpolated geoelectric fields at 04:47 UT using the Nearest Neighbor method. It uses the original field values for extrapolation therefore the same fields exist in the form of patches.

The Nearest Neighbor method does not give continuous or smoothly interpolated fields, but fields which are a repetition of the original data. To derive a smooth interpolation which looks more natural between MT sites another interpolation technique is adopted as shown in Figure 4.10. This technique, Biharmonic Spline, is based on smoothness and tension. Figure 4.10 shows the interpolation results using the Spline method. The two techniques are significantly different where very sparse MT data is available e.g., in the relatively low conductance regions on the west coast above $-39^{0}$ latitude. The Spline method generates smooth interpolated fields, as can be seen in lower and middle of island, but extrapolated fields in the northern part which are very unrealistic. In the north the geoelectric fields do not agree with any of the MT geoelectric fields. For example, based upon MT data, the geoelectric fields generally point in a north-west direction. The extrapolated fields in the northern region are perpendicular to this direction. The Nearest Neighbor method, however, gives relatively uniform geoelectric fields based upon the available 
MT data. Therefore, instead of choosing completely unverifiable results it is preferable to choose values based upon observed data. Therefore, the preferred method of interpolation is using Nearest Neighbor, although the use of extrapolated geoelectric fields in the calculation of GIC in the power network may likely to lead to significant errors. Therefore, an approach of using an equivalent circuit to represent the northern part of the network has been investigated.

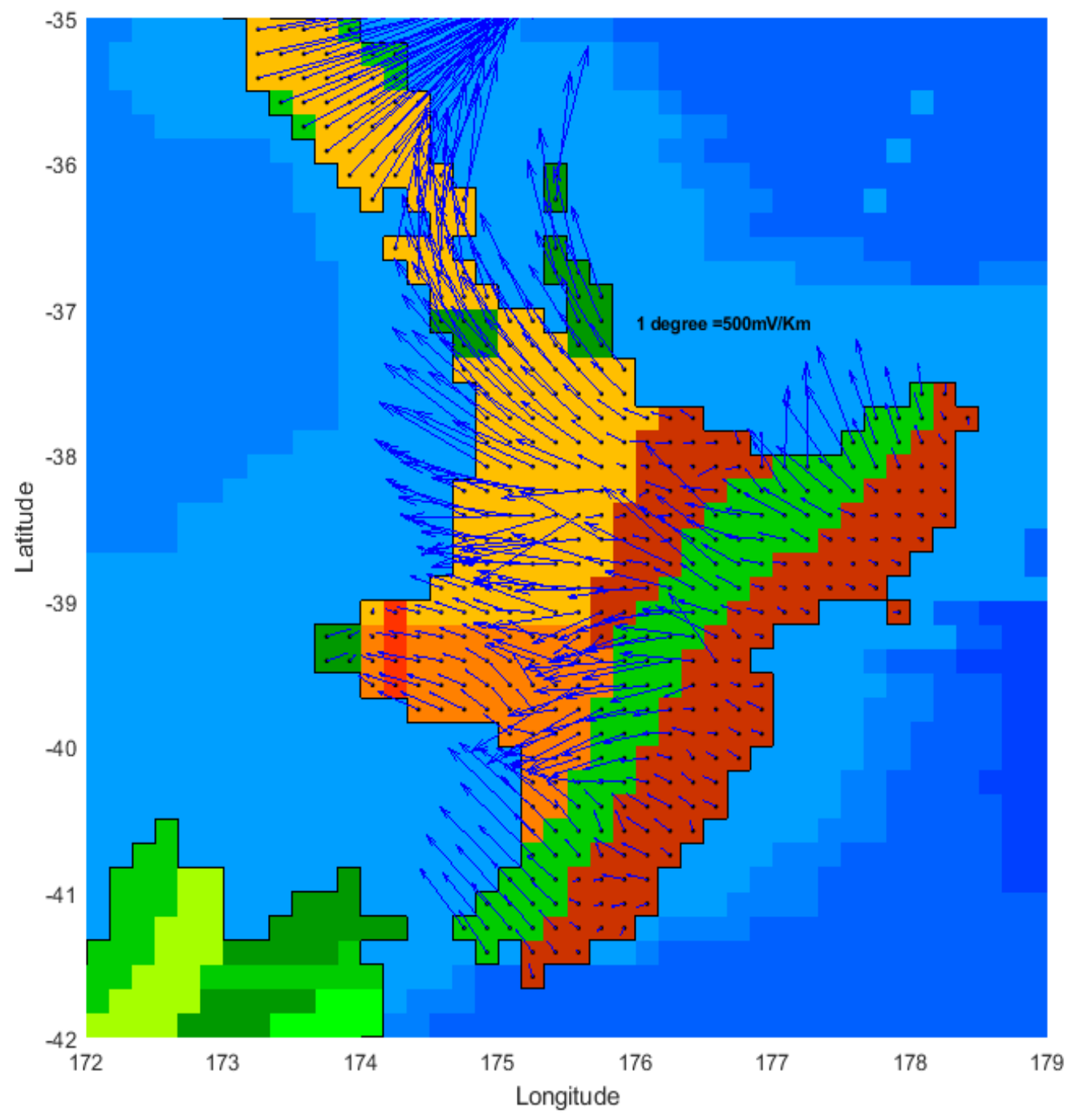

Figure 4. 10: Interpolated geoelectric fields at 04:47 UT using Biharmonic Spline method. This gives smooth fields between MT sites, but results are worst in northern part.

\subsection{Uniform Electric Field and Transformer Response}

Geoelectric fields are the input for GIC modelling. The local distribution, orientation and magnitudes of these fields determine the magnitude of GIC in the power grid system. The value and distribution of GIC in power network not only depends upon the geoelectric fields, but on 
the electrical parameters related to network itself. The key network parameters include resistance and length of power lines, earthing resistance and type of transformers and number of transformers within a substation (Divett et al, 2018). It is assumed here that network parameters remain unchanged and therefore we are more concerned with the spatial and temporal distribution of geoelectric fields. For example, when power lines and electric fields are in parallel maximum current is drawn into power lines and hence through transformers. The number of transformers within a substation is also an important factor as in a substation having many earthed transformers the total earthing current would be divided between transformers and appear as small GIC in an individual transformer. If a substation has only a single earthed transformer the whole line current would appear in the single transformer and lead to a large GIC. Similarly, the direction of current, which is shown in terms of positive and negative signs, also plays an important role in determining GIC in transformers. For example, if all lines towards a substation have same signs of currents it would add up to large GIC, whereas line currents with opposite signs subtract and give a small GIC.

Transformer level GIC refers to the current flowing through each winding of a transformer in the power network (Divett et al., 2018). To gain an initial understanding of the transformer response of the whole North Island power network a uniform electric field have been applied in different orientations and the transformer GIC calculated. In this way transformers have been identified which are more vulnerable to certain directions of geoelectric fields. Although real geoelectric fields are neither uniform in magnitude nor in direction, this approach can provide useful information about the response of the network to geoelectric field variations. For this purpose, a uniform electric field of $1 \mathrm{~V} / \mathrm{km}$ in different directions (east, north-east, north and north-west) has been used and the GIC computed for all transformers.

Figure 4.11 shows GIC (in Amperes) computed for all transformers within a substation for the whole North Island power network. Transformers with positive GIC are shown by blue bars and negative GIC transformers are shown by red bars. Since there are many transformers within a substation individual transformers are not named but all transformers within a substation are shown by tick marks and the name of the substation. The names of the substations are given in the order from south to north of the North Island. Following the nomenclature given in section 
2.5.2 of Chapter 2, the GIC through high-side and low-side windings of a transformers are given by adding $\mathrm{H}$ and $\mathrm{L}$ letters respectively, along with transformer names.

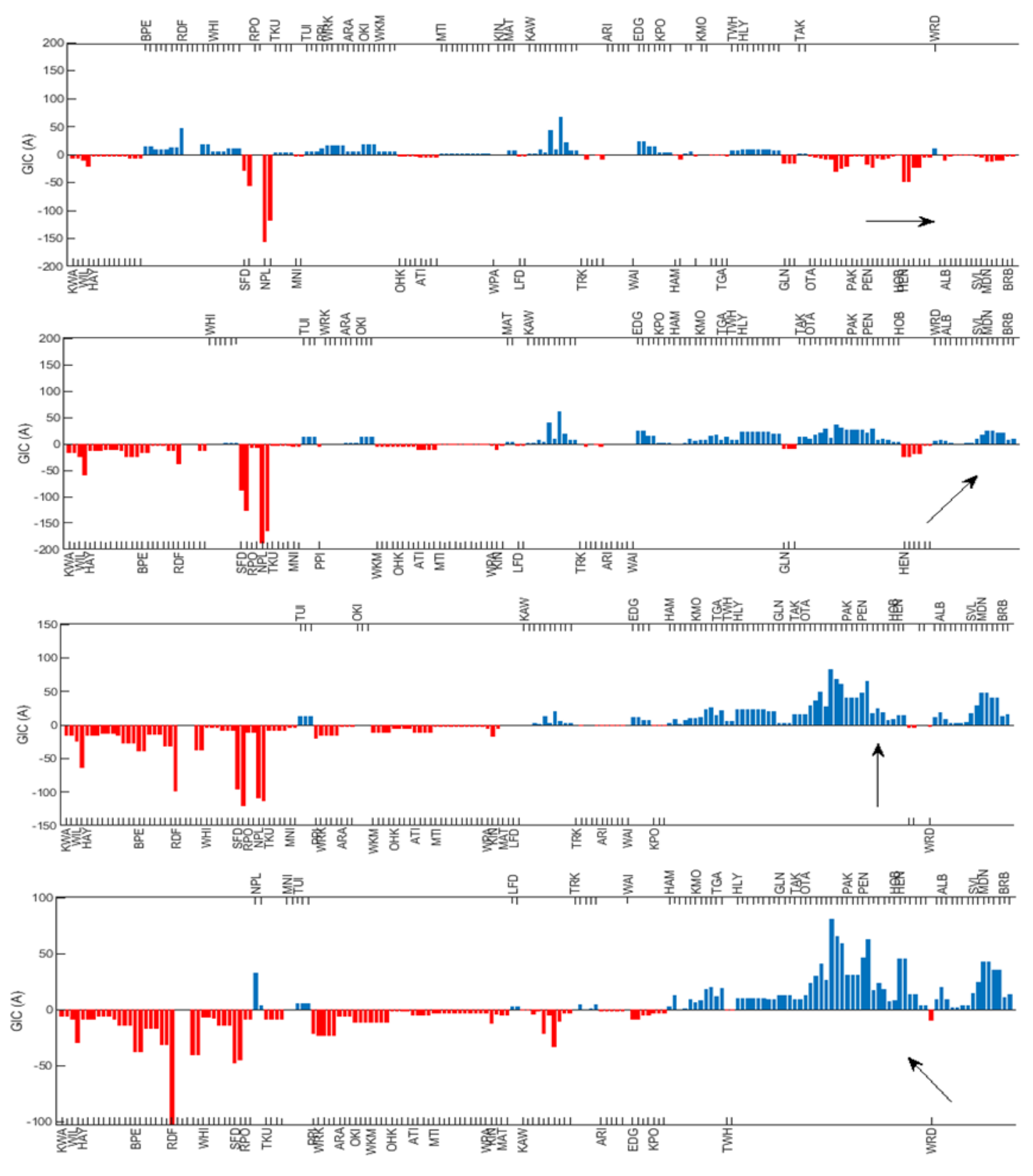

Figure 4. 11: Transformer GIC produced in the North Island power network of after applying a uniform electric field of $1 \mathrm{~V} / \mathrm{Km}$ in the direction shown by the arrow.

Figure 4.11 shows New Plymouth transformer (NPL-T8L), which lies on west coast, has the highest GIC magnitude of order -150 A for all orientations of the geoelectric field other than when the field is in a north-west direction (last panel). Stratford (SFD-T1OH), which is near the New 
Plymouth substation, also experiences significant GIC of -100 A when fields are in a north direction. The Redclyffe transformer (RDF-T1H), which lies on the east coast, experiences large GIC for north and north-west fields. In the central part of the island Kawerau (KAW-T12H) is the only transformer which experiences significant GIC, exceeding a magnitude of $50 \mathrm{~A}$ when fields are in an east direction. In the north, Otahuhu (OTA-T5L) and Penrose (PEN-T1OH) show significant GIC, but the magnitude is less than 100 A. The transformers in the south show large GIC with a negative sign whereas transformers in the north tend to show large positive GIC. This indicates the overall flow of current from one part of the network to the other part. Transformers in the south experience largest GIC for east and north-east fields when the transformer response is negligible in the north. Although, northern transformers also show significant GIC during north and north-west fields the magnitude is less than for transformers in the south. In summary, the transformers which experience large GIC using uniform fields include New Plymouth (NPL), Stratford (SFD), Redclyffe (RDF), Kawerau (KAW), and, in the north of the North Island Otahuhu (OTA) and Penrose (PEN).

Table 4.2

Currents in lines connected with NPL substation and transformer GIC.

\begin{tabular}{llllll}
\hline $\begin{array}{l}\text { New } \\
\text { Plymouth }\end{array}$ & $\begin{array}{l}\text { Connected } \\
\text { substations }\end{array}$ & $\begin{array}{l}\text { Number } \\
\text { of lines }\end{array}$ & $\begin{array}{l}\text { Network } \\
\text { Resistance }\end{array}$ & $\begin{array}{l}\text { Line length } \\
\text { (Km) }\end{array}$ & GIC (A) \\
\hline NPL110 & CST110 & 2 & 0.11 & 11 & -17.8 \\
NPL220 & SFD220 & 2 & 0.45 & 42 & -60.2
\end{tabular}

The magnitude of GIC in a transformer depends upon currents through lines connecting with neighboring substations. The connections of New Plymouth substation with other substations and the magnitude of line currents for an east directed geoelectric field are given in Table 4.2. Note that NPL has only one transformer and is connected to two substations Carrington Street (CST) and Stratford (SFD) through two lines with each substation. Both currents are towards the NPL substation, as indicated by negative sign, and therefore add up to give a large transformer GIC at NPL. Since there are two lines to both CST and SFD a factor a 2 needs to be multiplied to account for the total currents. The length of the power line and the orientation play an important role in producing large GIC. For example, the lines running from SFD is the main contributor and carries more current (-60.21 A) compared to lines from CST which carry only -17.82 A. 


\subsection{Uniform Electric Field and Substation Response}

The substation level GIC is the sum of GIC in all earthed transformers (Divett et al., 2018), therefore it can be calculated by adding the GIC of all earthed transformers as described in the above section. Substation GIC show how much current goes into or out of a substation and gives a much broader picture of the impact of geoelectric fields on a power network. This is the reason many studies include substation level GIC to see the impact during different geomagnetic storms (Nakamura et al., 2018; Beggan et al., 2015 and Blake et al., 2016). In a substation having a large number of earthed transformers the total GIC divides between all individual transformers resulting in small transformer GIC even though the total substation GIC may be large. Not all transformers in a substation are earthed e.g., in the case of autotransformers the windings are partly earthed contributing to substation GIC, but partly only exchanging currents between different voltage nodes and therefore not contributing into substation GIC. Substation GIC take into account the total current going through windings of all earthed transformers.

Substation GIC have been computed for the same uniform fields as for transformer GIC. Figure 4.12 shows substation-level GIC on a North Island map when a uniform electric field of $1 \mathrm{~V} / \mathrm{km}$ is used in the east direction. The color intensity shows the magnitude of GIC in substations and line currents. NPL substation (in red) shows the largest GIC exceeding -150 A. Kawerau (KAW) which lies close to the north coast also shows a large GIC value of $85 \mathrm{~A}$. In the upper part, the substation which is most effected is Henderson (HEN) where the GIC value reaches to $-110 \mathrm{~A}$. The line that goes from SFD to Huntly experience a large current $\sim 70 \mathrm{~A}$ as shown by the color. Similarly, lines extending from RDF into the middle and upper part of island also experience large currents.

Figure 4.13 shows substation-level GIC for all orientations (east, north-east, north and northwest) of a uniform electric field of $1 \mathrm{~V} / \mathrm{km}$. Substations that experience positive GIC are shown in blue and substations having negative GIC are shown in red. The Figure shows that the substation response is quite different from the transformer response. There were few individual transformers showing large GIC for all orientations, but the substation response is more diverse. New Plymouth substation shows a GIC magnitude exceeding -150 A for east and north-east fields. The same magnitude GIC can be seen at Huntly (HLY) for north and north-east fields. Field orientations produce large substation GIC in southern and northern substations equally but in 
opposite directions except for eastward electric fields. In the south Haywards (HAY), Bunnythorpe (BPE) and Redclyffe (RDF) show large GIC above $100 \mathrm{~A}$ and in the north Penrose (PEN), Otahuhu (OTA) and Henderson (HEN) show GIC above $100 \mathrm{~A}$. The analysis shows that large transformer level GIC at NPL, SFD, RDF and OTA cause large substation GIC. However, large substation GIC also depend upon the number of transformers as seen at HLY, HAY, HEN and BPE substations which have 8, 7, 4 and 4 earthed transformer nodes, respectively. The substation plots also show an equal amount of positive and negative current in substations, showing the flow of current from one end of the network to the other.

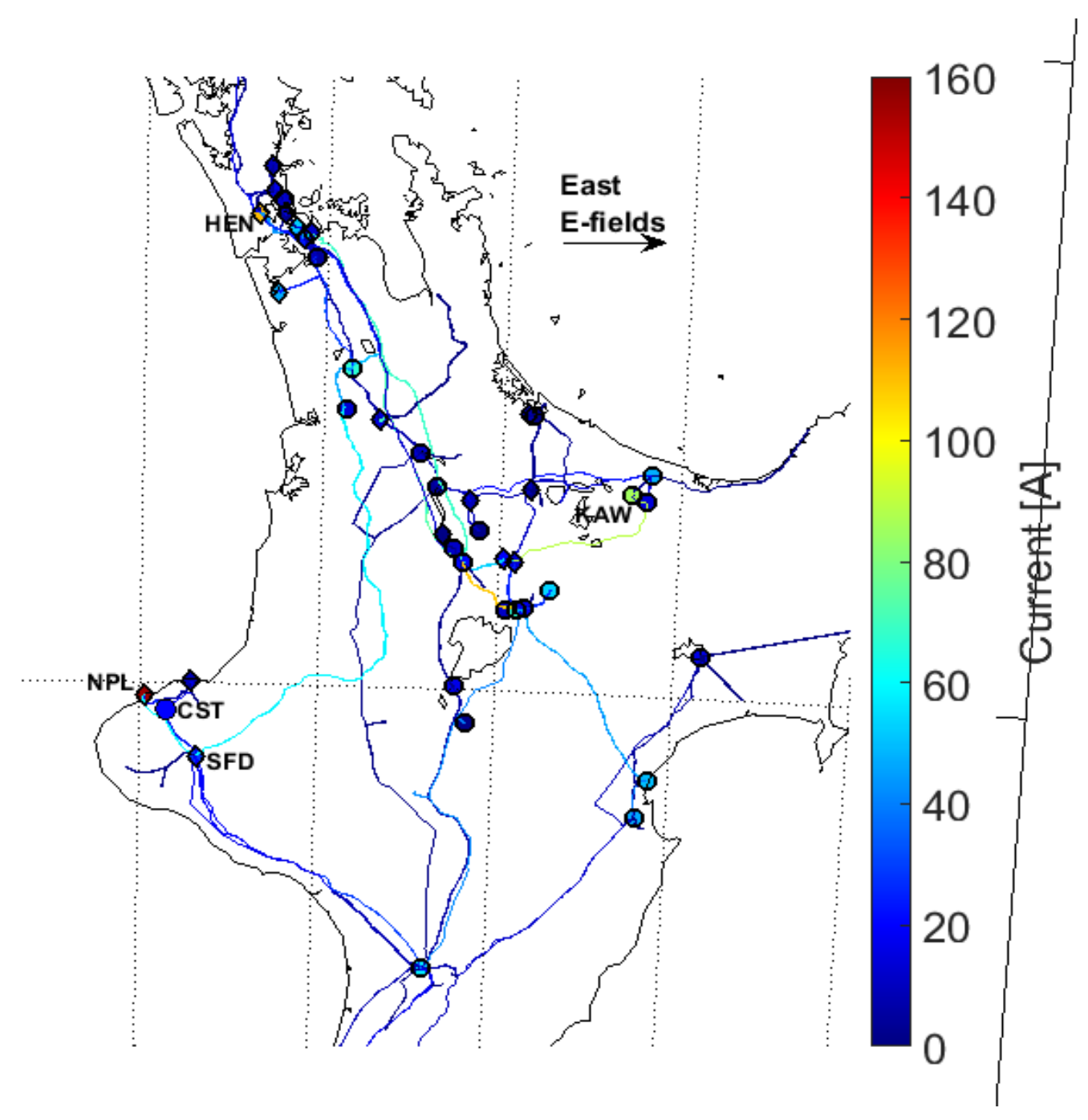

Figure 4. 12: Color map showing GIC at different substations and line currents for a uniform geoelectric field of $1 \mathrm{~V} / \mathrm{km}$ in the east direction. 

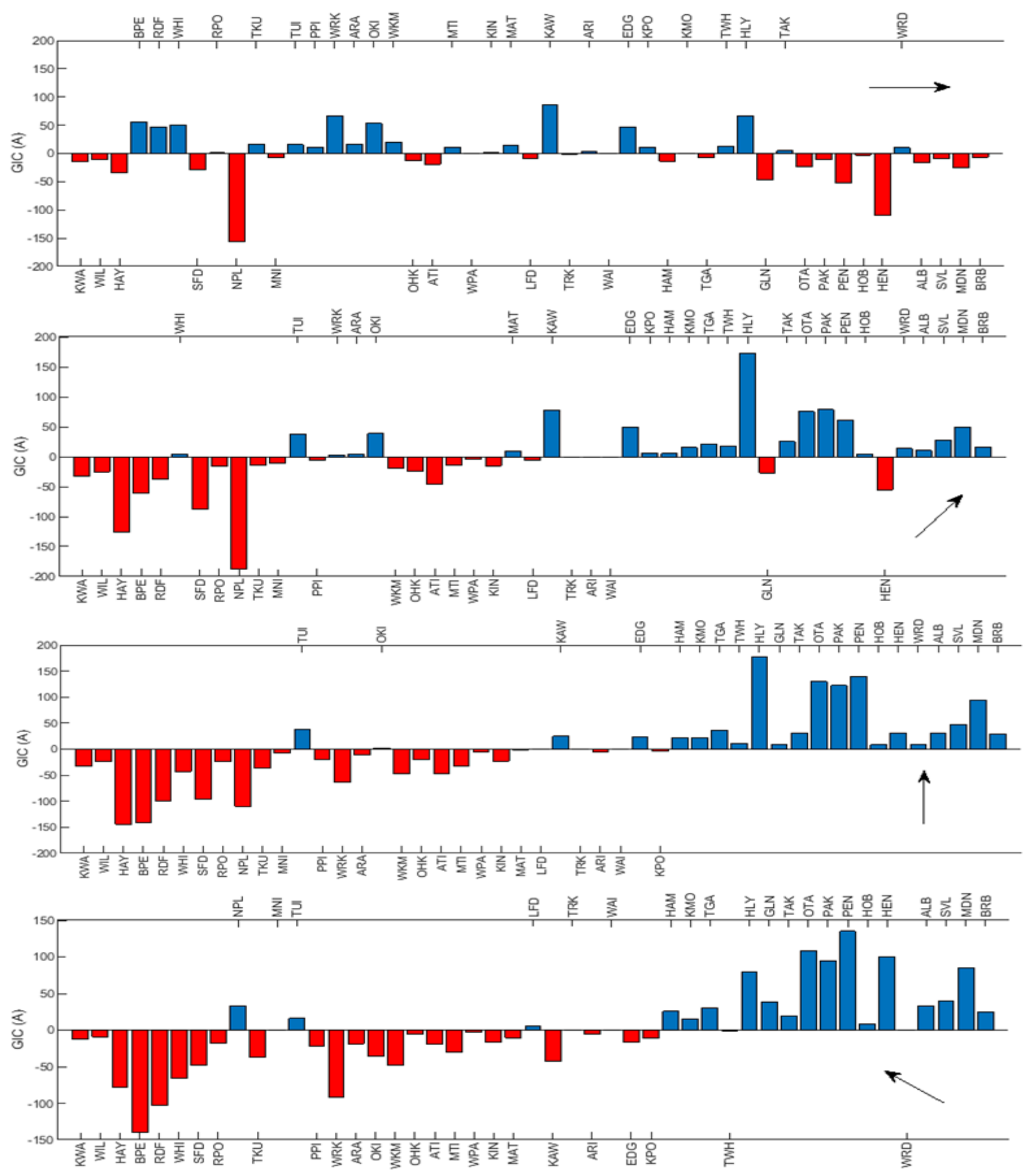

Figure 4. 13: GIC computed in all substations of the North Island while applying uniform electric field of $1 \mathrm{~V} / \mathrm{km}$ in four different directions shown by arrow. 


\subsection{Network Model and Equivalent Circuit Approach}

The North Island power network lies in higher mid-latitudes, between $34^{\circ}$ to $42^{\circ} \mathrm{S}$, where auroral effects are minimal compared to the South Island. The power network of the North Island is connected to the South Island only through a High Voltage DC link, and GIC cannot flow between the two networks (Mac Manus et al. 2017). Therefore, the power network of the North Island can be treated as isolated. The network is owned and operated by a single network operator, Transpower New Zealand Ltd. Transpower maintains records of the network characteristics. For the present study, all data of transmission line resistances, substation locations, and earthing resistances have been supplied by Transpower. The North Island high-voltage transmission network consists of transmission lines with four different voltage ranges: 50 or $66 \mathrm{kV}, 110 \mathrm{kV}$, $220 \mathrm{kV}$ and $400 \mathrm{kV}$. The North Island network is therefore effectively an isolated network of 64 nodes connected by 121 transmission lines. As discussed in the last section the network model requires information on geoelectric fields at each cell of the grid to compute GIC.

It has been seen that interpolation works well to fill gaps in geoelectric fields between MT sites but that the extrapolated geoelectric fields extending towards the northern part of the North Island are unrealistic. Since GIC can flow in or out of every part of network such unrealistic geoelectric fields would affect adversely the accuracy of calculation of GIC in the whole network. To avoid this an equivalent circuit approach has been explored to cover the area without any MT data. Different equivalent circuit approaches have been discussed by Boteler et. al., (2013) in terms of Thevenin's equivalent voltage and resistance values of a neighboring network. For example, the simplest approach is to ignore the neighboring network (in this case the northern part of North Island) and leave the connection as an open circuit. This involves no information from the neighboring network and the equivalent/Thevenin's circuit voltage and resistance would be $V_{t h}=0$ and $R_{t h}=\infty$. The most appropriate equivalent circuit approach uses line voltages connecting the two neighboring networks and line resistance i.e. $V_{t h}=V_{L}$ and $R_{t h}=$ $R_{L}$. This equivalent circuit has been verified by applying uniform geoelectric fields of different orientations.

To implement this approach, we have disconnected the part of network (the neighboring network) where no MT data are available. Figure 4.14 shows the network configuration after 
applying the equivalent circuit approach. The black dots show substations and green lines are usual network connections whereas the blue lines are of infinite resistance and treated as a neighboring network. The point of disconnection lies at Karapiro (KPO) substation (-37.92, 175.53) shown by the diamond in close-up view (Figure 4.14b).
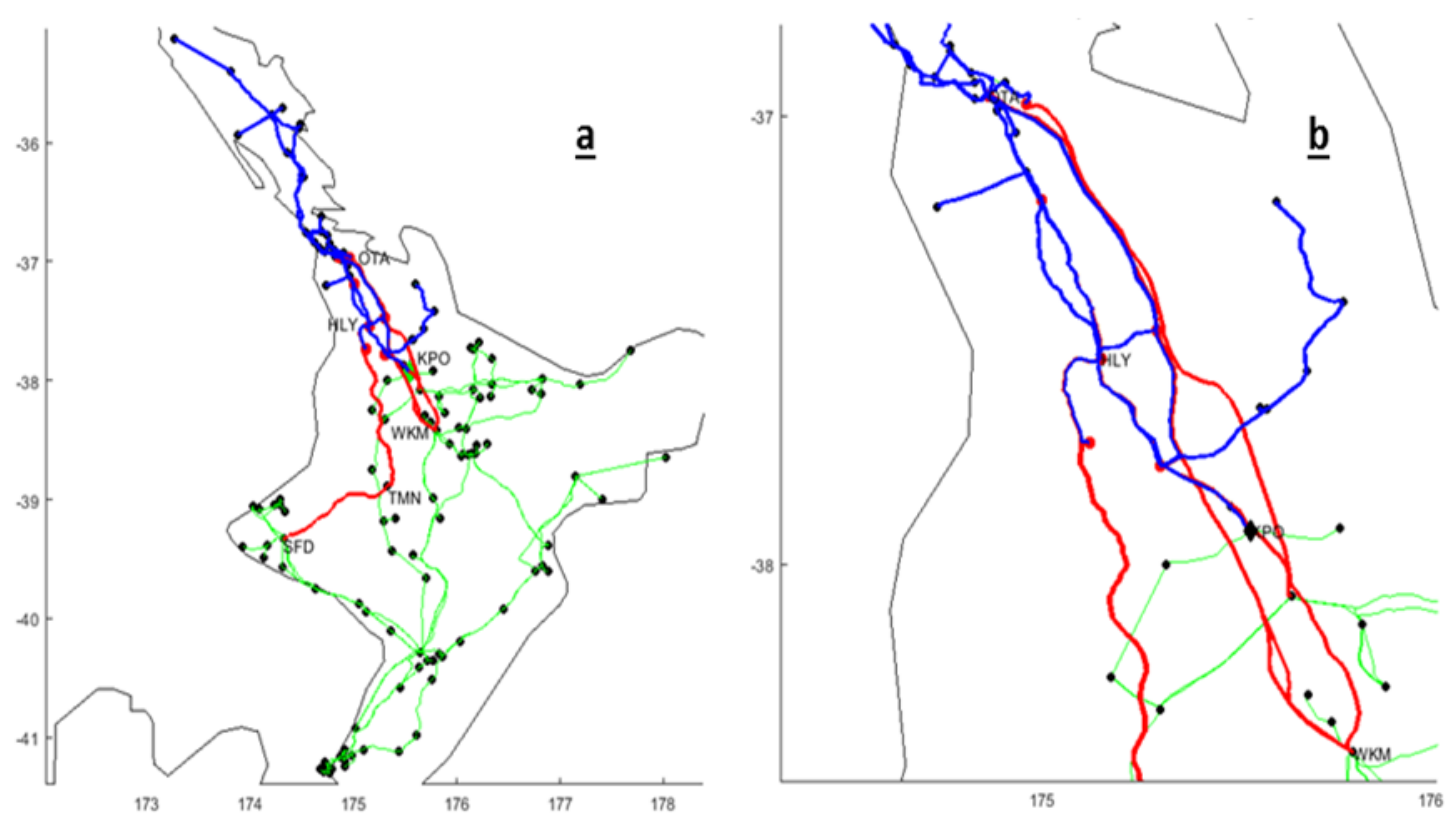

Figure 4. 14: (a) Equivalent Circuit configuration for the North Island power network. Green lines show normal connections with substations (shown by black dots). Blue lines show part of neighboring network and are regarded as having infinite resistances. The red lines are connections with normal resistance values except that their northern ends (shown by red dots) have been earthed. (b) shows close-up view of earthed connection substations.

There are, however, a few long power lines which originate from substations south of this substation. The power lines in question, from Arapuni (ARI), Whakamaru (WKM), Taumarunui (TMU) and Stratford (SFD) substations, extend towards the north where extrapolated geoelectric fields have been calculated. These lines, as shown by their substation names and voltage nodes, are given in Table 4.3. For the purposes of GIC calculation the northern ends of these lines have been earthed and these lines are shown as red lines in Figure 4.14. The importance of including these lines is that they connect the parts of the network to the north and south of Karapiro and, having long lengths, may have a significant effect on the lower part of network. 


\section{Table 4.3}

The lines for which northern ends (third column) have been earthed to isolate network of lower North Island.

\begin{tabular}{cccccc}
\hline $\begin{array}{c}\text { S. } \\
\text { No. }\end{array}$ & $\begin{array}{c}\text { Substation } \\
\text { node (From) }\end{array}$ & $\begin{array}{c}\text { Substation } \\
\text { node (To) }\end{array}$ & Resistance & $\begin{array}{c}\text { Line } \\
\text { length }\end{array}$ & $\begin{array}{c}\text { No. of } \\
\text { Lines }\end{array}$ \\
\hline 1 & ARI110 & BOB110 & 8.20 & 122 & 1 \\
2 & ARI110 & HAM110 & 3.28 & 47 & 2 \\
3 & WKM220 & OTA220 & 5.37 & 191 & 2 \\
4 & WKM220 & BHL220 & 0.86 & 185 & 2 \\
5 & WKM220 & HAM220 & 0.411 & 91 & 1 \\
6 & WKM220 & OHW220 & 0.53 & 118 & 1 \\
7 & TMN220 & TWH220 & 0.33 & 145 & 1 \\
8 & SFD220 & HLY220 & 0.64 & 280 & 1
\end{tabular}

To check the validity of this approach we have calculated GIC resulting from hypothetical uniform geoelectric fields of different orientations. First geoelectric fields of $1 \mathrm{~V} / \mathrm{km}$ in different orientations in intervals of 45 degrees are applied to whole North Island network and the resulting GIC computed. The same fields are then applied with the northern part of the network disconnected, as explained above, and earthing the power lines mentioned in Table 4.3. The calculated substation GIC extending up to Karapiro (KPO) are shown in Figure 4.15 for both calculations. It can be seen that disconnecting the network north of Karapiro, and grounding the lines listed in Table 4.3, has minimal effect on the calculation of GIC in the lower part of the network. The GIC are almost the same for all substations except for small differences for substations connected through the power lines mentioned above. That difference is well understood because these substations have been disconnected from the neighboring network. This implies that this approach can be used to calculate GIC from the geoelectric fields derived from the limited range of MT data. But it is important to note that, using this approach, only the lower part of the island can effectively be modelled as the upper part of network is regarded as a neighboring network. 

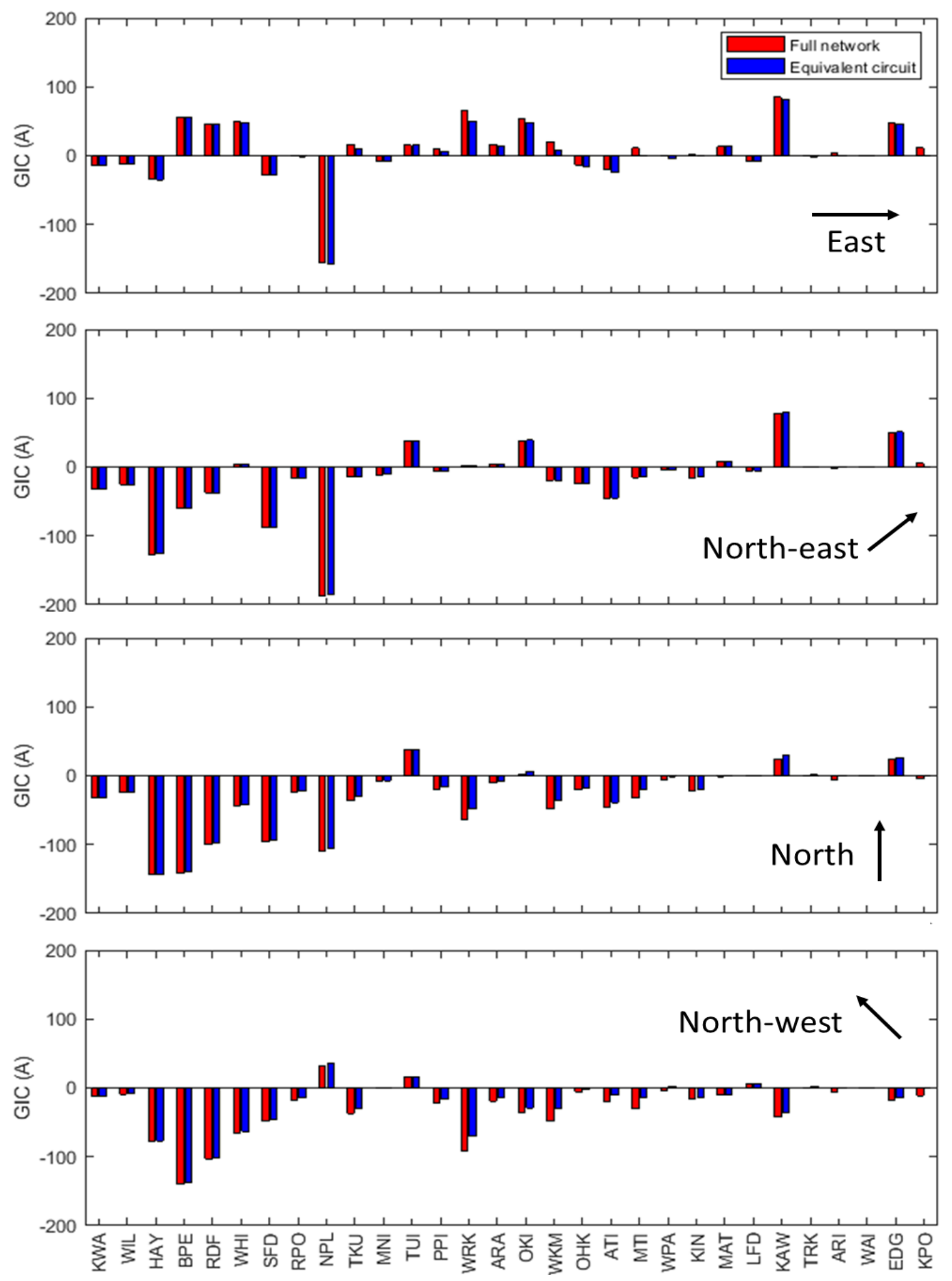

Figure 4. 15: A comparison of GIC calculated using the full network (red) and calculated using the equivalent circuit approach (blue) using uniform fields of $1 \mathrm{~V} / \mathrm{km}$ in different orientations as shown in respective panels. 


\subsection{MT Geoelectric Field and Transformer GIC}

The results of GIC computed using geoelectric fields derived from MT data are given in this section. After computation of the geoelectric field in each cell of the grid GIC are computed using the power network model. The network model of New Zealand used in this study was developed by Divett et al. (2018) to compute transformer level GIC. For details the reader is referred to Chapter 2. The network model computes transformer level GIC flowing through each winding of every transformer in the high-voltage transmission network. Transformer-level GIC are important for comparison with measurements, as GIC measurement devices are installed on individual transformers. Magnetic storms also pose a real threat to individual transformers in a power grid system.

One-minute discrete geoelectric fields of a total of 2048 data points (as shown in Figure 4.7) covering the St. Patrick's Day storm have been used to compute GIC. The sign convention for GIC of positive (out of substation/transformer) and negative (into a substation/transformer) is used. In the calculation for MT geoelectric fields only substations in the lower part of North Island are considered as the equivalent circuit approach has been used for the upper part of the network which is regarded as a neighboring network.

Figure 4.16 shows a snapshot of model GIC at 04:47 UT computed using the Nearest Neighbor geoelectric fields. This shows the response of the network during the Sudden Storm Commencement (SSC) when field values are maximum and generally in a north-west direction on average (Figure 4.9). Figure 4.16 shows that few substation transformers experience large GIC. The largest GIC are produced in transformers at Redclyffe (RDF-T1H) and New Plymouth (NPLT8L). For Redclyffe the GIC magnitude is $-10.97 \mathrm{~A}$ and for NPL it is $9.08 \mathrm{~A}$. Kawerau (KAW-T12H) is the only substation in the north-east part of network experiencing large GIC with a value of $4.5 \mathrm{~A}$. 


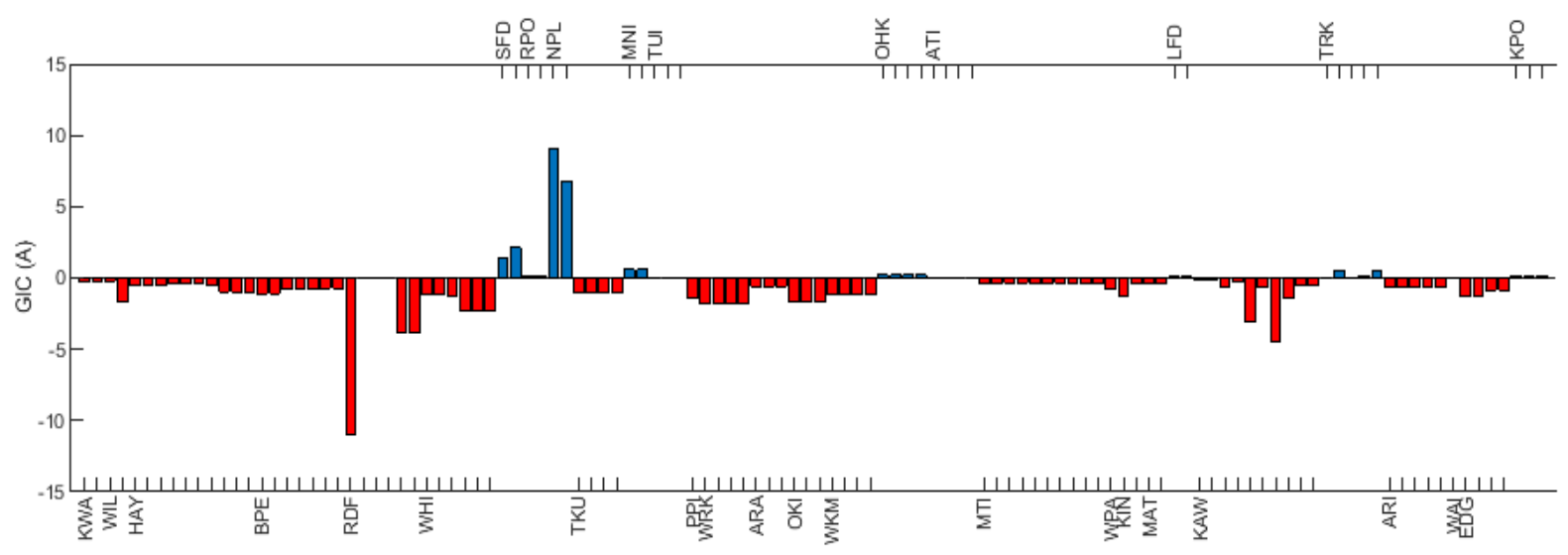

Figure 4. 16: MT model transformer GIC for all North Island substations during the SSC at 0447 UT. The Red bars show negative GIC values and blue bars show positive GIC values.
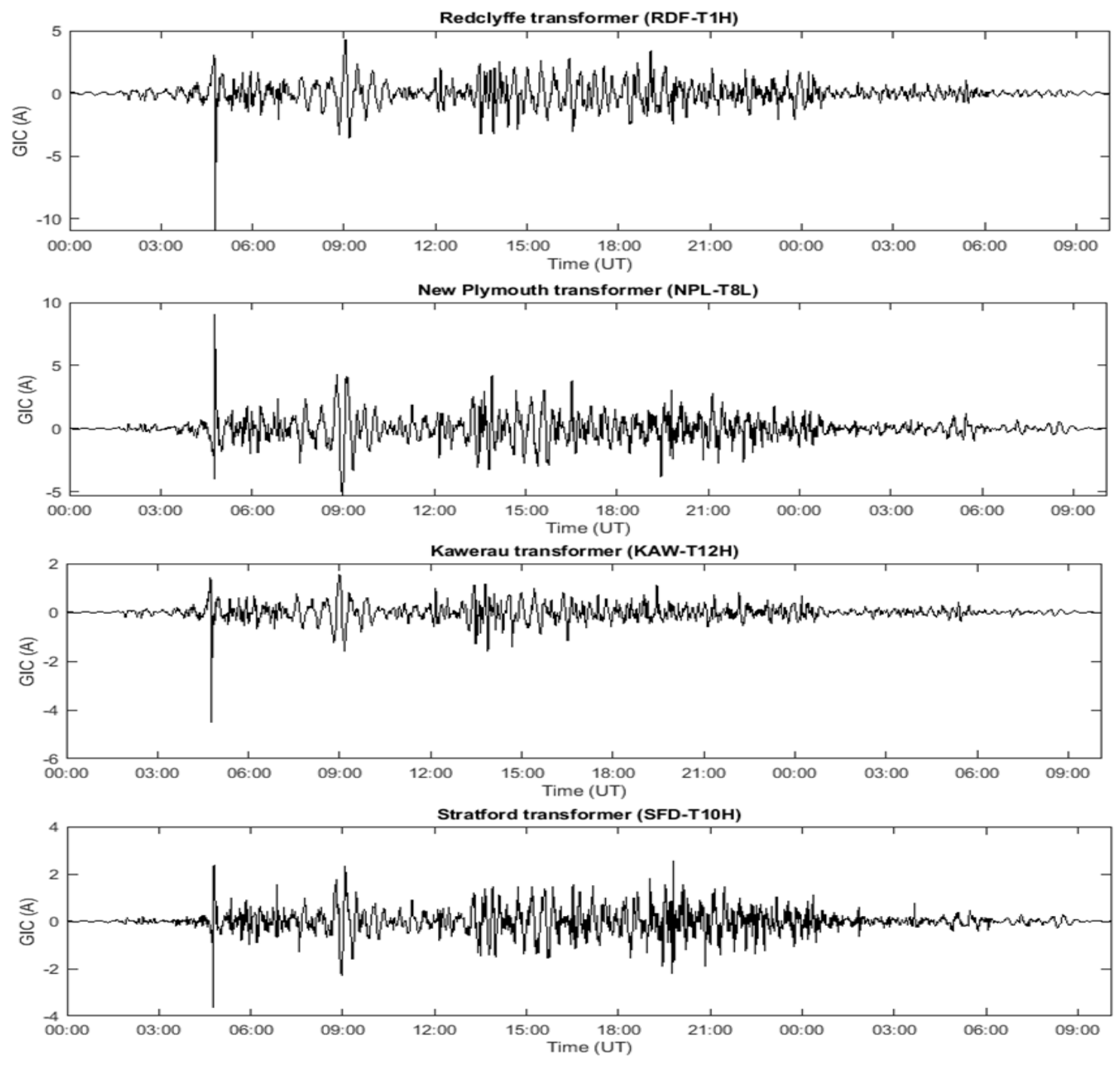

Figure 4. 17: Transformers (as mentioned on top of each panel) showing largest GIC during St. Patrick's Day storm calculated using MT geoelectric fields. All transformers show a strong peak of GIC at 04:47 UT (SSC). 
Figure 4.17 shows the time domain GIC for transformers experiencing the largest GIC during the St. Patrick's Day storm. Since the transformer GIC are maximum during the SSC these are the same transformers as discussed above.

1. Redclyffe (RDF-T1H)- (-39.55, 176.82)

2. New Plymouth (NPL-T8L)- (-39.06, 174.03)

3. Kawerau (KAW-T12H)- $(-38.08,176.72)$

4. Stratford (SFD-T10H)- $(-39.33,174.32)$

It is important to remember that these GIC are computed only in the period range of 2-30 minutes as permitted by the period range of the MT data. Therefore, lower frequency components are missing in the model GIC. The model GIC show large currents during the SSC which has also been observed in the measured data of all transformers of the South Island.

\subsection{MT Geoelectric Fields and Substation GIC}

As stated earlier, in a substation, different transformers could be earthed and the sum of GIC of all earthed transformers is known as the substation GIC. As they are the accumulative effect of all earthed transformers large substation GIC are not necessarily harmful, but these are significant in the context of showing the general pattern of current flow in different parts of the network. Substation level GIC have also been used in many studies of GIC modelling in different networks (Nakamura et al., 2018; Beggan et al., 2015 and Blake et al., 2016) during different magnetic storms, therefore substation level GIC determined here can also be useful for comparison purposes. Figure 4.18 shows substation GIC calculated using MT geoelectric fields at 04:47 UT. The following substations are identified as having the largest substation GIC. In order of magnitude:

1. Redclyffe (RDF)- $(-39.55,176.82)$

2. Whirinaki (WHI)- $(-39.38,176.89)$

3. New Plymouth (NPL)- $(-39.06,174.03)$

4. Wairakei (WRK)- $(-38.62,176.11)$

5. Kawerau (KAW)- $(-38.08,176.72)$ 


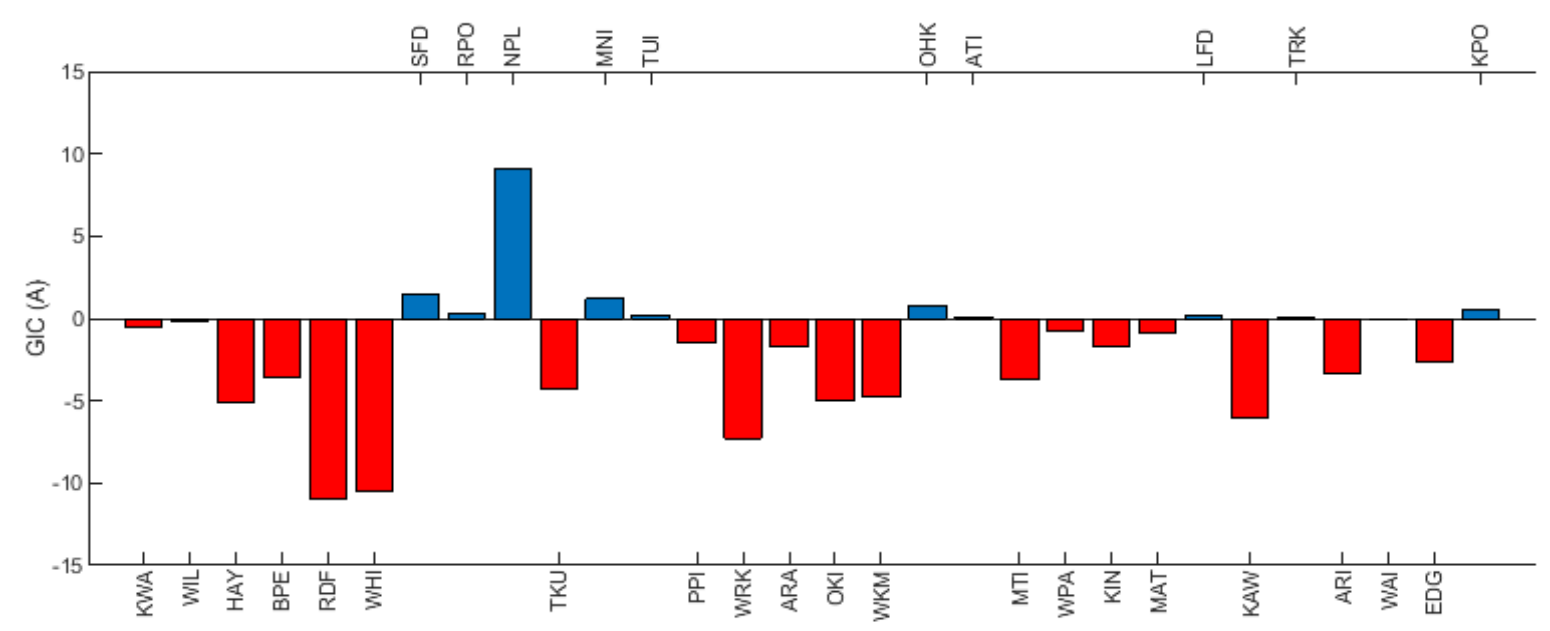

Figure 4. 18: Substation-level GIC. Two substations for which transformer-level GIC are very small are BPE and HAY, both in the lower North Island. WHI is located on the east coast.

Whirinaki has six earthed transformers, Wairakei has 4 earthed transformers and Kawerau has 8 earthed transformers. Redclyffe has 4 earthed transformers and New Plymouth has only 1 earthed transformer. Table 4.4 shows the corresponding GIC values in individual nodes for Redclyffe and Kawerau, and the total (substation) GIC at 04:47 UT. Figure 4.19 (a) shows a summary of transformer locations experiencing the largest GIC at 0447 UT during the St. Patrick's Day storm, and Figure 4.19 (b) shows show the largest substation level GIC. Different colors of circles show direction of current; blue is for positive current (out of substation) and red is for negative current (into the substation). The size of circle shows amount of current, and the largest circle corresponds to a GIC value of $\sim 11 \mathrm{~A}$.

\section{Table 4.4}

GIC in all earthed transformers of RDF and KAW substations, and the substation level GIC which is sum of GIC of all earthed transformers.

\begin{tabular}{cccc}
\hline $\begin{array}{c}\text { Redclyffe (RDF) } \\
\text { transformers }\end{array}$ & GIC (A) & $\begin{array}{c}\text { Kawerau (KAW) } \\
\text { transformers }\end{array}$ & GIC (A) \\
\hline RDF-T1H & -10.97 & KAW-T6H & -0.15 \\
RDF-T2H & -0.0085 & KAW-T7H & -0.15 \\
& & KAW-T8H & -0.64 \\
RDF-T3L & -0.0046 & KAW-T9H & -0.23 \\
& & KAW-T12L & -3.05 \\
RDF-T4L & -0.0046 & KAW-T13L & -0.69 \\
& & KAW-T11H & -0.51 \\
Substation GIC & -10.99 & KAW-T14H & -0.51 \\
& & Substation GIC & -5.98
\end{tabular}



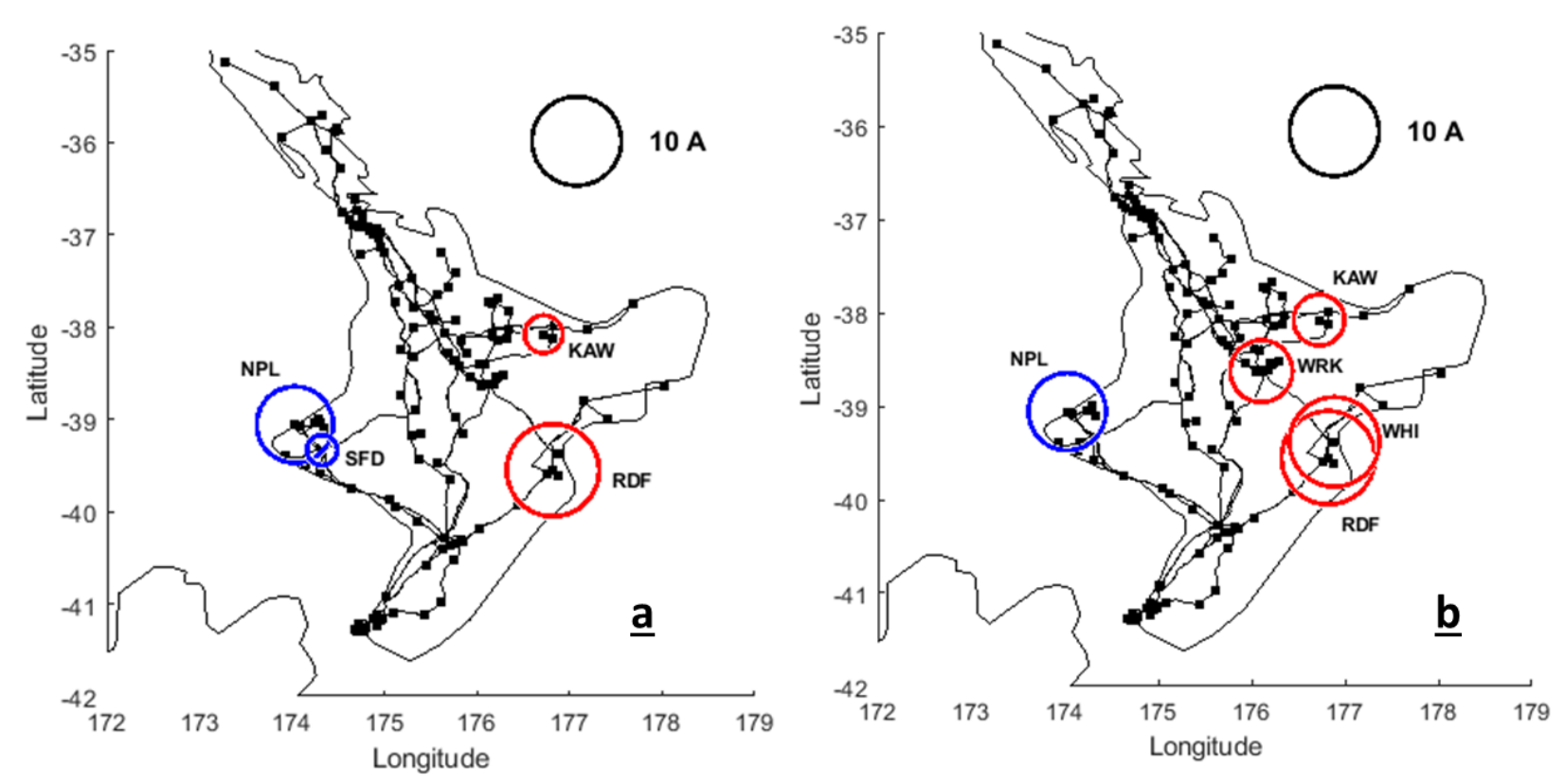

Figure 4. 19: MT model results. (a) Transformer locations experiencing the largest GIC and (b) locations of significant substation-level GIC during St. Patrick's Day storm. The size of circles is proportional to GIC magnitude.

\subsection{Comparison with Measured GIC}

Since there are no GIC measured data in the North Island which can be compared with model GIC, it is not possible to directly verify the MT GIC model results. But many GIC observations are available for South Island so a comparison is given here using GIC measured at Islington transformer (ISL-T6H). The comparison is given quantitatively by using the linear correlation coefficient $(r)$ and the performance parameter $(P)$. Although, the use of the correlation coefficient alone gives a good estimate of model results when compared with measured data, the additional use of the performance parameter allows inclusion of the scale difference between model and measured GIC. That is the reason several authors e.g., Torta et al. $(2014,2017)$; Ingham et al. (2017), have used performance parameter to test model results in GIC studies. See Section 3.5 for more details on the performance parameter. Both parameter values vary from 0 to 1 where 0 means no correlation and 1 means maximum/strongest correlation. Although negative values of $r$ show anticorrelation, this is not necessarily true in terms of the performance parameter. A total of 2048 data points (both for model and measured data) have been used to derive values of both parameters. 

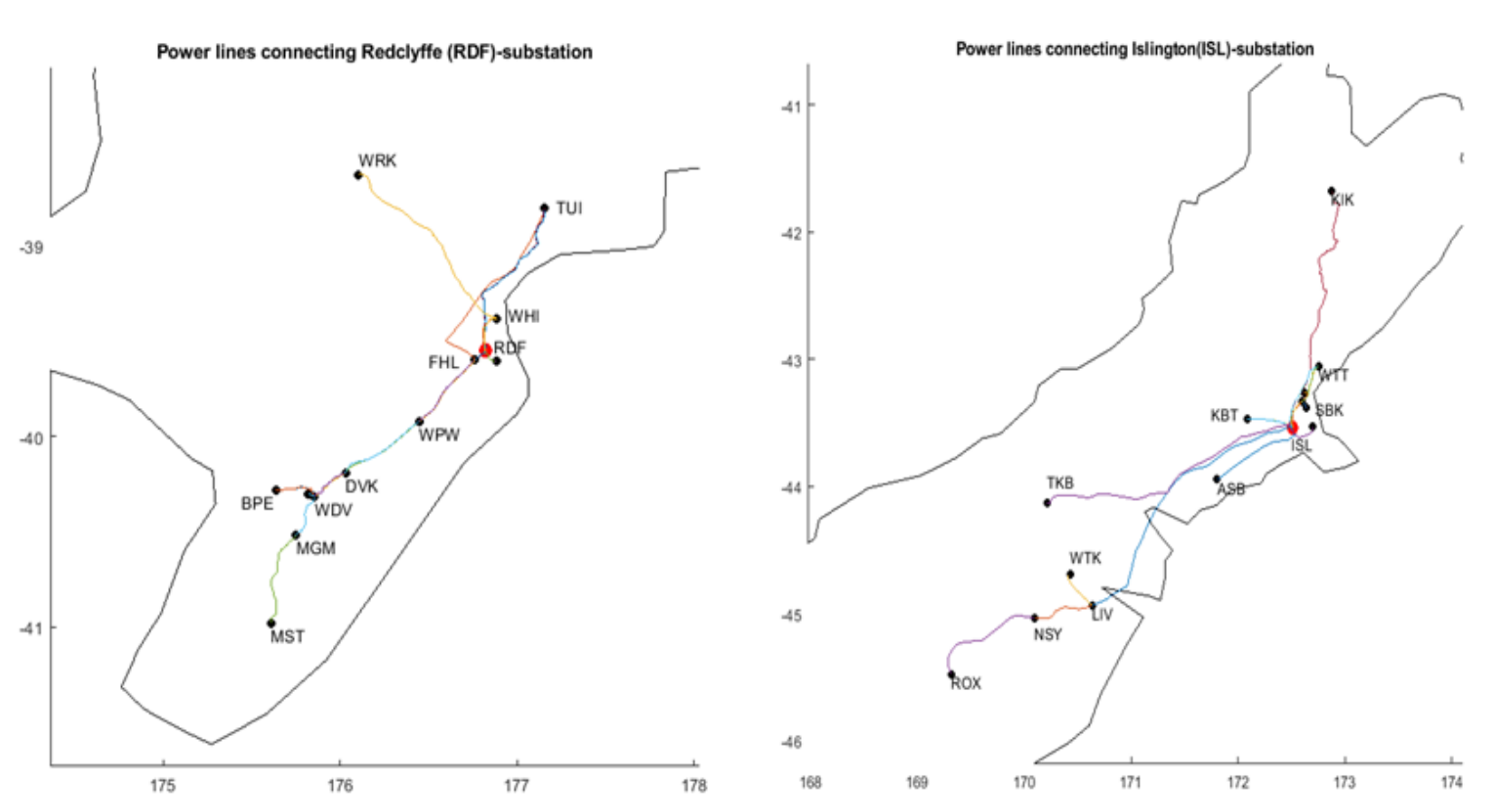

Figure 4. 20: The network configurations for transformers (RDF-T1H) of Redclyffe (RDF) substation in left panel, and transformer (ISL-T6H) of Islington substation in right panel. The red dots show RDF and ISL transformers connected to other substations by power lines.

Although the conductance map for the South Island is different to that for the North Island such a comparison might be helpful. Both RDF and ISL substations share some common features in the context of conductive structure and network connections. RDF and ISL are (almost) on the east coast of their respective islands and both are in relatively conductive regions bounded to the north-west by much more resistive regions. Both substations also share similar network configurations within the power network as can be seen in Figure 4.20. Both are connected to other substations by power lines extending in the north-east and south-west directions. Basically, RDF is connected to 5 substations through 8 lines, but not all these substations are earthed therefore GIC within these unearthed substations simply pass through. Power lines extending from these unearthed substations have also been shown. For example, to the south-west of Redclyffe, Waipawa (WPW), Dannevirke (DVK) and Woodville (WDV) are all unearthed, with the line ending at Bunnythorpe (BPE) which is earthed. The total length of line extending to BPE is $200 \mathrm{~km}$ and two other lines extending north from RDF are 136 and $96 \mathrm{~km}$ in length. Similarly, ISL is also connected with unearthed substations in a south-west direction extending through Livingstone (LIV) and Naseby (NSY) to Roxburgh (ROX) substation, with a total line length of 374 $\mathrm{km}$. One line to the north, which extends from ISL to Kikiwa (KIK) is $229 \mathrm{~km}$ in length. These common features make observed GIC at ISL the best for comparison with Redclyffe (RDF). 
Since the geoelectric fields calculated using MT data are only in the period range of 2-30 minutes Islington measured GIC have been filtered in this period range for comparison. Figure 4.21a shows individual time-domain plots for the Islington filtered data and two Redclyffe transformers RDF-T1H and RDF-T4H. A comparison is given for RDF-T1H transformer because it has comparable GIC amplitude with the measured data and it has $r=0.64$ and $P=0.30$. Figure 4.21 shows that the MT model GIC at RDF effectively reproduce the GIC variations of the observed data at ISL, particularly during the SSC as can be seen in the zoom view in Figure 4.21b. The model GIC has slightly bigger values than the observed GIC. Since the observed data is for the South Island quantitative analysis is difficult but the role of MT geoelectric fields in this short period range looks promising as seen during the SSC.
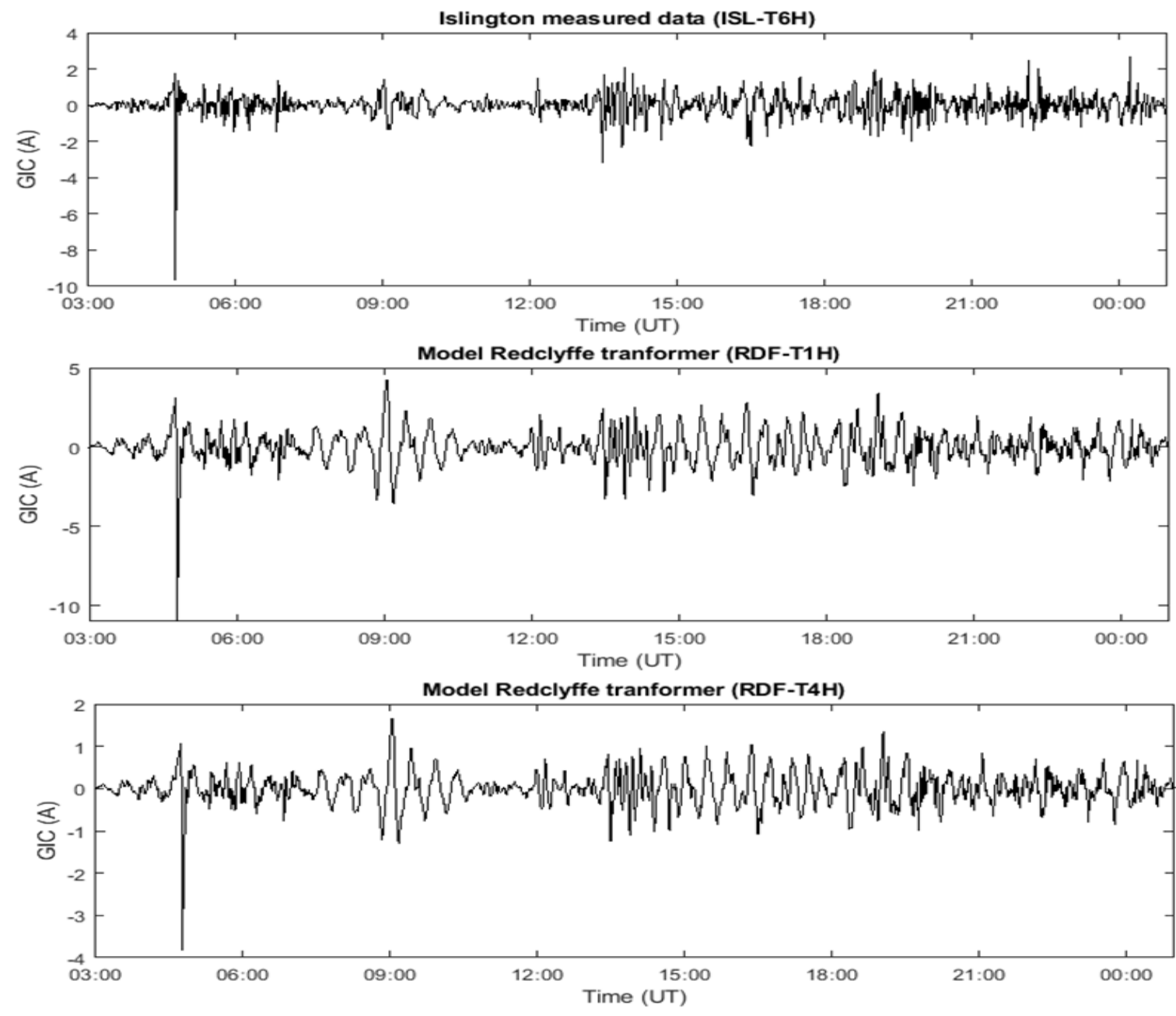

Figure 4. 21a: Individual time domain plots of measured GIC of Islington transformer and MT model GIC of two transformers of Redclyffe substation as mentioned on top of each panel. The model GIC shows different scale of magnitudes but the time variations are same. 

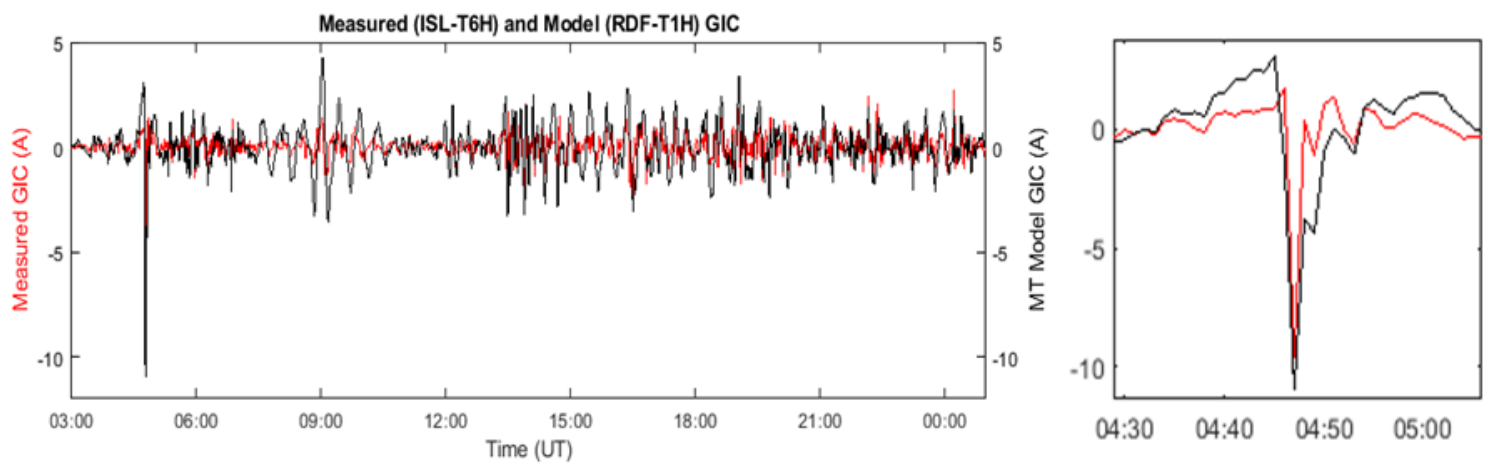

Figure 4.21b: A comparison of model GIC of Redclyffe transformer (RDF-T1H) with observed GIC at Islington (ISL-T6H). The correlation coefficient is 0.64. The model GIC effectively reproduces the observed GIC during the SSC as shown in close-up view (right-hand panel).

\subsection{Comparison with the Thin-sheet Conductance Model}

Previously, GIC modelling has been carried out using the thin-sheet conductance model geoelectric fields for the South Island of New Zealand (Divett et al, 2017, 2018). In this section geoelectric fields calculated for the North Island using the thin-sheet conductance model have been used to model GIC for comparison purpose with the MT modelling results. Figure 4.22 shows the geoelectric field at 0447 derived from the thin-sheet conductance model. The same scale $(500 \mathrm{mV} / \mathrm{km})$ as used in Figures 4.8-4.10 has been chosen to plot fields so these can be compared with the MT geoelectric fields.

Figure 4.22 shows that the thin-sheet model generates very smooth fields over the whole North Island. The largest field value $(73.89 \mathrm{mV} / \mathrm{km})$ occurs in the high resistive region in the southern part, and smallest field value $(11.56 \mathrm{mV} / \mathrm{km})$ occurs in the highly conductive region in the middle of the North Island. In comparison MT calculated fields vary much more significantly from region to region. For example, the MT geoelectric fields are large on the north coast to the east of Kawerau. These local variations consequently effect the computed GIC as can in fact be seen in GIC computed for Kawerau (KAW) substation (-38.08, 176.72). Temporal variations in the geoelectric fields are also not as abrupt, i.e., the fields do not change direction significantly, compared to the MT calculated fields. The thin-sheet fields are also smaller in magnitude compared to MT generated geoelectric fields. 


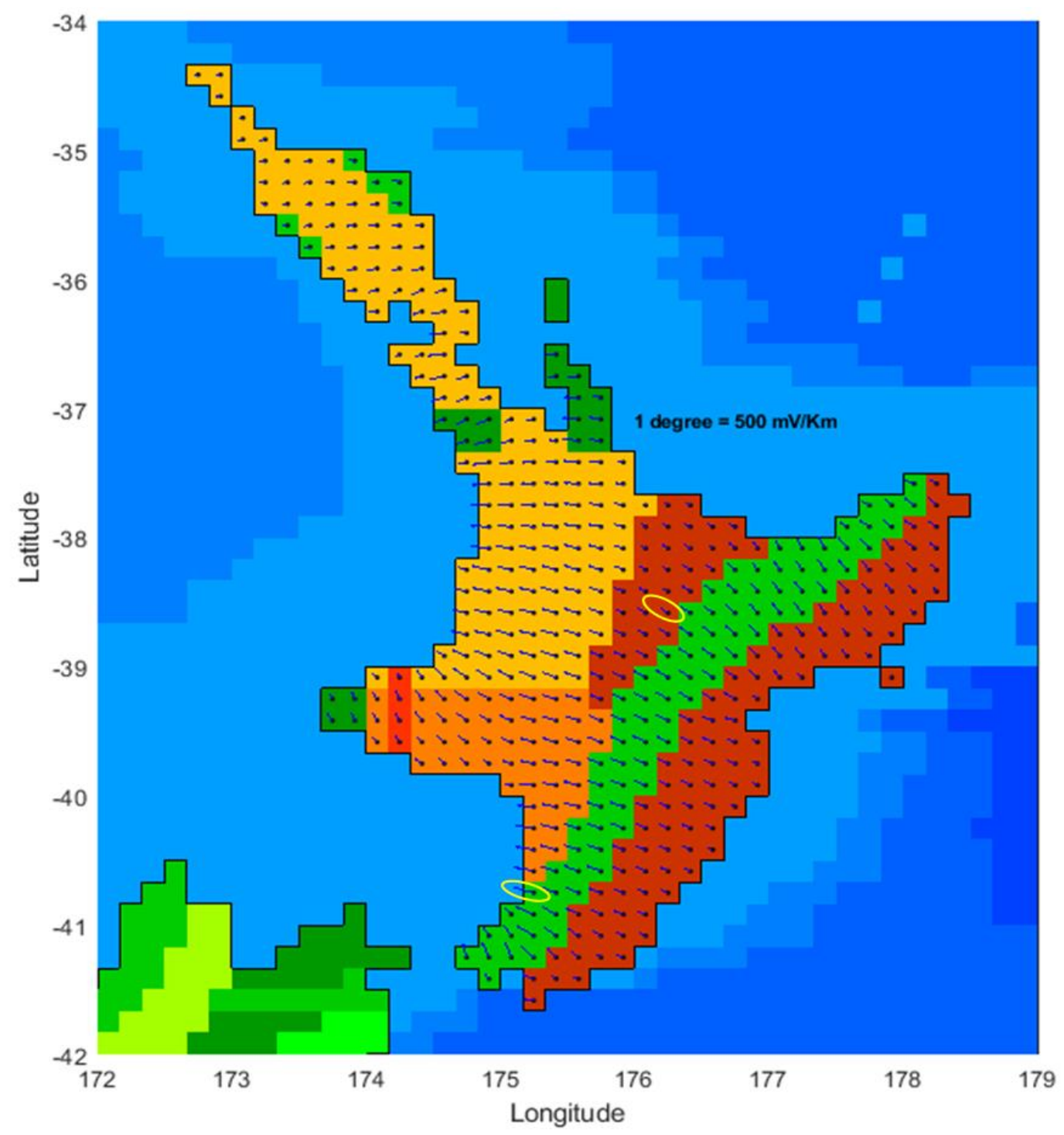

Figure 4. 22: Thin-sheet electric fields at 0447 UT during Sudden Storm Commencement. The geoelectric fields are on the same scale as used for MT geoelectric fields (Figure 4.8). The spatial distribution of geoelectric fields is smooth over different conductive regions. The yellow circles show location of largest and smallest geoelectric fields.

\subsubsection{Thin-sheet Geoelectric Field and Transformer GIC}

Thin-sheet computed GIC are presented in the same fashion as was done for the MT computed GIC. Since the thin-sheet geoelectric fields can be computed for whole of the North Island use of an equivalent circuit is not needed. For comparison purpose with the magnetotelluric GIC results, the thin-sheet GIC model results are also given for the lower part of the North Island up to Karapiro substation. The bar graph in Figure 4.23 shows a snapshot of GIC computed using the thin-sheet geoelectric fields at 04:47 UT. Redclyffe transformer (RDF-T1H) and New Plymouth transformer (NPL-T8L) experience the largest GIC during the SSC among all transformers and 
values are almost the same $\sim 4.0 \mathrm{~A}$ apart from the different sign of the currents. These are the same transformers as showed the largest GIC for the MT model, but the magnitudes of the currents are less than half those predicted by the MT data. No other transformers can be identified as experiencing significant GIC during the SSC.

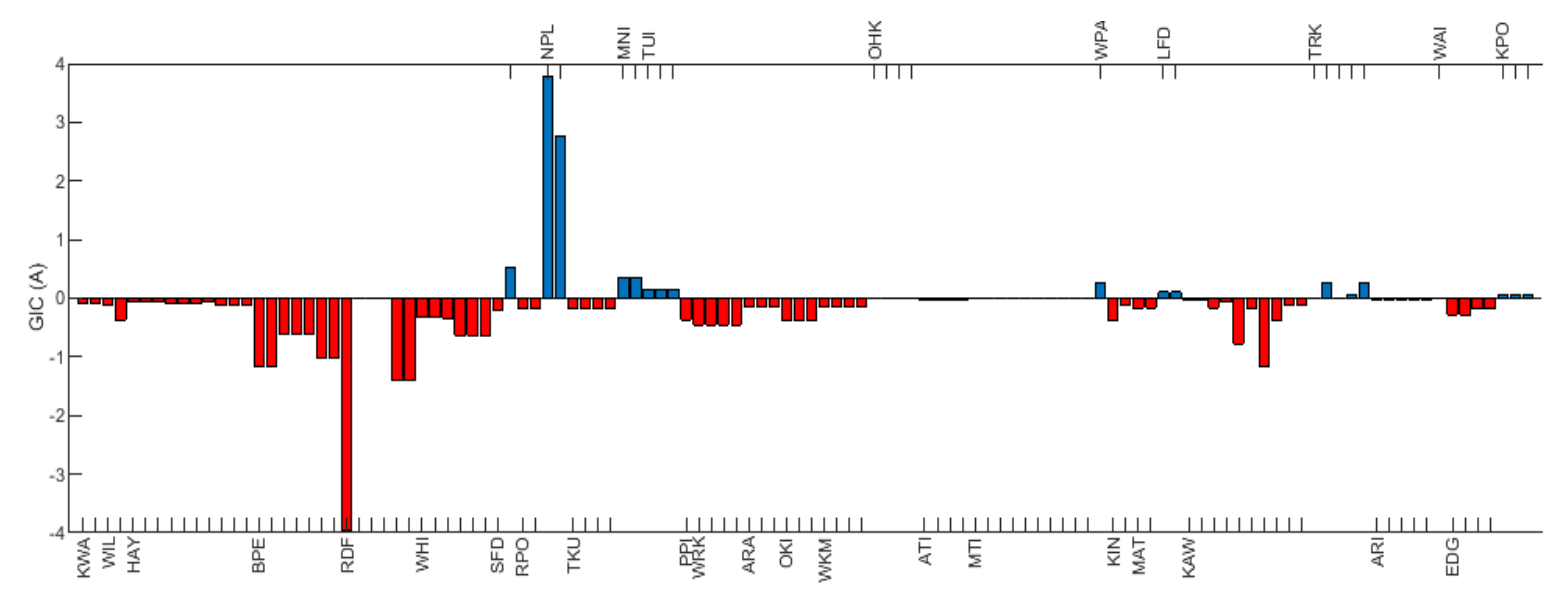

Figure 4. 23: Computed GIC during the Sudden Storm Commencement at 04:47 UT using TS conductance model.

Time domain GIC are shown in Figure 4.24 for transformers experiencing the largest GIC. There are four substations which experiences large GIC at transformer-level given in the order of magnitude.

1. New Plymouth (NPL-T8L)- (-39.06, 174.03)

2. Redclyffe (RDF-T1H)- (-39.55, 176.82)

3. Stratford (SFD-T10H)- $(-39.33,174.32)$

4. Kawerau (KAW-T12H)- $(-38.08,176.72)$

These are the same transformers as identified using the MT modelling technique. The similarity between locations with high GIC in both modelling approaches, given the very different electric fields in the two approaches, shows that the location of high GIC is predominantly due to the network topology rather than local variations in electric fields. It is also worth noting that the network model takes an average of the geoelectric fields of different conductance grid cells under the power lines and produces an averaged network repose to these geoelectric fields. However, the thin-sheet model GIC show a dominance of low frequency components as can be seen in the 
GIC amplitude variations of Figure 4.24. Although the thin-sheet model effectively reproduces low frequency components in GIC there are only very small GIC during the SSC. This suggests that the MT model can better be used for calculating shorter period GIC.
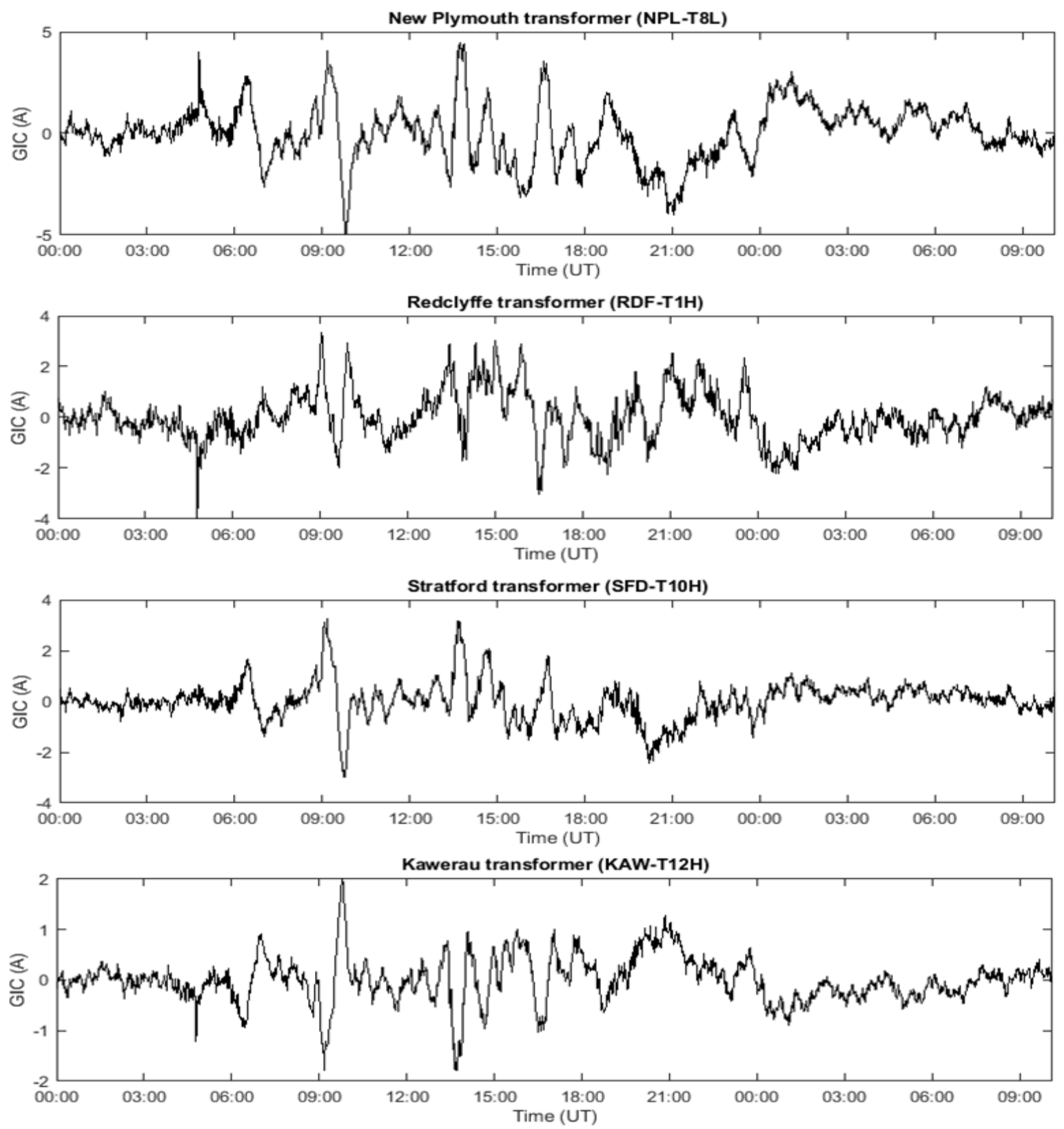

Figure 4. 24: Transformers having large GIC at transformer-level during the St. Patrick's Day storm using TS conductance model. The effect of SSC is not very strong in these modelling results. 


\subsubsection{Thin-sheet Geoelectric Field and Substation GIC}

Figure 4.25 shows substation GIC computed using the thin-sheet geoelectric fields at 04:47 UT. Bunnythorpe (BPE) substation shows the largest GIC. This is a different result from the MT model although for the rest of the substations the pattern is very similar. As for the thin-sheet model transformer GIC, the substation GIC also have smaller magnitude than in the MT model.

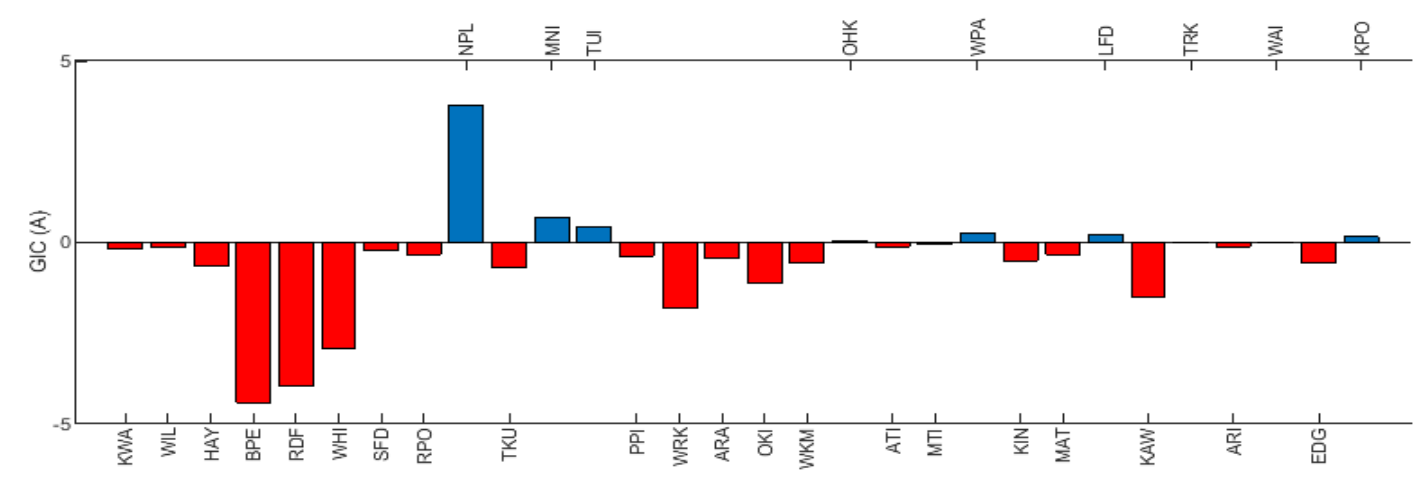

Figure 4. 25: Substation GIC at transformer-level during the St. Patrick's Day storm using TS conductance model.

Using the geoelectric fields for whole duration of magnetic storm the following substations have been identified having largest GIC (in order of magnitude)

1. Bunnythorpe (BPE)-( $-40.28,175.64)$

2. New Plymouth (NPL)- $(-39.06,174.03)$

3. Redclyffe (RDF)- $(-39.55,176.82)$

4. Haywards (HAY)- $(-41.15,174.98)$

The substations experiencing the largest GIC are different from the MT modelling results. Although NPL and RDF are the same substations, Bunnythorpe and Haywards did not appear in list of large substation GIC for the MT model. For the MT model the list included Wairakei (WRK) and Kawerau (KAW), both in the middle of the North Island. BPE and HAY are close to each other and lie in the south of the North Island. The large substation GIC at Haywards and Bunnythorpe are because there are many transformers within each substation: 7 and 4 earthed transformers, respectively. 4.26 (a) shows a summary of thin-sheet GIC modelling results during St. Patrick's 
Day storm for transformer experiencing the largest GIC, and Figure 4.26 (b) shows show the largest substation level GIC. The size of the circle is chosen on the same scale as was done for MT results shown in Figure 4.19. Blue and red circles show positive and negative currents, respectively.
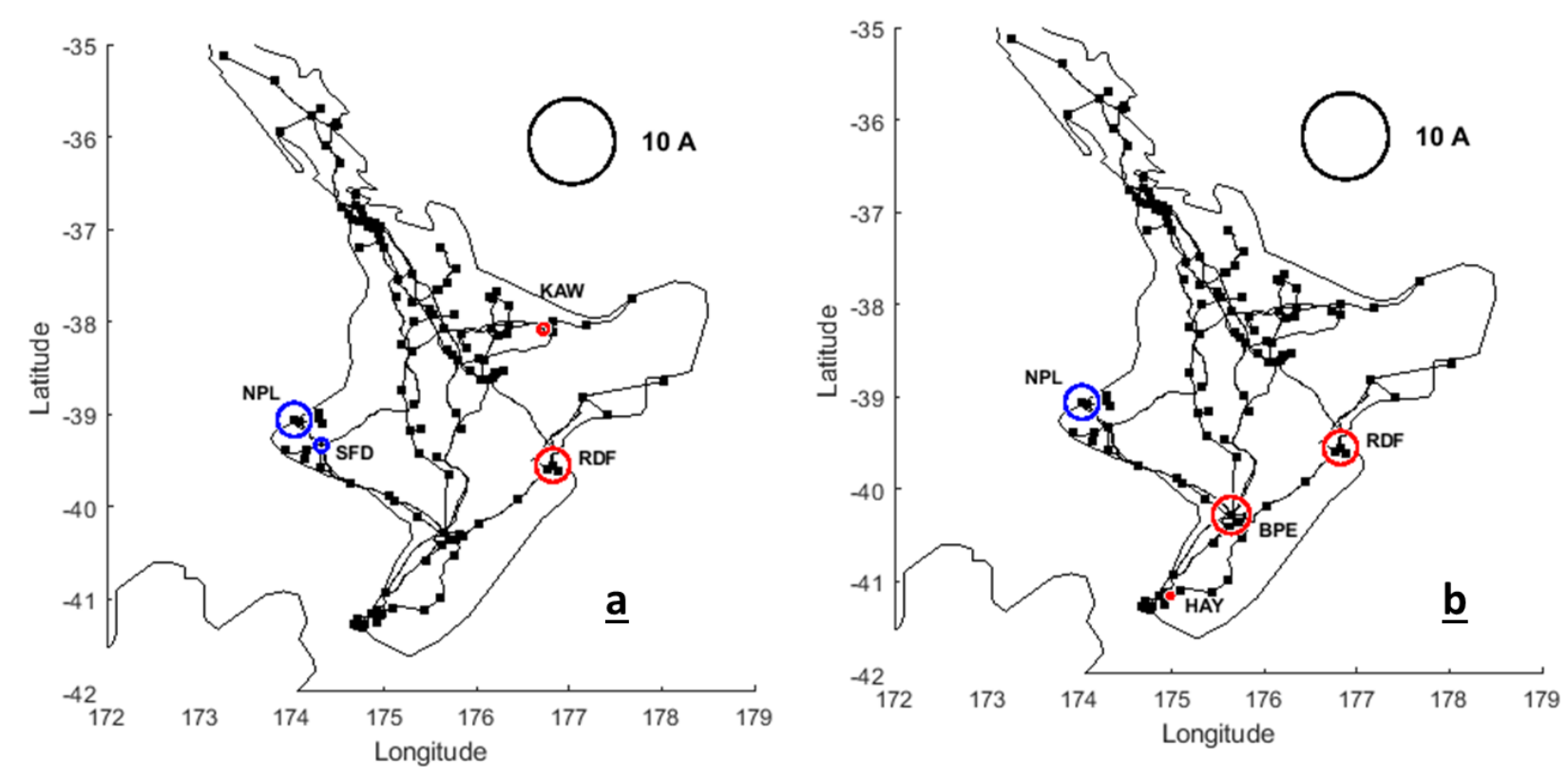

Figure 4. 26: Thin-sheet model results. (a) Transformer locations experiencing the largest GIC and (b) locations of significant substation-level GIC during St. Patrick's Day storm. The size of circles is proportional to GIC magnitude.

The different substation GIC results between techniques may reflect the different period range of the models and the pattern of currents in the power network. However, they are also due to the different geoelectric fields calculated by the two techniques as can be seen in Figure 4.9 and Figure 4.22. Although the geoelectric fields both in the MT and thin-sheet techniques at 0447 UT are largely in a northwest-southeast direction there are some significant differences in orientation. In particular, fields near BPE calculated from the MT data are more east-west oriented. Consideration of the GIC results from uniform electric fields (Figure 4.13) shows that geoelectric fields in this direction result in much smaller GIC at BPE than do northwest-southeast oriented fields. Thus, the differences appear primarily to be due to the fact that the MT fields are more local and diverse compared to the thin-sheet fields, showing the impact of localized geoelectric fields and how the galvanic distortion could impact the network model. 


\subsection{Discussion}

An alternative approach to GIC modelling has been discussed in this chapter. It uses geoelectric fields derived from MT data for the North Island. Since no GIC observations are available for the North Island, a direct comparison of model results is not possible. A comparison with the results calculated from the thin-sheet conductance model, and of GIC observations at ISL in South Island has been presented. Since no MT data was available in the upper part of North Island model GIC are restricted to substations in the lower part of North Island. Model GIC are also restricted to the shorter period range/high frequency (2-30 minutes) as permitted by the frequency range of the MT data. The MT model identifies two transformers, Redclyffe (RDF-T1H) and New Plymouth (NPL-T8L) as experiencing the largest GIC during the SSC given by $-10.97 \mathrm{~A}$ and $9.08 \mathrm{~A}$, respectively. RDF lies on east coast whereas NPL lies on the west coast of the North Island. Of the other transformers Kawerau (KAW-T12H) and Stratford (SFD-T10H) also show significant GIC of $\sim-4.5$ and -3.62 A respectively. The model GIC at the Redclyffe transformer is compared with observed data from Islington and this shows that the MT model effectively reproduces the observed GIC particularly during the SSC. GIC are many times larger during the SSC compared to the rest of the storm period. This feature is not evident in the thin-sheet conductance model GIC calculation. The thin-sheet model GIC do not have substantial GIC during the SSC but do have larger, varying, GIC during the main period of the magnetic storm. The primary reason is that the geoelectric fields in both models are different both spatially and temporarily. MT data give more detailed geoelectric fields, whereas the thin-sheet model produce geoelectric fields which are spatially smoother and seem to be an averaged result of the MT data. Similarly, geoelectric fields computed through MT data show more abrupt variations in time than does the thin-sheet conductance model. Although both models largely depict the same transformers as experiencing large GIC, substation GIC differ. For example, thin-sheet shows large substation GIC appearing in south part of the North Island (Bunnythorpe and Haywards) but these does not show up in MT modelling technique. Direct comparison of the MT and thin-sheet models is difficult in that both models produce GIC in different period ranges. 


\section{Chapter 5: GIC Modelling of North Island for the Magnetic Storm 20 November2003}

\subsection{Introduction}

Magnetic storms are produced by conditions in the magnetosphere and ionosphere in response to solar storms. The characteristics and features are largely controlled by solar wind parameters along with the fact that magnetospheric and ionospheric electrodynamics vary for different solar storms. Therefore, the GIC model results derived for the St. Patrick's Day magnetic storm cannot be generalized and other magnetic storms should also be considered for comprehensive analysis. In this context the magnetic storm of 20 November 2003 has been used to model GIC using both the MT and thin-sheet models. Spectral analysis of GIC data from Islington (ISL) also shows different spectral components during the magnetic storm of 2003 in comparison to the St. Patrick Day magnetic storm (details are given in Chapter 3). The magnetic storm of 2003 belongs to a different class of solar storm known as a magnetic cloud preceded by a shock (SMC) which is different from the St. Patrick's Day magnetic storm caused by SH+ICME. It occurred during extreme space weather events of October-November2003 showing strong magnetic fluctuations in EYR magnetic data. The $K p$ index stayed at a maximum of 9 for a long duration. GIC observed at Islington (ISL) showed strong low frequency components causing a saturation effect in transformers although GIC during the sudden storm commencement (SSC), unlike for the storm of 2015, were not prominent. Due to these different characteristics, it is of interest to model GIC for this storm and compare the results with measured data.

\subsection{The Solar Storm of 20 November2003}

There were a series of extreme magnetic storms during October-November 2003 (Ermolaev et al., 2005) during the declining phase (2003-2006) of solar cycle 23. In the declining phase there was a predominance of SMC (magnetic cloud preceded by a shock) producing storms, and the 
magnetic storm of 20 November2003 belongs to this type (Gonzalez et al., 2007). It has the largest value of $D s t$ index among all storms during this period, reaching a minimum value of -472 nT (Ermolaev et al., 2005) during the main phase of the storm. This is also indicated by the $K p$ index staying at its maximum value of 9 for a long period (15-21 UT) as indicated in Figure 5.1(a) (the data are from https://www.spaceweatherlive.com/). One-minute solar wind plasma and field parameters observed by ACE/WIND satellites downloaded from (https://omniweb.gsfc.nasa.gov/) are shown as Figure 5.1(b). The solar storm is categorized as a Magnetic Cloud preceded by a shock (sMC) (Echer et al., 2008; Yushkov et al., 2005) where the shock is shown by the dotted line and the boundaries of the magnetic cloud are shown by the arrow bars between solid vertical lines.

Solar parameters include the interplanetary magnetic field (IMF-B), the interplanetary magnetic field south component (IMF-Bz), the solar wind speed, proton density, temperature and solar dynamic pressure. The shock hit the magnetosphere before the magnetic cloud at $~ 08: 06$ UT when there was a sudden increase in the values of the solar parameters. The $K p$-index reached a value of 6 during the time of the shock. A subsequent sudden increase in magnetic field at Eyrewell observatory was observed reaching maximum values of $19580 \mathrm{nT}$ and $8364 \mathrm{nT}$ for north $(B x)$ and east $(B y)$ components respectively, as shown in Figure 5.2(a). The rate of change of the magnetic field was $81.9 \mathrm{nT} / \mathrm{min}$ and $17.5 \mathrm{nT} / \mathrm{min}$ for the $B x$ and $B y$ components respectively (Figure 5.2(b)), and a GIC of 5.6 A was measured in transformer T6H at Islington (Figure 5.2(c)). After the shock hit the magnetosphere, solar plasma and field parameter values continued increasing with some fluctuations until a smooth rotation in the IMF-Bz occurred, identified as the magnetic cloud (MC) (Gupta and Badruddin, 2009; Echer et al., 2008). The Kp reached a maximum during the storm's main phase. The geomagnetic storm has a long and monotonic development before the $K p$ index stays at maximum, and the maximum rate of change in magnetic field was observed. During the main phase, a continuum of large GIC values was observed with a total of 57 data points over a threshold of $\pm 5 \mathrm{~A}$. This shows large GIC occurring in the transformer for a long period of time during the main phase of the storm. The maximum current value is $-12.5 \mathrm{~A}$ in the one-minute interpolated GIC data. 

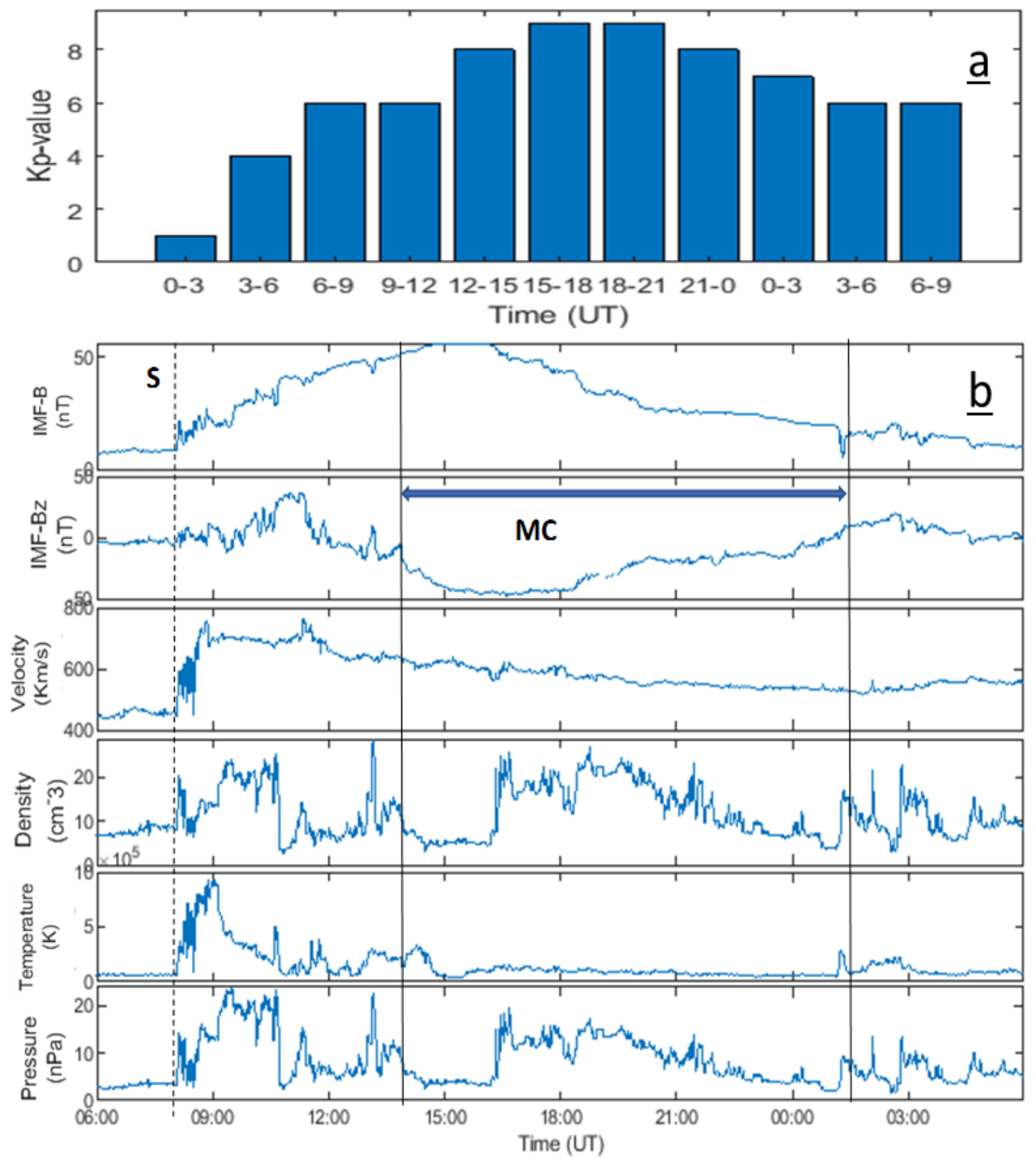

Figure 5. 1: (a) Kp-index values and (b) solar plasma and field parameters as observed by ACE satellite on 20 November2003. The dotted vertical line shows arrival of the shock and the horizontal arrow bar shows the boundaries of magnetic cloud (MC). 


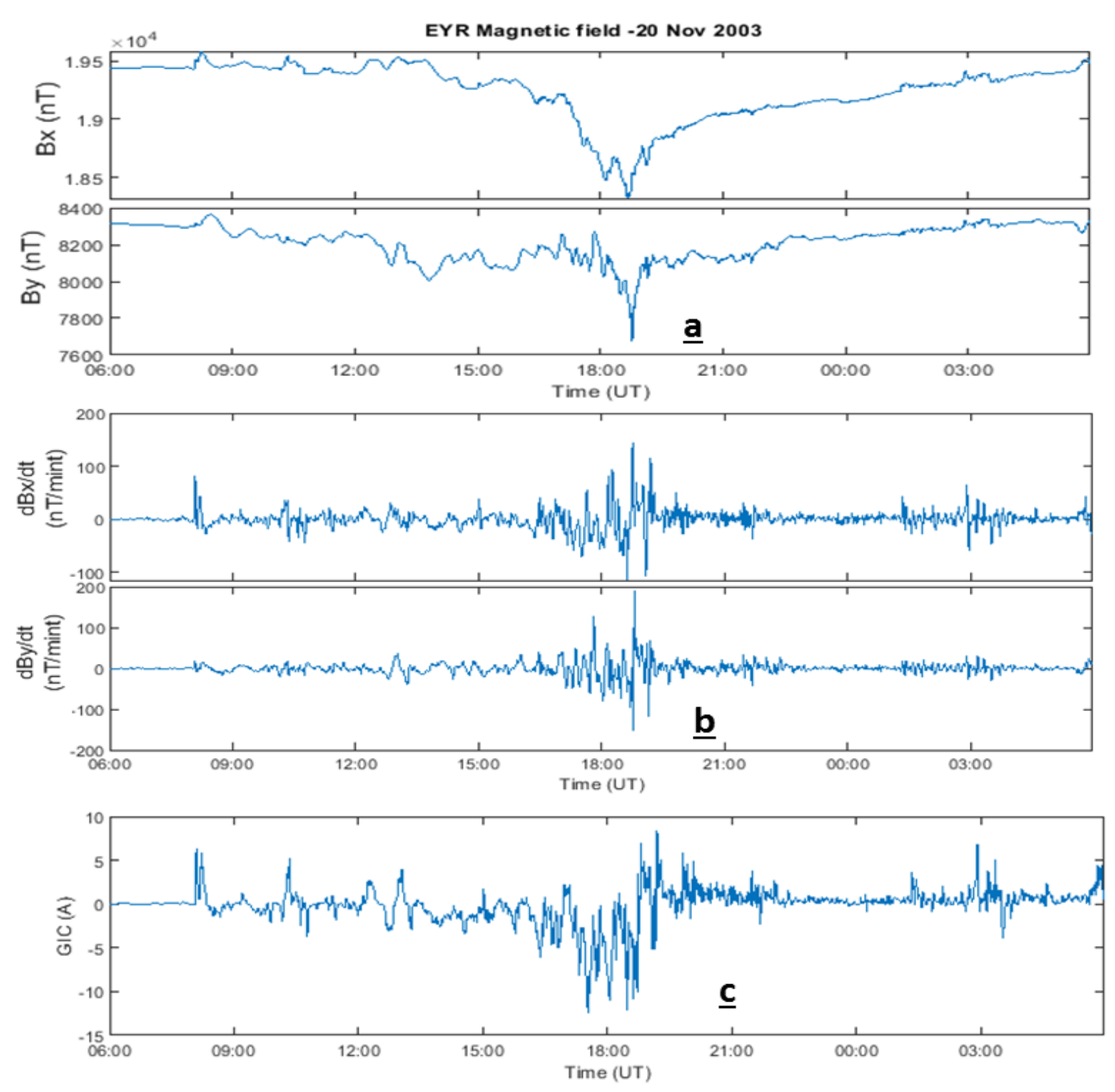

Figure 5. 2: (a) Magnetic field measured at EYR observatory, (b) time derivatives of the field, (c) One-minute averaged GIC in transformer T6H at Islington during the magnetic storm of 20 November2003.

\subsection{MT Model Geoelectric Field}

Geoelectric fields for the 2003 magnetic storm have been computed in the same way as was discussed for the St. Patrick's Day magnetic storm in the last chapter. Since no magnetic observatory data are available for the North Island one-minute magnetic data $\left(B_{x}\right.$ and $B_{y}$ components) at Eyrewell magnetic observatory are used. The validity of this assumption has been discussed in section 4.5 of Chapter 4 showing magnetic field variations at Eyrewell do indeed give a reasonable estimate of those in the North Island, although the accuracy of the agreement will vary from storm to storm with, on occasion, rates of change of the field in the lower North Island 
perhaps being only $60-70 \%$ of those at EYR (Mukhtar et al., 2020). The EYR data allows oneminute discrete geoelectric fields to be calculated, using the magnetotelluric response tensors, for 20 November2003 magnetic storm for all 115 MT sites. The computed geoelectric fields are in the period range of 2-30 minutes and the same procedure is adopted as for the St. Patrick's Day storm. Figure 5.3 shows resulting one-minute discrete geoelectric fields computed for an example MT site CAR-119 (-41.07 N, 175.64 E). The site location is also shown on the inset map and lies at the southern end of North Island. The site is in a relatively low resistive region. Strong geoelectric fields during the main phase of magnetic storm can be seen and these are about four time stronger than the St. Patrick's Day geoelectric fields calculated for the same site.

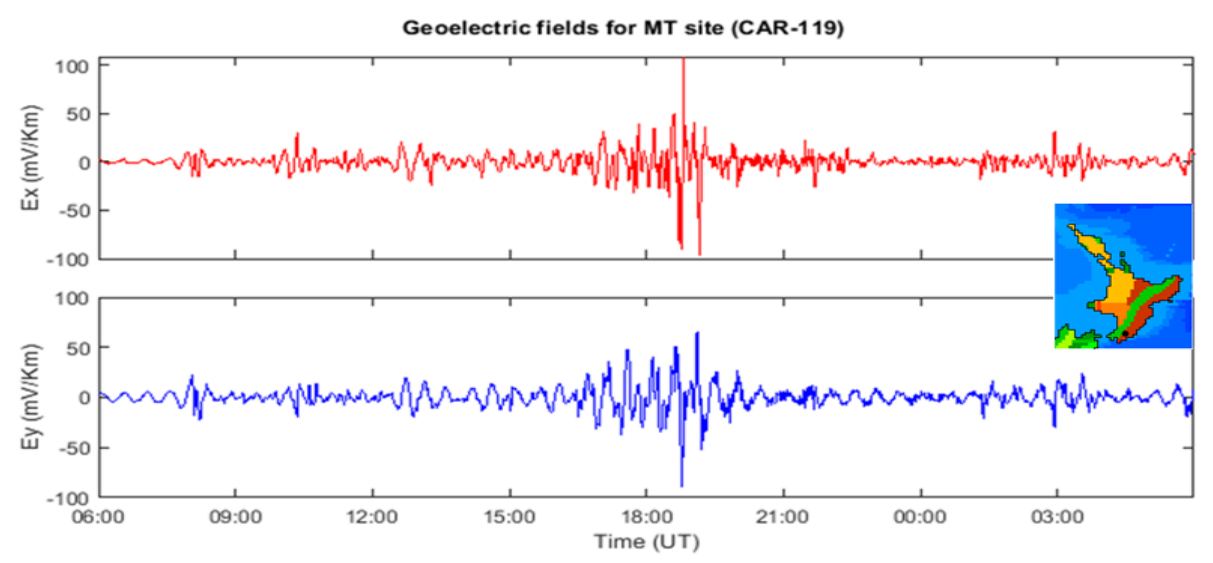

Figure 5. 3: Time domain geoelectric field north $\left(\boldsymbol{E}_{\boldsymbol{x}}\right)$ and east $\left(\boldsymbol{E}_{\boldsymbol{y}}\right)$ components computed using magnetotelluric response tensor of an MT site shown in the map.

Figure 5.4 shows the vector form of the geoelectric fields for all MT sites during the main phase of the magnetic storm at 1844 and 1847 UT when field values reach a maximum. The direction and magnitude of geoelectric fields is different during both instants. Figure 5.4(a) shows that at 1844 UT fields are mostly in a south-east direction and the lowest value of geoelectric field (7.95 $\mathrm{mV} / \mathrm{km}$ ) exists in the high conductive region in the center of North Island. The maximum field value $(495.5 \mathrm{mV} / \mathrm{km})$ is shown by the white arrow. Figure $5.4(\mathrm{~b})$ shows the field direction is changed to south-west at 1847 UT. The minimum field value $(47.14 \mathrm{mV} / \mathrm{km})$ again exists in the high conductive region of the central North Island, and the maximum field value $(1490.8 \mathrm{mV} / \mathrm{km})$ is again shown by the white arrow. Figure 5.4 illustrates the rapid temporal variations in geoelectric field calculated using MT data. 

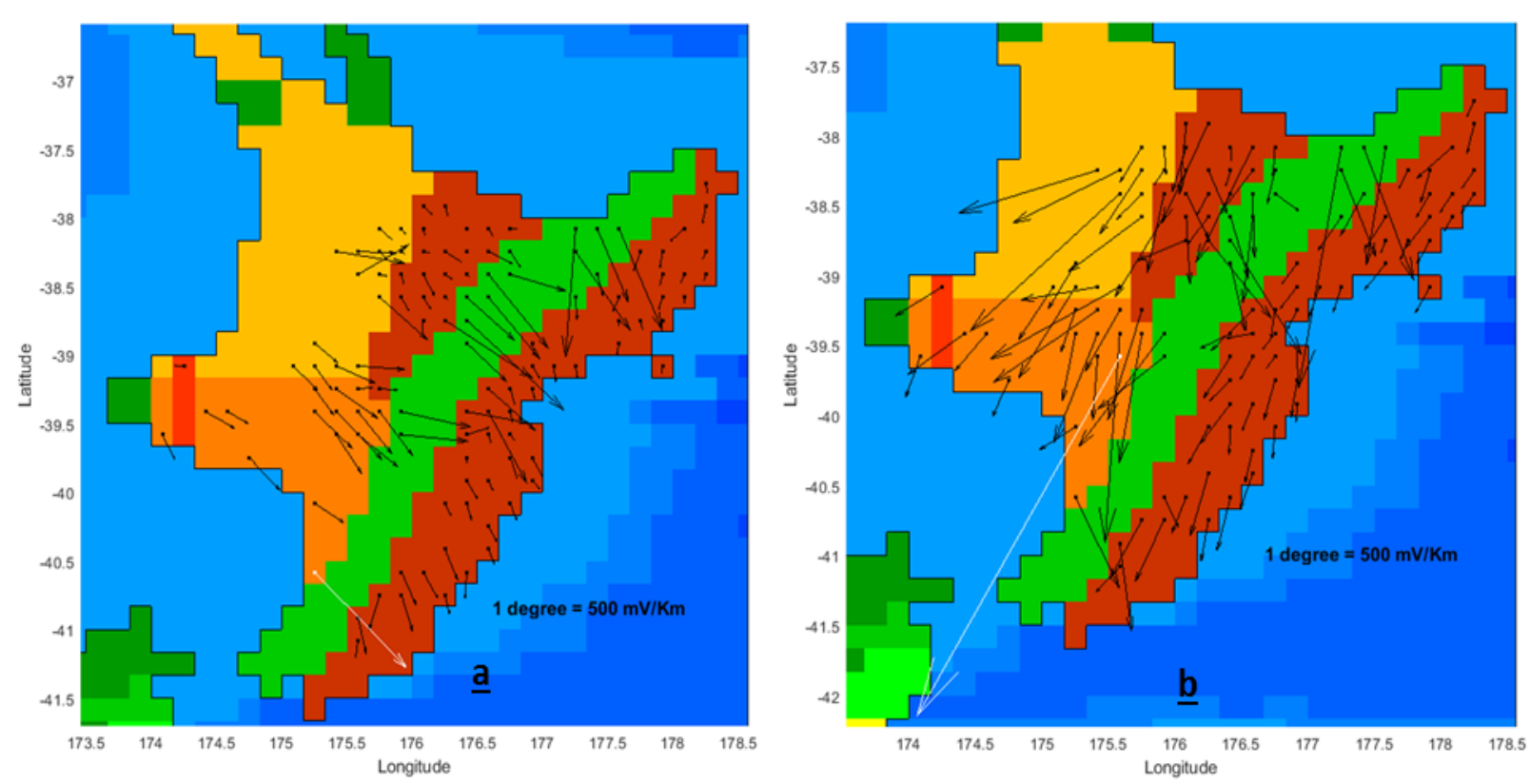

Figure 5. 4: Computed geoelectric fields vectors at the location of MT sites on the North Island grid at 1844 and 1847 UT during the magnetic storm of 20 November2003. The white arrows show the maximum fields.

As for analysis of the St. Patrick's Day storm, to fill empty grid cells the MT site data are interpolated/extrapolated using the Nearest Neighbor method (Ashraf et at., 2017). The resulting E-field vectors are given as Figure 5.5. Low resistive regions (red cells) have small geoelectric fields, while large geoelectric fields can be seen in the highly resistive (green) region. The cells with yellow shades have intermediate range geoelectric field values. The interpolation results are considered reliable in the east and center of the Island where the dense network of MT sites exists. In the west and, particularly, the north of the island, where MT data are sparse and the Nearest Neighbor method produces the same fields in all empty grid cells, the extrapolated geoelectric fields have been ignored and the equivalent circuit technique (Boteler et al., 2013), as presented in Chapter 4, has been used. 

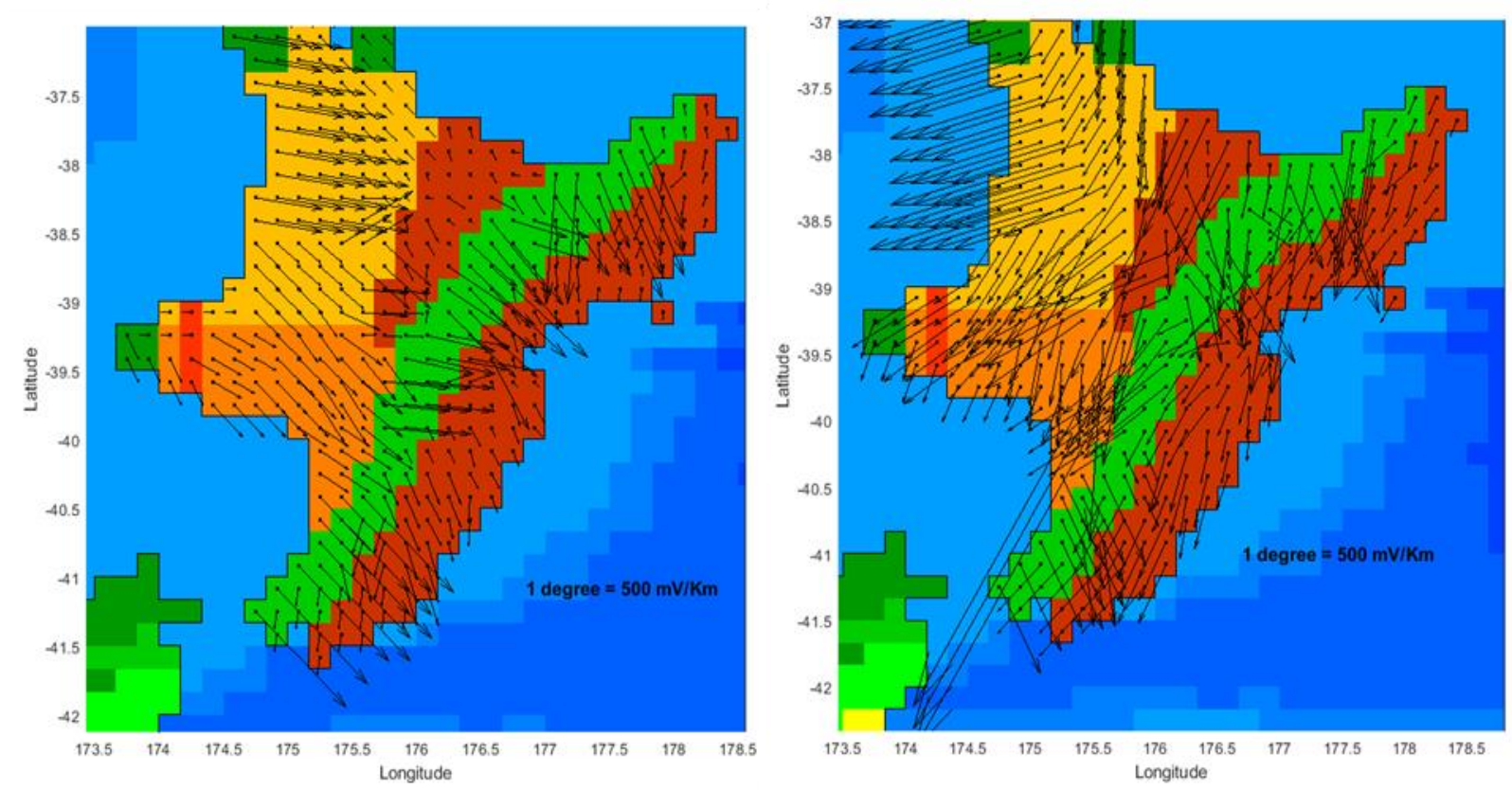

Figure 5. 5: Geoelectric fields after applying Nearest Neighbor interpolation technique to MT geoelectric fields shown in Figure 5.4.

\subsection{MT Geoelectric Field and Transformer GIC}

Transformer GIC and substation GIC have been computed following Divett et al. (2018) using the geoelectric fields derived from the MT data. The northern part of the network has been represented by an equivalent circuit (Boteler et al., 2013) as discussed in the previous chapter. Remembering that the network model computes GIC through high-side and low-side windings of individual transformer and the letters ' $\mathrm{H}$ ' and ' $\mathrm{L}$ ' have been used to distinguish GIC through them (Divett et al., 2018).

Figure 5.6 shows a snapshot of transformer GIC computed at 1844 and 1847 UT (during the main phase of the magnetic storm) for which geoelectric fields are also given in Figure 5.5. The bar plot shows the transformers of substations only up to Karapiro (KPO). Since field directions are quite different during these two times different GIC are produced. At 1844 UT the largest GIC flow through transformers $(\mathrm{T} 1 \mathrm{H}, \mathrm{T} 3 \mathrm{H}$ and $\mathrm{T} 4 \mathrm{H})$ at Redclyffe substation. Transformer $(\mathrm{T} 1 \mathrm{H})$ shows the largest value of $14.1 \mathrm{~A}$. No other substation can be identified as experiencing large GIC except Wilton T8H which has a GIC value of -5.03 A. At 1847 UT most geoelectric fields are in a southwest direction and produce the largest GIC in New Plymouth transformer, for which GIC values 
for T8L and T8H are given by -42.14 and $-36.37 \mathrm{~A}$, respectively. Redclyffe transformers do not show any significant response during this time. Transformer (T10) in Stratford can also be seen as showing a significant value of GIC of $\sim 25.31 \mathrm{~A}$.

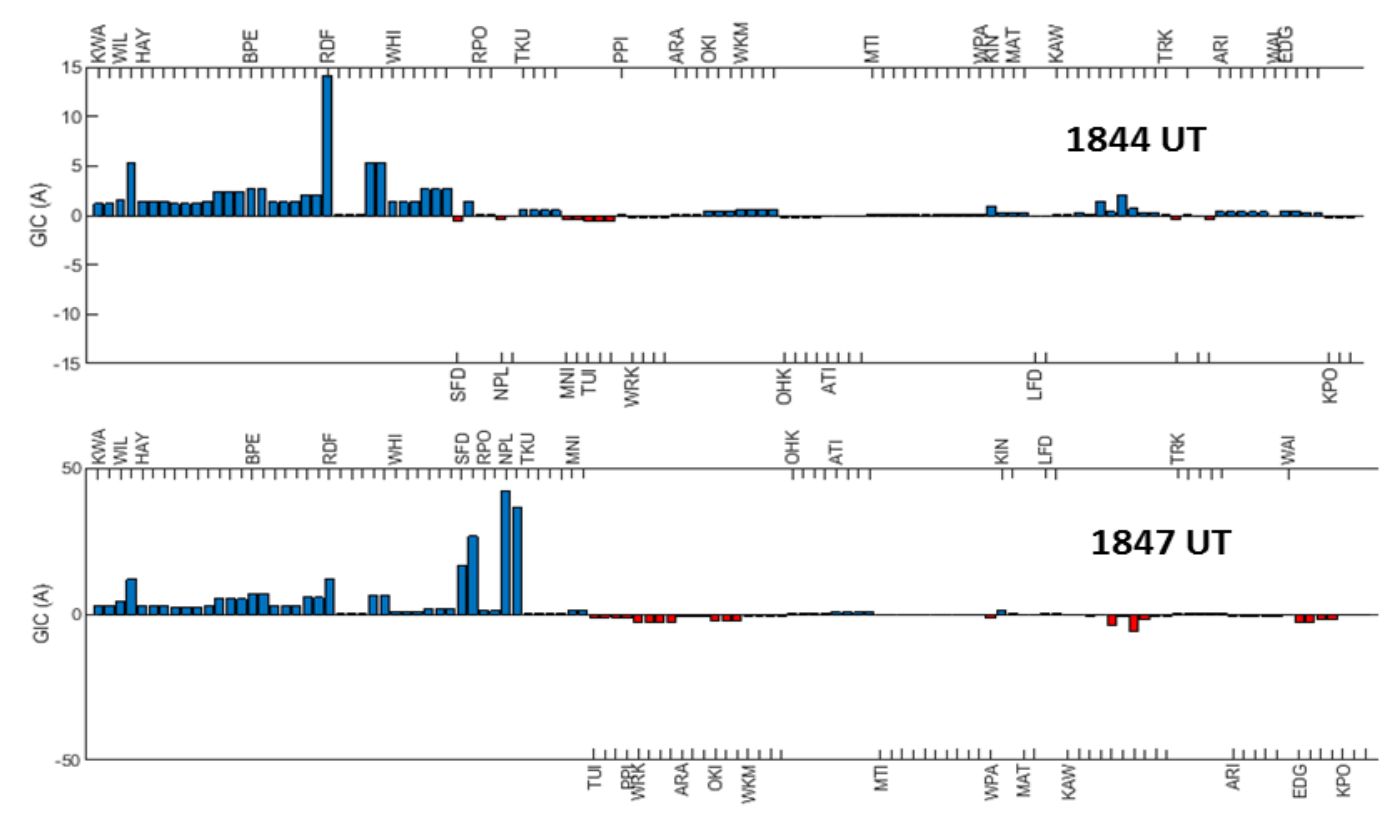

Figure 5. 6: Model transformer GIC at 1844 and 1847 UT using MT data. Redclyffe (RDF) and New Plymouth (NPL) transformers, on the horizontal axis, show largest response to geoelectric fields.

Since currents in power lines are sources of GIC in a transformer the corresponding current values in power lines for Redclyffe, New Plymouth and Stratford substations are given in Table 5.1. For visualization purpose the line currents and transformer GIC are also shown on the map of the network in Figure 5.7. Since there could be more than one transformers within a substation, the transformer having extreme GIC value is plotted per substation in Figure 5.7. The primary source of large GIC in Redclyffe T1 transformer at 1847 UT is the line connection of Redclyffe with Wairakei (WRK), highlighted in yellow. It has a length of $\sim 136 \mathrm{Km}$ and carries a current of $10 \mathrm{~A}$. The line connecting NPL to Stratford carries a large amount of current ( $18.2 \mathrm{~A}$ ) at 1847 UT. Note that this current originates from two long lines which connect SFD with Huntly (HLY) and Taumarunui (TMN). The HLY line is $\sim 280 \mathrm{~km}$ length and carry a current of $\sim 41 \mathrm{~A}$ whereas the TMN line is 109. $\mathrm{km}$ and carries current of $\sim 36 \mathrm{~A}$. 
Table 5.1

The line connection and the currents produced at 1844 UT and 1847 UT based on transformers experiencing large GIC.

\begin{tabular}{cccccccc}
\hline Node1 & Node2 & $\begin{array}{c}\text { Number } \\
\text { of Lines }\end{array}$ & Resistance & Voltage & $\begin{array}{c}\text { Length } \\
\text { (Km) }\end{array}$ & $\begin{array}{c}\text { GIC } \\
\mathbf{1 8 4 4} \text { UT }\end{array}$ & $\begin{array}{c}\text { GIC } \\
\mathbf{1 8 4 7} \text { UT }\end{array}$ \\
\hline RDF & TUI & 2 & 5.63 & 110 & 96 & 0.50 & 0.91 \\
RDF & WHI & 1 & 0.24 & 220 & 22 & 0.32 & 2.69 \\
RDF & WRK & 1 & 1.46 & 220 & 136 & 10.40 & 9.88 \\
RDF & WTU & 2 & 0.19 & 220 & 8 & 0 & 0 \\
RDF & FHL & 2 & 0.53 & 110 & 7 & 1.20 & -1.24 \\
& & & New Plymouth (NPL) & & & \\
\hline NPL & CST & 2 & 0.11 & 110 & 10 & -0.15 & 2.88 \\
NPL & SFD & 2 & 0.44 & 220 & 42 & -0.08 & 18.20 \\
& & & Stratford (SFD) & & & \\
\hline SFD & BRK & 3 & 2.04 & 220 & 96 & -4.89 & -4.70 \\
SFD & CST & 1 & 0.59 & 110 & 35 & 0.68 & -5.10 \\
SFD & HLY & 1 & 0.64 & 220 & 280 & 8.42 & 41.29 \\
SFD & HWA & 1 & 0.81 & 110 & 26 & -3.19 & -1.86 \\
SFD & KPA_e & 1 & 0.57 & 110 & 17 & 0 & 0 \\
SFD & MKT_e & 1 & 0.76 & 110 & 46 & 0.59 & -3.34 \\
SFD & NPL & 2 & 0.44 & 220 & 42 & 0.08 & -18.20 \\
SFD & OPK & 1 & 1.25 & 110 & 38 & 0 & 0 \\
SFD & TMN & 1 & 0.24 & 220 & 109 & 7.46 & 35.79 \\
& & & & & & &
\end{tabular}

In the above, GIC computed for two different times during the main phase of magnetic storm are discussed. To calculate GIC for the whole duration of the magnetic storm one-minute discrete geoelectric fields of a total 2048 data points have been used, for example as shown in Figure 5.3. The following transformers at different substations have been identified as experiencing large GIC.

1. New Plymouth transformer (NPL-T8L) - $(-39.06,174.03)$

2. Stratford transformer (SFD-T10H) - $(-39.33,174.32)$

3. Redclyffe transformer (RDF-T1H) - (-39.55, 176.82)

4. Wilton transformer (WIL-T8H) - $(-41.26,174.76)$

5. Kawerau transformer (KAW-T12H) - $(-38.08,176.72)$ 

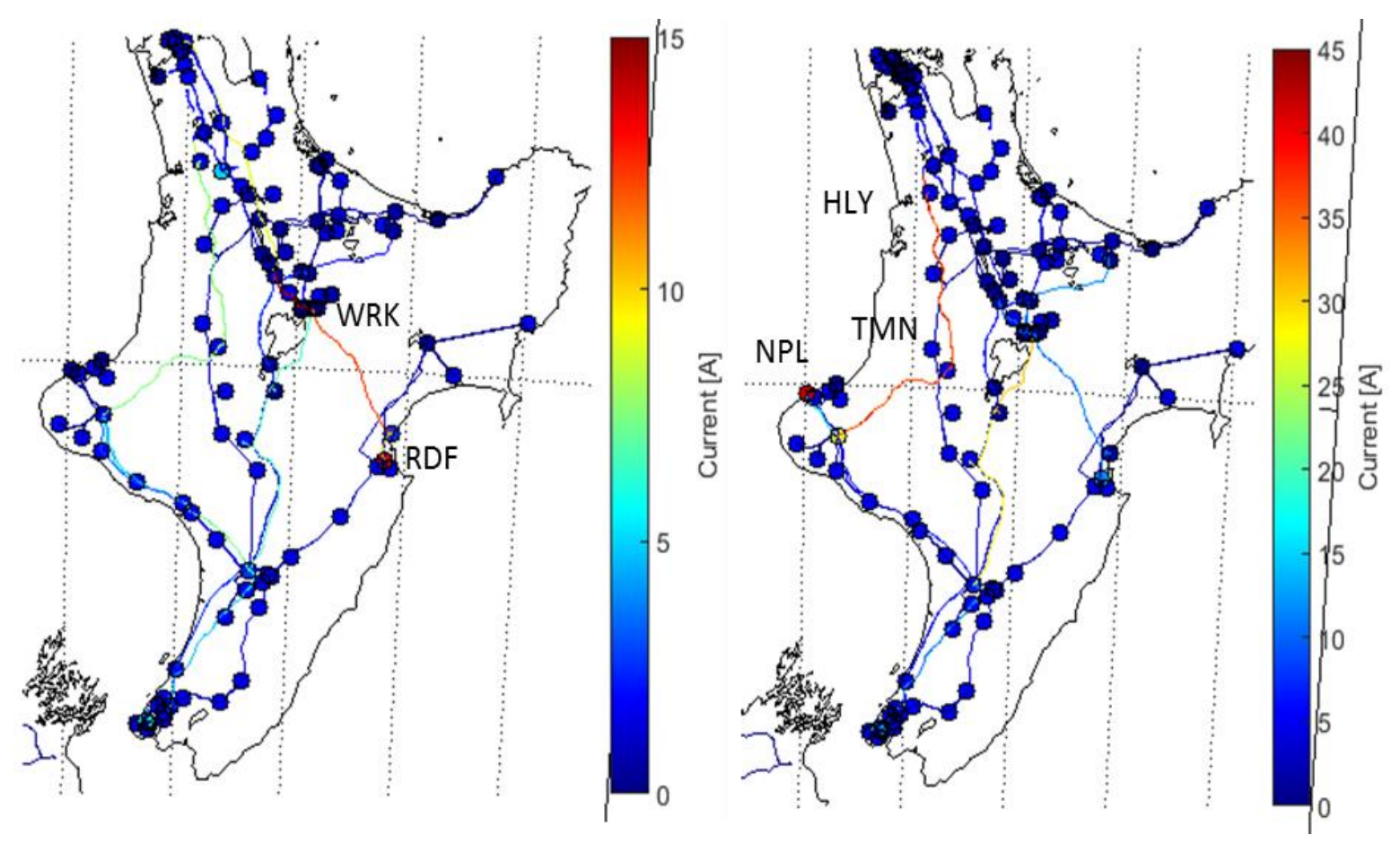

Figure 5. 7: Currents through power lines and transformers at 1844 and 1847 UT respectively. Left hand panel shows large GIC in RDF is caused by line (in red) connected with Wairakei (WRK) substation. Similarly, right hand panel shows large GIC in NPL transformer is caused by lines (in red) connected with Huntly (HLY) and Taumarunui (TMN) substations.

Figure 5.8 shows a plot of GIC for the above-mentioned transformers. These are almost the same transformers experiencing large GIC during the St. Patrick's Day magnetic storm but there is a difference in the order of GIC magnitude, and Wilton (WIL) transformer also appears in the list as experiencing significant GIC.

The New Plymouth transformer experiences the largest GIC and the peak value reaches to $40 \mathrm{~A}$ during the main phase of the magnetic storm. All other transformers have smaller GIC values. For example, Stratford (SFD-T10H) has a maximum value of $\sim 25 \mathrm{~A}$ and Redclyffe (RDF-1H) $20 \mathrm{~A}$. The GIC signatures/spectral components are different for different transformers. Between 1600 and 2000 UT New Plymouth transformer shows a single very strong peak significantly larger than GIC observed during the rest of this period. SFD, which is close to NPL, has a similar signature. In contrast, Redclyffe shows the same magnitude of variations during the whole of this period of the magnetic storm. The calculated GIC do not show very low frequency components because the MT geoelectric fields are restricted to the period range of 2-30 minutes. 
NPL-T8L

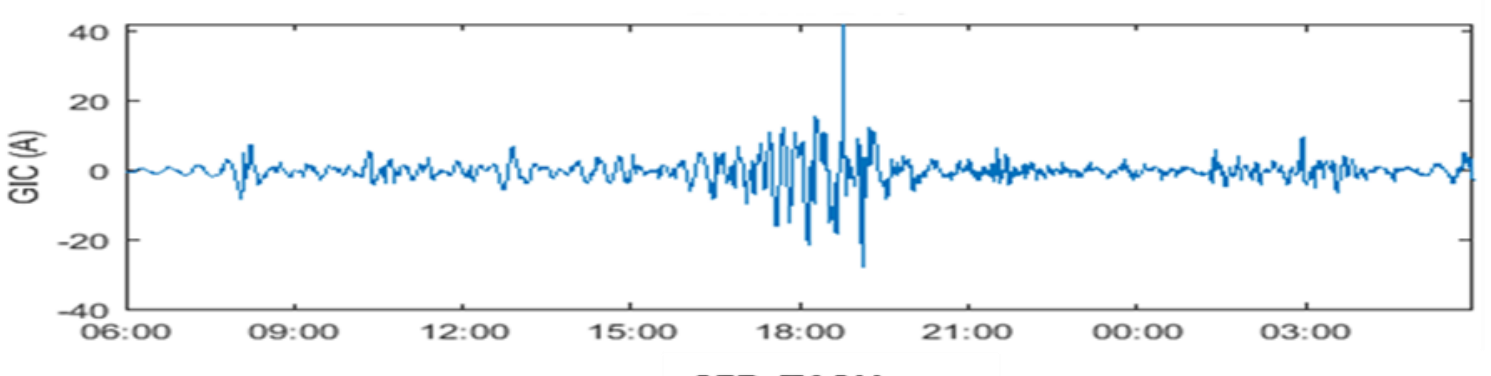

SFD-T10H
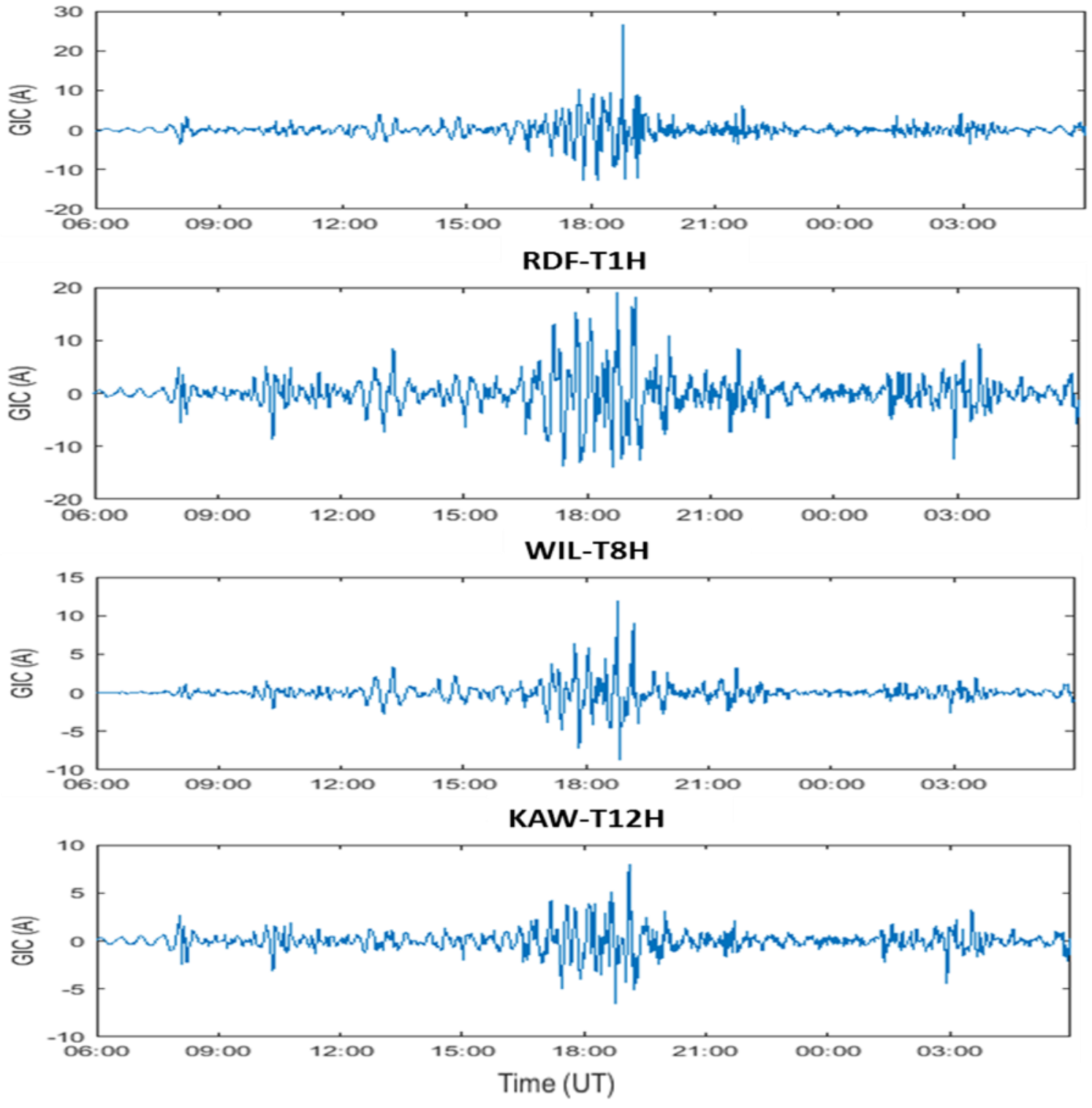

Figure 5. 8: Transformers (as shown on top of each panel) identified experiencing large GIC during the magnetic storm of 20 November2003 using MT modelling. Largest GIC occurs during the main phase of magnetic storm when magnetic field variations are maximum. 


\subsection{MT Model Substation GIC}

This section discuss substation level GIC. Remember that substation GIC is the total current going into or out of a substation due to all earthed transformers. Figure 5.9 shows a snapshot of substation GIC during 1844 and 1847 UT. The bar plot shows large currents go out of substations (positive sign) in the southern part of North Island, whereas small currents pass into the rest of the substations. As, in total, the same current should go into (negative sign) substations as goes out, Figure 5.9 indicates that, in general, current must pass into substations in the northern part of the Island (not shown in the Figure). During the whole magnetic storm, the following substations have been identified as having the largest earthing currents

1. New Plymouth (NPL) - $(-39.06,174.03)$

2. Haywards (HAY)- $(-41.15,174.98)$

3. Bunnythorpe (BPE)- $(-40.28,175.64)$

4. Redclyffe (RDF) - $(-39.55,176.82)$

5. Whirinaki (WHI)- $(-39.38,176.89)$

6. Stratford (SFD) - $(-39.33,174.32)$

7. Wairakei (WRK)- $(-38.62,176.11)$

8. Kawerau (KAW) - $(-38.08,176.72)$

This list of substations includes some new substations which did not appear for the substation level GIC prediction for the St. Patrick's Day magnetic storm. Most of the substations showing large GIC include those which have large transformer level GIC e.g., NPL, RDF and SFD etc. However, some substations do not show large transformer level GIC but do have large substation level GIC e.g., HAY, BPE and WRK. These are substations which have many earthed transformers. For example, Bunnythorpe has 6, Haywards has 10, Whirinaki has 6 and Wairakei has 4 earthed transformers. 

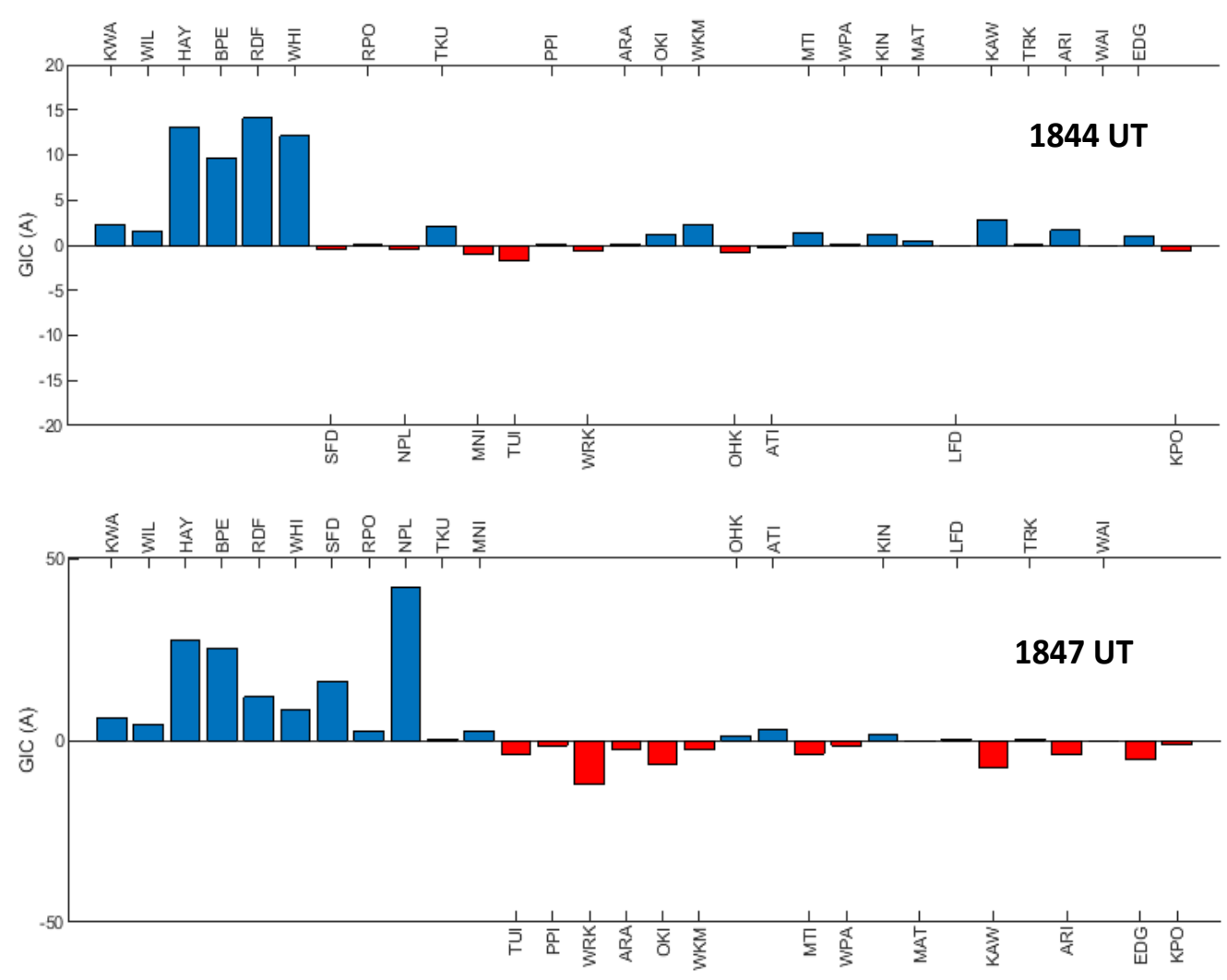

Figure 5. 9: Snapshot of substation GIC during two different times. Substation (on horizontal axis) showing largest GIC here also have large number of transformers.
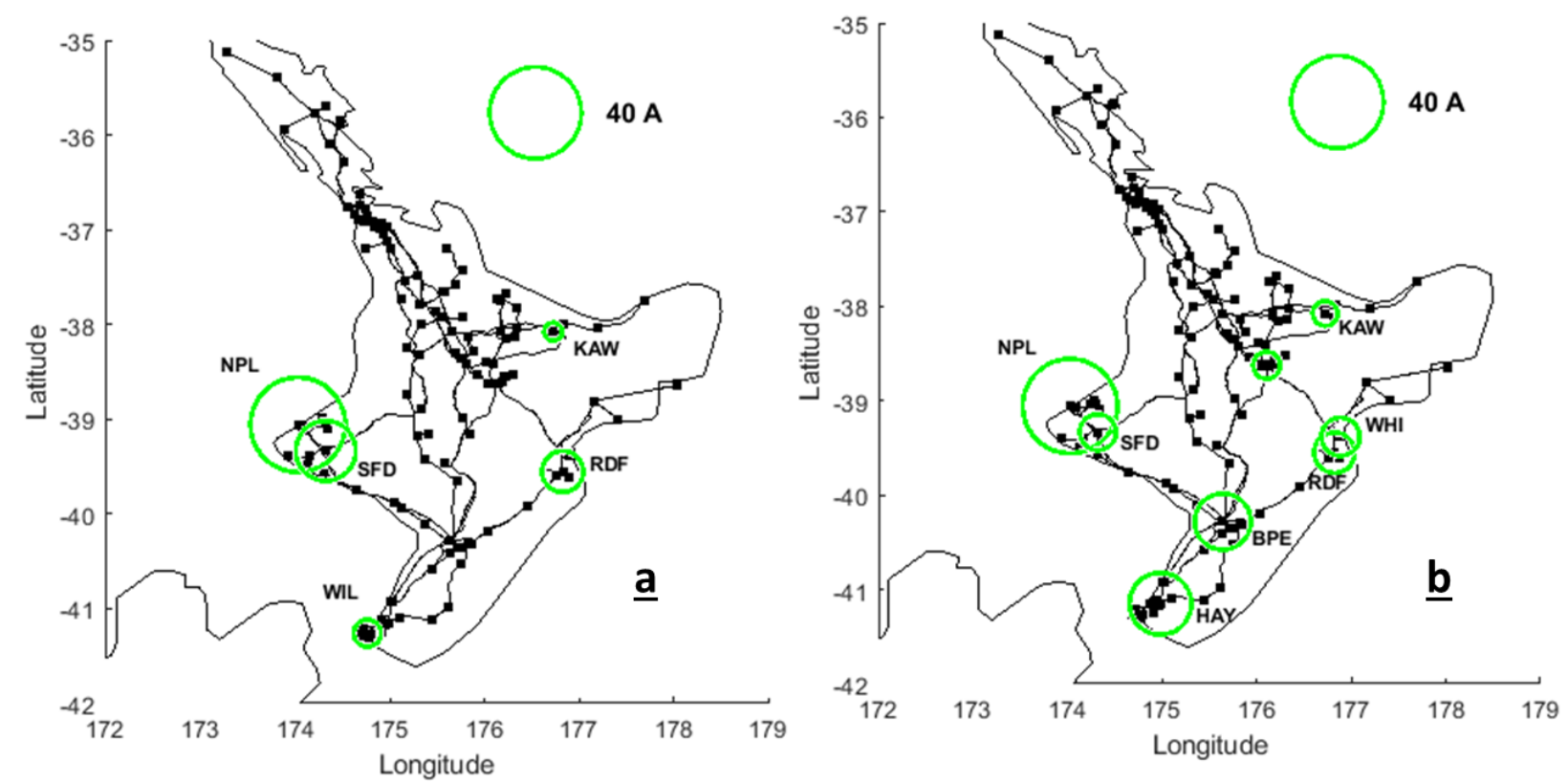

Figure 5. 10: MT model results. (a) Transformer locations experiencing the largest GIC and (b) locations of significant substation-level GIC during 20 November 2003 storm. The size of circles is proportional to GIC magnitude. 
The summary of results of MT technique for transformers and substations experiencing large GIC during 20 November 2003 magnetic storm is shown in Figure 5.10. The size of the circle corresponds to magnitude of GIC where the largest circle, for NPL, has a GIC magnitude of $42 \mathrm{~A}$. The direction of current is not shown because the maximum value of the GIC is not necessary at the same time for different transformers during the main phase of magnetic storm.

\subsection{Comparison of MT Model and Measured GIC}

It was seen in the previous chapter that model GIC at Redclyffe can be compared with observed GIC in transformer T6H at ISL. The same comparison is carried out for the 2003 magnetic storm. The measured GIC at ISL have been high-pass filtered (2-30 mins) to match the period range of the MT data and the time plot is shown in the first panel of Figure 5.11 (a). Since RDF substation consists of many transformers the GIC plot for two transformers (T4H and T1H) are shown in the second and third panels of the same Figure. Model GIC for both transformers show the same kind of variations except for a different scale of magnitude: transformer $(\mathrm{T} 1 \mathrm{H})$ has almost twice the magnitude of GIC as transformer (T4H). T1H is an earthed transformer and connected to low voltage node $(110 \mathrm{kV})$ whereas $\mathrm{T} 4 \mathrm{H}$ is upper phase earthed and shares connections with both the low $(110 \mathrm{kV})$ and high voltage nodes $(220 \mathrm{kV})$. The network configurations are almost the same and there is just a difference of magnitude. Thus, either transformer could be used for comparison with the measured data. Although $\mathrm{T} 1 \mathrm{H}$ transformer data has been used for comparison for the St. Patrick's Day magnetic storm, here we have compared T4H with the measured data as it is closer to the measured data in term of magnitude. Figure 5.11 (b) shows the comparison of RDF-T4H and ISL-T6H with the second panel showing a close-up view during the main phase of the magnetic storm. The correlation coefficient between model and observed GIC is 0.76 and the performance parameter is 0.31 . The plots show that the model effectively reproduces the measured variations of GIC data during most of magnetic storm. There are, however, some differences. For example, the model amplitude is smaller than the measured data around 17:32, 18:30 and 19:30 UT. As the MT data were available only at discrete periods and interpolation was adopted across the available MT period range, it is possible that these differences may be attributed to the wide gaps between measured periods in the MT data. 

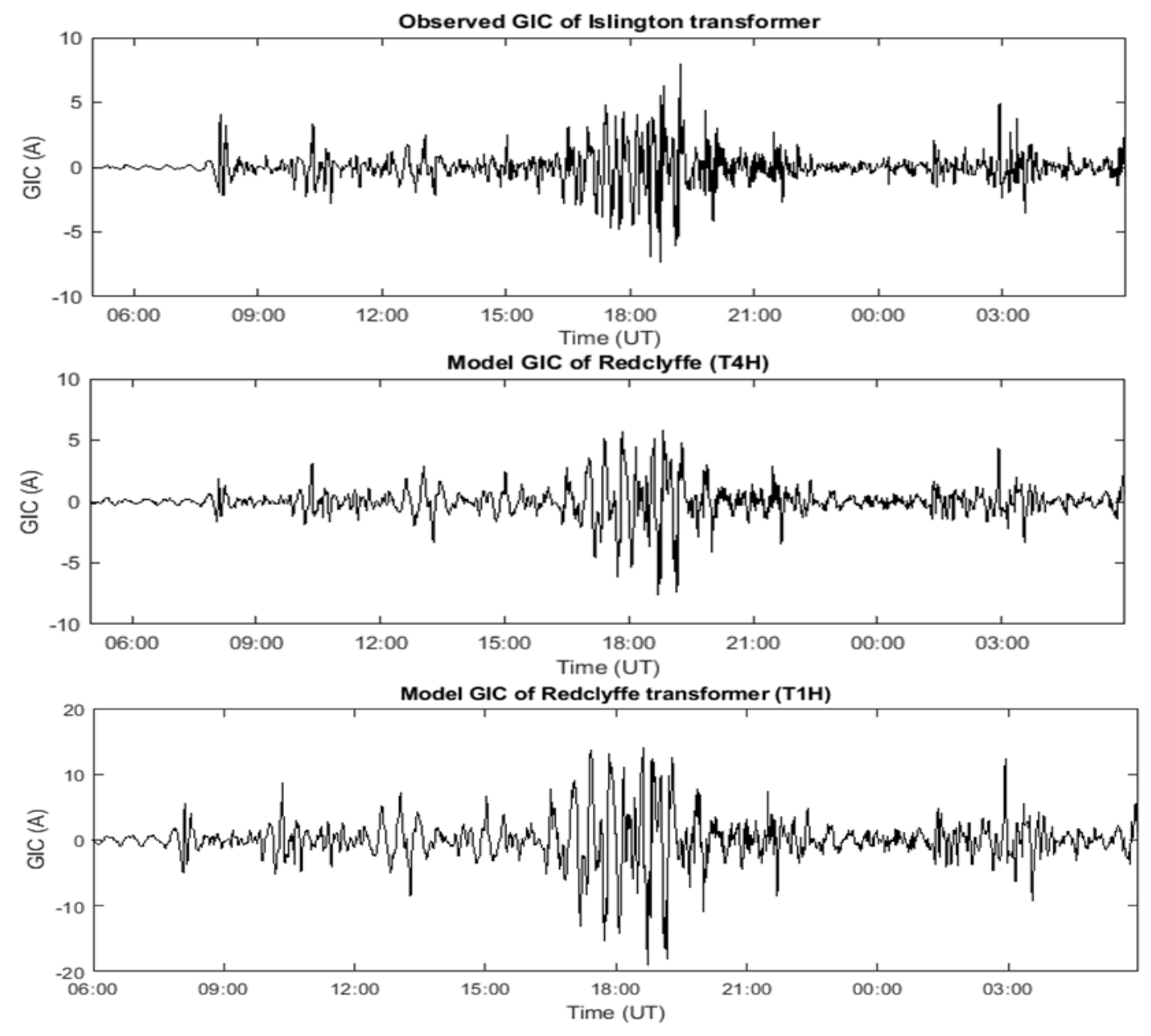

Figure 5. 11a: Individual plot of measured GIC of ISL transformer (T6H) in the period range of 230 minutes and the model GIC of Redclyffe transformers T4H and T1H.

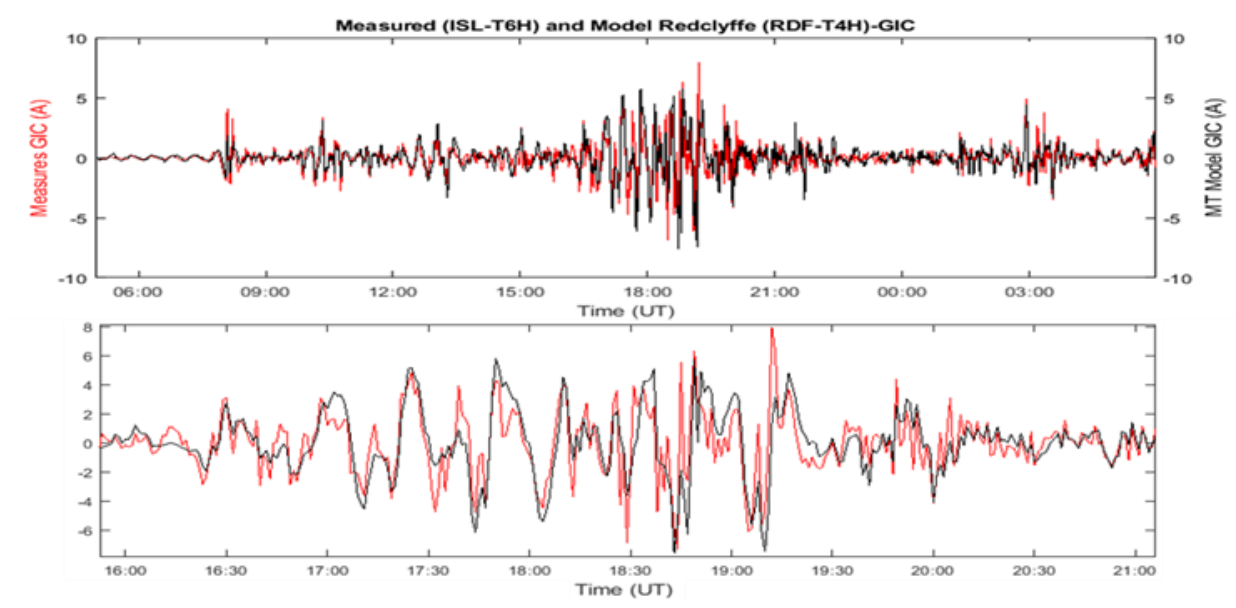

Figure 5.11b: Top panel shows model GIC of RDF T4H (black) and observed GIC of Islington (red). Bottom graph shows zoom in view during the main phase of magnetic storm. Model GIC effectively reproduces variations during most of magnetic storm $(r=0.76)$. 


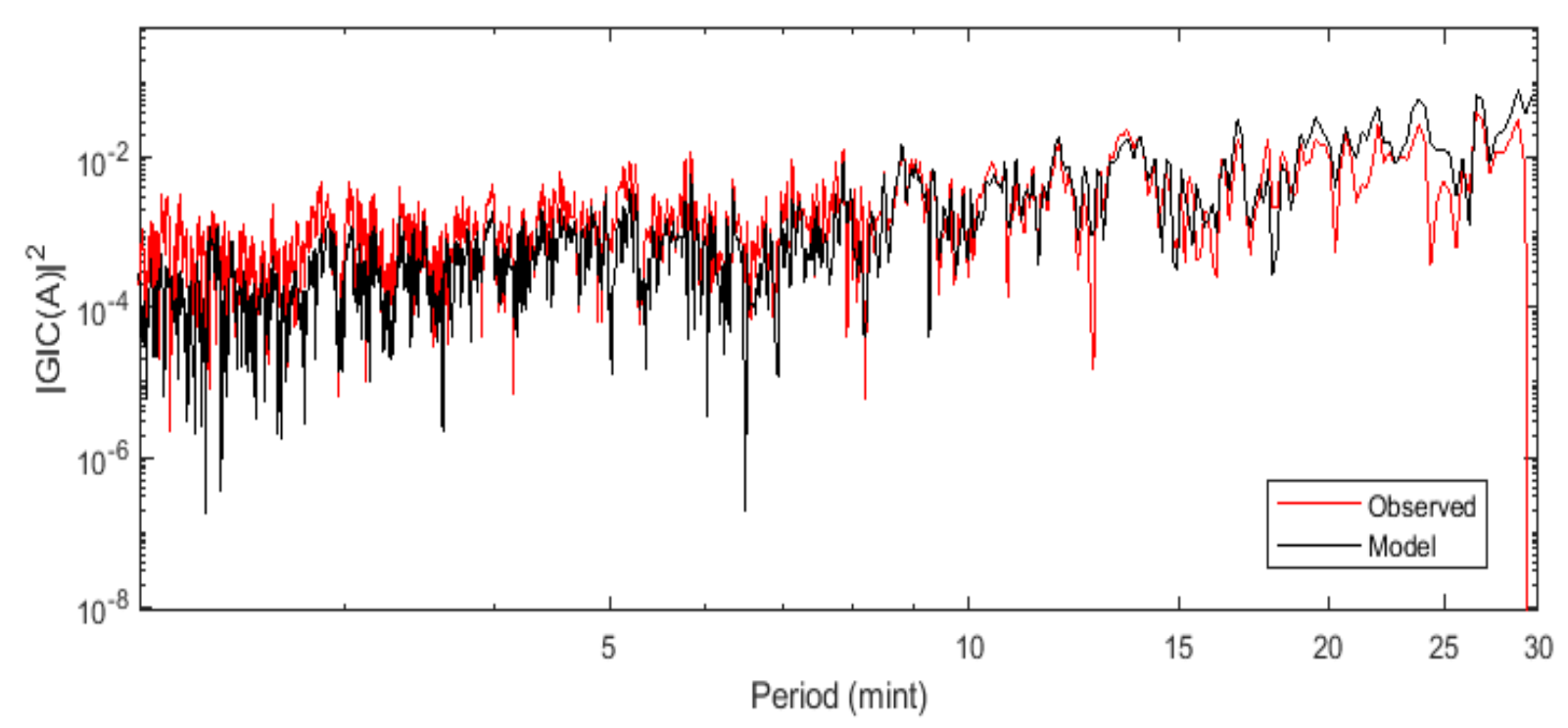

Figure 5.11c: Power spectrum comparison of measured and model GIC in the period range of 230 minutes.

A comparison of the power spectra of both model and observed GIC is also given in Figure 5.11(c). The horizontal axis shows the period range from 2-30 minutes and the vertical axis is the square of absolute magnitude of each frequency component. The model and measured GIC both show a gradual increase of spectral power density from shorter to longer periods. Model and observed data show a good match at longer periods starting from 8 minutes but during shorter periods the observed spectrum has stronger frequency components than the model data.

\subsection{Thin-sheet Model Geoelectric Field}

This section describes the geoelectric fields derived for the 20 November 2003 storm using the thin-sheet conductance model of Vasseur and Weidelt (1977) which has been used extensively for GIC modelling in the South Island e.g., Divett et al. (2017, 2018, 2020). The geoelectric fields have been derived using the thin-sheet conductance model for the same interval of EYR magnetic data shown in Figure 5.2. The thin-sheet model has been used for the whole resulting period range of the magnetic data (2-2048 minutes) therefore the computed geoelectric fields cover the same period range. Figure 5.12 shows the time domain geoelectric fields for the MT site CAR-119 (as shown for the MT data in Figure 5.3) calculated using the thin-sheet conductance model. 
TS Geoelectric fields at site (CAR-119)

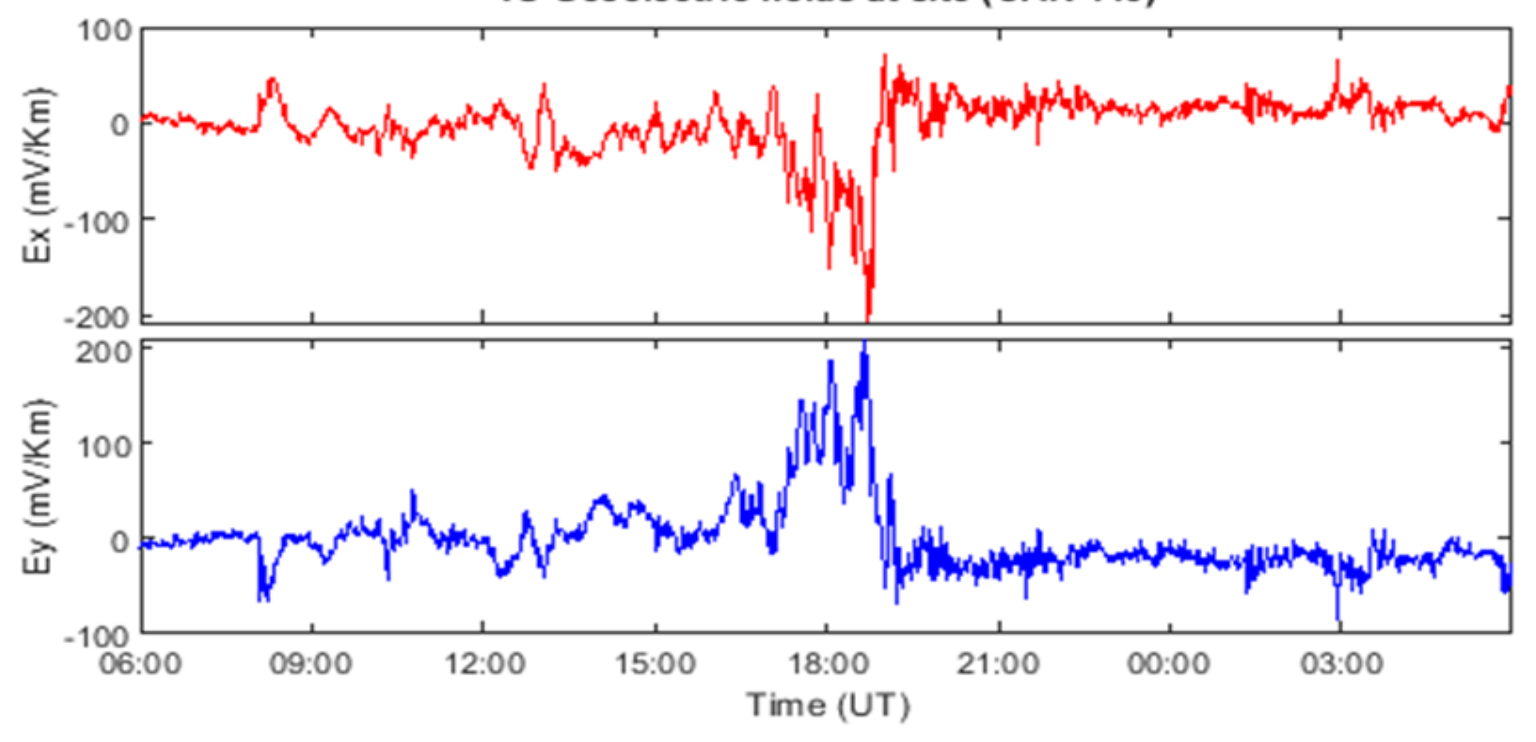

Figure 5. 12: One-minute discrete geoelectric fields using the TS conductance model for MT site CAR-119. Field values reach to $200 \mathrm{mV} / \mathrm{km}$ during the main phase of the magnetic storm and the existence of strong low frequency components is evident.

Strong low frequency variations in the amplitude of geoelectric fields exist during the main phase of the magnetic storm i.e., from 1500 to 2100 UT reaching a maximum value of $\sim 200 \mathrm{mV} / \mathrm{km}$. This is primarily due to large magnetic variations during the main phase of the magnetic storm. Comparison of Figures 5.3 and 5.12 suggests that both thin-sheet and MT electric fields have similar variations over their common period range. However, lower frequency variations exist in the thin-sheet fields only.

Figure 5.13 shows the vector form of the geoelectric fields superimposed on the thin-sheet model grid at 1844 and 1847 UT. The geoelectric fields have been plotted on the same scale of 500 $\mathrm{mV} / \mathrm{km}$ as was used for the MT fields. Figure 5.13 shows that at these times the thin-sheet fields are smaller in magnitude compared to the MT fields and vary smoothly throughout the North Island. Field values does not change significantly between different conductance regions. The field direction changes between the two times but not as significantly as for the MT fields. This is due to the inclusion of longer periods in the thin-sheet model. 

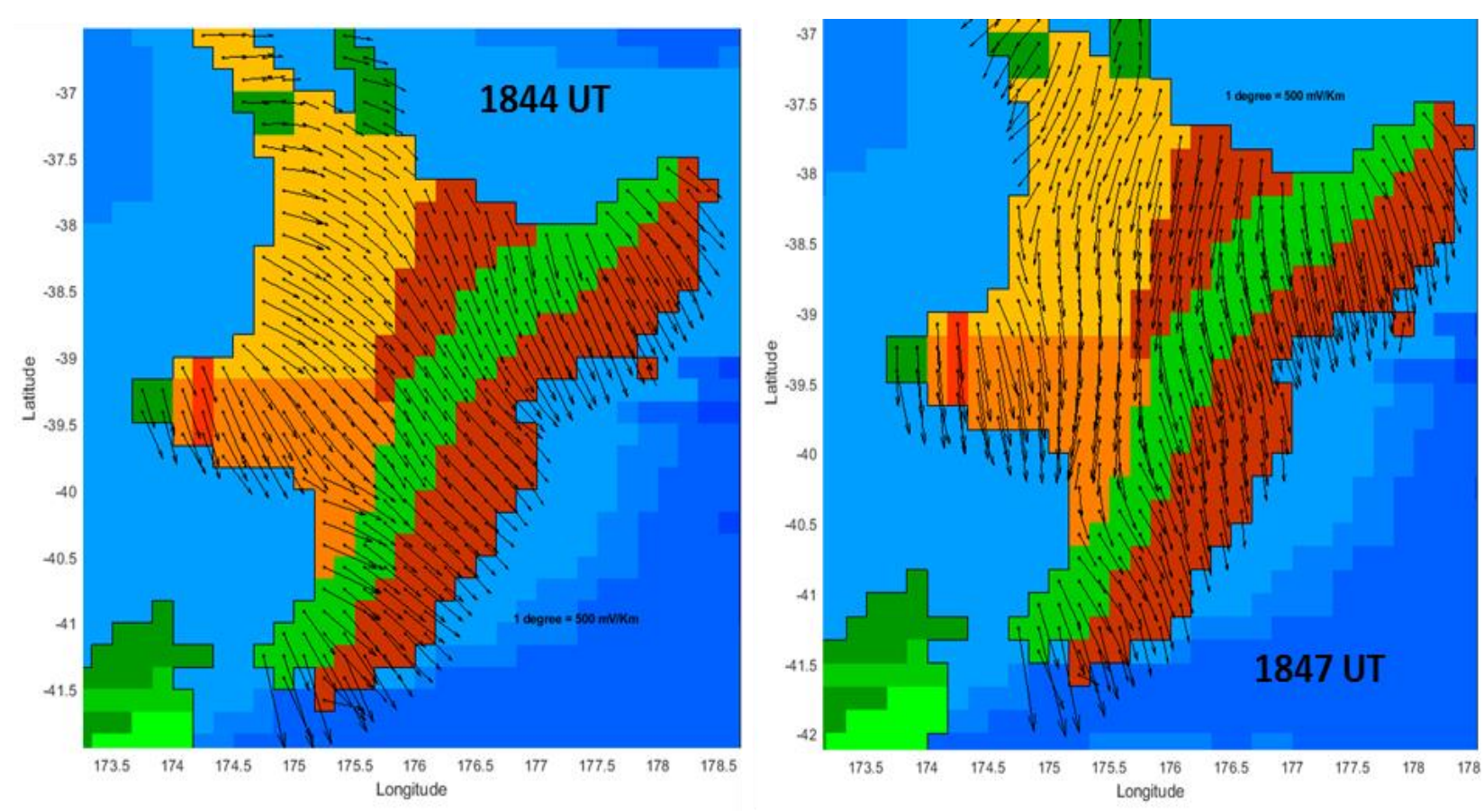

Figure 5. 13: Geoelectric field vectors using the thin-sheet conductance model at 1844 and 1847 UT. The smooth temporal variation is associated with long periods included in TS conductance model.

\subsection{Thin-sheet Model Transformer GIC}

Figure 5.14 shows transformer level GIC computed using the thin-sheet geoelectric fields at the two different times given in Figure 5.13. It can be seen that the transformer response is not the same as for the MT model results, again because the different period ranges covered by the models result in different GIC magnitudes. Redclyffe transformer shows the largest GIC at both times with values around 20 A. New Plymouth shows large GIC at 1844 UT given by -11.54 A. A Stratford transformer also shows strong GIC of 16.4 A at 1847 UT. These differences are partly due to the longer period range of the thin-sheet model, including the fact that the wider period range also smooths the field directions as seen in Figure 5.13 compared to Figure 5.5. 

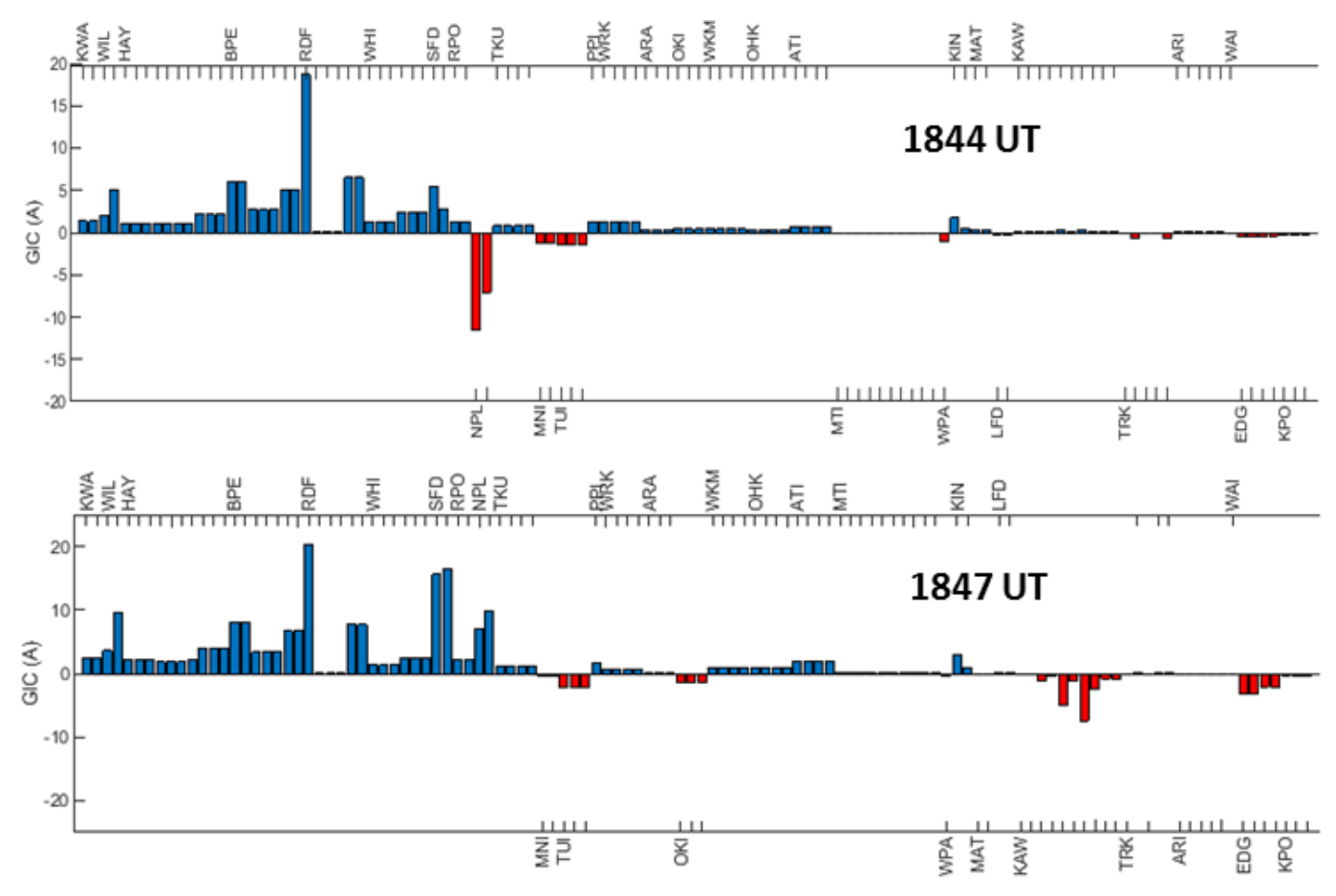

Figure 5. 14: Transformer level GIC at 18:44 and 18:47 UT extending up to Karapiro (KPO) of the North Island. Redclyffe (RDF) and New Plymouth (NPL) and Stratford (SFD) transformers can be seen experiencing the largest GIC.

To compute GIC for the whole duration of the magnetic storm, one-minute discrete geoelectric fields have been used. By plotting the GIC during the whole magnetic storm the following transformers have been identified as having the largest GIC. Time domain plots for these transformers are given in Figure 5.15.

1. Redclyffe transformer (RDF-T1H) - (-39.55, 176.82)

2. New Plymouth transformer (NPL-T8L) - $(-39.06,174.03)$

3. Stratford transformer (SFD-T10H) - $(-39.33,174.32)$

4. Wilton transformer (WIL-T8H) - $(-41.26,174.76)$

5. Bunnythorpe transformer (BPE-T3L)- $(-40.28,175.64)$

6. Kawerau transformer (KAW-T12H) - $(-38.08,176.72)$ 

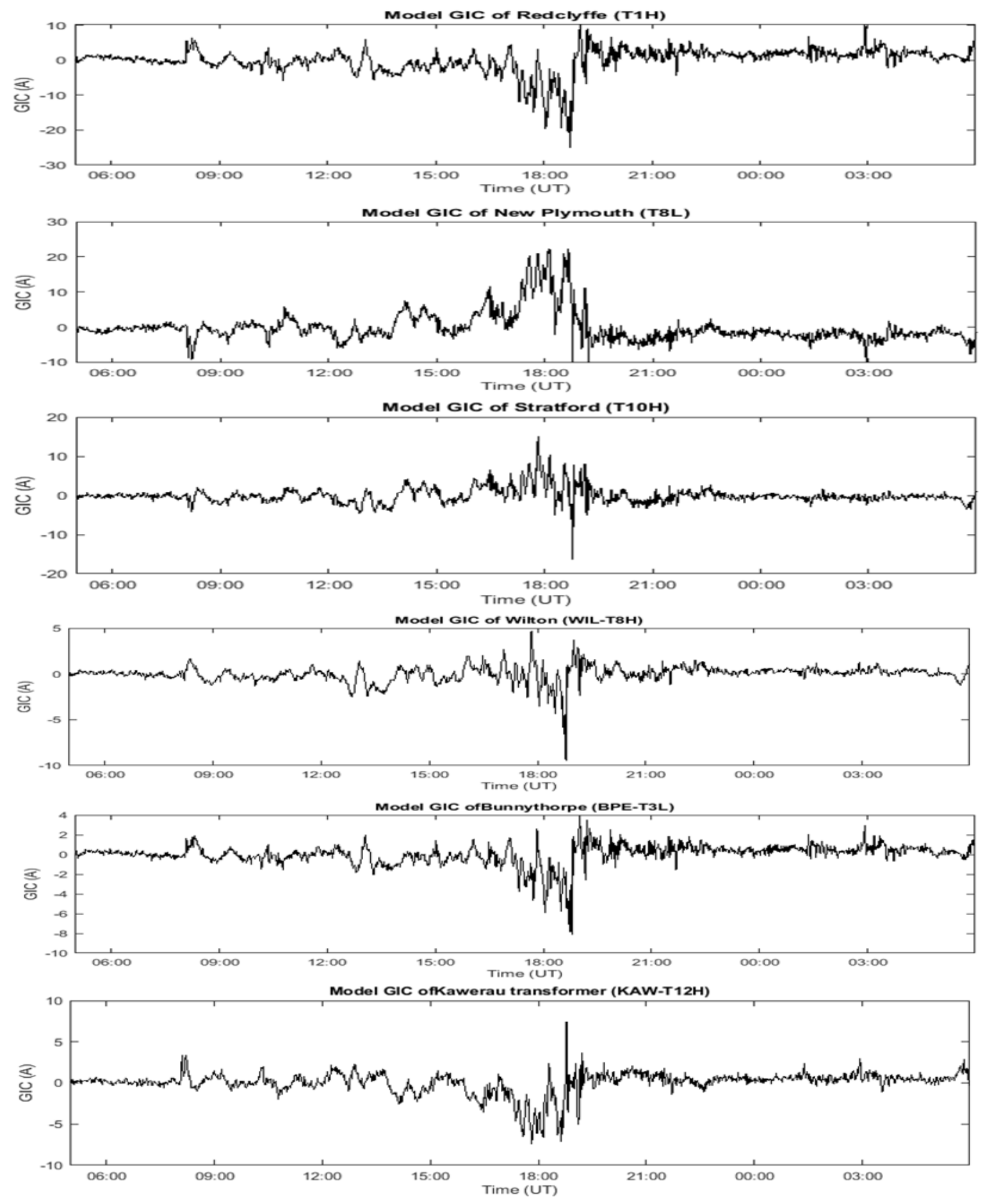

Figure 5. 15: Transformers experiencing large GIC using the TS conductance geoelectric fields. These show positive and negative current going through transformers at different times during the main phase of magnetic storm.

The above plots clearly show the existence of low frequency components in GIC resulting from the inclusion of low frequency variations in the thin-sheet model. Transformers identified as 
having the largest GIC during the magnetic storm are almost the same as were predicted for the St. Patrick's Day magnetic storm with the addition of two transformers in substations in the lower part of the North Island: Wilton (WIL) and Bunnythorpe (BPE), which also experience large GIC. Note that the plots shown in Figure 5.15 not only vary in GIC magnitude but also in the shape of the plots e.g., Redclyffe transformer shows smooth variations in GIC whereas the Stratford transformer has more abrupt GIC variations.

\subsection{Thin-sheet Model Substation GIC}

The following substations have been identified as having the largest GIC. The snapshot of substation GIC at 1844 and 1847 UT is given as Figure 5.16 .

1. Bunnythorpe (BPE) - $(-40.28,175.64)$

2. Redclyffe (RDF) - $(-39.55,176.82)$

3. New Plymouth (NPL) - $(-39.06,174.03)$

4. Haywards (HAY) - $(-41.15,174.98)$

5. Whirinaki (WHI) - $(-39.38,176.88)$

6. Stratford (SFD) - $(-39.33,174.32)$

Again, these are the almost same substations as was predicted for the St. Patrick's Day storm with minor changes in the order of experiencing largest GIC. It can be seen that substation GIC are widely distributed in different parts of the network. For example, Bunnythorpe and Haywards lie in the south, Redclyffe and Whirinaki are on the east coast and New Plymouth and Stratford on the west cost of the North Island.

The summary of results of thin-sheet technique for transformers and substations experiencing large GIC during 20 November 2003 magnetic storm is shown in Figure 5.17. The size of the circle corresponds to magnitude of GIC and the same scale is chosen as was for the Figure 5.10. The direction of current is not shown because the maximum value of the GIC is not necessary at the same time for different transformers during the main phase of magnetic storm. 

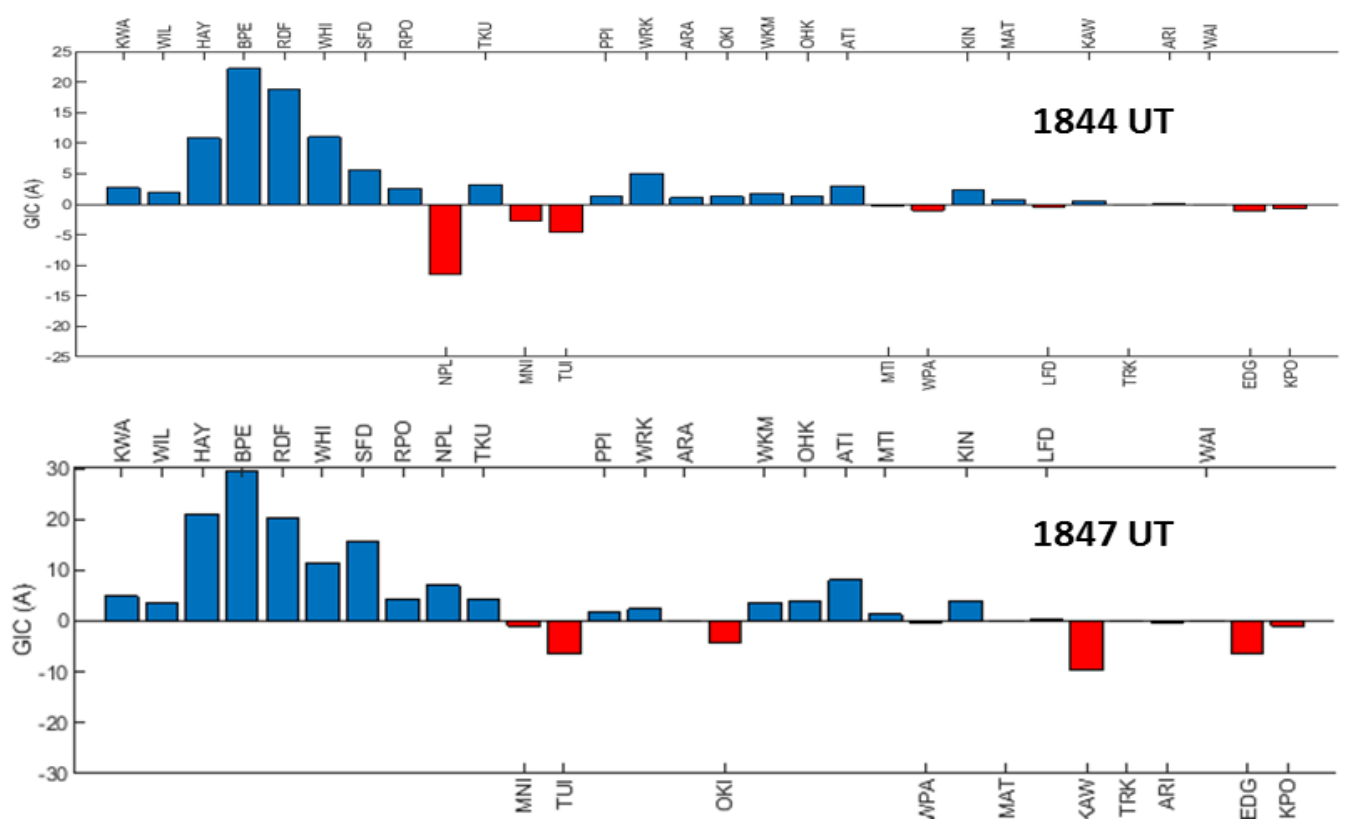

Figure 5. 16: Substation-level GIC at 1844 and 1847 UT using TS conductance model.
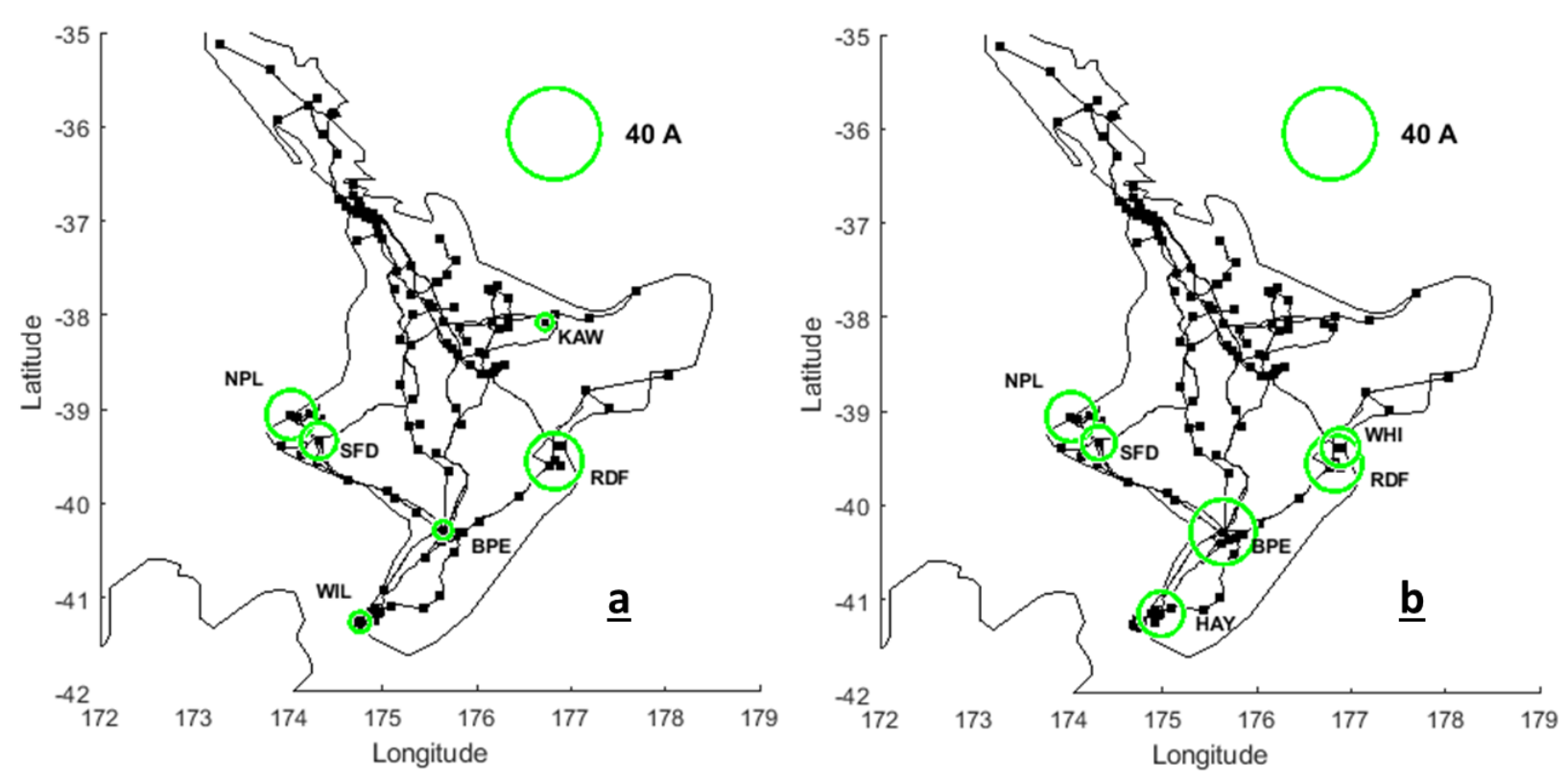

Figure 5. 17: Thin-sheet model results. (a) Transformer locations experiencing the largest GIC and (b) locations of significant substation-level GIC during 20 Nov 2003 storm. The size of circles is proportional to GIC magnitude and plotted on the same scale as was for the Figure 5.10. 


\subsection{Thin-sheet Model GIC and Measured Data Comparison}

As was done for the MT model GIC, the thin-sheet model GIC at Redclyffe (T4H) transformer have been compared with measured GIC for ISL-T6H. This is shown as Figure 5.18a for the full period range of the measured data. The thin-sheet model effectively reproduces the low frequency variations as seen in the observed data during most of the magnetic storm although the model amplitude is smaller than the measured data. There exists a prominent discrepancy in the amplitudes of model and observed GIC around 17:32 UT when ISL GIC has a value of $-12.48 \mathrm{~A}$ and RDF is only -4.26 A. However, RDF-T4H does not experience the largest GIC at Redclyffe substation, larger GIC occur in transformer T1H. The correlation coefficient between GIC at ISL and RDF-T4H is 0.82 and the performance parameter $P=0.39$. These are much better results than for the St. Patrick's Day magnetic storm.

The comparison of measured and model GIC power spectra is given in Figure 5.18b. This shows that the power density gradually increases at longer periods both in the measured and model spectrum. The thin-sheet power spectrum (black) has a good match with the measured spectrum (red) at longer periods compared to shorter periods. Since more power is embedded in longer periods of the GIC data the TS model reproduces GIC more effectively.

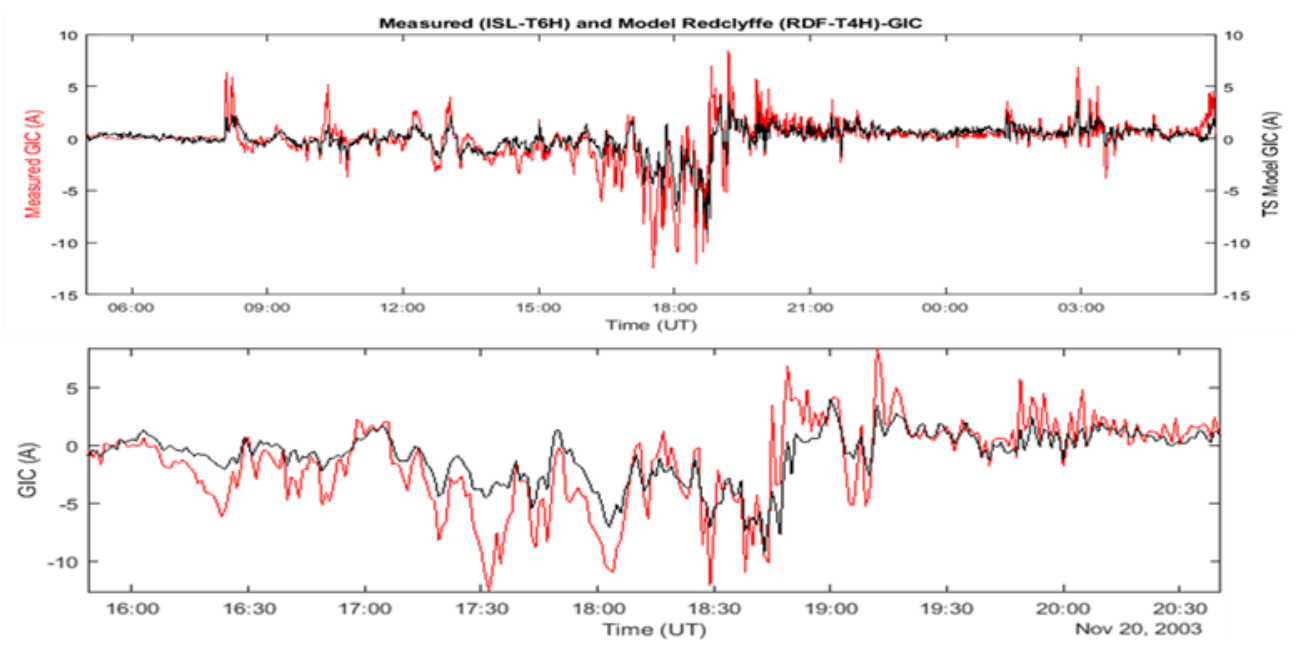

Figure 5. 18a: TS model GIC of RDF transformer and ISL measured GIC comparison shown in first panel ( $r=0.82)$. Bottom panel shows close-up view during large GIC fluctuations where TS model amplitudes have good match with measured data in low frequency components. 


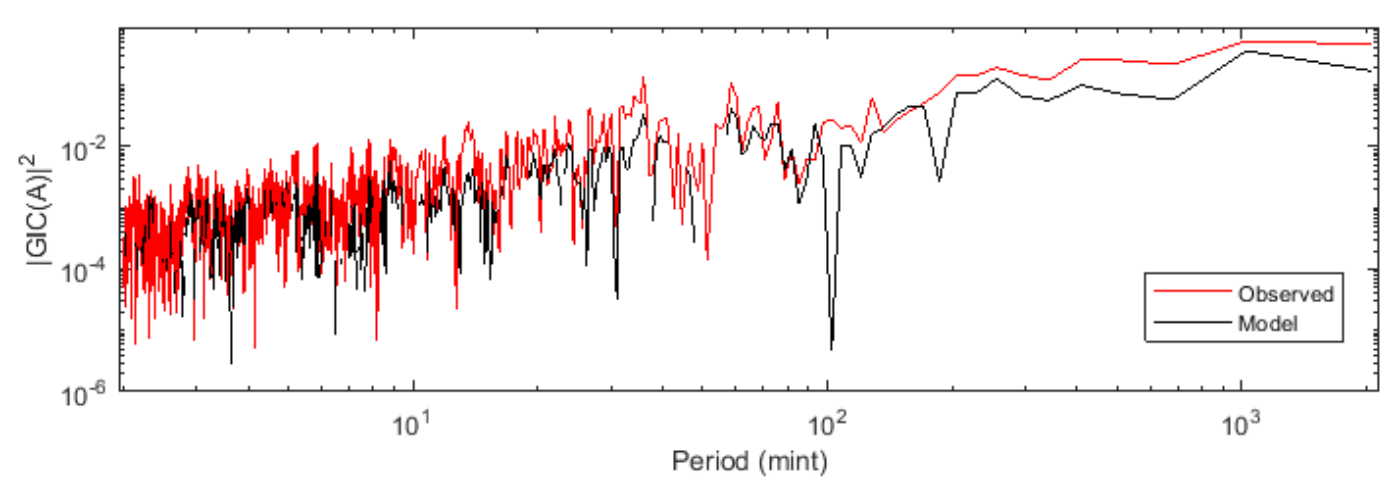

Figure 5.18b: A power spectrum comparison of measured and TS model GIC as given in Figure $5.18 a$.
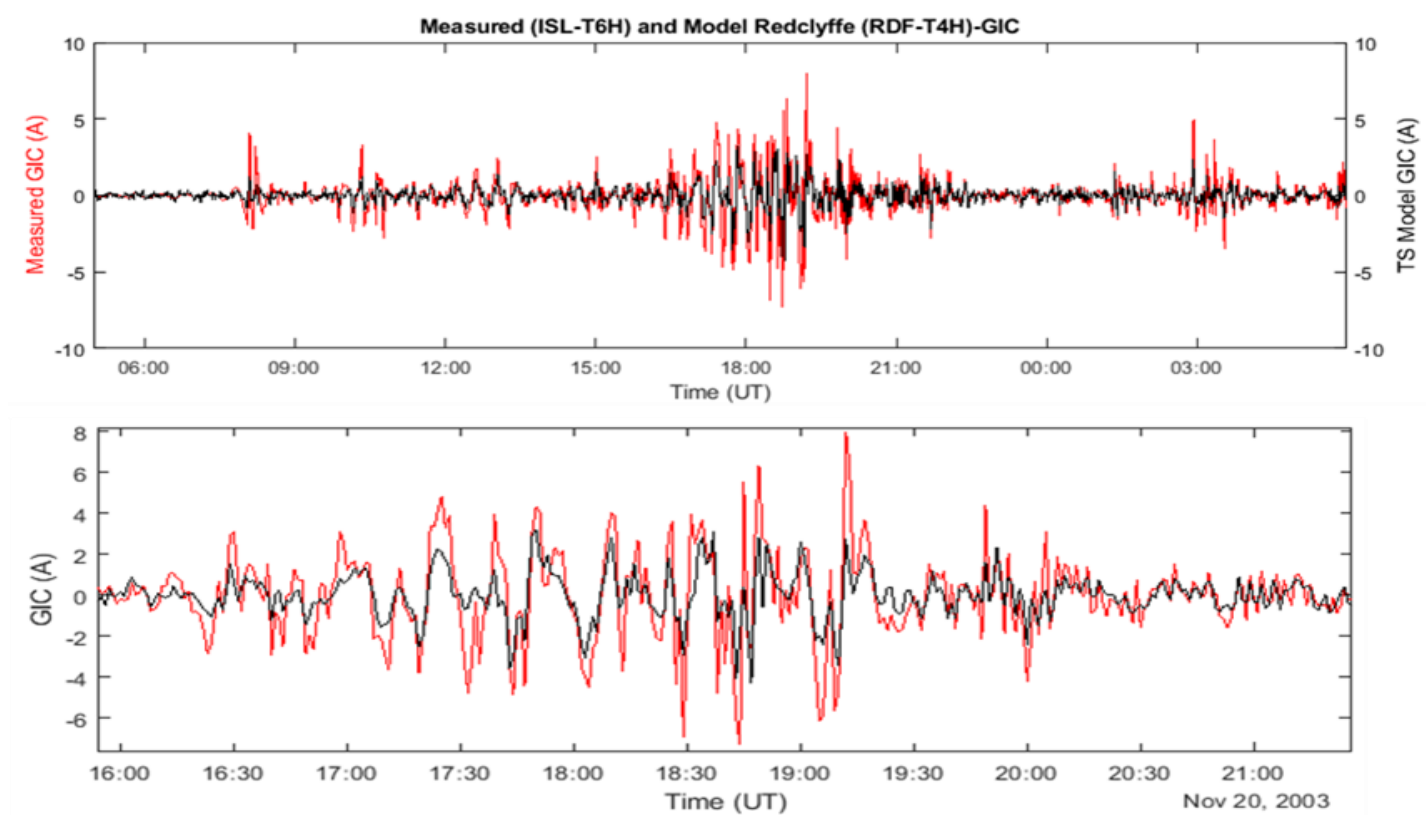

Figure 5.18c: TS model (black) and measured GIC (red) of ISL in shorter period range (2-30 minutes) as top panel. Bottom panel shows close-up view where TS model has smaller amplitudes as compared to measured data $(r=0.77)$.

A comparison in the period range of 2-30 minutes is also shown in Figure $5.18 \mathrm{c}$ to visualize the model GIC results in the high frequency range. It is evident that the thin-sheet model GIC have a magnitude about half of the observed values at ISL during the whole magnetic storm. The correlation coefficient is 0.77 and performance parameter is 0.32 . To see the effectiveness of the thin-sheet model at lower frequencies a comparison of model GIC with measured GIC at ISL only for periods longer than 30 minutes is shown in Figure 5.18d. The model GIC effectively produce the same amplitude variations during most of magnetic storm. An exception is at 17: 32 when the model GIC has very small value compared to the measured value. Since T1H transformer at 
Redclyffe has larger GIC than T4H transformer the amplitude of GIC does not seem to be a problem in using the thin-sheet model. The correlation coefficient is 0.84 and performance parameter is 0.44 . The correlation coefficient and performance parameter are better than when calculated for the shorter period range, showing that thin-sheet model GIC results are not as good in the shorter period range. The thin-sheet model is unable to reproduce same magnitude sharp peaks seen in the measured GIC and this is consistent with the limited frequency range at which thin-sheet model can be used (Divett et al., 2020).

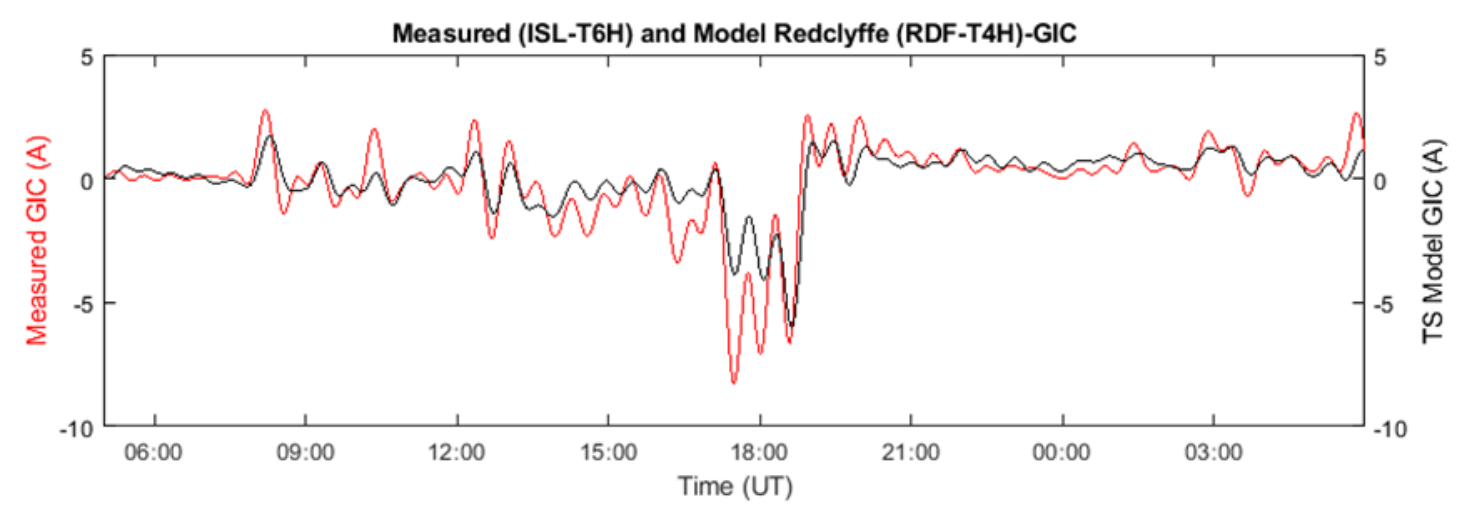

Figure 5.18d: Comparison of TS model GIC for RDF T1 with measured ISL GIC at longer periods starting from 30 minutes $(r=0.85)$.

\subsection{Discussion}

This chapter presents results of GIC modelling using MT data and the thin-sheet conductance model for the magnetic storm of 20 November2003. The magnetic storm was caused by a different type of solar interplanetary structure (magnetic cloud preceded by a shock), and the spectral characteristics of measured GIC at Islington are also different from the previously discussed magnetic storm of St. Patrick's Day 2015. In the context of transformers experiencing large GIC both techniques (i.e., MT and TS) give almost the same results. New Plymouth (NPLT8L), Stratford (SFD-10H) and Redclyffe (RDF-T1H) are the most susceptible to this magnetic storm. These are also the same transformers that showed large GIC during the St. Patrick's Day storm. Thus, the transformers experiencing large GIC do not vary much between both magnetic storms using either of the techniques. The difference in scale of magnitude of model GIC during 
MT and thin-sheet techniques is mainly due to the different period range covered by the two techniques.

In terms of substation GIC the results using MT modelling technique differ between the St. Patrick's Day storm and the 2003 storm. For the 2003 magnetic storm two substations from the lower part of the North Island, Bunnythorpe and Haywards show large substation GIC. Both MT and thin-sheet models show same substations experiencing large substation level GIC although the order of magnitude they appear is different during both techniques. Therefore, unlike transformer level GIC, substation GIC may be different during different storms and due to different modelling technique.

The MT model GIC for Redclyffe transformer (RDF-T4H) is also compared with Islington (ISL-T6H) observed data in the period range of 2-30 minutes. The model reproduces largely the same variations, particularly during the main phase of the magnetic storm. The correlation coefficient is 0.76 and performance parameter is 0.31 . The thin-sheet model GIC have also been compared with the measured ISL transformer data. The thin-sheet model effectively reproduces the low frequency components seen in the measured data, with values of correlation coefficient and performance parameters of 0.82 and 0.39 , respectively. This shows that the thin-sheet model works well for lower frequencies whereas the MT modelling results are more promising than the thin-sheet model in the high frequency range.

Overall, unlike the St. Patrick's Day magnetic storm, the thin-sheet model results are much better when compared with ISL transformer data during the magnetic storm of 20 November2003. In the St. Patrick's Day storm, there was one single spike of strong GIC ( -10.5 A) during the SSC and no significant GIC for rest of storm. Whereas in the later storm, GIC during the SSC are not prominent, but strong magnetic fluctuations occur during the main phase and there are $57 \mathrm{GIC}$ data points above a threshold of $5 \mathrm{~A}$, demonstrating the existence of strong low frequency components. Since the thin-sheet model is valid over a longer period range it may more effectively reproduce these observed GIC. During the St. Patrick's Day magnetic storm, when high frequency component dominates, the thin-sheet is unable to reproduce such variations and the MT model gives better results. 


\section{Chapter 6: MT Data GIC Modelling for the South Island During St. Patrick's Day 2015 Storm}

\subsection{Introduction}

Despite the lack of actual measurements of GIC, GIC modelling for the North Island using MT data has been successful in identifying those substations/transformers potentially at risk. There is therefore motivation to apply the same technique to the South Island where there is a lot of measured GIC data. The aim of this work is to utilize the available MT data from the South Island so that the model results can be validated through the measured data. The thin-sheet conductance model of Vasseur and Weidelt (1977) has been used effectively for GIC modelling for the South Island (Divett et al., 2017; 2018) but comes with some serious limitations. As discusses in Chapter 2, it is valid over only a limited period range and is therefore unable to reproduce the complete range of variations in model GIC. It also has spatial limitations as the model itself is a crude approximation of variations in conductive structure and is not able to produce locally accurate surface geoelectric fields. This has been demonstrated by comparing the results of predictions of GIC for the South Island between the thin-sheet model (Divett et al., $2017,2018,2020$ ), and a transfer function analysis (Ingham et al., 2017) which is independent of the thin-sheet model. There are significant differences in the predictions for GIC in the lower South Island (Divett et al., 2020).

Therefore, as was done for the North Island, this chapter describes the approach for computing geoelectric fields for the South Island based on magnetotelluric (MT) sounding data. As for the North Island we have initially considered the magnetic field variation during the St. Patrick's Day magnetic storm (17 March2015). Furthermore, in the South Island MT sites are scattered mainly in three groups and there are large gaps with no MT data. Thus, again as for the North Island, spatial interpolation is performed to fill gaps in the data. With the big gaps in the available MT data the possibility of utilization of Thevenin's Equivalent circuit approach given by Boteler et al. 
(2013), has also been discussed. The modelling results from the MT data have been compared both with measured GIC data, and those from the thin-sheet conductance model.

\subsection{Magnetotelluric (MT) Data in the South Island}

Unlike the North Island, the South Island has very sparse MT measurements. Figure 6.1 shows the sites of selected MT measurements (black dots) superimposed on the thin-sheet conductance map of New Zealand used by Divett et al. (2017). There is a total of 42 cells which can be filled using available MT data. Although some cells contain multiple MT sites, in Figure 6.1 each grid cell is represented by a single MT site. The MT site distribution can broadly be divided into sites in three main regions from south to north as also given in Table 6.1.

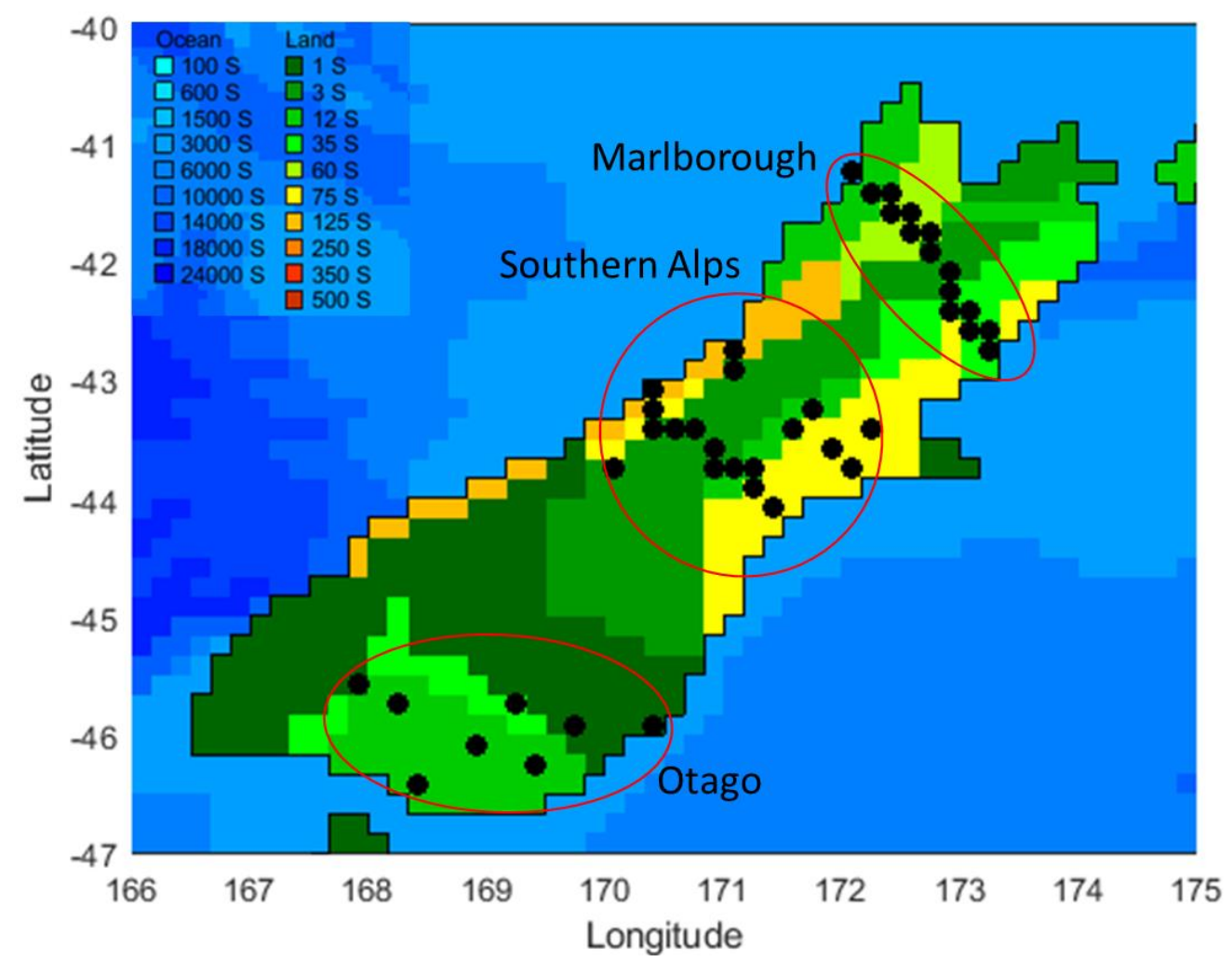

Figure 6. 1: Locations of MT sites superimposed on the conductance map of South Island. Sites are distributed in three main groups. The conductivity structure varies from medium to high resistive regions based upon shades of yellow and green, respectively.

Furthest south, Southland/ Otago (shown by shades of green on the conductance map) is a region mainly of medium to low conductance. There is a total of $8 \mathrm{MT}$ sites with a broad range of periods varying from 2- 83 minutes. Measurements at these sites were made as part of this project and 
are described in the Appendix. The second group of MT sites is in the center of the South Island. The majority of these sites were measured as part of a geophysical transect of the Southern Alps (Wannamaker et al. 2002). Ingham (1995, 1996a, 1996b) also reported MT measurements from a line of sites slightly further to the north. This region has a range of conductance variations from the east to west coasts shown by shades of yellow and green. Relatively high conductance (shown by shades of yellow) exists at both the east and west coast whereas a highly resistive region exists between them. Mostly MT sites are in the period range of 2-30 minutes but a few sites in the high conductive region have longer period range of 2-60 minutes.

The third group of MT measurements is in the north of the island and were reported by Wannamaker et al. (2009) as part of a study of the southern end of the Hikurangi subduction zone in the Marlborough region of the South Island. Although there are significant differences in the geology the conductance variations in this region are similar to those in the central region. The total number of MT sites across Marlborough is 15 and these have a shorter period range of 2-15 minutes. The detailed period range of all MT sites is given in Table 6.1. Since the Earth response function is best represented in the frequency domain, as was done for the North Island study, time domain magnetic field data during the St. Patrick's Day Storm is converted into the frequency domain. Again, the shortest period of MT data equivalent to the interval of the time series of magnetic field is 120 seconds.

\section{Table 6.1}

Details of magnetotelluric data available for the South Island.

\begin{tabular}{ccccc}
\hline $\begin{array}{c}\text { S. } \\
\text { No. }\end{array}$ & MT sites region & $\begin{array}{c}\text { No. of } \\
\text { MT sites }\end{array}$ & $\begin{array}{c}\text { Period range } \\
\text { (minutes) }\end{array}$ & $\begin{array}{c}\text { Discrete } \\
\text { periods }\end{array}$ \\
\hline 1 & Otago/Southland & 6 & $2.098-83.531$ & 9 \\
& & 1 & $2.098-209.82$ & 11 \\
& & 1 & $2.098-332.54$ & 12 \\
2 & \multirow{2}{*}{ Southern Alps } & 15 & $2.730-29.127$ & 8 \\
& & 3 & $1.92-60.86$ & 9 \\
& & 1 & $1.92-19.25$ & 9 \\
3 & Marlborough & 15 & $1.896-15.165$ & 7
\end{tabular}




\subsection{St. Patrick's Day Magnetic Storm (2015) and the South Island measured GIC}

GIC have been computed for the St. Patrick's Day 2015 magnetic storm for the South Island in the same fashion as was done for the North Island. The advantage in the South Island is that there are plenty of GIC measurements to compare with and validate the MT modelling technique. As stated in first chapter the GIC measurements were initially intended to monitor currents in the Earth return path of the high-voltage DC (HVDC) link, therefore the impact of the HVDC earth return currents from the data have been removed prior to use of the data for GIC studies (Mac Manus et al., 2017). The St. Patrick's Day storm has also been discussed in most GIC modelling studies for the South Island (Divett et al., 2018; Mac Manus et al., 2017; Rodger et al., 2017). The principal features of the storm were described previously in the discussion of modelling of North Island GIC in Chapter 4. The horizontal magnetic field components at Eyrewell (EYR) magnetic observatory and the time derivative of these shown are shown again in Figure 6.2 for comparison purposes with measured GIC data. One-minute interpolated GIC data at Islington (ISL-T6H), South Dunedin (SDN-T2H), and Halfway Bush (HWB-T4L) transformers are shown in Figure 6.3, where the letters ' $\mathrm{H}$ ' and ' $\mathrm{L}$ ' along with transformer names show high and low voltage windings of a transformer as described previously. The data span around 1.5 days with a one-minute sampling interval (2048 total data points) starting from 0000 (UT) 17 March 2015.

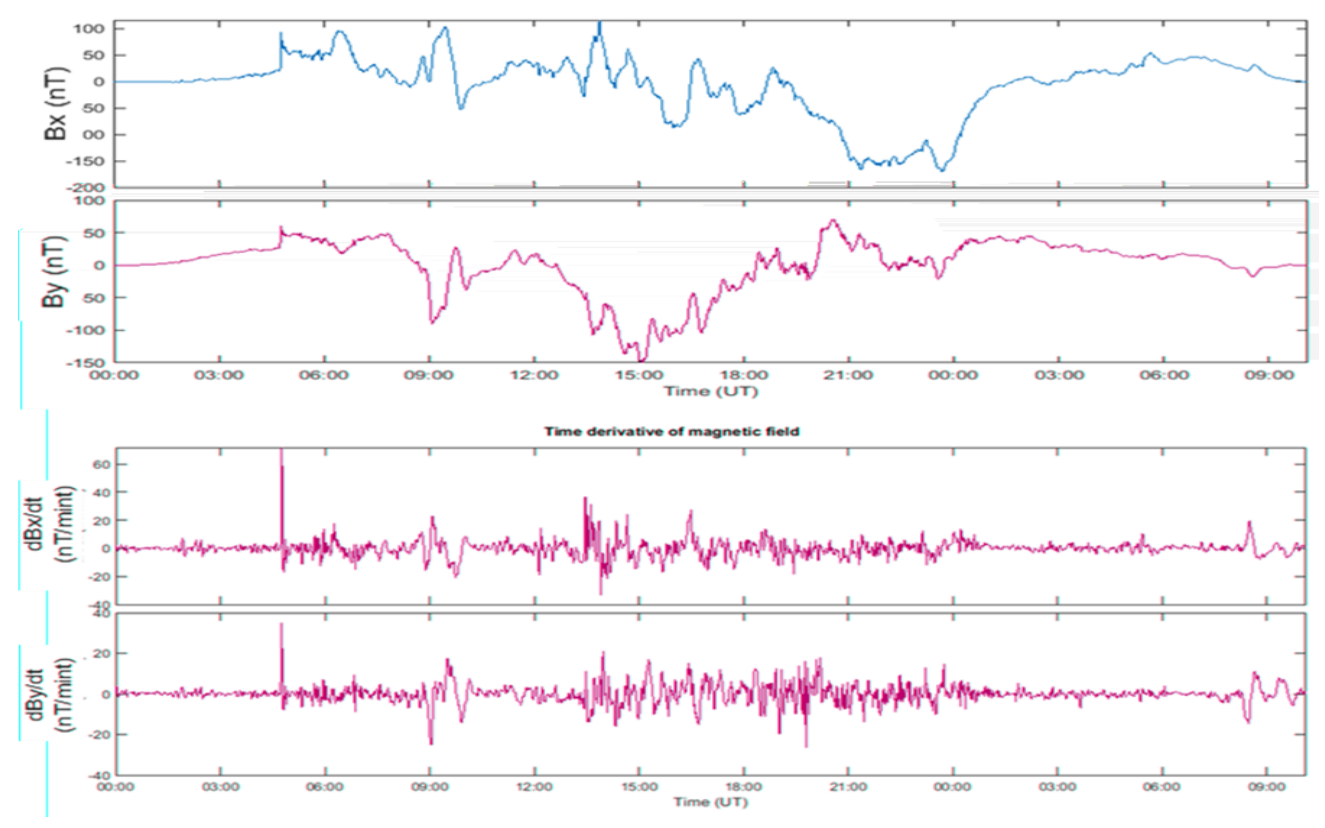

Figure 6. 2: Eyrewell magnetic observatory data of the horizontal field components (top panel) and their time derivative (bottom panel) during the St. Patrick's Day storm, 2015. 

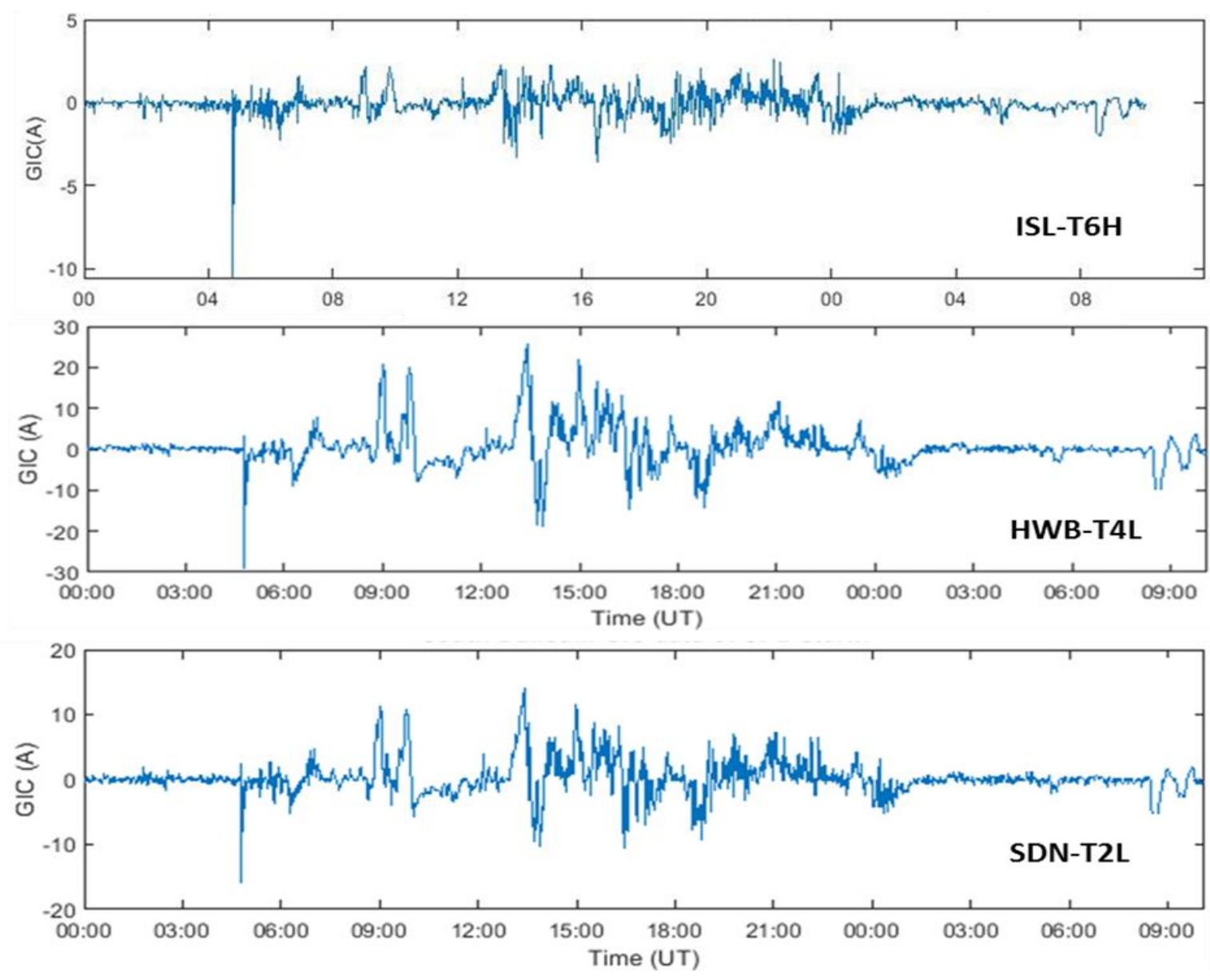

Figure 6. 3: One-minute interpolated GIC measured data at Islington (ISL-T6H), Halfway Bush (HWB-T4L) and South Dunedin (SDN-T2L) transformers, respectively during the St. Patrick's Day storm.

As previously described the St. Patrick's Day magnetic storm of 2015 was caused by a solar storm classified as SH+ICME- sheath followed by non-magnetic cloud ICME (Rawat et. al. 2018; Kozyreva et al., 2018). The interplanetary shock associated with the storm hit the magnetosphere at 0446 UT inducing the Sudden Storm Commencement (SSC) as can be seen in magnetic field data of Figure 6.2. The maximum rate of change of magnetic field observed during the SSC was $100 \mathrm{nT} / \mathrm{mint}$ and $40 \mathrm{nT} / \mathrm{mint}$ for the $\mathrm{Bx}$ and By components of the magnetic field, respectively. At the same time, the largest GIC were observed at transformers at Islington (ISL), South Dunedin (SDN) and Halfway Bush (HWB) reaching values of approximately $-10.5,-18.0$ and $-30.0 \mathrm{~A}$ respectively as shown in Figure 6.3. During the main phase of the storm the South Dunedin transformer (SDN-T2H) and the HWB transformer (HWB-T4L) showed strong low frequency components in contrast to the ISL transformer (ISL-T6H). 


\subsection{Calculation of Geoelectric Field}

The geoelectric fields have been computed using the same procedure as was adopted for the North Island:

- the magnetic field data have been Fast Fourier Transformed (FFT) into the frequency/period domain

- $\quad$ the MT data have been interpolated to all the frequencies given by the FFT by fitting a $3^{\text {rd }}$ order polynomial to the real and imaginary parts of each impedance tensor element

- $\quad$ the standard MT equations relating electric fields to magnetic fields through the impedance tensor have been applied to give the electric field at each frequency

- $\quad$ the resulting frequency domain electric fields have had an inverse-FFT applied to give the electric field time series.
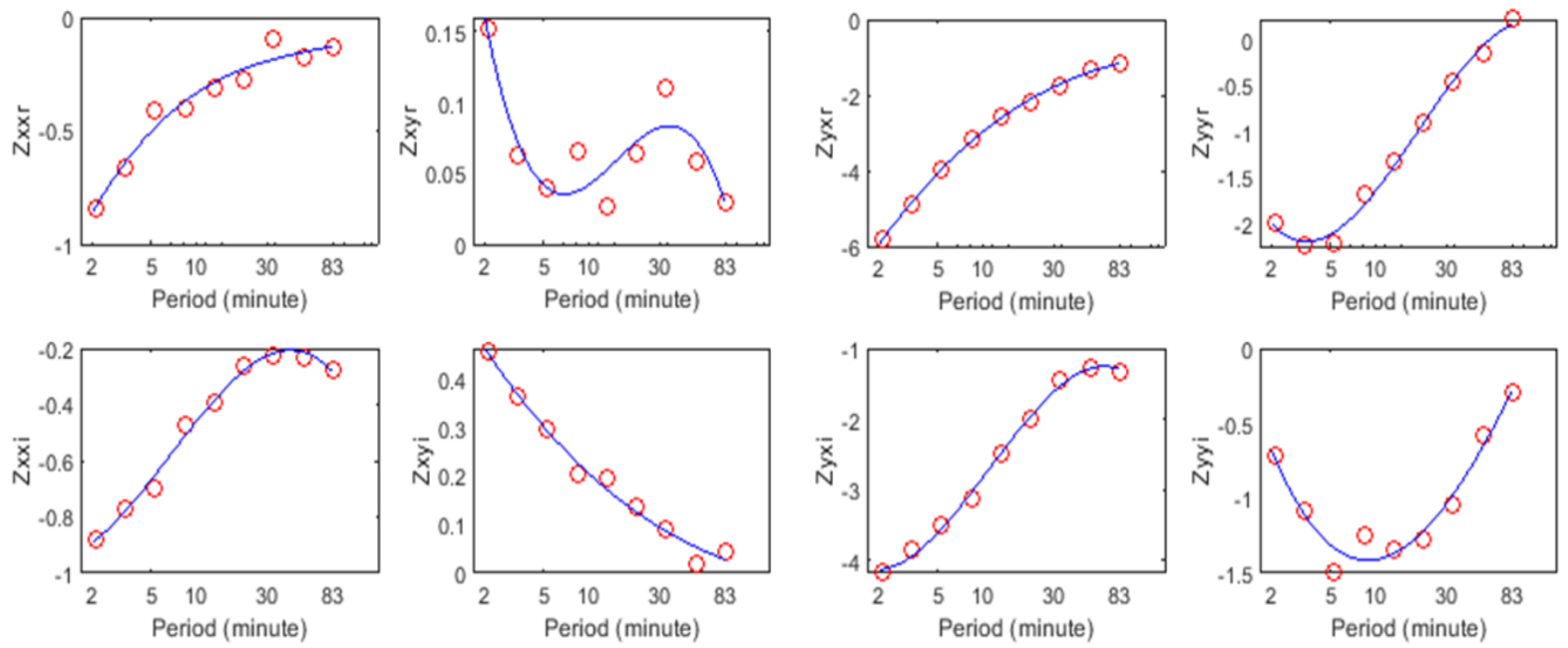

Figure 6. 4: Plot of real and imaginary components of the magnetotelluric response function against period range for an MT site. Red circles show discrete MT periods and blue line is a thirddegree polynomial fit.

Figure 6.4 shows plots of the real and imaginary parts of $Z_{x x}, Z_{x y}, Z_{y x}$, and $Z_{y y}$ at one MT site in Southland $(-45.84,169.81)$. The red circles show the discrete periods of the given MT site where the shortest period is 2 minutes and the longest period is $~ 83$ minutes. These periods have been 
interpolated using a third-degree polynomial (blue curve) so that values of the components of the impedance tensor can be estimated for all periods/frequencies. Following the steps mentioned above the corresponding time domain real-valued geoelectric fields for the MT site have been calculated using the magnetic data from EYR observatory. Figure 6.5 shows the north $\left(\boldsymbol{E}_{\boldsymbol{x}}\right)$ and east $\left(\boldsymbol{E}_{\boldsymbol{y}}\right)$ components of the geoelectric field at the Southland site. The vector sum of both components of the electric field gives the total electric field.

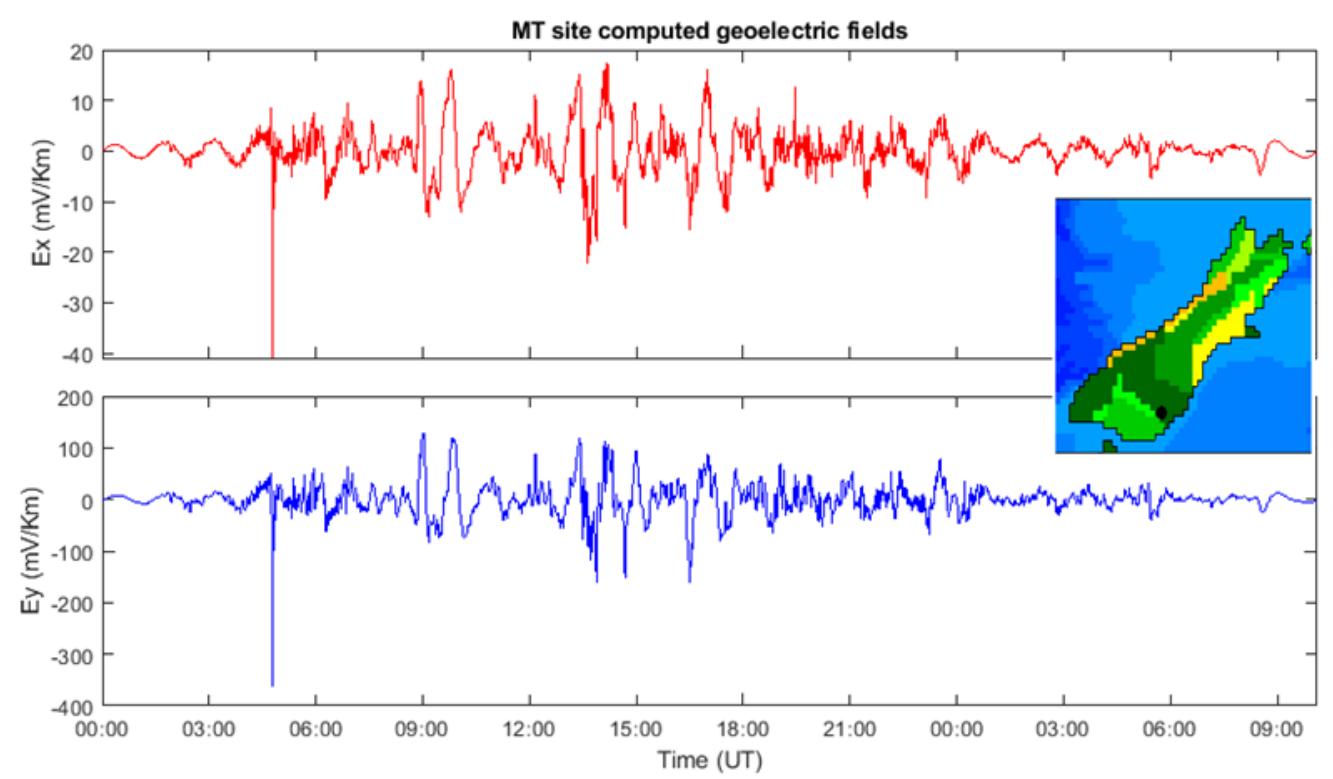

Figure 6. 5: Resulting geoelectric field for an MT site in Southland (shown as dot in the inset picture) depicting an abrupt change in geoelectric field value during the SSC of the solar storm.

\subsection{Spatial Interpolation of Geoelectric Field}

MT sites in the South Island are very sparse compared to MT data in the North Island, and, as indicated above, these exist in three groups with wide gaps between them. The application of MT data for calculating geoelectric fields requires a dense array of measurements (Ingham et al., 2017). To compute the GIC using the power network model horizontal geoelectric fields $\left(\boldsymbol{E}_{\boldsymbol{x}}, \boldsymbol{E}_{\boldsymbol{y}}\right)$ at each cell of the grid are necessary or, at least, where the substations and power lines are located. The South Island thin-sheet grid consists of a total of 616 cells covering the whole island among which only 42 cells can be filled using MT data. Figure 6.6 shows a vector representation of the computed geoelectric fields at MT sites at 0447 UT when the geoelectric fields have maximum value during the SSC. This shows that geoelectric fields vary both in magnitude and direction throughout the South Island. The minimum geoelectric field $(7.22 \mathrm{mV} / \mathrm{km})$ is on the 
Canterbury Plains $(-43.75,172.08)$ near Islington and has a direction to the south, whereas the maximum geoelectric field $(1110.9 \mathrm{mV} / \mathrm{km})$ is in the Nelson region in the north-west of the South Island and has a north-west direction. Since only EYR magnetic data is available from the South Island it has been used to compute the geoelectric fields throughout the South Island. As has been seen, the validity of this assumption was tested by Divett et al., (2020) for the South Island for the St Patrick's Day magnetic storm-2015. The geoelectric fields calculated using the EYR magnetic field, showed only minor differences when compared to those calculated using a spatially varying field.

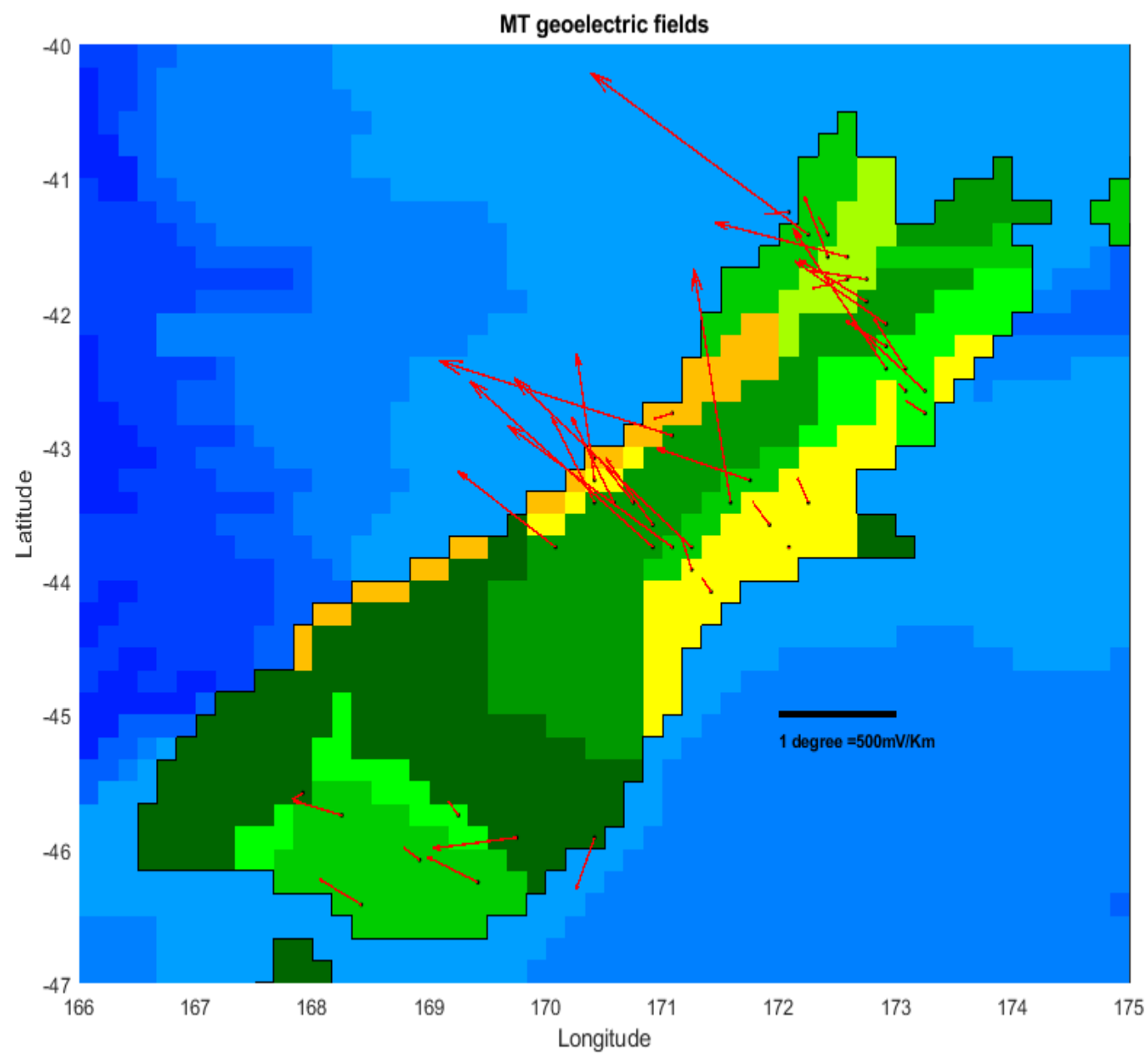

Figure 6. 6: Resultant vector geoelectric fields calculated from the MT data at 0447 UT. The smallest field value is on the east coast whereas the largest field value is in the north-west.

As was discussed in Chapter 4, although different interpolation techniques can be used, the Nearest Neighbor technique works well to fill data gaps the MT sites. Therefore, the Nearest Neighbor technique is again used to interpolate MT geoelectric fields as shown in Figure 6.7. 


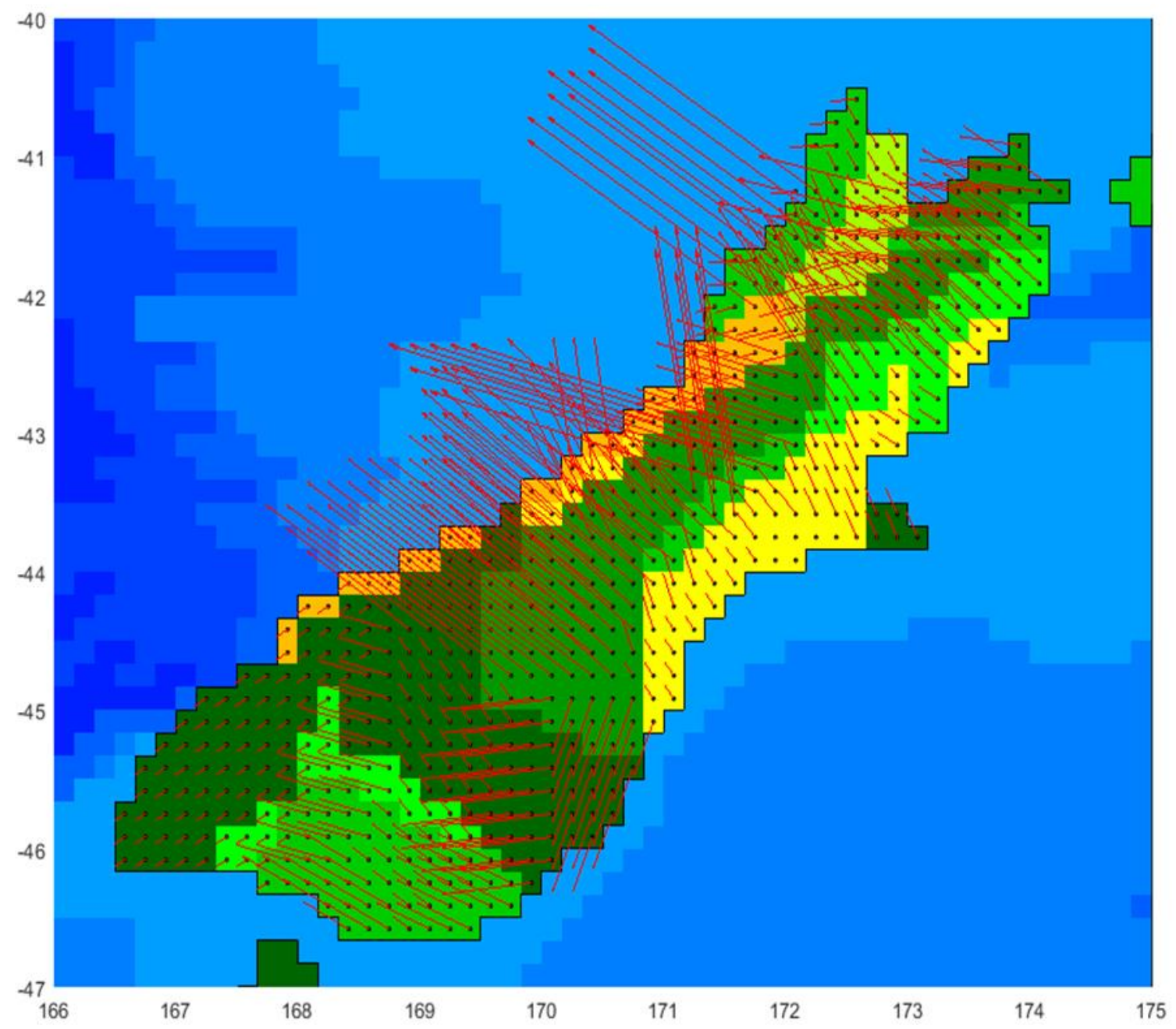

Figure 6. 7: Interpolated geoelectric fields at 0447 UT calculated using the Nearest Neighbor method. NN uses original field values for extrapolation therefore the same fields exist in form of patches. A large area of the South Island is interpolated with very little information and this could affect GIC modelling results.

In the Nearest Neighbor method fields do not vary smoothly and, unlike the North Island where there are many more MT sites, with the small number of sites in the South Island fields repeat over large areas. Therefore, in an attempt to generate smooth fields between the MT sites, another technique was explored. Delaunay Triangulation is an interpolation technique that is often used in applications such as terrain modelling when a shape must be divided into triangles (Berg et al. 2000; Bonner and Schultz, 2017). The Delaunay Triangulation method has been explored and implemented on our data using the Scattered Interpolant tool, available in MATLAB. This technique gives smoother fields between MT sites but at the north and south ends of the South Island results are considered to be unreliable. For example, Figure 6.8 shows that in the 
regions not between MT sites interpolation gives fields for which the direction reverses over short distances. Based upon these results it remains preferable to use the Nearest Neighbor interpolated geoelectric fields for computation of GIC.

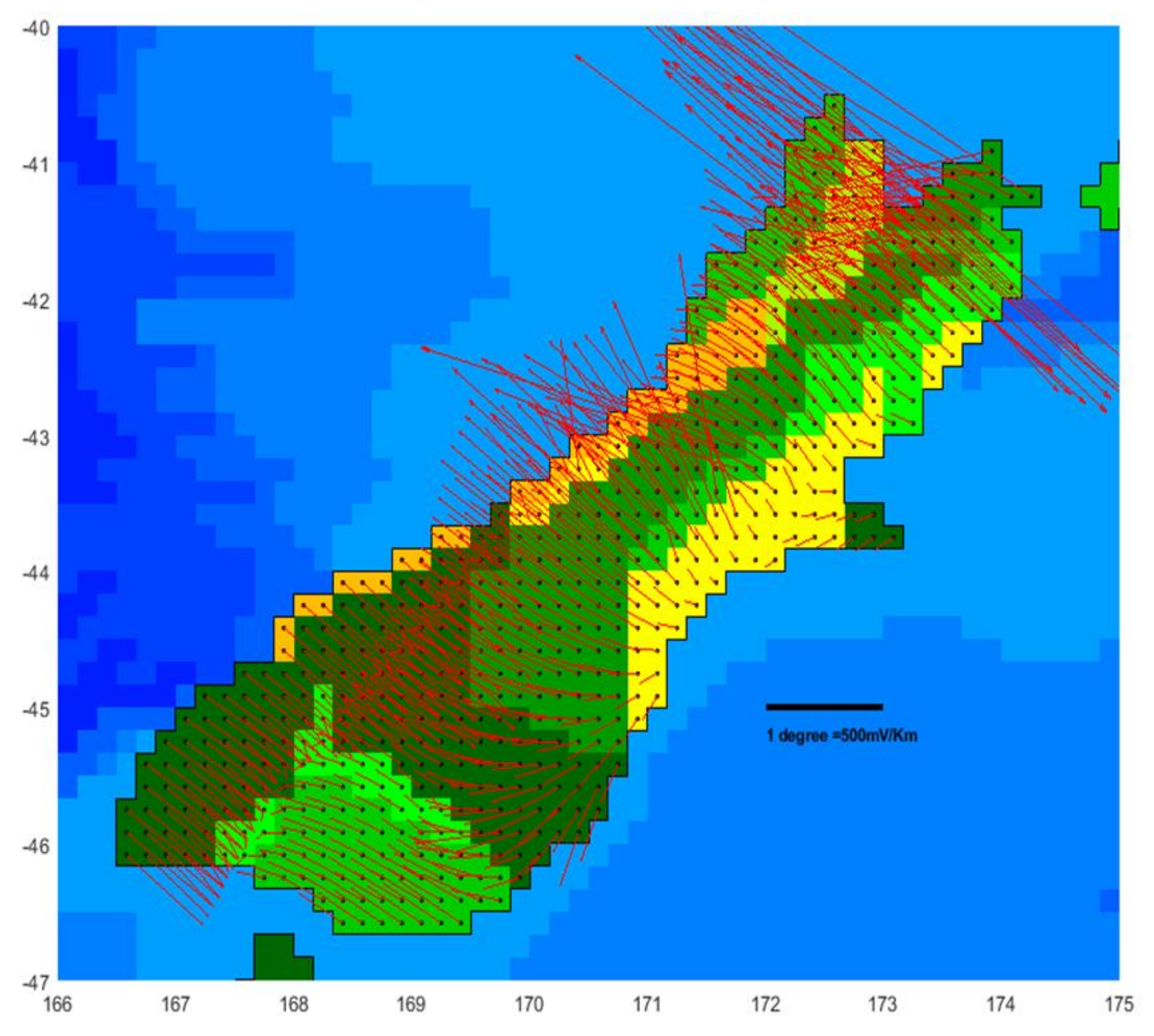

Figure 6. 8: Interpolated geoelectric fields at 0447 UT using the Delaunay triangulation method. This gives smooth fields between MT sites but results are unreliable elsewhere.

\subsection{Uniform Electric Field and Transformer Response}

The local distribution, orientation and magnitudes of geoelectric fields primarily determine the GIC in the power grid system (Pirjola, 2009; Boteler, 2014). As was done for the North Island, it is useful to estimate the network repose to uniform fields in particular directions. Since the network 
model gives information about both transformer GIC and substations GIC (Divett et al., 2018) both levels of GIC are discussed here in response to uniform geoelectric fields. Figures 6.9 to 6.12 show transformer level GIC for the South Island power network when a uniform electric field of $1 \mathrm{~V} / \mathrm{km}$ is assumed in four orientations (east, north, northeast and northwest). The South Dunedin transformer (SDN-T2H) response is significant for all directions of geoelectric fields and reaches a maximum value of $\sim \pm 80 \mathrm{~A}$ when the electric field is in an east and northwest direction as shown in Figures 6.9 and 6.12 respectively. This is the maximum value of GIC experienced by any transformer in the power network. For these two orientations the response of other transformers is not as significant except for Halfway Bush transformer (HWB-T6L) which also experiences significant GIC of \pm 35 A for east and northwest fields. South Dunedin and Halfway Bush are close to each other lying on the east coast at Dunedin and share direct line connections with each other. SDN has only one earthed transformer, and HWB has three earthed transformers and two high side transformer windings. Figure $6.9 \mathrm{~b}$ shows currents/GIC in transformers and power lines for an eastward oriented geoelectric field. Since a substation may have many transformers, the one transformer per substation having maximum GIC value is shown. Figure $6.9 \mathrm{~b}$ shows that line connections to SDN substation have alignments which are generally parallel to east and north-west geoelectric fields and this alignment produces large GIC at SDN. SDN has line connections with HWB and Three Mile Hill (TMH) substations which both carry large positive currents of $\sim 19$ and $\sim 60$ A respectively, giving rise to the large transformer GIC.

North and northeast electric fields produce significantly different GIC to the east and northwest fields. Since these fields are approximately aligned to north-south oriented power lines in the network large currents result in power lines extending in the north-south direction. Figure 6.10a shows that a north directed field produces the largest GIC (of -40 A) in both Tiwai (T1H and T2H) in the south, and the opposite GIC (40 A) at Stoke (T7L) which is in the north of the South Island. Tiwai has two earthed transformers, whereas Stoke has 4 earthed transformers. Figure 6.10b shows line currents and transformer GIC. It shows that northward directed fields produce large currents in power lines having a north-south orientation (green and red colors). For example, the line connection $(220 \mathrm{kV}$ ) of Stoke with Kikiwa (KIK) substation through a south extended line carries the largest current of $\sim 40 \mathrm{~A}$. Kikiwa is further connected to Islington by a southward 
oriented line carrying a large current as shown by the green color line. Similarly, Tiwai (TWI) line connection with Invercargill (INV) extends to the north carrying a current of -19 A. Although, a red line can be seen in the center of the South Island, which connects Cromwell (CML) and Twizel (TWZ), and carries a current of $\sim 65 \mathrm{~A}$, there is no large transformer GIC as there is another line from Clyde (CYD) to CML which carries almost the same amount of current but is in the opposite direction, therefore cancelling each other's effect.

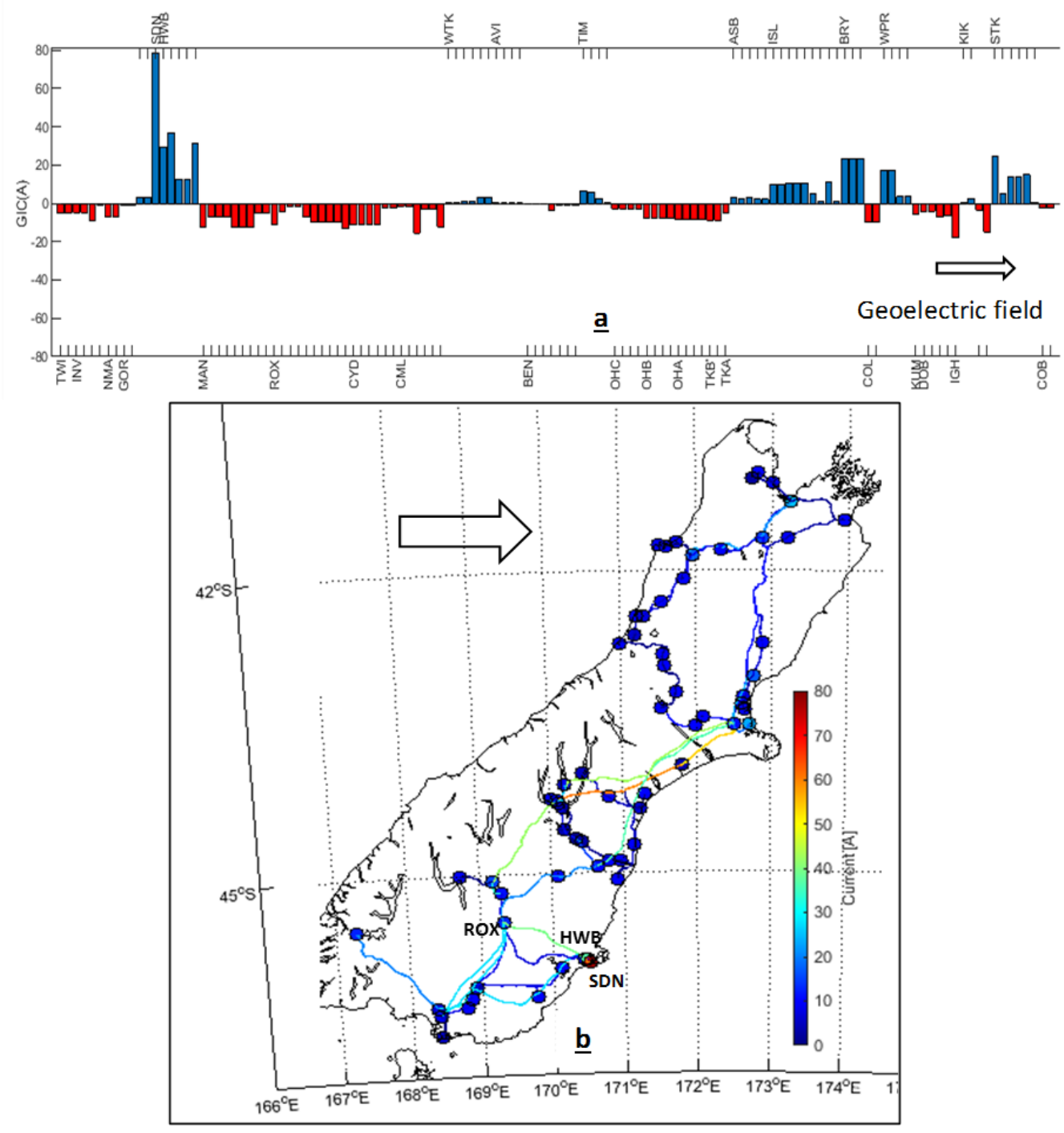

Figure 6. 9: (a) Transformer level GIC produced in the South Island power network by a uniform electric field of $1 \mathrm{~V} / \mathrm{km}$ in east direction. (b) Line currents and the transformer GIC shown on the map where current magnitude is given by the color bar.

The above analysis shows that South Dunedin transformer (SDN-T2H) is the most susceptible in the South Island network for most field directions. Halfway Bush transformer T6L which shares connections with SDN also shows as having significant GIC. Transformers in the north and south 
of the South Island which include Stoke (STK) and Tiwai (TWI), respectively, also show a large GIC response when fields are in north and north-east directions.
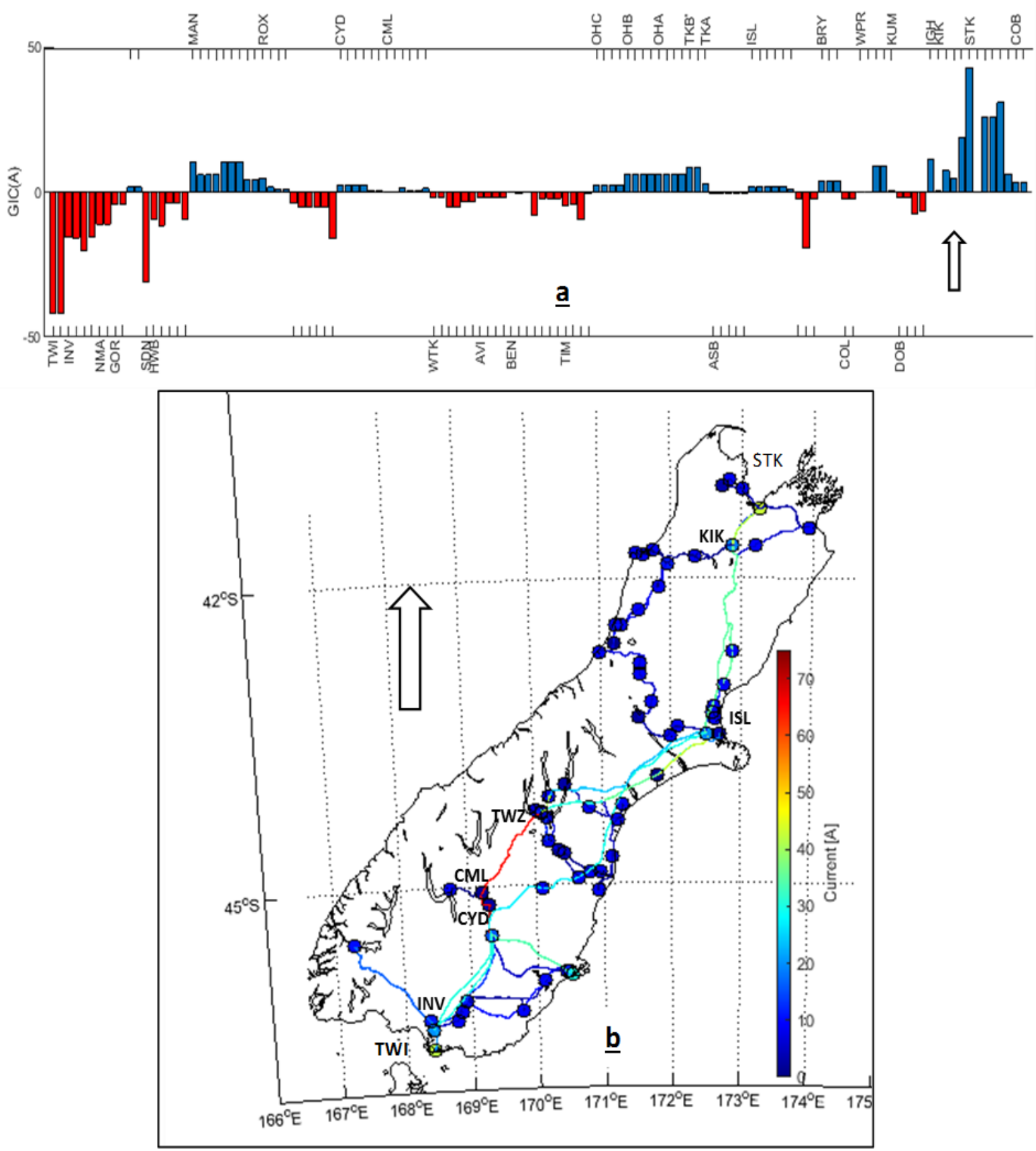

Figure 6. 10: (a) Transformer level GIC produced in the South Island power network by a uniform electric field of $1 \mathrm{~V} / \mathrm{km}$ in north direction. (b) Line currents and the transformer GIC shown on the map where current magnitude is given by the color bar. 


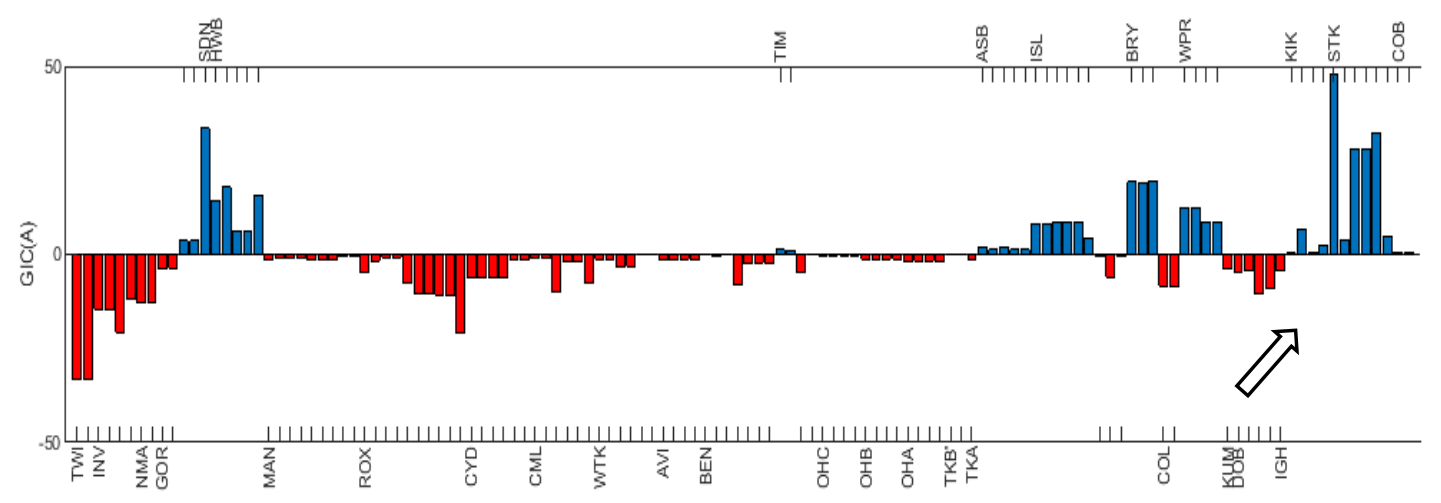

Figure 6. 11: Transformer level GIC produced in the South Island power network by a uniform electric field of $1 \mathrm{~V} / \mathrm{km}$ in northeast direction.

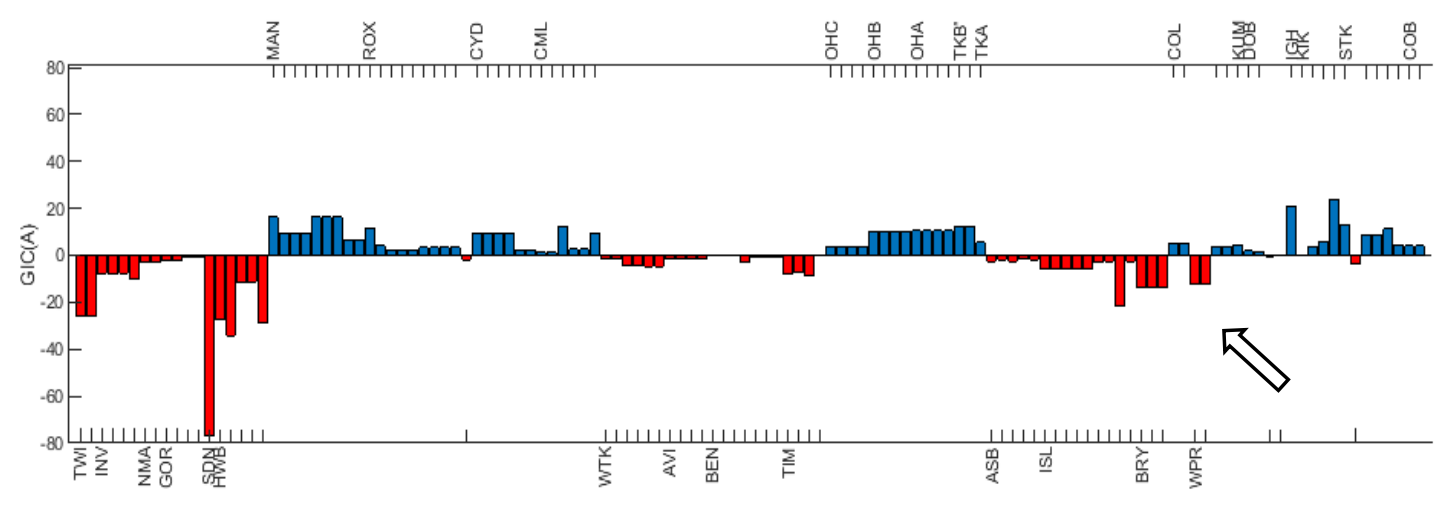

Figure 6. 12: Transformer level GIC produced in the South Island power network by a uniform electric field of $1 \mathrm{~V} / \mathrm{km}$ in northwest direction.

\subsection{Uniform Electric Field and Substation Response}

The substation-level GIC response to uniform geoelectric fields is shown in Figure 6.13. The top panel gives information on GIC in all South Island substations whereas the bottom panel shows the same with information about currents going through power lines. The color bar on the righthand side shows the magnitude of current going through lines and substations. Remembering that a substation GIC is the sum of currents through all earthed transformer nodes at the substation (Divett et al., 2017; 2018) the substation GIC have a quite different pattern to the transformer-level GIC. It is important to remember that although upper phase earthed nodes are 
transformer windings that do experience GIC, they only exchange currents in different voltage levels so effectively are not earthed. Therefore, substation GIC are only summed for all earthed windings of transformers excluding the GIC through high side windings of the transformers within the substation. As discussed earlier a large substation level GIC is not necessarily an indication of harmful current to transformers, but are significant in identifying the pattern and flow of current in different parts of the network. Figure 6.13 shows many substations as having large GIC including the substation which experiences large transformer GIC i.e., SDN. In the section on transformer GIC it was seen that a substation with many transformers usually have smaller transformer level GIC as the total current is divided among all transformers, whereas the substation GIC can be significant due to the large number of earthed transformers. This can be seen for Islington substation which has 9 earthed transformers. South Dunedin has the largest transformer GIC, which is also earthed, and therefore it also has large substation level GIC.

For an eastward electric field (Figure 6.13a) the substations which show large GIC (reaching \pm 100 A) include South Dunedin (SDN), Halfway Bush (HWB), Manapouri (MAN), Islington (ISL), Bromley (BRY) and Stoke (STK). SDN and HWB also have large transformer GIC whereas the other substations have a large number of earthed transformers. Manapouri, Islington, Bromley and Stoke have 9, 9, 3 and 4 earthed transformers, respectively. All these substations have only earthed transformers expect for Stoke, which additionally has two side transformer windings. The same substations also show large GIC for a north-west (Figure 6.13d) directed field with small differences in magnitude. For north and north-east fields TWI, MAN, STK and ROX substations shows significant response as shown in Figure 6.13b and Figure 6.13c, respectively.

The positive and negative GIC at different substations also provide useful information about current paths from one part of the network or substation to other substations. Since the total current is conserved the current going out of substations must equal that going into other substations. For example, in the southern part of the network, for an eastward field current goes out of the HWB and SDN (positive sign) and into the MAN and ROX (negative sign) substations. Whereas in the northern part positive currents from ISL, BRY and STK go into substations in the center of the South Island including Ohau-A and Tekapo. A similar pattern can be seen for other orientations of the geoelectric field. 

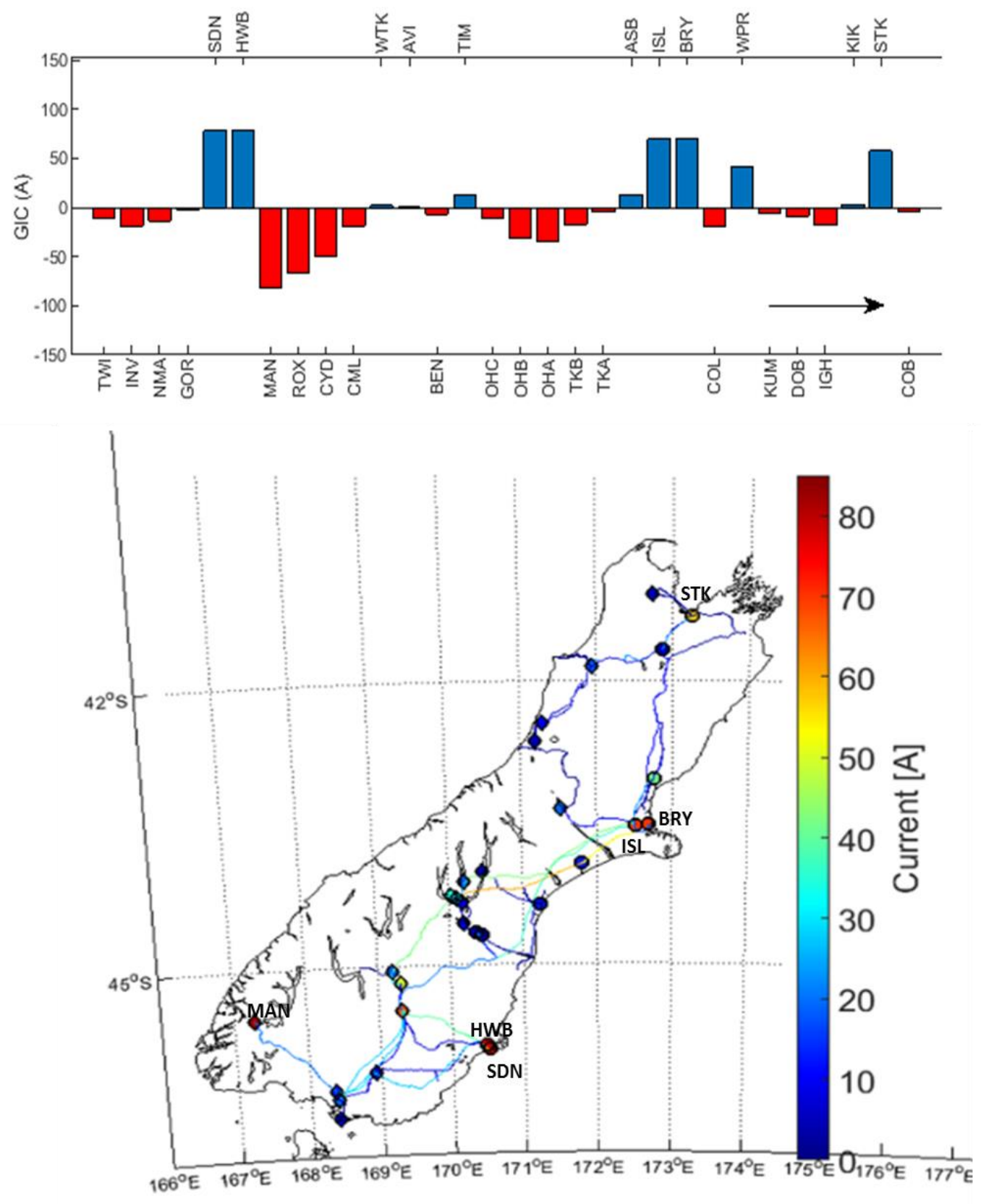

Figure 6. 13a: Substation GIC produced in the South Island power network for uniform electric field of $1 \mathrm{~V} / \mathrm{km}$ in an east direction. The right-hand panel shows the location of substations and currents in power lines. 

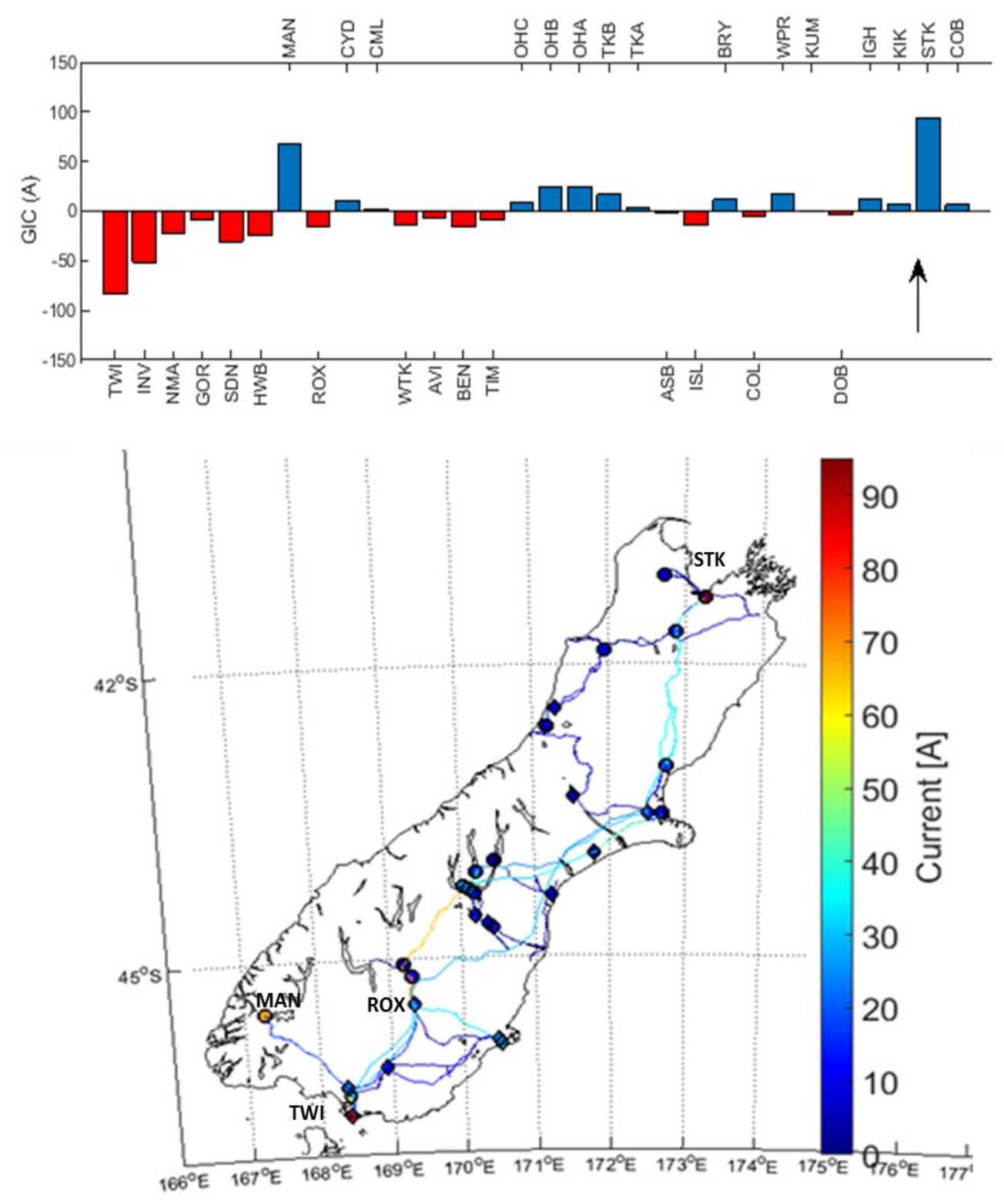

Figure 6.13b: Substation GIC produced in the South Island power network for uniform electric field of $1 \mathrm{~V} / \mathrm{km}$ in a north direction. The right-hand panel shows the location of substations and currents in power lines. 

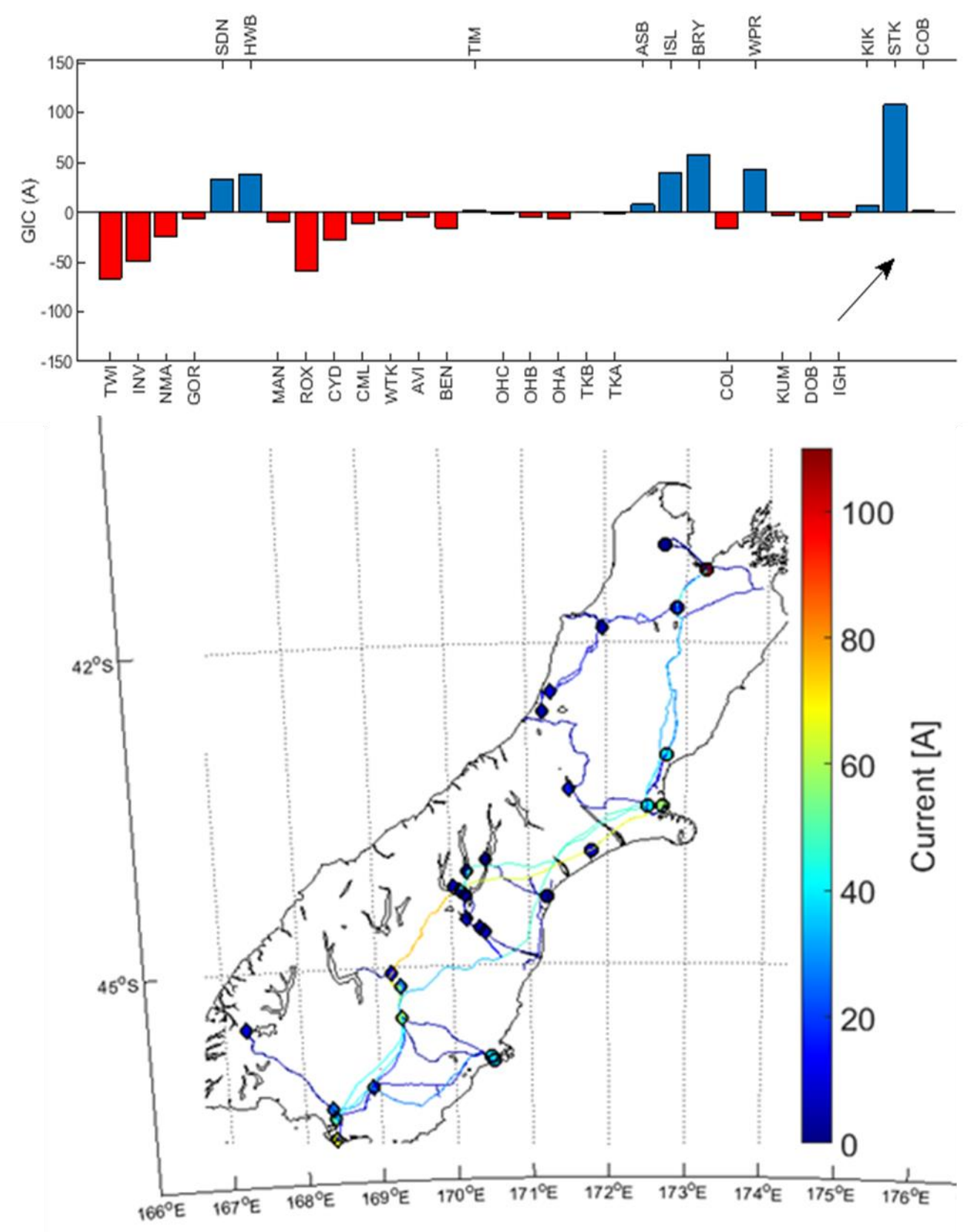

Figure 6.13c: Substation GIC produced in the South Island power network for uniform electric field of $1 \mathrm{~V} / \mathrm{km}$ in a northeast direction. The right-hand panel shows the location of substations and currents in power lines. 


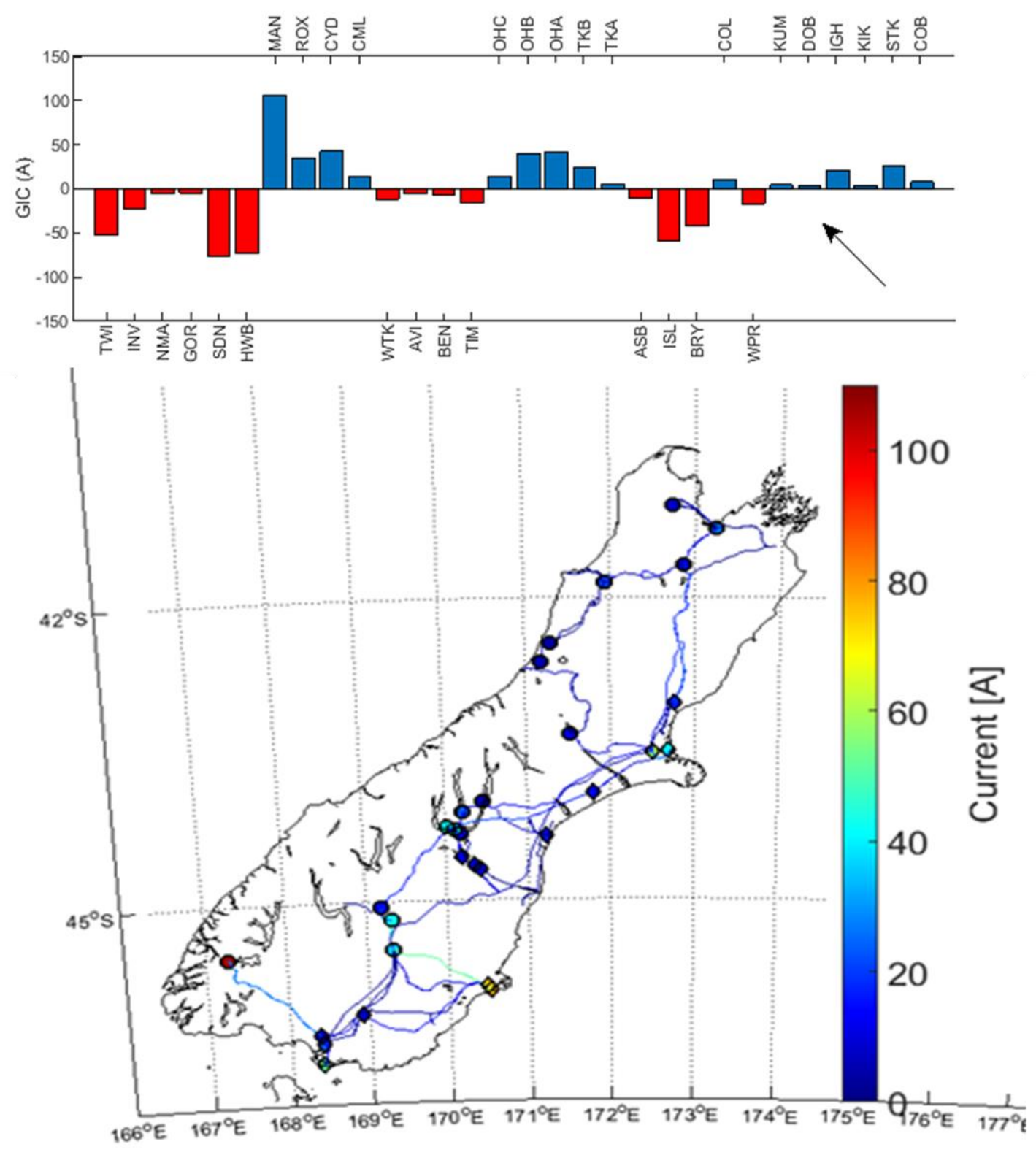

Figure 6.13d: Substation GIC produced in the South Island power network for uniform electric field of $1 \mathrm{~V} / \mathrm{km}$ in a northwest direction. The right-hand panel shows the location of substations and currents in power lines.

\subsection{South Island Network Model and Equivalent Circuit Approach}

For the North Island it has been seen that the equivalent circuit approach can be utilized where the interpolation of MT geoelectric fields is very poor. Following Boteler et. al. (2013) and the application of the technique in the North Island the most appropriate equivalent circuit approach 
is that which uses line voltages connecting the two neighboring networks and line resistance i.e. $V_{t h}=V_{L}$ and $R_{t h}=R_{L}$. The same equivalent circuit technique is also explored for the South Island network where interpolation results are not very reliable. The South Island high-voltage transmission network consists of transmission lines at three different voltage ranges from 50 or $66 \mathrm{kV}, 110 \mathrm{kV}$, and $220 \mathrm{kV}$. The network consists of 64 substations connected by 121 transmission lines (for further details on the South Island network see Chapter 2).

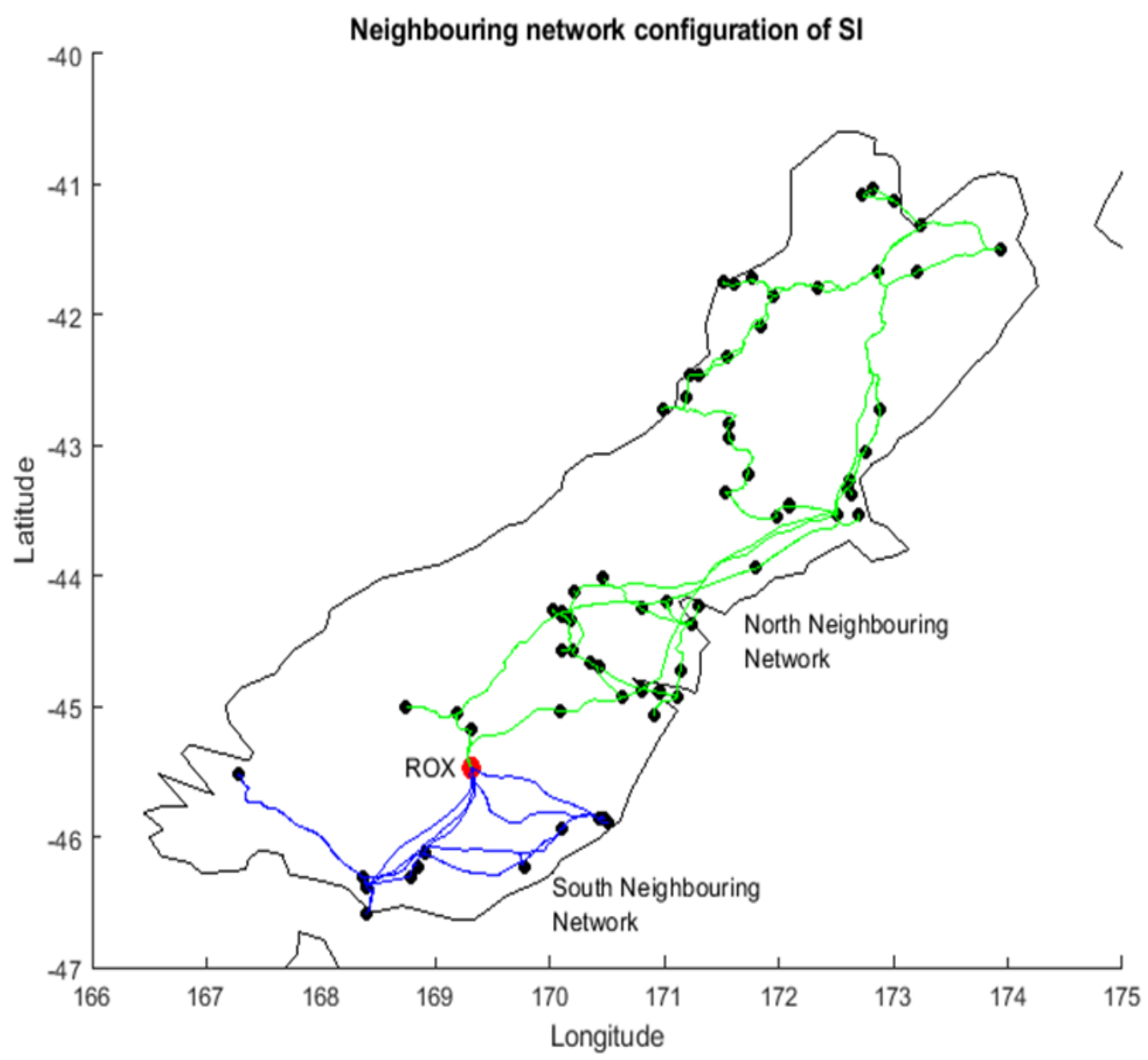

Figure 6. 14: The application of the approach of using two neighboring networks for the South Island power network. All connection points at Roxburgh (ROX) substation shown as a red dot have been earthed to isolate both parts of the networks. The blue lines and green lines now represent two independent networks without any electric connection between them.

To implement the equivalent circuit technique, we have earthed all connections at Roxburgh. That means no GIC flow between the southern and northern parts of the network shown by blue and green lines respectively in Figure 6.14. By earthing all lines GIC will go to ground at Roxburgh and not flow to any of the neighboring parts of the network. 

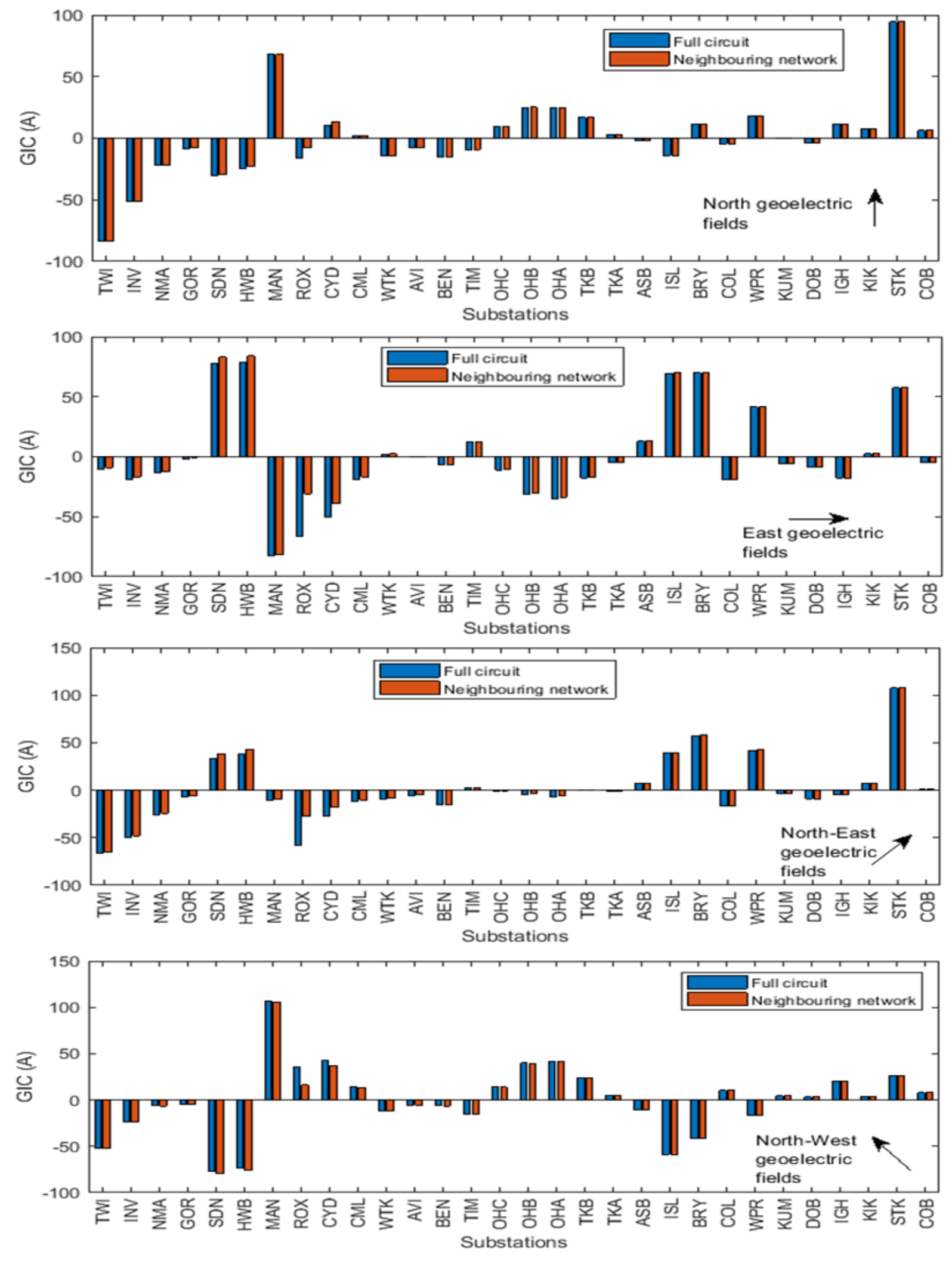

Figure 6. 15: A comparison of GIC calculated using full network (orange) and neighboring network/ equivalent circuit approach (blue) using uniform fields of $1 \mathrm{~V} / \mathrm{km}$ at different orientations as shown in respective panels. 
To check the validity of this approach we have calculated GIC resulting from hypothetical uniform geoelectric fields of different orientations. Firstly, geoelectric fields of $1 \mathrm{~V} / \mathrm{km}$ in different orientations in intervals of 45 degrees are applied to the whole South Island network and the resulting GIC computed. The same fields are then applied with all connections earthed at Roxburgh. The results are shown in Figure 6.15. For all orientations of the electric field using the equivalent circuit approach does not make a significant difference to GIC at any substation except Roxburgh, where the magnitude of GIC are reduced by about 50\%, and at some of the nearby substations such as Clyde (CYD) and Cromwell (CML). This suggests that, even given the large gaps in MT data which require interpolation across them, the full network can be effectively used in this study without having the need for an equivalent circuit network model. It must be noted that although an equivalent circuit is not used, it is only in Otago/Southland (Figure 6.1), and possibly in the central part of the South Island, that the spatial distribution of MT sites is sufficient to give meaningful interpolation of geoelectric fields. Where power lines cross the large gaps between areas with MT sites, the interpolation of electric fields is potentially inaccurate and calculated GIC which depends upon these fields should be treated with caution.

\subsection{GIC Results Using MT Data}

The results of GIC computed using geoelectric fields derived from the MT data are presented in this section. The geoelectric fields given in Figure 6.7, which uses Nearest Neighbor interpolation, have been used to compute GIC for the South Island power network. The bars in Figure 6.16 show GIC through all transformers in all substations at 0447 UT. It shows that South Dunedin transformer (SDN-T2H) experiences the largest GIC of -13.5 A. South Dunedin substation lies on the south-east coast of the South Island and has only one earthed transformer. Kikiwa (KIK-T2H) and Inangahua (IGH-T1H) which lie in the north of the Island experience large GIC of $9.5 \mathrm{~A}$. Halfway Bush transformer (HWB-T6L) which lies close to South Dunedin also experiences significant GIC of value -7.5 A. In the middle of the South Island, transformers at Tekapo (TKB$\mathrm{T} 3 \mathrm{H}$ ) and Timaru (TIM-T5H) experience medium level GIC in the range of $\pm 5 \mathrm{~A}$, respectively. Many transformers at other substations experience medium-level GIC including Islington and Ohau $(\mathrm{OHA})$. As previously described, current is designated as positive (out of substation) or negative (into a substation) (Divett et al., 2017). 


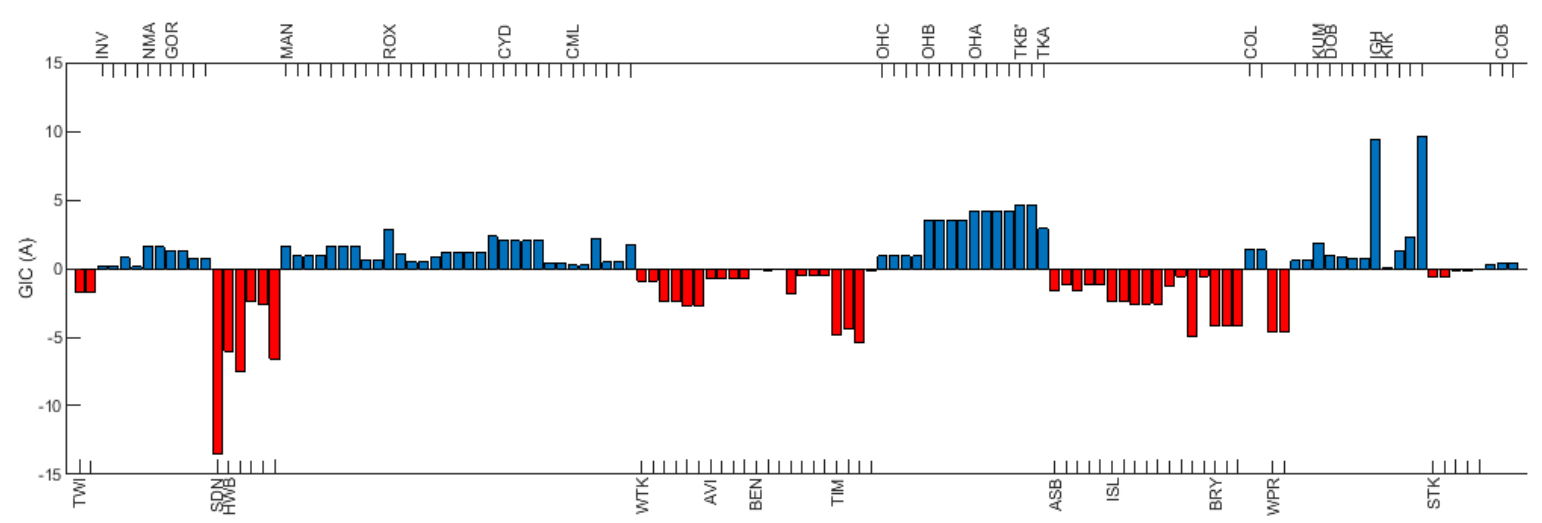

Figure 6. 16: Transformer level GIC in the South Island power network during the Sudden Storm Commencement (SSC) using MT computed geoelectric fields.

The currents through transformer windings is mainly controlled by currents going through power lines, whereas the magnitude of the line current depends upon line integral of the geoelectric fields along the path of the power line. The line currents for two substations SDN and ISL at 0447 UT are given in Table 6.2. South Dunedin high voltage node (SDN220) is connected to Halfway Bush (HWB220) and Three Mile Hill (TMH220) and both lines carry currents towards SDN, as shown in the table by a negative sign, giving rise to a large GIC at SDN. The line connecting SDN to Three Mile Hill (TMH) carries a large amount of current (-10.57 A). For Islington, the line connection from Islington high voltage node (ISL220) to Tekapo B (TKB220) carries the largest current of $-7.46 \mathrm{~A}$, as the line passes through strong geoelectric fields in the middle of the South Island. The line connecting Islington with Livingstone (LIV) has only a small amount of current 4.38 A. Livingstone lies close to the east coast south of Islington, and, while the line length is almost the same as that to Tekapo, the geoelectric fields are normal to the line and thus result in only a small amount of current. The information on line currents is important in the context that the MT data are very sparse and where interpolation results are not very reliable it is possible to gain some indication of GIC from the line currents.

One-minute discrete geoelectric fields for a total of 2048 data points covering the St. Patrick's Day storm have been used to compute time domain GIC for the South Island using the network model. Figure 6.17 shows time domain GIC computed for the whole St. Patrick's Day storm for four transformers that have been identified as experiencing large GIC during the storm. These are 
1. South Dunedin (SDN-T2H)- $(-45.89,170.50)$

2. Inangahua (IGH-T1H)- $(-41.85,171.95)$

3. Kikiwa (KIK-T2H)- $(-41.67,172.87)$

4. Halfway Bush (HWB-T6L)- $(-45.85,170.47)$

\section{Table 6.2}

Line connection and currents for different substations experiencing large transformer level GIC.

\begin{tabular}{|c|c|c|c|c|c|c|}
\hline Node1 & Node2 & $\begin{array}{c}\text { No. of } \\
\text { lines }\end{array}$ & $\begin{array}{c}\text { Network } \\
\text { Resistance }\end{array}$ & Voltage & $\begin{array}{c}\text { Length } \\
(\mathrm{Km})\end{array}$ & $\begin{array}{c}\text { GIC } \\
0447 \text { UT }\end{array}$ \\
\hline \multicolumn{7}{|c|}{ South Dunedin (SDN) } \\
\hline SDN220 & HWB220 & 1 & 0.26 & 220 & 12 & -2.92 \\
\hline SDN220 & TMH220 & 1 & 0.18 & 220 & 8 & -10.57 \\
\hline \multicolumn{7}{|c|}{ Islington (ISL) } \\
\hline ISL066 & KBT_e & 2 & 1.85 & 66 & 37 & -2.47 \\
\hline ISL220 & KIK220 & 1 & 4.90 & 220 & 230 & -3.10 \\
\hline ISL220 & LIV220 & 1 & 3.16 & 220 & 233 & -4.38 \\
\hline ISL066 & SBK066 & 2 & 1.47 & 66 & 25 & -0.56 \\
\hline ISL220 & TKB220 & 1 & 3.01 & 220 & 213 & -7.46 \\
\hline ISL220 & WTT_e & 2 & 1.12 & 220 & 61 & 0.43 \\
\hline ISL220 & ASB220 & 1 & 0.85 & 220 & 79 & -5.64 \\
\hline ISL220 & BRY220 & 1 & 0.30 & 220 & 28 & 5.90 \\
\hline
\end{tabular}

Figure 6.17 shows that during the SSC current is negative for both transformers SDN-T2H and HWB-T6L, whereas it is positive for transformers IGH-T1H and KIK-T2H which lie further north. This is, according to conservation of charge, showing that the current flows from the north to south of the power network. South Dunedin and Halfway Bush transformers show the existence of low frequency components in the model GIC data. This results from the fact that MT data for this region cover an extended period range from 2-83 minutes compared to other parts of the South Island. For example, Inangahua and Kikiwa have only high frequency components in their GIC as all MT sites in the Marlborough region have a shorter period range of 2-15 minutes. Usually, strong geoelectric fields are responsible for large GIC in transformers, but it also depends upon the number of transformers in a substation as if there are more transformers within a substation current will split between them. For example, South Dunedin and Inangahua show 
large GIC because they have only one earthed transformer. Kikiwa has two earthed transformers whereas Halfway Bush and Islington transformers show smaller GIC because they have three and six earthed transformers, respectively. The strong geoelectric fields in the Marlborough region also significantly enhance the GIC for IGH and KIK substations.
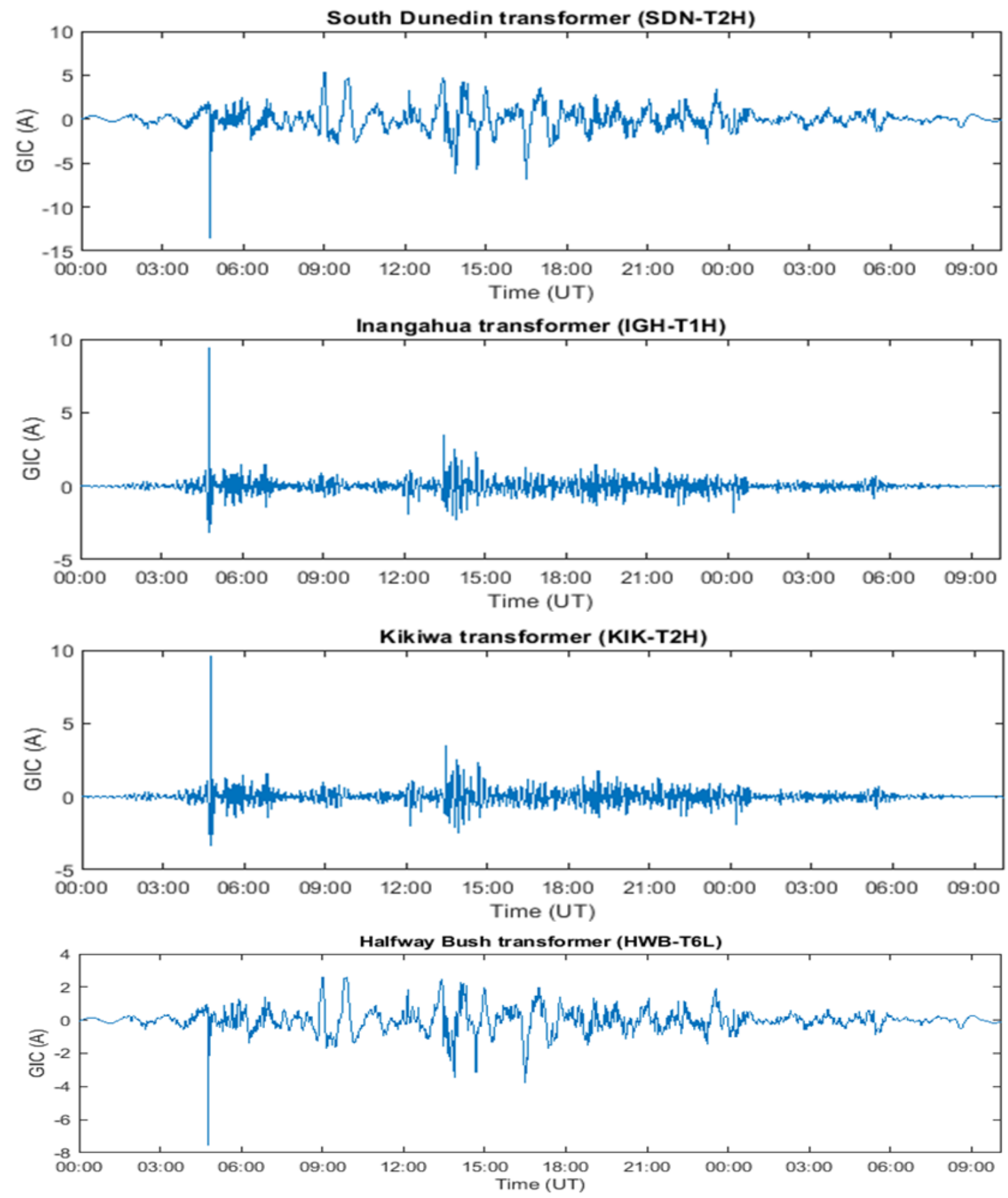

Figure 6. 17: MT GIC modelling results showing transformers (as shown on top of each panel) experiencing the largest GIC during St. Patrick's Day storm. 

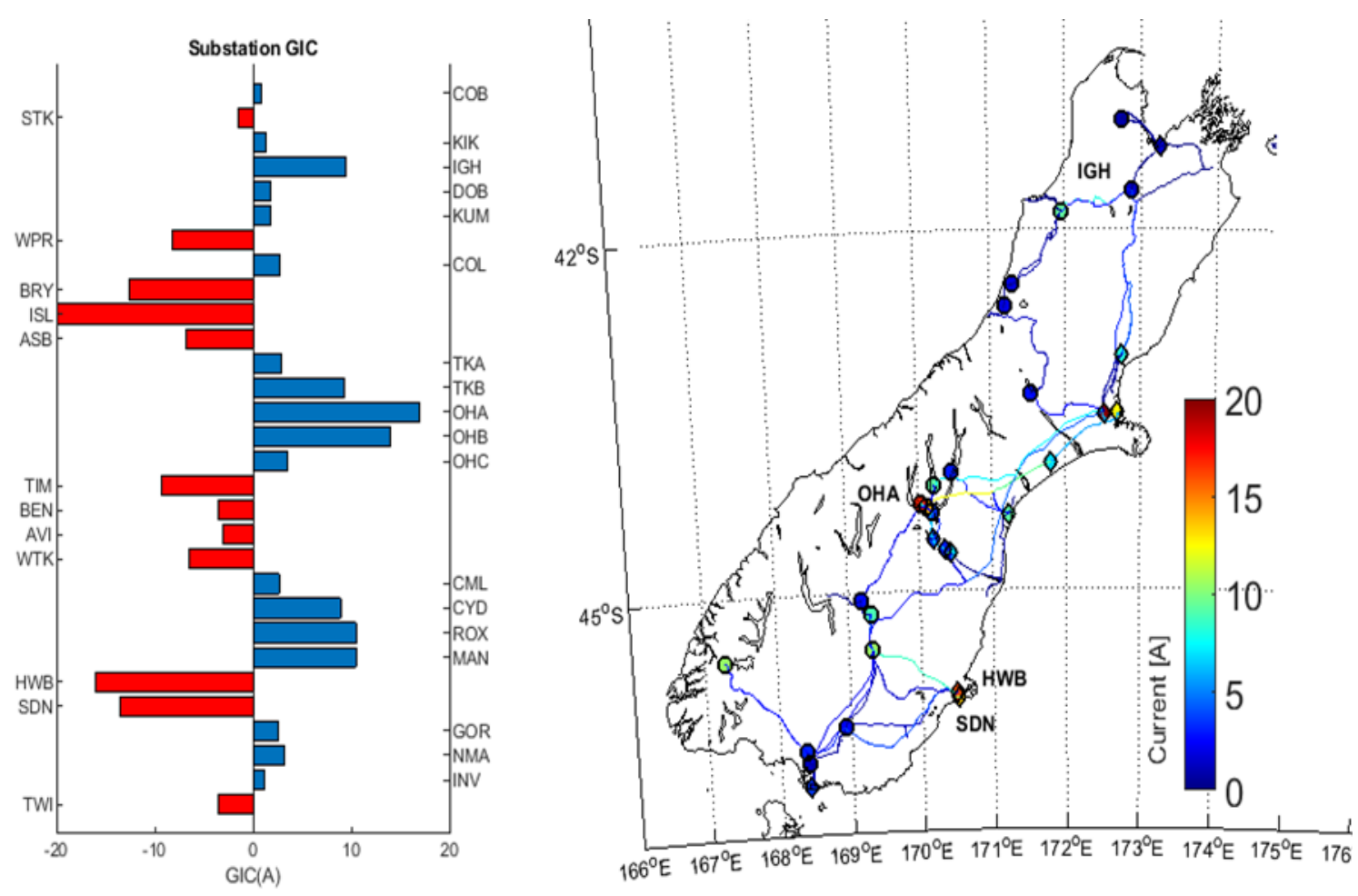

Figure 6. 18: Substation-level GIC magnitude during Sudden Storm Commencement (SSC) at 0447 UT (left hand panel) and location of substations and power line currents (right hand panel).

In the above, GIC through individual transformers have been discussed which is important in that observed GIC are measured at transformer level. The network model also computes the substation-level GIC which is the sum of GIC through all earthed transformers (Divett et al., 2018). Figure 6.18 shows substation level GIC at 0447 UT. The left-hand panel shows the magnitude of GIC and the right-hand panel shows locations of substations and current intensity, both at substations and in power lines, in different colors. Substations which experience the largest GIC include:

1. Islington (ISL)- $(-43.53,172.51)$

2. Ohau A (OHA)- $(-44.26,170.02)$

3. Halfway Bush (HWB)- $(-45.85,170.47)$

4. South Dunedin (SDN)- $(-45.89,170.50)$ 
Of these Islington has 9, Ohau A has 4 and Halfway Bush has 3 earthed transformers. It can also be seen that Kikiwa (KIK) substation shows large transformer GIC but small substation GIC, the reason being that although a large current flows through the high side transformer winding that connects two high voltage transmission lines, the current does not flow to the Earth. Based on the MT modelling results Figure 6.19 shows the locations experiencing large GIC during the St. Patrick's Day storm at transformer level (in red) and substation level GIC (in blue).
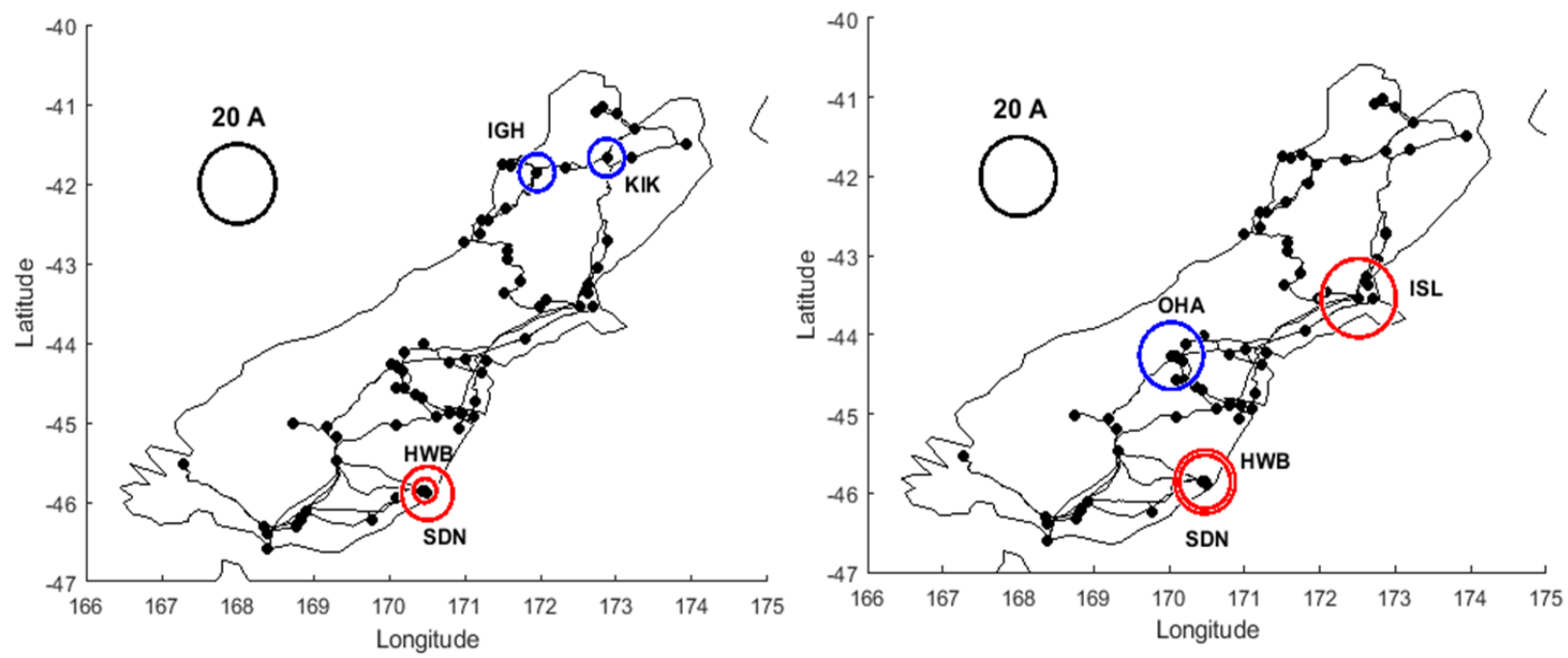

Figure 6. 19: Left panel shows transformers experiencing large GIC and right panel shows large substations level GIC for St. Patrick's Day storm.

\subsection{Comparison with Measured GIC}

As mentioned earlier, in the South Island GIC are measured on many transformers. Although data are not measured at a uniform sampling interval a one-minute average or interpolated GIC has been used for comparison with the model results. Islington, South Dunedin and Halfway Bush GIC have been compared with the MT model GIC. Although measured GIC data at Manapouri and Invercargill are also available these have not been compared as the interpolation of geoelectric fields is not particularly good in the region around these substations.

Figure 6.20a shows one-minute discrete time domain measured and model GIC for Islington transformer (ISL-T6H). Since all of the MT sites in the region around Islington have a shorter period range the measured GIC have been filtered in the period range of 2-30 minutes for 
comparison. The model GIC has small magnitude $(-2.56 \mathrm{~A})$ in comparison with the observed data $(-9.62$ A) during the SSC. However, the MT model effectively reproduces variations in the observed data, for example as shown in the right-hand panel of Figure 6.20b. The correlation coefficient $(r)$ between measured and model values has a value of 0.86 and the performance parameter $(P)$ is 0.22 (Ingham et al., 2017; Torta et al., 2014; 2017).

The comparison of model and measured GIC shows a large difference in magnitude between measured and model GIC. This has also been observed by Divett et al. $(2018,2020)$ using thinsheet geoelectric fields. Raw GIC measurements are also available for other transformers (T3H, $\mathrm{T7H}$ and $\mathrm{T9H}$ ) at the Islington substation during the SSC. The measured raw GIC values corresponding to these transformers are $-6.4,-5.7$ and $-2.2 \mathrm{~A}$, respectively. As these transformers (ISLT3H, ISLT7H, and ISLT9H) have the same network configuration as ISL-T6H GIC values should not differ from ISL-T6H (Divett et al., 2018).
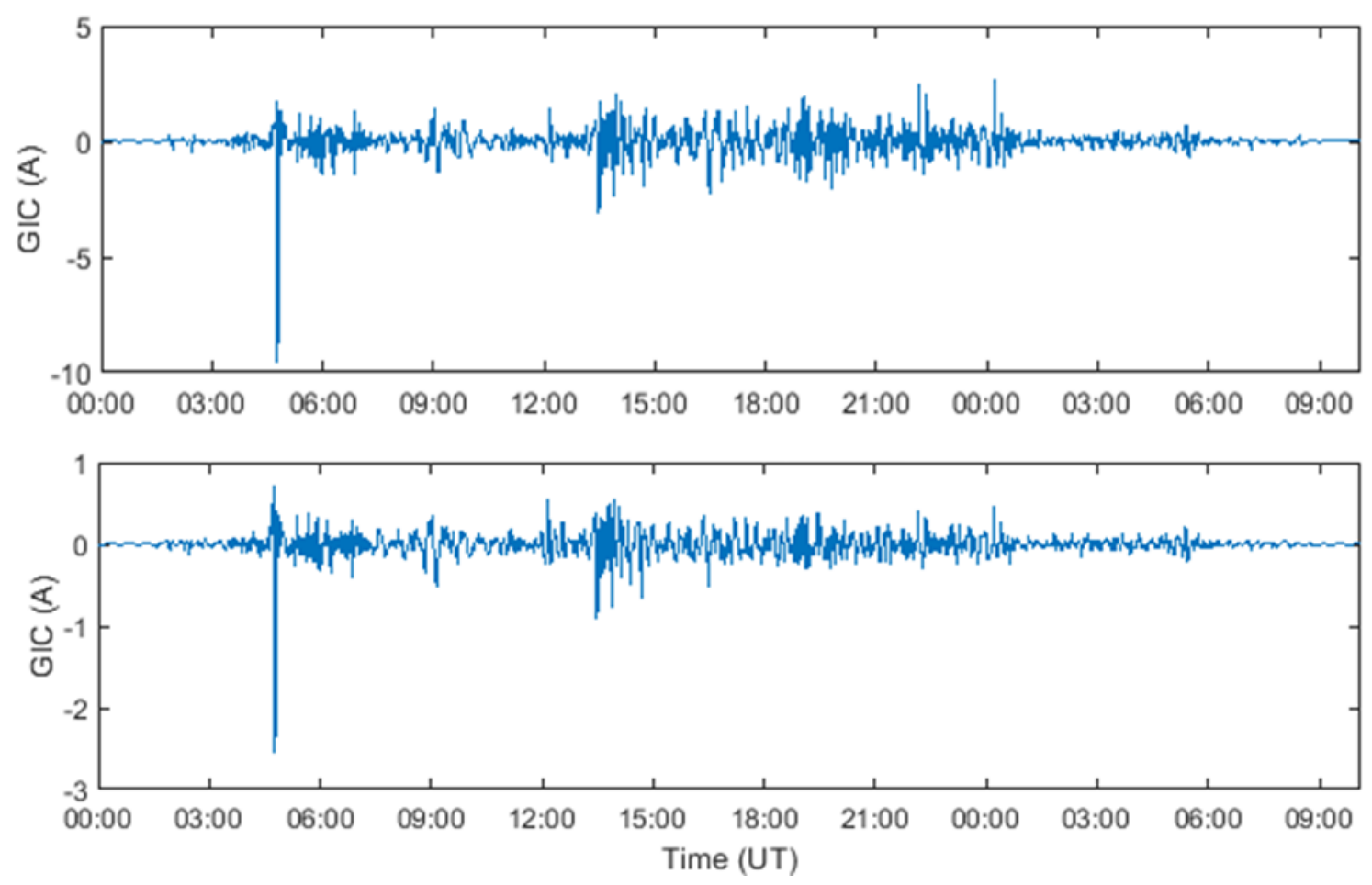

Figure 6. 20a: Top: observed GIC in Islington transform ISL-T6H in the period range of 2-30 minutes. Bottom: MT model GIC. The variations are quite similar both in observed and model GIC but the magnitude of model GIC is almost four times smaller than observed GIC. 

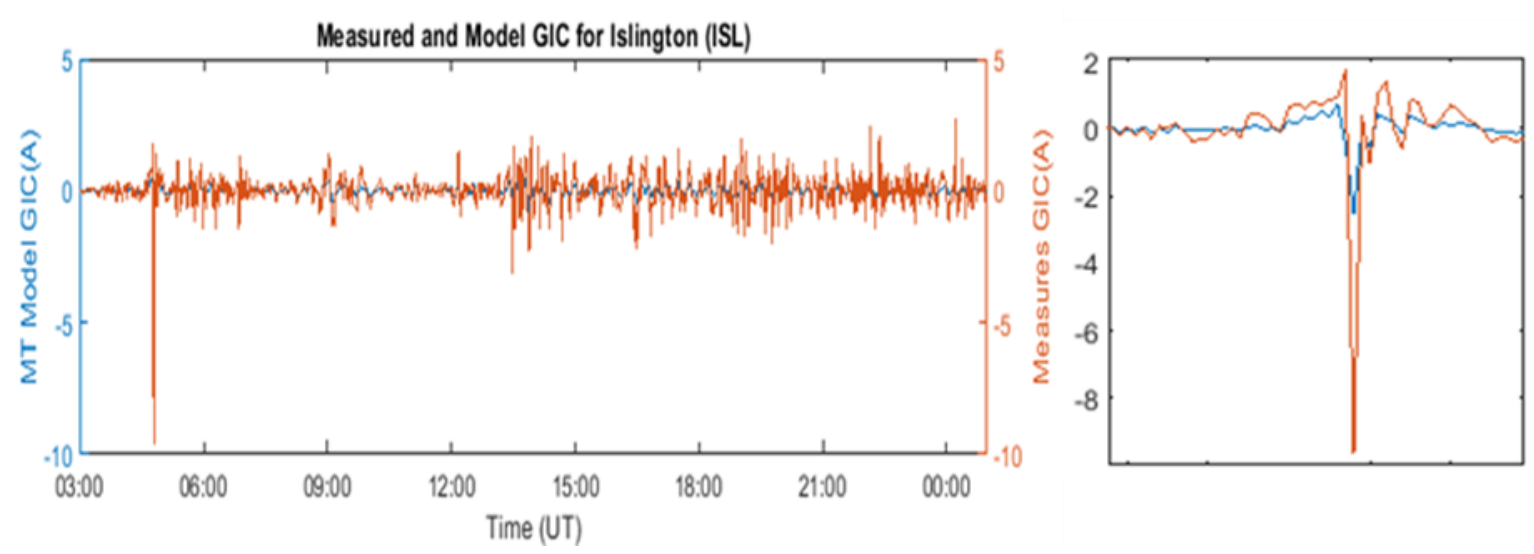

Figure 6.20b: A comparison of model and observed GIC in Islington transform ISL-T6H showing correlation coefficient of 0.86. Although the model GIC is very small in magnitude but it effectively reproduces GIC time variations during both the SSC (on right) and the rest of the storm.

It is interesting to speculate about this small magnitude of model GIC in (ISL-T6H) in terms of interpolated geoelectric fields that extend to the south from the ISL substations. The part of the network which extends both north and south from Islington has MT sites only in a low resistive region. In fact, as was indicated previously, the lowest geoelectric field value given by the MT data lies near Islington. Interpolation extends these small geoelectric fields in a way which may be unrealistic, giving a small value of model GIC. How this affects ISL-T6H can perhaps be understood with the help of Figure 6.21, showing line connections of the Islington high voltage node (ISL220) with six other substations (KIK, TKB, LIV, ASB, BRY and WTT). The red lines show currents towards ISL (negative sign) which contribute to GIC in the ISL transformers whereas blue lines show currents away from ISL (positive sign) which act to reduce the GIC (see Table 6.2 for values). The line connecting Islington with Tekapo-B carries the largest current of $-7.46 \mathrm{~A}$, as the line passes through strong geoelectric fields in the middle of the South Island. The line connection with Livingstone (LIV) has only small amount of current (-4.38 A). Livingstone lies on the east coast and, while the line length is almost the same as that to Tekapo, the interpolated geoelectric fields are both very small, and nearly perpendicular to the line, therefore giving a small amount of current. Clearly, a better interpolation technique based on more MT data in this region may help to better model GIC for Islington. 


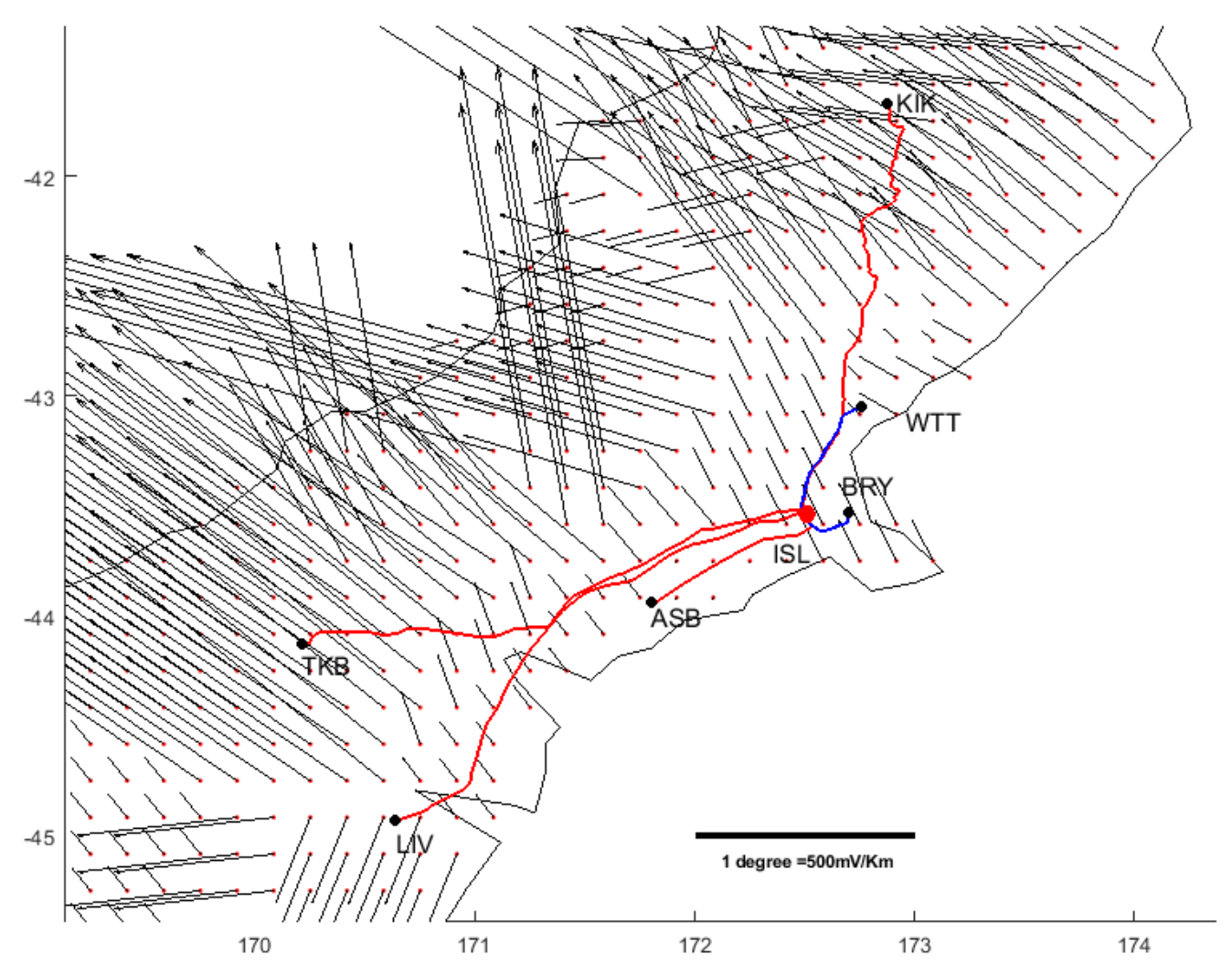

Figure 6. 21: How interpolation affects the GIC results at Islington transformer (ISL-T6H).

South Dunedin transformer (SDN-T2H) GIC data during the St. Patrick's Day storm is also available for comparison with the model result. Figure 6.22a shows individual plots of observed and model GIC for South Dunedin transformer (SDN-T2H). Measured data show the largest GIC during SSC and large GIC variations for the rest of the magnetic storm. The model GIC also has maximum value during the SSC but the GIC is not as large as the measured data for the rest of storm. The magnitude of measured GIC at SSC is $-14.36 \mathrm{~A}$ and the model value is $-13.48 \mathrm{~A}$. The plots of both model and measured GIC together are given in Figure 6.22b. The model data for SDN show a better agreement with the measured data compared to Islington. The correlation coefficient is 0.79 and performance parameter is 0.37 . The measured data have been filtered in the period range of 2-85 minutes as most of MT data in Otago/Southland exist in this longer period range (see Table 6.1 for reference). 

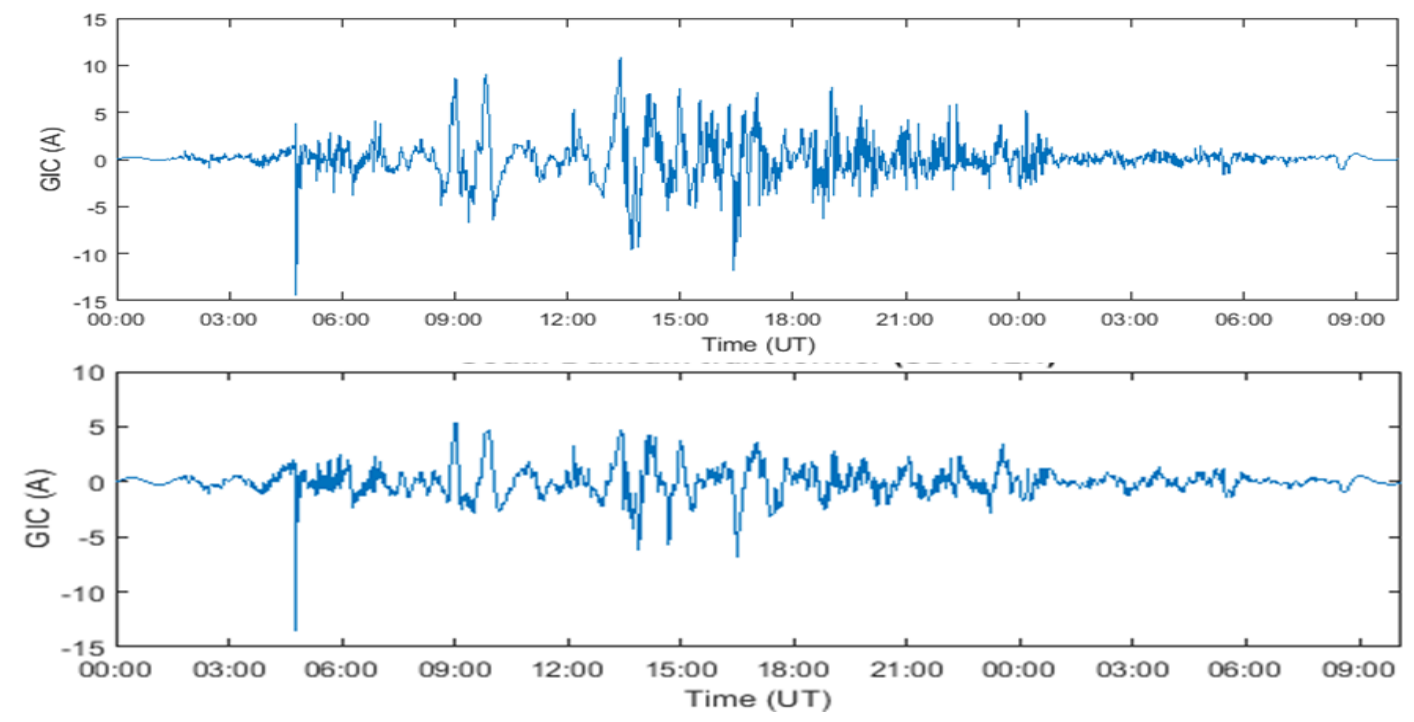

Figure 6. 22a: Top: observed GIC in South Dunedin transform SDN-T2H in the period range of 283 minutes; bottom MT model GIC. The model GIC match very well both in magnitude and time variations.
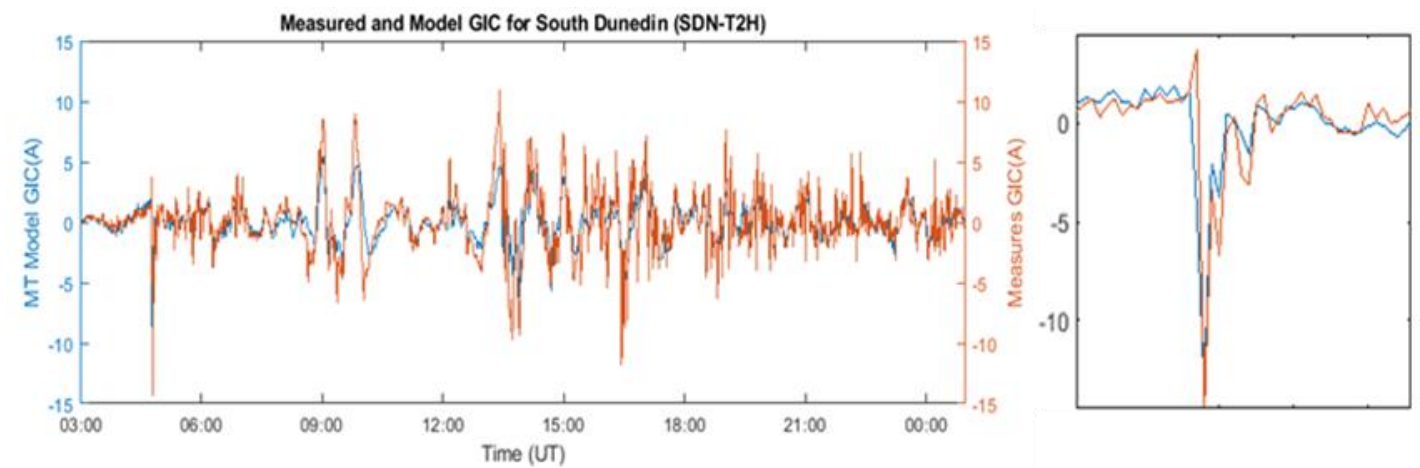

Figure 6.22b: A comparison of model and observed GIC in South Dunedin transform SDN-T2H showing correlation coefficient of 0.79 . The model GIC effectively reproduces variations during both the SSC (on right) and the rest of storm.

Figure 6.23 shows observed and model GIC in Halfway Bush transformer (HWB-T4L) in the period range of 2-85 minutes. Of all the South Island transformers, Halfway Bush shows the largest observed GIC with the peak value of -25.85 A during SSC. The observed GIC also have very strong low frequency components reaching a value of $20 \mathrm{~A}$. In contrast the model GIC has very small magnitude with the peak GIC value during SSC only $-6.01 \mathrm{~A}$, a fraction of the measured GIC. The value of $r$ is 0.83 and $P=0.13$. Understanding the reason why the modelled GIC is lower than the measured GIC at HWBT4 is more speculative. Halfway Bush substation has 3 earthed 
transformers and 2 high side transformer windings, but measured GIC is only available for one earthed transformer (T4L). South Dunedin and Halfway Bush are very close to each other so this discrepancy in results cannot be attributed to the size of geoelectric fields. Divett et al. (2020) discussed a possible cause attributed to the effect of geoelectric fields along the path of line connections to HWB substation. However, high spatial resolution MT data would be required to test this.
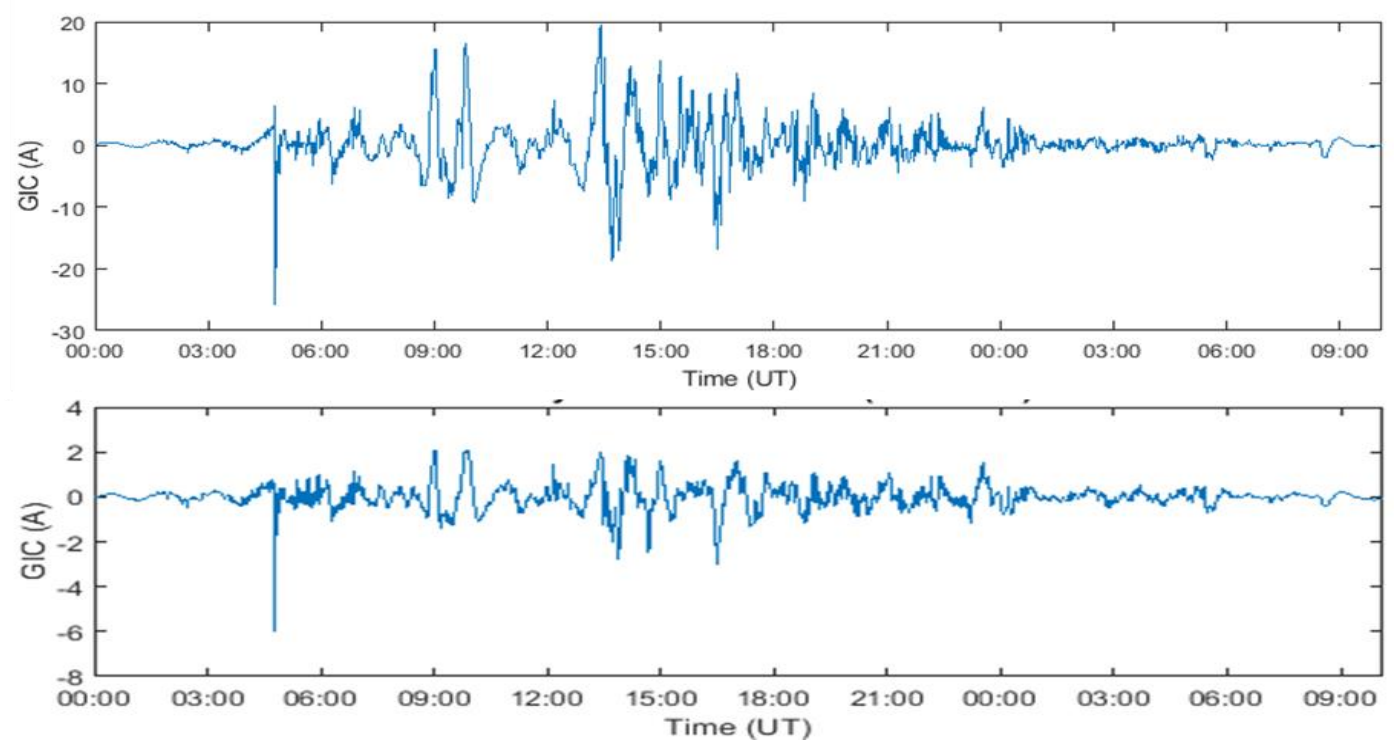

Figure 6. 23a: Top: observed GIC in Halfway Bush transform HWB-T4L in the period range of 283 minutes; bottom MT model GIC. The model GIC has very small magnitude compared to the observed GIC.

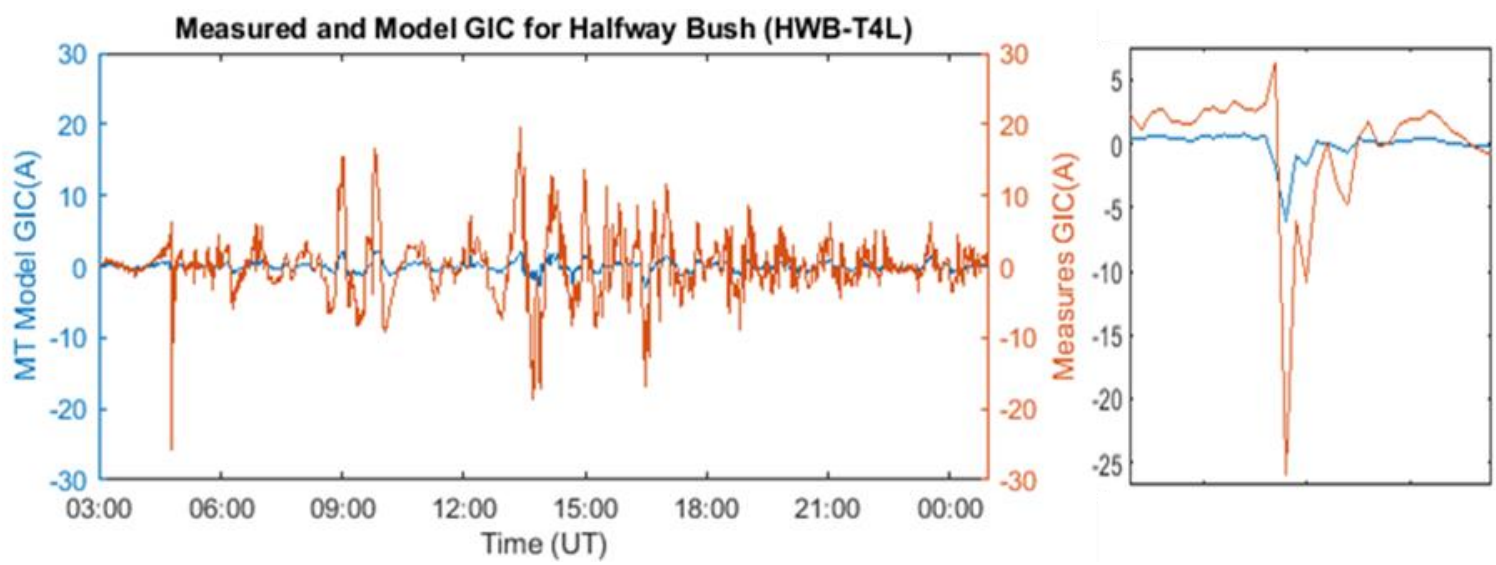

Figure 6.23b: A comparison of model and observed GIC in Halfway Bush transform HWB-T4L showing correlation coefficient of 0.83 . The model GIC during the SSC is very small compared to measured data. 


\subsection{Geoelectric Fields Using Thin-sheet Conductance Model}

In the preceding section we have used geoelectric fields derived from MT data for GIC modelling, and results have been compared with observed data at different transformers. In this section geoelectric fields derived from the thin-sheet conductance model have been used to model GIC. Figure 6.24 shows the geoelectric fields derived from the thin-sheet conductance model at 0447 UT. The scale of geoelectric fields is $500 \mathrm{mV} / \mathrm{km}$ which is the same as was used for MT geoelectric fields shown in Figure 6.7. The thin-sheet geoelectric vectors do not show much variation in direction and magnitude and remain almost uniform over different conductance regions. This pattern is quite different to that for the MT geoelectric fields and the magnitude of geoelectric fields is also smaller than MT geoelectric fields.

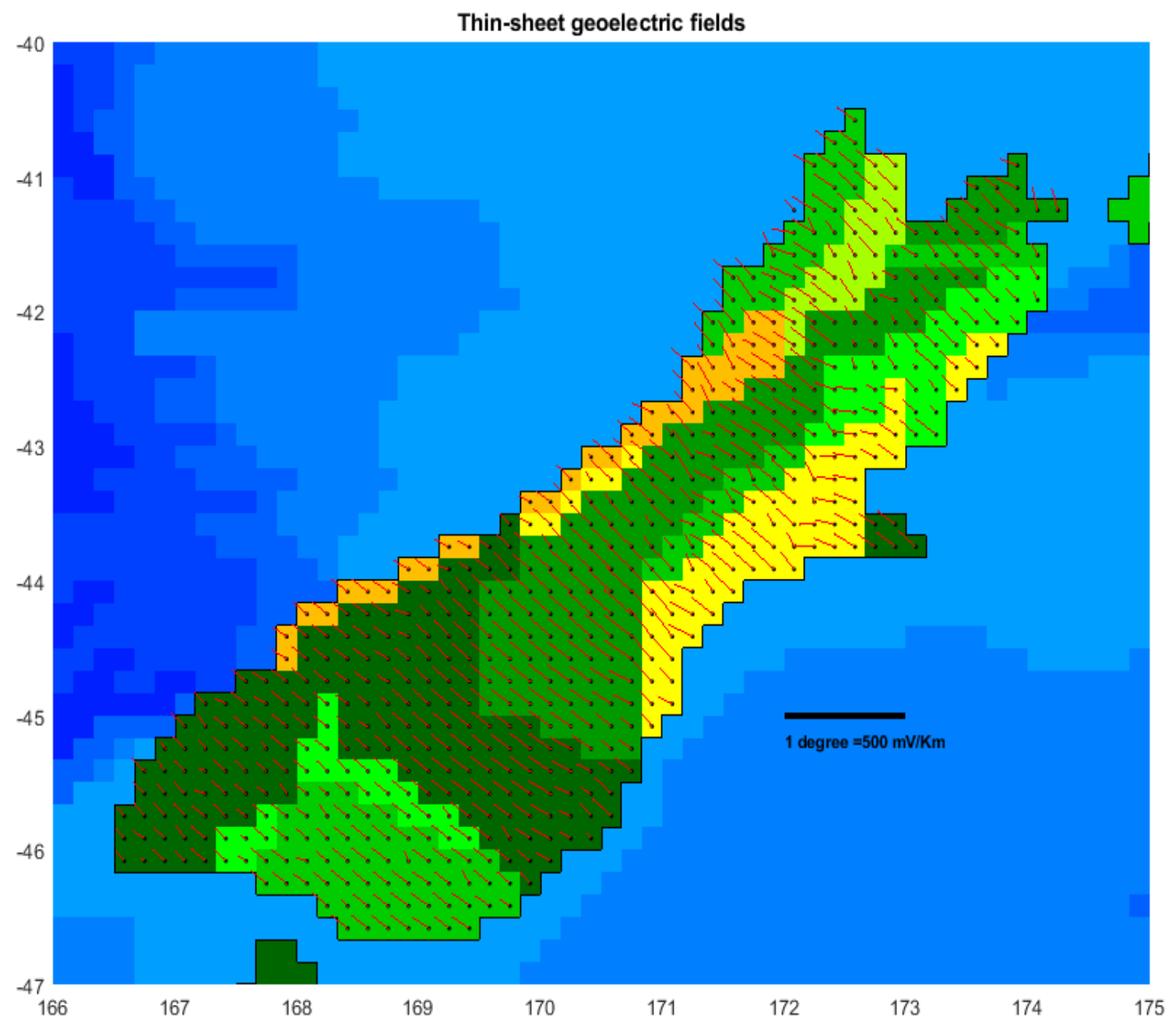

Figure 6. 24: TS electric fields computed during the Sudden Storm Commencement at 0447 UT. 


\subsubsection{Model GIC using Thin-sheet Geoelectric fields}

The network model has been used to compute GIC for all transformers to estimate the response of transformers during the SSC. Figure 6.25 shows a bar graph representation of computed GIC at 0447 UT. It is evident that the pattern is almost the same as for the MT model except that the magnitudes of GIC are smaller. For example, the highest GIC is seen in South Dunedin transformer (SDN-T2H) of value -6.20 A whereas this was -13.48 A for the MT model. Other transformers which show large GIC include HWB-T6L (-2.86 A), IGH-T1H (2.05 A) and KIK-T2H (2.38 A) which are the same transformers as for the MT model predictions. However, there is a difference in the ratio of GIC magnitude at IGH-T1H and $\mathrm{KIK}-\mathrm{T} 2 \mathrm{H}$ in relation to HWB-T6L. For example, the thinsheet GIC at HWB-T6H is almost three times the values at IGH-T1H and KIK-T2H, whereas in the MT model GIC in IGH-T1H and KIK-T2H are more than half the value of HWB-T6L. This can be associated to strong geoelectric fields in the north of the South Island calculated using the MT data.

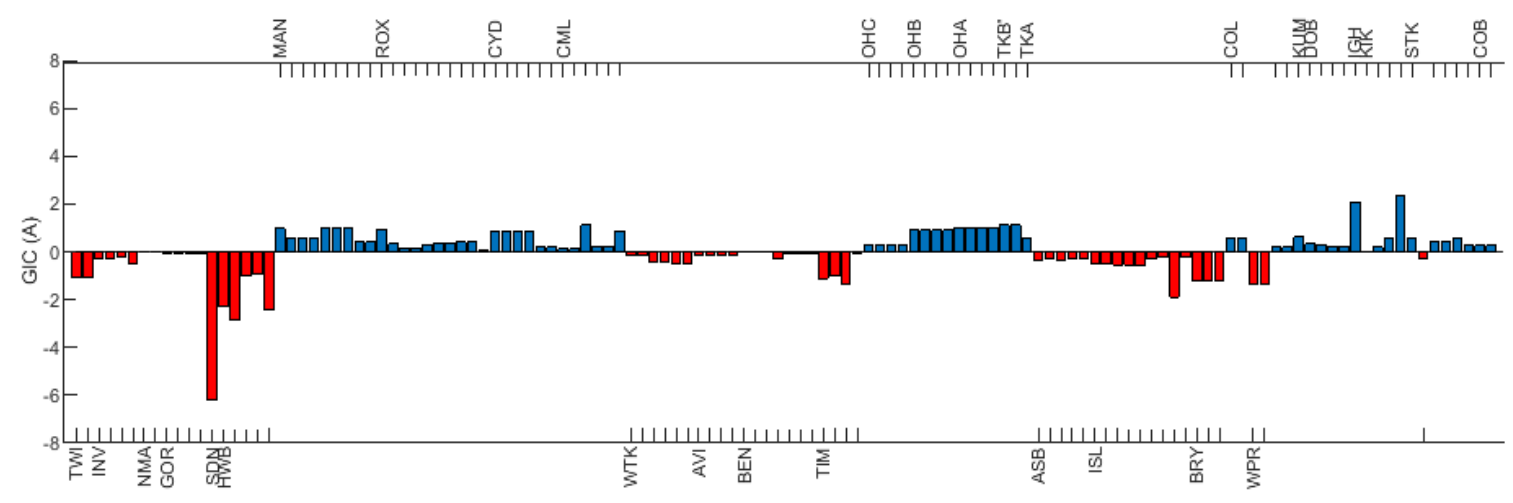

Figure 6. 25: Transformer GIC during Sudden Storm Commencement at 0447 UT using the thinsheet conductance model.

The network model has been used to compute transformer and substation level GIC using oneminute discrete time domain thin-sheet geoelectric fields. The total data points are 2048 which encompasses around 1.5 days so covering the full St. Patrick's Day magnetic variations. Four transformers have been identified experiencing large GIC which are the same as was predicted by the MT model, these are 
1. South Dunedin (SDN-T2H)- $(-45.89,170.50)$

2. Halfway Bush (HWB-T6L)- $(-45.85,170.47)$

3. Kikiwa (KIK-T2H)- $(-41.67,172.87)$

4. Inangahua (IGH-T1H)- $(-41.85,171.95)$
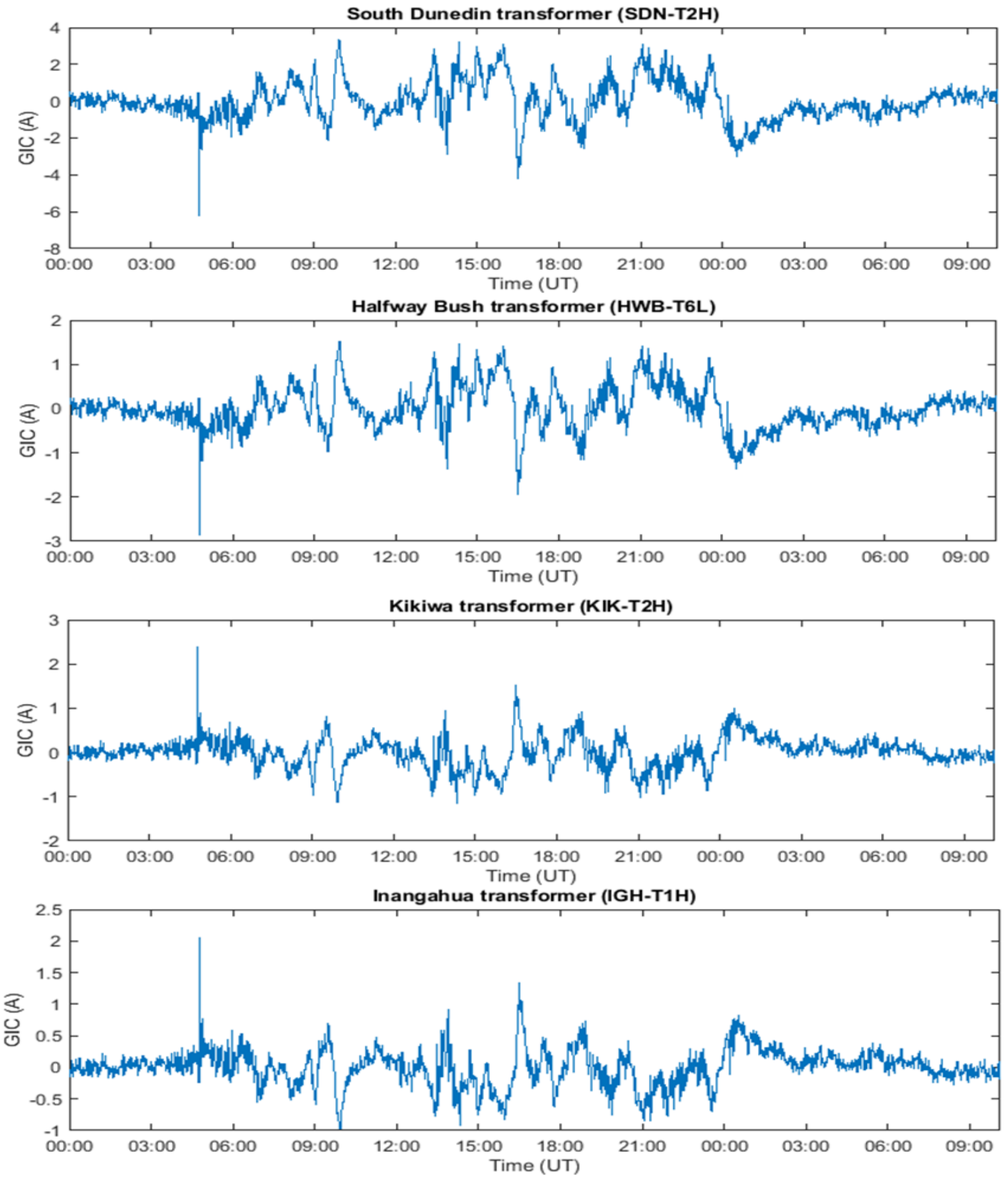

Figure 6. 26: Thin-sheet conductance model results of transformer having largest GIC during St. Patrick's Day storm. 
A time plot of GIC for the above mentioned transformers is given as Figure 6.26. We have selected a time range starting from 17 March to 0900 UT of 18 March which effectively encompass all GIC variations during the magnetic storm.

The snapshot of substation GIC for the thin-sheet geoelectric fields (0447 UT) is also given as Figure 6.27. Since the network GIC response is maximum during the SSC the above substations also show the largest GIC during the whole duration of the storm.

1. Manapouri (MAN)- $(-45.52,167.28)$

2. South Dunedin (SDN)- $(-45.89,170.50)$

3. Halfway Bush (HWB)- $(-45.85,170.47)$

4. Islington (ISL)- $(-43.53,172.51)$

SDN and HWB substations show the largest GIC as they also have large transformer level GIC whereas MAN and ISL show large substation-level GIC because of a large number of earthed transformers. Manapouri and Islington both have 9 earthed transformer windings. The MT model does not show Manapouri as experiencing large substation GIC because of the poor interpolation of geoelectric fields in that region.

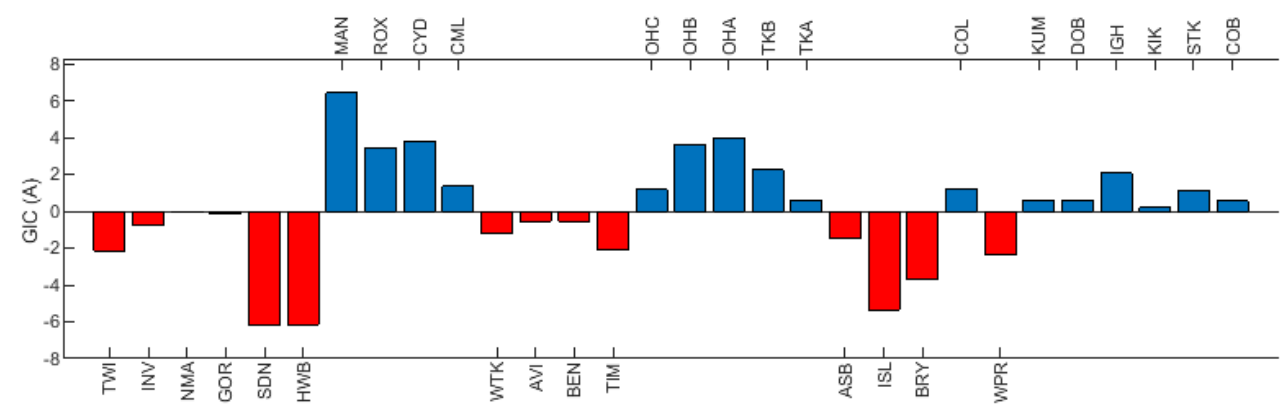

Figure 6. 27: Substation level GIC at 0447 UT using thin-sheet conductance model during St. Patrick's Day storm. 


\subsubsection{Thin-sheet Model GIC Comparison with Measured Data}

The thin-sheet model results have also been compared with the measured transformer data. The measured data of one-minute interpolated GIC of four transformers (ISL-T6H, HWB-T4L, SDN$\mathrm{T} 2 \mathrm{H}$ and MAN-T6H) have been used for this purpose. The thin-sheet GIC show smaller magnitude compared to the observed data during the whole magnetic storm and particularly during the SSC. Although the thin-sheet model does effectively reproduce longer period variations this is consistent with the high frequency limitation on the validity of the thin-sheet modelling technique. Since the thin-sheet model covers a broad range of frequencies the measured data have not been filtered.

Figure 6.28a shows individual plots of measured and model GIC in Islington transformer T6H. The measured data shows a magnitude 4 times larger than the thin-sheet model during the SSC. For the rest of the storm the measured data are at least twice the model data. Both plots are shown together using different scales as Figure $6.28 \mathrm{~b}$ where the panel on right hand side shows a magnified view during the SSC. The thin-sheet model reproduces GIC variations at low frequencies most of the time, but results are not good during the SSC. The correlation coefficient is 0.66 and performance parameter 0.08 between model and measured data.

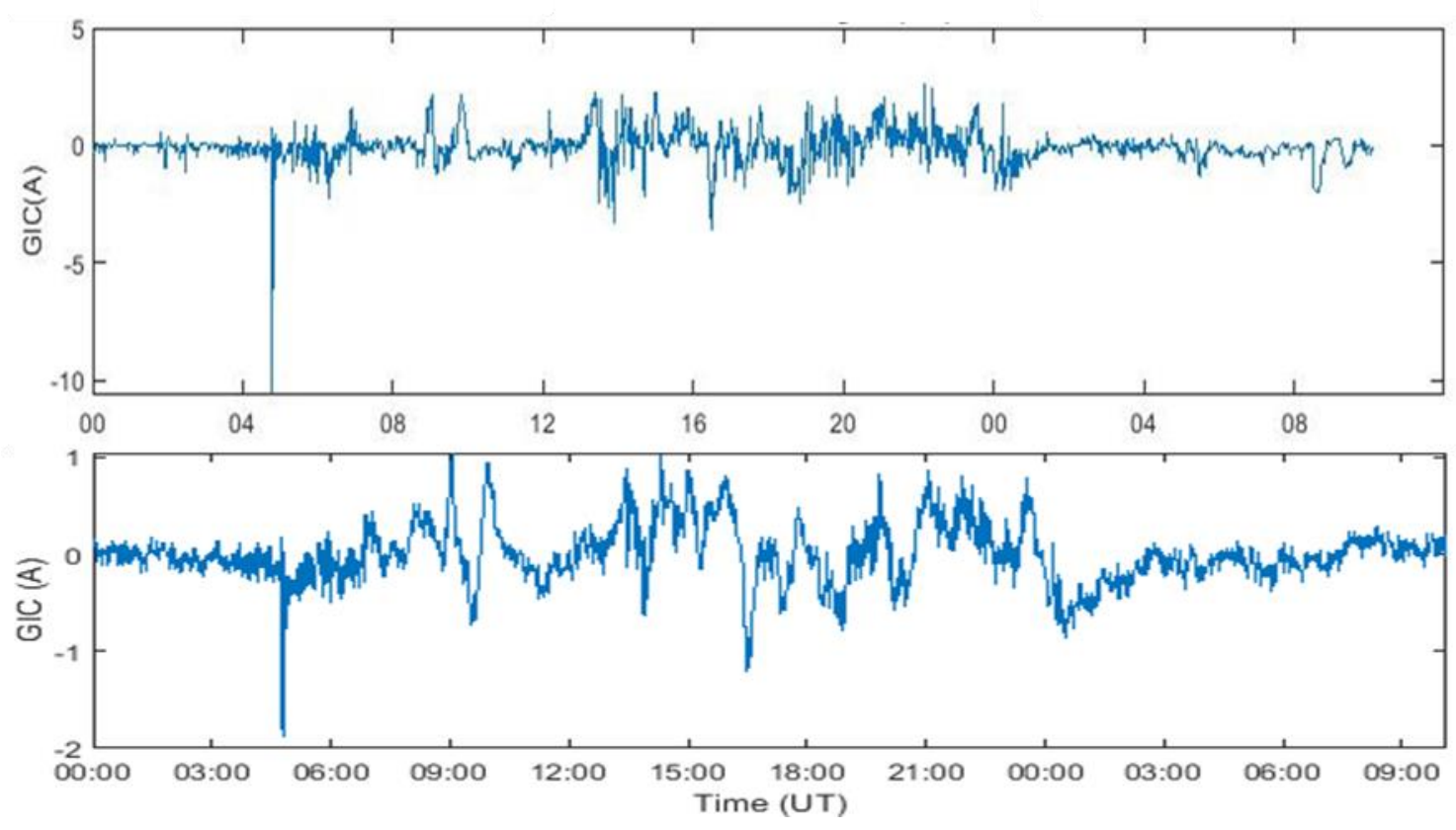

Figure 6. 28a: Top: observed GIC in Islington transform ISL-T6H, bottom: TS model GIC. 

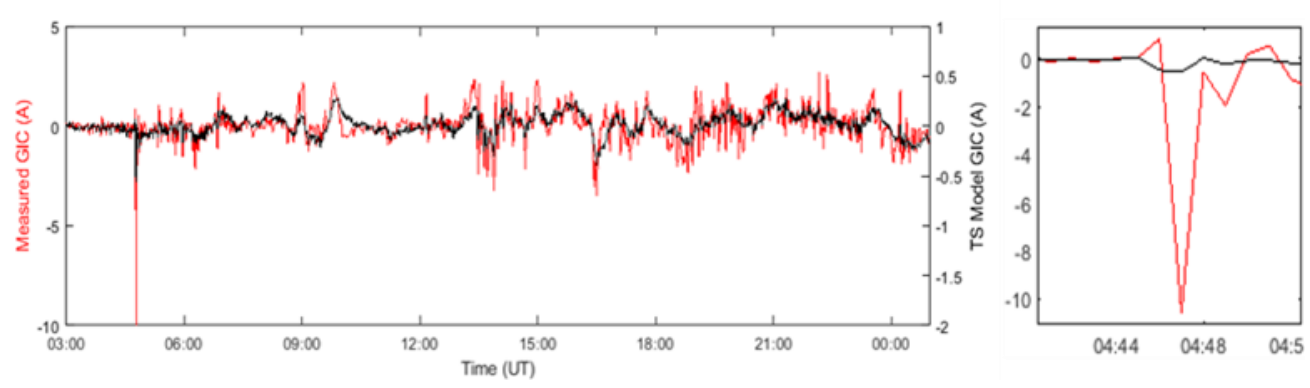

Figure 6.28b: A comparison of TS model and observed GIC in Islington transform ISL-T6H ( $r=0.66)$.

Figure 6.29a shows individual plots of measured and model GIC in South Dunedin transformer$\mathrm{T} 2 \mathrm{H}$. The measured data show about twice the GIC magnitude compared to the model GIC during the whole magnetic storm. Both plots are shown together on the same scale as Figure $6.29 \mathrm{~b}$ where the panel on right hand side shows a magnified view during the SSC. The correlation coefficient is 0.62 and performance parameter 0.19 between model and measured data.

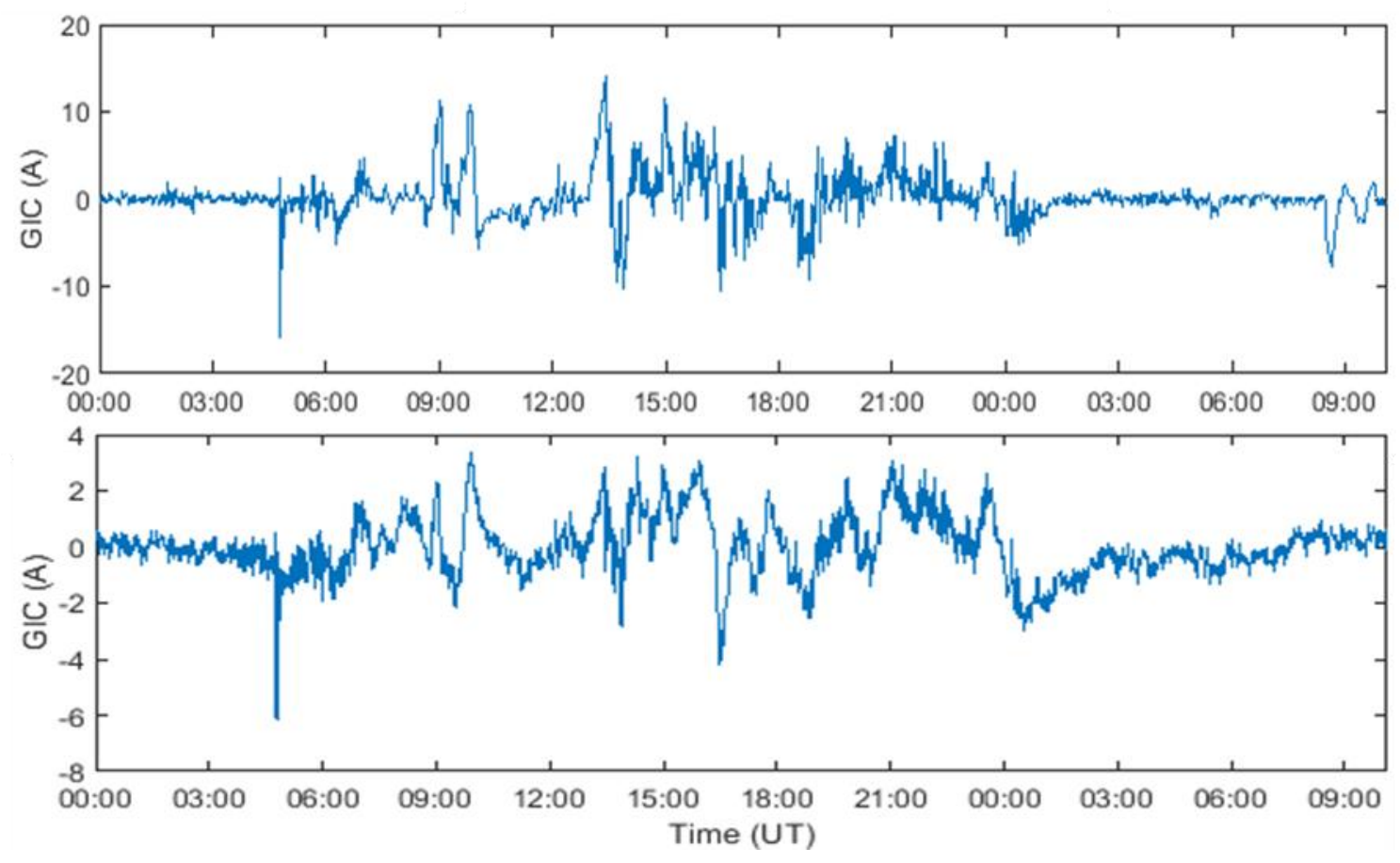

Figure 6. 29a: Top: observed GIC in South Dunedin transform-T2H; bottom TS model GIC. 

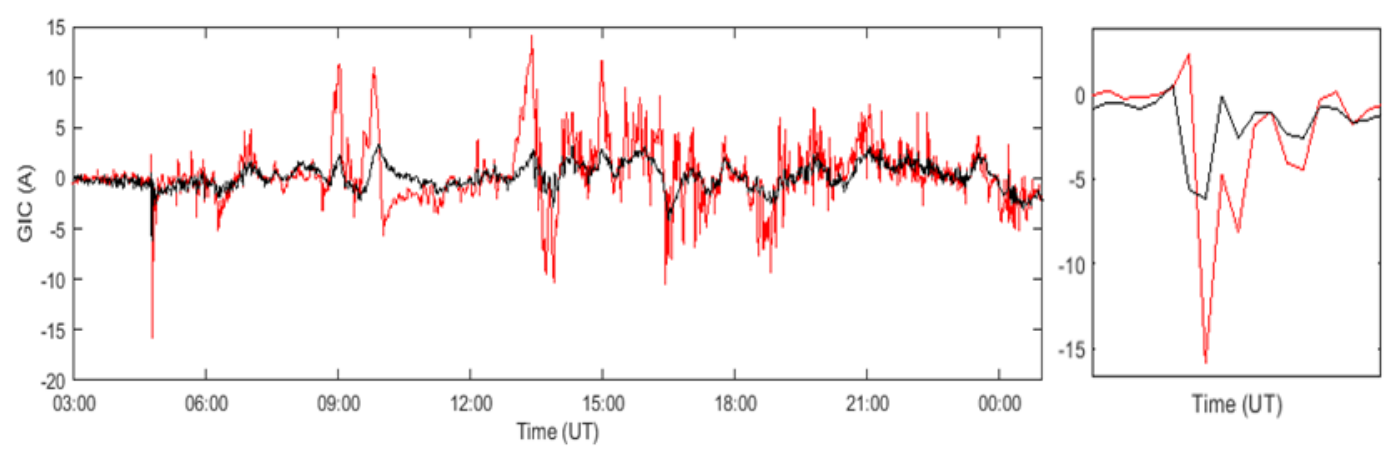

Figure 6.29b: A comparison of TS model and observed GIC in South Dunedin transform-T2H $(r=0.61)$.

Figure 6.30a shows individual plots of measured and model GIC for Halfway Bush transformer T4L. Here the measured data are at least 10 times the GIC magnitude compared to the thin-sheet model GIC during the whole magnetic storm. In Figure $6.30 \mathrm{~b}$ both plots are shown together with different scales. This shows that the thin-sheet model reproduces GIC variations at low frequencies but not during the SSC. The correlation coefficient is 0.63 and performance parameter 0.05 between model and measured data.
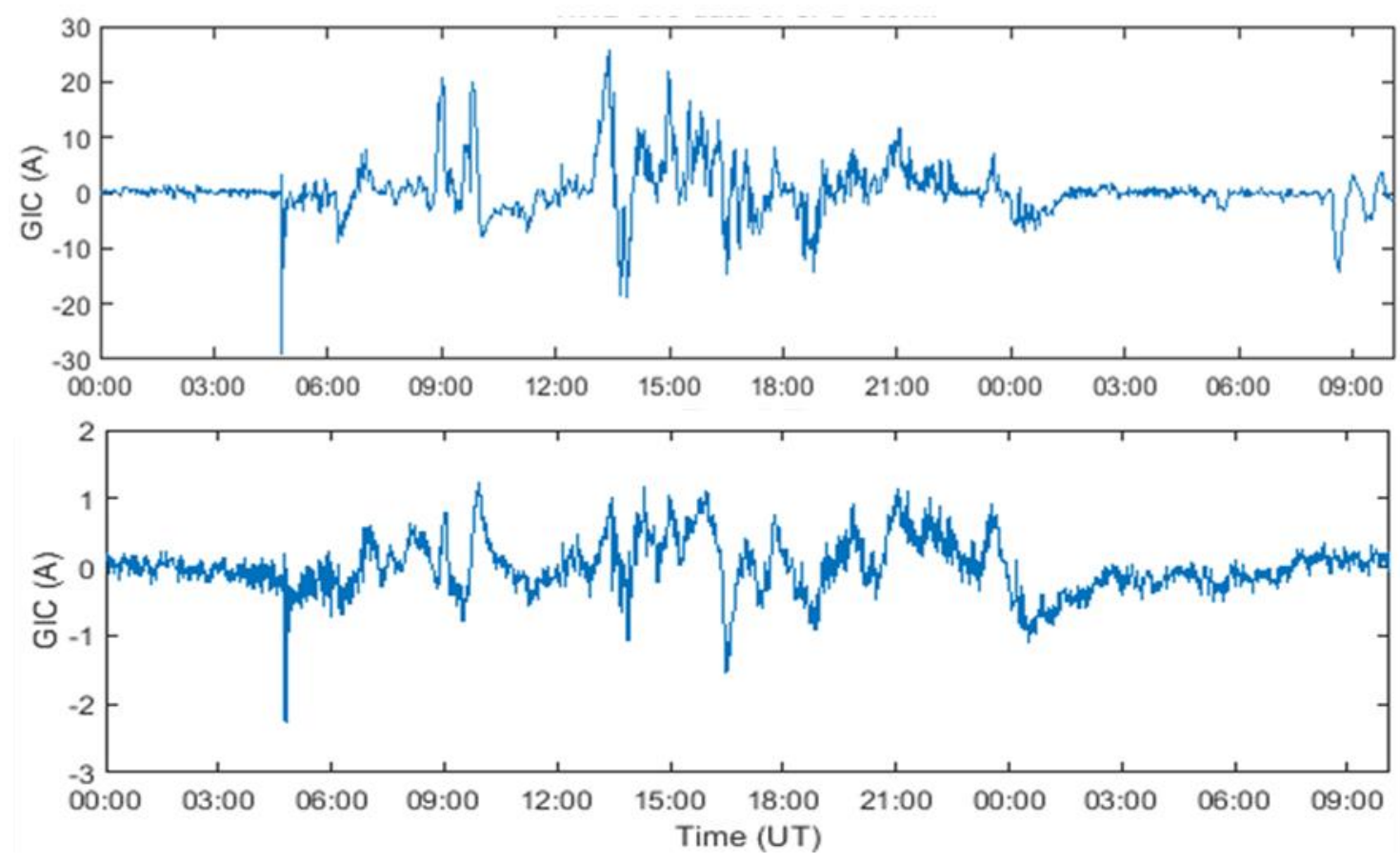

Figure 6. 30a: Top: observed GIC in Halfway Bush transform HWB-T4L; bottom TS model GIC. 


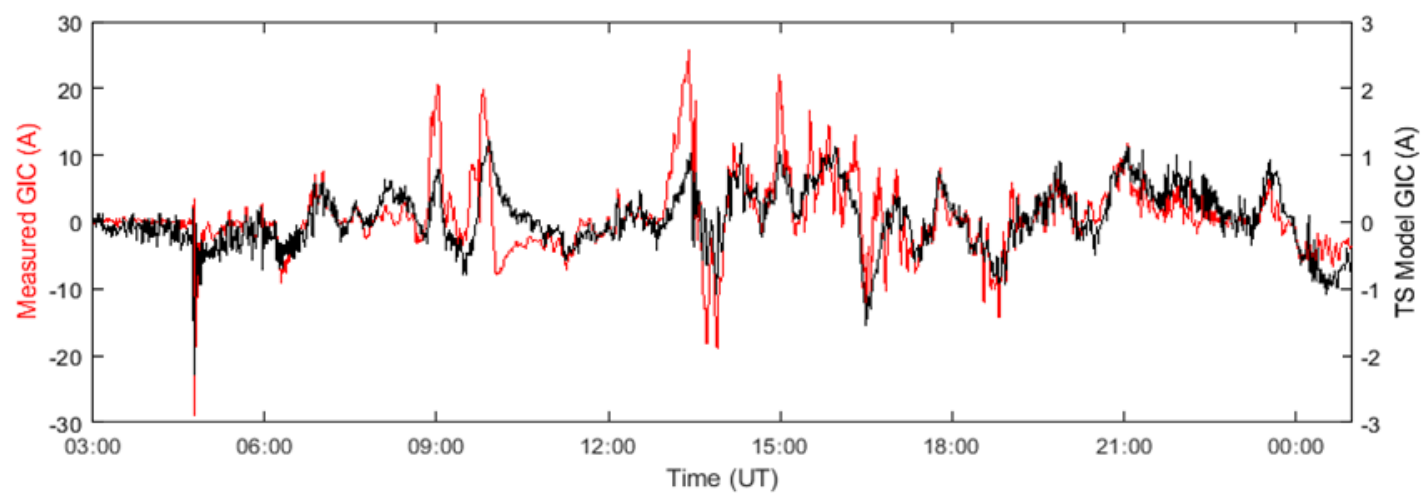

Figure 6.30b: A comparison of TS model and observed GIC of Halfway Bush transform HWB-T4 where both plots are on different scale $(r=0.64)$.

Figure6.31a shows plots of measured and model GIC in Manapouri transformer T6H. Again, the measured data are about three times the GIC magnitude of the model GIC ( $1 \mathrm{~A}$ ) during the whole magnetic storm. The thin-sheet model reproduces GIC variations at low frequencies during most of magnetic storm. The correlation coefficient is 0.67 and performance parameter 0.19 between model and measured data.
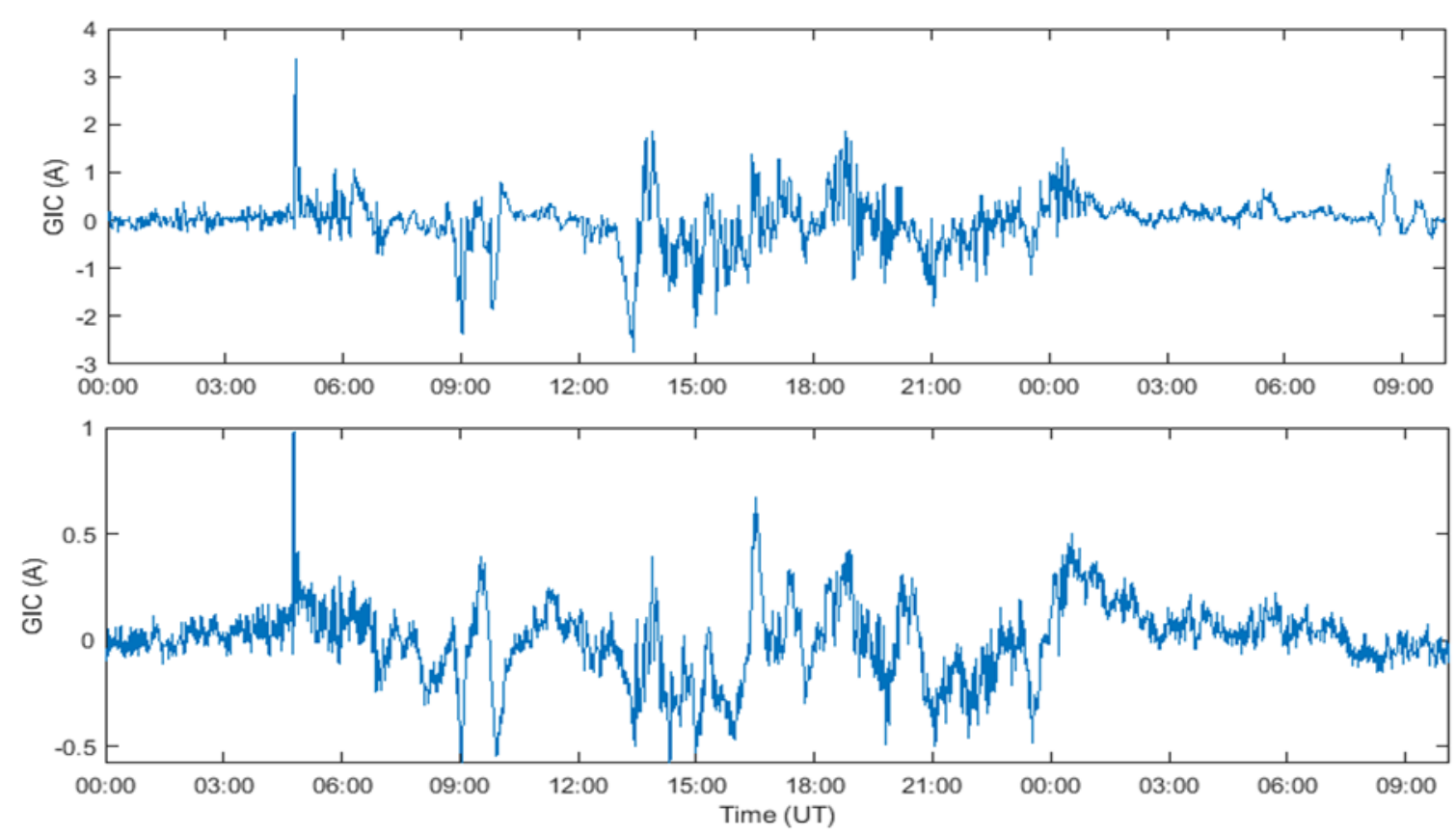

Figure 6. 31a: Top: observed GIC in Manapouri transform MAN-T6H; bottom TS model GIC. 


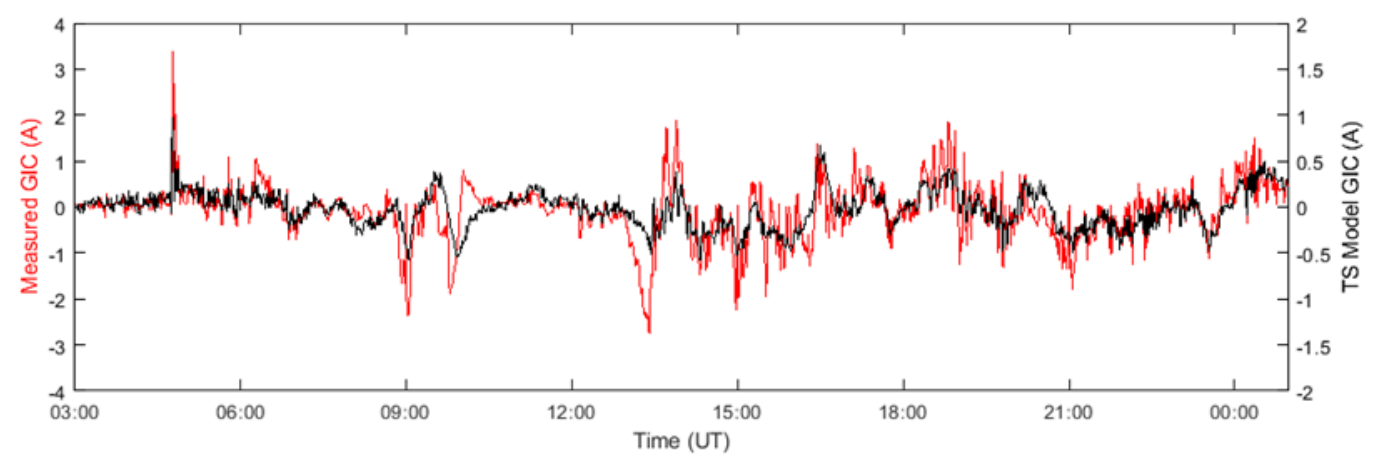

Figure 6.31b: A comparison of TS model and observed GIC of Manapouri transform MAN-T6H where both plots are on different scale $(r=0.68)$.

\subsection{Discussion}

Chapter 4 showed GIC modelling using MT data for the North Island for the St. Patrick's Day storm of 2015 , and in this chapter the same procedure has been extended to model GIC for the South Island power network. Although, the availability of measured GIC provides the opportunity for comparison with model results, the South Island has very sparse MT data. This makes it difficult to realistically interpolate geoelectric fields across the whole Island. Despite these limitations, the implementation of MT data for GIC modelling has produced encouraging results. The MT model GIC effectively reproduces GIC variations seen in the observed data particularly during the Sudden Storm Commencement. The MT model results have been compared with measured GIC at SDN-T2H, HWB-T4L and ISL-T6H transformers. The MT model GIC for South Dunedin transformer (SDN-T2H) has the best match with the observed data both in scale of magnitude and time variations. There is only a difference of $1 \mathrm{~A}$ between model and observed data during the SSC. Islington transformer (ISL-T6H) model GIC have a smaller magnitude compared to observed GIC, the model GIC has only -2.56 A while the measured GIC is -9.62 A during the SSC. Despite the small magnitude, model GIC time variations match well with observed data during the whole duration of the magnetic storm. The interpolated geoelectric fields are small in the network domain around Islington substation which does not seems realistic, and it is anticipated that improved MT coverage would lead to better prediction for Islington transformer GIC. 
Similarly, Halfway Bush transformer HWB-T4L, which has the largest measured GIC of all measured GIC during the St. Patrick's Day storm. The model GIC again predict only small magnitude. For example, the measured data showed the largest GIC of -25.85 A during the SSC whereas it this has GIC of only -6.01 A when using the MT model. South Dunedin and Halfway Bush substations are very close to each other and in the same grid cell of the thin-sheet conductance map, therefore the discrepancy in HWB model GIC results has not been explored further in terms of distribution of geoelectric fields. It is suspected that it is related to some change in network parameters over time that needs to be further explored.

Both the MT model and the thin-sheet model show the same transformers experiencing the largest GIC during the St. Patrick's Day storm. These include South Dunedin (T2H), Halfway Bush (T6L), Kikiwa $(\mathrm{T} 2 \mathrm{H})$ and Inangahua $(\mathrm{T} 1 \mathrm{H})$. The thin-sheet model effectively reproduces low frequency components seen in the measured GIC, but the results are very poor during the SSC which is consistent with the limitations in the applicable period range of the thin-sheet model. For example, for the SDN transformer where the MT model is best the thin-sheet can only produce half of the measured GIC during the SSC. The MT model has better results particularly during the SSC and it has a higher correlation coefficient and performance parameter during the whole duration of the magnetic storm. In terms of substation GIC the substations consisting of large number of earthed transformers, show large GIC e.g., Islington and Manapouri, along with South Dunedin and Halfway Bush, which have large transformer GIC. A comparison of substation GIC for both techniques was also not possible because the interpolation results of MT data around Manapouri region were not reliable. 


\section{Chapter 7: GIC Modelling for the South Island for the Magnetic Storm 20 November2003}

\subsection{Introduction}

The GIC modelling results for two different magnetic storms are not generally the same, as seen during GIC modelling using MT data for North Island. Therefore, the magnetic storm of 20 November2003 has been used to model South Island GIC, using MT data, to assess the reliability of the model results. The St. Patrick's Day magnetic storm and the 20 November2003 magnetic storm were caused by different interplanetary magnetic field structures as discussed in detail during GIC modelling for the North Island in Chapter 5. The Eyrewell (EYR) magnetic field data and GIC data for Islington (ISL) transformer were also given there as Figure 5.2. Spectral analysis of the GIC data for Islington showed different GIC signatures for the two storms. Since the measured GIC data at Islington are available for this storm it provides an opportunity to compare the modelling results with measured data.

\subsection{MT Geoelectric Field}

The magnetic data ( $\boldsymbol{B}_{\boldsymbol{x}}$ and $\boldsymbol{B}_{\boldsymbol{y}}$ components at EYR) along with magnetotelluric response tensor have been used to compute time domain geoelectric fields for all MT sites in the South Island in the same way as was discussed in previous chapters. Since the magnetic field data of only one magnetic observatory (EYR) is available this data have been used to model geoelectric fields throughout the South Island. The computed geoelectric fields are in a different period range based upon the period range of the MT data (refer to Chapter 6 for the detail of the range of periods). Figure 7.1 shows a comparison of geoelectric fields $\left(\boldsymbol{E}_{\boldsymbol{x}}, \boldsymbol{E}_{\boldsymbol{y}}\right)$ computed for two MT sites which have different period ranges during the magnetic storm of 2003. Figure 7.1a shows resulting geoelectric fields for an MT site located in Otago $(-45.84,169.81)$ in the period range of 2.1-83.5 minutes. Figure 7.1b shows geoelectric fields for another MT site in Marlborough ($42.76,173.31$ ) which has a comparatively shorter period range of 1.9-15.16 minutes. The shorter 
period range MT site shows only high frequency components. At both sites the geoelectric fields reach maximum amplitude during the main phase of the magnetic storm and the field values are about four time larger than for the St. Patrick's Day storm.

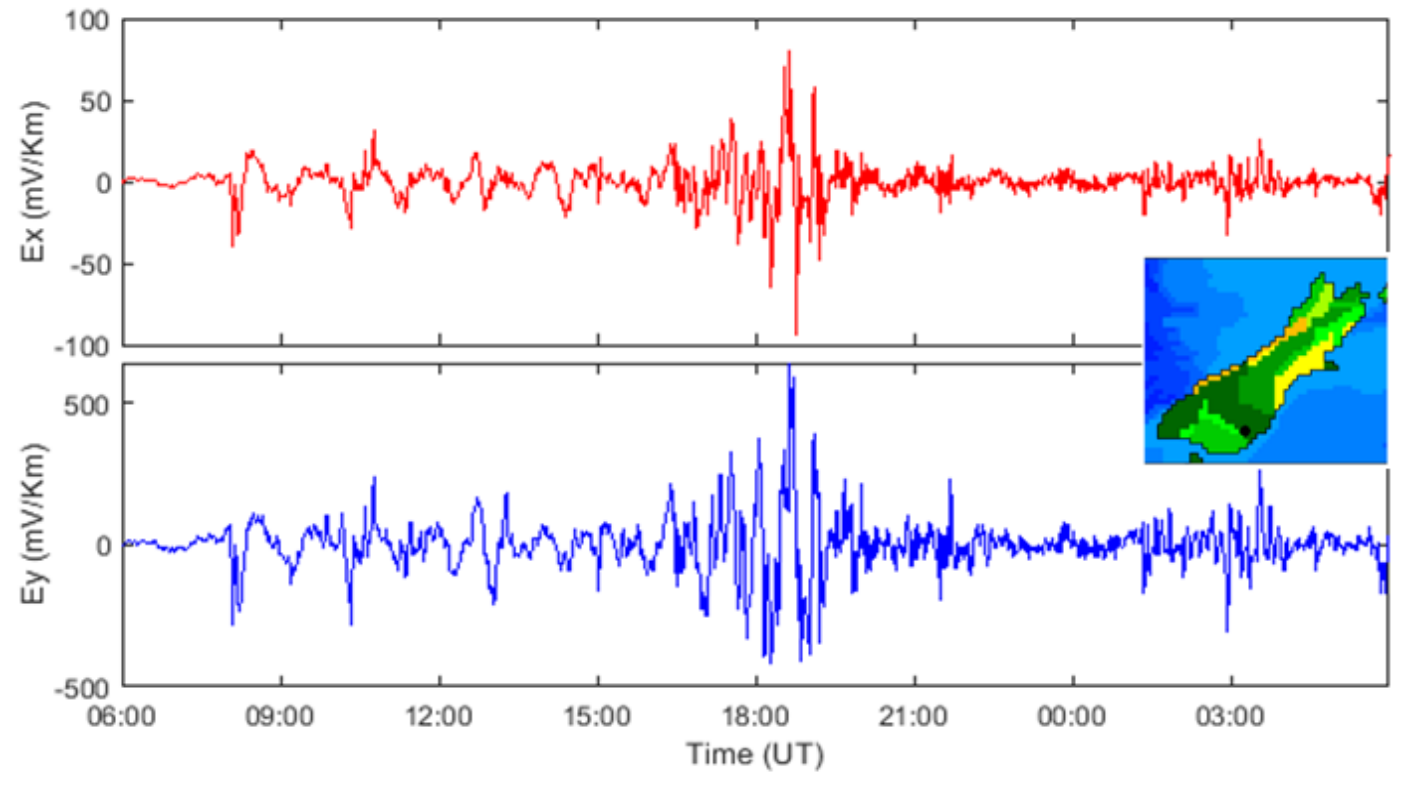

Figure 7. 1a: Time domain geoelectric field computed using the impedance response tensor of an MT site in Otago (also shown by dot on the map) during magnetic storm of 20 November2003.

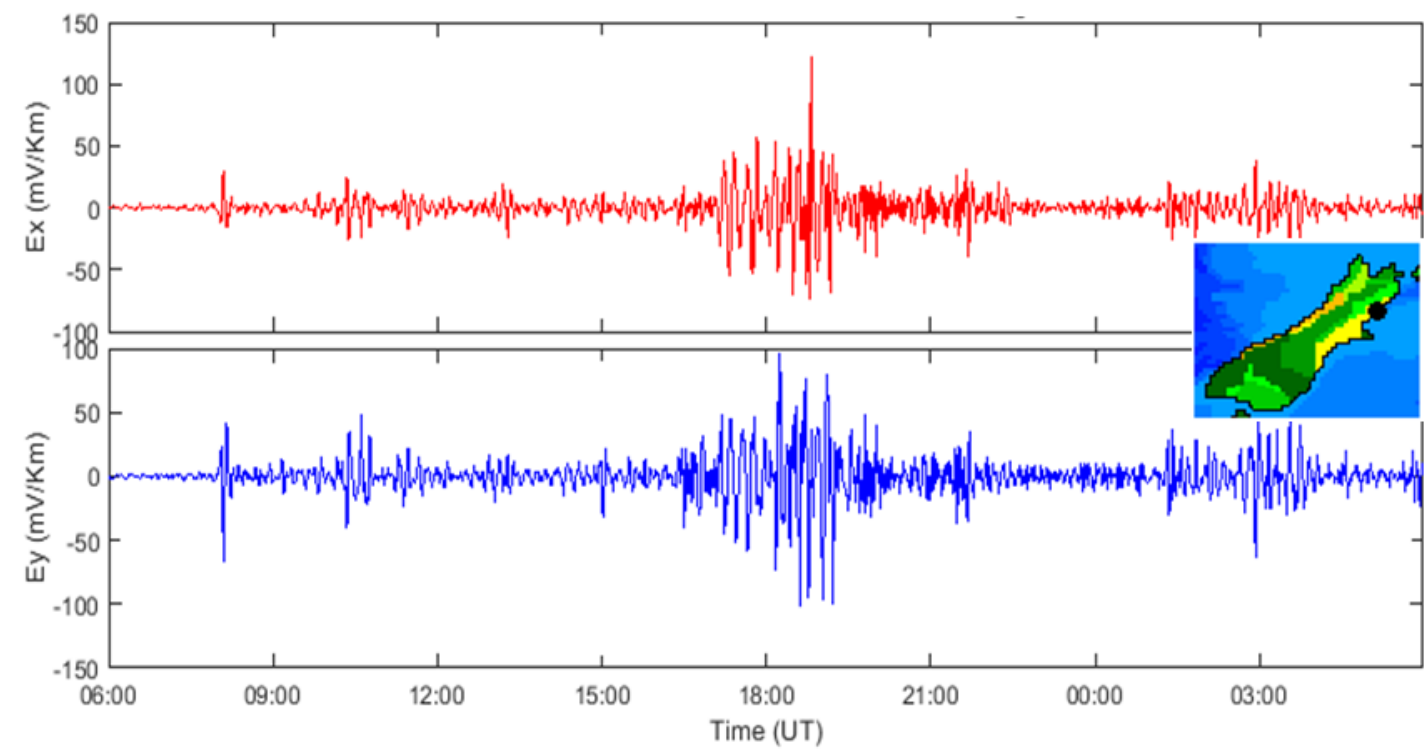

Figure 7.1b: Computed geoelectric field for MT site in the region of Marlborough covering the comparatively shorter period (1.9-15.2 minutes). 
The snapshot of geoelectric fields corresponding to all MT sites at 18:44 and 18:47 UT during the storm is given in Figure 7.2, where each arrow in a cell is the vector sum of the north $\left(\boldsymbol{E}_{\boldsymbol{x}}\right)$ and east $\left(\boldsymbol{E}_{\boldsymbol{y}}\right)$ fields.
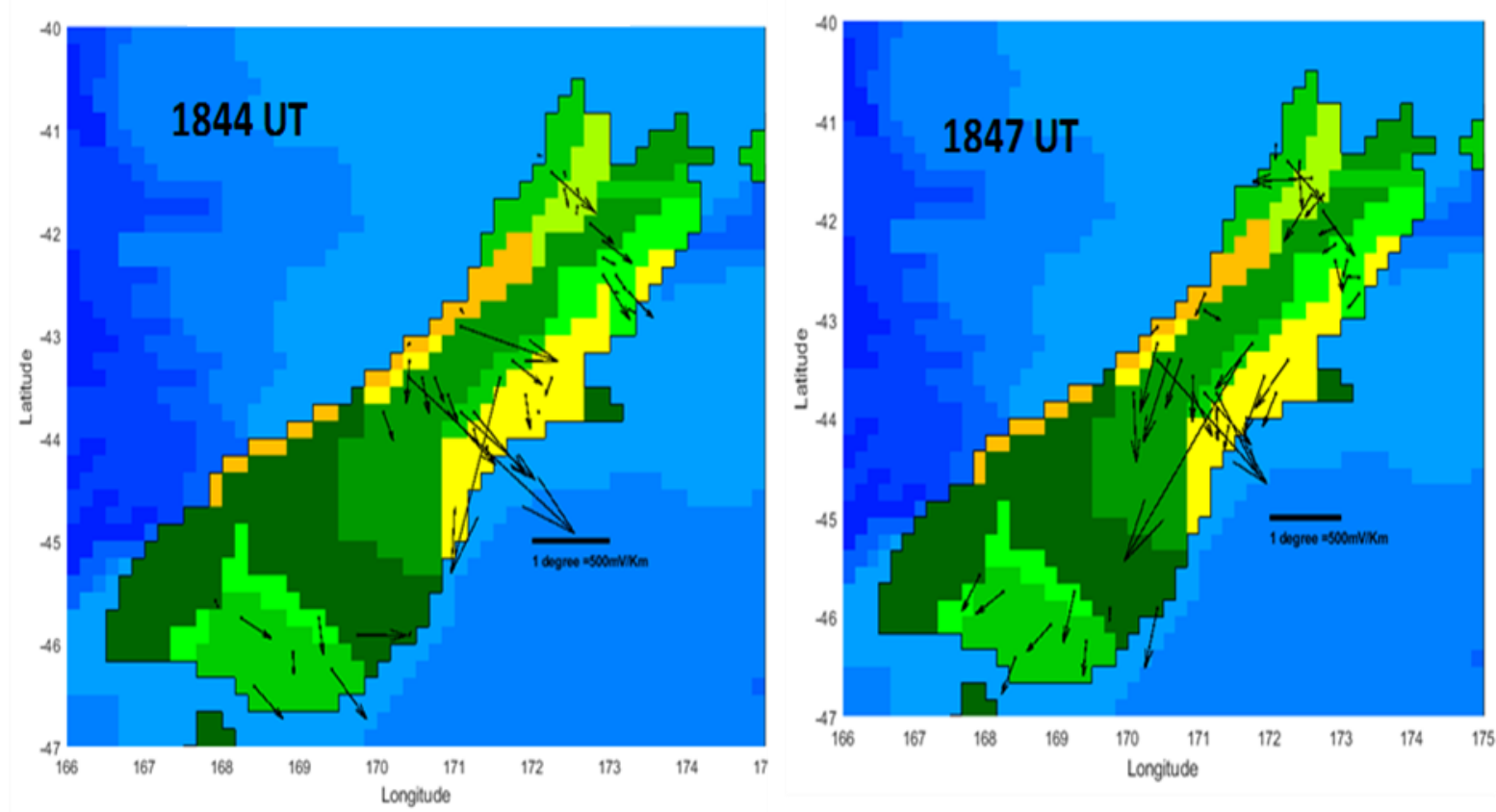

Figure 7. 2: Computed geoelectric fields vectors at the locations of MT sites on the South Island grid at 1844 and 1847 UT during the magnetic storm of 20 November2003.
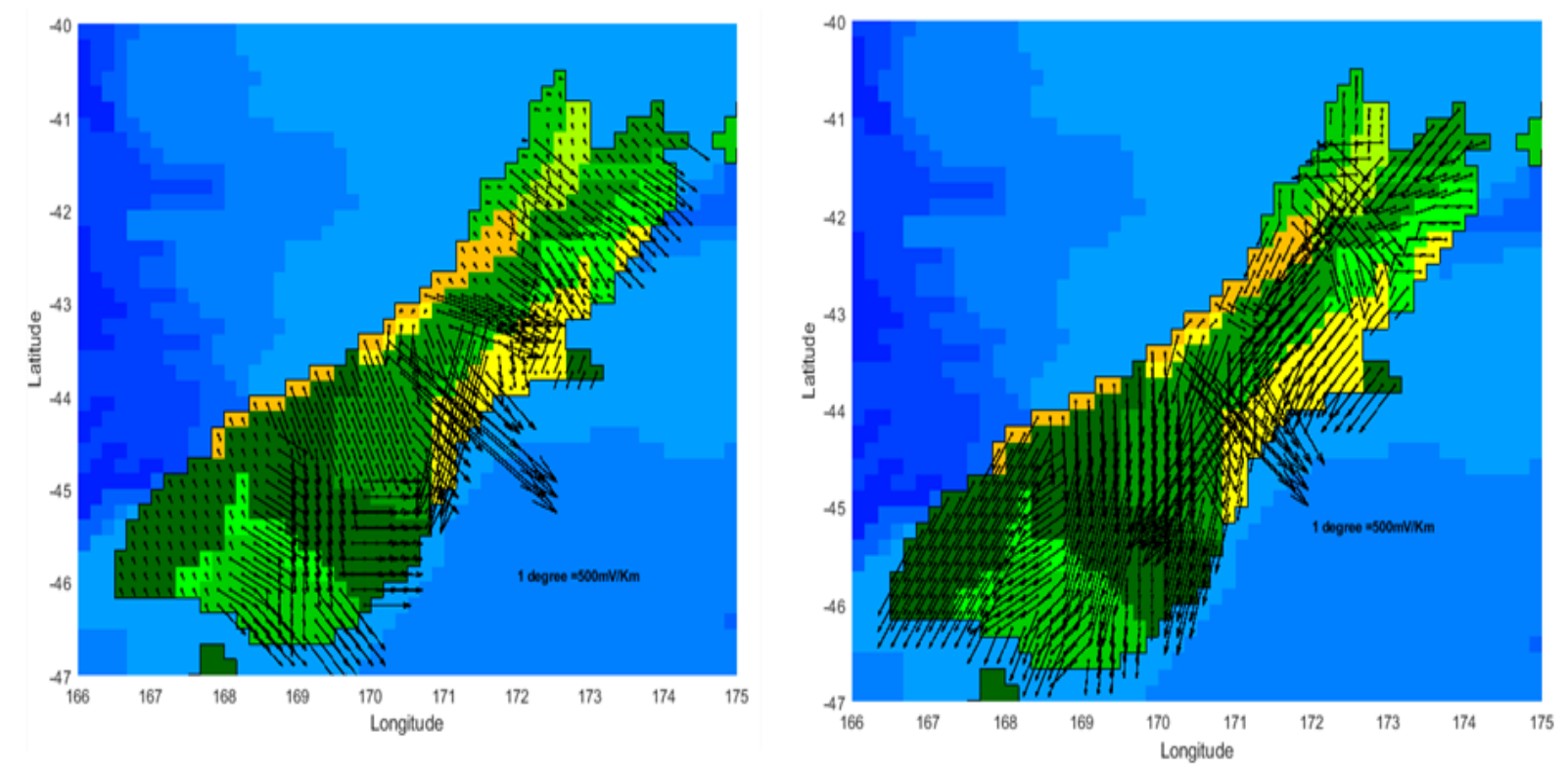

Figure 7. 3: Geoelectric fields after applying Nearest Neighbor interpolation technique to MT fields shown in Figure 7.2. Nearest Neighbor fills the empty cells using the given field values. 
The geoelectric fields vary both in direction and magnitude in different conductance regions of the South Island. Although these variations are consistent in different conductance regions, the MT data show more diverse variations that are not evident from the conductance map. The Southern Alps regions shows the largest field values compared to the Southland/Otago and Marlborough regions. Comparison of fields during both times also shows that the response of MT sites varies during the magnetic storm.

Figure 7.2 shows that MT data for the South Island is very sparse having wide gaps between MT sites. The large gaps between MT sites makes interpolation results more uncertain. To fill the empty grid cells, as was done previously, the Nearest Neighbor interpolation technique (Ashraf et al., 2017; Berg et al., 2000) is used. Figure 7.3 shows the results of interpolation where empty cells in the grid have been filled based upon values of the available geoelectric fields. The plots show that due to the gaps between sites the empty cells are filled with original MT fields. Since MT sites are small in number the effect of differences between individual sites results in areas of the South Island where fields are the same but differ from those in adjacent areas. Even so, as discussed in previous chapters, these interpolation results are regarded as better than other interpolation techniques.

\subsection{Transformer Level GIC Modelling Using MT Data}

The results of GIC computed using the geoelectric fields derived from the MT data are given in this section. As was discussed in the previous chapter, the use of uniform electric fields across the South Island showed there to be no difference in calculated GIC between using the whole network in the calculation and using an equivalent circuit to represent part of it. Thus, the network response has been calculated without the consideration of any equivalent circuit. Figure 7.4 shows transformer-level GIC at 1844 and 1847 UT using the interpolated geoelectric fields. The network response is quite different during the two times. At 1844 UT South Dunedin (T2H) and Halfway Bush (T6L) transformers show the largest GIC which reach values of 14.3 and $7.5 \mathrm{~A}$ respectively, whereas at 1847 large GIC appear in the southern and northern parts of the network in transformers including Tiwai (TWI-T2H) 8 and Stoke (STK-T7L) -7 A, respectively.

Since currents in the lines connecting the different substations are responsible for GIC in transformers, the current values in Amperes in different lines are given as Table 7.1. The large 
GIC in the SDN transformer is due to the line that is connected to Three Mile Hill (TMH) which carries a current of -11.06 A. Similarly, for HWB transformer the line connection with TMH carries the largest current of magnitude $14.58 \mathrm{~A}$. The large GIC in the Stoke transformer is due to a 220 kV line connecting Stoke (STK) to Kikiwa (KIK) and carries a current of -6.63 A. Tiwai (TWI) which lies at very southern end of the power network, shows large GIC due to the addition of line currents from Invercargill (INV) and North Makarewa (NMA) substations.

The information about line currents may also be important in the context that since MT data are very sparse, we can look for line currents where interpolation results are not very reliable. The location of transformers and line currents are shown on the map in the Figure 7.5. Different colors show the magnitude of current through transformers and lines. Since there can be many transformers within a substation the single transformer with maximum GIC value is chosen for plotting purpose.
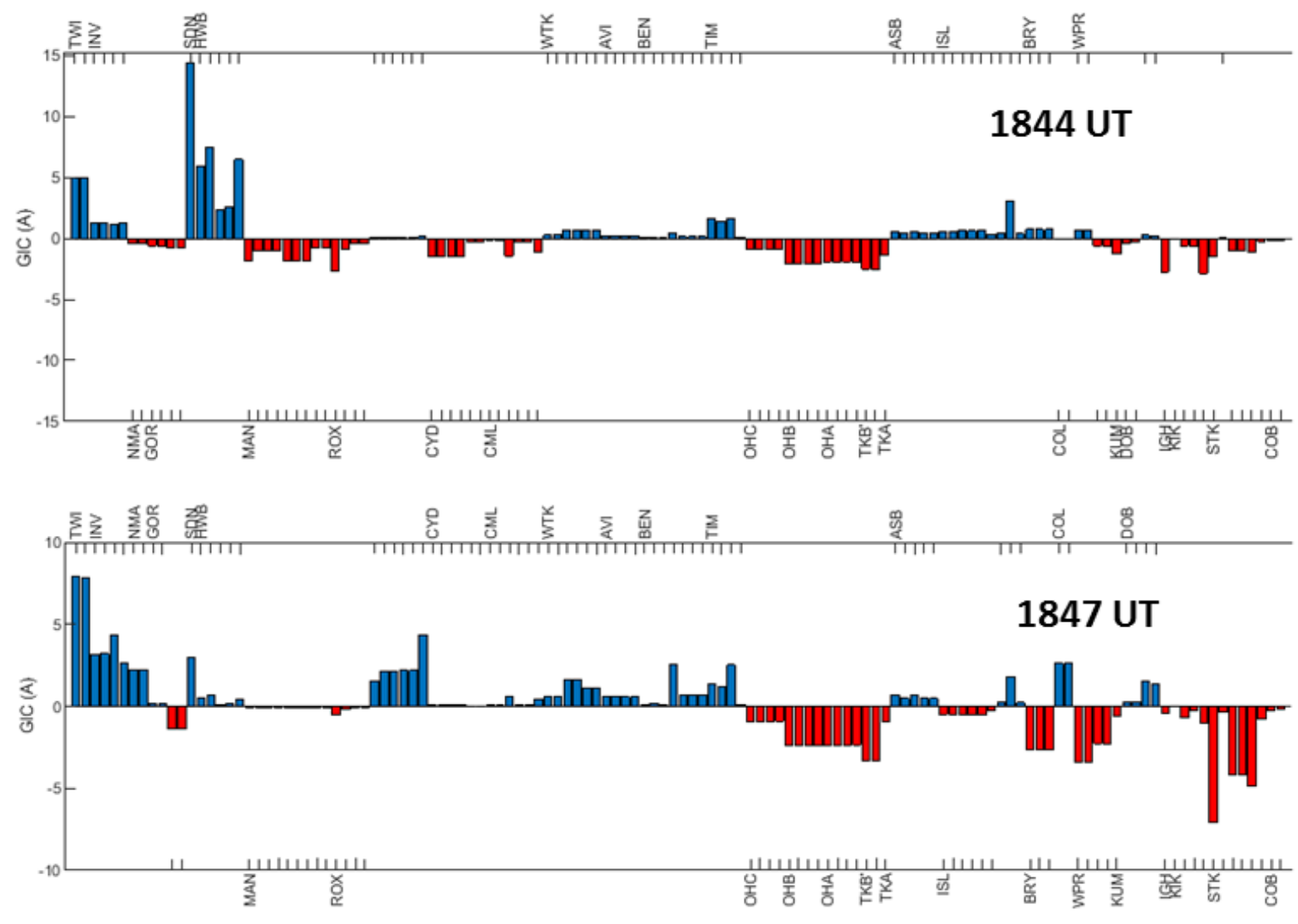

Figure 7. 4: Transformer level GIC at 1844 and 1847 UT calculated using MT data. 
The first panel (1844 UT) of Figure 7.5 shows SDN transformer (in red) experiencing the largest GIC. The line connecting SDN to TMH ( $11.06 \mathrm{~A}$ ), is mainly responsible for the SDN large GIC although the line cannot be seen in the figure as TMH substation is very close to SDN. The line that connects TMH to Roxburgh (ROX) can be seen in yellow carrying a large current of $-10.8 \mathrm{~A}$. In the second panel (1847 UT), the largest current is due to a line (shown in red) that connects Cromwell (CML) to Twizel (TWZ) and carries a current of $\sim 19$ A. As almost same current flows towards other substations without going into the transformers, the transformer level GIC is not very significant for either CML or TWZ. Tiwai (TWI) and Stoke (STK) show up significant transformer GIC ( 7 A) as can be seen in tones of yellow and cyan at the southern and northern ends of the South Island, respectively. The information on line currents is very useful in identification of current flow in the network leading to production of large GIC in certain transformers/substations of the network. This could effectively be used as a mitigation tactic showing that disconnecting a few transmission lines can significantly reduce GIC in the whole network.

Table 7.1

Line connections and their currents for transformers experiencing large GIC.

\begin{tabular}{lllllll}
\hline Node-1 & Node-2 & Resistance & $\begin{array}{l}\text { Line length } \\
(\mathbf{k m})\end{array}$ & $\begin{array}{l}\text { No. of } \\
\text { Lines }\end{array}$ & $\begin{array}{l}\text { GIC } \\
\mathbf{1 8 4 4}\end{array}$ & $\begin{array}{l}\text { GIC } \\
\mathbf{1 8 4 7}\end{array}$ \\
\hline \multicolumn{7}{c}{ South Dunedin (SDN) } \\
\hline SDN220 & HWB220 & 0.26 & 12 & 1 & 3.25 & 0.94 \\
SDN220 & TMH220 & 0.18 & 8 & 1 & 11.06 & 2.04 \\
& \multicolumn{7}{c}{ Halfway Bush (HWB) } \\
\hline HWB110 & ROX110 & 7.29 & 126 & 2 & 1.83 & 0.82 \\
HWB220 & SDN220 & 0.26 & 12 & 1 & -3.25 & -0.94 \\
HWB220 & TMH220 & 0.079 & 4 & 1 & 14.58 & 1.59 \\
HWB110 & BWK110 & 2.48 & 35 & 1 & 0.74 & -1.00 \\
& & \multicolumn{7}{c}{ Stoke (STK) } & & & \\
\hline STK110 & BLN110 & 2.15 & 76 & 2 & -0.09 & -0.44 \\
STK110 & KIK110 & 3.66 & 53 & 1 & -0.45 & -2.10 \\
STK220 & KIK220 & 1.12 & 52 & 2 & -1.58 & -6.63 \\
STK066 & MOT066 & 2.91 & 29 & 2 & 0.14 & 0.21
\end{tabular}



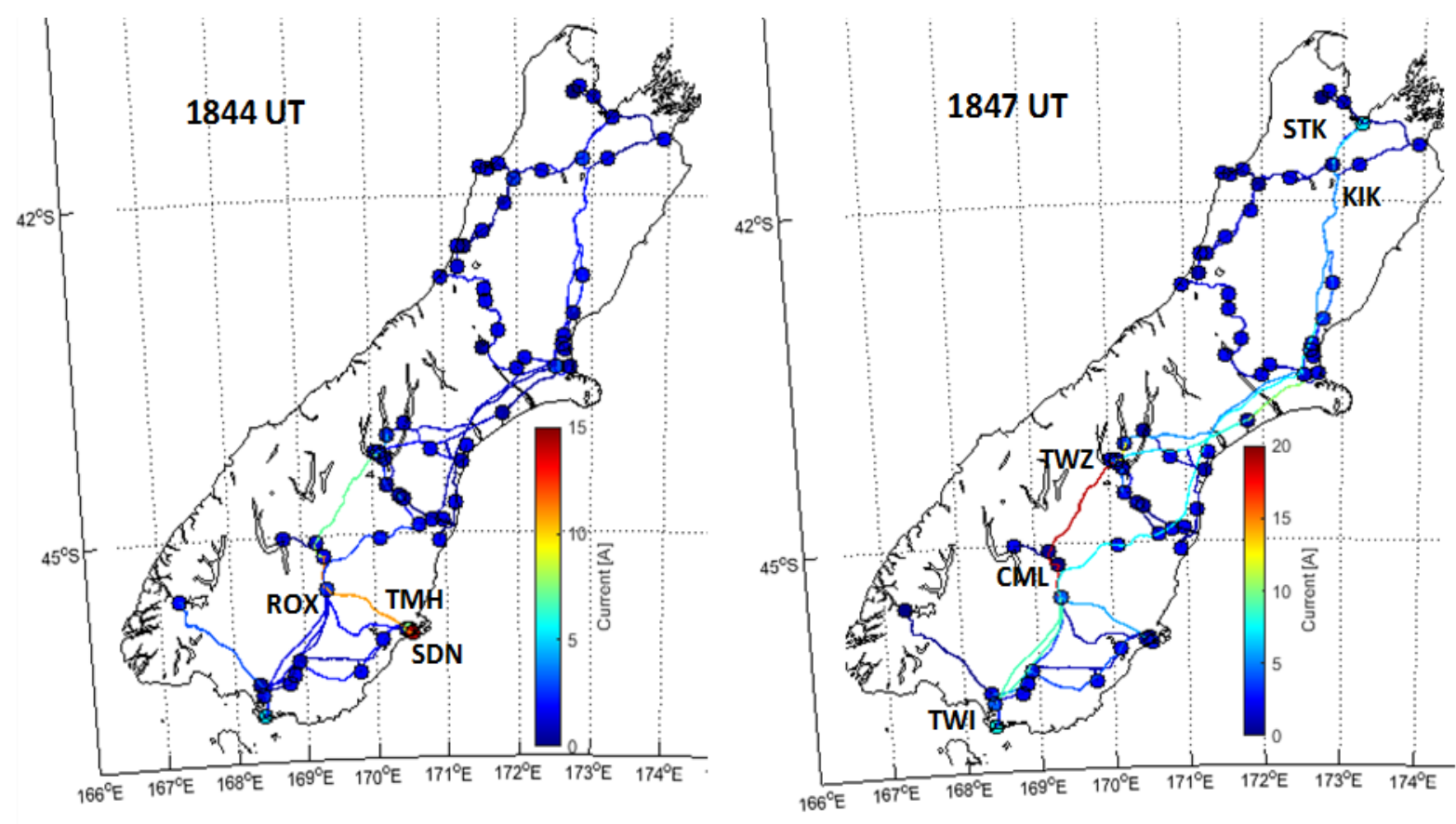

Figure 7. 5: Color intensity plot of transformers and line currents at 1844 and 1847 UT. Transformer and line currents are on the same scale where red color shows highest current and blue color shows small currents.

One-minute discrete geoelectric fields for a total 2048 data points (almost 1.5 days) covering the whole magnetic storm have been used to compute time domain GIC for all transformers in the South Island power network. The following transformers experience the largest GIC

1. South Dunedin (SDN-T2H)- $(-45.89,170.50)$

2. Halfway Bush (HWB-T6L)- $(-45.85,170.47)$

3. Kikiwa (KIK-T2H)- $(-41.67,172.87)$

4. Inangahua (IGH-T1H)- $(-41.85,171.95)$

A 24-hour duration time plot (0600 UT 20 November to 0600 UT 21 November2003) of these transformers is shown in Figure 7.6. The largest GIC for the South Dunedin transformer (SDN$\mathrm{T} 2 \mathrm{H}$ ) reaches a maximum value of $24.7 \mathrm{~A}$ whereas for Halfway Bush (HWB-T6L) the maximum value is 13.7 A. HWB and SDN both show longer period variations in their GIC whereas IGH and KIK only show shorter periods. This is primarily because the MT sites have different period ranges 
which are reflected in their GIC plots. Note that these are the same transformers experiencing large GIC as was seen for the St. Patrick's Day magnetic storm.
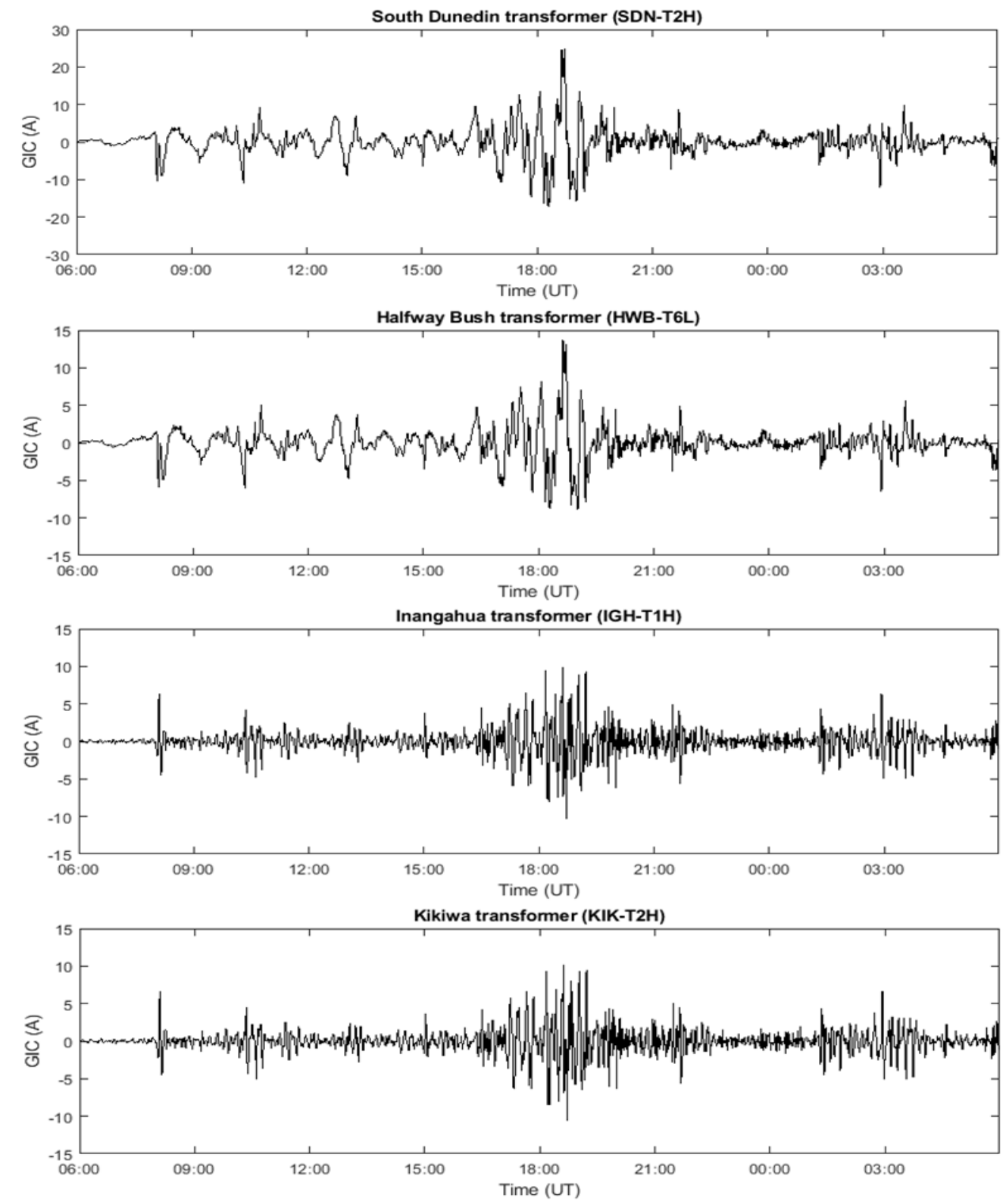

Figure 7. 6: Transformers identified (shown top of each panel) experiencing large GIC during the magnetic storm of 20 November 2003 using MT modelling. Significant GIC occur during the main phase of the magnetic storm when the magnetic field variations are maximum. 


\subsection{MT Model and Substation GIC}

In the above the transformer response has been given at 1844 and 1847 . The corresponding substation level GIC is shown in Figure 7.7. Substations experiencing large transformer level GIC also have large substation level GIC, these include SDN, HWB, TWI and STK.

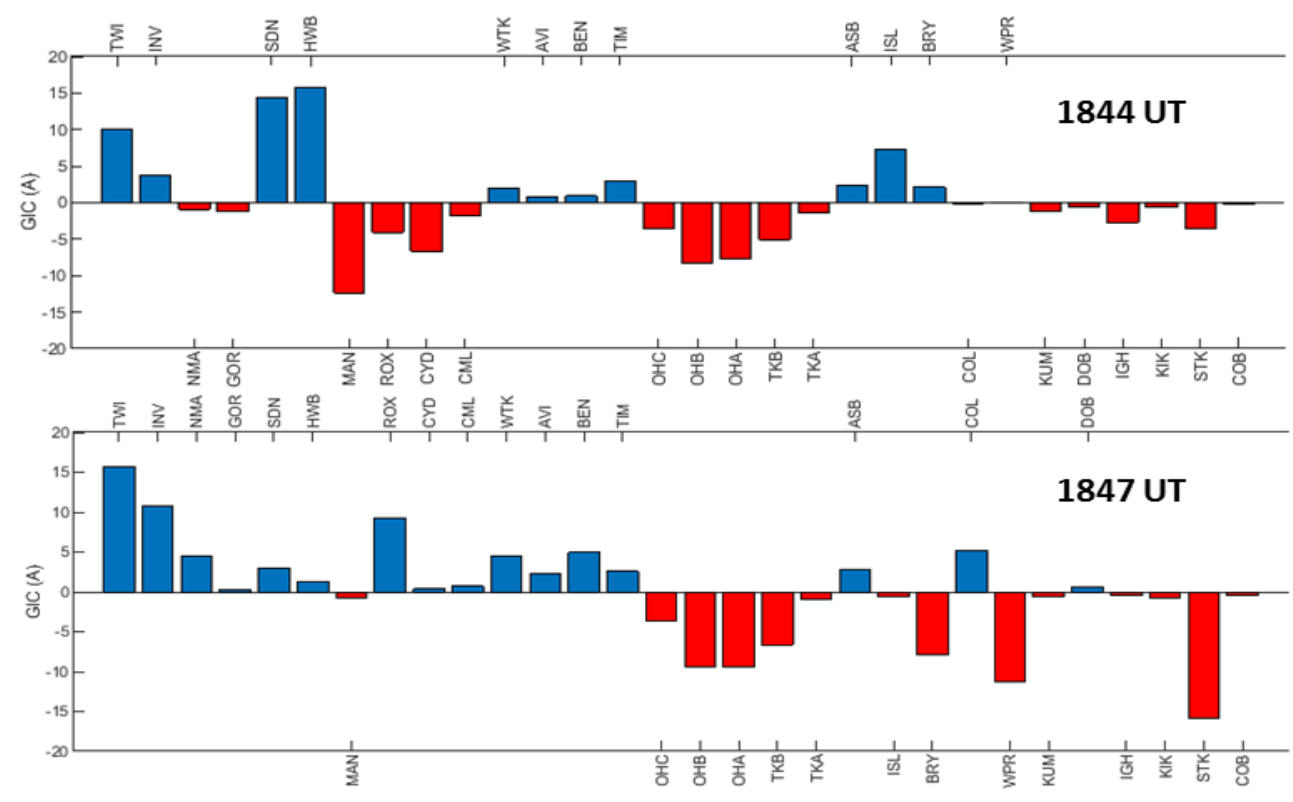

Figure 7. 7: Substation level GIC at 1844 and 1847 UT using MT model.

Time domain computed GIC for all 2048 data points during the magnetic storm show that the following substations have the largest GIC

1. Halfway Bush (HWB)- $(-45.85,170.47)$

2. South Dunedin (SDN)- $(-45.89,170.50)$

3. Ohau-A (OHA)- $(-44.26,170.02)$

4. Islington (ISL)- $(-43.53,172.51)$

5. Stoke (STK)- $(-41.31,173.24)$

HWB and SDN have the largest substation GIC because they have largest earthed transformerlevel GIC. The other three substations (Ohau-A, Islington and Stoke) have large substation GIC 
because they have many earther transformers within the substation. Islington has 9, Ohau-A and Stoke each has 4 earthed transformer nodes. These substation GIC results are somewhat different from those for the St. Patrick's Day magnetic storm for which Islington showed the largest substation GIC, and SDN did not appear in the list of large substation GIC.

Based upon the MT derived GIC for the whole South Island power network, Figure 7.8 shows locations of large transformer level GIC and large substation level GIC during the magnetic storm of 2003. Comparing this with Figure 6.19 for the St. Patrick's Day Storm the main difference is that for the 2003 storm Stoke (STK) also appeared as experiencing large substation level GIC.
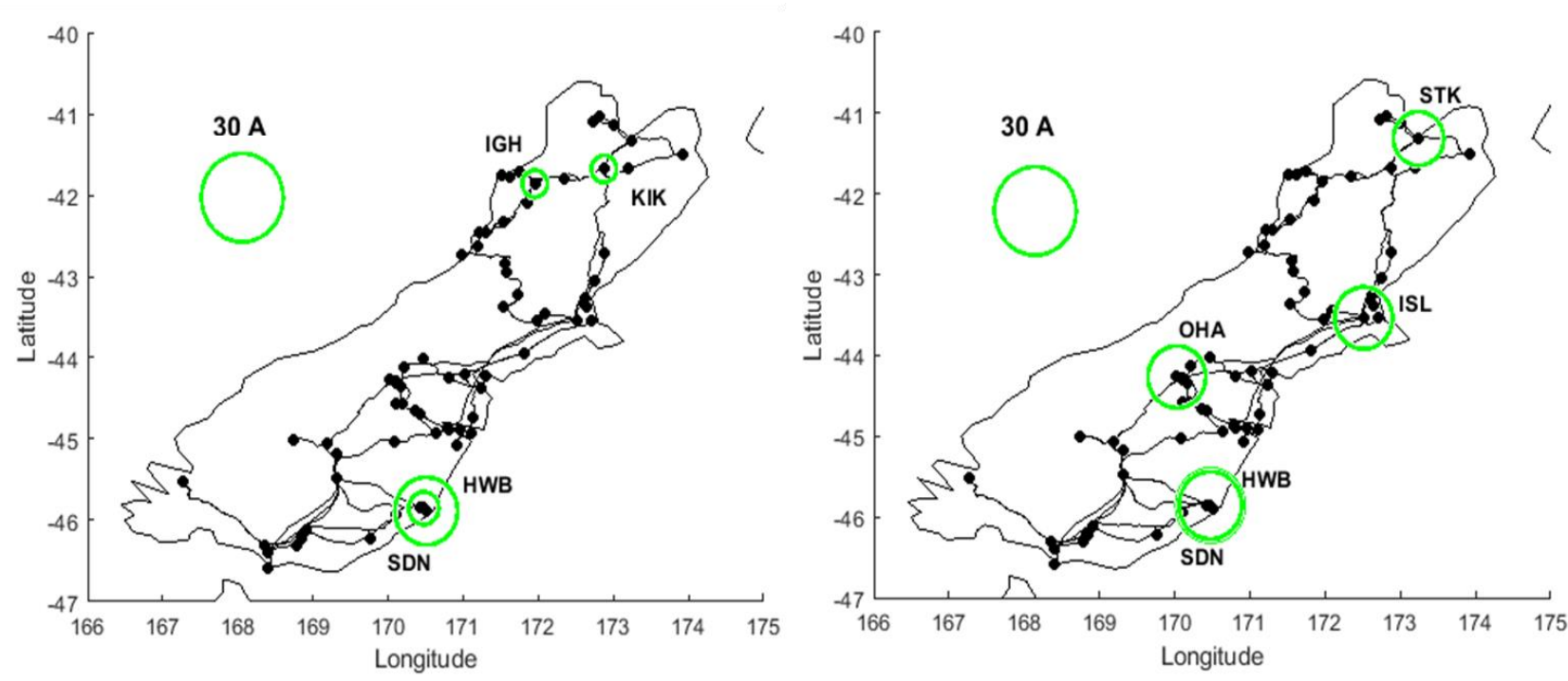

Figure 7. 8: Left panel (red circles) shows transformers experiencing largest GIC whereas right panel shows (blue circles) substations experiencing largest GIC. The size of the circle on scale of maximum GIC magnitude.

\subsection{MT Model Comparison with Measured GIC}

The model GIC calculated using the MT data has been compared with one-minute interpolated measured GIC at Islington transformer T6H. We can only compare with Islington as the Islington transformer data is the only reliable data available during this year of the solar cycle 23. Although GIC observations of Ohau (OHA) substations were also available they are not reliable enough to be compared with model GIC. 
Figure 7.9a shows individual time domain plots of both measured and model GIC for ISL-T6H. The measured GIC have been filtered in the period range of 2-30 minutes as that is the period range of the MT sites near Islington. The time variations of the model GIC are close to those of the measured data, but the model GIC have very small magnitude compared to the measured GIC. The maximum GIC value in the measured GIC is $\sim 8 \mathrm{~A}$ whereas for the model GIC it is $\sim 3 \mathrm{~A}$. Figure $7.9 \mathrm{~b}$ shows the measured (red) and model (black) GIC on the same plot. The close-up view in the lower panel shows that, although of smaller magnitude, the model effectively reproduces the measured time variations. The correlation coefficient is 0.83 and performance parameter is 0.29 . The same thing is seen in a frequency domain through a spectral comparison of measured and model GIC in Figure 7.9c. The measured GIC show large amplitudes of frequency components compared to the model GIC.
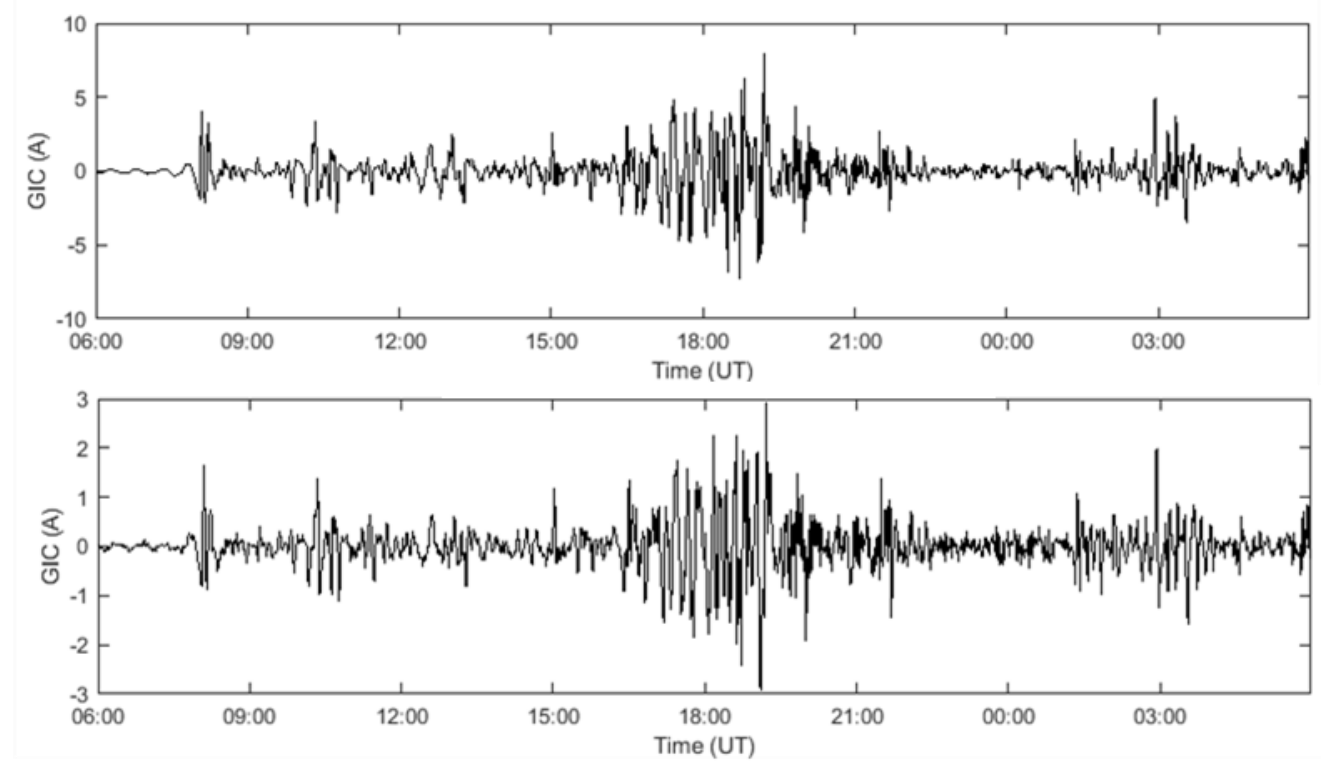

Figure 7. 9a: Plots of measured (upper panel) and model GIC calculated using MT data (lower panel) of transformer-T6H at Islington. 

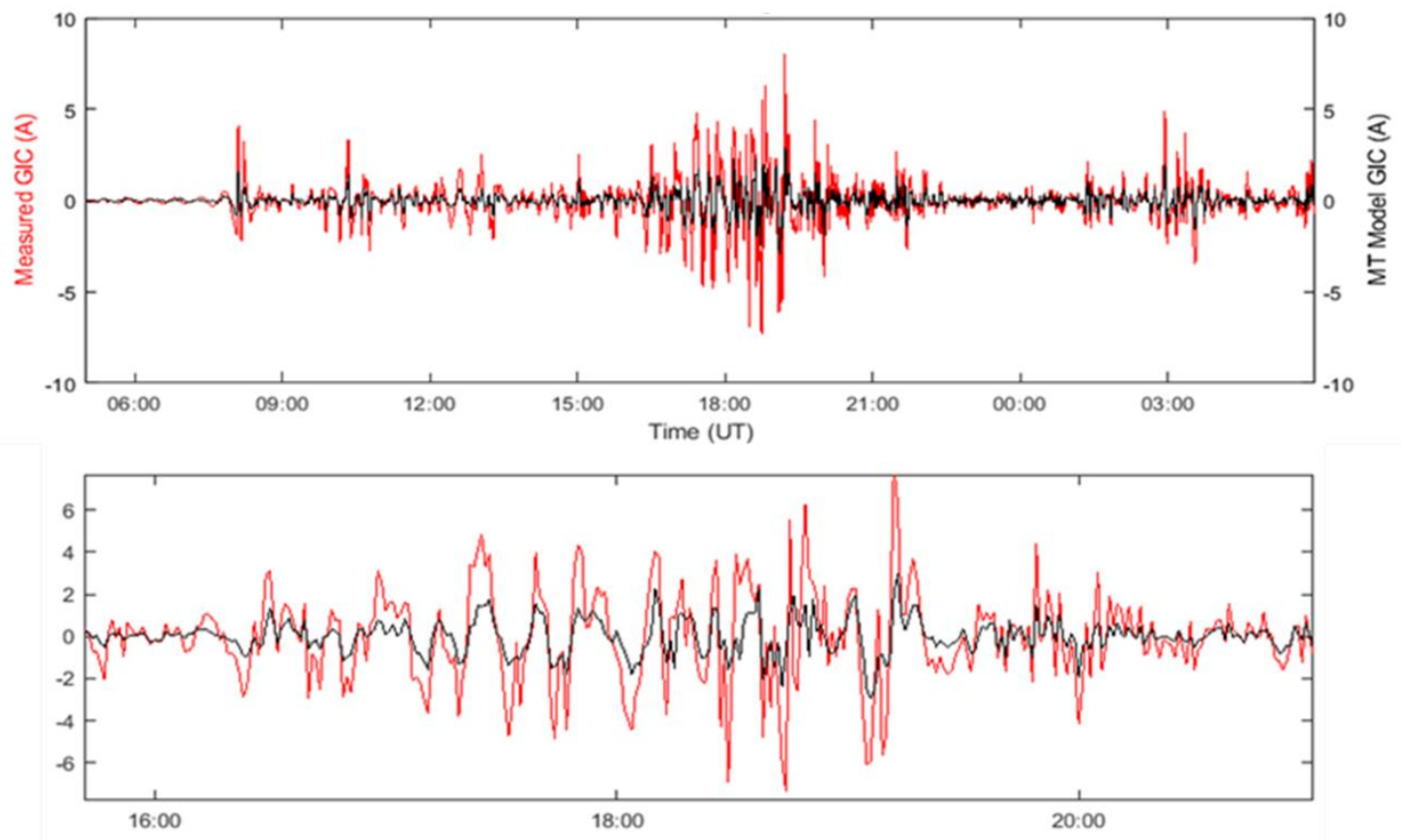

Figure 7.9b: A comparison plot of measured (red) and model (black) GIC at Islington T6H. The bottom panel shows a zoom view during the main phase of the magnetic storm ( $r=0.84)$.

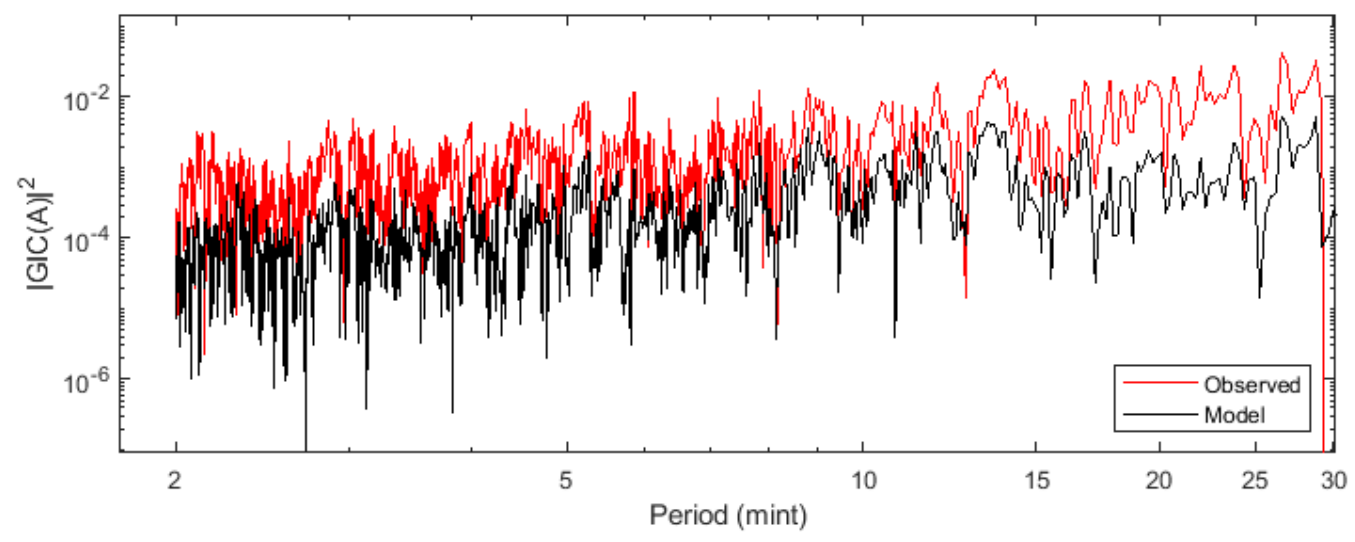

Figure 7.9c: A spectral comparison of measured and model GIC.

\subsection{Thin-Sheet Model Geoelectric Field}

Geoelectric fields can also be derived using the thin-sheet conductance model as was discussed in detail by Divett et al. $(2017,2018)$ using the thin-sheet technique of Vasseur and Weidelt (1977). In this section geoelectric fields derived from the thin-sheet model have been treated in same way as was done for those calculated from the MT data. Figure 7.10 shows a plot of north 
and east components of geoelectric field at the same location in Otago for which MT geoelectric fields were shown in Figure 7.1. The thin-sheet geoelectric fields show a strong amplitude during the main phase of the magnetic storm when the maximum magnitude reaches around 300 $\mathrm{mV} / \mathrm{km}$ at 18:32 UT. This amplitude value is much larger than observed during the St. Patrick's Day magnetic storm. The thin-sheet fields also show the existence of strong low frequency components, although high frequency components are not as evident.

To visualize the spatial distribution of geoelectric fields at 1844 and 1847 UT, as the geoelectric fields can be computed at each cell of the conductance grid using the thin-sheet conductance model Figure 7.11 shows the thin-sheet E-field vectors for all grid cells in the South Island. The Figure shows that the thin-sheet fields are smooth both in magnitude and direction, and, unlike the MT derived geoelectric fields, do not show substantial variations in different conductance regions. The temporal variation also does not change significantly as can be seen in Figure 7.11. One reason for this is because the thin-sheet geoelectric fields are calculated in a longer period range.

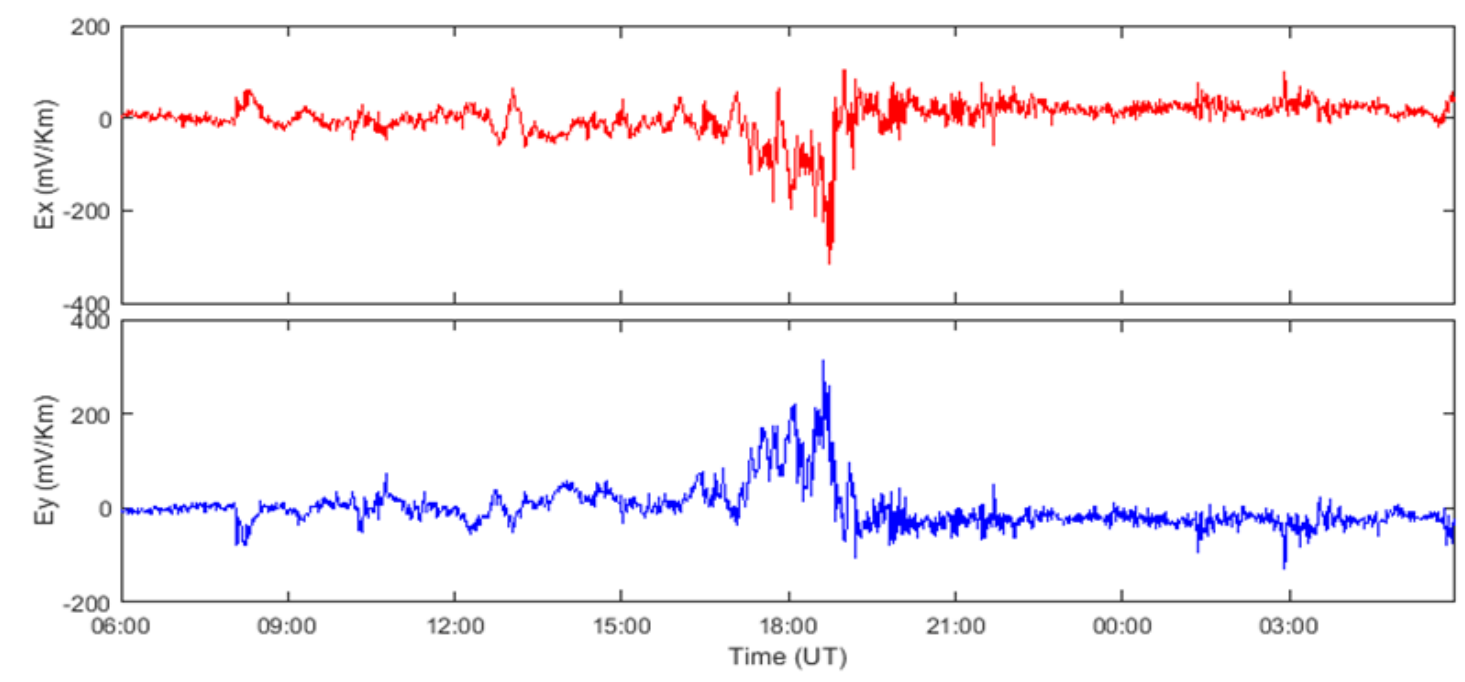

Figure 7. 10: TS computed horizontal components of the geoelectric field for a site in Otago during the magnetic storm of 2003. 

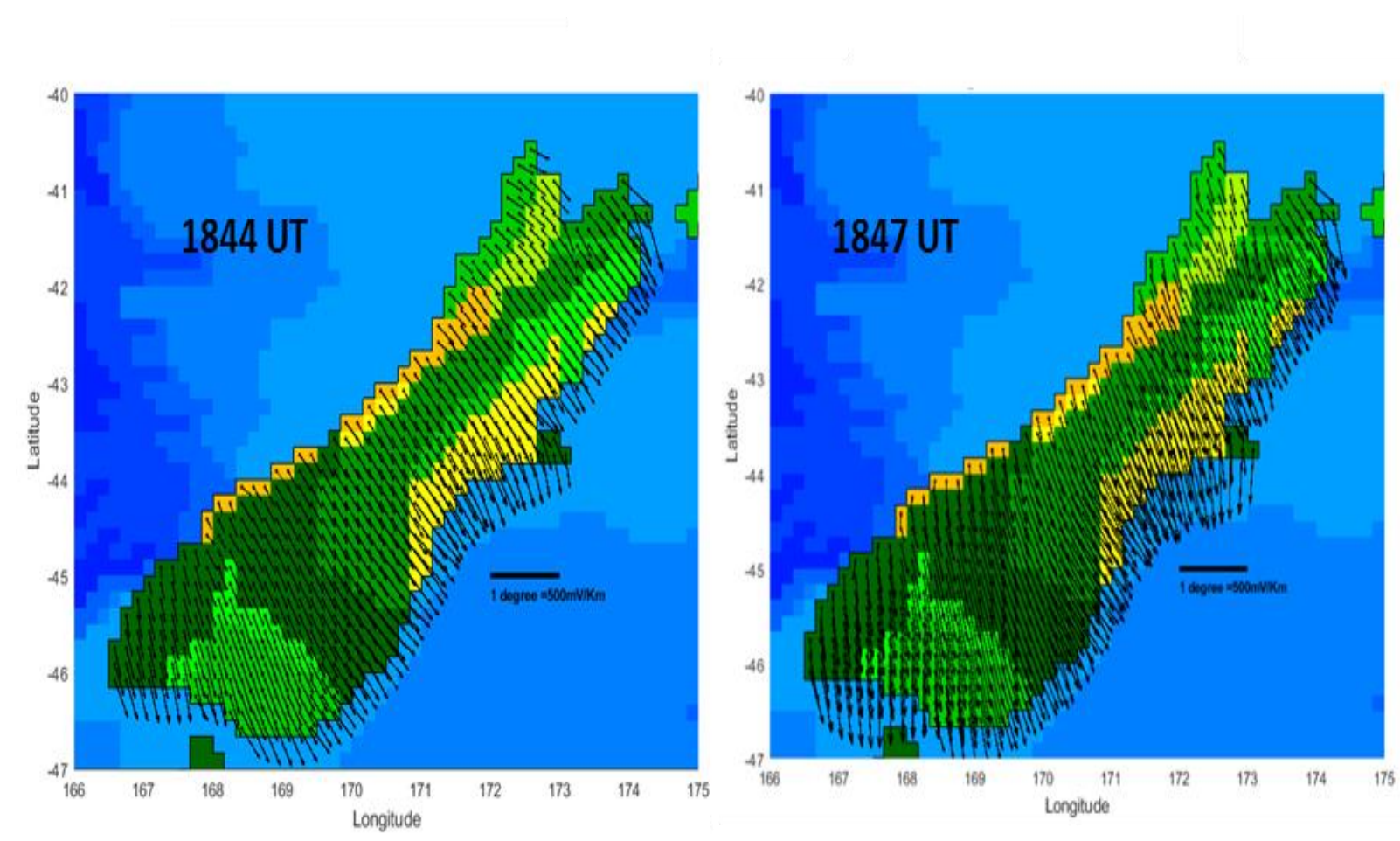

Figure 7. 11: Thin-sheet geoelectric field vectors on conductance map of the South Island at 1844 and 1847 UT, respectively.

\subsection{Transformer Level GIC Modelling Using the Thin-sheet Model}

Thin-sheet geoelectric fields have been used to compute GIC in the power network of the South Island. Figure 7.12 shows a snapshot of transformer-level GIC computed using the thin-sheet fields given in Figure 7.11. The transformer response is almost the same at both times. For example, South Dunedin (SDN) transformer has the largest GIC of 14.18 and 16.39 A, respectively. At 1847 transformers at Tiwai (TWI) and Stoke (STK) show an increase in their GIC values compared to for the MT model GIC. This is primarily because of the longer period range of thinsheet geoelectric fields compared to the shorter period range of the MT data.

Using one-minute discrete geoelectric field of total 2048 data points time GIC has been computed for all transformers in the South Island. The following four transformers have been identified as experiencing the largest GIC

1. South Dunedin (SDN-T2H)- $(-45.89,170.50)$

2. Halfway Bush (HWB-T6L)- $(-45.85,170.47)$ 
3. Tiwai (TWI-T2H)- $(-46.57,168.39)$

4. Kikiwa (KIK-T2H)- $(-41.67,172.87)$
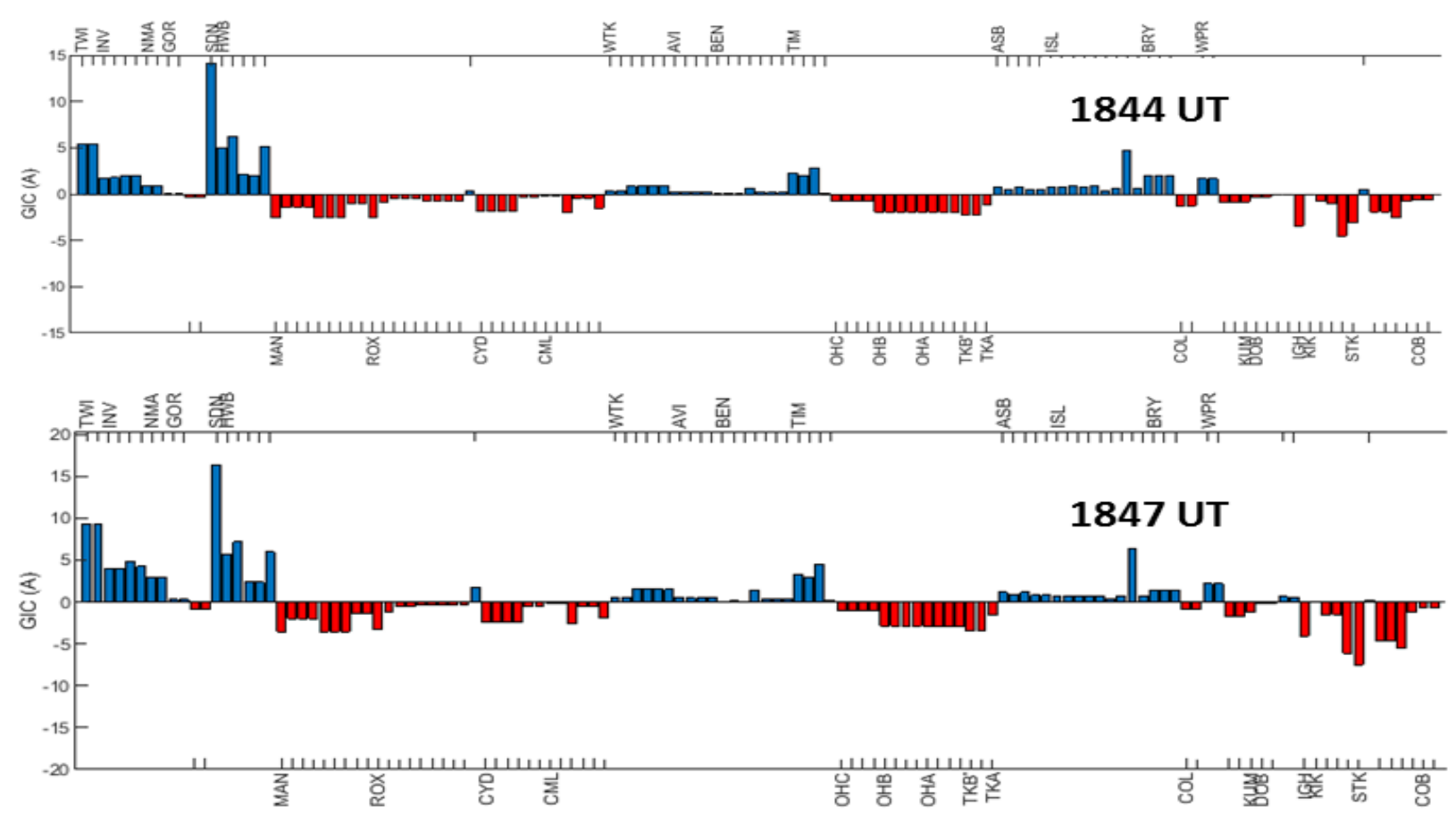

Figure 7. 12: Transformer GIC at 1844 and 1847 UT respectively using TS geoelectric fields.

South Dunedin transformer-T2H experiences the largest GIC in the whole South Island network using the thin-sheet model. These are almost the same results as were predicted using the MT model except that for the thin-sheet model except that Tiwai-T2H shows up in place of Inangahua-T1H transformer. The time plot GIC for these transformers is shown as Figure 7.13. The GIC for SDN is significantly higher, exceeding a value of $\sim 25 \mathrm{~A}$, compared to the rest of these three transformers which only show GIC less than $15 \mathrm{~A}$. 

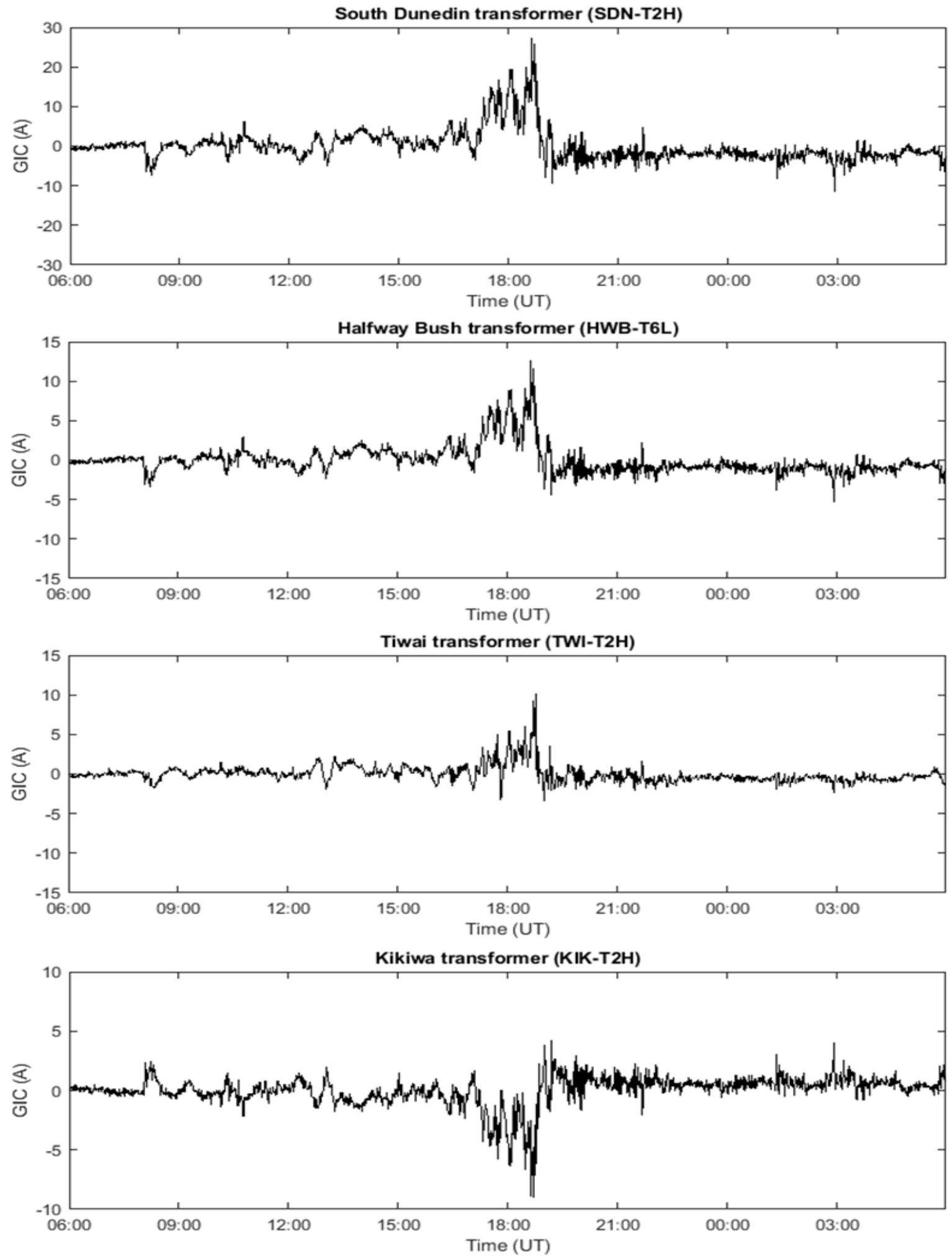

Figure 7. 13: Transformers identified (as shown in top of each panel) experiencing large GIC during the magnetic storm of 20 November 2003 using TS fields. The existence of low frequency components is quite evident using the TS model. 


\subsection{Substation GIC Using the Thin-sheet Model}

The snapshot of substation GIC for the thin-sheet geoelectric fields (1844 and 1847 UT) is shown in Figure 7.14.

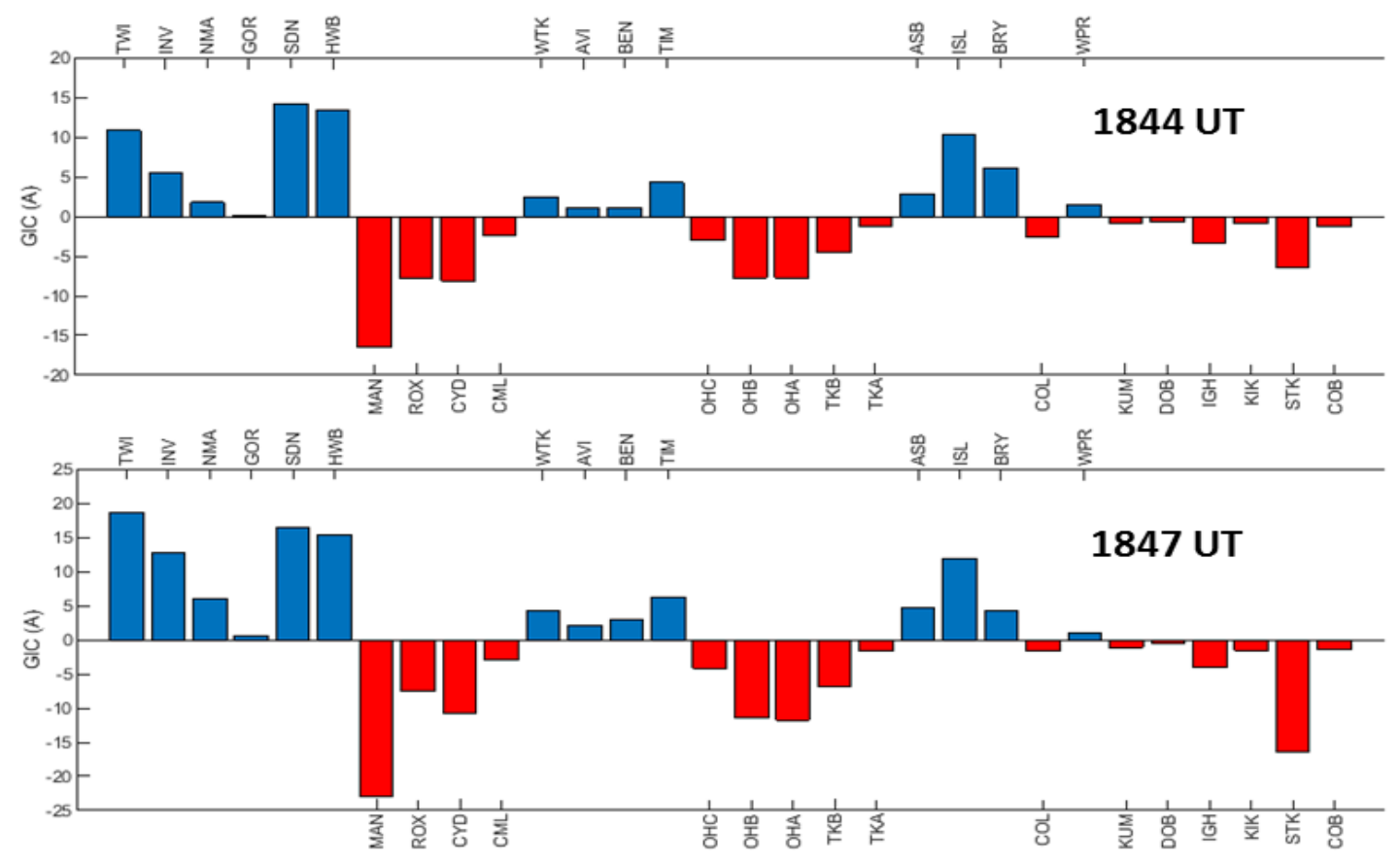

Figure 7. 14: Substations identified as experiencing large GIC during the magnetic storm of 20 November 2003 using TS geoelectric fields.

Using the one-minute discrete geoelectric fields for whole duration of the magnetic storm the following substations show the largest GIC using the thin-sheet model

1. Manapouri (MAN)- $(-45.52,167.28)$

2. South Dunedin (SDN)- $(-45.89,170.50)$

3. Halfway Bush (HWB)- $(-45.85,170.47)$

4. Islington (ISL)- $(-43.53,172.51)$

These differ slightly from the MT modelling results. For example, although SDN, HWB and ISL are the same, Manapouri substation does not show up in the MT model GIC. One reason for this is 
that, due to lack of MT data, MT geoelectric fields were interpolated/extrapolated as being very small around Manapouri.

\subsection{Comparison of ISL Measured and Thin-sheet Model GIC}

The modelled thin-sheet transformer GIC at Islington (T6H) are compared with the measured ISL data for the 20 November2003 storm. The individual plots of model and measured GIC are shown as Figure 7.15a. Since the model GIC are very small in magnitude compared to the measured data they are shown on different scales in Figure 7.15b. The model and measured GIC time variations are very similar to each other during most of the time period except around 1735 UT when the model amplitude is small while the measured GIC reaches its maximum value. There is clear discrepancy in magnitude between the model and measured data with the measured GIC having a largest value of -12.48 whereas the largest model value is only $-2.3 \mathrm{~A}$. ISI-T6H is not the transformer in Islington substation showing the largest GIC as T6L shows the largest GIC for which the value reaches to $-8.18 \mathrm{~A}$. The correlation coefficient is 0.84 and performance parameter is 0.13 between measured and model GIC.
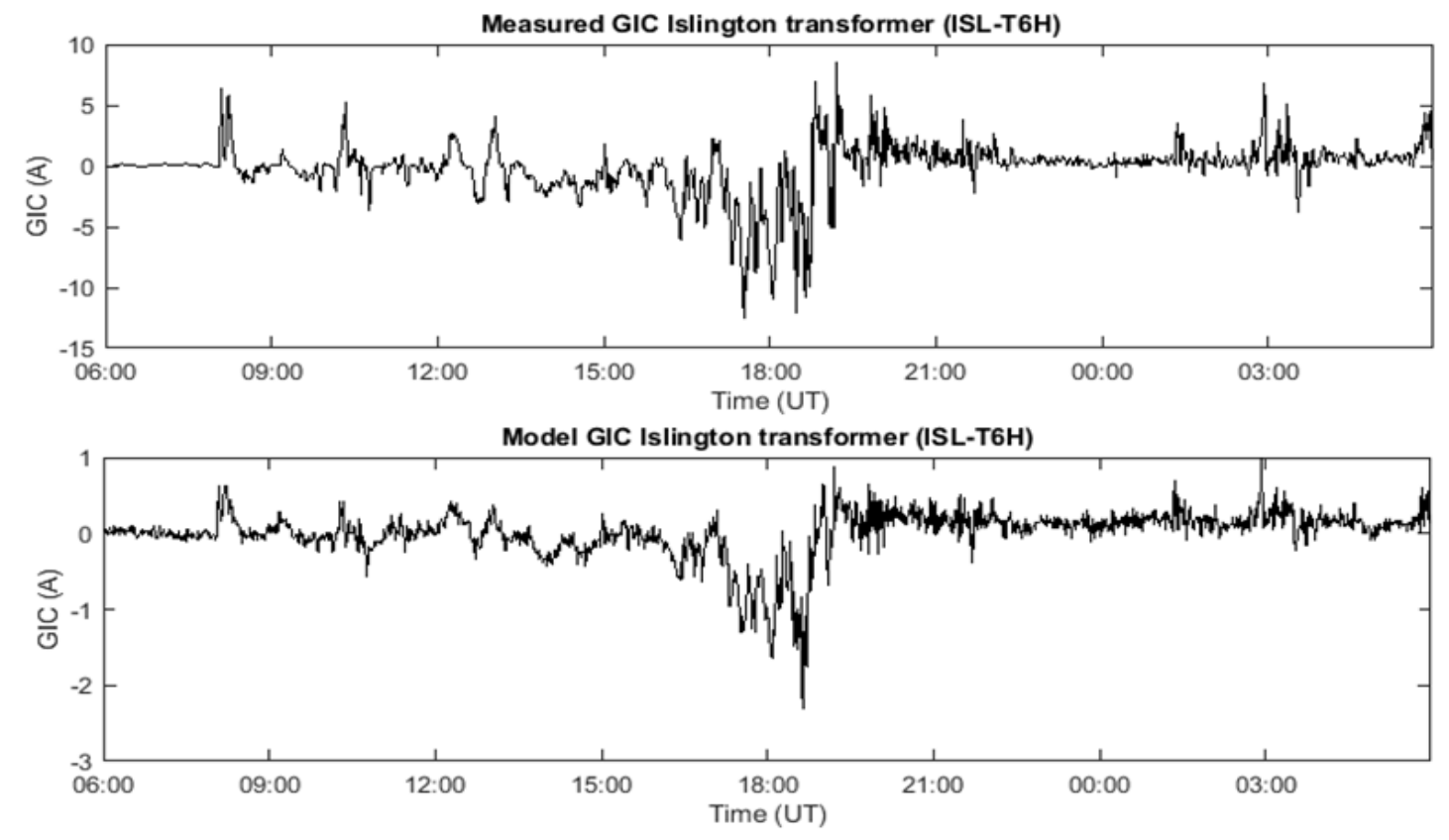

Figure 7. 15a: Individual plots of measured (top panel) and model (bottom panel) GIC of Islington transformer (ISL-T6H). 


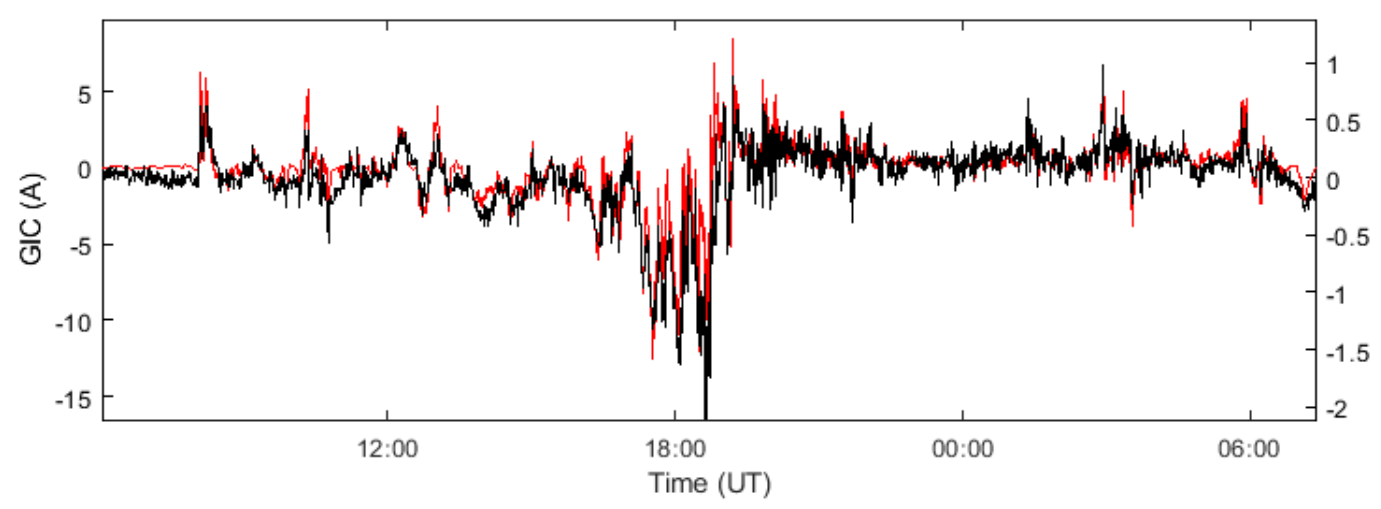

Figure 7.15b: Comparison of measured (red) and thin-sheet model (black) GIC. Although, model GIC has very small magnitude as compared to measured data, but it effectively reproduces variations particularly in longer period range $(r=0.84)$.

\subsection{Discussion}

Chapter 6 showed GIC modelling results using MT data and the thin-sheet conductance model for the South Island for the St. Patrick's Day storm of 2015, and in this chapter the same idea has been extended to model GIC for the 20 November2003 magnetic storm.

In the context of transformers experiencing large GIC both techniques (i.e., MT and TS) give almost the same results. These include three transformers in Otago/Southland: South Dunedin (SDN-T2H), Halfway Bush (HWB-T6L) and Tiwai (TWI-T2H). Two transformers further north, Kikiwa (KIK-T2H) and Inangahua (IGH-T1H) are susceptible to magnetic storms. South Dunedin experiences the largest GIC of magnitude 24.7 and 27.9 A in MT and thin-sheet modelling results, respectively. In all other transformers the largest GIC values are in the range of 10-15 A. These are also the same transformers that showed large GIC during the St. Patrick's Day storm. Thus, the transformers experiencing large GIC results do not vary much between magnetic storms or using either of the modelling techniques. The scale of magnitude is also the same in both modelling techniques which is a different result from the previously considered magnetic storm. The reason for this is attributed to the existence of strong low frequency components in the measured GIC and the fact that the thin-sheet model works well in the low frequency range. Since there were few very rapid variations as occurred in the St. Patrick's Day storm the MT data gives almost the same results as the thin-sheet model. 
The MT model results have been compared with the observed GIC data for ISL-T6H transformer in the period range of 2-30 minutes. The model GIC have smaller magnitude compared to observed GIC e.g., model GIC have a maximum magnitude of 3 A while the measured GIC reach $8 \mathrm{~A}$. Despite the small magnitude, the model variations match well with the observed data particularly during the main phase of the magnetic storm, with correlation coefficient and performance parameter of 0.83 and 0.29 respectively. The interpolated geoelectric fields are possibly unrealistically small in the region of Islington substation and it is anticipated that more MT measurements might lead to a better prediction for Islington transformer GIC. The thin-sheet model also effectively reproduces time variations during the main phase of the magnetic storm with a correlation coefficient of 0.84 and performance parameter of 0.13 . This shows that the thin-sheet model works well at lower frequencies when the GIC spectrum of observed data shows the existence of strong low frequency components. Unlike the thin-sheet results for the St. Patrick's Day magnetic storm, the model results are much better when compared with ISL transformer, during the magnetic storm of 20 November 2003. 


\section{Chapter 8: Discussion and Conclusions}

The principal aim of the work described in this thesis has been to compare two potential modelling techniques for assessing the risk to New Zealand's power transmission network posed by GIC. The availability of a long record of measured GIC data at multiple locations in the South Island provides an opportunity for models of GIC to be assessed against actual measurements. Previous such modelling (Divett et al., 2017, 2018, 2020) has been based on the thin-sheet technique of Vassuer and Wiedelt (1977) and has concentrated solely on the South Island. The work discussed here has looked at the following techniques.

(1) Single site modelling of GIC at Islington (ISL) near Christchurch, the location with the longest record of GIC measurements.

(2) The use of magnetotelluric (MT) impedance tensor data to assess the likelihood of significant GIC in the North Island power network.

(3) Similarly, the use of MT data to model GIC in the southern part of the South Island.

(4) Thin-sheet (TS) GIC for both the North and South Islands and comparison with the MT GIC models.

Discussion and critical analysis of these aspects follows in the following sections.

\subsection{Single Site Modelling at ISL}

The GIC record for T6 transformer at Islington (ISL) is the longest record of measured GIC in New Zealand. A single station modelling approach has been followed to model ISL GIC using the measured GIC at ISL and measured magnetic data from Eyrewell (EYR) geomagnetic observatory during major geomagnetic storms. In this retrospective approach, time domain geoelectric fields have been determined using a model of uniform conductivity, and the network constants $(a, b)$ (equation 3.5) have been determined using linear regression relation. The network constant 
values essentially remained stable during all the magnetic storms considered, showing that the average values of these constants could be used to model GIC effectively, given a knowledge of magnetic data and assuming there are no changes to the transmission network. However, a change in sign of the network constants between 2002-2006 and 2011-2015 was noted. It is believed that this results from a change in the local network configuration.

A discrepancy in spectral content (i.e., the relative amplitudes of low and high frequency components) was also seen during many of geomagnetic storms with a dominance of high frequency components for many geomagnetic storms during 2011-2015 which was not observed during 2002-2006. No such significant change in spectral content in the Eyrewell (EYR) magnetic observatory data was seen. Calculation of slope values of the power spectrum showed that the amplitudes of high frequency components of GIC measurements increased during 2011-2015, whereas no such change was seen in the magnetic field power spectra (Figure 3.6 and 3.7). These differences in spectral content meant that the use a single set of network constants did not provide equally satisfactory modelling of measured GIC for the whole dataset. To develop a better model for all magnetic storms two sets of network constant $\left(a_{1}, b_{1}\right)$ and $\left(a_{2}, b_{2}\right)$ have been used for the low and high frequency components, respectively. Periods shorter than 13 minutes are considered as high frequencies with the longer periods taken as low frequencies. Using this approach, model GIC reproduce the measured GIC for all years regardless of the dominance of spectral components. The accuracy of model GIC was determined using the correlation coefficient $(r)$ and performance parameter $(P)$ (Torta et al., 2014) over the whole duration of each magnetic storm. This model gives a correlation coefficient of greater than $70 \%$ for all magnetic storms. Although the single station model reproduces an overall variation in GIC seen in the measured data, it does not reproduce extreme GIC values during many of the geomagnetic storms. This shows the limitation of the single station model where the linear constants $(a, b)$ give the average response of the network to geoelectric fields and underestimate extreme GIC values. Geoelectric fields calculated using magnetotelluric response tensors for different substations gave very different fields to those calculated from uniform conductivity models. This, and the improvement in modeling GIC but using different network constants for different frequency bands, suggests that more detailed conductivity models or the use of MT determined geoelectric fields should be used for further GIC modelling. 
To see if the spectral discrepancy is local and only exists in ISL GIC data, GIC data from other substations (SDN, HWB and INV) were explored. This suggested that the spectral discrepancy seen in ISL GIC data is local and does not prevail in other substations. This reinforces the assumption that the changes between 2002-2006 and 2011-2015 are due to local changes in the network. It was also seen that the spectral content of measured GIC at INV, HWB and SDN is significantly different from that at ISL. This shows that consideration of the full network is an important aspect of GIC modelling.

Four primary interplanetary structures, ICME, SH+ICME, SH and CIR were identified as resulting in magnetic storms during Solar Cycles 23 and 24. The spectral discrepancy in the ISL GIC could not be associated with any of these interplanetary structures. For example, ICME dominated during 2002-2006 whereas SH+ICME dominated during 2010-2016, but GIC spectral components could not be associated with these dominating structures. Among solar wind parameters hourly mean values of solar wind electric field $\left(E_{y}\right)$ and IMF- $B$ field showed some correlation with hourly maximum GIC at ISL. Solar wind proton density and flow pressure had lower correlation whereas solar wind speed and temperature showed no correlation. ISL GIC also showed that the southward $B z$ component has a critical role, being associated with $\sim 75 \%$ of large GIC ( $\geq \pm 4 A)$ during 20 major geomagnetic storms in 2002-2015.

\subsection{Modelling North Island GIC}

Previously, to determine GIC at different substations a thin-sheet conductance model has been used to determine the geoelectric field in the South Island using the magnetic field data from Eyrewell (EYR) observatory. However, geoelectric fields derived from the thin-sheet conductance model have limitations both in spatial variations and the frequencies involved in producing large GIC. Apart from these numerical constraints, most of conductivity models on which the conductance model is based do not include the effects of near-surface distortions. Therefore, geoelectric fields computed by directly using the magnetotelluric response tensor are expected to give more realistic geoelectric fields in response to magnetic variations. This was demonstrated by showing thin-sheet and MT computed geoelectric fields for a single frequency both for the North and the South Islands (Figure 2.15 and 2.17). As MT data are generally of limited frequency range, varying from survey to survey, the computation of geoelectric fields in 
the vicinity of a power network and model GIC would be restricted to the period range permitted by MT data. A dense array of magnetotelluric data would also be required.

The North Island has many MT measurements, consisting of 115 sites in grid cells in the thinsheet model, at different locations. These are largely limited to of the southern and eastern parts of the North Island while the north-western part has few sites (Figure 4.1). To use MT data to model GIC for the North Island network an equivalent circuit approach (Boteler et al., 2013) was followed by considering the northern part of the network as a neighboring network. This was done by earthing all substation connection near Huntly (HLY) substation so that no GIC can flow between neighboring networks. To fill the gaps between MT sites a Nearest Neighbor interpolation was used (Figure 4.9). This enabled GIC to be modelled for the lower North Island substations and transformers for both the St. Patrick's Day Storm of 2015 and for a storm in November 2003. It was demonstrated for the St. Patrick's Day storm that large GIC in the period range of 2-30 minutes showed at two transformers, Redclyffe (RDF-T1H) and New Plymouth (NPL-T8L). Kawerau (KAW-T12H) and Stratford (SFD-T10H) also showed significant, but smaller, GIC. The thin-sheet GIC model showed the same transformers as experiencing large GIC but the magnitude of GIC was significantly smaller than given by the MT model, particularly during the Sudden Storm Commencement (SSC).

In terms of substation-level GIC the MT and thin-sheet models results were slightly different. While the MT model showed NPL, RDF, WHI, WRK and KAW as experiencing large GIC the thinsheet model shows NPL, RDF, BPE and HAY. Apart from that substation GIC contains information on GIC from all earthed transformers at the substation, the primary reason is also that frequency range of both models is not the same.

The model GIC at Redclyffe (RDF) substation has been compared with measured GIC at ISL. The two substations, although in different islands, have similar network configurations and magnetic data from same magnetic observatory was used. ISL data showed the largest GIC as occurring during SSC when the GIC value is quite high -9 A. Through the rest of the storm the GIC stayed less than \pm 5 A. The MT model GIC for Redclyffe transformer (RDF-T1H) matched well with the ISL GIC showing that the model effectively reproduces the observed GIC, particularly during the SSC. The striking feature of the MT model is that it effectively reproduced GIC during the SSC when 
GIC are many times larger compared to the rest of the storm period. This feature was not evident in the thin-sheet GIC which did not have substantial GIC during the SSC but did have larger, varying, GIC during the main phase of the magnetic storm.

ISL GIC data for the St. Patrick's Day magnetic storm only showed a strong peak during the SSC therefore another geomagnetic storm, that of 20 November 2003, was considered depicting different GIC spectral variations. This storm shows strong fluctuations during the main phase of storm demonstrating the existence of strong low frequency components. Both the MT and thinsheet models showed the same transformers as experiencing large GIC as for the St. Patrick's Day Storm, suggesting that the same North Island transformers are likely to experience large GIC during magnetic storms.

The thin-sheet model GIC for this storm also effectively reproduced the low frequency components seen in the measured data from ISL. This suggests that the thin-sheet model works well for lower frequencies compared with the MT model. This is consistent with the valid frequency range of thin-sheet model. The MT modelling results, which are also limited in frequency range, give promising comparisons with measured GIC, and are much better than the thin-sheet model in the high frequency range.

In terms of substation-level GIC, the MT modelling technique showed differences between the St. Patrick's Day storm and the 2003 storm. For the 2003 magnetic storm substations in lower North Island (e.g., Bunnythorpe) experienced large GIC.

The following principal conclusions regarding GIC in the North Island can be drawn. Firstly, the MT model shows significant GIC as occurring at several substations in the lower part of the North Island during the St. Patrick's Day Storm during the SSC. Both the MT and thin-sheet models show that calculated GIC resulting from the 20 November 2003 storm may be experienced at a few substations. In the context of what might be expected during a major space weather event, such as has been estimated by Ingham et al. (2017) and Rodger et al. (2017), major disruption of the North Island transmission network could therefore be anticipated during extreme storms. Secondly, although the use of an equivalent circuit does not allow GIC calculation from the MT data for the northern part of the North Island network, calculation using uniform electric fields suggests that substations around Auckland, New Zealand's largest city, may also experience risk. 
As seen in the analysis of THD by Rodger et al. (2020), and supported by GIC calculated from the thin-sheet model, it is apparent that longer period variations of the magnetic field are likely to result in significant GIC which may be sustained over long periods of time.

\subsection{Modelling South Island GIC}

Although the South Island is ideal for GIC modelling because of the availability of measured GIC data the main hindrance to applying MT data to model GIC is that MT data in the South Island are relatively sparse. There are only a total 42 of MT data measurement sites in cells in the conductance model, and the MT data exist only in three main groups. However, the same MT GIC modelling technique has been applied to the South Island as model results can be compared with measured GIC. The same two magnetic storms were considered for GIC modelling using the MT and thin-sheet models. The MT and thin-sheet model results were compared with measured oneminute interpolated GIC data from SDN-T2H, HWB-T4L and ISL-T6H transformers. Although large GIC has also been seen in transformers in the northern part of the South Island e.g., KIK and IGH, the model results cannot not be compared due to no availability of measured data.

A major difference between modelling for the North Island and the South Island relates to use of an equivalent circuit. Despite the much sparser MT data in the South Island, test calculations of GIC using uniform electric fields in the South Island showed that calculations using the whole South Island network gave essentially the same results as those using an equivalent circuit for that part of the South Island north of Roxburgh (ROX). Thus, GIC were calculated for all substations/transformers even though the distribution of MT sites means that the interpolation of geoelectric fields gave very uncertain results over much of the South Island. Only in Otago/Southland is the distribution of the (few) MT sites sufficiently non-linear to lend some confidence to the interpolation.

Both the MT and thin-sheet models showed the same transformers as experiencing large GIC during the St. Patrick's Day storm. These included South Dunedin (T2H) and Halfway Bush (T6L) and two from much further north in the South Island - Kikiwa (T2H) and Inangahua (IGH-T1H). The thin-sheet model effectively reproduced the low frequency components, but the results were very poor during the SSC. The MT model was better, particularly during the SSC, but also had a higher correlation coefficient $(r)$ and performance parameter $(P)$ during the whole 
duration of the magnetic storm. The MT GIC at SDN-T2H matched best with the observed data both in magnitude and in terms of time variations. The MT model GIC for Islington transformer (ISL-T6H) had much smaller magnitude compared to observed GIC. Despite the small magnitude the model variations matched well with the observed data during the whole duration of the magnetic storm. It is anticipated that improved MT coverage would lead to better prediction for Islington transformer GIC. At Halfway Bush (HWB) transformer HWB-T4L showed the largest observed GIC among all transformers but neither the MT nor thin-sheet models could reproduce such large GIC. However, measured GIC at South Dunedin are well modelled. South Dunedin and Halfway Bush substations are very close to each other and in the same grid cell of the thin-sheet map and are therefore given the same geoelectric field in the model calculation. The discrepancy in HWB model GIC results is suspected to be related to some difference in network parameters over time which needs to be further explored. The thin-sheet model GIC also gave smaller magnitudes than observed during the SSC. Both model results therefore disagreed with measured data both for ISL and HWB transformers.

In terms of substation GIC, substations consisting of a large number of earthed transformers, showed large GIC. These included Islington (ISL) and Manapouri (MAN), along with South Dunedin (SN) and Halfway Bush (HWB) which had the largest transformer GIC.

For the 20 November2003 magnetic storm, in the context of transformers experiencing large GIC both techniques (MT and thin-sheet models) gave almost the same results as for the St. Patrick's Day storm. These included three transformers in Otago/Southland - South Dunedin (SDN-T2H), Halfway Bush (HWB-T6L) and Tiwai (TWI-T2H) - and two transformers further north - Kikiwa (KIK$\mathrm{T} 2 \mathrm{H}$ ) and Inangahua (IGH-T1H). Both the MT and thin-sheet models gave the largest GIC for SDN transformer Thus, the transformers experiencing large GIC results did not vary much between magnetic storms and using either of the modelling techniques. The scale of magnitude of GIC was also the same for both modelling techniques. This was a different result from the St. Patrick's Day storm and is attributed to the existence of strong amplitudes of low frequency components seen in measured GIC, and the fact that the thin-sheet model works well in the low frequency range as is permitted by the numerical constraints on the thin-sheet conductance model. 
The MT model results were compared with measured GIC at ISL-T6H transformer. Again, apart from the scale difference in both model and measured GIC, the MT model effectively reproduced the model GIC. The thin-sheet model also effectively reproduced the time variations during the main phase of the magnetic storm. This emphasized that the thin-sheet model works well at lower frequencies for which the GIC spectrum of observed data shows the existence of strong low frequency components. Unlike the thin-sheet results for the St. Patrick's Day magnetic storm, the model results are much better when compared with ISL transformer during the magnetic storm of 20 November2003.

An interesting aspect of the use of the network model is that large currents generally occur simultaneously in different parts of the network producing large GIC. For example, during both considered storms, transformers experiencing the largest GIC in the southern part of the South Island are South Dunedin (T2H) and Halfway Bush (T6L), while in the northern part Kikiwa (T2H) and Inangahua $(\mathrm{T} 1 \mathrm{H})$. A similar pattern of observation can be made for the North Island network. This demonstrates the dependence on the complete network configuration such that transformers can experience large GIC regardless of the local geoelectric fields.

A major difference of magnitude between the model and measured GIC for Halfway Bush (HWBT6L) and Islington (ISL-T6H) has been seen. Both MT and thin-sheet model geoelectric fields gives comparatively very small GIC magnitude in both these transformers when compared with the measured GIC, although time variations are well modelled, giving a high correlation coefficient and poor performance parameter. These differences at HWB have also been discussed by Divett et al., (2020) in terms of both geoelectric fields and network parameters, where it was pointed out that the SSC is modelled poorly by the thin-sheet model due to the limited range of valid frequencies in the thin-sheet model. For ISL-T6H, the difference is believed to be due to poor MT data interpolation of geoelectric fields around ISL. This needs to be explored further.

\subsection{Comparison with GIC studies in Other Countries}

The GIC modelling for the North Island provides the opportunity to compare results with those found for other island nations, for example Japan, the United Kingdom and Ireland. Such comparison needs to take into consideration that both the North Island of New Zealand and Japan lie at mid-latitude (35-41 S for New Zealand, 30-45 N for Japan), while the British Isles are 
at higher latitudes where space weather impacts have been explored more widely. It is also important that quite apart from the geographic position of the network, the conductivity structure of the local ground and topology of the network are unique factors controlling the magnitude of the GIC in a network. For example, the largest GIC for the North Island calculated using MT data during the two storms studied are $10 \mathrm{~A}$ and $40 \mathrm{~A}$ for New Plymouth. Similar kinds of geomagnetic storms in the Irish network are calculated to give significantly smaller GIC even though the network lies at higher latitudes. The Irish network is also comparatively simpler than North Island network having different line orientations and network topology. Thus, a real comparison of modelling results for the North Island with those for other networks is not possible. However, some GIC modelling features identified in other studies can be seen to be closely related to North Island GIC results, and these are discussed below.

1. North Island GIC modelling shows that large GIC mostly occur at the corners or end points of the network, for example at New Plymouth, Redclyffe and Bunnythorpe. These results are consistent with the general trend of large GIC in many networks not only for island nations e.g., Japan (Nakamura et al., 2018), UK (Beggan et al., 2015) and Ireland (Blake et al., 2016)., but also in locations such as in Scandinavia (Wik et al. 2008, 2009; Mylyss et al., 2014).

2. Substations experiencing large GIC in a network are not necessarily the same during two different magnetic storms as shown by the North Island GIC results for the St Patrick's Day 2015 and the 20 November 2003 magnetic storms. Similar kinds of results have been seen by Blake et al., (2016) in the Irish network for two different storms.

3. The impact of the local geoelectric field has been explored by use of magnetotelluric data and compared with the more uniform geoelectric fields resulting from thin-sheet modelling. This shows that the more detailed, and uneven, distribution of geoelectric fields has a profound impact on the pattern of GIC in the North Island network. This result is consistent with that found by Nakamura et al. (2018) in part of the Japanese.

4. Although it has been seen that large GIC tend to occur at end points of a network, this is not always the case. For example, the use of thin-sheet modelling has shown that large GIC do not appear at substations in the extreme north of the North Island network such as Silverdale (SVL), Marsden (MDN) and Bream Bay (BRB). Substations experiencing large GIC lie south of these 
corner substations and in a denser part of the network e.g., Huntly (HLY), Penrose (PEN) and Henderson (HEN). These results are again consistent with results for the Japanese network where a few long, extended transmission lines are not seen to produce large GIC at end substations compared to in denser parts of the network (Nakamura et al., 2018).

5. Although a similar kind of analysis can also be conducted for the South Island, the limited MT data of the South Island does not allow the kind of results as have been discussed for the North Island. South Island MT data is only sparse, but interpolation results down southwest coast near Manapouri are very poor. Therefore, substation level model GIC results using MT data are not reliable. However, the substations identified experiencing large GIC are consistent with other network GIC results as discussed above for the North Island. Thin-sheet model results shows substations experiencing large GIC lie at the corners of the network, including Manapouri (MAN), South Dunedin (SDN), Halfway Bush (HWB) and Islington (ISL).

\subsection{Summary and Suggestions}

The magnetotelluric GIC modelling technique has shown promising results both for the North and South Islands networks and performs better than the thin-sheet technique particularly at higher frequencies. This study has identified some steps that should be addressed to evolve the MT model for better results. These points may be helpful in further GIC modelling. Firstly, a dense array of MT data in the vicinity of power network/transmission lines is necessary to capture the geoelectric fields effecting the network. This is more important in areas with no previous MT survey, and in locations with abrupt conductivity contrasts or adjacent to the land-ocean interface. However, the use of the equivalent circuit technique to represent different parts of the network means that it may be unnecessary to have complete coverage with MT sites. Further investigation of areas where this technique can and cannot be applied successfully is needed.

An implication of the limited period range of the MT data, and the potential ability of the thinsheet model to better predict longer period GIC, is that improved broadband modelling of GIC might be achieved by combining the two techniques into a single prediction. The limitations of these both techniques have been discussed by modelling GIC during two different magnetic storms i.e., the St Patrick's Day storm and the 20 November2003 storm. The magnetotelluric data derived GIC is largely restricted to the shorter period range, mostly 2-30 minutes, and therefore 
model results cannot be compared fully with the measured data. Similarly, the validity of thinsheet results is in comparatively longer periods and thus the thin-sheet model can only be compared with measured GIC in the longer period range. Combining the geoelectric fields derived by the individual techniques would give geoelectric fields across a broader period range which could be used to model GIC. The computed GIC can then be compared to measured GIC over a broader range than for the MT and thin-sheet techniques separately. This approach seems a straightforward solution to modelling GIC over a broad period range. Where longer period range measurements are available for MT data geoelectric fields calculated from these could also be compared with thin-sheet geoelectric fields. If there is found to be consistency in the fields, gaps in the MT data at other sites could be filled by taking into account impedance tensor estimates calculated from the thin-sheet model. Implementation of this approach should be explored in future studies. As the thin-sheet models show that GIC with longer periods may be important future MT surveys should be extended to significantly longer periods.

A uniform magnetic field based on the Eyrewell (EYR) magnetic observatory data was used in GIC modelling for both for North and South Islands. It is likely that the magnetic field variations vary significantly between higher latitudes of the South Island, near the auroral zone, and lower latitudes of the North Island, and possibly with longitude. Therefore, interpolated magnetic fields based on multiple magnetic field measurements may give much better modelling results. This becomes more important at higher latitudes of the South Island near the substation like Halfway Bush and Invercargill. 


\section{Appendix-Maxwell's Equations}

Like any other electromagnetic phenomenon, the geoelectric fields are based on Maxwell's equations. In geo-electromagnetism the displacement currents can be ignored based on frequencies $(w)$ and conductivities $(\sigma)$ involved i.e., $\sigma \gg w \varepsilon_{0}$ (Backus et al., 1996). It can also be assumed that no source currents exist within the Earth i.e., $(\nabla . j=0)$, the resulting equations would be

\begin{tabular}{|c|c|c|}
\hline Maxwell's Equations & General form & Geo-Electromagnetism \\
\hline $\begin{array}{c}\text { Gauss's Law of } \\
\text { Electrostatics }\end{array}$ & $\boldsymbol{\nabla} \cdot \boldsymbol{E}=\rho / \varepsilon_{o}$ & $\boldsymbol{\nabla} \cdot \boldsymbol{E}=0$ \\
\hline $\begin{array}{c}\text { Gauss's Law of } \\
\text { Magnetostatics }\end{array}$ & $\boldsymbol{\nabla} \cdot \boldsymbol{B}=0$ & $\boldsymbol{\nabla} \cdot \boldsymbol{B}=0$ \\
\hline $\begin{array}{c}\text { Faraday's Law } \\
\text { Ampere's Law with } \\
\text { Maxwell's modification }\end{array}$ & $\boldsymbol{\nabla} \times \boldsymbol{E}=-\frac{\partial \boldsymbol{B}}{\partial t}$ & $\boldsymbol{\nabla} \times \boldsymbol{E}=-\frac{\partial \boldsymbol{B}}{\partial t}$ \\
\hline
\end{tabular}

It can further be simplified if time dependence of $\boldsymbol{E}$ and $\boldsymbol{B}$ is considered as sinusoidal i.e., $e^{i \omega t}$. This would lead to have diffusion equation for electric and magnetic fields

$$
\begin{aligned}
& \nabla^{2} \boldsymbol{E}=i w \mu_{0} \sigma \boldsymbol{E} \\
& \nabla^{2} \boldsymbol{B}=i w \mu_{0} \sigma \boldsymbol{B}
\end{aligned}
$$

showing that source fields (due to ionospheric currents) diffuse through the Earth and their energy is dissipated exponentially. 


\section{Geoelectric Fields}

According to Helmholtz's theorem any general field that goes to zero at infinity can be decomposed into two fields, implying that a realistic geoelectric field can be written as

$$
E=-\nabla \emptyset+\nabla \times A
$$

where $\varnothing$ is scalar function and $\boldsymbol{A}$ is vector function. It shows geoelectric fields are nonconservative due to component $(\boldsymbol{\nabla} \times \boldsymbol{A})$, and so to compute voltage in a power line integration has to be performed along the path of transmission line.

\section{Kirchhoff's Law}

In power network substations are considered as nodes, and for any node say A, Kirchhoff's current Law can be applied stating that sum of currents going into the node $(A)$ is equal to sum of currents going out of the node (A). If there are $n$ number of lines connections with $\mathrm{A}$ then it could be written as

$$
\begin{gathered}
\sum_{k=1}^{n} I_{k}=0 \\
I_{1}+I_{2}+I_{3} \ldots . \quad+I_{n}=0
\end{gathered}
$$

Similarly, in any loop formed by the substations/ nodes connected by transmission lines the sum of all potential differences around that loop is always zero.

$$
\begin{gathered}
\sum_{k=1}^{m} V_{k}=0 \\
V_{1}+V_{2}+V_{3} \ldots+\quad+V_{m}=0
\end{gathered}
$$

\section{Ohm's Law}

It states that current $(I)$ produced in a transmission line of a network depends upon voltage difference $(V)$ and resistance $(R)$ of the line. 


$$
I=V / R
$$

Voltage difference between nodes of a straight power line is given by product of line length $(L)$ and magnitude of geoelectric field $(E)$ underneath the line.

$$
V=\boldsymbol{E} . \boldsymbol{L}
$$

\section{Vector Identities}

Following vector identities have been used to solved vector equations.

$$
\begin{gathered}
\boldsymbol{\nabla} \times(\boldsymbol{\nabla} \times \boldsymbol{A}) \equiv \nabla(\nabla \cdot \boldsymbol{A})-\nabla^{2} \boldsymbol{A} \\
\boldsymbol{\nabla}(\boldsymbol{\nabla} \cdot \boldsymbol{A}) \equiv 0 \\
\boldsymbol{\nabla} \times(\boldsymbol{\nabla} \emptyset) \equiv 0
\end{gathered}
$$




\section{Appendix - MT Measurements}

The following MT measurements were made as part of this project.

South Island sites

\begin{tabular}{|c|c|c|c|c|}
\hline Site & Latitude & Longitude & Start & End \\
\hline OS101 & -45.84723 & 169.81756 & $12 / 02 / 2019$ & $15 / 02 / 2019$ \\
\hline OS102 & -45.71218 & 169.25999 & $12 / 02 / 2019$ & $15 / 02 / 2019$ \\
\hline OS103 & -46.07565 & 168.85680 & $16 / 02 / 2019$ & $19 / 02 / 2019$ \\
\hline OS104 & -46.22269 & 169.42960 & $16 / 02 / 2019$ & $19 / 02 / 2019$ \\
\hline OS105 & $-45,68894$ & 168.31924 & $27 / 04 / 2019$ & $30 / 04 / 2019$ \\
\hline OS106 & -45.51304 & 167.84141 & $27 / 04 / 2019$ & $30 / 04 / 2019$ \\
\hline OS107 & -45.90581 & 167.95354 & $30 / 04 / 2019$ & $03 / 05 / 2019$ \\
\hline OS108 & -46.38009 & 168.42803 & $01 / 05 / 2019$ & $04 / 05 / 2019$ \\
\hline OS109 & -45.84569 & 170.48283 & $06 / 05 / 2019$ & $15 / 05 / 2019$ \\
\hline
\end{tabular}

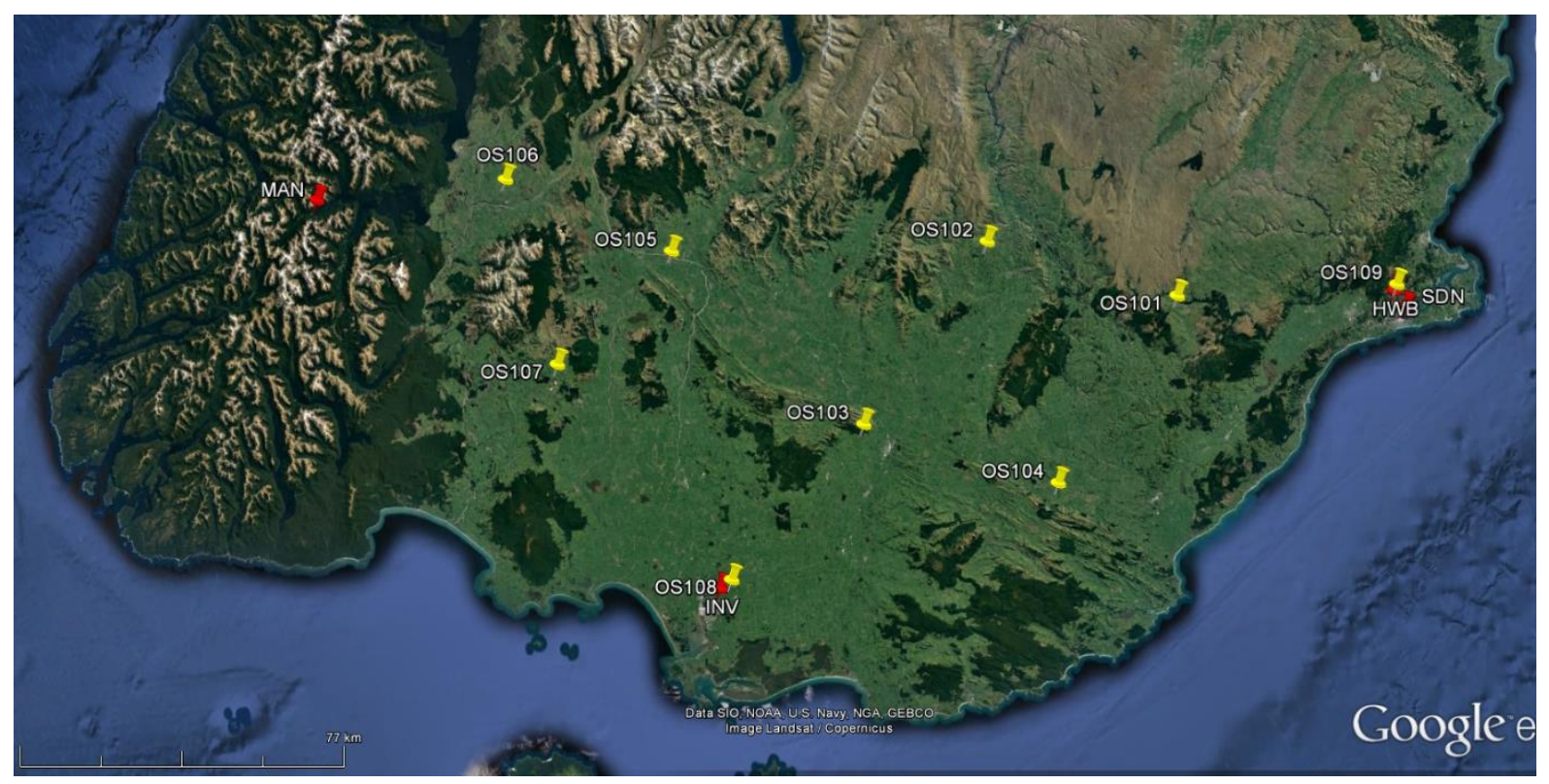

Figure A1: Locations of MT sites in Otago/Southland measured as part of this project. 
North Island sites

\begin{tabular}{|c|c|c|c|c|}
\hline Site & Latitude & Longitude & Start & End \\
\hline NI100 & -40.55442 & 175.20610 & $18 / 01 / 2019$ & $22 / 01 / 2019$ \\
\hline NI101 & -41.11540 & 175.11832 & $1 / 11 / 2019$ & $4 / 11 / 2019$ \\
\hline NI102 & -39.82102 & 174.82375 & $7 / 11 / 2019$ & $11 / 11 / 2019$ \\
\hline NI103 & -40.08227 & 175.28758 & $7 / 11 / 2019$ & $11 / 11 / 2019$ \\
\hline NI104 & -39.55140 & 174.15466 & $11 / 11 / 2019$ & $14 / 11 / 2019$ \\
\hline NI105 & -39.03851 & 174.21696 & $11 / 11 / 2019$ & $14 / 11 / 2019$ \\
\hline
\end{tabular}

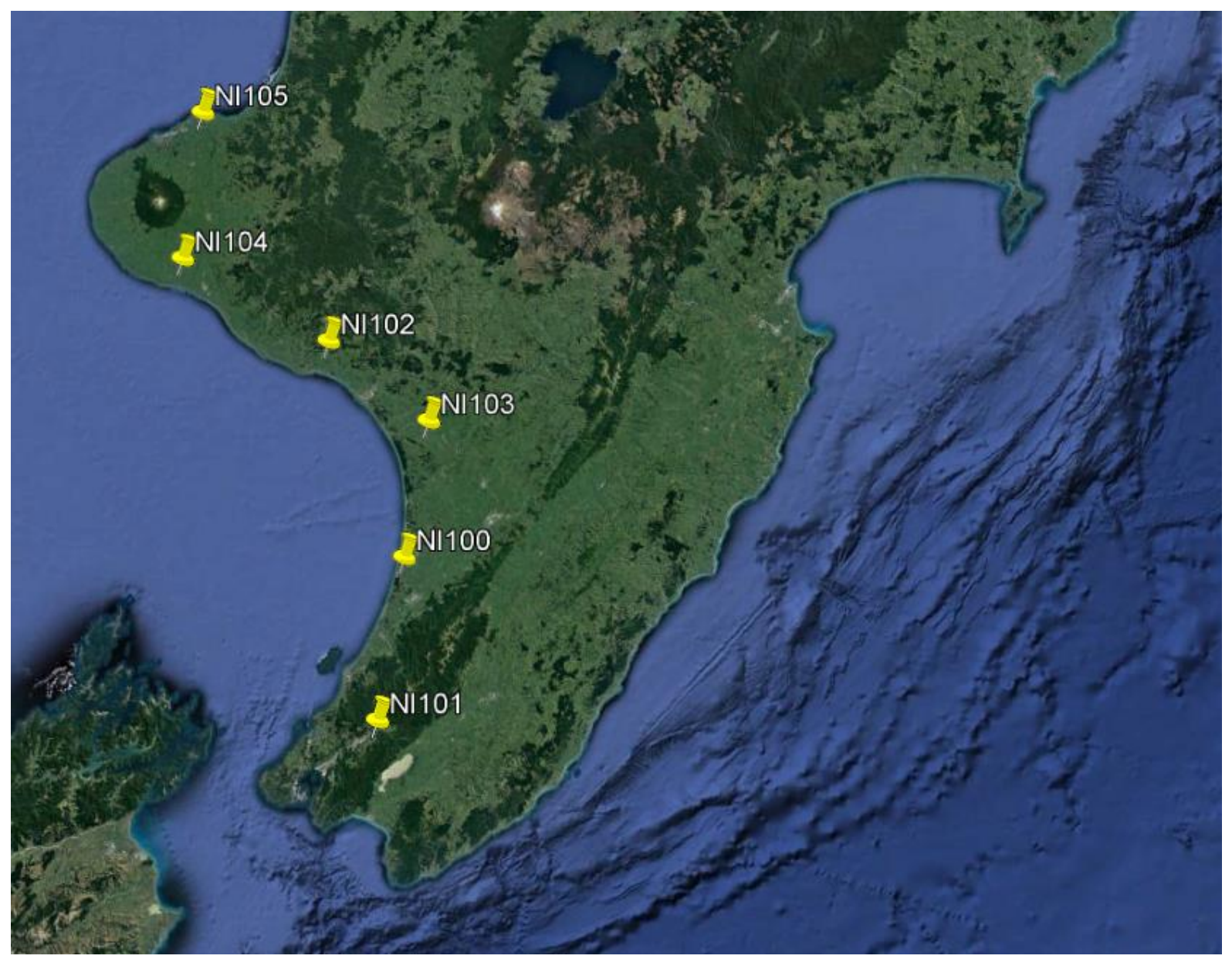

Figure A2: Locations of MT sites in the lower North Island measured as part of this project.

\section{Equipment}

Magnetometers -Bartington Mag-03MSESL100 3 component fluxgate magnetometer 
Electrodes - MCMiller RE-5C Cu/CuSO 4

\section{Datalogger - Campbell Scientific CR6}
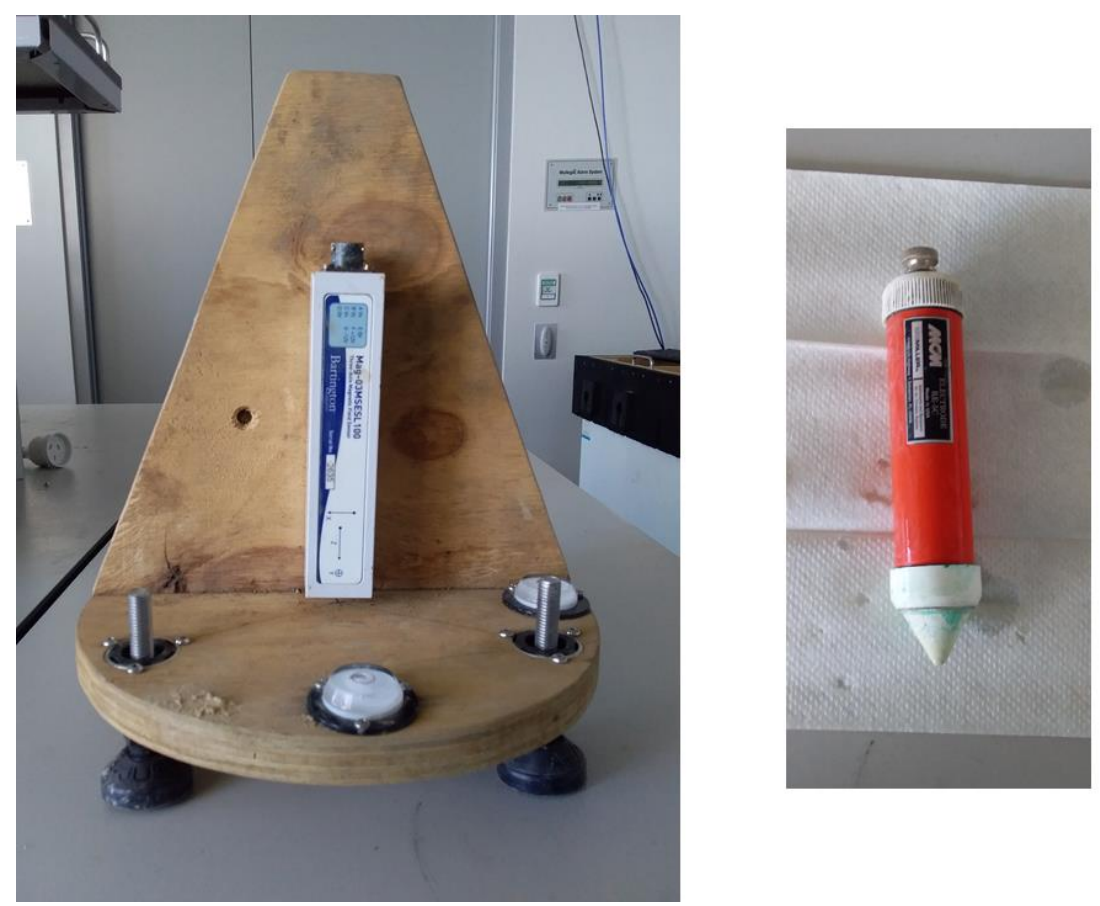

Figure A3: Left - Bartington 3-component magnetometer on mounting; right - MCMiller $\mathrm{Cu} / \mathrm{CuSO}_{4}$ electrode.

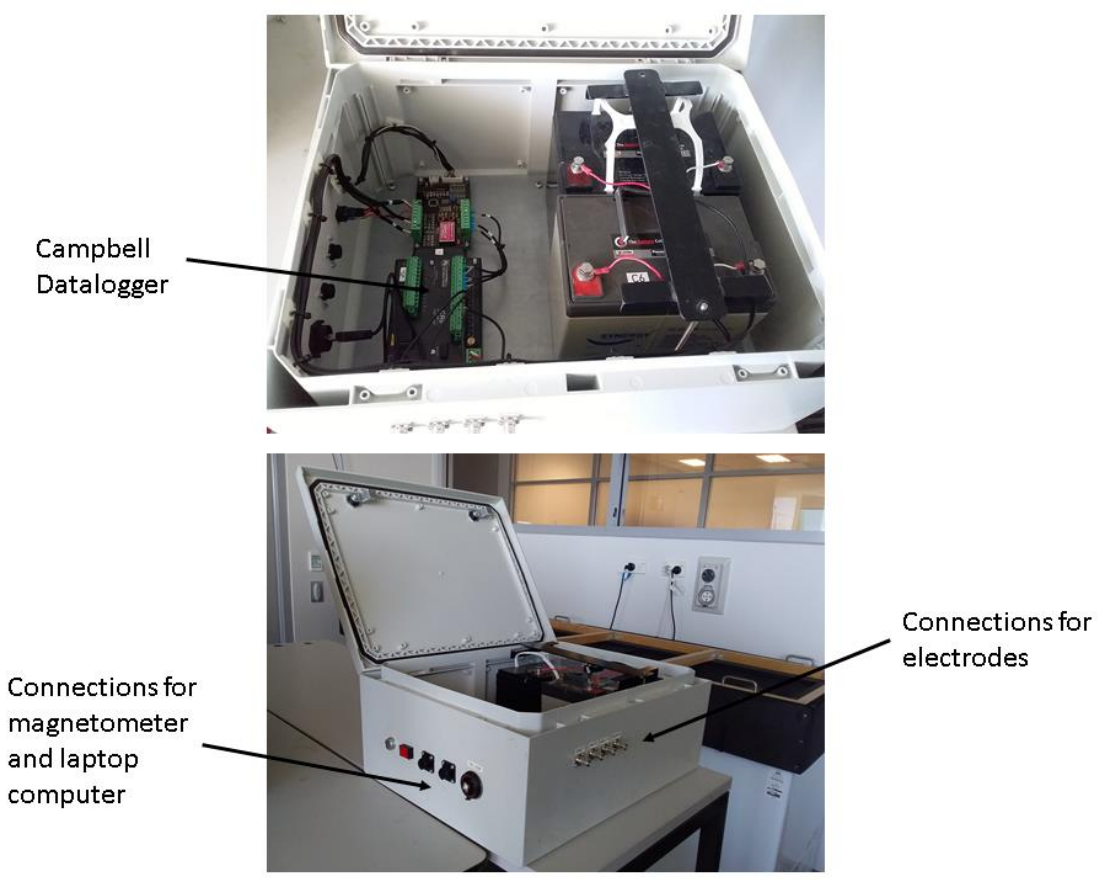

Figure A4: Top - internal view of datalogger box; bottom external view showing Magnetometer, electrode and computer connections. 


\section{Field installation}
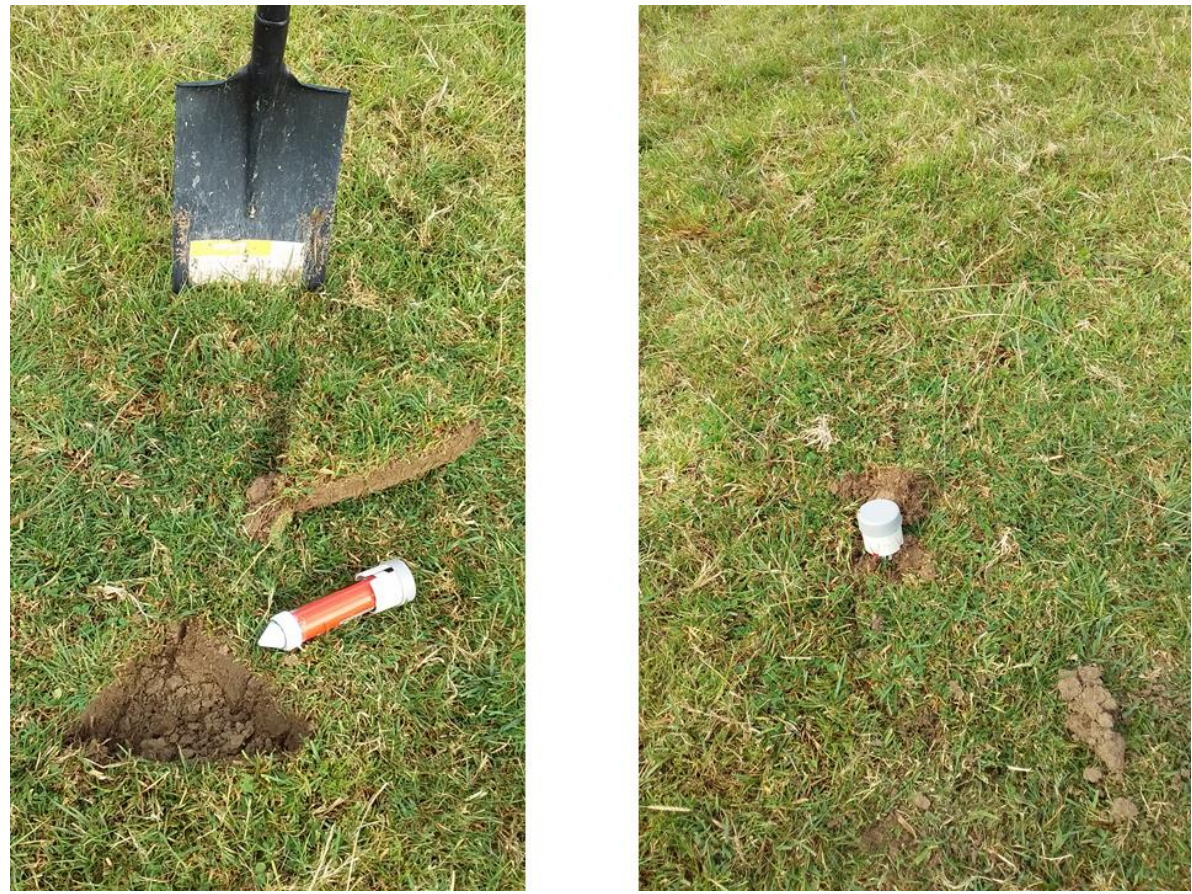

Figure A5: $\mathrm{Cu} / \mathrm{CuSO} 4$ electrode prepared to be installed and post installation.

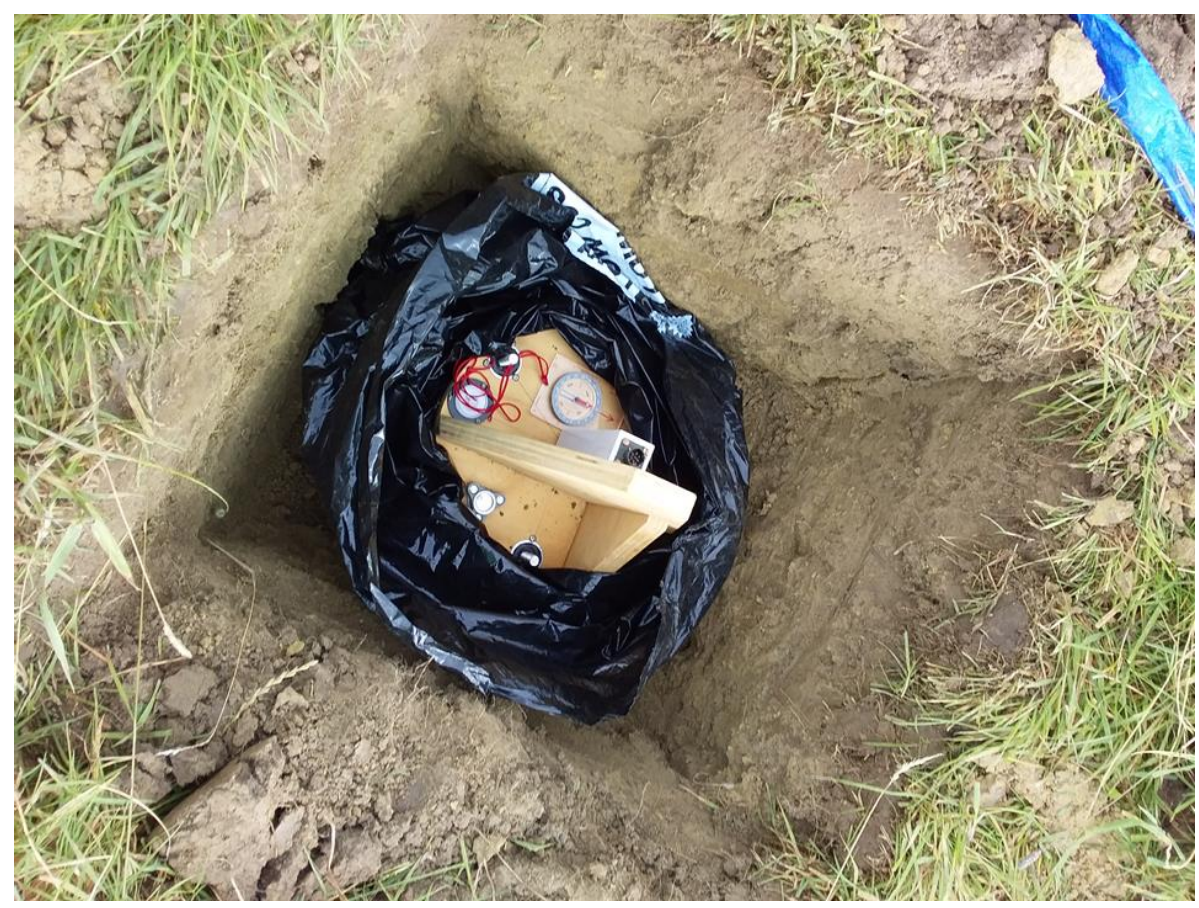

Figure A6: 3-component magnetometer ready for burial in hole. 

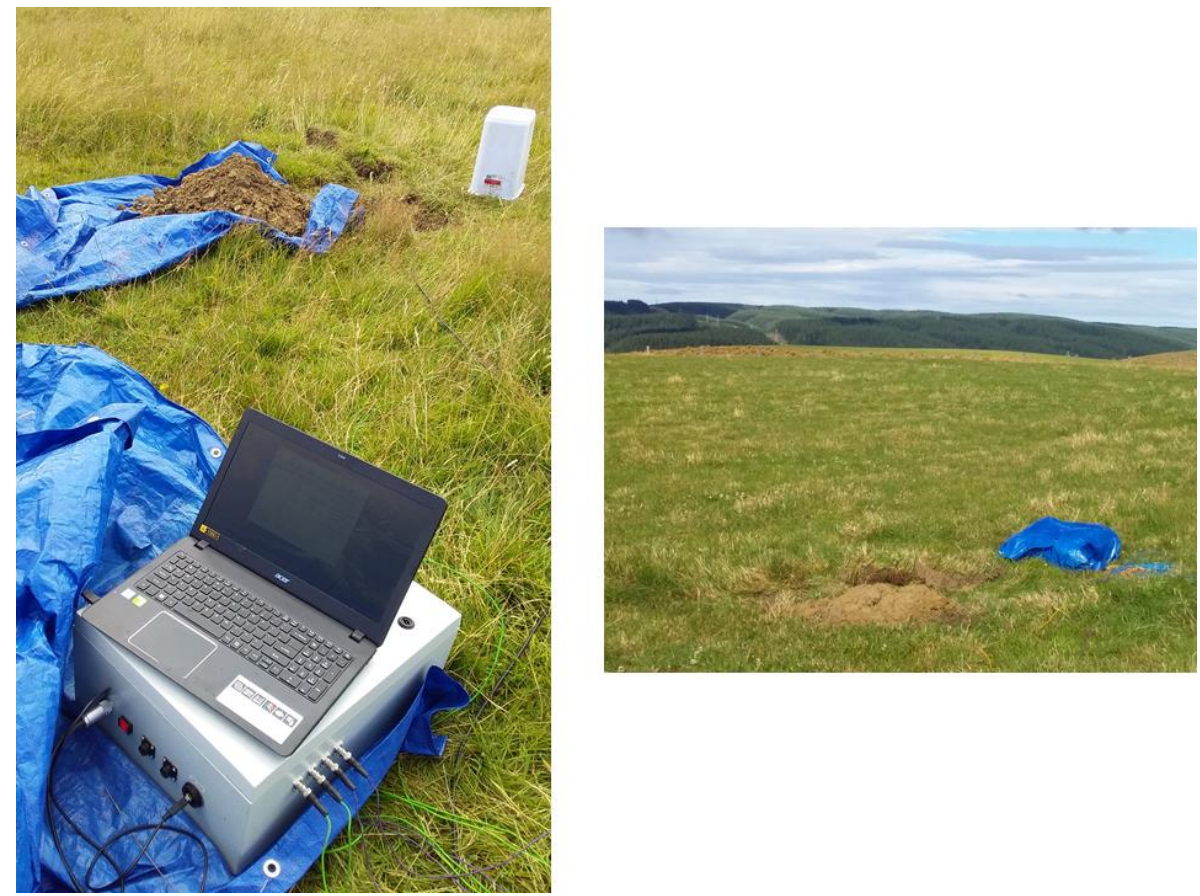

Figure A7: General view of field set-up in preparation and after turn-on. 


\section{Appendix-Paper Published}

A paper is published in an International Journal from this study and is given as attachment. The title of the paper is, 'Calculation of GIC in the North Island of New Zealand Using MT Data and Thin-Sheet Modeling'. 


\section{References}

Arajärvi, E., Pirjola, R., \& Viljanen, A. (2011). Effects of neutral point reactors and series capacitors on geomagnetically induced currents in a high-voltage electric power transmission system. Space Weather, 9(11). https://doi.org/10.1029/2011sw000715

Ashraf, I., Hur, S., \& Park, Y. (2017). An Investigation of Interpolation Techniques to Generate 2D Intensity Image From LIDAR Data. IEEE Access, 5, 8250-8260. https://doi.org/10.1109/access.2017.2699686

Augusto, C. R. A., Navia, C. E., de Oliveira, M. N., Nepomuceno, A. A., Raulin, J. P., Tueros, E., de Mendonça, R. R. S., Fauth, A. C., Vieira de Souza, H., Kopenkin, V., \& Sinzi, T. (2018). The 2015 Summer Solstice Storm: One of the Major Geomagnetic Storms of Solar Cycle 24 Observed at Ground Level. Solar Physics, 293(5), 1-28. https://doi.org/10.1007/s11207-018-1303-8

Backus, G., George, B., Parker, R., Parker, R., \& Constable, C. (1996). Foundations of Geomagnetism. Cambridge University Press.

Bailey, R. L., Halbedl, T. S., Schattauer, I., Achleitner, G., \& Leonhardt, R. (2018). Validating GIC Models With Measurements in Austria: Evaluation of Accuracy and Sensitivity to Input Parameters. Space Weather, 16(7), 887-902. https://doi.org/10.1029/2018sw001842

Bailey, R. L., Halbedl, T. S., Schattauer, I., Römer, A., Achleitner, G., Beggan, C. D., Wesztergom, V., Egli, R., \& Leonhardt, R. (2017). Modelling geomagnetically induced currents in midlatitude Central Europe using a thin-sheet approach. Annales Geophysicae, 35(3), 751-761. https://doi.org/10.5194/angeo-35-751-2017

Bedrosian, P. A., \& Love, J. J. (2015). Mapping geoelectric fields during magnetic storms: Synthetic analysis of empirical United States impedances. Geophysical Research Letters, 42(23), 160-170. https://doi.org/10.1002/2015g1066636 
Beggan, C. D. (2015). Sensitivity of geomagnetically induced currents to varying auroral electrojet and conductivity models. Earth, Planets and Space, 67(1), 1-12. https://doi.org/10.1186/s40623-014-0168-9

Beggan, C. D., Beamish, D., Richards, A., Kelly, G. S., \& P. Thomson, A. W. (2013). Prediction of extreme geomagnetically induced currents in the UK high-voltage network. Space Weather, 11(7), 407-419. https://doi.org/10.1002/swe.20065

Béland, J. and K. Small (2004). Space weather effects on power transmission systems: The Cases of Hydro-Québec and Transpower New Zealand Ltd. Effects of space weather on technology infrastructure, Springer: 287-299.

Berg, D. M., Krefeld, V. M., Overmars, M., \& Schwarzkopf, O. (2000). Computational Geometry: Algorithms and Applications, Second Edition (2nd ed.). Springer.

Bertrand, E. A., Caldwell, T. G., Hill, G. J., Bennie, S. L., \& Soengkono, S. (2013). Magnetotelluric imaging of the Ohaaki geothermal system, New Zealand: Implications for locating basement permeability. Journal of Volcanology and Geothermal Research, 268, 36-45. https://doi.org/10.1016/j.jvolgeores.2013.10.010

Bertrand, E. A., Caldwell, T. G., Hill, G. J., Wallin, E. L., Bennie, S. L., Cozens, N., Onacha, S. A., Ryan, G. A., Walter, C., Zaino, A., \& Wameyo, P. (2012). Magnetotelluric imaging of upper-crustal convection plumes beneath the Taupo Volcanic Zone, New Zealand. Geophysical Research Letters, 39(2). https://doi.org/10.1029/2011g1050177

Blake, S. P., Gallagher, P. T., McCauley, J., Jones, A. G., Hogg, C., Campanyà, J., Beggan, C. D., Thomson, A. W. P., Kelly, G. S., \& Bell, D. (2016). Geomagnetically induced currents in the Irish power network during geomagnetic storms. Space Weather, 14(12), 1136-1154. https://doi.org/10.1002/2016sw001534

Bothmer, V., \& Daglis, I. A. (2007). Space Weather: Physics and Effects. Berlin, Heidelberg: Springer Berlin Heidelberg. https://doi.org/10.1063/1.2825074

Bolduc, L. (2002). GIC observations and studies in the Hydro-Québec power system. Journal of Atmospheric and Solar-Terrestrial Physics, 64(16), 1793-1802. https://doi.org/10.1016/s1364-6826(02)00128-1 
Bolduc, L., Granger, M., Pare, G., Saintonge, J., \& Brophy, L. (2005). Development of a DC Current-Blocking Device for Transformer Neutrals. IEEE Transactions on Power Delivery, 20(1), 163-168. https://doi.org/10.1109/tpwrd.2004.835437

Bolduc, L., Langlois, P., Boteler, D., \& Pirjola, R. (1998). A study of geoelectromagnetic disturbances in Quebec. I. General results. IEEE Transactions on Power Delivery, 13(4), 1251-1256. https://doi.org/10.1109/61.714492

Bonner, L. R., \& Schultz, A. (2017). Rapid prediction of electric fields associated with geomagnetically induced currents in the presence of three-dimensional ground structure: Projection of remote magnetic observatory data through magnetotelluric impedance tensors. Space Weather, 15(1), 204-227. https://doi.org/10.1002/2016sw001535

Borovsky, J. E., \& Denton, M. H. (2006). Differences between CME-driven storms and CIRdriven storms. Journal of Geophysical Research, 111(A7). https://doi.org/10.1029/2005ja011447

Boteler, D. H. (2001). Space weather effects on power systems. Washington DC American Geophysical Union Geophysical Monograph Series, 125, 347-352.

Boteler D. H. (2013). The use of linear superposition in modelling geomagnetically induced currents. IEEE Power \& Energy Society General Meeting, Vancouver, BC, 2013, pp. 1-5, https://doi: 10.1109/PESMG.2013.6672717.

Boteler, D. H., Lackey, A. J. C., Marti, L., \& Shelemy, S. (2013). Equivalent circuits for modelling geomagnetically induced currents from a neighbouring network. In Paper 002195 presented at IEEE Power \& Energy Society GM, Vancouver, 21-25 July. IEEE. https://doi.org/10.1109/PESMG.2013.6672982

Boteler, D. H. (2014). Methodology for simulation of geomagnetically induced currents in power systems. Journal of Space Weather and Space Climate, 4, A21. https://doi.org/10.1051/swsc/2014018

Boteler, D. H. (1999). Calculating the voltages induced in technological systems during a geomagnetic disturbance. IEEE Transactions on Electromagnetic Compatibility, 41(4), 398-402. https://doi.org/10.1109/15.809834 
Boteler, D. H. (2006). The super storms of August/September 1859 and their effects on the telegraph system. Advances in Space Research, 38(2), 159-172. https://doi.org/10.1016/j.asr.2006.01.013

Boteler, D. H. (2019). A 21st Century View of the March 1989 Magnetic Storm. Space Weather, 17(10), 1427-1441. https://doi.org/10.1029/2019sw002278

Boteler, D. H., \& Pirjola, R. J. (1998a). Modelling geomagnetically induced currents produced by realistic and uniform electric fields. IEEE Transactions on Power Delivery, 13(4), 1303-1308. https://doi.org/10.1109/61.714500

Boteler, D. H., \& Pirjola, R. J. (1998b). The complex-image method for calculating the magnetic and electric fields produced at the surface of the Earth by the auroral electrojet. Geophysical Journal International, Volume 132, Issue 1, January 1998, Pages 3140, https://doi.org/10.1046/j.1365-246x.1998.00388.x

Boteler, D. H., \& Pirjola, R. J. (2014). Comparison of methods for modelling geomagnetically induced currents. Annales Geophysicae, 32(9), 1177-1187. https://doi.org/10.5194/angeo-32-1177-2014

Boteler, D. H., \& Pirjola, R. J. (2017). Modeling geomagnetically induced currents. Space Weather, 15(1), 258-276. https://doi.org/10.1002/2016sw001499

Backus G, Parker R, and Constable C (1996) Foundations of Geomagnetism. Cambridge: Cambridge University Press.

Burstinghaus, E. J., Saha, T. K., Yumoto, K., Marshall, R. A., \& Waters, C. L. (2013, July). The importance of non-uniform geoelectric fields in calculating GIC distributions. In 2013 IEEE Power \& Energy Society General Meeting (pp. 1-5). IEEE. https://doi:10.1109/PESMG.2013.6672289.

Cagniard, L. (1953). Basic theory of the magneto-telluric method of geophysical prospecting. Geophysics, 18(3), 605-635. https://doi.org/10.1190/1.1437915

Carrington, R. C. (1859). Description of a Singular Appearance seen in the Sun on September 1, 1859. Monthly Notices of the Royal Astronomical Society, 20(1), 13-15. https://doi.org/10.1093/mnras/20.1.13 
Carter, B. A., Yizengaw, E., Pradipta, R., Weygand, J. M., Piersanti, M., Pulkkinen, A., Moldwin, M. B., Norman, R., \& Zhang, K. (2016). Geomagnetically induced currents around the world during the 17 March 2015 storm. Journal of Geophysical Research: Space Physics, 121(10), 10,496-10,507. https://doi.org/10.1002/2016ja023344

Cassidy, J., Ingham, M., Locke, C. A., \& Bibby, H. (2009). Subsurface structure across the axis of the Tongariro Volcanic Centre, New Zealand. Journal of Volcanology and Geothermal Research, 179(3-4), 233-240. https://doi.org/10.1016/j.jvolgeores.2008.11.017

Chave, A. D. (2012), Estimation of the magnetotelluric response function, in The Magnetotelluric Method, edited by A. D. Chave and A. G. Jones, pp. 165-218, Cambridge Univ. Press, Cambridge, U. K.

Clilverd, M. A., Rodger, C. J., Brundell, J. B., Dalzell, M., Martin, I., Mac Manus, D. H., Thomson, N. R., Petersen, T., \& Obana, Y. (2018). Long-Lasting Geomagnetically Induced Currents and Harmonic Distortion Observed in New Zealand During the 7-8 September 2017 Disturbed Period. Space Weather, 16(6), 704-717. https://doi.org/10.1029/2018sw001822

Cliver, E. W., \& Svalgaard, L. (2004). The 1859 Solar-Terrestrial Disturbance And the Current Limits of Extreme Space Weather Activity. Solar Physics, 224(1-2), 407-422. https://doi.org/10.1007/s11207-005-4980-z

Constable, S. (2015). Geomagnetic Induction Studies. Treatise on Geophysics, 219-254. https://doi.org/10.1016/b978-0-444-53802-4.00101-9

Davidson, W. F. (1940), The magnetic storm of March 24, 1940-Effects in the power system, Edison Electric Institute Bulletin, July 1940, 365-366 and 374.

Divett, T., Ingham, M., Beggan, C. D., Richardson, G. S., Rodger, C. J., Thomson, A. W. P., \& Dalzell, M. (2017). Modeling Geoelectric Fields and Geomagnetically Induced Currents Around New Zealand to Explore GIC in the South Island's Electrical Transmission Network. Space Weather, 15(10), 1396-1412. https://doi.org/10.1002/2017sw001697

Divett, T., Mac Manus, D. H., Richardson, G. S., Beggan, C. D., Rodger, C. J., Ingham, M., Clarke, E., Thomson, A. W. P., Dalzell, M., \& Obana, Y. (2020). Geomagnetically 
Induced Current Model Validation From New Zealand's South Island. Space Weather, 18(8). https://doi.org/10.1029/2020sw002494

Divett, T., Richardson, G. S., Beggan, C. D., Rodger, C. J., Boteler, D. H., Ingham, M., Mac Manus, D. H., Thomson, A. W. P., \& Dalzell, M. (2018). Transformer-Level Modeling of Geomagnetically Induced Currents in New Zealand's South Island. Space Weather, 16(6), 718-735. https://doi.org/10.1029/2018sw001814

Echer, E., Gonzalez, W. D., Tsurutani, B. T., \& Gonzalez, A. L. C. (2008). Interplanetary conditions causing intense geomagnetic storms (Dst $\leq-100 \mathrm{nT}$ ) during solar cycle 23 (1996-2006). Journal of Geophysical Research: Space Physics, 113(A5). https://doi.org/10.1029/2007ja012744

Echer, E., Tsurutani, B. T., \& Gonzalez, W. D. (2013). Interplanetary origins of moderate (-100 nT Journal of Geophysical Research: Space Physics, 118(1), 385-392. https://doi.org/10.1029/2012ja018086

Ellis, S., Heise, W., Kissling, W., Villamor, P., \& Schreurs, G. (2014). The effect of crustal melt on rift dynamics: case study of the Taupo Volcanic Zone. New Zealand Journal of Geology and Geophysics, 57(4), 453-458. https://doi.org/10.1080/00288306.2014.972961

Ermolaev, Y. I., et al. (2005). Solar and Heliospheric Disturbances that Resulted in the Strongest Magnetic Storm of November 20, 2003. Geomagnetism Aeronomy, Vol. 45, pp. 23-50.

Ferguson, I. J. (2012), Instrumentation and field procedure, in The Magnetotelluric Method, edited by A.D. Chave and A.G. Jones, pp. 421-479, Cambridge Univ. Press, Cambridge, U. K.

Gannon, J. L., L. Trichtchenko, and P. Fernberg (2012), United States regional GIC hazard assessment, Abstract SM21D-08 presented at 2012 Fall Meeting, AGU, San Francisco, Calif., 3-7 Dec.

Gaunt C. T. and Coetzee G. (2007). Transformer failures in regions incorrectly considered to have low GIC-risk. IEEE Lausanne Power Tech, Lausanne, 2007, pp. 807-812, doi: 10.1109/PCT.2007.4538419. 
Gilbert, J. L. (2015). Simplified Techniques for Treating the Ocean-Land Interface for Geomagnetically Induced Electric Fields. IEEE Transactions on Electromagnetic Compatibility, 57(4), 688-692. https://doi.org/10.1109/temc.2015.2453196

Girgis, R. S., \& Vedante, K. B. (2015). Impact of GICs on Power Transformers: Overheating is not the real issue. IEEE Electrification Magazine, 3(4), 8-12. https://doi.org/10.1109/mele.2015.2480355

Goldberg, S., \& Rotstein, Y. (1982). A simple form of presentation of magnetotelluric data using the Bostick transform. Geophysical Prospecting, 30(2), 211-216. https://doi.org/10.1111/j.1365-2478.1982.tb01299.x

Gonzalez, W. D., Echer, E., Clua-Gonzalez, A. L., \& Tsurutani, B. T. (2007). Interplanetary origin of intense geomagnetic storms (DstGeophysical Research Letters, 34(6). https://doi.org/10.1029/2006g1028879

Gonzalez, W. D., Joselyn, J. A., Kamide, Y., Kroehl, H. W., Rostoker, G., Tsurutani, B. T., \& Vasyliunas, V. M. (1994). What is a geomagnetic storm? Journal of Geophysical Research, 99(A4), 5771. https://doi.org/10.1029/93ja02867

Gopalswamy, N., Yashiro, S., Xie, H., Akiyama, S., \& Mäkelä, P. (2015). Properties and geoeffectiveness of magnetic clouds during solar cycles 23 and 24. Journal of Geophysical Research: Space Physics, 120(11), 9221-9245. https://doi.org/10.1002/2015ja021446

Gupta, V., \& Badruddin. (2009). Interplanetary structures and solar wind behaviour during major geomagnetic perturbations. Journal of Atmospheric and Solar-Terrestrial Physics, 71(89), 885-896. https://doi.org/10.1016/j.jastp.2009.02.004

Häkkinen, L. and Pirjola, R.: 1986, Calculation of electric and magnetic fields due to an electrojet current system above a layered earth, Geophysica 22, 31-44.

Heise, W., Caldwell, T. G., Bibby, H. M., \& Bannister, S. C. (2008). Three-dimensional modelling of magnetotelluric data from the Rotokawa geothermal field, Taupo Volcanic Zone, New Zealand. Geophysical Journal International, 173(2), 740-750. https://doi.org/10.1111/j.1365-246x.2008.03737.x 
Heise, W., Caldwell, T. G., Bibby, H. M., \& Bennie, S. L. (2010). Three-dimensional electrical resistivity image of magma beneath an active continental rift, Taupo Volcanic Zone, New Zealand. Geophysical Research Letters, 37(10). https://doi.org/10.1029/2010g1043110

Heise, W., Caldwell, T. G., Hill, G. J., Bennie, S. L., Wallin, E., \& Bertrand, E. A. (2012). Magnetotelluric imaging of fluid processes at the subduction interface of the Hikurangi margin, New Zealand. Geophysical Research Letters, 39(4). https://doi.org/10.1029/2011g1050150

Heise, W., Bertrand, E. A., Caldwell, T. G., Hill, G. J., Palmer, N., \& Bennie, S. L. (2014). 2-D magnetotelluric imaging of the Rotorua and Waimangu geothermal fields. In Proceedings 36th New Zealand Geothermal Workshop, 24-26 November 2014, Auckland, New Zealand (p. 2). Auckland, N.Z: Univ. of Auckland.

Huttunen, K. E. J. (2002). Variability of magnetospheric storms driven by different solar wind perturbations. Journal of Geophysical Research, 107(A7). https://doi.org/10.1029/2001ja900171

Huttunen, K. E. J., Kilpua, S. P., Pulkkinen, A., Viljanen, A., \& Tanskanen, E. (2008). Solar wind drivers of large geomagnetically induced currents during the solar cycle 23. Space Weather, 6(10). https://doi.org/10.1029/2007sw000374

Ingham, M. (1996a). Magnetotelluric soundings across the South Island of New Zealand: electrical structure associated with the orogen of the Southern Alps. Geophysical Journal International, 124(1), 134-148. https://doi.org/10.1111/j.1365-246x.1996.tb06358.x

Ingham, M. (1996b). Magnetotelluric soundings across the southern Alps orogen, South Island of New Zealand: data presentation and preliminary interpretation. Physics of the Earth and Planetary Interiors, 94(3-4), 291-306. https://doi.org/10.1016/0031-9201(95)030972

Ingham, M. (2005). Deep electrical structure of the Central Volcanic Region and Taupo Volcanic Zone, New Zealand. Earth, Planets and Space, 57(7), 591-603. https://doi.org/10.1186/bf03351838 
Ingham, M. R. (1995). Electrical structure along a transect of the central South Island, New Zealand. New Zealand Journal of Geology and Geophysics, 38(4), 559-563. https://doi.org/10.1080/00288306.1995.9514683

Ingham, M. R., Bibby, H. M., Heise, W., Jones, K. A., Cairns, P., Dravitzki, S., Bennie, S. L., Caldwell, T. G., \& Ogawa, Y. (2009). A magnetotelluric study of Mount Ruapehu volcano, New Zealand. Geophysical Journal International, 179(2), 887-904. https://doi.org/10.1111/j.1365-246x.2009.04317.x

Ingham, M., \& Rodger, C. J. (2018). Telluric Field Variations as Drivers of Variations in Cathodic Protection Potential on a Natural Gas Pipeline in New Zealand. Space Weather, 16(9), 1396-1409. https://doi.org/10.1029/2018sw001985

Ingham, M., Rodger, C. J., Divett, T., Dalzell, M., \& Petersen, T. (2017). Assessment of GIC Based On Transfer Function Analysis. Space Weather, 15(12), 1615-1627. https://doi.org/10.1002/2017sw001707

Ingham, M., Whaler, K., \& McKnight, D. (2001). Magnetotelluric sounding of the Hikurangi Margin, New Zealand. Geophysical Journal International, 144(2), 343-355. https://doi.org/10.1046/j.0956-540x.2000.01330.x

Jiracek, G. R. (1990). Near-surface and topographic distortions in electromagnetic induction. Surveys in Geophysics, 11(2-3), 163-203. https://doi.org/10.1007/bf01901659

Kamide, Y., \& Kusano, K. (2015). No Major Solar Flares but the Largest Geomagnetic Storm in the Present Solar Cycle. Space Weather, 13(6), 365-367. https://doi.org/10.1002/2015sw001213

Kappenman J. G. (2007). Geomagnetic disturbances and impacts upon power system operation. in Electrical Power Engineering Handbook, FL, Boca Raton:CRC/IEEE, pp. 16-1-16-22, 2007.

Kappenman, J. G., Zanetti, L. J., \& Radasky, W. A. (1997). Geomagnetic storm forecasts and the power industry. Eos, Transactions American Geophysical Union, 78(4), 37. https://doi.org/10.1029/97eo00022 
Kataoka, R., \& Pulkkinen, A. (2008). Geomagnetically induced currents during intense storms driven by coronal mass ejections and corotating interacting regions. Journal of Geophysical Research: Space Physics, 113(A3). https://doi.org/10.1029/2007ja012487

Kelbert, A., Balch, C. C., Pulkkinen, A., Egbert, G. D., Love, J. J., Rigler, E. J., \& Fujii, I. (2017). Methodology for time-domain estimation of storm time geoelectric fields using the 3-D magnetotelluric response tensors. Space Weather, 15(7), 874-894. https://doi.org/10.1002/2017sw001594

Kelly, G. S., Viljanen, A., Beggan, C. D., \& Thomson, A. W. P. (2017). Understanding GIC in the UK and French high-voltage transmission systems during severe magnetic storms. Space Weather, 15(1), 99-114. https://doi.org/10.1002/2016sw001469

Kertz, W., 1954. Modelle für erdmagnetisch induzierte elektrische Ströme im Untergrund. Nachr. Akad. Wiss. Göttingen, Math.-phys. Kl. Vol. IIa, pp. 101-110.

Koen J. and Gaunt T. (2003). Geomagnetically induced currents in the Southern African electricity transmission network. IEEE Bologna Power Tech Conference Proceedings, , Bologna, Italy, 2003, pp. 7 pp. Vol.1-, doi: 10.1109/PTC.2003.1304165.

Kozyreva, O. V., Pilipenko, V. A., Belakhovsky, V. B., \& Sakharov, Y. A. (2018). Ground geomagnetic field and GIC response to March 17, 2015, storm. Earth, Planets and Space, 70(1). https://doi.org/10.1186/s40623-018-0933-2

Lehtinen, M., \& Pirjola, R. (1985). Currents produced in earthed conductor networks by geomagnetically-induced electric fields. Annales Geophysicae, 3, 479-484.

Liu, C.-M., Liu, L.-G., Pirjola, R., \& Wang, Z.-Z. (2009). Calculation of geomagnetically induced currents in mid- to low-latitude power grids based on the plane wave method: A preliminary case study. Space Weather, 7(4). https://doi.org/10.1029/2008sw000439

Liu, C., Wang, X., Wang. H. and Zhao, H. (2018). Quantitative influence of coast effect on geomagnetically induced currents in power grids: a case study. J. Space Weather Space Clim., 8, A60. https://doi.org/10.1051/swsc/2018046

Longley, P. A., Goodchild, M. F., Maguire, D. J., \& Rhind, D. W. (2005). Geographic Information Systems and Science (2nd ed.). Wiley. 
Love, J. J., Lucas, G. M., Kelbert, A., \& Bedrosian, P. A. (2018). Geoelectric Hazard Maps for the Mid-Atlantic United States: 100 Year Extreme Values and the 1989 Magnetic Storm. Geophysical Research Letters, 45(1), 5-14. https://doi.org/10.1002/2017g1076042

Mac Manus, D. H., Rodger, C. J., Dalzell, M., Thomson, A. W. P., Clilverd, M. A., Petersen, T., Wolf, M. M., Thomson, N. R., \& Divett, T. (2017). Long-term geomagnetically induced current observations in New Zealand: Earth return corrections and geomagnetic field driver. Space Weather, 15(8), 1020-1038. https://doi.org/10.1002/2017sw001635

Marsal, S., Torta, J. M., Segarra, A., \& Araki, T. (2017). Use of spherical elementary currents to map the polar current systems associated with the geomagnetic sudden commencements on 2013 and 2015 St. Patrick’s Day storms. Journal of Geophysical Research: Space Physics, 122(1), 194-211. https://doi.org/10.1002/2016ja023166

Marshall, R. A., Dalzell, M., Waters, C. L., Goldthorpe, P., \& Smith, E. A. (2012). Geomagnetically induced currents in the New Zealand power network. Space Weather, 10(8). https://doi.org/10.1029/2012sw000806

McKay, A. J., \& Whaler, K. A. (2006). The electric field in northern England and southern Scotland: implications for geomagnetically induced currents. Geophysical Journal International, 167(2), 613-625. https://doi.org/10.1111/j.1365-246x.2006.03128.x

McKay, A. J. (2003). Geoelectric fields and geomagnetically induced currents in the United Kingdom (PhD Thesis). Retrieved from (https://era.ed.ac.uk/handle/1842/639). University of Edinburgh.

McLoughlin, C., Ingham, M., Whaler, K., \& McKnight, D. (2002). A magnetotelluric transect of the Wairarapa region, New Zealand. New Zealand Journal of Geology and Geophysics, 45(2), 257-269. https://doi.org/10.1080/00288306.2002.9514972

Miyoshi, Y., \& Kataoka, R. (2005). Ring current ions and radiation belt electrons during geomagnetic storms driven by coronal mass ejections and corotating interaction regions. Geophysical Research Letters, 32(21). https://doi.org/10.1029/2005g1024590 
Molinski, T. S. (2002). Why utilities respect geomagnetically induced currents. Journal of Atmospheric and Solar-Terrestrial Physics, 64(16), 1765-1778. https://doi.org/10.1016/s1364-6826(02)00126-8

Myllys, M., Viljanen, A., Rui, Y. A., \& Ohnstad, T. M. (2014). Geomagnetically induced currents in Norway: the northernmost high-voltage power grid in the world. Journal of Space Weather and Space Climate, 4, A10. https://doi.org/10.1051/swsc/2014007

Nakamura, S., Ebihara, Y., Fujita, S., Goto, T., Yamada, N., Watari, S., \& Omura, Y. (2018). Time Domain Simulation of Geomagnetically Induced Current (GIC) Flowing in 500-kV Power Grid in Japan Including a Three-Dimensional Ground Inhomogeneity. Space Weather, 16(12), 1946-1959. https://doi.org/10.1029/2018sw002004

Navia, C. E., de Oliveira, M. N., \& Augusto, C. R. A. (2018). The highest geomagnetic storms of the solar cycle observed at ground level. In P. J. Sallis (Ed.), Extreme Weather. London: Intech Open. https://doi.org/10.5772/intechopen.75688

Ngwira, C. M., McKinnell, L.-A., \& Cilliers, P. J. (2011). Geomagnetic activity indicators for geomagnetically induced current studies in South Africa. Advances in Space Research, 48(3), 529-534. https://doi.org/10.1016/j.asr.2011.03.042

Overbye, T. J., Shetye, K. S., Hutchins, T. R., Qiu, Q., \& Weber, J. D. (2013). Power Grid Sensitivity Analysis of Geomagnetically Induced Currents. IEEE Transactions on Power Systems, 28(4), 4821-4828. https://doi.org/10.1109/tpwrs.2013.2274624

Pirjola, R. (1985). Electromagnetic induction in the earth by an electrojet current system harmonic in time and space, Geophysica 21, 145-159.

Pirjola, R. (2002), Review on the calculation of surface electric and magnetic fields and of geomagnetically induced currents in ground-based technological systems, Surveys in Geophysics., 23, 71-90, doi:10.1023/A:1014816009303.

Pirjola, R. (2009). Properties of matrices included in the calculation of geomagnetically induced currents (GICs) in power systems and introduction of a test model for GIC computation algorithms. Earth, Planets and Space, 61(2), 263-272. https://doi.org/10.1186/bf03352906 
Pirjola, R. (2013). Practical model applicable to investigating the coast effect on the geoelectric field in connection with studies of geomagnetically induced currents. Advances in Applied Physics, 1, 9-28. https://doi.org/10.12988/aap.2013.13002

Pirjola, R., \& Viljanen, A. (1998). Complex image method for calculating electric and magnetic fields produced by an auroral electrojet of finite length. Annales Geophysicae, 16(11), 1434-1444. https://doi.org/10.1007/s00585-998-1434-6

Pulkkinen, A., Hesse, M., Kuznetsova, M., \& Rastätter, L. (2007). First-principles modeling of geomagnetically induced electromagnetic fields and currents from upstream solar wind to the surface of the Earth. Annales Geophysicae, 25(4), 881-893. https://doi.org/10.5194/angeo-25-881-2007

Pulkkinen, A., Viljanen, A., Pajunpää, K., \& Pirjola, R. (2001). Recordings and occurrence of geomagnetically induced currents in the Finnish natural gas pipeline network. Journal of Applied Geophysics, 48(4), 219-231. https://doi.org/10.1016/s0926-9851(01)00108-2

Pulkkinen, A., Lindahl, S., Viljanen, A., \& Pirjola, R. (2005). Geomagnetic storm of 29-31 October 2003: Geomagnetically induced currents and their relation to problems in the Swedish high-voltage power transmission system. Space Weather, 3(8). https://doi.org/10.1029/2004sw000123

Rawat, R., Echer, E., \& Gonzalez, W. D. (2018). How Different Are the Solar WindInterplanetary Conditions and the Consequent Geomagnetic Activity During the Ascending and Early Descending Phases of the Solar Cycles 23 and 24? Journal of Geophysical Research: Space Physics, 123(8), 6621-6638. https://doi.org/10.1029/2018ja025683

Rodger, C. J., Clilverd, M. A., Mac Manus, D. H., Martin, I., Dalzell, M., Brundell, J. B., Divett, T., Thomson, N. R., Petersen, T., Obana, Y., \& Watson, N. R. (2020). Geomagnetically Induced Currents and Harmonic Distortion: Storm-Time Observations From New Zealand. Space Weather, 18(3). https://doi.org/10.1029/2019sw002387

Rodger, C. J., Mac Manus, D. H., Dalzell, M., Thomson, A. W. P., Clarke, E., Petersen, T., Clilverd, M. A., \& Divett, T. (2017). Long-Term Geomagnetically Induced Current 
Observations From New Zealand: Peak Current Estimates for Extreme Geomagnetic Storms. Space Weather, 15(11), 1447-1460. https://doi.org/10.1002/2017sw001691

Simpson, F., \& Bahr, K. (2005). Practical Magnetotellurics. Cambridge University Press.

Stagpoole, V. M., Bennie, S. L., Bibby, H. M., Dravitzki, S., \& Ingham, M. R. (2009). Deep structure of a major subduction back thrust: Magneto-telluric investigations of the Taranaki Fault, New Zealand. Tectonophysics, 463(1-4), 77-85. https://doi.org/10.1016/j.tecto.2008.09.035

Torta, J. M., Marcuello, A., Campanyà, J., Marsal, S., Queralt, P., \& Ledo, J. (2017). Improving the modeling of geomagnetically induced currents in Spain. Space Weather, 15(5), 691703. https://doi.org/10.1002/2017sw001628

Torta, J. M., Marsal, S., \& Quintana, M. (2014). Assessing the hazard from geomagnetically induced currents to the entire high-voltage power network in Spain. Earth, Planets and Space, 66(1). https://doi.org/10.1186/1880-5981-66-87

Trichtchenko, L., Boteler, D. H., Trichtchenko, L., \& Boteler, D. H. (2004). Modeling Geomagnetically Induced Currents Using Geomagnetic Indices and Data. IEEE Transactions on Plasma Science, 32(4), 1459-1467. https://doi.org/10.1109/tps.2004.830993

Trichtchenko, L. and Boteler D. H. (2006). Response of Power Systems to the Temporal Characteristics of Geomagnetic Storms. Canadian Conference on Electrical and Computer Engineering, Ottawa, Ont., 2006, pp. 390-393, doi: 10.1109/CCECE.2006.277733.

Trivedi, N. B., Vitorello, Í,, Kabata, W., Dutra, S. L. G., Padilha, A. L., Bologna, M. S., de Pádua, M. B., Soares, A. P., Luz, G. S., Pinto, F. A., Pirjola, R., \& Viljanen, A. (2007). Geomagnetically induced currents in an electric power transmission system at low latitudes in Brazil: A case study. Space Weather, 5(4). https://doi.org/10.1029/2006sw000282

Vasseur, G., \& Weidelt, P. (1977). Bimodal electromagnetic induction in non-uniform thin sheets with an application to the northern Pyrenean induction anomaly. Geophysical 
Journal International, 51(3), 669-690. https://doi.org/10.1111/j.1365246x.1977.tb04213.x

Viljanen, A. (1998). Relation of geomagnetically induced currents and local geomagnetic variations. IEEE Transactions on Power Delivery, 13(4), 1285-1290. https://doi.org/10.1109/61.714497

Viljanen, A., Nevanlinna, H., Pajunpää, K., \& Pulkkinen, A. (2001). Time derivative of the horizontal geomagnetic field as an activity indicator. Annales Geophysicae, 19(9), 11071118. https://doi.org/10.5194/angeo-19-1107-2001

Viljanen, A., \& Pirjola, R. (1989). Statistics on geomagnetically-induced currents in the Finnish $400 \mathrm{kV}$ power system based on recordings of geomagnetic variations. Journal of Geomagnetism and Geoelectricity, 41(4), 411-420. https://doi.org/10.5636/jgg.41.411

Viljanen, A., \& Pirjola, R. (1994). Geomagnetically induced currents in the Finnish high-voltage power system. Surveys in Geophysics, 15(4), 383-408. https://doi.org/10.1007/bf00665999

Viljanen, A., Pirjola, R., Prácser, E., Ahmadzai, S., \& Singh, V. (2013). Geomagnetically induced currents in Europe: Characteristics based on a local power grid model. Space Weather, 11(10), 575-584. https://doi.org/10.1002/swe.20098

Viljanen, A., Pulkkinen, A., Amm, O., Pirjola, R., \& Korja, T. (2004). Fast computation of the geoelectric field using the method of elementary current systems and planar Earth models. Annales Geophysicae, 22(1), 101-113. https://doi.org/10.5194/angeo-22-1012004

Wait, J. R. (1980). Electromagnetic surface impedance for a layered earth for general excitation. Radio Science, 15(1), 129-134. https://doi.org/10.1029/rs015i001p00129

Wannamaker, P. E. (2002). Fluid generation and pathways beneath an active compressional orogen, the New Zealand Southern Alps, inferred from magnetotelluric data. Journal of Geophysical Research, 107(B6). https://doi.org/10.1029/2001jb000186

Wannamaker, P. E., Caldwell, T. G., Jiracek, G. R., Maris, V., Hill, G. J., Ogawa, Y., Bibby, H. M., Bennie, S. L., \& Heise, W. (2009). Fluid and deformation regime of an advancing 
subduction system at Marlborough, New Zealand. Nature, 460(7256), 733-736. https://doi.org/10.1038/nature08204

Watari, S. (2017). Geomagnetic storms of cycle 24 and their solar sources. Earth, Planets and Space, 69(1). https://doi.org/10.1186/s40623-017-0653-z

Watari, S., Kunitake, M., Kitamura, K., Hori, T., Kikuchi, T., Shiokawa, K., Nishitani, N., Kataoka, R., Kamide, Y., Aso, T., Watanabe, Y., \& Tsuneta, Y. (2009). Measurements of geomagnetically induced current in a power grid in Hokkaido, Japan. Space Weather, 7(3). https://doi.org/10.1029/2008sw000417

Wei, L. H., Homeier, N., \& Gannon, J. L. (2013). Surface electric fields for North America during historical geomagnetic storms. Space Weather, 11(8), 451-462. https://doi.org/10.1002/swe.20073

Wik, M., Pirjola, R., Lundstedt, H., Viljanen, A., Wintoft, P., \& Pulkkinen, A. (2009). Space weather events in July 1982 and October 2003 and the effects of geomagnetically induced currents on Swedish technical systems. Annales Geophysicae, 27(4), 1775-1787. https://doi.org/10.5194/angeo-27-1775-2009

Wik, M., Viljanen, A., Pirjola, R., Pulkkinen, A., Wintoft, P., \& Lundstedt, H. (2008). Calculation of geomagnetically induced currents in the $400 \mathrm{kV}$ power grid in southern Sweden. Space Weather, 6(7). https://doi.org/10.1029/2007sw000343

Zheng K. et al., (2013). Effects of System Characteristics on Geomagnetically Induced Currents, in IEEE Transactions on Power Delivery, vol. 29, no. 2, pp. 890-898, April 2014, doi: 10.1109/TPWRD.2013.2281191. 